\title{
Asymmetric Mannich Reaction of Dicarbonyls with $\alpha$-Amido Sulfones Catalyzed by Cinchona Alkaloids and Synthesis of Chiral Dihydropyrimidones
}

\author{
Sha Lou, Peng Dai, and Scott E. Schaus* \\ Department of Chemistry, Center for Chemical Methodology and Library Development at \\ Boston University (CMLD-BU), Life Science and Engineering Building, Boston University \\ 24 Cummington Street, Boston, Massachusetts, 02215 (USA)
}

\section{Supporting Information}

\section{Table of Contents}

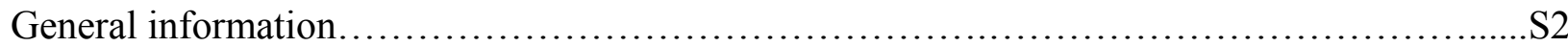

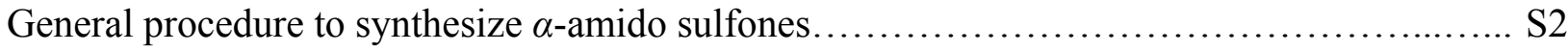

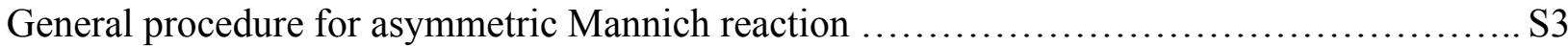

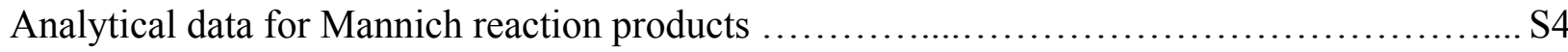

Absolute stereochemistry determination of Mannich reaction products...................S21

General procedure for chiral dihydropyrimidones synthesis..........................S26

Analytical data for chiral dihydropyrimidones library $\ldots \ldots \ldots \ldots \ldots \ldots \ldots \ldots \ldots \ldots \ldots \ldots . . .527$

Analysis of trace Pd in dihydropyrimidones by ICP-MS .................................. 43

General procedure for synthesis of tetrahydropyrimidones ............................ S44

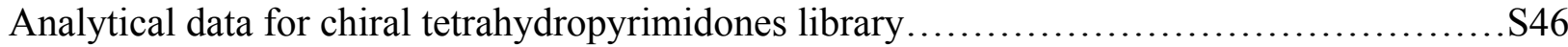

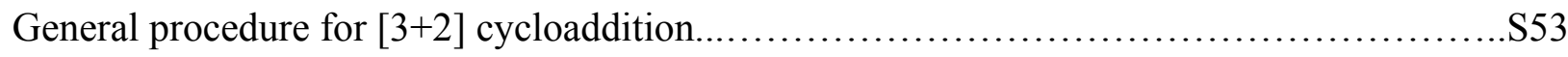

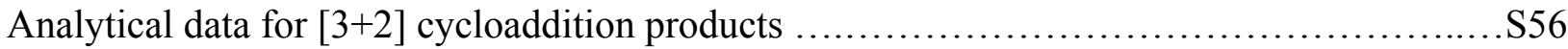

Procedures and analytical data of intermediates for intramolecular cycloaddition.............S60

Selected NMR Spectra........................................................ S66

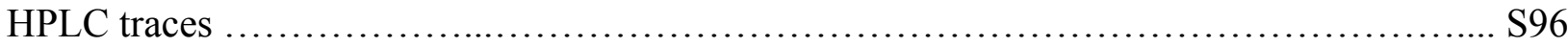


General Information. All ${ }^{1} \mathrm{H}$ NMR, and ${ }^{13} \mathrm{C}$ NMR spectra were recorded using Varian Unity Plus $400\left(93.94 \mathrm{kG},{ }^{1} \mathrm{H} 400 \mathrm{MHz}\right)$ or Varian Gemini $300\left(70.5 \mathrm{kG},{ }^{13} \mathrm{C} 75 \mathrm{MHz}\right)$ spectrometers at ambient temperature in $\mathrm{CDCl}_{3}$. Chemical shifts are reported in parts per million as follows: chemical shift, multiplicity $(\mathrm{s}=$ singlet, $\mathrm{d}=$ doublet, $\mathrm{t}=$ triplet, $\mathrm{q}=$ quartet, $\mathrm{m}=$ multiplet, $\mathrm{br}=$ broad), coupling constant, and integration. Infrared spectra were recorded on a Nicolet Nexus 670 FT-IR ESP spectrophotometer. Optical rotations were recorded on an AUTOPOL III digital polarimeter at $589 \mathrm{~nm}$, and were reported as $[\alpha]_{\mathrm{D}}$ (concentration in grams $/ 100 \mathrm{~mL}$ solvent). Analytical thin layer chromatography was performed using EMD $0.25 \mathrm{~mm}$ silica gel $60-\mathrm{F}$ plates. Flash column chromatography was performed on Sorbent Technologies $60 \AA$ silica gel. Chiral HPLC analysis was performed using an Agilent 1100 series HPLC or Waters Breeze HPLC System with a diode array detector. Chiral columns include Chiralcel ${ }^{\circledR}$ OD (Chiral Technologies Inc., $24 \mathrm{~cm} \times 4.6 \mathrm{~mm}$ I.D.), Chiralcel ${ }^{\circledR} \mathrm{OD}-\mathrm{H}$ (Chiral Technologies Inc., $15 \mathrm{~cm} \times 4.6 \mathrm{~mm}$ I.D.), Chiralpak ${ }^{\circledR} \mathrm{AD}-\mathrm{H}$ (Chiral Technologies Inc., $15 \mathrm{~cm} \times 4.6 \mathrm{~mm}$ I.D.) and $(R, R)$-Whelk-O 1 (Regis ${ }^{\circledR}$ Technologies Inc., $25 \mathrm{~cm} \times 4.6 \mathrm{~mm}$ I.D.) columns. All Mannich reactions were performed under air with magnetic stirring. Hydrogenation was performed on $\mathrm{H}-\mathrm{Cube}$ system supported by Thales Nanotechnology, Inc. Reaction solvents were obtained from a dry solvent system (alumina). $\alpha$ Amido sulfones were prepared according to the published procedure. ${ }^{1}$ Allyl carbamate was prepared according to the published procedure. ${ }^{2}$ The $(+)$-cinchonine, quinine, quinidine and other reagents were used as received from Sigma-Aldrich, Alfa Aesar, Lancaster, and Acros unless otherwise noted.

\section{General procedure to synthesize $\alpha$-amido sulfones}

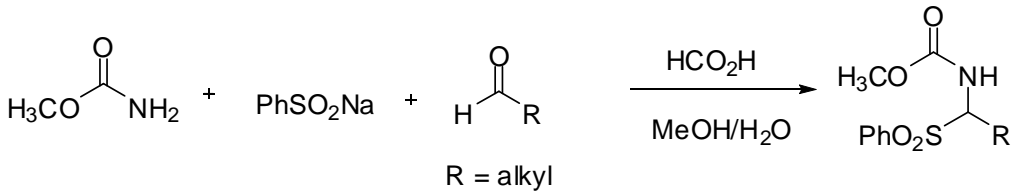

Method A for aliphatic $\boldsymbol{\alpha}$-amido sulfones: To a $250 \mathrm{~mL}$ one-neck round-bottom flask was chared with a stir bar, sodium benzenesulfinate $(3.0 \mathrm{~g}, 15 \mathrm{mmol})$, methyl carbamate $(1.12 \mathrm{~g}, 15$ $\mathrm{mmol})$, methanol $(10 \mathrm{~mL})$ and water $(20 \mathrm{~mL})$. Aldehyde $(10 \mathrm{mmol})$ and formic acid $(1.9 \mathrm{~mL}, 50$ mmol) were added successfully. The reaction mixture was stirred for two days. The solution was extracted with $\mathrm{CH}_{2} \mathrm{Cl}_{2}(3 \times 50 \mathrm{~mL})$. The combined organic layers was dried over sodium sulfate and filtered. The filtrate was concentrated under reduced pressure and the residue was purified by flash chromatography over silica gel (elution with $20 \%-40 \%$ ethyl acetate in hexanes) to afford the corresponding $\alpha$-amido sulfones as white solids.

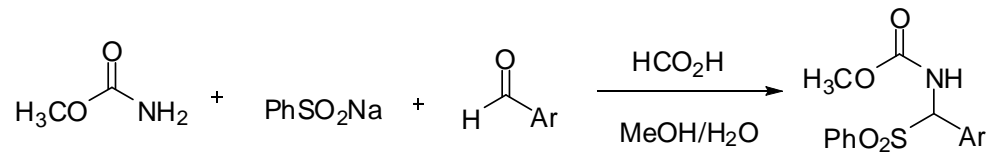

Method B for aromatic $\boldsymbol{\alpha}$-amido sulfones: A $250 \mathrm{~mL}$ one neck round bottom flask was charged with a stir bar, sodium benzenesulfinate $(3.0 \mathrm{~g}, 15 \mathrm{mmol})$, methyl carbamate $(1.12 \mathrm{~g}, 15 \mathrm{mmol})$, methanol $(10 \mathrm{~mL})$ and water $(20 \mathrm{~mL})$. Aldehyder $(10 \mathrm{mmol})$ and formic acid $(50 \mathrm{mmol})$ were added sucessfully. The reaction mixture was stirred overnight. The white precipitate was filtered, washed with petroleum ether and azeotropically dried with toluene. The product was used in the Mannich reaction without further purification. 


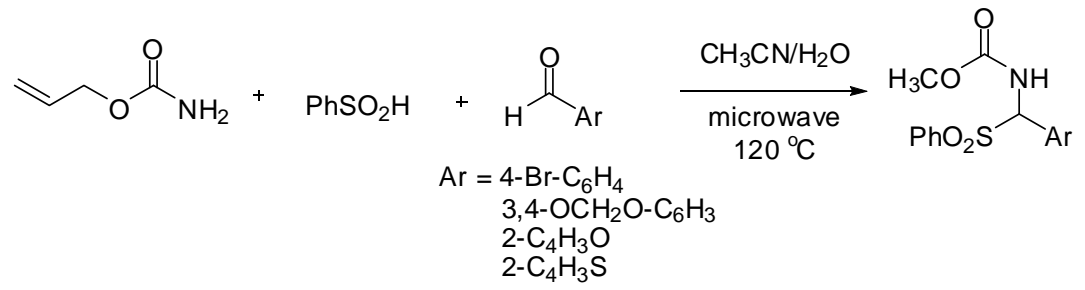

Method $\mathbf{C}$ for electron rich aromatic $\boldsymbol{\alpha}$-amido sulfones: To a heavy-walled microwave tube was charged with stir bar, allyl carbamate $(202 \mathrm{mg}, 2.0 \mathrm{mmol})$, benzenesulfinic acid (280 $\mathrm{mg}$, $2.0 \mathrm{mmol})$, aldehyde $(1.0 \mathrm{mmol}), 4.0 \mathrm{~mL}$ acetonitrile and 1 drop of water. The solution was subjected to microwave irradiation $(300 \mathrm{~W})$ at $120{ }^{\circ} \mathrm{C}$ for $30 \mathrm{~min}$ and then stirred at room temperature for 4 hours. The reaction solution was concentrated under reduced pressure and diluted with water. The resulting white precipitate was filtered, washed with petroleum ether and azeotropically dried with toluene. The product was used in the Mannich reaction without further purification.

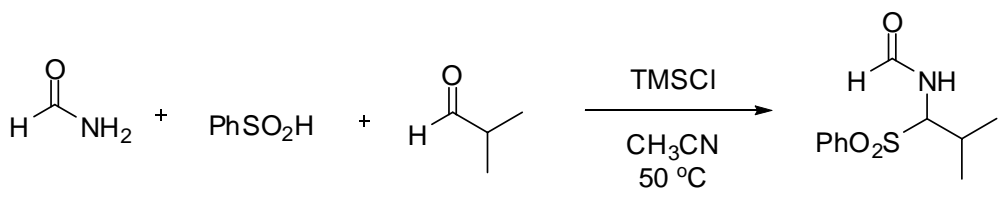

Method D for $\boldsymbol{\alpha}$-formamido sulfones: To a $50 \mathrm{~mL}$ one-neck round-bottom flask was charged with a stir bar, benzenesulfinic acid $(853 \mathrm{mg}, 6.0 \mathrm{mmol})$, formamide (338 $\mathrm{mg}, 7.5 \mathrm{mmol})$, acetonitrile $(20 \mathrm{~mL})$ and aldehyde $(3.0 \mathrm{mmol})$. TMSCl $(0.38 \mathrm{~mL}, 3 \mathrm{mmol})$ was added slowly into reaction mixture. The reaction solution was heated and stirred at $50{ }^{\circ} \mathrm{C}$ for 5 hours. The solution was concentrated under reduced pressure and the residue was purified by flash chromatography over silica gel (elution with $20 \%-40 \%$ ethyl acetate in hexanes) to afford the corresponding $\alpha$ formamido sulfones as white solids.

\section{Preparation of aqueous sodium carbonate solution for Mannich reactions.}

Sodium carbonate $(5.0 \mathrm{~g})$ was dissolved in deionized water $(100 \mathrm{~mL})$. The resulting solution was saturated with sodium chloride.

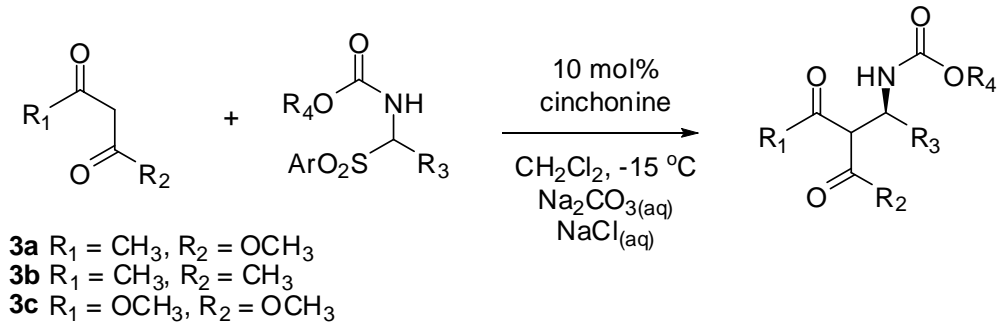

General procedure for asymmetric Mannich reaction of dicarbonyls with $\alpha$-amido sulfones.

To a $25 \mathrm{~mL}$ one-neck round-bottom flask equipped with stir bar was charged with (+)cinchonine $(16.0 \mathrm{mg}, 0.05 \mathrm{mmol}), \alpha$-amido sulfone $(0.50 \mathrm{mmol})$ and $\mathrm{CH}_{2} \mathrm{Cl}_{2}(5.0 \mathrm{~mL})$. The reaction solution was cooled to $-15{ }^{\circ} \mathrm{C}$. The Dicarbonyl compound $(1.50 \mathrm{mmol})$ and aqueous $\mathrm{Na}_{2} \mathrm{CO}_{3} / \mathrm{NaCl}$ solution $\left(5.0 \mathrm{~mL}, 5 \mathrm{wt} \% \mathrm{Na} \mathrm{Na}_{2} \mathrm{CO}_{3}\right.$ in deionized water saturated with $\mathrm{NaCl}$ ) was slowly added into reaction mixture successfully. The resulting biphasic solution was vigorously stirred at $-15{ }^{\circ} \mathrm{C}$ for 48 hours and diluted with $\mathrm{CH}_{2} \mathrm{Cl}_{2}(10 \mathrm{~mL})$ and $\mathrm{H}_{2} \mathrm{O}(10 \mathrm{~mL})$. The organic layer was quickly separated and the aqueous phase was extracted with $\mathrm{CH}_{2} \mathrm{Cl}_{2}(2 \times 10 \mathrm{~mL})$. The 
combined organic layers were dried over sodium sulfate, filtered and concentrated under reduced pressure. The resulting residue was purified by flash chromatography over silica gel (elution with $15 \%-40 \%$ ethyl acetate in hexanes) to afford the enantioenriched Mannich product.

\section{General Procedure for preparation of racemic Mannich products.}

To a $25 \mathrm{~mL}$ one neck round bottom flask equipped with stir bar was charged with DABCO (11.0 $\mathrm{mg}, 0.10 \mathrm{mmol}), \alpha$-amido sulfone $(0.5 \mathrm{mmol})$ and $\mathrm{CH}_{2} \mathrm{Cl}_{2}(5.0 \mathrm{~mL})$. The dicarbonyl compound $(1.50 \mathrm{mmol})$ and $5 \%$ sodium carbonate aquous solution $(5.0 \mathrm{~mL})$ were added successfully. The reaction mixture was stirred at room temperature for 48 hours. $\mathrm{CH}_{2} \mathrm{Cl}_{2}(10 \mathrm{~mL})$ and water $(10$ $\mathrm{mL})$ were added to dilute the solution. The organic layer was separated and the aqueous phase was extracted with $\mathrm{CH}_{2} \mathrm{Cl}_{2}(2 \times 10 \mathrm{~mL})$. The combined organic layers were dried over sodium sulfate, filtered and concentrated under reduced pressure. The resulting residue was purified by flash chromatography over silica gel (elution with $15 \%-40 \%$ ethyl acetate in hexanes) to afford the racemic Mannich product.

\section{General procedure for the preparation of $(Z)$-enamines $(8 \mathbf{a}-8 \mathbf{p}, 9 \mathbf{a}-91){ }^{3}$}

An oven dried $10 \mathrm{~mL}$ round- bottom flask was charged with $\mathrm{Yb}(\mathrm{OTf})_{3}(2.0 \mathrm{mg}, 0.003 \mathrm{mmol})$, flamed dried under high vacuum and purged with nitrogen. The flask was cooled to room temperature and charged with the $\beta$-ketone ester Mannich addition product $(0.30 \mathrm{mmol})$. Trimethyl orthoformate $(1 \mathrm{~mL})$ and benzyl amine $(0.070 \mathrm{~mL}, 0.60 \mathrm{mmol})$ were added sucessfully. The solution was stirred at room temperature under nitrogen for $4.0 \mathrm{~h}$. The reaction mixture was subjected directly to the flash chromatography over silica gel (elution with $15-20 \%$ ethyl acetate in hexanes) to give the enamines 8a-8p, 9a-9l.

\section{8a: methyl $(S, Z)-4$-(methoxycarbonyl)-5-(benzylamino)-1-phenylhex-4-en-3-ylcarbamate}<smiles>COC(=O)N[C@@H](CCc1ccccc1)/C(C(=O)OC)=C(/C)NCc1ccccc1</smiles>

Yield: $81 \mathrm{mg}, 71 \%$; er: 95:5; HPLC Analysis, $t_{\mathrm{r}}$ major: 14.5 min., $\mathrm{t}_{\mathrm{r}}$ minor: 18.0 min., [Chiralcel ${ }^{\circledR} \mathrm{OD}-\mathrm{H}$ column, Hexanes:IPA = 98:2, $1.0 \mathrm{~mL} / \mathrm{min}] ;{ }^{1} \mathbf{H}$ NMR $(400 \mathrm{MHz}$, $\left.\mathrm{CDCl}_{3}\right): \delta 9.81(\mathrm{t}, J=6.0 \mathrm{~Hz}, 1 \mathrm{H}), 7.34(\mathrm{~m}, 2 \mathrm{H}), 7.24(\mathrm{~m}$, $5 \mathrm{H}), 7.15(\mathrm{~m}, 3 \mathrm{H}), 5.58(\mathrm{~d}, J=9.6 \mathrm{~Hz}, 1 \mathrm{H}), 4.46(\mathrm{dd}, J=$ $15.2,9.6 \mathrm{~Hz}, 1 \mathrm{H}), 4.39(\mathrm{~d}, J=6.0 \mathrm{~Hz}, 2 \mathrm{H}), 3.69(\mathrm{~s}, 3 \mathrm{H})$, $3.63(\mathrm{~s}, 3 \mathrm{H}), 2.56(\mathrm{t}, J=7.6 \mathrm{~Hz}, 2 \mathrm{H}), 2.14(\mathrm{~m}, 1 \mathrm{H}), 1.95(\mathrm{~m}$, $1 \mathrm{H}), 1.93(\mathrm{~s}, 3 \mathrm{H}) .{ }^{13} \mathrm{C}$ NMR $\left(75.0 \mathrm{MHz}, \mathrm{CDCl}_{3}\right)$ : $\delta$ 166.7, $157.8,152.9,142.1,134.7,125.1,124.8,124.7,124.6$, $123.7,123.2,122.0,90.1,48.1,46.5,45.7,43.6,33.4,31.0$, 18.9; IR (thin film, $\mathrm{cm}^{-1}$ ): 3333, 3050, 2953, 1720, 1657, $1580,1498,1230,1081 ;[\alpha]^{23} \mathbf{D}=-33.7^{\circ}\left(\mathrm{c}=1.0, \mathrm{CHCl}_{3}\right)$. HRMS $\left(\mathrm{CI} / \mathrm{NH}_{3}\right) \mathrm{m} / \mathrm{z}$ : calc'd for $(\mathrm{M}+\mathrm{H})^{+} \mathrm{C}_{23} \mathrm{H}_{29} \mathrm{~N}_{2} \mathrm{O}_{4}$ : 397.2049; found: 397.2081 .

\section{8b: methyl $(S, Z)-3-($ methoxycarbonyl)-4-(benzylamino)-1-phenylpent-3-en-2-ylcarbamate}


<smiles>COC(=O)N[C@@H](Cc1ccccc1)/C(C(=O)OC)=C(/C)NCc1ccccc1</smiles>

Yield: $99 \mathrm{mg}, 84 \%$; er: 95:5; HPLC Analysis, $\mathrm{t}_{\mathrm{r}}$ major: 25.5 min., $\mathrm{t}_{\mathrm{r}}$ minor: $31.5 \mathrm{~min}$., [Chiralcel ${ }^{\circledR} \mathrm{OD}$ column, Hexanes:IPA = 98:2, $1.0 \mathrm{~mL} / \mathrm{min}] ;{ }^{1} \mathbf{H}$ NMR (400 MHz, $\left.\mathrm{CDCl}_{3}\right): \delta 9.77(\mathrm{br}, 1 \mathrm{H}), 7.25-6.92(\mathrm{~m}, 10 \mathrm{H}), 5.73(\mathrm{~d}, J=$ $10.4 \mathrm{~Hz}, 1 \mathrm{H}), 4.77(\mathrm{~m}, 1 \mathrm{H}), 4.25(\mathrm{~d}, J=4 \mathrm{~Hz}, 2 \mathrm{H}), 3.74(\mathrm{~s}$, $3 \mathrm{H}), 3.58(\mathrm{~s}, 3 \mathrm{H}), 2.96(\mathrm{~m}, 2 \mathrm{H}), 2.13(\mathrm{~s}, 3 \mathrm{H}) .{ }^{13} \mathbf{C}$ NMR $\left(75.0 \mathrm{MHz}, \mathrm{CDCl}_{3}\right): \delta 171.3,162.0,158.0,143.5,128.7$, $129.8,129.0,128.3,127.4,126.7,89.7,52.1,47.2$, 45.7, 42.2, 32.9, 15.9.; IR (thin film, $\mathrm{cm}^{-1}$ ): 3345, 3040, 2953, $1715,1650,1581,1488,1250,1029 ;[\alpha]^{23}{ }_{D}=-34.5^{\circ}(\mathrm{c}=$ 1.0, $\left.\mathrm{CHCl}_{3}\right)$. HRMS $\left(\mathrm{CI} / \mathrm{NH}_{3}\right) \mathrm{m} / \mathrm{z}$ : calc'd for $(\mathrm{M}+\mathrm{Na})^{+}$ $\mathrm{C}_{22} \mathrm{H}_{26} \mathrm{~N}_{2} \mathrm{NaO}_{4}$ : 405.1790; found: 405.1754 .

8c: methyl ( $S, Z)-4-(m e t h o x y c a r b o n y l)-5-(b e n z y l a m i n o)-2-m e t h y l h e x-4-e n-3-y l c a r b a m a t e$<smiles>COC(=O)N[C@@H](C(=O)OC)C(C)C(C)NCc1ccccc1</smiles>
Yield: $73 \mathrm{mg}, 73 \%$; er: 96.5:3.5; HPLC Analysis, $\mathrm{t}_{\mathrm{r}}$ major: 15.9 min., $\mathrm{t}_{\mathrm{r}}$ minor: $19.4 \mathrm{~min}$., [Chiralcel ${ }^{\circledR} \mathrm{OD}-\mathrm{H}$ column, Hexanes:IPA = 98:2, $1.0 \mathrm{~mL} / \mathrm{min}] ;{ }^{1} \mathbf{H}$ NMR (400 MHz, $\left.\mathrm{CDCl}_{3}\right): \delta 9.85(\mathrm{t}, J=5.6 \mathrm{~Hz}, 1 \mathrm{H}), 7.31(\mathrm{~m}, 2 \mathrm{H}), 7.24(\mathrm{~m}$, $3 \mathrm{H}), 5.50(\mathrm{~d}, J=10 \mathrm{~Hz}, 1 \mathrm{H}), 4.43(\mathrm{~d}, J=5.6 \mathrm{~Hz}, 2 \mathrm{H}), 4.27$ $(\mathrm{t}, J=10 \mathrm{~Hz}, 1 \mathrm{H}), 3.68(\mathrm{~s}, 3 \mathrm{H}), 3.61(\mathrm{~s}, 3 \mathrm{H}), 2.05(\mathrm{~s}, 3 \mathrm{H})$, $1.85(\mathrm{~m}, 1 \mathrm{H}), 0.98(\mathrm{t}, J=6.0 \mathrm{~Hz}, 6 \mathrm{H}) .{ }^{13} \mathbf{C}$ NMR $(75.0 \mathrm{MHz}$, $\left.\mathrm{CDCl}_{3}\right): \delta .169 .3,162.4,155.2,142.1,129.2,128.3,126.1$, 95.2, 52.3, 48.1, 46.2, 32.5, 18.5, 15.4; IR (thin film, $\mathrm{cm}^{-1}$ ): $3415,3035,2930,1720,1564,1470,1380,1215 ;[\alpha]^{\mathbf{2 3}}{ }_{\mathbf{D}}=$ $-38.0^{\circ}\left(\mathrm{c}=1.0, \mathrm{CHCl}_{3}\right)$. HRMS $\left(\mathrm{CI} / \mathrm{NH}_{3}\right) \mathrm{m} / \mathrm{z}$ : calc'd for $(\mathrm{M}+\mathrm{H})^{+} \mathrm{C}_{18} \mathrm{H}_{27} \mathrm{~N}_{2} \mathrm{O}_{4}$ : 335.1971; found: 335.1998.

8d: methyl (S,Z)-2-(methoxycarbonyl)-3-(benzylamino)-1-cyclohexylbut-2-nylcarbamate.<smiles></smiles>
Yield: $93 \mathrm{mg}$, 84\%; er: 82:8; HPLC Analysis, $\mathrm{t}_{\mathrm{r}}$ major: 12.4 min., $\mathrm{t}_{\mathrm{r}}$ minor: 16.6 min., [Chiralcel ${ }^{\circledR} \mathrm{OD}-\mathrm{H}$ column, Hexanes:IPA = 98:2, $1.0 \mathrm{~mL} / \mathrm{min}] ;{ }^{1} \mathbf{H}$ NMR $(400 \mathrm{MHz}$, $\left.\mathrm{CDCl}_{3}\right): \delta 9.83(\mathrm{t}, \mathrm{J}=5.6 \mathrm{~Hz}, 1 \mathrm{H}), 7.32(\mathrm{~m}, 2 \mathrm{H}), 7.24(\mathrm{~m}$, $3 \mathrm{H}), 5.50(\mathrm{~d}, J=10 \mathrm{~Hz}, 1 \mathrm{H}), 4.43(\mathrm{~d}, J=5.6 \mathrm{~Hz}, 2 \mathrm{H}), 4.27$ $(\mathrm{t}, J=10 \mathrm{~Hz}, 1 \mathrm{H}), 3.68(\mathrm{~s}, 3 \mathrm{H}), 3.61(\mathrm{~s}, 3 \mathrm{H}), 2.11(\mathrm{~s}, 3 \mathrm{H})$, $1.92(\mathrm{~m}, 1 \mathrm{H}), 1.65(\mathrm{~m}, 4 \mathrm{H}), 1.13(\mathrm{~m}, 4 \mathrm{H}), 0.86(\mathrm{~m}, 1 \mathrm{H})$, $0.72(\mathrm{~m}, 1 \mathrm{H}) .{ }^{13} \mathbf{C}$ NMR $\left(75.0 \mathrm{MHz}, \mathrm{CDCl}_{3}\right): \delta 169.7$, $160.5,152.7,140.6,134.7,129.0,128.2,126.7,97.1,50.4$, 48.3, 47.5, 45.4, 34.3, 26.8, 26.2, 18.7; IR (thin film, $\mathbf{c m}^{-1}$ ): $3440,3020,2944,1715,1435,1380,1222 ;[\alpha]^{23}=-32.2^{\circ}$ $\left(\mathrm{c}=1.0, \mathrm{CHCl}_{3}\right)$. HRMS $\left(\mathrm{CI} / \mathrm{NH}_{3}\right) \mathrm{m} / \mathrm{z}$ : calc'd for $(\mathrm{M}+\mathrm{H})^{+}$ $\mathrm{C}_{21} \mathrm{H}_{31} \mathrm{~N}_{2} \mathrm{O}_{4}$ : 375.2284; found: 375.2267.

8e: methyl (S,Z)-3-(methoxycarbonyl)-2-(benzylamino)-6-methylhept-2-en-4-ylcarbamate 
<smiles>COC(=O)N[C@@H](CC(C)C)/C(C)=C(\C)NCc1ccccc1</smiles>

Yield: 78 mg, 75\%; er: 93.5:6.5; HPLC Analysis, $t_{\mathrm{r}}$ major: 11.2 min., $\mathrm{t}_{\mathrm{r}}$ minor: 14.5 min., [Chiralcel ${ }^{\circledR} \mathrm{OD}-\mathrm{H}$ column, Hexanes:IPA = 98:2, $1.0 \mathrm{~mL} / \mathrm{min}] ;{ }^{1} \mathbf{H}$ NMR $(400 \mathrm{MHz}$, $\left.\mathrm{CDCl}_{3}\right): \delta 9.80(\mathrm{t}, J=5.6 \mathrm{~Hz}, 1 \mathrm{H}), 7.24-7.35(5 \mathrm{H}), 5.90(\mathrm{~d}$, $J=10 . .0 \mathrm{~Hz}, 1 \mathrm{H}), 4.72(\mathrm{~m}, 1 \mathrm{H}), 4.45(\mathrm{~d}, J=5.6 \mathrm{~Hz}, 2 \mathrm{H})$, $3.70(\mathrm{~s}, 3 \mathrm{H}), 3.61(\mathrm{~s}, 3 \mathrm{H}), 2.11(\mathrm{~s}, 3 \mathrm{H}), 1.74(\mathrm{~m}, 1 \mathrm{H}), 1.55$ $(\mathrm{m}, 1 \mathrm{H}), 1.39(\mathrm{~m}, 1 \mathrm{H}), 0.92(\mathrm{~d}, J=6.3 \mathrm{~Hz}, 3 \mathrm{H}), 0.90(\mathrm{~d}, J=$ $6.3 \mathrm{~Hz}, 3 \mathrm{H} .{ }^{13} \mathbf{C}$ NMR $\left(75.0 \mathrm{MHz}, \mathrm{CDCl}_{3}\right): \delta 170.4,160.8$, $156.2,139.4,129.3,127.8,127.3,95.3,50.4,48.3,45.4$, 25.9, 23.4, 22.8, 15.6; IR (thin film, $\mathbf{c m}^{-1}$ ): 3335, 2948, $1718,1660,1430,1367,1044 ;[\alpha]^{23}{ }_{\mathrm{D}}=-28.3^{\circ}(\mathrm{c}=1.0$, $\left.\mathrm{CHCl}_{3}\right)$. HRMS $\left(\mathrm{CI} / \mathrm{NH}_{3}\right) \quad \mathrm{m} / \mathrm{z}$ : calc'd for $(\mathrm{M}+\mathrm{H})^{+}$ $\mathrm{C}_{19} \mathrm{H}_{28} \mathrm{~N}_{2} \mathrm{O}_{4}$ : 348.2049; found: 348.2083.

8f: methyl( $R, Z)-3-($ methoxycarbonyl)-4-(benzylamino)-1-(benzyloxy)pent-3-en-2-yl carbamate<smiles>COC(=O)N[C@@H](COCc1ccccc1)C(C(=O)OC)=C(C)NCc1ccccc1</smiles>

Yield: $96 \mathrm{mg}$, 78\%; er: 97.5:2.5; HPLC Analysis, $\mathrm{t}_{\mathrm{r}}$ major: 7.3 min., $\mathrm{t}_{\mathrm{r}}$ minor: 8.9 min., [Chiralcel ${ }^{\circledR} \mathrm{OD}-\mathrm{H}$ column, Hexanes:IPA = 95:5, $1.0 \mathrm{~mL} / \mathrm{min}]$; ${ }^{1} \mathbf{H}$ NMR $(400 \mathrm{MHz}$, $\left.\mathrm{CDCl}_{3}\right): \delta 9.84(\mathrm{br}, 1 \mathrm{H}), 7.30-7.15(\mathrm{~m}, 10 \mathrm{H}), 5.53(\mathrm{~d}, J=$ $9.6 \mathrm{~Hz}, 1 \mathrm{H}), 4.90(\mathrm{dd}, J=17.2,7.2 \mathrm{~Hz}, 1 \mathrm{H}), 4.45$ (s, 2H), 4.43-4.37 (m, 4H), 3.59 (s, 3H), $3.56(\mathrm{~s}, 3 \mathrm{H}), 2.09$ (s, 3H); ${ }^{13}$ C NMR (75.0 MHz, $\left.\mathrm{CDCl}_{3}\right): \delta 170.4,162.7,157.0,138.7$, $138.5,129.1,128.5,128.0,127.7,127.6,127.1,91.4,72.8$, 72.4, 52.2, 50.5, 49.6, 47.6, 15.8. IR (thin film, $\mathrm{cm}^{-1}$ ): 3430 , 3255, 2933, 1715, 1649, 1553, 1484, 1350, 1200, 1003; $[\alpha]^{23}{ }_{\mathbf{D}}=-47.3^{\circ}\left(\mathrm{c}=1.0, \mathrm{CHCl}_{3}\right)$. HRMS $\left(\mathrm{CI} / \mathrm{NH}_{3}\right) \mathrm{m} / \mathrm{z}$ : calc'd for $(\mathrm{M}+\mathrm{H})^{+} \mathrm{C}_{23} \mathrm{H}_{29} \mathrm{~N}_{2} \mathrm{O}_{5}$ : 413.2076; found: 413.2055.

8g: methyl (Z,4S,6R)-3-(methoxycarbonyl)-2-(benzylamino)-6,10-dimethylundeca-2,9-dien4-ylcarbamate.<smiles>COC(=O)N[C@@H](CC(C)CCC=C(C)C)C(C(=O)OC)=C(C)NCc1ccccc1</smiles>

Yield: $100 \mathrm{mg}, 81 \%$; er: 97:3; ${ }^{\mathbf{1}} \mathbf{H}$ NMR (400 MHz, $\left.\mathrm{CDCl}_{3}\right): \delta 9.80(\mathrm{t}, \mathrm{J}=6 \mathrm{~Hz}, 1 \mathrm{H}), 7.33(\mathrm{~m}, 2 \mathrm{H}), 7.24(\mathrm{~m}$, $3 \mathrm{H}), 5.51(\mathrm{~d}, J=10 \mathrm{~Hz}, 1 \mathrm{H}), 5.06(\mathrm{t}, J=10 \mathrm{~Hz}, 1 \mathrm{H}), 4.72$ (dd, $J=17.2,7.2 \mathrm{~Hz}, 1 \mathrm{H}), 4.45$ (d, $J=6 \mathrm{~Hz}, 2 \mathrm{H}), 3.71(\mathrm{~s}$, $3 \mathrm{H}), 3.60(\mathrm{~s}, 3 \mathrm{H}), 2.17(\mathrm{~s}, 3 \mathrm{H}), 1.92(\mathrm{~m}, 2 \mathrm{H}), 1.70(\mathrm{~m}, 1 \mathrm{H})$, $1.67(\mathrm{~s}, 3 \mathrm{H}), 1.58(\mathrm{~s}, 3 \mathrm{H}), 1.52(\mathrm{~m}, 1 \mathrm{H}), 1.34(\mathrm{~m}, 2 \mathrm{H}), 1.12$ $(\mathrm{m}, 1 \mathrm{H}), 0.87(\mathrm{~d}, J=6.4 \mathrm{~Hz}, 3 \mathrm{H}) .{ }^{13} \mathbf{C}$ NMR $(75.0 \mathrm{MHz}$, $\left.\mathrm{CDCl}_{3}\right): \delta 170.8,161.3,156.7,137.2,131.3,129.0,128.9$, 127.6, 127.1, 125.0, 124.8, 95.7, 52.0, 48.5, 47.6, 43.1, 41.5, 37.2, 30.3, 29.7, 25.9, 25.6, 20.3, 17.9, 15.7; IR (thin film, $\left.\mathbf{c m}^{-1}\right): 3441,3030,2943,1717,1659,1550,1494$, $1380,1220,1014 ;[\alpha]^{23}{ }_{\mathbf{D}}=-21.3^{\mathrm{o}}\left(\mathrm{c}=1.0, \mathrm{CHCl}_{3}\right)$.

Compound $8 \mathrm{~h}, 8 \mathrm{j}, 80$ and $8 \mathrm{p}$ were isolated in $>82 \%$ yields and their spectropic data are in agreement with previously reported compounds. ${ }^{3}$ 


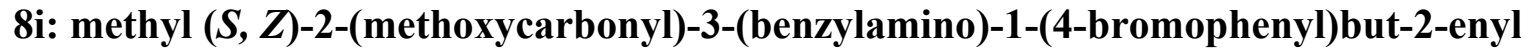
carbamate<smiles></smiles>

Yield: $111 \mathrm{mg}$, 83\%; er: 95:5; HPLC Analysis, $\mathrm{t}_{\mathrm{r}}$ major: 12.3 min., $\mathrm{t}_{\mathrm{r}}$ minor: $16.9 \mathrm{~min}$., [Chiralcel ${ }^{\circledR} \mathrm{OD}$ column, Hexanes:IPA = 95:5, $1.0 \mathrm{~mL} / \mathrm{min}] ;{ }^{1} \mathbf{H}$ NMR $(400 \mathrm{MHz}$, $\left.\mathrm{CDCl}_{3}\right): \delta 9.84(\mathrm{br}, 1 \mathrm{H}), 7.28(\mathrm{~m}, 3 \mathrm{H}), 7.23(\mathrm{~m}, 3 \mathrm{H}), 7.05(\mathrm{~d}$, $J=8.4 \mathrm{~Hz}, 2 \mathrm{H}), 5.82(\mathrm{~s}, 2 \mathrm{H}), 4.44$ (d, $J=5.6 \mathrm{~Hz}, 2 \mathrm{H}), 3.65$ (s, 3H), $3.40(\mathrm{~s}, 3 \mathrm{H}), 2.14(\mathrm{~s}, 3 \mathrm{H}) .{ }^{13} \mathrm{C}$ NMR $(75.0 \mathrm{MHz}$, $\left.\mathrm{CDCl}_{3}\right): \delta 172.2,162.1,157.5,146.5,140.2,131.2,129.2$, $127.8,127.3,127.1,121.1,94.1,52.4,52.2,50.5,47.7$, 15.8; IR (thin film, $\mathrm{cm}^{-1}$ ): 3330, 3011, 2945, 1715, 1630, $1581,1477,1250,1095,1003 ;[\alpha]^{23}{ }_{D}=-82.5^{\circ}(\mathrm{c}=1.0$, $\left.\mathrm{CHCl}_{3}\right)$. HRMS $\left(\mathrm{CI} / \mathrm{NH}_{3}\right) \mathrm{m} / \mathrm{z}$ : calc'd for $(\mathrm{M}+\mathrm{Na})^{+}$ $\mathrm{C}_{21} \mathrm{H}_{23} \mathrm{BrN}_{2} \mathrm{NaO}_{4}$ : 469.0739; found: 469.0770 .

8k: methyl ( $S, Z)-2-($ methoxycarbonyl)-3-(benzylamino)-1-(3-(trifluoromethyl)phenyl)but2-enylcarbamate<smiles></smiles>

Yield: $109 \mathrm{mg}$, 84\%; er: 96:4; HPLC Analysis, $t_{\mathrm{r}}$ major: 11.2 min., $\mathrm{t}_{\mathrm{r}}$ minor: 14.5 min., [Chiralcel ${ }^{\mathbb{B}} \mathrm{OD}$ column, Hexanes:IPA = 95:5, $1.0 \mathrm{~mL} / \mathrm{min}] ;{ }^{1} \mathbf{H}$ NMR $(400 \mathrm{MHz}$, $\left.\mathrm{CDCl}_{3}\right): \delta 9.93(\mathrm{t}, J=5.2 \mathrm{~Hz}, 1 \mathrm{H}), 7.50(\mathrm{~s}, 1 \mathrm{H}), 7.42(\mathrm{t}, J=$ $6.8 \mathrm{~Hz}, 2 \mathrm{H}), 7.36(\mathrm{t}, J=8.0 \mathrm{~Hz}, 3 \mathrm{H}), 7.28(\mathrm{~m}, 3 \mathrm{H}), 5.97(\mathrm{~d}$, $J=9.6 \mathrm{~Hz}, 1 \mathrm{H}), 5.92(\mathrm{~d}, J=9.6 \mathrm{~Hz}, 1 \mathrm{H}), 4.53(\mathrm{~d}, J=4.6$ $\mathrm{Hz}, 2 \mathrm{H}), 3.73$ (s, 3H), 3.44 (s, 3H), 2.23 (s, 3H). ${ }^{13} \mathbf{C}$ NMR $\left(75.0 \mathrm{MHz}, \mathrm{CDCl}_{3}\right): \delta 170.0,162.2,157.3,144.9,138.4$, $129.2,128.7,127.8,127.0,123.3,122.5,94.1,52.4,51.5$, 50.4, 47.7, 15.7; IR (thin film, $\mathrm{cm}^{-1}$ ): 3450, 3340, 2955, $1720,1651,1497,1339,1250,1135,1085 ;[\alpha]^{23}{ }_{\mathbf{D}}=-57.5^{\circ}$ $\left(\mathrm{c}=1.0, \mathrm{CHCl}_{3}\right)$. HRMS $\left(\mathrm{CI} / \mathrm{NH}_{3}\right) \mathrm{m} / \mathrm{z}$ : calc'd for $(\mathrm{M})^{+}$ $\mathrm{C}_{22} \mathrm{H}_{23} \mathrm{~F}_{3} \mathrm{~N}_{2} \mathrm{O}_{4}$ : 436.1610; found: 436.1649 .

81: methyl (S, Z)-2-(methoxycarbonyl)-3-(benzylamino)-1-p-tolylbut-2-enylcarbamate<smiles>COC(=O)N[C@@H](C(C)=C(C)NCc1ccccc1)c1ccc(C)cc1</smiles>

Yield: $110 \mathrm{mg}$, 96\%; er: 95:5; HPLC Analysis, $\mathrm{t}_{\mathrm{r}}$ major: 14.5 min., $\mathrm{t}_{\mathrm{r}}$ minor: $18.0 \mathrm{~min}$., [Chiralcel ${ }^{\mathbb{B}} \mathrm{OD}$ column, Hexanes:IPA = 95:5, $1.0 \mathrm{~mL} / \mathrm{min}] ;{ }^{1} \mathbf{H}$ NMR (400 MHz, $\mathrm{CDCl}_{3}$ ): $\delta 9.91$ (br, $\left.1 \mathrm{H}\right), 7.06-7.40(9 \mathrm{H}), 5.90(\mathrm{~d}, J=10.4$, $2 \mathrm{H}), 5.25(\mathrm{~d}, J=10.4 \mathrm{~Hz}, 1 \mathrm{H}), 4.49(\mathrm{~d}, J=5.6 \mathrm{~Hz}, 2 \mathrm{H})$, 3.71(s, 3H), 3.45(s, 3H), 2.33(s, 3H), 2.19(s, 3H); ${ }^{13}$ C NMR $\left(75.0 \mathrm{MHz}, \mathrm{CDCl}_{3}\right): \delta 170.1,161.6,156.4,142.8,138.4$, $135.5,130.0,129.2,129.1,128.9,127.6,127.1,125.8$, 125.7. 94.3, 52.1, 50.1 , 47.3, 21.5, 15.4; IR (thin film, $\mathrm{cm}^{-1}$ ): 3390, 2949, 1719, 1651, 1593, 1497, 1454, 1251, 1192; $[\alpha]^{23}{ }_{\mathrm{D}}=-45.8^{\circ}\left(\mathrm{c}=1.0, \mathrm{CHCl}_{3}\right)$. HRMS $\left(\mathrm{CI} / \mathrm{NH}_{3}\right) \mathrm{m} / \mathrm{z}$ : calc'd for $(\mathrm{M}+\mathrm{H})^{+} \mathrm{C}_{22} \mathrm{H}_{27} \mathrm{~N}_{2} \mathrm{O}_{4}$ : 383.1971; found: 383.1933 . 
8m: methyl $(S, Z)-2-($ methoxycarbonyl)-3-(benzylamino)-1-(3-methoxyphenyl)but-2-enyl carbamate<smiles>COC(=O)N[C@@H](C(C)=NCc1ccccc1)c1cccc(OC)c1</smiles>

Yield: $102 \mathrm{mg}, 86 \%$; er: 95.5:4.5; HPLC Analysis, $\mathrm{t}_{\mathrm{r}}$ major: 11.5 min., $\mathrm{t}_{\mathrm{r}}$ minor: 15.3 min., [Chiralcel ${ }^{\circledR} \mathrm{OD}$ column, Hexanes:IPA = 95:5, $1.0 \mathrm{~mL} / \mathrm{min}] ;{ }^{1} \mathbf{H}$ NMR $(400$ $\left.\mathrm{MHz}, \mathrm{CDCl}_{3}\right): \delta 9.92(\mathrm{t}, J=5.4 \mathrm{~Hz}, 1 \mathrm{H}), 7.16-7.40(9 \mathrm{H})$, $5.95(\mathrm{~d}, J=10.0 \mathrm{~Hz}, 1 \mathrm{H}), 5.25(\mathrm{~d}, J=10 \mathrm{~Hz}, 1 \mathrm{H}), 4.53(\mathrm{~d}, J$ $=6.0 \mathrm{~Hz}, 1 \mathrm{H}), 3.78(\mathrm{~s}, 3 \mathrm{H}), 3.61(\mathrm{~s}, 3 \mathrm{H}), 3.48(\mathrm{~s}, 3 \mathrm{H}), 2.18$ $(\mathrm{s}, 3 \mathrm{H}) ;{ }^{13} \mathrm{C}$ NMR $\left(75.0 \mathrm{MHz}, \mathrm{CDCl}_{3}\right): \delta 170.4,161.9$, $159.8,156.5,145.5,138.6,129.2,129.1,127.7,127.1,94.5$, 65.9, 55.4, 52.5, 50.5, 47.6, 15.7; IR (thin film, $\mathrm{cm}^{-1}$ ): 3331, $2933,1715,1652,1583,1350,1280,1005 ;[\alpha]^{23}{ }_{\mathbf{D}}=-53.2^{\circ}$ $\left(\mathrm{c}=1.0, \mathrm{CHCl}_{3}\right)$. HRMS $\left(\mathrm{CI} / \mathrm{NH}_{3}\right) \mathrm{m} / \mathrm{z}$ : calc'd for $(\mathrm{M}+\mathrm{H})^{+}$ $\mathrm{C}_{22} \mathrm{H}_{27} \mathrm{~N}_{2} \mathrm{O}_{5}$ : 399.1920; found: 399.1964 .

8n: methyl $(S, Z)-2-($ methoxycarbonyl)-1-(benzo[d][1,3]dioxol-5-yl)-3-(benzylamino)but-2enylcarbamate.<smiles></smiles>

Yield: $101 \mathrm{mg}, 82 \%$; er: 95.5:4.5; HPLC Analysis, $\mathrm{t}_{\mathrm{r}}$ major: 14.5 min., $t_{\mathrm{r}}$ minor: 18.0 min., [Chiralcel ${ }^{\circledR} \mathrm{OD}$ column, Hexanes:IPA = 95:5, $1.0 \mathrm{~mL} / \mathrm{min}] ;{ }^{1} \mathbf{H}$ NMR $(400$ $\left.\mathrm{MHz}, \mathrm{CDCl}_{3}\right): \delta 9.89(\mathrm{t}, J=5.5 \mathrm{~Hz}, 1 \mathrm{H}), 7.25-7.38(\mathrm{~m}, 5 \mathrm{H})$, 6.70-6.77 (m, 3H), $5.85(\mathrm{~m}, 3 \mathrm{H}), 5.25(\mathrm{~d}, J=10 \mathrm{~Hz}, 1 \mathrm{H})$, $4.50(\mathrm{~d}, J=5.5 \mathrm{~Hz}, 2 \mathrm{H}), 3.51(\mathrm{~s}, 3 \mathrm{H}), 2.18(\mathrm{~s}, 3 \mathrm{H}) ;{ }^{13} \mathrm{C}$ NMR $\left(75.0 \mathrm{MHz}, \mathrm{CDCl}_{3}\right): \delta 170.4,162.0,156.4,142.8$, $138.4,135.5,129.2,129.1,128.9,127.7,127.1,125.2$, 120.5, 101.1, 52.3, 50.6, 47.7, 15.7; IR (thin film, $\mathbf{c m}^{-1}$ ): $3347,2935,1717,1667,1580,1490,1330,1257,1006$; $[\alpha]^{23}{ }_{D}=-27.5^{\circ}\left(\mathrm{c}=1.0, \mathrm{CHCl}_{3}\right)$ HRMS $\left(\mathrm{CI} / \mathrm{NH}_{3}\right) \mathrm{m} / \mathrm{z}$ : calc'd for $(\mathrm{M}+\mathrm{H})^{+} \mathrm{C}_{22} \mathrm{H}_{25} \mathrm{~N}_{2} \mathrm{O}_{6}$ : 413.1713; found: 413.1772 .

For $9 \mathrm{a}-9 \mathrm{~h}$, the condensation reactions were run in $0.2 \mathrm{mmol}$ scale.

9a: $(S, Z)$-methyl 3-(allyloxycarbonylamino)-2-(1-(benzylamino)ethylidene)-5-phenyl pentanoate<smiles>C=CCOC(=O)N[C@@H](CCc1ccccc1)/C(C(=O)OC)=C(\C)NCc1ccccc1</smiles>

Yield: 76mg, 81\%; er: 95:5; HPLC analysis, $t_{r}$ major: 4.7 min, $t_{r}$ minor: 5.5 min, (Chiralcel ${ }^{\circledR} \mathrm{OD}-\mathrm{H}$ Column, Hexane:IPA $=95: 5,1.0 \mathrm{~mL} / \mathrm{min}) ;{ }^{1} \mathbf{H}$ NMR $(400 \mathrm{MHz}$, $\left.\mathrm{CDCl}_{3}\right): \delta 9.85(\mathrm{t}, J=5.5 \mathrm{~Hz}, 1 \mathrm{H}), 7.12-7.38(10 \mathrm{H}), 5.94$ $(\mathrm{m}, 1 \mathrm{H}), 5.68(\mathrm{~d}, J=10.2 \mathrm{~Hz}, 1 \mathrm{H}), 5.31(\mathrm{~d}, J=17.2 \mathrm{~Hz}$, $1 \mathrm{H}), 5.21(\mathrm{~d}, J=11.0 \mathrm{~Hz}, 1 \mathrm{H}), 4.68(\mathrm{~m}, 1 \mathrm{H}), 4.59$ (dd, $J=$ $13.3,5.5 \mathrm{~Hz}, 1 \mathrm{H}), 4.51(\mathrm{dd}, J=13.3,5.5 \mathrm{~Hz}, 1 \mathrm{H}), 4.40(\mathrm{~d}, J$ $=6.3 \mathrm{~Hz}, 2 \mathrm{H}), 3.71(\mathrm{~s}, 3 \mathrm{H}), 2.59(\mathrm{t}, J=7.8 \mathrm{~Hz}, 2 \mathrm{H}), 2.18$ $(\mathrm{m}, 1 \mathrm{H}), 2.00(\mathrm{~m}, 1 \mathrm{H}), 1.94(\mathrm{~s}, 3 \mathrm{H}) ;{ }^{13} \mathrm{C}$ NMR $(75.0 \mathrm{MHz}$, $\left.\mathrm{CDCl}_{3}\right): \delta 170.6,161.7,156.1,142.1,138.7,133.4,129.0$, $128.7,128.5,127.6,127.1,126.0,117.8,94.0,66.6,50.5$, 49.6, 47.5, 37.4, 33.4, 15.5; IR (thin film, $\mathrm{cm}^{-1}$ ): 3328, 
3030, 2943, 1715, 1646, 1590, 1498, 1235, 1091, 1041; $[\alpha]^{23}=-38.6^{\circ}\left(\mathrm{c}=1.9, \mathrm{CHCl}_{3}\right)$.

9b: (S,Z)-methyl 2-(1-(allyloxycarbonylamino)-2-phenylethyl)-3-(benzylamino)but-2-enoate<smiles>C=CCOC(=O)N[C@@H](Cc1ccccc1)/C(C(=O)OC)=C(\C)NCc1ccccc1</smiles>
Yield: $64 \mathrm{mg}, 78 \%$; er: 84.5:5.5; HPLC analysis, $t_{\mathrm{r}}$ major: $6.3 \mathrm{~min}, \mathrm{t}_{\mathrm{r}}$ minor: $6.9 \mathrm{~min}$, (Chiralcel OD Column, Hexane:IPA $=95: 5,1.0 \mathrm{~mL} / \mathrm{min}) ;{ }^{1} \mathbf{H}$ NMR $(400 \mathrm{MHz}$, $\left.\mathrm{CDCl}_{3}\right): \delta 9.85(\mathrm{t}, J=5.5 \mathrm{~Hz}, 1 \mathrm{H}), 7.00-7.32(10 \mathrm{H}), 5.85-$ $5.98(2 \mathrm{H}), 5.29(\mathrm{~d}, J=17.2 \mathrm{~Hz}, 1 \mathrm{H}), 5.21(\mathrm{~d}, J=10.2 \mathrm{~Hz}$, $1 \mathrm{H}), 4.85(\mathrm{~m}, 1 \mathrm{H}), 4.58$ (dd, $J=13.3,5.5 \mathrm{~Hz}, 1 \mathrm{H}), 4.51$ (dd, $J=13.3,5.5 \mathrm{~Hz}, 1 \mathrm{H}), 4.31(\mathrm{~m}, 2 \mathrm{H}), 3.81(\mathrm{~s}, 3 \mathrm{H}), 3.04(\mathrm{~m}$, 2H), $1.64(\mathrm{~s}, 3 \mathrm{H}) ;{ }^{13} \mathrm{C}$ NMR $\left(75.0 \mathrm{MHz}, \mathrm{CDCl}_{3}\right): \delta 170.4$, $162.5,129.8,129.1,129.0,128.3,127.4,126.7,126.3$, 117.9, 65.6, 51.8, 50.6, 47.2, 42.2, 15.3; IR (thin film, $\mathrm{cm}^{-}$ $\left.{ }^{1}\right): 3031,2944,1715,1647,1591,1498,1235,1040 ;[\boldsymbol{\alpha}]^{\mathbf{2 3}}{ }_{\mathbf{D}}$ $=-37.4^{\mathrm{o}}\left(\mathrm{c}=1.1, \mathrm{CHCl}_{3}\right)$.

9c: $(R, Z)$-methyl 2-(1-(allyloxycarbonylamino)-2-phenoxyethyl)-3-(benzylamino)but-2enoate<smiles>C=CCOC(=O)N[C@@H](COc1ccccc1)/C(C(=O)OC)=C(\C)NCc1ccccc1</smiles>

Yield: $66 \mathrm{mg}, 76 \%$; er: 95:5; HPLC analysis, $\mathrm{t}_{\mathrm{r}}$ major: 12.9 min, $t_{r}$ minor: 18.5 min, (Chiralcel ${ }^{\circledR}$ OD-H Column, Hexane:IPA $=96: 4,1.0 \mathrm{~mL} / \mathrm{min}) ;{ }^{1} \mathbf{H}$ NMR $(400 \mathrm{MHz}$, $\left.\mathrm{CDCl}_{3}\right): \delta 9.93(\mathrm{t}, J=5.5 \mathrm{~Hz}, 1 \mathrm{H}), 7.22-7.36(10 \mathrm{H}), 5.92$ $(\mathrm{m}, 1 \mathrm{H}), 5.66(\mathrm{~d}, J=10.2 \mathrm{~Hz}, 1 \mathrm{H}), 5.31(\mathrm{~d}, J=16.4 \mathrm{~Hz}$, $1 \mathrm{H}), 5.20$ (d, $J=10.2 \mathrm{~Hz}, 1 \mathrm{H}), 4.99(\mathrm{~m}, 1 \mathrm{H}), 4.59$ (dd, $J=$ $13.3,5.5 \mathrm{~Hz}, 1 \mathrm{H}), 4.54-4.51(3 \mathrm{H}), 4.44(\mathrm{~d}, J=6.3 \mathrm{~Hz}, 2 \mathrm{H})$, $3.69(\mathrm{dd}, J=9.4,8.6 \mathrm{~Hz}, 1 \mathrm{H}), 3.64(\mathrm{~s}, 3 \mathrm{H}), 3.52(\mathrm{dd}, J=$ 9.4, 7.0 Hz, 1H), $2.16(\mathrm{~s}, 3 \mathrm{H}) ;{ }^{13} \mathbf{C}$ NMR $(75.0 \mathrm{MHz}$, $\left.\mathrm{CDCl}_{3}\right): \delta 170.4,162.7,156.2,138.7,138.5,133.3,129.1$, $128.7,128.5,127.69,127.65,127.0,117.9,91.4,72.8,72.4$, 65.7, 51.5, 49.6, 47.6, 15.8; IR (thin film, $\mathrm{cm}^{-1}$ ): 3261, $2945,1717,1649,1593,1498,1240,1087,1003 ;[\alpha]^{23}{ }_{\mathbf{D}}=$ $-42.4^{\circ}\left(\mathrm{c}=1.2, \mathrm{CHCl}_{3}\right)$.

9d: (S,Z)-methyl 3-(allyloxycarbonylamino)-2-(1-(benzylamino)ethylidene)-5-

\section{methylhexanoate}<smiles>C=CCOC(=O)N[C@@H](CCC(C)C)/C(C(=O)OC)=C(\C)NCc1ccccc1</smiles>

Yield: $55 \mathrm{mg}, 73 \%$; er: 94:6; HPLC analysis, $\mathrm{t}_{\mathrm{r}}$ major: 10.6 min, $t_{r}$ minor: 12.4 min, (Chiralcel OD Column, Hexane:IPA $=96: 4,1.0 \mathrm{~mL} / \mathrm{min}) ;{ }^{1} \mathbf{H}$ NMR $(400 \mathrm{MHz}$, $\left.\mathrm{CDCl}_{3}\right): \delta 9.77(\mathrm{t}, J=5.5 \mathrm{~Hz}, 1 \mathrm{H}), 7.22-7.36(5 \mathrm{H}), 5.92(\mathrm{~m}$, $1 \mathrm{H}), 5.58(\mathrm{~d}, J=10.2 \mathrm{~Hz}, 1 \mathrm{H}), 5.29(\mathrm{~d}, J=17.2 \mathrm{~Hz}, 1 \mathrm{H})$, $5.22(\mathrm{~d}, J=11.0 \mathrm{~Hz}, 1 \mathrm{H}), 4.72(\mathrm{~m}, 1 \mathrm{H}), 4.57$ (dd, $J=13.3$, $5.5 \mathrm{~Hz}, 1 \mathrm{H}), 4.49$ (dd, $J=13.3,5.5 \mathrm{~Hz}, 1 \mathrm{H}), 4.43$ (d, $J=5.5$ $\mathrm{Hz}, 2 \mathrm{H}), 3.70(\mathrm{~s}, 3 \mathrm{H}), 1.74(\mathrm{~m}, 1 \mathrm{H}), 1.54(\mathrm{~m}, 1 \mathrm{H}), 1.39(\mathrm{~m}$, $1 \mathrm{H}), 0.90(\mathrm{~d}, J=6.3 \mathrm{~Hz}, 3 \mathrm{H}), 0.88(\mathrm{~d}, J=6.3 \mathrm{~Hz}, 3 \mathrm{H}) ;{ }^{13} \mathrm{C}$ NMR $\left(75.0 \mathrm{MHz}, \mathrm{CDCl}_{3}\right): \delta 170.8,161.0,156.0,138.7$, 
$133.5,129.0,127.6,127.1,117.7,95.1,65.5,50.4,48.3$, 47.5, 45.4, 25.7, 23.3, 22.7, 15.6; IR (thin film, $\mathrm{cm}^{-1}$ ): 3327 , $2954,1717,1650,1506,1442,1226,1044 ;[\alpha]^{23}=-30.5^{\circ}$ $\left(\mathrm{c}=2.0, \mathrm{CHCl}_{3}\right)$.

9e: $(S, Z)$-methyl 2-((allyloxycarbonylamino) (phenyl) methyl)-3-(benzylamino)but-2-enoate<smiles>C=CCOC(=O)N[C@H](C(C)=O)/C(C)=N/Cc1ccccc1</smiles>

Yield: $64 \mathrm{mg}, 81 \%$; er: 95.5:4.5; HPLC analysis, $\mathrm{t}_{\mathrm{r}}$ major: 8.5 min, $\mathrm{t}_{\mathrm{r}}$ minor: $9.7 \mathrm{~min}$, (Chiralcel ${ }^{\circledR} \mathrm{OD}-\mathrm{H}$ Column, Hexane:IPA $=96: 4,1.0 \mathrm{~mL} / \mathrm{min}$ ); The spectra of this compound has been reported before. ${ }^{3}$

9f: (S, Z)-methyl 2-((allyloxycarbonylamino)(4-bromophenyl)methyl)-3-(benzylamino)but2-enoate<smiles>C=CCOC(=O)NC(C(C)=O)C(C)=O</smiles>

Yield: $76 \mathrm{mg}, 80 \%$; er: 95:5; HPLC analysis, $\mathrm{t}_{\mathrm{r}}$ major: 8.5 $\min , \quad t_{r}$ minor: 9.6 min, (Chiralcel ${ }^{\circledR} \mathrm{OD}-\mathrm{H}$ Column, Hexane:IPA $=96: 4,1.0 \mathrm{~mL} / \mathrm{min}) ;{ }^{1} \mathbf{H}$ NMR $(400 \mathrm{MHz}$, $\left.\mathrm{CDCl}_{3}\right): \delta 9.91(\mathrm{t}, J=5.5 \mathrm{~Hz}, 1 \mathrm{H}), 7.10-7.42(9 \mathrm{H}), 5.89-$ $6.02(3 \mathrm{H}), 5.35(\mathrm{~d}, J=17.2 \mathrm{~Hz}, 1 \mathrm{H}), 5.26(\mathrm{~d}, J=9.4 \mathrm{~Hz}$, $1 \mathrm{H}), 4.65(\mathrm{dd}, J=12.5,5.5 \mathrm{~Hz}, 1 \mathrm{H}), 4.58(\mathrm{dd}, J=12.5,5.5$ $\mathrm{Hz}, 1 \mathrm{H}), 4.51(\mathrm{~d}, J=6.3 \mathrm{~Hz}, 2 \mathrm{H}), 3.46(\mathrm{~s}, 3 \mathrm{H}), 2.20(\mathrm{~s}, 3 \mathrm{H})$; ${ }^{13}$ C NMR (75.0 MHz, $\left.\mathrm{CDCl}_{3}\right): \delta 156.4,142.8,138.4,133.1$, $131.5,131.2,129.1,127.9,127.8,127.6,127.4,127.1$, $118.2,66.0,52.2,50.5,47.7,15.7$; IR (thin film, $\mathrm{cm}^{-1}$ ): 3324, 3031, 2945, 1716, 1650, 1591, 1492, 1260, 1086, $1005 ;[\boldsymbol{\alpha}]^{\mathbf{2 3}}{ }_{\mathbf{D}}=-24.9^{\circ}\left(\mathrm{c}=1.3, \mathrm{CHCl}_{3}\right)$.

9g: (S, Z)-methyl 2-((allyloxycarbonylamino)(3-fluorophenyl)methyl)-3-(benzylamino)but-

2-enoate<smiles>C=CCOC(=O)N[C@H](C(C)=O)/C(C)=N/Cc1ccccc1</smiles>

Yield: $68 \mathrm{mg}, 83 \%$; er: 95:5; HPLC analysis, $\mathrm{t}_{\mathrm{r}}$ major: 10.2 min, $t_{r}$ minor: 11.9 min, (Chiralcel ${ }^{\circledR}$ OD-H Column, Hexane:IPA $=96: 4,1.0 \mathrm{~mL} / \mathrm{min}) ;{ }^{1} \mathbf{H}$ NMR $(400 \mathrm{MHz}$, $\left.\mathrm{CDCl}_{3}\right): \delta 9.92(\mathrm{t}, J=5.5 \mathrm{~Hz}, 1 \mathrm{H}), 7.18-7.40(9 \mathrm{H}), 5.91-$ $6.04(3 \mathrm{H}), 5.35(\mathrm{~d}, J=17.2 \mathrm{~Hz}, 1 \mathrm{H}), 5.26(\mathrm{~d}, J=10.2 \mathrm{~Hz}$, $1 \mathrm{H}), 4.66(\mathrm{dd}, J=13.3,5.5 \mathrm{~Hz}, 1 \mathrm{H}), 4.58(\mathrm{dd}, J=13.3,6.3$ $\mathrm{Hz}, 1 \mathrm{H}), 4.51(\mathrm{~d}, J=6.3 \mathrm{~Hz}, 2 \mathrm{H}), 3.46(\mathrm{~s}, 3 \mathrm{H}), 2.21(\mathrm{~s}, 3 \mathrm{H})$; ${ }^{13}$ C NMR (75.0 MHz, $\left.\mathrm{CDCl}_{3}\right)$ : $\delta 169.9,164.1,161.8,161.7$, $156.2,146.5,146.4,138.2,132.9,129.4,129.3,128.9$, $127.5,126.8,121.0,117.9,113.0,112.8,112.6,112.4,94.0$, 77.3, 77.0, 76.7, 65.7, 52.0, 50.2, 47.4, 15.4; IR (thin film, $\left.\mathrm{cm}^{-1}\right): 3323,3030,2943,1716,1650,1591,1495,1261$, $1087 ;[\boldsymbol{\alpha}]^{\mathbf{2 3}}{ }_{\mathbf{D}}=-31.6^{\circ}\left(\mathrm{c}=1.2, \mathrm{CHCl}_{3}\right)$.

9h: (S, Z)-methyl 2-((allyloxycarbonylamino)(3-(trifluoromethyl)phenyl)methyl)-3(benzylamino)but-2-enoate 
<smiles>C=CCOC(=O)N[C@H](C(C(=O)OC)=C(C)NCc1ccccc1)c1cccc(C(F)(F)F)c1</smiles>

Yield: $76 \mathrm{mg}, 82 \%$; er: 95.5:4.5; HPLC analysis, $\mathrm{t}_{\mathrm{r}}$ major: 4.7 min, $t_{r}$ minor: 5.5 min, (Chiralcel ${ }^{\circledR} \mathrm{OD}-\mathrm{H}$ Column, Hexane:IPA $=95: 5,1.0 \mathrm{~mL} / \mathrm{min}) ;{ }^{1} \mathbf{H}$ NMR $(400 \mathrm{MHz}$, $\left.\mathrm{CDCl}_{3}\right): \delta 9.94(\mathrm{t}, J=5.5 \mathrm{~Hz}, 1 \mathrm{H}), 7.27-7.52(9 \mathrm{H}), 5.95-$ $6.01(3 \mathrm{H}), 5.37(\mathrm{~d}, J=17.2 \mathrm{~Hz}, 1 \mathrm{H}), 5.27(\mathrm{~d}, J=10.2 \mathrm{~Hz}$, $1 \mathrm{H}), 4.57-4.69(2 \mathrm{H}), 4.53(\mathrm{~d}, J=5.4 \mathrm{~Hz}, 2 \mathrm{H}), 3.45(\mathrm{~s}, 3 \mathrm{H})$, $2.23(\mathrm{~s}, 3 \mathrm{H}) ;{ }^{13} \mathbf{C}$ NMR $\left(75.0 \mathrm{MHz}, \mathrm{CDCl}_{3}\right): \delta 170.0,162.3$, $156.5,144.9,138.4,133.1,129.2,128.8,128.7,128.4$, $127.8,127.0,123.3,122.5,118.3,94.1,66.1,52.4,50.5$, 47.7, 15.8; IR (thin film, $\mathrm{cm}^{-1}$ ): 3331, 2945, 1717, 1651, $1592,1496,1329,1242,1166,1124,1075 ;[\alpha]^{23}=-31.5^{\circ}$ $\left(\mathrm{c}=1.3, \mathrm{CHCl}_{3}\right)$.

9i: (S, Z)-methyl 2-((allyloxycarbonylamino)(p-tolyl)methyl)-3-(benzylamino)but-2-enoate<smiles>C=CCOC(=O)N[C@H](C(C)=C(C)NCc1ccccc1)c1ccc(C)cc1</smiles>
Yield: $69 \mathrm{mg}, 84 \%$; er: 95:5; HPLC analysis, $\mathrm{t}_{\mathrm{r}}$ major: 18.5 min, $t_{r}$ minor: 19.6 min, (Chiralcel ${ }^{\circledR}$ OD-H Column, Hexane:IPA $=98: 2,1.0 \mathrm{~mL} / \mathrm{min}) ;{ }^{1} \mathbf{H}$ NMR $(400 \mathrm{MHz}$, $\left.\mathrm{CDCl}_{3}\right): \delta 9.91(\mathrm{t}, J=5.5 \mathrm{~Hz}, 1 \mathrm{H}), 7.06-7.40(9 \mathrm{H}), 5.90-$ $6.04(3 \mathrm{H}), 5.35(\mathrm{~d}, J=17.2 \mathrm{~Hz}, 1 \mathrm{H}), 5.25(\mathrm{~d}, J=10.2 \mathrm{~Hz}$, $1 \mathrm{H}), 4.65(\mathrm{dd}, J=13.3,5.5 \mathrm{~Hz}, 1 \mathrm{H}), 4.58(\mathrm{dd}, J=13.3,5.5$ $\mathrm{Hz}, 1 \mathrm{H}), 4.51(\mathrm{~d}, J=5.5 \mathrm{~Hz}, 2 \mathrm{H}), 3.48(\mathrm{~s}, 3 \mathrm{H}), 2.31(\mathrm{~S}$, $3 \mathrm{H}), 2.20(\mathrm{~s}, 3 \mathrm{H}) ;{ }^{13} \mathbf{C}$ NMR $\left(75.0 \mathrm{MHz}, \mathrm{CDCl}_{3}\right): \delta 156.4$, $142.8,138.4,135.5,130.0,129.2,129.1,128.9,127.6$, $127.1,125.8,125.7,118.2,66.0,52.2,50.5,47.6,21.2$, 15.7; IR (thin film, $\mathrm{cm}^{-1}$ ): 3335, 3031, 2943, 1716, 1649, $1592,1497,1256,1192,1088 ;[\boldsymbol{\alpha}]^{\mathbf{2 3}}{ }_{\mathbf{D}}=-53.1^{\circ}(\mathrm{c}=1.9$, $\left.\mathrm{CHCl}_{3}\right)$.

9j: (S,Z)-methyl 2-((allyloxycarbonylamino)(3-methoxyphenyl)methyl)-3-(benzylamino) but-2-enoate<smiles>C=CCOC(=O)N[C@@H](C(C)=NCc1ccccc1)c1cccc(OC)c1</smiles>

Yield: $68 \mathrm{mg}, 80 \%$; er: 94:6; HPLC analysis, $\mathrm{t}_{\mathrm{r}}$ major: 13.8 min, $t_{r}$ minor: 17.4 min, (Chiralcel ${ }^{\circledR} \mathrm{OD}-\mathrm{H}$ Column, Hexane:IPA $=96: 4,1.0 \mathrm{~mL} / \mathrm{min}) ;{ }^{1} \mathbf{H}$ NMR (400 MHz, $\left.\mathrm{CDCl}_{3}\right): \delta 9.92(\mathrm{t}, J=5.5 \mathrm{~Hz}, 1 \mathrm{H}), 7.16-7.40(9 \mathrm{H}), 5.92-$ $6.04(3 \mathrm{H}), 5.35(\mathrm{~d}, J=17.2 \mathrm{~Hz}, 1 \mathrm{H}), 5.25(\mathrm{~d}, J=10.2 \mathrm{~Hz}$, $1 \mathrm{H}), 4.66(\mathrm{dd}, J=13.3,6.3 \mathrm{~Hz}, 1 \mathrm{H}), 4.58(\mathrm{dd}, J=13.3,6.3$ $\mathrm{Hz}, 1 \mathrm{H}), 4.50(\mathrm{~d}, J=6.3 \mathrm{~Hz}, 2 \mathrm{H}), 3.78(\mathrm{~s}, 3 \mathrm{H}), 3.48(\mathrm{~s}, 3 \mathrm{H})$, $2.20(\mathrm{~s}, 3 \mathrm{H}) ;{ }^{13} \mathbf{C}$ NMR $\left(75.0 \mathrm{MHz}, \mathrm{CDCl}_{3}\right): \delta 170.4,161.9$, $159.8,156.5,145.5,138.6,133.3,129.2,129.1,127.7$, $127.1,118.3,118.0,112.1,111.3,94.5,65.9,55.4,52.5$, 50.5, 47.6, 15.7; IR (thin film, $\left.\mathrm{cm}^{-1}\right): 3321,2945,1714$, $1650,1593,1495,1265 ;[\boldsymbol{\alpha}]^{23}{ }_{\mathbf{D}}=-23.7^{\circ}\left(\mathrm{c}=1.2, \mathrm{CHCl}_{3}\right)$.

9k: (S,Z)-methyl 2-((allyloxycarbonylamino)(benzo[d][1,3]dioxol-5-yl)methyl)-3-(benzylamino)but-2-enoate 
<smiles>C=CCOC(=O)N[C@H](C(C(=O)OC)=C(C)NCc1ccccc1)c1ccc2c(c1)OCO2</smiles>

Yield: $71 \mathrm{mg}, 81 \%$, ee: $93: 7$; HPLC analysis, $\mathrm{t}_{\mathrm{r}}$ major: 8.8 min, $t_{\mathrm{r}}$ minor: $10.0 \mathrm{~min}$, (Chiralcel OD Column, Hexane:IPA $=95: 5,1.0 \mathrm{~mL} / \mathrm{min}) ;{ }^{1} \mathbf{H} \mathbf{N M R}\left(400 \mathrm{MHz}, \mathrm{CDCl}_{3}\right): \delta 9.89(\mathrm{t}$, $J=5.5 \mathrm{~Hz}, 1 \mathrm{H}), 7.25-7.38(6 \mathrm{H}), 6.70-6.77(2 \mathrm{H}), 5.85-6.04$ $(5 \mathrm{H}), 5.35(\mathrm{~d}, J=17.2 \mathrm{~Hz}, 1 \mathrm{H}), 5.25(\mathrm{~d}, J=10.2 \mathrm{~Hz}, 1 \mathrm{H})$, 4.64 (dd, $J=13.3,5.5 \mathrm{~Hz}, 1 \mathrm{H}), 4.58$ (dd, $J=13.3,5.5 \mathrm{~Hz}$, $1 \mathrm{H}), 4.50(\mathrm{~d}, J=5.5 \mathrm{~Hz}, 2 \mathrm{H}), 3.51(\mathrm{~s}, 3 \mathrm{H}), 2.18(\mathrm{~s}, 3 \mathrm{H}) ;{ }^{13} \mathrm{C}$ NMR (75.0 $\left.\mathrm{MHz}, \mathrm{CDCl}_{3}\right): \delta 156.4,142.8,138.4,135.5$, $130.1,129.2$, 129.1, 128.9, 127.7, 127.1, 125.2, 120.5, 108.0, 106.7, 101.1, 65.9, 52.3, 50.6, 47.7, 15.7; IR (thin film, $\mathrm{cm}^{-}$ $\left.{ }^{1}\right): 3327,2924,1715,1649,1591,1492,1237,1036 ;[\alpha]^{\mathbf{2 3}}{ }_{\mathbf{D}}=$ $-22.3^{\circ}\left(\mathrm{c}=1.2, \mathrm{CHCl}_{3}\right)$.

91: $(R, Z)$-methyl 2-((allyloxycarbonylamino)(furan-2-yl)methyl)-3-(benzylamino)but-2enoate<smiles>C=CCOC(=O)N[C@H](C(C)=O)/C(C)=N/Cc1ccccc1</smiles>

Yield: $62 \mathrm{mg}, 81 \%$; er: 95:5; HPLC analysis, $\mathrm{t}_{\mathrm{r}}$ major: 11.1 min, $t_{r}$ minor: 15.1 min, (Chiralcel ${ }^{\circledR}$ OD-H Column, Hexane:IPA $=96: 4,1.0 \mathrm{~mL} / \mathrm{min}) ;{ }^{1} \mathbf{H}$ NMR $(400 \mathrm{MHz}$, $\left.\mathrm{CDCl}_{3}\right): \delta 9.92(\mathrm{t}, J=5.5 \mathrm{~Hz}, 1 \mathrm{H}), 7.25-7.38(6 \mathrm{H}), 6.27(\mathrm{~m}$, $1 \mathrm{H}), 5.90-6.04(3 \mathrm{H}), 5.34(\mathrm{~d}, J=17.2 \mathrm{~Hz}, 1 \mathrm{H}), 5.23(\mathrm{~d}, J=$ $9.4 \mathrm{~Hz}, 1 \mathrm{H}), 4.64$ (dd, $J=13.3,5.5 \mathrm{~Hz}, 1 \mathrm{H}), 4.56$ (dd, $J=$ 13.3, $6.3 \mathrm{~Hz}, 1 \mathrm{H}), 4.49$ (d, $J=5.5 \mathrm{~Hz}, 2 \mathrm{H}), 3.58(\mathrm{~s}, 3 \mathrm{H})$, $2.21(\mathrm{~s}, 3 \mathrm{H}) ;{ }^{13} \mathbf{C}$ NMR $\left(75.0 \mathrm{MHz}, \mathrm{CDCl}_{3}\right): \delta 161.9,156.0$, $141.1,132.9,131.7,128.9,127.5,126.9,117.8,117.7$, $117.2,117.0,116.6,115.4,110.2,65.7,50.3,48.2,47.4$, 15.4; IR (thin film, $\mathrm{cm}^{-1}$ ): 3329, 2924, 1717, 1649, 1591, $1497,1238,1086 ;[\boldsymbol{\alpha}]^{23} \mathbf{D}=-6.5^{\circ}\left(\mathrm{c}=1.0, \mathrm{CHCl}_{3}\right)$.

12a: methyl (3S)-4-(methoxycarbonyl)-5-oxo-1-phenylheptan-3-ylcarbamate.<smiles>CCC(=O)C(C(=O)OC)[C@H](CCc1ccccc1)NC(=O)OC</smiles>
Yield: $137 \mathrm{mg}, 86 \%$. er: 96:4. HPLC analysis, $t_{\mathrm{r}}$ major: $10.7 \mathrm{~min}, 12.8 \mathrm{~min} \mathrm{t}_{\mathrm{r}}$ minor: $8.8 \mathrm{~min}$, [ Chiralpac AD-H column, Hexanes:IPA $95: 5,1.0 \mathrm{~mL} / \mathrm{min}^{1} \mathbf{H}$ NMR (400 $\mathrm{MHz}, \mathrm{CDCl}_{3}$, both diastereromer were reported): $\delta 7.36(\mathrm{~m}$, 4H), $7.25(\mathrm{~m}, 6 \mathrm{H}), 5.79(\mathrm{~d}, J=9.6 \mathrm{~Hz}, 1 \mathrm{H}), 5.48$ (d, $J=9.6$ $\mathrm{Hz}, 1 \mathrm{H}), 4.45(\mathrm{~m}, 1 \mathrm{H}), 4.33(\mathrm{~m}, 1 \mathrm{H}), 3.82(\mathrm{~d}, J=3.2 \mathrm{~Hz}$, $1 \mathrm{H}), 3.80(\mathrm{~s}, 3 \mathrm{H}), 3.79(\mathrm{~s}, 3 \mathrm{H}), 3.76(\mathrm{~d}, J=3.6 \mathrm{~Hz}, 1 \mathrm{H})$, $3.73(\mathrm{~s}, 3 \mathrm{H}), 3.72(\mathrm{~s}, 3 \mathrm{H}), 2.84-2.63(\mathrm{~m}, 4 \mathrm{H}), 2.49(\mathrm{~m}, 4 \mathrm{H})$, $2.03(\mathrm{~m}, 2 \mathrm{H}), 1.88(\mathrm{~m}, 2 \mathrm{H}), 1.06(\mathrm{~m}, 6 \mathrm{H}) .{ }^{13} \mathbf{C}$ NMR $(75.0$ $\mathrm{MHz}, \mathrm{CDCl}_{3}$, both diastereomers were reported): $\delta 206.1$, $204.5,170.0,169.1,157.1,155.2,141.2,140.2,129.5$, $129.4,128.6,127,5,126.2,61.7,61.0,51.1,50.3,37.4$, $36.1,35.2,33.0,32.8,32.6,7.7$. IR (thin film, $\mathbf{c m}^{-1}$ ): 3363, 3003, 2960, 1750, 1701, 1519, 1444, 1356, 1302, 1228,$1047 ;[\alpha]^{23} \mathbf{D}=-7.5^{\circ}\left(\mathrm{c}=1.0, \mathrm{CHCl}_{3}\right)$.

12b: methyl (3S)-2-(methoxycarbonyl)-1-oxo-1,5-diphenylpentan-3-ylcarbamate. 
<smiles>COC(=O)N[C@@H](CCc1ccccc1)C(C(=O)OC)C(=O)c1ccccc1</smiles>

Yield: $177 \mathrm{mg}, 96 \%$. er: 92.5:7.5. HPLC analysis, $\mathrm{t}_{\mathrm{r}}$ major: 11.9, $16.3 \mathrm{~min}, \mathrm{t}_{\mathrm{r}}$ minor: $14.0 \mathrm{~min}$, [ Chiralpac AD-H column, Hexanes:IPA $95: 5,1.0 \mathrm{~mL} / \mathrm{min}{ }^{1} \mathbf{H}$ NMR (400 $\mathrm{MHz}, \mathrm{CDCl}_{3}$, both diastereromer were reported): $\delta 7.91(\mathrm{~d}$, $J=7.2 \mathrm{~Hz}, 2 \mathrm{H}), 7.79(\mathrm{~d}, J=8.0 \mathrm{~Hz}, 2 \mathrm{H}), 7.57(\mathrm{~m}, 2 \mathrm{H}), 7.44$ $(\mathrm{m}, 4 \mathrm{H}), 7.27(\mathrm{~m}, 2 \mathrm{H}), 7.21(\mathrm{~m}, 5 \mathrm{H}), 5.11(\mathrm{~m}, 3 \mathrm{H}), 5.75(\mathrm{~d}$, $J=9.6 \mathrm{~Hz}, 1 \mathrm{H}), 5.61(\mathrm{~d}, J=9.2 \mathrm{~Hz}, 1 \mathrm{H}), 4.65(\mathrm{t}, J=4.4$ $\mathrm{Hz}, 2 \mathrm{H}), 4.34(\mathrm{~m}, 2 \mathrm{H}), 4.15(\mathrm{~m}, 4 \mathrm{H}), 3.67$ (s, 3H), 3.60 (s, $3 \mathrm{H}), 2.74(\mathrm{~m}, 3 \mathrm{H}), 2.57(\mathrm{~m}, 1 \mathrm{H}), 2.11(\mathrm{~m}, 2 \mathrm{H}), 1.90(\mathrm{~m}$, 2H), $1.16(\mathrm{~m}, 6 \mathrm{H}) .{ }^{13} \mathbf{C}$ NMR $\left(75.0 \mathrm{MHz}, \mathrm{CDCl}_{3}\right.$, both diastereomers were reported): $\delta 195.3,192.8,169.1,168.6$, $157.1,156.8,141.2,134.1,133.9,130.6,129.5,129.2$, $129.1,129.0,128.8,128.7,128.6,128.5,128.4,127.3$, $126.5,126.3,126.2,62.0,61.8,57.2,56.5,52.4,51.7,51.1$, $35.5,34.8,33.1,32.8,32.8,14.2,14.1$. IR (thin film, $\mathbf{c m}^{-}$ $\left.{ }^{1}\right): 3337,2954,1712,1722,1508,1450,1361,1242,1047$; $[\alpha]^{23}=-20.3^{\circ}\left(\mathrm{c}=1.0, \mathrm{CHCl}_{3}\right)$.

12c: methyl (3S)-4-(methoxycarbonyl)-6-methoxy-5-oxo-1-phenylhexan-3-ylcarbamate<smiles>COCC(=O)C(C(=O)OC)[C@H](CCc1ccccc1)NC(=O)OC</smiles>

Yield: $143 \mathrm{mg}, 85 \%$. er: 95:5. HPLC analysis, $t_{r}$ major: $17.0 \mathrm{~min}, \mathrm{t}_{\mathrm{r}}$ minor: $18.3 \mathrm{~min}$, [ Chiralpac AD-H column, Hexanes:IPA $95: 5,1.0 \mathrm{~mL} / \mathrm{min}^{1} \mathbf{H}$ NMR (400 MHz, $\mathrm{CDCl}_{3}$, both diastereromer were reported): $\delta 7.25(\mathrm{~m}, 4 \mathrm{H})$, $7.18(\mathrm{~m}, 6 \mathrm{H}), 5.68(\mathrm{~d}, J=10.4 \mathrm{~Hz}, 1 \mathrm{H}), 5.50(\mathrm{~d}, J=10 \mathrm{~Hz}$, $1 \mathrm{H}), 4.38(\mathrm{~m}, 1 \mathrm{H}), 4.29(\mathrm{~m}, 1 \mathrm{H}), 4.11(\mathrm{~m}, 1 \mathrm{H}), 4.04(\mathrm{~d}, J=$ $4.4 \mathrm{~Hz}, 1 \mathrm{H}), 3.96(\mathrm{~m}, 3 \mathrm{H}), 3.71(\mathrm{~s}, 3 \mathrm{H}), 3.70(\mathrm{~s}, 3 \mathrm{H}), 3.65$ $(\mathrm{s}, 3 \mathrm{H}), 3.63(\mathrm{~s}, 3 \mathrm{H}), 3.37(\mathrm{~s}, 3 \mathrm{H}), 3.35(\mathrm{~s}, 3 \mathrm{H}), 2.77-2.58$ $(\mathrm{m}, 4 \mathrm{H}), 2.01(\mathrm{~m}, 2 \mathrm{H}), 1.81(\mathrm{~m}, 2 \mathrm{H}) .{ }^{13} \mathbf{C}$ NMR $(75.0 \mathrm{MHz}$, $\mathrm{CDCl}_{3}$, both diastereomers were reported): $\delta$ 204.5, 203.4, 169.6, 168.5, 157.2, 156.9, 141.3, 141.1, 128.7, 126.3, 77.6, $77.0,59.6,58.2,57.0,52.9,52.6,50.8,49.6,36.2,35.4$, 32.9, 32.8. IR (thin film, $\mathbf{c m}^{-1}$ ): 3363, 3003, 2960, 1750, $1701,1519,1444,1356,1302,1228,1047 ;[\alpha]^{23}{ }^{2}=-4.3^{\circ}(\mathrm{c}$ $\left.=1.0, \mathrm{CHCl}_{3}\right)$.

For 14a-14f, reactions were run in 4.0 mmol scale.

14a: (S)-Allyl 4-acetyl-5-oxo-1-phenylhexan-3-ylcarbamate<smiles>C=CCOC(=O)N[C@@H](CCc1ccccc1)C(C(C)=O)C(C)=O</smiles>

Yield: $1.12 \mathrm{~g}, 88 \%$, er: 95:5; HPLC analysis, $\mathrm{t}_{\mathrm{r}}$ minor: 9.4 min, $t_{r}$ major: 12.3 min, (ChiralPak ${ }^{\circledR} \mathrm{AD}-\mathrm{H}$ Column, Hexane:IPA = 95:5, $1.0 \mathrm{~mL} / \mathrm{min}) ;{ }^{1} \mathbf{H}$ NMR (400 MHz, $\left.\mathrm{CDCl}_{3}\right): \delta 7.16-7.32(5 \mathrm{H}), 5.90(\mathrm{~m}, 1 \mathrm{H}), 5.61(\mathrm{~d}, J=10.2$ $\mathrm{Hz}, 1 \mathrm{H}), 5.25(\mathrm{~m}, 2 \mathrm{H}), 4.54(\mathrm{~m}, 2 \mathrm{H}), 4.35(\mathrm{~m}, 1 \mathrm{H}), 3.88(\mathrm{~d}$, $J=4.7 \mathrm{~Hz}, 1 \mathrm{H}), 2.74(\mathrm{~m}, 1 \mathrm{H}), 2.61(\mathrm{~m}, 1 \mathrm{H}), 2.24(\mathrm{~s}, 3 \mathrm{H})$, $2.10(\mathrm{~s}, 3 \mathrm{H}), 1.96(\mathrm{~m}, 1 \mathrm{H}), 1.74(\mathrm{~m}, 1 \mathrm{H}) ;{ }^{13} \mathbf{C}$ NMR $(75.0$ $\left.\mathrm{MHz}, \mathrm{CDCl}_{3}\right): \delta$ 205.2, 203.8, 156.3, 141.1, 132.8, 128.8, 
128.6, 126.4, 117.9, 69.6, 65.9, 50.8, 35.9, 33.0, 31.1, 30.1;

IR (thin film, $\mathrm{cm}^{-1}$ ): 3323, 1720, 1694, 1536, 1363, 1278, $1149,1060,700 ;[\alpha]^{23} \mathbf{D}=-56.2^{\circ}\left(\mathrm{c}=1.0, \mathrm{CHCl}_{3}\right)$.

Enantiomer of 10a was obtained as a white crystal $89 \%$ yield and 95:5 er from a reaction catalyzed by cinchonidine at $-20{ }^{\circ} \mathrm{C}$ to

$-15^{\circ} \mathrm{C}$ for $48 \mathrm{~h}$.

14b: (R)-Allyl 2-acetyl-3-oxo-1-phenylbutylcarbamate<smiles>C=CCOC(=O)NC(C(C)=O)C(C)=O</smiles>

Yield: $1.05 \mathrm{~g}, 91 \%$; er: 96.5:3.5; HPLC analysis, $\mathrm{t}_{\mathrm{r}}$ minor: 9.0 min, $t_{\mathrm{r}}$ major: $9.8 \mathrm{~min}$, (ChiralPak ${ }^{\circledR} \mathrm{AD}-\mathrm{H}$ Column, Hexane:IPA $=95: 5,1.0 \mathrm{~mL} / \mathrm{min}) ;{ }^{1} \mathbf{H}$ NMR $(400 \mathrm{MHz}$, $\left.\mathrm{CDCl}_{3}\right): \delta 7.21-7.34(5 \mathrm{H}), 6.22(\mathrm{~d}, J=9.4 \mathrm{~Hz}, 1 \mathrm{H}), 5.83(\mathrm{~m}$, $1 \mathrm{H}), 5.53(\mathrm{dd}, J=8.6,7.8 \mathrm{~Hz}, 1 \mathrm{H}), 5.23(\mathrm{~d}, J=17.2 \mathrm{~Hz}$, $2 \mathrm{H}), 5.15(\mathrm{~d}, J=10.2 \mathrm{~Hz}, 1 \mathrm{H}), 4.50(\mathrm{~m}, 2 \mathrm{H}), 4.26(\mathrm{~d}, J=$ 7.0 Hz, 1H), $2.15(\mathrm{~s}, 3 \mathrm{H}), 2.10(\mathrm{~s}, 3 \mathrm{H}) ;{ }^{13} \mathbf{C}$ NMR $(75.0$ $\left.\mathrm{MHz}, \mathrm{CDCl}_{3}\right): \delta 204.6,202.4,155.9,139.7,132.7,129.0$, 128.1, 126.7, 118.0, 71.8, 66.1, 54.5, 30.6, 30.3; IR (thin film, $\left.\mathrm{cm}^{-1}\right): 3320,1727,1693,1538,1360,1262,1148$, 1049,$702 ;[\boldsymbol{\alpha}]^{23}{ }_{\mathbf{D}}=+8.4^{\circ}\left(\mathrm{c}=1.0, \mathrm{CHCl}_{3}\right)$.

Enantiomer of 10b was obtained as a white crystal $90 \%$ yield and 95.5:4.5 er from a reaction catalyzed by cinchonidine at $-15{ }^{\circ} \mathrm{C}$ for $16 \mathrm{~h}$.

14c: (R)-Allyl 2-acetyl-1-(4-bromophenyl)-3-oxobutylcarbamate<smiles>C=CCOC(=O)NC(C(C)=O)C(C)=O</smiles>

Yield: 1.32 g, 90\%; er: 95.5:4.5; HPLC analysis, $t_{\mathrm{r}}$ minor: 8.5 min, $t_{r}$ major: $9.6 \mathrm{~min}$, (ChiralPak ${ }^{\circledR} \mathrm{AD}-\mathrm{H}$ Column, Hexane:IPA $=95: 5,1.0 \mathrm{~mL} / \mathrm{min}) ;{ }^{1} \mathbf{H}$ NMR $(400 \mathrm{MHz}$, $\left.\mathrm{CDCl}_{3}\right): \delta 7.45(\mathrm{~d}, J=8.6 \mathrm{~Hz}, 2 \mathrm{H}), 7.17(\mathrm{~d}, J=8.6 \mathrm{~Hz}, 2 \mathrm{H})$, $6.09(\mathrm{~d}, J=8.6 \mathrm{~Hz}, 1 \mathrm{H}), 5.85(\mathrm{~m}, 1 \mathrm{H}), 5.47(\mathrm{t}, J=7.8 \mathrm{~Hz}$, $1 \mathrm{H}), 5.26(\mathrm{~d}, J=17.2 \mathrm{~Hz}, 2 \mathrm{H}), 5.20(\mathrm{~d}, J=11.0 \mathrm{~Hz}, 1 \mathrm{H})$, $4.52(\mathrm{~d}, J=5.5 \mathrm{~Hz}, 2 \mathrm{H}), 4.19(\mathrm{~d}, J=6.3 \mathrm{~Hz}, 1 \mathrm{H}), 2.23$ (s, $3 \mathrm{H}), 2.11(\mathrm{~s}, 3 \mathrm{H}) ;{ }^{13} \mathbf{C}$ NMR $\left(75.0 \mathrm{MHz}, \mathrm{CDCl}_{3}\right): \delta 206.8$, $204.5,158.8,138.8,132.6,132.2,128.4,122.1,118.2,71.2$, 66.2, 53.9, 30.9, 30.2; IR (thin film, $\mathrm{cm}^{-1}$ ): 3321, 1726, $1694,1531,1414,1361,1252,1149,1010,826 ;[\alpha]^{23} \mathbf{D}=$ $+9.9^{\circ}\left(\mathrm{c}=1.0, \mathrm{CHCl}_{3}\right)$.

Enantiomer of 10c was obtained as a white crystal $90 \%$ yield and 95:5 er from a reaction catalyzed by cinchonidine at $-15{ }^{\circ} \mathrm{C}$ for $16 \mathrm{~h}$.

14d: (R)-Allyl 2-acetyl-3-oxo-1-(3-(trifluoromethyl)phenyl)butylcarbamate 
<smiles>C=CCOC(=O)NC(C(C)=O)C(C)=O</smiles>

Yield: 1.24 g, 87 \%; er: 96:4; HPLC analysis, $t_{\mathrm{r}}$ minor: $10.8 \mathrm{~min}, \mathrm{t}_{\mathrm{r}}$ major: $12.1 \mathrm{~min}$, (ChiralPak ${ }^{\circledR} \mathrm{AD}-\mathrm{H}$ Column, Hexane:IPA $=95: 5,1.0 \mathrm{~mL} / \mathrm{min}) ;{ }^{1} \mathbf{H}$ NMR $(400 \mathrm{MHz}$, $\left.\mathrm{CDCl}_{3}\right): \delta 7.42-7.56(4 \mathrm{H}), 6.25(\mathrm{~d}, J=8.6 \mathrm{~Hz}, 1 \mathrm{H}), 5.85(\mathrm{~m}$, $1 \mathrm{H}), 5.58(\mathrm{dd}, J=8.6,7.0 \mathrm{~Hz}, 1 \mathrm{H}), 5.26(\mathrm{~d}, J=17.2 \mathrm{~Hz}$, $2 \mathrm{H}), 5.19(\mathrm{~d}, J=11.0 \mathrm{~Hz}, 1 \mathrm{H}), 4.53(\mathrm{~d}, J=5.5 \mathrm{~Hz}, 2 \mathrm{H})$, $4.26(\mathrm{~d}, J=6.3 \mathrm{~Hz}, 1 \mathrm{H}), 2.25(\mathrm{~s}, 3 \mathrm{H}), 2.11(\mathrm{~s}, 3 \mathrm{H}) ;{ }^{13} \mathrm{C}$ NMR $\left(75.0 \mathrm{MHz}, \mathrm{CDCl}_{3}\right): \delta$ 204.3, 201.8, 155.6, 132.2, $130.0,129.3,125.1,124.7,123.1,118.0,70.7,66.1,53.6$, 30.7, 29.9; IR (thin film, $\mathrm{cm}^{-1}$ ): 3320, 1721, 1699, 1530, $1329,1260,1163,1126,1074,705 ;[\alpha]^{23}{ }_{D}=-10.9^{\circ}(\mathrm{c}=$ $\left.1.0, \mathrm{CHCl}_{3}\right)$.

Enantiomer of 10d was obtained as a white crystal $86 \%$ yield and $91 \%$ er from a reaction catalyzed by cinchonidine at $-15{ }^{\circ} \mathrm{C}$ for $16 \mathrm{~h}$.

\section{4e: (R)-Allyl 2-acetyl-1-(furan-2-yl)-3-oxobutylcarbamate}<smiles>C=CCOC(=O)NC(C(C)=O)C(C)=O</smiles>

Yield: $982 \mathrm{mg}, 88 \%$; er: 95.5:4.5; HPLC analysis, $t_{r}$ minor: $9.0 \mathrm{~min}, \mathrm{t}_{\mathrm{r}}$ major: $10.2 \mathrm{~min}$, $\left(\right.$ ChiralPak $^{\circledR} \mathrm{AD}-\mathrm{H}$ Column, Hexane:IPA = 95:5, $1.0 \mathrm{~mL} / \mathrm{min}) ;{ }^{1} \mathbf{H}$ NMR $(400$ $\left.\mathrm{MHz}, \mathrm{CDCl}_{3}\right): \delta 7.17(\mathrm{~d}, J=3.9 \mathrm{~Hz}, 1 \mathrm{H}), 6.86-6.93(2 \mathrm{H})$, $6.13(\mathrm{~d}, J=8.6 \mathrm{~Hz}, 1 \mathrm{H}), 5.85(\mathrm{~m}, 1 \mathrm{H}), 5.77(\mathrm{dd}, J=8.6,6.3$ $\mathrm{Hz}, 1 \mathrm{H}), 5.16-5.27(2 \mathrm{H}), 4.53(\mathrm{~m}, 2 \mathrm{H}), 4.33(\mathrm{~d}, J=6.3 \mathrm{~Hz}$, 1H), $2.22(\mathrm{~s}, 3 \mathrm{H}), 2.17(\mathrm{~s}, 3 \mathrm{H}) ;{ }^{13} \mathbf{C}$ NMR $(75.0 \mathrm{MHz}$, $\left.\mathrm{CDCl}_{3}\right): \delta 204.1,202.0,155.8,143.4,132.6,127.3,125.3$, $125.2,118.1,71.7,66.2,50.6,30.7,30.2 ;$ IR (thin film, $\mathrm{cm}^{-}$ $\left.{ }^{1}\right): 3349,1727,1702,1526,1268 ;[\boldsymbol{\alpha}]^{23}{ }^{D}=+24.0^{\circ}(\mathrm{c}=1.0$, $\left.\mathrm{CHCl}_{3}\right)$.

Enantiomer of 10e was obtained as a white crystal $87 \%$ yield and 95:5 er from a reaction catalyzed by cinchonidine at $-15{ }^{\circ} \mathrm{C}$ for $16 \mathrm{~h}$.

\section{4f: (R)-Allyl 2-acetyl-3-oxo-1-(thiophen-2-yl)butylcarbamate}<smiles>C=CCOC(=O)NC(C(C)=O)C(C)=O</smiles>

Yield: $1.01 \mathrm{~g}$, 86\%; er: 97:4; HPLC analysis, $\mathrm{t}_{\mathrm{r}}$ minor: 14.0 min, $\mathrm{t}_{\mathrm{r}}$ major: $14.8 \mathrm{~min}$, (ChiralPak ${ }^{\circledR} \mathrm{AD}-\mathrm{H}$ Column, Hexane:IPA = 95:5, $1.0 \mathrm{~mL} / \mathrm{min}) ;{ }^{1} \mathbf{H}$ NMR (400 MHz, $\left.\mathrm{CDCl}_{3}\right): \delta 7.27(\mathrm{~d}, J=1.6 \mathrm{~Hz}, 1 \mathrm{H}), 6.28(\mathrm{dd}, J=3.1,1.6$ $\mathrm{Hz}, 1 \mathrm{H}), 6.19$ (d, $J=3.1 \mathrm{~Hz}, 1 \mathrm{H}), 5.95$ (d, $J=9.4 \mathrm{~Hz}, 1 \mathrm{H})$, $5.85(\mathrm{~m}, 1 \mathrm{H}), 5.59(\mathrm{dd}, J=9.4,7.0 \mathrm{~Hz}, 1 \mathrm{H}), 5.16-5.30(2 \mathrm{H})$, $4.53(\mathrm{~m}, 2 \mathrm{H}), 4.35(\mathrm{~d}, J=7.0 \mathrm{~Hz}, 1 \mathrm{H}), 2.23(\mathrm{~s}, 3 \mathrm{H}), 2.17$ (s, $3 \mathrm{H}) ;{ }^{13} \mathrm{C}$ NMR $\left(75.0 \mathrm{MHz}, \mathrm{CDCl}_{3}\right): \delta$ 204.0, 202.2, 155.9, 152.2, 142.3, 132.6, 118.1, 111.0, 107.4, 68.7, 66.2, 48.8, 30.4, 30.0; IR (thin film, $\mathrm{cm}^{-1}$ ): 3329, 1725, 1697, 1526, 1361, 1264, 1149, 1048, 707; $[\boldsymbol{\alpha}]^{23}{ }_{\mathbf{D}}=+20.3^{\circ}(\mathrm{c}=1.0$, 


\section{$\left.\mathrm{CHCl}_{3}\right)$.}

Enantiomer of $\mathbf{1 0 f}$ was obtained as a white crystal $86 \%$ yield and $95.5: 4.5$ er from a reaction catalyzed by cinchonidine at $-15^{\circ} \mathrm{C}$ for $16 \mathrm{~h}$.

\section{8a: methyl (S)-1,1-di(methoxycarbonyl)-4-phenylbutan-2-ylcarbamate}<smiles>COC(=O)N[C@@H](CCc1ccccc1)C(C(=O)OC)C(=O)OC</smiles>

Yield: $70 \mathrm{mg}, 86 \%$. er: 96:4. HPLC analysis, $\mathrm{t}_{\mathrm{r}}$ major: 8.42 $\min , t_{r}$ minor: 8.95 min, [Chiralcel ${ }^{\circledR} \mathrm{OD}-\mathrm{H}$ column, Hexanes:IPA $90: 10,1.0 \mathrm{~mL} / \mathrm{min}] .{ }^{1} \mathbf{H}$ NMR (400 MHz, $\left.\mathrm{CDCl}_{3}\right): \delta 7.16-7.32(\mathrm{~m}, 5 \mathrm{H}), 5.64(\mathrm{~d}, J=10.2 \mathrm{~Hz}, 1 \mathrm{H})$, $4.35(\mathrm{~m}, 1 \mathrm{H}), 3.88(\mathrm{~d}, J=4.7 \mathrm{~Hz}, 1 \mathrm{H}), 3.76(\mathrm{~s}, 3 \mathrm{H}), 3.65(\mathrm{~s}$, $3 \mathrm{H}), 2.74(\mathrm{~m}, 1 \mathrm{H}), 2.61(\mathrm{~m}, 1 \mathrm{H}), 1.96(\mathrm{~m}, 1 \mathrm{H}), 1.74(\mathrm{~m}$, $1 \mathrm{H}) ;{ }^{13} \mathbf{C}$ NMR $\left(75.0 \mathrm{MHz}, \mathrm{CDCl}_{3}\right): \delta 168.5,167.4,156.3$, $141.1,132.8,128.8,128.6,126.4,117.9,56.7,54.2,53.2$, 52.8, 52.5, 35.9, 30.1. IR (thin film, $\mathbf{~ c m}^{-1}$ ): 3350, 1720, 1488, 1230, 1117; $[\alpha]^{23}{ }_{\mathrm{D}}=-37.2^{\circ}\left(\mathrm{c}=1.0, \mathrm{CHCl}_{3}\right)$. HRMS $\left(\mathrm{CI} / \mathrm{NH}_{3}\right) \mathrm{m} / \mathrm{z}$ : calc'd for $(\mathrm{M}+\mathrm{H})^{+} \mathrm{C}_{16} \mathrm{H}_{21} \mathrm{NO}_{6}$ : 324.1369; found: 323.1403 .

\section{8b: methyl (S)-1,1-di(methoxycarbonyl)undecan-2-ylcarbamate}

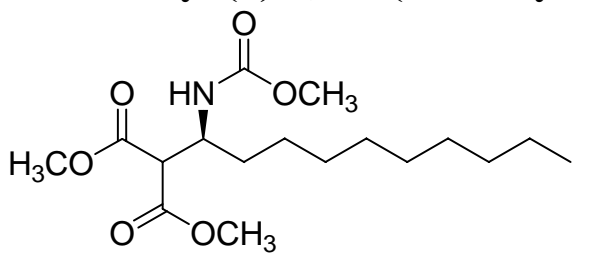

Yield: $61 \mathrm{mg}, 71 \%$. er: 95:5. HPLC analysis, $t_{\mathrm{r}}$ major: $14.8 \mathrm{~min}, \mathrm{t}_{\mathrm{r}}$ minor: $11.2 \mathrm{~min}$, [Chiralcel ${ }^{\circledR} \mathrm{OD}-\mathrm{H}$ column, Hexanes:IPA $95: 5,1.0 \mathrm{~mL} / \mathrm{min}] .{ }^{1} \mathbf{H}$ NMR (400 MHz, $\left.\mathrm{CDCl}_{3}\right): \delta 5.67(\mathrm{~d}, J=10.0 \mathrm{~Hz}, 1 \mathrm{H}), 4.38(\mathrm{~m}, 1 \mathrm{H}), 3.84(\mathrm{~d}$, $J=4.8 \mathrm{~Hz}, 1 \mathrm{H}), 3.78(\mathrm{~s}, 3 \mathrm{H}), 3.66(\mathrm{~s}, 3 \mathrm{H}), 1.74(\mathrm{~m}, 1 \mathrm{H})$, $1.29(\mathrm{~m}, 14 \mathrm{H}), 0.98(\mathrm{t}, \mathrm{J}=4.4 \mathrm{~Hz}, 3 \mathrm{H}) ;{ }^{13} \mathbf{C}$ NMR $(75.0$ $\left.\mathrm{MHz} \mathrm{CDCl}_{3}\right): \delta 168.7,167.3,156.5,56.7,54.2,53.2,52.8$, 52.5, 31.1, 29.7, 29.4, 24.5, 22.6, 14.1. IR (thin film, $\mathbf{c m}^{-}$ 1): $3350,2935,1730,1715,1496,1150 ;[\alpha]^{23}{ }_{D}=-5.0^{\circ}(\mathrm{c}=$ 1.0, $\left.\mathrm{CHCl}_{3}\right)$. HRMS $\left(\mathrm{CI} / \mathrm{NH}_{3}\right) \mathrm{m} / \mathrm{z}$ : calc'd for $(\mathrm{M}+\mathrm{H})^{+}$ $\mathrm{C}_{17} \mathrm{H}_{32} \mathrm{NO}_{6}$ : 346.2230; found: 346.2257 .

18c: methyl (R)-1,1-di(methoxycarbonyl)-3-(benzyloxy)propan-2-ylcarbamate<smiles>COC(=O)N[C@@H](COCc1ccccc1)C(C(=O)OC)C(=O)OC</smiles>

Yield: $69 \mathrm{mg}, 82 \%$. er: 95.5:4.5. HPLC analysis, $\mathrm{t}_{\mathrm{r}}$ major: $35.5 \mathrm{~min}, \mathrm{t}_{\mathrm{r}}$ minor: $41.7 \mathrm{~min}$, [Chiralcel ${ }^{\circledR} \mathrm{OD}-\mathrm{H}$ column, Hexanes:IPA 99 : 1, $1.0 \mathrm{~mL} / \mathrm{min}] .{ }^{1} \mathbf{H}$ NMR $(400 \mathrm{MHz}$, $\left.\mathrm{CDCl}_{3}\right): \delta 7.30(\mathrm{~m}, 5 \mathrm{H}), 5.75(\mathrm{~d}, J=8.0 \mathrm{~Hz}, 1 \mathrm{H}), 5.31(\mathrm{br}$, $1 \mathrm{H}), 4.55(\mathrm{~m}, 2 \mathrm{H}), 3.87(\mathrm{~d}, J=4.4 \mathrm{~Hz}, 1 \mathrm{H}), 3.71(\mathrm{~s}, 3 \mathrm{H})$, $3.68(\mathrm{~s}, 3 \mathrm{H}), 3.62(\mathrm{~s}, 3 \mathrm{H}), 3.60(\mathrm{~m}, 1 \mathrm{H}), 3.50(\mathrm{~m}, 1 \mathrm{H}) . ;{ }^{13} \mathrm{C}$ NMR (75.0 MHz, $\left.\mathrm{CDCl}_{3}\right): \delta 169.5,167.3,155.7,137.1$, $129.7,129.2,128.9,75.5,72.4,56.5,54.3,53.2,52.5,43.1$. IR (thin film, $\mathrm{cm}^{-1}$ ): 3350, 2935, 1730, 1715, 1496, 1150; $[\alpha]^{23}{ }_{\mathbf{D}}=-12.3^{\circ}\left(\mathrm{c}=1.0, \mathrm{CHCl}_{3}\right)$. HRMS $\left(\mathrm{CI} / \mathrm{NH}_{3}\right) \mathrm{m} / \mathrm{z}$ : calc'd for $(\mathrm{M}+\mathrm{H})^{+} \mathrm{C}_{16} \mathrm{H}_{22} \mathrm{NO}_{7}$ : 340.1396; found: 340.1439 . 
18e: methyl (R)-2,2-di(methoxycarbonyl)-1-phenylethylcarbamate<smiles>COC(=O)N[C@H](c1ccccc1)C(C(=O)OC)C(=O)OC</smiles>

Yield: $122 \mathrm{mg}$, 83\%; er: 95:5; HPLC Analysis, $\mathrm{t}_{\mathrm{r}}$ major: 9.2 min., $\mathrm{t}_{\mathrm{r}}$ minor: $12.7 \mathrm{~min}$., [Chiralcel ${ }^{\mathbb{B}} \mathrm{OD}-\mathrm{H}$ column, Hexanes:IPA $=95: 5,1.0 \mathrm{~mL} / \mathrm{min}] ;{ }^{1} \mathbf{H}$ NMR $(400 \mathrm{MHz}$, $\left.\mathrm{CDCl}_{3}\right): \delta 7.33-7.22(\mathrm{~m}, 5 \mathrm{H}), 6.39(\mathrm{~d}, \mathrm{~J}=8.4 \mathrm{~Hz}, 1 \mathrm{H})$, $5.51(\mathrm{dd}, \mathrm{J}=8.4,4.0 \mathrm{~Hz}, 1 \mathrm{H}), 3.92(\mathrm{~d}, \mathrm{~J}=4.0 \mathrm{~Hz}, 1 \mathrm{H})$, $3.73(\mathrm{~s}, 3 \mathrm{H}), 3.65(\mathrm{~s}, 3 \mathrm{H}), 3.63$ (s, 3H). ${ }^{13} \mathbf{C}$ NMR $(75.0$ $\left.\mathrm{MHz}, \mathrm{CDCl}_{3}\right): \delta 168.6,167.6,156.6,139.3,128.9,128.1$, 126.4, 56.7, 54.2, 53.2, 52.8, 52.5.; IR (thin film, $\mathrm{cm}^{-1}$ ): $3387,1720,1500,1220,1130 ;[\alpha]^{23}{ }_{D}=-15.3^{\circ}(\mathrm{c}=1.0$, $\left.\mathrm{CHCl}_{3}\right)$. HRMS (CI/NH3) m/z: calc'd for $(\mathrm{M}+\mathrm{H})^{+}$ $\mathrm{C}_{14} \mathrm{H}_{18} \mathrm{NO}_{6}$ : 396.1056; found: 296.1065 . HRMS $\left(\mathrm{CI} / \mathrm{NH}_{3}\right)$ $\mathrm{m} / \mathrm{z}$ : calc'd for $(\mathrm{M}+\mathrm{H})^{+} \mathrm{C}_{14} \mathrm{H}_{18} \mathrm{NO}_{6}$ : 296.1134; found: 296.1147.

18f: methyl (R)-2,2-di(methoxycarbonyl)-1-(4-bromophenyl)ethylcarbamate<smiles>COC(=O)N[C@@H](c1ccc(Br)cc1)C(C(=O)OC)C(=O)OC</smiles>

Yield: $89 \mathrm{mg}$, 95\%. er: 95:5. HPLC analysis, $\mathrm{t}_{\mathrm{r}}$ major: 11.0 min, $t_{\mathrm{r}}$ minor: $18.0 \mathrm{~min}$, [Chiralcel ${ }^{\circledR} \mathrm{OD}-\mathrm{H}$ column, Hexanes:IPA $98: 2,1.0 \mathrm{~mL} / \mathrm{min}] .{ }^{1} \mathbf{H}$ NMR $(400 \mathrm{MHz}$, $\left.\mathrm{CDCl}_{3}\right): \delta 7.44(\mathrm{~d}, J=8.4 \mathrm{~Hz}, 2 \mathrm{H}), 7.18(\mathrm{~d}, J=8.4 \mathrm{~Hz}, 2 \mathrm{H})$, $6.38(\mathrm{~d}, J=6.4 \mathrm{~Hz}, 1 \mathrm{H}), 5.44(\mathrm{br}, 1 \mathrm{H}), 3.87(\mathrm{~d}, J=3.2 \mathrm{~Hz}$, $1 \mathrm{H}), 3.75(\mathrm{~s}, 3 \mathrm{H}), 3.65$ (s, 6H). ${ }^{13} \mathbf{C}$ NMR $(75.0 \mathrm{MHz}$, $\left.\mathrm{CDCl}_{3}\right): \delta 168.5,167.3,156.5,138.5,132.1,128.2,122.1$, 56.4, 53.7, 53.0, 52.7. IR (thin film, $\mathrm{cm}^{-1}$ ): 3350, 1717, 1496, 1230, 1120; $[\alpha]^{23}{ }_{\mathbf{D}}=-25.1^{\circ}\left(\mathrm{c}=1.0, \mathrm{CHCl}_{3}\right)$. HRMS $\left(\mathrm{CI} / \mathrm{NH}_{3}\right) \mathrm{m} / \mathrm{z}$ : calc'd for $(\mathrm{M}+\mathrm{H})^{+} \mathrm{C}_{14} \mathrm{H}_{17} \mathrm{BrNO}_{6}$ : 374.0239; found: 374.0288 .

19a: (S)-1,1-di(methoxycarbonyl)-3-methylbutan-2-ylformate<smiles>COC(=O)C(C(=O)OC)[C@H](NC=O)C(C)C</smiles>

Yield: $55 \mathrm{mg}, 72 \%$. er: 98:2. HPLC analysis, $\mathrm{t}_{\mathrm{r}}$ major: 17.2 min, $\mathrm{t}_{\mathrm{r}}$ minor: 23.0 min, $[(R, R)$-Whelk-O 1 column, Hexanes:IPA $95: 5,1.0 \mathrm{~mL} / \mathrm{min}^{1} \mathbf{H}$ NMR $(400 \mathrm{MHz}$, $\left.\mathrm{CDCl}_{3}\right): \delta 8.18(\mathrm{~s}, 1 \mathrm{H}), 4.35(\mathrm{~m}, 1 \mathrm{H}), 3.72(\mathrm{~s}, 3 \mathrm{H}), 3.67(\mathrm{~s}$, $3 \mathrm{H}), 3.50(\mathrm{~d}, J=8.4 \mathrm{~Hz}, 1 \mathrm{H}), 1.65(\mathrm{~m}, 1 \mathrm{H}), 0.92$ (t, $J=8.0$ $\mathrm{Hz}, 6 \mathrm{H}) .{ }^{13} \mathbf{C}$ NMR $\left(75.0 \mathrm{MHz}, \mathrm{CDCl}_{3}\right): \delta 168.3,166.2$, 160.9, 53.3, 53.0, 52.4, 44.0, 31.8, 19.9, 19.8. IR (thin film, $\left.\mathrm{cm}^{-1}\right): 3355,2935,1720,1670,1444,1110 ;[\alpha]^{23}{ }_{\mathbf{D}}=-17.6^{\circ}$ $\left(\mathrm{c}=1.0, \mathrm{CHCl}_{3}\right)$. HRMS $\left(\mathrm{CI} / \mathrm{NH}_{3}\right) \mathrm{m} / \mathrm{z}$ : calc'd for $(\mathrm{M}+\mathrm{H})^{+}$ $\mathrm{C}_{10} \mathrm{H}_{17} \mathrm{NO}_{5}$ : 231.1107; found: 231.1155 .

19b: (S)-1,1-di(methoxycarbonyl)-3-(2,4,5-trifluorophenyl)propan-2-ylformate 
<smiles>CN[C@@H](Cc1cc(F)c(F)cc1F)C(C(=O)OC)C(=O)OC</smiles>

Yield: $61 \mathrm{mg}, 73 \%$. er: 95:5. HPLC analysis, $\mathrm{t}_{\mathrm{r}}$ major: 17.2 min, $\mathrm{t}_{\mathrm{r}}$ minor: $23.0 \mathrm{~min}$, [[(R,R)-Whelk-O 1, Hexanes:IPA $95: 5,1.0 \mathrm{~mL} / \mathrm{min} .{ }^{1} \mathbf{H} \mathbf{N M R}\left(400 \mathrm{MHz}, \mathrm{CDCl}_{3}\right): \delta 8.27$ $(\mathrm{s}, 1 \mathrm{H}), 7.31(\mathrm{~d}, \mathrm{~J}=9.6 \mathrm{~Hz}, 1 \mathrm{H}), 7.17(\mathrm{dd}, J=15.6,10.4 \mathrm{~Hz}$, $1 \mathrm{H}), 6.96(\mathrm{~m}, 1 \mathrm{H}), 6.02(\mathrm{dd}, J=9.2,4.4 \mathrm{~Hz}, 1 \mathrm{H}), 3.98(\mathrm{~d}, J$ $=4.4 \mathrm{~Hz}, 1 \mathrm{H}), 3.78(\mathrm{~s}, 3 \mathrm{H}), 3.74(\mathrm{~d}, J=9.2 \mathrm{~Hz}, 2 \mathrm{H}), 3.69$ $(\mathrm{s}, 3 \mathrm{H}),{ }^{13} \mathrm{C}$ NMR (75.0 MHz, $\left.\mathrm{CDCl}_{3}\right): \delta 168.3,167.2$, $160.7,157.8,148.4,145.3,123.8,106.6,53.3,53.0,52.4$, 43.6, 29.8. IR (thin film, $\mathrm{cm}^{-1}$ ): 3343, 2940, 1720, 1680, 1440, 1135; $[\alpha]^{23}{ }_{\mathbf{D}}=-22.8^{\circ}\left(\mathrm{c}=1.0, \mathrm{CHCl}_{3}\right)$. HRMS $\left(\mathrm{CI} / \mathrm{NH}_{3}\right) \mathrm{m} / \mathrm{z}$ : calc'd for $(\mathrm{M}+\mathrm{H})^{+} \mathrm{C}_{14} \mathrm{H}_{15} \mathrm{~F}_{3} \mathrm{NO}_{5}$ : 334.0902; found: 334.0945 .

19c: (R)-2,2-di(methoxycarbonyl)-1-phenylethylformate<smiles>COC(=O)C(C(=O)OC)[C@H](NC=O)c1ccccc1</smiles>

Yield: $63 \mathrm{mg}$, 95\%. er: 99:1. HPLC analysis, $\mathrm{t}_{\mathrm{r}}$ major: 14.2 min, $t_{r}$ minor: 17.1 min, [ChiralPak ${ }^{\circledR} \mathrm{AD}-\mathrm{H}$ column, Hexanes:IPA $90: 10,1.0 \mathrm{~mL} / \mathrm{min}] .{ }^{1} \mathbf{H}$ NMR $(400 \mathrm{MHz}$, $\left.\mathrm{CDCl}_{3}\right): \delta 8.29(\mathrm{~s}, 1 \mathrm{H}), 7.30(\mathrm{~m}, 6 \mathrm{H}), 5.90(\mathrm{dd}, J=9.6,4.0$ $\mathrm{Hz}, 1 \mathrm{H}), 3.97$ (d, $J=4.8 \mathrm{~Hz}, 1 \mathrm{H}), 3.77(\mathrm{~s}, 3 \mathrm{H}), 3.67$ (s, 3H). ${ }^{13}$ C NMR (75.0 MHz, $\left.\mathrm{CDCl}_{3}\right): \delta 168.9,167.6,160.6,138.5$, $129.0,128.2,126.4,56.1,53.3,53.0,50.5$. IR (thin film, $\left.\mathrm{cm}^{-1}\right)$ : 3287, 3033, 2955, 1742, 1677, 1502, 1438, 1260, $1160 ;[\alpha]^{23}{ }^{2}=-25.0^{\circ}\left(\mathrm{c}=1.0, \mathrm{CHCl}_{3}\right) . \operatorname{HRMS}\left(\mathrm{CI} / \mathrm{NH}_{3}\right)$ $\mathrm{m} / \mathrm{z}$ : calc'd for $(\mathrm{M})^{+} \mathrm{C}_{13} \mathrm{H}_{15} \mathrm{NO}_{5}$ : 265.0950; found: 265.0978 .

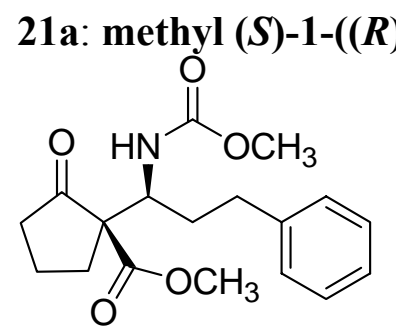

Yield: $158 \mathrm{mg}$ 91\% er: $97.5 \cdot 2.5$ HPLC analysis, $t_{\mathrm{r}}$ major: 9.4 min, $t_{r}$ minor: $10.3 \mathrm{~min}$, [ChiralPak ${ }^{\circledR} \mathrm{AD}-\mathrm{H}$ column, Hexanes:IPA 95 : 5, $1.0 \mathrm{~mL} / \mathrm{min}^{1} \mathbf{H}$ NMR (400 MHz, $\mathrm{CDCl}_{3}$, major diastereromer was reported): $\delta 7.23(\mathrm{~m}, 2 \mathrm{H})$, $7.15(\mathrm{~m}, 3 \mathrm{H}), 5.35(\mathrm{~d}, J=10.4 \mathrm{~Hz}, 1 \mathrm{H}), 3.97(\mathrm{td}, J=10.4$, $2.0 \mathrm{~Hz}, 1 \mathrm{H}), 3.67(\mathrm{~s}, 3 \mathrm{H}), 3.65(\mathrm{~s}, 3 \mathrm{H}), 2.77(\mathrm{~m}, 1 \mathrm{H}), 2.55$ $(\mathrm{m}, 1 \mathrm{H}), 2.45(\mathrm{~m}, 1 \mathrm{H}), 2.33(\mathrm{~m}, 1 \mathrm{H}), 1.90(\mathrm{~m}, 5 \mathrm{H}), 1.71(\mathrm{~m}$, 1H). ${ }^{13} \mathbf{C}$ NMR $\left(75.0 \mathrm{MHz}, \mathrm{CDCl}_{3}\right.$, major diastereromer was reported): $\delta 212.2,170.8,157.7,141.5,128.7,128.6$, $126.2,64.4,53.1,52.8,52.5,37.9,34.0,33.2,32.1,19.2$. IR (thin film, $\mathrm{cm}^{-1}$ ): 3332, 2945, 1730, 1537, 1450, 1358, 1241,$1042 ;[\alpha]^{23}{ }_{\mathbf{D}}=-25.6^{\circ}\left(\mathrm{c}=1.0, \mathrm{CHCl}_{3}\right)$.

21b: methyl (S)-1-((S)-1-acetyl-2-oxocyclopentyl)-3-phenylpropylcarbamate 
<smiles>COC(=O)N[C@@H](CCc1ccccc1)C1(C(C)=O)CCCC1=O</smiles>

Yield: $145 \mathrm{mg}, 95 \%$. er: 98.5:1.5. HPLC analysis, $\mathrm{t}_{\mathrm{r}}$ major: 13.7 min, $t_{r}$ minor: $11.3 \mathrm{~min}$, [ChiralPak ${ }^{\circledR} \mathrm{AD}-\mathrm{H}$ column, Hexanes:IPA $95: 5,1.0 \mathrm{~mL} / \mathrm{min}]{ }^{1} \mathbf{H}$ NMR (400 MHz, $\mathrm{CDCl}_{3}$, major diastereromer was reported): $\delta 7.27(\mathrm{~m}, 2 \mathrm{H})$, $7.17(\mathrm{~m}, 3 \mathrm{H}), 4.68(\mathrm{~d}, J=10.8 \mathrm{~Hz}, 1 \mathrm{H}), 4.43(\mathrm{td}, J=10.8$, $2.0 \mathrm{~Hz}, 1 \mathrm{H}), 3.65(\mathrm{~s}, 3 \mathrm{H}), 2.73(\mathrm{~m}, 1 \mathrm{H}), 2.63(\mathrm{~m}, 1 \mathrm{H}), 2.55$ $(\mathrm{m}, 1 \mathrm{H}), 2.28(\mathrm{~m}, 1 \mathrm{H}), 2.18(\mathrm{~s}, 3 \mathrm{H}), 1.83(\mathrm{~m}, 5 \mathrm{H}), 1.58(\mathrm{~m}$, 1H). ${ }^{13} \mathbf{C}$ NMR (75.0 MHz, $\left.\mathrm{CDCl}_{3}\right): \delta 214.3,203.9,157.2$, $141.3,128.7,128.7,126.4,73.4,53.8,52.6,38.9,34.8$, 33.2, 28.0, 26.4, 19.4. IR (thin film, $\mathrm{cm}^{-1}$ ): 3388, 3344, $2965,1715,1540,1228,1190,1159,1043 ;[\alpha]^{23}{ }_{\mathbf{D}}^{=}+77.4^{\circ}$ $\left(\mathrm{c}=1.0, \mathrm{CHCl}_{3}\right)$.

21c: methyl $(S)-1-((S)-3$-acetyl-tetrahydro-2-oxofuran-3-yl)-3-phenylpropylcarbamate<smiles>COC(=O)C1(C(C)=O)CCOC1=O</smiles>
Yield: $133 \mathrm{mg}, 84 \%$. er: 98.5:1.5. HPLC analysis, $\mathrm{t}_{\mathrm{r}}$ major: 13.7 min, $\mathrm{t}_{\mathrm{r}}$ minor: $11.3 \mathrm{~min}$, [ChiralPak ${ }^{\circledR} \mathrm{AD}-\mathrm{H}$ column, Hexanes:IPA $95: 5,1.0 \mathrm{~mL} / \mathrm{min}^{1} \mathbf{H}$ NMR (400 MHz, $\mathrm{CDCl}_{3}$, major diastereromer was reported): $\delta 7.24(\mathrm{~m}, 2 \mathrm{H})$, $7.15(\mathrm{~m}, 3 \mathrm{H}), 4.55(\mathrm{~m}, 2 \mathrm{H}), 4.24(\mathrm{td}, J=10.4,2.0 \mathrm{~Hz}, 1 \mathrm{H})$, $4.05(\mathrm{dd}, J=12.4,8.0 \mathrm{~Hz}, 1 \mathrm{H}), 3.67(\mathrm{~s}, 3 \mathrm{H}), 2.73(\mathrm{~m}, 1 \mathrm{H})$, $2.63(\mathrm{~m}, 1 \mathrm{H}), 2.55(\mathrm{~m}, 1 \mathrm{H}), 2.28(\mathrm{~m}, 1 \mathrm{H}), 1.83(\mathrm{~m}, 2 \mathrm{H}) .{ }^{13} \mathrm{C}$ NMR $\left(75.0 \mathrm{MHz}, \mathrm{CDCl}_{3}\right): \delta 200.9,175.3,155.5,138.7$, $129.3,128.7,127.9,67.3,66.2,56.7,52.6,34.5,30.4,25.5$, 24.2. IR (thin film, $\mathrm{cm}^{-1}$ ): 3327, 2956, 2922 1760, 1716, $1537,1361,1248,1170,1028 ;[\alpha]^{23}{ }_{\mathbf{D}}=-15.6^{\circ}(\mathrm{c}=1.0$, $\left.\mathrm{CHCl}_{3}\right)$.

23a: tert-butyl $(S, Z)$-2-(methoxycarbonyl)-3-(benzylamino)-1-phenylbut-2-enylcarbamate<smiles>CCOC(=O)N[C@H](C(C(=O)OC)=C(C)NCc1ccccc1)c1ccccc1</smiles>
Yield: $77 \mathrm{mg}, 84 \%$. er: 98:2. HPLC analysis, $\mathrm{t}_{\mathrm{r}}$ major: 15.8 $\min , \quad \mathrm{t}_{\mathrm{r}}$ minor: $14.6 \mathrm{~min},[(R, R)-$ Whelk-O 1 column, Hexanes:IPA 95 : 5, $1.0 \mathrm{~mL} / \mathrm{min}^{1} \mathbf{H}$ NMR $(400 \mathrm{MHz}$, $\left.\mathrm{CDCl}_{3}\right): \delta 9.82(\mathrm{br}, 1 \mathrm{H}), 7.30-7.09(\mathrm{~m}, 10 \mathrm{H}), 5.85(\mathrm{~d}, \mathrm{~J}=$ $9.6 \mathrm{~Hz}, 1 \mathrm{H}), 5.69(\mathrm{~d}, J=9.6 \mathrm{~Hz}, 1 \mathrm{H}), 4.43(\mathrm{~d}, J=5.2 \mathrm{~Hz}$, 2H), 3.39 (s, 3H), 2.13 (s, 3H), 1.37 (s, 9H). ${ }^{13}$ C NMR $\left(75.0 \mathrm{MHz}, \mathrm{CDCl}_{3}\right)$ : $\delta 170.6,161.8,156.1,144.1,138.7$, $129.1,128.9,128.8,128.1,127.7,127.1,126.3,126.2$, $125.8,95.0,79.4,51.8,51.5,50.4,47.6,28.7,28.5,15.8$. IR (thin film, $\mathrm{cm}^{-1}$ ): $3446,3395,2949,1717,1651,1593,1497$, 1455,$1239 ;[\alpha]^{23} \mathbf{D}=-18.2^{\circ}\left(\mathrm{c}=1.0, \mathrm{CHCl}_{3}\right)$.

23b: tert-butyl (R)-3-oxo-2-acyl1-phenylbutylcarbamate 
<smiles>CCCCOC(=O)NC(C(C)=O)C(C)=O</smiles>

Yield: $147 \mathrm{mg}$, 97\%. er: 97.5:2.5. HPLC analysis, $\mathrm{t}_{\mathrm{r}}$ major: $18.3 \mathrm{~min}, \quad \mathrm{t}_{\mathrm{r}}$ minor: $17.1 \mathrm{~min}, \quad\left[\right.$ ChiralPak ${ }^{\circledR} \mathrm{AD}-\mathrm{H}$, Hexanes:IPA $99: 1,1.0 \mathrm{~mL} / \mathrm{min}^{1} \mathbf{H}$ NMR $(400 \mathrm{MHz}$, $\left.\mathrm{CDCl}_{3}\right): \delta 7.26(\mathrm{~m}, 5 \mathrm{H}), 5.82(\mathrm{br}, 1 \mathrm{H}), 5.48(\mathrm{br}, 1 \mathrm{H}), 4.20$ $(\mathrm{d}, J=8.8 \mathrm{~Hz}, 1 \mathrm{H}), 2.15(\mathrm{~s}, 3 \mathrm{H}), 2.10(\mathrm{~s}, 3 \mathrm{H}), 1.37(\mathrm{~s}, 9 \mathrm{H})$. ${ }^{13}$ C NMR (75.0 MHz, $\left.\mathrm{CDCl}_{3}\right): \delta 204.9,202.8,155.4,140.1$, $129.0,127.4,126.6,125.9,80.3,71.9,53.9,28.6,28.4$.

23d: tert-butyl $(S, Z)-4-($ methoxycarbonyl)-5-(benzylamino)-1-phenylhex-4-en-3ylcarbamate<smiles>COC(=O)N[C@H](CCc1ccccc1)C(C(=O)OC)=C(C)NCc1ccccc1</smiles>

Yield: $84 \mathrm{mg}, 81 \%$. er: 95:5. HPLC analysis, $\mathrm{t}_{\mathrm{r}}$ major: 30.5 $\min , \quad \mathrm{t}_{\mathrm{r}}$ minor: $29.0 \mathrm{~min}, \quad[(R, R)-$ Whelk-O 1column, Hexanes:IPA $98: 2,1.0 \mathrm{~mL} / \mathrm{min}^{1} \mathbf{H}$ NMR (400 MHz, $\left.\mathrm{CDCl}_{3}\right): \delta 9.75(\mathrm{br}, 1 \mathrm{H}), 7.30-7.07(\mathrm{~m}, 10 \mathrm{H}), 5.36(\mathrm{~d}, J=$ $9.6 \mathrm{~Hz}, 1 \mathrm{H}), 4.75(\mathrm{~m}, 1 \mathrm{H}), 4.35(\mathrm{~d}, J=6.0 \mathrm{~Hz}, 2 \mathrm{H}), 3.28(\mathrm{~s}$, $3 \mathrm{H}), 2.63(\mathrm{~m}, 2 \mathrm{H}), 1.90(\mathrm{~s}, 3 \mathrm{H}), 1.87(\mathrm{~m}, 1 \mathrm{H}), 1.77(\mathrm{~m}, 1 \mathrm{H})$, 1.39 (s, 9H). ${ }^{13} \mathrm{C}$ NMR (75.0 MHz, $\left.\mathrm{CDCl}_{3}\right): \delta 169.4,161.5$, $154.5,146.6,135.7,127.4,127.3,127.2,126.4,125.8$, $125.0,93.7,54.3,47.7,46.3,36.4,30.3,27.3,14.4$. IR (thin film, $\left.\mathrm{cm}^{-1}\right): 3451,3390,2949,1719,1651,1593,1497$, $1454,1251,1192 ;[\alpha]^{23}{ }_{\mathbf{D}}=-36.1^{\mathrm{o}}\left(\mathrm{c}=1.0, \mathrm{CHCl}_{3}\right)$.

23e: tert-butyl (S)-5-oxo-4-acyl-1-phenylhexan-3-ylcarbamate<smiles>CC(=O)OC(=O)N[C@@H](CCc1ccccc1)C(C(C)=O)C(C)=O</smiles>

Yield: $150 \mathrm{mg}, 90 \%$ er: 95:5. HPLC analysis, $\mathrm{t}_{\mathrm{r}}$ major: 8.5 $\min , \quad \mathrm{t}_{\mathrm{r}}$ minor: $6.1 \mathrm{~min},[(R, R)-$ Whelk-O 1 column, Hexanes:IPA $85: 15,1.0 \mathrm{~mL} / \mathrm{min}^{1} \mathbf{H}$ NMR (400 MHz, $\left.\mathrm{CDCl}_{3}\right): \delta 7.30-7.15(\mathrm{~m}, 5 \mathrm{H}), 5.40(\mathrm{~d}, J=10 \mathrm{~Hz}, 1 \mathrm{H}), 4.32$ (br, 1H), 3.83 (d, $J=4.4 \mathrm{~Hz}, 1 \mathrm{H}), 2.81-2.55$ (m, 2H), 2.22 (s, 3H), 2.09(s, 3H), $1.42(\mathrm{~s}, 9 \mathrm{H}) .{ }^{13} \mathrm{C}$ NMR $(75.0 \mathrm{MHz}$, $\left.\mathrm{CDCl}_{3}\right): \delta 205.3,204.0,155.9,141.3,128.8,128.5,126.3$, 79.8, 70.0, 50.3, 36.0, 32.9, 30.9, 30.2, 28.5. IR (thin film, $\left.\mathrm{cm}^{-1}\right): 3446,3390,2951,1720,1650,1591,1499,1453$, $1253,1193,1090 ;[\alpha]^{23}{ }_{\mathbf{D}}=-19.3^{\circ}\left(\mathrm{c}=1.0, \mathrm{CHCl}_{3}\right)$. 


\section{Absolute and relative stereochemistry determination.}

\section{Absolute stereochemistry determination for $8 a-8 p, 9 a-91 .^{3}$}
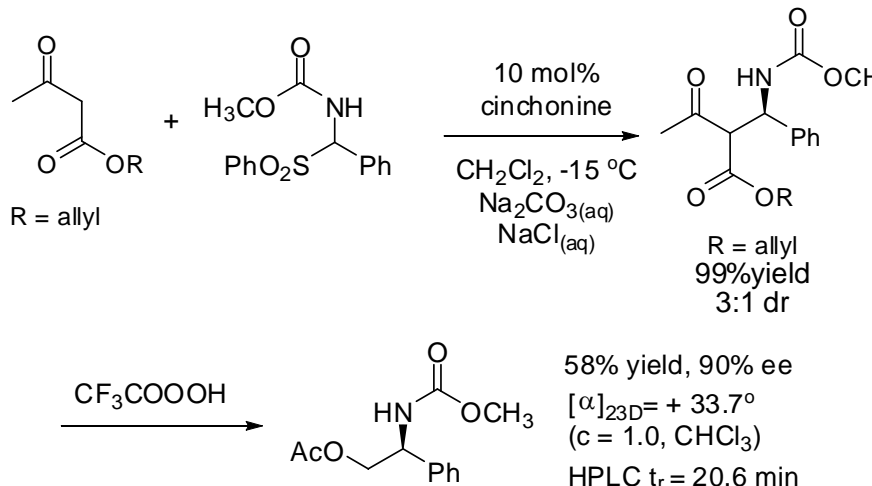

$58 \%$ yield, $90 \%$ ee

$[\alpha]_{23 \mathrm{D}}=+33.7^{\circ}$

(c= 1.0, $\mathrm{CHCl}_{3}$ )

$\mathrm{HPLC} \mathrm{t}_{\mathrm{r}}=20.6 \mathrm{~min}$

An oven dried $17 \times 110 \mathrm{~mm}$ round bottom reaction vessel was charged with allyl palladium chloride dimer (3.6 $\mathrm{mg}, 0.010 \mathrm{mmol})$ and $( \pm)$-2,3-O-Isopropylidene-2,3-dihydroxy-1,4bis(diphenylphosphino)butane $(( \pm) \mathrm{DIOP}) 2(10 \mathrm{mg}, 0.020 \mathrm{mmol})$. The Pd catalyst and ligand were dissolved in $\mathrm{CH}_{2} \mathrm{Cl}_{2}(0.5 \mathrm{~mL})$ and stirred at room temperature for 10 minutes. Methyl acetoacetate $(0.045 \mathrm{~mL}, 0.40 \mathrm{mmol})$ and Mannich reaction product $(0.20 \mathrm{mmol})$ were added successively. The reaction solution was stirred at room temperature for $3 \mathrm{~h}$ and subjected directly to flash chromatography over silica gel (elution with $30 \%-50 \%$ ethyl acetate in hexanes) to afford $(R)$-methyl 3-oxo-1-phenylbutylcarbamate

(R)-Acetic acid 2-methoxycarbonylamino-2-phenyl-ethyl ester. To a suspension of sodiumpercarbonate $(636 \mathrm{mg}, 4.0 \mathrm{mmol})$ in $\mathrm{CH}_{2} \mathrm{Cl}_{2}$ was added trifluoroacetic anhydride $(924 \mathrm{mg}$, $4.4 \mathrm{mmol}$ ) over $3 \mathrm{~min}$. The mixture was stirred at room temperature for $15 \mathrm{~min}$. The solution becameslightly cloudy and was transferred via syringe to the solution of $(R)$-(3-Oxo-1-phenylbutyl)-carbamic acid methyl ester $(86 \mathrm{mg}, 0.37 \mathrm{mmol})$ in $\mathrm{CH}_{2} \mathrm{Cl}_{2}(3 \mathrm{~mL})$. The reaction mixture wasstirred overnight until the starting material was not detectable by TLC. The reaction wasquenched by the addition of saturated $\mathrm{Na}_{2} \mathrm{~S}_{2} \mathrm{O}_{3}$ solution $(5 \mathrm{~mL})$. The organic layer was extracted with $\mathrm{CH}_{2} \mathrm{Cl}_{2}(3 \times 10 \mathrm{~mL})$. The combined organic layers were dried over $\mathrm{Na}_{2} \mathrm{SO}_{4}$, filtered, and concentrated under reduced pressure. The residue was further purified by flash chromatography on silica gel (elution with $30 \%$ ethyl acetate in hexanes) to give the product 50 mg (58\% yield). Yield: $50 \mathrm{mg}$, 58\%. ee: $86 \%$. HPLC analysis, tr minor: $15.1 \mathrm{~min}$, tr major: 20.6 min, $[(R, R)$-Whelk-O 1 column, Hexanes:IPA, 85:15, $1.5 \mathrm{~mL} / \mathrm{min}] .{ }^{1} \mathbf{H}$ NMR $\left(400 \mathrm{MHz}, \mathrm{CDCl}_{3}\right)$ : $\delta$ 7.33-7.22 (m, 5H), 5.43 (br, 1H), 4.97 (br, $1 \mathrm{H}), 4.30$ (dd, $J=11,6.0 \mathrm{~Hz}, 1 \mathrm{H}), 4.24-4.20$ (dd, $J$ $=11,6.0 \mathrm{~Hz}, 1 \mathrm{H}), 3.63(\mathrm{~s}, 3 \mathrm{H}), 2.01(\mathrm{~s}, 3 \mathrm{H}) .{ }^{13} \mathbf{C} \mathbf{~ N M R}\left(75.0 \mathrm{MHz}, \mathrm{CDCl}_{3}\right): \delta 170.9,156.2$, 138.5, 128.7, 127.9, 126.4, 66.3, 54.3, 52.2, 20.7. IR (thin film, cm-1): 3328, 3030, 2952, 1712, 1700, 1538, 1455, 1360, 1258, 1057, 700. HRMS: calc'd for $(\mathrm{M}+\mathrm{H})+\mathrm{C}_{12} \mathrm{H}_{15} \mathrm{NO}_{4}$ : 238.1035; found: $238.1076 .[\alpha]_{23 \mathrm{D}}=+37.5\left(\mathrm{c}=1.1, \mathrm{CHCl}_{3}\right)$. 


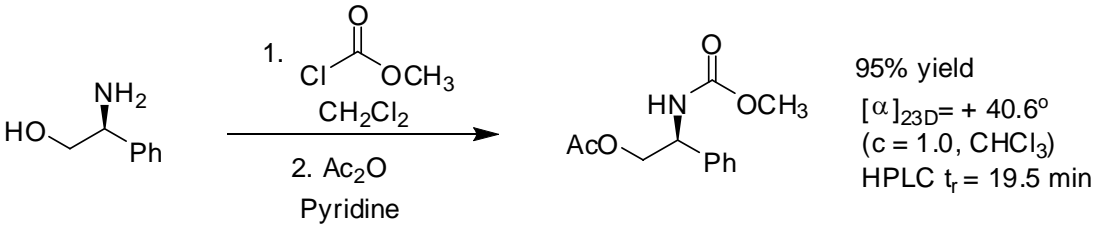

$(R)$-Acetic acid 2-methoxycarbonylamino-2-phenyl-ethyl ester. To a solution of $(R)$-2-amino2-phenylethanol (137 mg, $1.0 \mathrm{mmol})$ in $2 \mathrm{~mL} \mathrm{CH}_{2} \mathrm{Cl}_{2}$ was added a solution of methyl chloroformate $(0.070 \mathrm{~mL}, 0.80 \mathrm{mmol})$ in $1 \mathrm{~mL} \mathrm{CH} 2 \mathrm{Cl}_{2}$. The reaction was stirred at room temperature for $2 \mathrm{~h}$ and was quenched by the addition of saturated $\mathrm{NaHCO}_{3}$ aqueous solution ( 5 $\mathrm{mL})$. The organic layer was extracted with $\mathrm{CH}_{2} \mathrm{Cl}_{2}(3 \times 10 \mathrm{~mL})$. The combined organic layers were dried over $\mathrm{Na}_{2} \mathrm{SO}_{4}$, filtered, and concentrated under reduced pressure. The residue was purified by flash chromatography over silica gel (elution with $40 \%$ ethyl acetate in hexanes) to give the (2-Hydroxy-1-phenyl-ethyl)-carbamic acid methyl ester (135 $\mathrm{mg}, 70 \%$ yield). The product $(60 \mathrm{mg}, 0.30 \mathrm{mmol})$ was treated with acetic anhydride $(0.036 \mathrm{~mL}, 0.36 \mathrm{mmol})$ and stirred in $2 \mathrm{~mL}$ pyridine until the alcohol was not detectable by TLC. The reaction solution was subjected directly to flash chromatography on silica gel (elution with $30 \%$ ethyl acetate in hexanes) to give the product (52 mg, 71\% yield). HPLC analysis, tr: $19.5 \mathrm{~min},[(R, R)-$ Whelk-O 1 column, Hexanes:IPA, 85:15, $1.5 \mathrm{~mL} / \mathrm{min}] .{ }^{1} \mathbf{H}$ NMR $\left(400 \mathrm{MHz}, \mathrm{CDCl}_{3}\right): \delta 7.33-7.22(\mathrm{~m}, 5 \mathrm{H})$, 5.43 (br, 1H), 4.97 (br, 1H), 4.30 (dd, $J=11,6.0 \mathrm{~Hz}, 1 \mathrm{H}), 4.24-4.20$ (dd, $J=11,6.0 \mathrm{~Hz}, 1 \mathrm{H}$ ), $3.63(\mathrm{~s}, 3 \mathrm{H}), 2.01(\mathrm{~s}, 3 \mathrm{H}) .{ }^{13} \mathbf{C}$ NMR $\left(75.0 \mathrm{MHz}, \mathrm{CDCl}_{3}\right): \delta 170.9,156.2,138.5,128.7,127.9$, 126.4, 66.3, 54.3, 52.2, 20.7. IR (thin film, cm-1): 3328, 3030,2952, 1712, 1700, 1538, 1455, 1360, 1258, 1057, 700. HRMS: calc'd for $(\mathrm{M}+\mathrm{H})+\mathrm{C}_{12} \mathrm{H}_{15} \mathrm{NO}_{4}$ : 238.1035; found: 238.1076. $[\alpha]_{23 \mathrm{D}}=+40.6\left(\mathrm{c}=1.0, \mathrm{CHCl}_{3}\right)$.

\section{$Z / E$ configuration determination for $8 \mathbf{a}-8 \mathbf{p}, 9 \mathbf{a}-91$}

The $(Z)$ configuration of enamines 8a-8p, 9a-9l were determined by ${ }^{1} \mathrm{H}-\mathrm{NMR}$ analogy of reported compounds. ${ }^{4}$

The absolute stereochemistry of 14a-14f, $\mathbf{2 3 b}$ and $\mathbf{2 3 d}$ was determined via comparison of HPLC retention times and optical rotations with reported compounds. ${ }^{5}$

The absolute stereochemistry of 18a-h, 19a-c and 23c was determined via comparison of HPLC retention times and optical rotations with reported compounds. ${ }^{6}$

Determination of absolute and relative stereochemistry of $\mathbf{2 1 \mathbf { b } ^ { 7 }}$
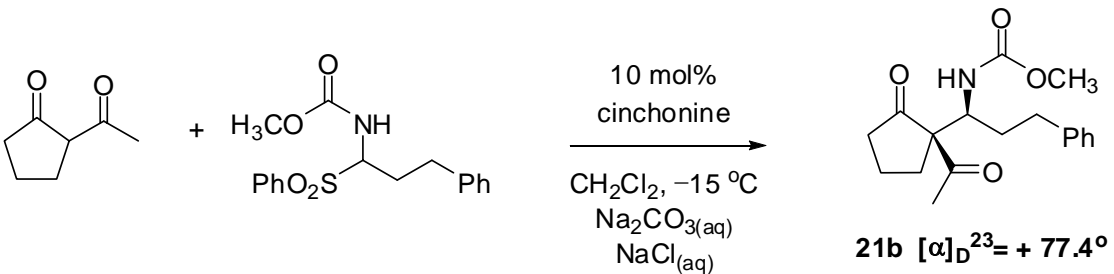

$t_{\text {major }}=13.8 \mathrm{~min}$

HPLC analysis: ChiralPak AD-H column

Hexanes:IPA $95: 5,1.0 \mathrm{~mL} / \mathrm{min}$

To a $25 \mathrm{~mL}$ one-neck round-bottom flask equipped with stir bar was charged with (+)cinchonine $(16.0 \mathrm{mg}, 0.05 \mathrm{mmol}), \alpha$-amido sulfone $(0.50 \mathrm{mmol})$ and $\mathrm{CH}_{2} \mathrm{Cl}_{2}(5.0 \mathrm{~mL})$. The 
reaction solution was cooled to $-15{ }^{\circ} \mathrm{C}$. 2-Acetylcyclopentanone $(1.50 \mathrm{mmol})$ and aqueous $\mathrm{Na}_{2} \mathrm{CO}_{3} / \mathrm{NaCl}$ solution $\left(5.0 \mathrm{~mL}, 5 \mathrm{wt} \% \mathrm{Na} \mathrm{Na}_{2} \mathrm{CO}_{3}\right.$ in deionized water saturated with $\mathrm{NaCl}$ ) was slowly added into reaction mixture successfully. The resulting biphasic solution was vigorously stirred at $-15{ }^{\circ} \mathrm{C}$ for 48 hours and diluted with $\mathrm{CH}_{2} \mathrm{Cl}_{2}(10 \mathrm{~mL})$ and $\mathrm{H}_{2} \mathrm{O}(10 \mathrm{~mL})$. The organic layer was quickly separated and the aqueous phase was extracted with $\mathrm{CH}_{2} \mathrm{Cl}_{2}(2 \times 10 \mathrm{~mL})$. The combined organic layers were dried over sodium sulfate, filtered and concentrated under reduced pressure. The resulting residue was purified by flash chromatography over silica gel (elution with $15 \%-40 \%$ ethyl acetate in hexanes) to afford the enantioenriched Mannich product.

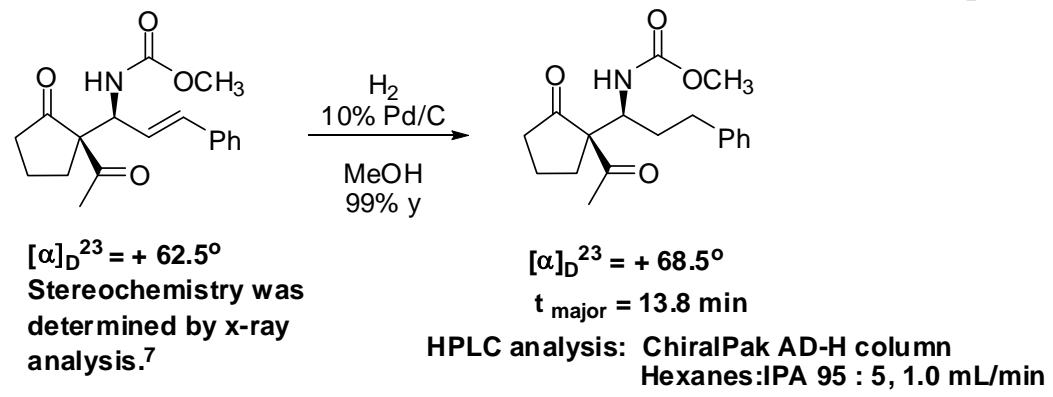

$\mathrm{H}$-cube system was charged with $\mathrm{Pd} / \mathrm{C}$ CatCart column and was heated to $50{ }^{\circ} \mathrm{C}$. The hydrogen pressure was set to 50 bars. [(E)- $(S)-1-((S)$-1-Acetyl-2-oxo-cyclopentyl)-3-phenyl-allyl]carbamic acid methyl ester $(50 \mathrm{mg}, 0.158 \mathrm{mmol})$ was dissolved in methanol $(5.0 \mathrm{~mL})$, and the solution was pumped through the H-Cube system with a flow rate of $1 \mathrm{~mL} / \mathrm{min}$. The collected solution was concentrated under reduced pressure to give pure reduced product $50 \mathrm{mg}(99 \%$ yield). ${ }^{1} \mathbf{H}$ NMR was identical with $\mathbf{2 1 b}$.

Relative Stereochemistry of [(E)- $(S)-1-((S)$-1-Acetyl-2-oxo-cyclopentyl)-3-phenyl-allyl]carbamic acid methyl ester: Crystals suitable for x-ray analysis were obtained by slow evaporation from methylene chloride and hexanes. ${ }^{7}$

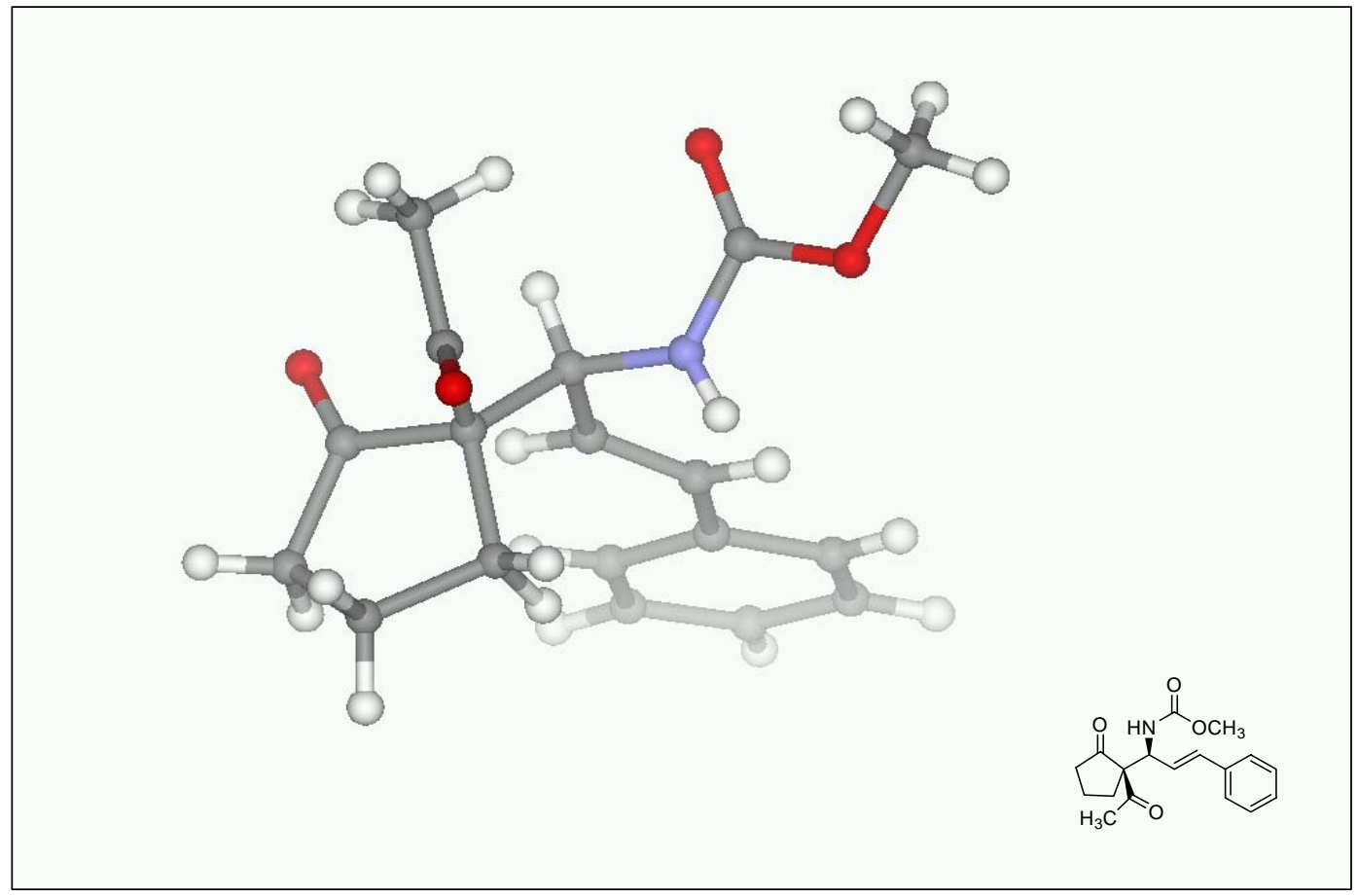


Table 1. Crystal data and structure refinement. ${ }^{7}$

Identification code

Empirical formula

Formula weight

Temperature

Wavelength

Crystal system

Space group

Unit cell dimensions

Volume

Z

Density (calculated)

Absorption coefficient

$\mathrm{F}(000)$

Crystal size

Theta range for data collection

Index ranges

Reflections collected

Independent reflections

Completeness to theta $=28.28^{\circ}$

Absorption correction

Max. and min. transmission

Refinement method

Data / restraints / parameters

Goodness-of-fit on $\mathrm{F}^{2}$

Final $R$ indices [I $>2 \operatorname{sigma}(\mathrm{I})]$

$\mathrm{R}$ indices (all data)

Absolute structure parameter

Largest diff. peak and hole 20c

$\mathrm{C} 18 \mathrm{H} 21 \mathrm{~N} \mathrm{O} 4$

315.36

173(2) K

$0.71073 \AA$

Orthorhombic

P2(1)2(1)2(1)

$\mathrm{a}=6.3666(4) \AA \quad \alpha=90^{\circ}$.

$\mathrm{b}=15.3404(9) \AA \quad \beta=90^{\circ}$.

$\mathrm{c}=17.1852(13) \AA \quad \gamma=90^{\circ}$.

1678.41(19) $\AA^{3}$

4

$1.248 \mathrm{Mg} / \mathrm{m}^{3}$

$0.088 \mathrm{~mm}^{-1}$

672

$0.40 \times 0.10 \times 0.05 \mathrm{~mm}^{3}$

1.78 to $28.28^{\circ}$.

$-8<=\mathrm{h}<=8,-20<=\mathrm{k}<=20,-22<=\mathrm{l}<=15$

12635

$4169[\mathrm{R}(\mathrm{int})=0.0264]$

$99.9 \%$

Semi-empirical from equivalents

0.9956 and 0.9656

Full-matrix least-squares on $\mathrm{F}^{2}$

4169 / 0 / 292

1.018

$\mathrm{R} 1=0.0331, \mathrm{wR} 2=0.0785$

$\mathrm{R} 1=0.0455, \mathrm{wR} 2=0.0835$

$1.0(8)$

0.177 and -0.157 e. $\AA^{-3}$ 
Table 2. Atomic coordinates $\left(\mathrm{x} 10^{4}\right)$ and equivalent isotropic displacement parameters $\left(\AA^{2} \mathrm{x}\right.$ $\left.10^{3}\right)$. $U(\mathrm{eq})$ is defined as one third of the trace of the orthogonalized $\mathrm{Uij}^{\mathrm{ij}}$ tensor. ${ }^{7}$

\begin{tabular}{|c|c|c|c|c|}
\hline $\mathrm{O}(1)$ & $8617(1)$ & $2714(1)$ & 1781(1) & $30(1)$ \\
\hline $\mathrm{O}(2)$ & $3778(2)$ & 2189(1) & $162(1)$ & $36(1)$ \\
\hline $\mathrm{O}(3)$ & $3372(2)$ & $309(1)$ & $2247(1)$ & $37(1)$ \\
\hline $\mathrm{O}(4)$ & $-43(2)$ & $692(1)$ & 2101(1) & $33(1)$ \\
\hline $\mathrm{N}(1)$ & $2353(2)$ & $1726(1)$ & $2097(1)$ & $23(1)$ \\
\hline$C(1)$ & $4414(2)$ & $2128(1)$ & $2169(1)$ & $21(1)$ \\
\hline$C(2)$ & $4915(2)$ & $2659(1)$ & $1422(1)$ & $19(1)$ \\
\hline$C(3)$ & $7121(2)$ & $3072(1)$ & 1491(1) & $20(1)$ \\
\hline$C(4)$ & $7048(2)$ & $3995(1)$ & $1202(1)$ & $26(1)$ \\
\hline$C(5)$ & $4926(2)$ & $4074(1)$ & $804(1)$ & $29(1)$ \\
\hline$C(6)$ & $3517(2)$ & $3452(1)$ & $1268(1)$ & $24(1)$ \\
\hline$C(7)$ & $4876(2)$ & $2031(1)$ & $715(1)$ & $23(1)$ \\
\hline$C(8)$ & $6182(2)$ & $1225(1)$ & $761(1)$ & $29(1)$ \\
\hline$C(9)$ & $4634(2)$ & $2672(1)$ & 2894(1) & $24(1)$ \\
\hline$C(10)$ & $3224(2)$ & $2788(1)$ & $3450(1)$ & $24(1)$ \\
\hline $\mathrm{C}(11)$ & $3587(2)$ & $3264(1)$ & $4184(1)$ & $25(1)$ \\
\hline$C(12)$ & 2092(3) & $3213(1)$ & $4775(1)$ & $32(1)$ \\
\hline$C(13)$ & $2455(3)$ & $3598(1)$ & $5494(1)$ & $41(1)$ \\
\hline$C(14)$ & $4290(3)$ & $4041(1)$ & $5632(1)$ & $42(1)$ \\
\hline$C(15)$ & $5775(3)$ & $4119(1)$ & $5048(1)$ & $40(1)$ \\
\hline$C(16)$ & $5411(2)$ & $3737(1)$ & $4327(1)$ & $34(1)$ \\
\hline$C(17)$ & 2039(2) & $866(1)$ & $2157(1)$ & $24(1)$ \\
\hline$C(18)$ & $-613(3)$ & $-212(1)$ & 2039(1) & $46(1)$ \\
\hline
\end{tabular}


General procedure for synthesis and purification of dihydropyrimidones library:

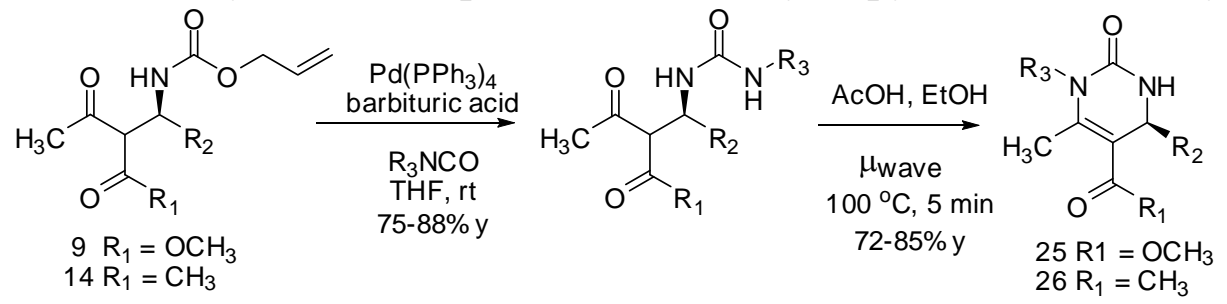

To an oven dried $5 \mathrm{~mL}$ vial was charged with $\mathrm{Pd}\left(\mathrm{PPh}_{3}\right)_{4}(8 \mathrm{mg}, 0.01 \mathrm{mmol})$ and $1 \mathrm{~mL}$ THF, isocyanate $(0.4 \mathrm{mmol})$ and 1,3-dimethylbarbituric acid $(31 \mathrm{mg}, 0.2 \mathrm{mmol})$ successfully. A solution of the Mannich adduct $(0.2 \mathrm{mmol})$ in $0.5 \mathrm{~mL}$ THF was added dropwise. The solution was stirred at room temperature until disappearance of the starring material by TLC. The reaction mixture was then concentrated under reduced pressure and purified by Combi-flash column chromatograpy on silica gel to afford urea intermediate. In some cases methyl nitroacetate was used instead of 1,2-dimethylbarbituric acid as allyl acceptor for easier purification.

The urea intermediate was then dissolved in $0.2 \mathrm{~mL}$ acetic acid/ethanol (3:1) and the solution was degassed and flushed with Argon. The solution was subjected to microwave irradiation $(150 \mathrm{~W})$ at $100{ }^{\circ} \mathrm{C}$ for $5 \mathrm{~min}$. The solvent was removed under reduced pressure and residue was purified by Combi-flash column chromatograpy on silica gel to afford the crude dihydropyrimidone. To scavenge the Pd residue, the dihydropyrimidone was dissolved in $5 \mathrm{~mL}$ dichloromethane and then PS-TMT resin (20mg) was added. The mixture was shaken for $2 \mathrm{~h}$ and then filtered. The resin was washed with $\mathrm{CH}_{2} \mathrm{Cl}_{2}(2 \times 5 \mathrm{~mL})$, ethylacetate $(2 \times 5 \mathrm{~mL})$. The combined organic layers were concentrated under reduced pressure, dissolved in $800 \mu \mathrm{L}$ DMSO and purified by preparative reverse phase HPLC (fraction triggering was based on calculated exact mass). The pure fractions were combined and the solvent was removed under reduced pressure to afford the pure dihydropyrimidone product.

\section{Characterization of selected compounds was listed as below:}

$(S)$-25Ab: $(S)$-methyl 1-ethyl-6-methyl-2-oxo-4-phenyl-1,2,3,4-tetrahydropyrimidine-5carboxylate<smiles>CCN1C(=O)NC(c2ccccc2)C(C(=O)OC)=C1C</smiles>

er $=95.5: 4.5$; HPLC analysis, $\mathrm{t}_{\mathrm{r}}$ major $7.0 \mathrm{~min}, \mathrm{t}_{\mathrm{r}}$ minor: $8.2 \mathrm{~min}$, (ChiralPak OD Column, Hexane:IPA = 90:10, $1.0 \mathrm{~mL} / \mathrm{min}) ;{ }^{1} \mathbf{H}$ NMR $\left(400 \mathrm{MHz}, \mathrm{CDCl}_{3}\right): \delta 7.20-7.34(5 \mathrm{H}), 5.89(\mathrm{~d}, J=3.1 \mathrm{~Hz}, 1 \mathrm{H}), 5.35(\mathrm{~d}$, $J=3.1 \mathrm{~Hz}, 1 \mathrm{H}), 3.89(\mathrm{~m}, 1 \mathrm{H}), 3.73(\mathrm{~m}, .1 \mathrm{H}), 3.63(\mathrm{~s}, 3 \mathrm{H}), 2.53(\mathrm{~s}$, $3 \mathrm{H}), 1.20(\mathrm{t}, J=7.0 \mathrm{~Hz}, 3 \mathrm{H}) ;{ }^{13} \mathbf{C}$ NMR $\left(100 \mathrm{MHz}, \mathrm{CDCl}_{3}\right): \delta 166.6$, $153.5,148.8,143.3,128.7,127.8,126.1,104.1,53.9,51.3,37.8,16.0$, 14.9; IR (thin film, $\mathrm{cm}^{-1}$ ): $3233,2929,1685,1621,1390,1229,1189$, $1150,1178,761,699 ;[\boldsymbol{\alpha}]^{23}{ }_{\mathrm{D}}=-45.2^{\circ}\left(\mathrm{c}=2.0, \mathrm{CHCl}_{3}\right)$; HRMS (CI$\mathrm{NH}_{3}$ ) calculated for $\mathrm{C}_{15} \mathrm{H}_{19} \mathrm{~N}_{2} \mathrm{O}_{3}(\mathrm{M}+\mathrm{H}): 275.1390$, found: 275.1381 .

(R)-25Ab: $(R)$-methyl 1-ethyl-6-methyl-2-oxo-4-phenyl-1,2,3,4-tetrahydropyrimidine-5carboxylate 
<smiles>CCN1C(=O)N[C@H](c2ccccc2)C(C(=O)OC)=C1C</smiles>

er $=96: 4$; HPLC analysis, $t_{r}$ minor: 6.9 min, $t_{r}$ major: $8.0 \mathrm{~min}$, (ChiralPak OD Column, Hexane:IPA = 90:10, $1.0 \mathrm{~mL} / \mathrm{min}$ ); $[\boldsymbol{\alpha}]^{\mathbf{2 3}}{ }_{\mathbf{D}}=+$ $46.0^{\circ}\left(\mathrm{c}=1.0, \mathrm{CHCl}_{3}\right) ; \mathrm{MS}(\mathrm{ESI}+)$ calculated for $\mathrm{C} 15 \mathrm{H} 19 \mathrm{~N} 2 \mathrm{O} 3$ $(\mathrm{M}+\mathrm{H}): 275.1$, found: 274.9 .

(S)-26Ab: (S)-5-acetyl-1-ethyl-6-methyl-4-phenyl-3,4-dihydropyrimidin-2(1H)-one<smiles>CCN1C(=O)NC(c2ccccc2)C(C(C)=O)=C1C</smiles>

er $=95.5: 4.5 ;$ HPLC analysis, $\mathrm{t}_{\mathrm{r}}$ major: $10.2 \mathrm{~min}, \mathrm{t}_{\mathrm{r}}$ major: $10.9 \mathrm{~min}$, (ChiralPak OD Column, Hexane:IPA = 90:10, $1.0 \mathrm{~mL} / \mathrm{min}) ;{ }^{1} \mathbf{H}$ NMR $\left(400 \mathrm{MHz}, \mathrm{CDCl}_{3}\right): \delta 7.19-7.32(5 \mathrm{H}), 6.51(\mathrm{~d}, J=2.4 \mathrm{~Hz}, 1 \mathrm{H}), 5.28(\mathrm{~d}$, $J=2.4 \mathrm{~Hz}, 1 \mathrm{H}), 3.86(\mathrm{~m}, 1 \mathrm{H}), 3.71(\mathrm{~m}, .1 \mathrm{H}), 2.48(\mathrm{~s}, 3 \mathrm{H}), 2.10(\mathrm{~s}$, $3 \mathrm{H}), 1.17(\mathrm{t}, J=7.0 \mathrm{~Hz}, 3 \mathrm{H}) ;{ }^{13} \mathbf{C}$ NMR (100 MHz, $\left.\mathrm{CDCl}_{3}\right): \delta 196.7$, $153.2,147.7,142.3,128.9,128.0,126.3,112.9,54.5,37.6,30.3,16.5$, 14.9; IR (thin film, $\mathrm{cm}^{-1}$ ): 3280, 2925, 1690, 1660, 1379, 1230, 701; $[\boldsymbol{\alpha}]^{\mathbf{2 3}}{ }_{\mathbf{D}}=-51.3^{\mathrm{o}}\left(\mathrm{c}=1.2, \mathrm{CHCl}_{3}\right)$; HRMS $\left(\mathrm{CI}-\mathrm{NH}_{3}\right)$ calculated for $\mathrm{C}_{15} \mathrm{H}_{19} \mathrm{~N}_{2} \mathrm{O}_{2}(\mathrm{M}+\mathrm{H}): 259.1441$, found: 259.1426 .

(S)-26Ba: (S)-5-acetyl-1-benzyl-4-(4-bromophenyl)-6-methyl-3,4-dihydropyrimidin-2(1H)one

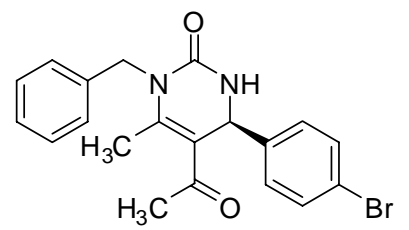

er $=95: 5$; HPLC analysis, $\mathrm{t}_{\mathrm{r}}$ major: $12.3 \mathrm{~min}, \mathrm{t}_{\mathrm{r}}$ minor: $21.1 \mathrm{~min}$, (ChiralPak OD Column, Hexane:IPA = 90:10, $1.0 \mathrm{~mL} / \mathrm{min}) ;{ }^{1} \mathbf{H}$ NMR $\left(400 \mathrm{MHz}, \mathrm{CDCl}_{3}\right): \delta 6.99-7.46(10 \mathrm{H}), 5.31(\mathrm{~d}, J=3.1 \mathrm{~Hz}, 1 \mathrm{H}), 5.21$ $(\mathrm{d}, J=16.4 \mathrm{~Hz}, 1 \mathrm{H}), 4.75(\mathrm{~d}, J=16.4 \mathrm{~Hz}, 1 \mathrm{H}), 2.36(\mathrm{~s}, 3 \mathrm{H}), 2.10$ (s, $3 \mathrm{H}) ;{ }^{13} \mathrm{C}$ NMR $\left(100 \mathrm{MHz}, \mathrm{CDCl}_{3}\right): \delta 196.4,154.1,148.3,141.1$, $137.5,131.9,128.7,128.2,127.3,126.3,121.9,113.5,53.3,45.7,30.3$, 17.1; IR (thin film, $\mathrm{cm}^{-1}$ ): 3236, 2923, 1692, 1665, 1210, 701; $[\boldsymbol{\alpha}]^{\mathbf{2 3}} \mathbf{\mathbf { D }}=$ $-26.2^{\circ}\left(\mathrm{c}=1.2, \mathrm{CHCl}_{3}\right)$; HRMS $\left(\mathrm{CI}-\mathrm{NH}_{3}\right)$ calculated for $\mathrm{C}_{20} \mathrm{H}_{20} \mathrm{BrN}_{2} \mathrm{O}_{2}$ $(\mathrm{M}+\mathrm{H}): 399.0703$, found: 399.0689 .

$(R)-26 B a:(R)-5$-acetyl-1-benzyl-4-(4-bromophenyl)-6-methyl-3,4-dihydropyrimidin-2(1H)one<smiles>CC(=O)C1=C(C)N(Cc2ccccc2)C(=O)N[C@H]1c1ccc(Br)cc1</smiles>

er $=95.5: 4.5$; HPLC analysis, $\mathrm{t}_{\mathrm{r}}$ minor: $12.5 \mathrm{~min}$, $\mathrm{t}_{\mathrm{r}}$ major: $21.0 \mathrm{~min}$, (ChiralPak OD Column, Hexane:IPA = 90:10, $1.0 \mathrm{~mL} / \mathrm{min}$ ); $[\boldsymbol{\alpha}]^{\mathbf{2 3}}{ }_{\mathbf{D}}=+$ $27.1^{\circ}\left(\mathrm{c}=1.1, \mathrm{CHCl}_{3}\right)$; MS $(\mathrm{ESI}+)$ calculated for $\mathrm{C}_{20} \mathrm{H}_{20} \mathrm{BrN}_{2} \mathrm{O}_{2}$ $(\mathrm{M}+\mathrm{H}): 399.1$, found: 398.8 .

(S)-25Fc: (S)-methyl 1-(2-ethoxy-2-oxoethyl)-6-methyl-2-oxo-4-phenethyl-1,2,3,4tetrahydropyrimidine-5-carboxylate 


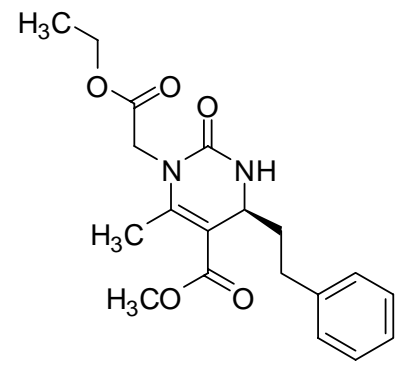

er $=95: 5$; HPLC analysis, $t_{r}$ major: $12.4 \mathrm{~min}, \mathrm{t}_{\mathrm{r}}$ minor: $17.8 \mathrm{~min}$, (ChiralPak OD Column, Hexane:IPA = 90:10, $1.0 \mathrm{~mL} / \mathrm{min}) ;{ }^{1} \mathbf{H}$ NMR $\left(400 \mathrm{MHz}, \mathrm{CDCl}_{3}\right): \delta 7.14-7.30(5 \mathrm{H}), 6.85(\mathrm{~d}, J=3.1 \mathrm{~Hz}, 1 \mathrm{H}), 4.61(\mathrm{~d}$, $J=18.0 \mathrm{~Hz}, 1 \mathrm{H}), 4.36(\mathrm{~d}, J=18.0 \mathrm{~Hz}, 1 \mathrm{H}), 4.26(\mathrm{~m}, 1 \mathrm{H}), 4.18(\mathrm{q}, J=$ $7.0 \mathrm{~Hz}, 2 \mathrm{H}), 3.64(\mathrm{~s}, 3 \mathrm{H}), 2.76(\mathrm{~m}, 1 \mathrm{H}), 2.62(\mathrm{~m}, 1 \mathrm{H}), 2.38(\mathrm{~s}, 3 \mathrm{H})$, $1.96(\mathrm{~m}, 1 \mathrm{H}), 1.86(\mathrm{~m}, 1 \mathrm{H}), 1.23(\mathrm{t}, J=7.0 \mathrm{~Hz}, 3 \mathrm{H}) ;{ }^{13} \mathbf{C}$ NMR $(100$ $\left.\mathrm{MHz}, \mathrm{CDCl}_{3}\right): \delta 169.1,166.3,154.2,148.3,141.1,128.3,128.2,125.7$, 105.1, 61.4, 51.2, 49.4, 44.0, 37.6, 30.6, 15.8, 14.0; IR (thin film, $\mathrm{cm}^{-}$ $\left.{ }^{1}\right): 3235,2950,2925,1747,1689,1630,1393,1192,1114,701 ;[\alpha]^{\mathbf{2 3}}{ }_{\mathbf{D}}=$ $-17.4^{\mathrm{o}}\left(\mathrm{c}=1.1, \mathrm{CHCl}_{3}\right)$; HRMS $\left(\mathrm{CI}-\mathrm{NH}_{3}\right)$ calculated for $\mathrm{C}_{20} \mathrm{H}_{20} \mathrm{BrN}_{2} \mathrm{O}_{2}$ $(\mathrm{M}+\mathrm{H}): 361.1758$, found: 361.1742 .

(S)-26Ce: (S)-5-acetyl-1-(2-methoxyethyl)-6-methyl-4-(3-(trifluoromethyl)phenyl)-3,4dihydropyrimidin-2(1H)-one

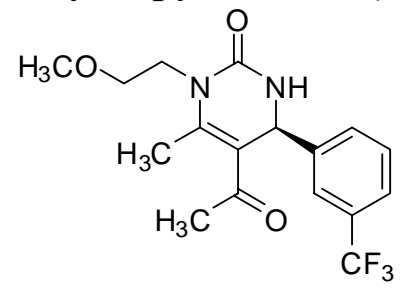

er $=95.5: 4.5$; HPLC analysis, $\mathrm{t}_{\mathrm{r}}$ major: $12.8 \mathrm{~min}, \mathrm{t}_{\mathrm{r}}$ minor: $19.8 \mathrm{~min}$, (ChiralPak OD Column, Hexane:IPA = 90:10, $1.0 \mathrm{~mL} / \mathrm{min}) ;{ }^{1} \mathbf{H}$ NMR $\left(400 \mathrm{MHz}, \mathrm{CDCl}_{3}\right): \delta 7.29-7.65(5 \mathrm{H}), 5.30(\mathrm{~d}, J=3.1 \mathrm{~Hz}, 1 \mathrm{H}), 4.14$ (dt, $J=14.9,4.7 \mathrm{~Hz}, 1 \mathrm{H}), 3.67$ (ddd, $J=14.9,7.8,3.9 \mathrm{~Hz}, 1 \mathrm{H}), 3.48$ $(\mathrm{m}, 1 \mathrm{H}), 3.27(\mathrm{~m}, 1 \mathrm{H}), 3.18(\mathrm{~s}, 3 \mathrm{H}), 2.43(\mathrm{~s}, 3 \mathrm{H}), 2.09(\mathrm{~s}, 3 \mathrm{H}) ;{ }^{13} \mathbf{C}$ NMR $\left(100 \mathrm{MHz}, \mathrm{CDCl}_{3}\right): \delta 196.2,153.5,149.3,143.5,129.7,129.1$, 124.4 (q), 123.2 (q), 112.7, 71.2, 58.5, 53.4, 41.7, 30.3, 17.1; IR (thin film, $\left.\mathrm{cm}^{-1}\right): 3277,2924,1692,1664,1589,1384,1195,1011 ;[\boldsymbol{\alpha}]^{\mathbf{2 3}}{ }_{\mathbf{D}}=$ $54.3^{\circ}\left(\mathrm{c}=1.3, \mathrm{CHCl}_{3}\right)$; HRMS $\left(\mathrm{CI}-\mathrm{NH}_{3}\right)$ calculated for $\mathrm{C}_{20} \mathrm{H}_{20} \mathrm{BrN}_{2} \mathrm{O}_{2}$ $(\mathrm{M}+\mathrm{H}): 357.1421$, found: 357.1414 .

(S)-25Ea: (S)-methyl 1-benzyl-6-methyl-2-oxo-4-(thiophen-2-yl)-1,2,3,4tetrahydropyrimidine-5-carboxylate

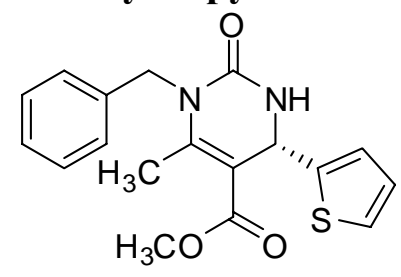

er $=96: 4$; HPLC analysis, $t_{r}$ major: $6.6 \mathrm{~min}, \mathrm{t}_{\mathrm{r}}$ minor: $7.5 \mathrm{~min}$, (ChiralPak OD Column, Hexane:IPA = 80:20, $1.0 \mathrm{~mL} / \mathrm{min}) ;{ }^{1} \mathbf{H}$ NMR $\left(400 \mathrm{MHz}, \mathrm{CDCl}_{3}\right): \delta 6.90-7.28(9 \mathrm{H}), 5.70(\mathrm{~d}, J=3.1 \mathrm{~Hz}, 1 \mathrm{H}), 5.21(\mathrm{~d}$, $J=16.4 \mathrm{~Hz}, 1 \mathrm{H}), 4.84(\mathrm{~d}, J=16.4 \mathrm{~Hz}, 1 \mathrm{H}), 3.72(\mathrm{~s}, 3 \mathrm{H}), 2.42(\mathrm{~s}, 3 \mathrm{H})$;

${ }^{13}$ C NMR $\left(100 \mathrm{MHz}, \mathrm{CDCl}_{3}\right): \delta 166.0,154.9,149.7,147.1,137.5$, $128.6,127.1,126.7,126.1,124.8,123.9,105.1,51.4,49.3,46.0,16.3$; IR (thin film, $\mathrm{cm}^{-1}$ ): 3258, 2925, 1688, 1626, 1234, 763; MS (ESI+) calculated for $\mathrm{C}_{18} \mathrm{H}_{19} \mathrm{~N}_{2} \mathrm{O}_{3} \mathrm{~S}(\mathrm{M}+1) 343.1$, found 342.8 .

(S)-26Bb: (S)-5-acetyl-4-(4-bromophenyl)-1-ethyl-6-methyl-3,4-dihydropyrimidin-2(1H)one<smiles>CCN1C(=O)NC(c2ccc(Br)cc2)C(C(C)=O)=C1C</smiles>

er $=95: 5$; HPLC analysis, $t_{r}$ major: $9.4 \mathrm{~min}, \mathrm{t}_{\mathrm{r}}$ minor: $13.4 \mathrm{~min}$, (ChiralPak OD Column, Hexane:IPA = 90:10, $1.0 \mathrm{~mL} / \mathrm{min}$ ); ${ }^{1} \mathbf{H}$ NMR $\left(400 \mathrm{MHz}, \mathrm{CDCl}_{3}\right): \delta 7.40(\mathrm{~d}, J=8.6 \mathrm{~Hz}, 2 \mathrm{H}), 7.07$ (d, $J=8.6 \mathrm{~Hz}$, $2 \mathrm{H}), 6.65(\mathrm{~d}, J=2.4 \mathrm{~Hz}, 1 \mathrm{H}), 5.26(\mathrm{~d}, J=2.4 \mathrm{~Hz}, 1 \mathrm{H}), 3.85(\mathrm{~m}, 1 \mathrm{H})$, $3.69(\mathrm{~m}, .1 \mathrm{H}), 2.46(\mathrm{~s}, 3 \mathrm{H}), 2.12(\mathrm{~s}, 3 \mathrm{H}), 1.16(\mathrm{t}, J=7.0 \mathrm{~Hz}, 3 \mathrm{H}) ;{ }^{13} \mathrm{C}$ NMR $\left(100 \mathrm{MHz}, \mathrm{CDCl}_{3}\right): \delta 196.4,153.3,147.9,141.4,132.0,128.0$, 122.0, 113.0, 53.7, 37.8, 30.4, 16.6, 14.9; MS (ESI+) calculated for $\mathrm{C}_{15} \mathrm{H}_{18} \mathrm{BrN}_{2} \mathrm{O}_{2}(\mathrm{M}+1) 337.1$, found 337.0. 
(S)-26Bc: (S)-ethyl 2-(5-acetyl-4-(4-bromophenyl)-6-methyl-2-oxo-3,4-dihydropyrimidin1(2H)-yl)- acetate

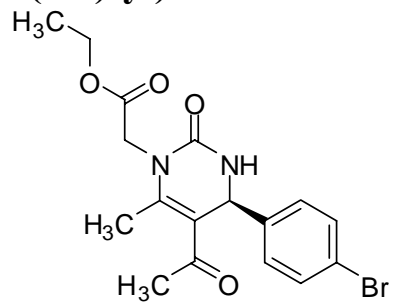

er $=95: 5$; HPLC analysis, $\mathrm{t}_{\mathrm{r}}$ major: $19.0 \mathrm{~min}, \mathrm{t}_{\mathrm{r}}$ minor: $23.7 \mathrm{~min}$, (ChiralPak OD Column, Hexane:IPA = 90:10, $1.0 \mathrm{~mL} / \mathrm{min}) ;{ }^{1} \mathbf{H}$ NMR $\left(400 \mathrm{MHz}, \mathrm{CDCl}_{3}\right): \delta 7.42(\mathrm{~d}, J=8.6 \mathrm{~Hz}, 2 \mathrm{H}), 7.21(\mathrm{~d}, J=8.6 \mathrm{~Hz}$, $2 \mathrm{H}), 6.79(\mathrm{~d}, J=3.1 \mathrm{~Hz}, 1 \mathrm{H}), 5.28(\mathrm{~d}, J=3.1 \mathrm{~Hz}, 1 \mathrm{H}), 4.63(\mathrm{~d}, J=$ $18.0 \mathrm{~Hz}, 1 \mathrm{H}), 4.33(\mathrm{~d}, J=18.0 \mathrm{~Hz}, 1 \mathrm{H}), 4.21(\mathrm{q}, J=7.0 \mathrm{~Hz}, 2 \mathrm{H}), 2.34$ $(\mathrm{s}, 3 \mathrm{H}), 2.11(\mathrm{~s}, 3 \mathrm{H}), 1.27(\mathrm{t}, J=7.0 \mathrm{~Hz}, 3 \mathrm{H}) ;{ }^{13} \mathrm{C}$ NMR $(100 \mathrm{MHz}$, $\left.\mathrm{CDCl}_{3}\right): \delta 196.6,169.1,152.9,146.6,141.1,132.0,128.5,122.1$, $113.3,61.7,54.1,44.0,30.4,16.7,14.1$; MS (ESI+) calculated for $\mathrm{C}_{17} \mathrm{H}_{20} \mathrm{BrN}_{2} \mathrm{O}_{4}(\mathrm{M}+1)$ 395.1, found 395.0.

(R)-25Eb: $(R)$-methyl 1-ethyl-6-methyl-2-oxo-4-(thiophen-2-yl)-1,2,3,4tetrahydropyrimidine-5-carboxylate

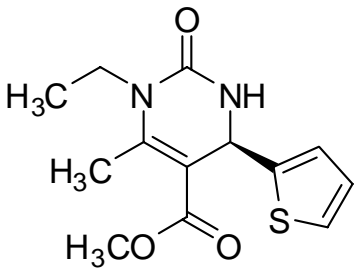

er $=95.5: 4.5 ;$ HPLC analysis, $\mathrm{t}_{\mathrm{r}}$ minor: $4.7 \mathrm{~min}, \mathrm{t}_{\mathrm{r}}$ major: $5.7 \mathrm{~min}$, (ChiralPak OD Column, Hexane:IPA = 80:20, $1.0 \mathrm{~mL} / \mathrm{min}) ;{ }^{1} \mathbf{H}$ NMR $\left(400 \mathrm{MHz}, \mathrm{CDCl}_{3}\right): \delta 7.15(\mathrm{~d}, J=5.5 \mathrm{~Hz}, 1 \mathrm{H}), 6.87-6.93(2 \mathrm{H}), 6.63(\mathrm{~d}$, $J=3.1 \mathrm{~Hz}, 1 \mathrm{H}), 5.61(\mathrm{~d}, J=3.1 \mathrm{~Hz}, 1 \mathrm{H}), 3.90(\mathrm{~m}, 1 \mathrm{H}), 3.70(\mathrm{~s}, 3 \mathrm{H})$, $3.68(\mathrm{~m}, .1 \mathrm{H}), 2.52(\mathrm{~s}, 3 \mathrm{H}), 1.19(\mathrm{t}, J=7.0 \mathrm{~Hz}, 3 \mathrm{H}) ;{ }^{13} \mathrm{C}$ NMR (100 $\left.\mathrm{MHz} \mathrm{CDCl}_{3}\right): \delta 166.1,154.1,149.3,147.3,145.3,126.7,124.6,123.7$, 104.6, 51.3, 49.2, 37.8, 15.8, 14.9; MS (ESI+) calculated for $\mathrm{C}_{13} \mathrm{H}_{17} \mathrm{~N}_{2} \mathrm{O}_{3} \mathrm{~S}(\mathrm{M}+1) 281.1$, found 280.9.

(S)-25Af: (S)-methyl 1-(cyclopropylmethyl)-6-methyl-2-oxo-4-phenyl-1,2,3,4tetrahydropyrimidine-5-carboxylate<smiles>COC(=O)C1=C(C)N(CC2CC2)C(=O)N[C@H]1c1ccccc1</smiles>

${ }^{1} \mathbf{H}$ NMR $\left(400 \mathrm{MHz}, \mathrm{CDCl}_{3}\right): \delta$ 7.21-7.31 $(5 \mathrm{H}), 5.96(\mathrm{~d}, J=3.1 \mathrm{~Hz}$, $1 \mathrm{H}), 5.32(\mathrm{~d}, J=3.1 \mathrm{~Hz}, 1 \mathrm{H}), 3.82(\mathrm{dd}, J=14.9,7.8 \mathrm{~Hz}, 1 \mathrm{H}), 3.62(\mathrm{~s}$, $3 \mathrm{H}), 3.56(\mathrm{~d}, J=14.9,5.5 \mathrm{~Hz}, 1 \mathrm{H}), 2.54(\mathrm{~s}, 3 \mathrm{H}), 0.95(\mathrm{~m}, 1 \mathrm{H}), 0.45(\mathrm{~m}$, $2 \mathrm{H}), 0.29(\mathrm{~m}, 2 \mathrm{H}) ;{ }^{13} \mathrm{C}$ NMR $\left(100 \mathrm{MHz}, \mathrm{CDCl}_{3}\right): \delta$ 166.6, 154.1, $148.9,143.3,128.6,127.8,126.3,105.1,53.9,51.3,46.2,16.4,11.4$, 3.9; MS (ESI+) calculated for $\mathrm{C}_{17} \mathrm{H}_{21} \mathrm{~N}_{2} \mathrm{O}_{3}(\mathrm{M}+1)$ 301.1, found 300.9.

(R)-25Ac: $(R)$-methyl 1-(2-ethoxy-2-oxoethyl)-6-methyl-2-oxo-4-phenyl-1,2,3,4tetrahydropyrimidine-5-carboxylate

$\mathrm{H}_{3} \mathrm{C}$<smiles>CCOC(=O)CN1C(=O)N[C@H](c2ccccc2)C(C(=O)OC)=C1C</smiles>

${ }^{1}$ H NMR $\left(400 \mathrm{MHz}, \mathrm{CDCl}_{3}\right): \delta 7.22-7.38(5 \mathrm{H}), 5.86(\mathrm{~d}, J=3.1 \mathrm{~Hz}$, $1 \mathrm{H}), 5.37(\mathrm{~d}, J=3.1 \mathrm{~Hz}, 1 \mathrm{H}), 4.63(\mathrm{~d}, J=18.0 \mathrm{~Hz}, 1 \mathrm{H}), 4.40(\mathrm{~d}, J=$ $18.0 \mathrm{~Hz}, 1 \mathrm{H}), 4.23(\mathrm{q}, J=7.0 \mathrm{~Hz}, 2 \mathrm{H}), 3.62(\mathrm{~s}, 3 \mathrm{H}), 2.42(\mathrm{~s}, 3 \mathrm{H}), 1.28$ $(\mathrm{t}, J=7.0 \mathrm{~Hz}, 3 \mathrm{H}) ;{ }^{13} \mathrm{C}$ NMR $\left(100 \mathrm{MHz}, \mathrm{CDCl}_{3}\right): \delta 169.1,166.3$, $152.9,147.7,143.1,128.7,127.9,126.6,104.6,61.6,54.4,51.3,44.2$, $16.2,14.1$; MS (ESI+) calculated for $\mathrm{C}_{17} \mathrm{H}_{21} \mathrm{~N}_{2} \mathrm{O}_{5}(\mathrm{M}+1) 333.1$, found 332.9 .

(S)-26Ac: (S)-ethyl 2-(5-acetyl-6-methyl-2-oxo-4-phenyl-3,4-dihydropyrimidin-1(2H)yl)acetate 
<smiles>CCOC(=O)CN1C(=O)NC(c2ccccc2)C(C(C)=O)=C1C</smiles>

${ }^{1}$ H NMR (400 MHz, $\left.\mathrm{CDCl}_{3}\right): \delta 7.22-7.32(5 \mathrm{H}), 6.64(\mathrm{~d}, J=3.1 \mathrm{~Hz}$, $1 \mathrm{H}), 5.31(\mathrm{~d}, J=3.1 \mathrm{~Hz}, 1 \mathrm{H}), 4.57(\mathrm{~d}, J=18.0 \mathrm{~Hz}, 1 \mathrm{H}), 4.36(\mathrm{~d}, J=$ $18.0 \mathrm{~Hz}, 1 \mathrm{H}), 4.18$ (q, $J=7.0 \mathrm{~Hz}, 2 \mathrm{H}), 2.33$ (s, 3H), 2.07 (s, 3H), 1.24 $(\mathrm{t}, J=7.0 \mathrm{~Hz}, 3 \mathrm{H}) ;{ }^{13} \mathbf{C}$ NMR $\left(100 \mathrm{MHz}, \mathrm{CDCl}_{3}\right): \delta 196.9,169.1$, $152.8,146.3,142.0,128.9,128.1,126.7,113.2,61.5,54.8,44.0,30.2$, 16.5, 14.0; MS (ESI+) calculated for $\mathrm{C}_{17} \mathrm{H}_{21} \mathrm{~N}_{2} \mathrm{O}_{4}(\mathrm{M}+1)$ 317.1, found 317.0 .

(S)-26Af: (S)-5-acetyl-1-(cyclopropylmethyl)-6-methyl-4-phenyl-3,4-dihydropyrimidin2(1H)-one<smiles>CC(=O)C1=C(C)N(CC2CC2)C(=O)NC1c1ccccc1</smiles>

${ }^{1} \mathbf{H}$ NMR (400 MHz, $\left.\mathrm{CDCl}_{3}\right): \delta$ 7.28-7.34 $(5 \mathrm{H}), 6.44(\mathrm{~d}, J=3.1 \mathrm{~Hz}$, $1 \mathrm{H}), 5.31(\mathrm{~d}, J=3.1 \mathrm{~Hz}, 1 \mathrm{H}), 3.86(\mathrm{dd}, J=14.9,7.8 \mathrm{~Hz}, 1 \mathrm{H}), 3.60(\mathrm{dd}$, $J=14.9,6.3 \mathrm{~Hz}, 1 \mathrm{H}), 2.54(\mathrm{~s}, 3 \mathrm{H}), 2.16(\mathrm{~s}, 3 \mathrm{H}), 1.00(\mathrm{~m}, 1 \mathrm{H}), 0.52(\mathrm{~m}$, $1 \mathrm{H}), 0.44(\mathrm{~m}, 1 \mathrm{H}), 0.33(\mathrm{~m}, 1 \mathrm{H}), 0.28(\mathrm{~m}, 1 \mathrm{H}) ;{ }^{13} \mathbf{C}$ NMR $(100 \mathrm{MHz}$, $\left.\mathrm{CDCl}_{3}\right): \delta 196.8,153.9,147.8,142.2,128.9,128.1,126.5,113.7,54.6$, $46.1,30.3,16.9,11.3,4.0,3.9$; MS (ESI+) calculated for $\mathrm{C}_{17} \mathrm{H}_{21} \mathrm{~N}_{2} \mathrm{O}_{2}$ $(\mathrm{M}+1)$ 285.2, found 285.1.

(S)-25Ad: (S)-methyl 1-(furan-2-ylmethyl)-6-methyl-2-oxo-4-phenyl-1,2,3,4tetrahydropyrimidine-5-carboxylate<smiles>COC(=O)C1=C(C)N(Cc2ccco2)C(=O)N[C@H]1c1ccccc1</smiles>

${ }^{1} \mathbf{H}$ NMR (400 MHz, $\left.\mathrm{CDCl}_{3}\right): \delta 7.32(\mathrm{~d}, J=2.3 \mathrm{~Hz}, 1 \mathrm{H}), 7.11-7.23$ $(5 \mathrm{H}), 6.45(\mathrm{~d}, J=3.2 \mathrm{~Hz}, 1 \mathrm{H}), 6.30(\mathrm{dd}, J=3.1,2.3 \mathrm{~Hz}, 1 \mathrm{H}), 6.18(\mathrm{~d}, J$ $=3.1 \mathrm{~Hz}, 1 \mathrm{H}), 5.34(\mathrm{~d}, J=3.2 \mathrm{~Hz}, 1 \mathrm{H}), 5.21(\mathrm{~d}, J=16.4 \mathrm{~Hz}, 1 \mathrm{H}), 4.76$ $(\mathrm{d}, J=16.4 \mathrm{~Hz}, 1 \mathrm{H}), 3.62(\mathrm{~s}, 3 \mathrm{H}), 2.59(\mathrm{~s}, 3 \mathrm{H}) ;{ }^{13} \mathrm{C}$ NMR $(100 \mathrm{MHz}$, $\left.\mathrm{CDCl}_{3}\right): \delta 166.4,153.8,150.9,148.4,142.9,141.9,128.6,127.7$, $126.2,110.5,108.0,105.1,53.9,51.3,39.0,16.2 ;$ MS (ESI+) calculated for $\mathrm{C}_{18} \mathrm{H}_{19} \mathrm{~N}_{2} \mathrm{O}_{4}(\mathrm{M}+1) 327.1$, found 326.9.

(S)-25Ae: (S)-methyl 1-(2-methoxyethyl)-6-methyl-2-oxo-4-phenyl-1,2,3,4tetrahydropyrimidine-5-carboxylate<smiles>COCCN1C(=O)NC(c2ccccc2)C(C(=O)OC)=C1C</smiles>

${ }^{1}$ H NMR $\left(400 \mathrm{MHz}, \mathrm{CDCl}_{3}\right): \delta 7.24-7.33(\mathrm{~m}, 5 \mathrm{H}), 6.61(\mathrm{~d}, J=3.1 \mathrm{~Hz}$, $1 \mathrm{H}), 5.29(\mathrm{~d}, J=3.1 \mathrm{~Hz}, 1 \mathrm{H}), 4.14(\mathrm{dt}, J=14.8,4.7 \mathrm{~Hz}, 1 \mathrm{H}), 3.72$ (ddd, $J=14.9,7.0,3.9 \mathrm{~Hz}, 1 \mathrm{H}), 3.59$ (s, 3H), 3.45 (m, 1H), 3.37 (m , $1 \mathrm{H}), 3.25(\mathrm{~s}, 3 \mathrm{H}), 2.52(\mathrm{~s}, 3 \mathrm{H}) ;{ }^{13} \mathbf{C}$ NMR $\left(100 \mathrm{MHz}, \mathrm{CDCl}_{3}\right): \delta 166.5$, $153.6,149.7,143.3,128.4,127.4,126.2,104.0,71.3,58.7,53.6,51.1$, 41.9, 16.4; MS (ESI+) calculated for $\mathrm{C}_{16} \mathrm{H}_{21} \mathrm{~N}_{2} \mathrm{O}_{4}(\mathrm{M}+1) 305.1$, found 304.9 .

(S)-26Ae: (S)-5-acetyl-1-(2-methoxyethyl)-6-methyl-4-phenyl-3,4-dihydropyrimidin-2(1H)one 


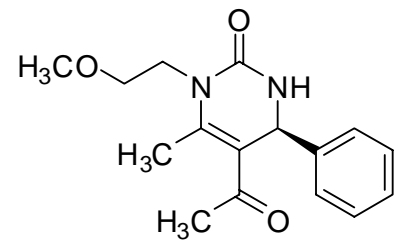

${ }^{1} \mathbf{H}$ NMR $\left(400 \mathrm{MHz}, \mathrm{CDCl}_{3}\right): \delta 7.23-7.31(\mathrm{~m}, 5 \mathrm{H}), 6.39(\mathrm{~d}, J=3.1 \mathrm{~Hz}$, $1 \mathrm{H}), 5.25(\mathrm{~d}, J=3.1 \mathrm{~Hz}, 1 \mathrm{H}), 4.15(\mathrm{dt}, J=14.8,4.7 \mathrm{~Hz}, 1 \mathrm{H}), 3.76$ (ddd, $J=14.8,7.8,3.9 \mathrm{~Hz}, 1 \mathrm{H}), 3.48(\mathrm{dt}, J=10.2,4.7 \mathrm{~Hz}, 1 \mathrm{H}), 3.38$ (ddd, $J=10.2,7.8,3.9 \mathrm{~Hz}, 1 \mathrm{H}), 3.28(\mathrm{~s}, 3 \mathrm{H}), 2.48(\mathrm{~s}, 3 \mathrm{H}), 2.10(\mathrm{~s}, 3 \mathrm{H})$; ${ }^{13}$ C NMR $\left(100 \mathrm{MHz}, \mathrm{CDCl}_{3}\right): \delta 196.8,153.4,148.4,142.3,128.9$, 128.0, 126.5, 113.2, 71.4, 58.8, 54.7, 42.0, 30.4, 17.0; MS (ESI+) calculated for $\mathrm{C}_{16} \mathrm{H}_{21} \mathrm{~N}_{2} \mathrm{O}_{3}(\mathrm{M}+1)$ 289.2, found 289.1.

(S)-25Ah: (S)-methyl 6-methyl-2-oxo-4-phenyl-1-(3,4,5-trimethoxyphenethyl)-1,2,3,4tetrahydropyrimidine-5-carboxylate

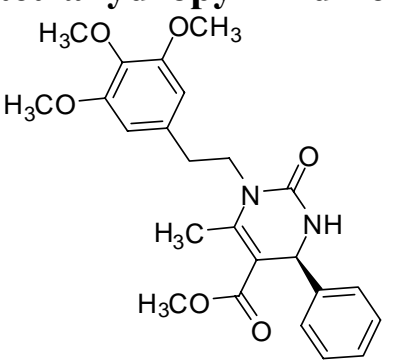

${ }^{1}$ H NMR (400 MHz, $\left.\mathrm{CDCl}_{3}\right): \delta$ 7.18-7.30 (m, 5H), $6.42(\mathrm{~s}, 2 \mathrm{H}), 6.06$ $(\mathrm{d}, J=3.1 \mathrm{~Hz}, 1 \mathrm{H}), 5.37(\mathrm{~d}, J=3.1 \mathrm{~Hz}, 1 \mathrm{H}), 4.11(\mathrm{~m}, 1 \mathrm{H}), 3.81(\mathrm{~s}$, $3 \mathrm{H}), 3.80(\mathrm{~s}, 6 \mathrm{H}), 3.80(\mathrm{~m}, 1 \mathrm{H}), 3.63(\mathrm{~s}, 3 \mathrm{H}), 2.81(\mathrm{~m}, 2 \mathrm{H}), 2.50(\mathrm{~s}$, $3 \mathrm{H}) ;{ }^{13} \mathrm{C}$ NMR $\left(100 \mathrm{MHz}, \mathrm{CDCl}_{3}\right): \delta 166.5,153.6,153.2,148.3$, 143.2, 134.0, 128.7, 127.8, 126.1, 105.6, 104.5, 60.8, 56.0, 53.9, 51.3, 44.1, 36.3, 16.2; MS (ESI+) calculated for $\mathrm{C}_{24} \mathrm{H}_{29} \mathrm{~N}_{2} \mathrm{O}_{6}(\mathrm{M}+1)$ 441.2, found 441.1 .

(S)-26Ad: (S)-5-acetyl-1-(furan-2-ylmethyl)-6-methyl-4-phenyl-3,4-dihydropyrimidin2(1H)-one<smiles>CC(=O)C1=C(C)N(Cc2ccco2)C(=O)N[C@H]1c1ccccc1</smiles>

${ }^{1}$ H NMR $\left(400 \mathrm{MHz}, \mathrm{CDCl}_{3}\right): \delta 7.11-7.33(7 \mathrm{H}), 6.29(\mathrm{dd}, J=3.1,2.3$ $\mathrm{Hz}, 1 \mathrm{H}), 6.19$ (d, $J=3.1 \mathrm{~Hz}, 1 \mathrm{H}), 5.29$ (d, $J=3.2 \mathrm{~Hz}, 1 \mathrm{H}), 5.16$ (d, $J=$ $16.4 \mathrm{~Hz}, 1 \mathrm{H}), 4.74(\mathrm{~d}, J=16.4 \mathrm{~Hz}, 1 \mathrm{H}), 2.52$ (s, 3H), 2.10 (s, 3H); MS (ESI+) calculated for $\mathrm{C}_{18} \mathrm{H}_{19} \mathrm{~N}_{2} \mathrm{O}_{3}(\mathrm{M}+1) 311.1$, found 311.0 .

(S)-25Ag: (S)-methyl 6-methyl-1-((R)-2-methylbutyl)-2-oxo-4-phenyl-1,2,3,4tetrahydropyrimidine-5-carboxylate<smiles>CC[C@H](C)CN1C(=O)N[C@H](c2ccccc2)C(C(=O)OC)=C1C</smiles>

${ }^{1} \mathbf{H}$ NMR (400 MHz, $\left.\mathrm{CDCl}_{3}\right): \delta$ 7.21-7.34 (5H), $5.86(\mathrm{~d}, J=3.1 \mathrm{~Hz}$, $1 \mathrm{H}), 5.41(\mathrm{~d}, J=3.1 \mathrm{~Hz}, 1 \mathrm{H}), 3.98(\mathrm{~m}, 1 \mathrm{H}), 3.66(\mathrm{~s}, 3 \mathrm{H}), 3.28(\mathrm{~m}$, $1 \mathrm{H}), 2.51(\mathrm{~s}, 3 \mathrm{H}), 1.55(\mathrm{~m}, 1 \mathrm{H}), 1.28(\mathrm{~m}, 2 \mathrm{H}), 0.85(\mathrm{t}, J=7.8 \mathrm{~Hz}, 3 \mathrm{H})$, $0.73(\mathrm{~d}, J=6.4 \mathrm{~Hz}, 3 \mathrm{H}) ; \mathrm{MS}(\mathrm{ESI}+)$ calculated for $\mathrm{C}_{18} \mathrm{H}_{25} \mathrm{~N}_{2} \mathrm{O}_{3}(\mathrm{M}+1)$ 317.2, found 317.1 .

(S)-26Aa: (S)-5-acetyl-1-benzyl-6-methyl-4-phenyl-3,4-dihydropyrimidin-2(1H)-one<smiles>CC(=O)C1=C(C)N(Cc2ccccc2)C(=O)NC1c1ccccc1</smiles>

${ }^{1}$ H NMR $\left(400 \mathrm{MHz}, \mathrm{CDCl}_{3}\right): \delta 7.08-7.33(10 \mathrm{H}), 6.19(\mathrm{~d}, J=3.1 \mathrm{~Hz}$, $1 \mathrm{H}), 5.35(\mathrm{~d}, J=3.1 \mathrm{~Hz}, 1 \mathrm{H}), 5.19(\mathrm{~d}, J=16.4 \mathrm{~Hz}, 1 \mathrm{H}), 4.80(\mathrm{~d}, J=$ $16.4 \mathrm{~Hz}, 1 \mathrm{H}), 2.36(\mathrm{~s}, 3 \mathrm{H}), 2.11(\mathrm{~s}, 3 \mathrm{H})$; MS (ESI+) calculated for $\mathrm{C}_{20} \mathrm{H}_{21} \mathrm{~N}_{2} \mathrm{O}_{2}(\mathrm{M}+1)$ 321.2, found 321.2.

$(R)-26 A h:(R)-5$-acetyl-6-methyl-4-phenyl-1-(3,4,5-trimethoxyphenethyl)-3,4dihydropyrimidin-2(1H)-one 


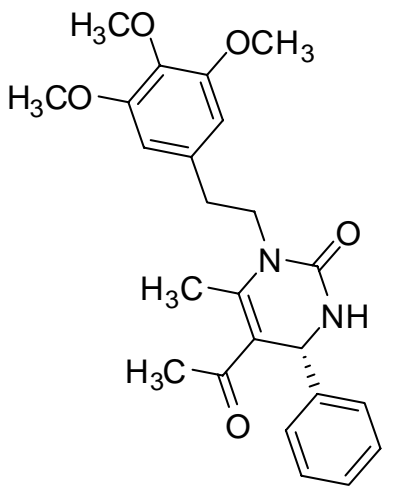

${ }^{1}$ H NMR (400 MHz, $\left.\mathrm{CDCl}_{3}\right): \delta$ 7.18-7.29 (5H), $6.40(\mathrm{~s}, 2 \mathrm{H}), 6.25(\mathrm{~d}, J$ $=3.1 \mathrm{~Hz}, 1 \mathrm{H}), 5.33(\mathrm{~d}, J=3.1 \mathrm{~Hz}, 1 \mathrm{H}), 3.80(\mathrm{~s}, 3 \mathrm{H}), 3.79(\mathrm{~s}, 6 \mathrm{H}), 3.80$ (m, 2H), 2.80 (m, 2H), 2.43 (s, 3H), 2.10 (s, 3H); ${ }^{13}$ C NMR (100 MHz, $\left.\mathrm{CDCl}_{3}\right): \delta 196.9,153.23,153.19,142.2,133.9,129.0,128.8,128.1$, $126.3,126.1,113.4,105.6,60.8,56.0,54.6,44.1,36.3,16.8$; MS (ESI+) calculated for $\mathrm{C}_{24} \mathrm{H}_{29} \mathrm{~N}_{2} \mathrm{O}_{5}(\mathrm{M}+1) 425.2$, found 425.1 .

(S)-25Ba: (S)-methyl 1-benzyl-4-(4-bromophenyl)-6-methyl-2-oxo-1,2,3,4tetrahydropyrimidine-5-carboxylate

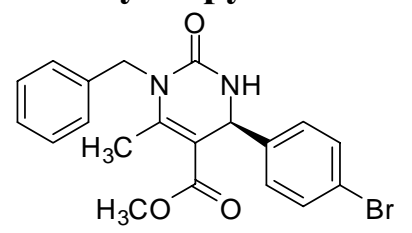

${ }^{1}$ H NMR $\left(400 \mathrm{MHz}, \mathrm{CDCl}_{3}\right): \delta$ 7.04-7.40 $(9 \mathrm{H}), 6.40(\mathrm{~d}, J=3.1 \mathrm{~Hz}$, $1 \mathrm{H}), 5.39(\mathrm{~d}, J=3.1 \mathrm{~Hz}, 1 \mathrm{H}), 5.21(\mathrm{~d}, J=16.4 \mathrm{~Hz}, 1 \mathrm{H}), 4.81(\mathrm{~d}, J=$ $16.4 \mathrm{~Hz}, 1 \mathrm{H}), 3.61(\mathrm{~s}, 3 \mathrm{H}), 2.44(\mathrm{~s}, 3 \mathrm{H}) ;{ }^{13} \mathbf{C ~ N M R}\left(100 \mathrm{MHz}, \mathrm{CDCl}_{3}\right)$ : $\delta 166.3,153.9,149.6,142.0,137.7,131.9,131.7,128.7,128.0,127.3$, $126.4,121.7,53.2,51.4,45.9,16.5$; MS (ESI+) calculated for $\mathrm{C}_{20} \mathrm{H}_{20} \mathrm{BrN}_{2} \mathrm{O}_{3}(\mathrm{M}+1)$ 415.1, found 415.0.

(S)-26Ba: (S)-5-acetyl-1-benzyl-4-(4-bromophenyl)-6-methyl-3,4-dihydropyrimidin-2(1H)one<smiles>CC(=O)C1=C(C)N(Cc2ccccc2)C(=O)NC1c1ccc(Br)cc1</smiles>

${ }^{1} \mathbf{H}$ NMR $\left(400 \mathrm{MHz}, \mathrm{CDCl}_{3}\right): \delta$ 6.99-7.46 $(10 \mathrm{H}), 5.31(\mathrm{~d}, J=3.1 \mathrm{~Hz}$, $1 \mathrm{H}), 5.21(\mathrm{~d}, J=16.4 \mathrm{~Hz}, 1 \mathrm{H}), 4.75(\mathrm{~d}, J=16.4 \mathrm{~Hz}, 1 \mathrm{H}), 2.36(\mathrm{~s}, 3 \mathrm{H})$, $2.10(\mathrm{~s}, 3 \mathrm{H}) ;{ }^{13} \mathrm{C}$ NMR $\left(100 \mathrm{MHz}, \mathrm{CDCl}_{3}\right): \delta 196.4,154.1,148.3$, $141.1,137.5,131.9,128.7,128.2,127.3,126.3,121.9,113.5,53.3$, 45.7, 30.3, 17.1; MS (ESI+) calculated for $\mathrm{C}_{20} \mathrm{H}_{20} \mathrm{BrN}_{2} \mathrm{O}_{2} 399.1$, found 399.0 .

(S)-25Bb: (S)-methyl 4-(4-bromophenyl)-1-ethyl-6-methyl-2-oxo-1,2,3,4tetrahydropyrimidine-5-carboxylate<smiles>CCN1C(=O)NC(c2ccc(Br)cc2)C(C(=O)OC)=C1C</smiles>

${ }^{1}$ H NMR (400 MHz, $\left.\mathrm{CDCl}_{3}\right): \delta 7.38(\mathrm{~d}, J=7.8 \mathrm{~Hz}, 2 \mathrm{H}), 7.08(\mathrm{~d}, J=$ $7.8 \mathrm{~Hz}, 2 \mathrm{H}), 6.33(\mathrm{~d}, J=3.1 \mathrm{~Hz}, 1 \mathrm{H}), 5.30(\mathrm{~d}, J=3.1 \mathrm{~Hz}, 1 \mathrm{H}), 3.86(\mathrm{~m}$, $1 \mathrm{H}), 3.70(\mathrm{~m}, .1 \mathrm{H}), 3.62(\mathrm{~s}, 3 \mathrm{H}), 2.52(\mathrm{~s}, 3 \mathrm{H}), 1.16(\mathrm{t}, J=7.0 \mathrm{~Hz}, 3 \mathrm{H})$; ${ }^{13} \mathbf{C}$ NMR $\left(100 \mathrm{MHz}, \mathrm{CDCl}_{3}\right): \delta 166.4,153.4,149.2,142.4,131.7$, $127.9,121.6,103.6,53.2,51.3,37.8,16.0,14.9$; MS (ESI+) calculated for $\mathrm{C}_{15} \mathrm{H}_{18} \mathrm{BrN}_{2} \mathrm{O}_{3}(\mathrm{M}+1) 353.0$, found 352.9.

(S)-25Bc: (S)-methyl 4-(4-bromophenyl)-1-(2-ethoxy-2-oxoethyl)-6-methyl-2-oxo-1,2,3,4tetrahydropyrimidine-5-carboxylate 


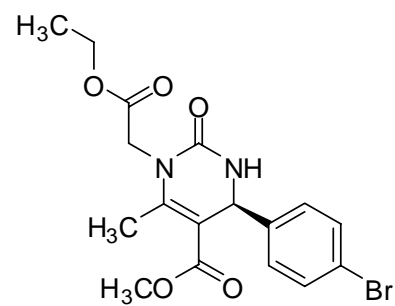

${ }^{1}$ H NMR (400 MHz, $\left.\mathrm{CDCl}_{3}\right): \delta 7.41(\mathrm{~d}, J=8.6 \mathrm{~Hz}, 2 \mathrm{H}), 7.24(\mathrm{~d}, J=$ $8.6 \mathrm{~Hz}, 2 \mathrm{H}), 6.17(\mathrm{~d}, J=3.1 \mathrm{~Hz}, 1 \mathrm{H}), 5.33(\mathrm{~d}, J=3.1 \mathrm{~Hz}, 1 \mathrm{H}), 4.68(\mathrm{~d}$, $J=18.0 \mathrm{~Hz}, 1 \mathrm{H}), 4.35(\mathrm{~d}, J=18.0 \mathrm{~Hz}, 1 \mathrm{H}), 4.22(\mathrm{q}, J=7.0 \mathrm{~Hz}, 2 \mathrm{H})$, $3.62(\mathrm{~s}, 3 \mathrm{H}), 2.42(\mathrm{~s}, 3 \mathrm{H}), 1.28(\mathrm{t}, J=7.0 \mathrm{~Hz}, 3 \mathrm{H}) ;{ }^{13} \mathbf{C}$ NMR (100 $\left.\mathrm{MHz}_{\mathrm{CDCl}}\right): \delta 169.1,166.1,152.9,148.1,142.2,131.8,128.4,121.8$, $104.2,61.7,53.8,51.4,44.1,16.2,14.1$; MS (ESI+) calculated for $\mathrm{C}_{17} \mathrm{H}_{20} \mathrm{BrN}_{2} \mathrm{O}_{5} 411.1(\mathrm{M}+1)$, found 411.0.

(S)-25Bf: (S)-methyl 4-(4-bromophenyl)-1-(cyclopropylmethyl)-6-methyl-2-oxo-1,2,3,4tetrahydropyrimidine-5-carboxylate

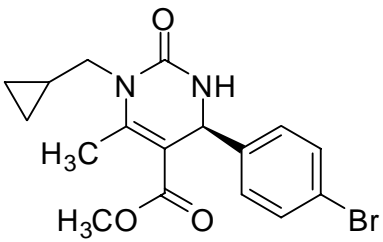

${ }^{1}$ H NMR $\left(400 \mathrm{MHz}, \mathrm{CDCl}_{3}\right): \delta 7.41(\mathrm{~d}, J=8.6 \mathrm{~Hz}, 2 \mathrm{H}), 7.18(\mathrm{~d}, J=$ $8.6 \mathrm{~Hz}, 2 \mathrm{H}), 6.63(\mathrm{~d}, J=3.1 \mathrm{~Hz}, 1 \mathrm{H}), 5.31(\mathrm{~d}, J=3.1 \mathrm{~Hz}, 1 \mathrm{H}), 3.83$ $(\mathrm{dd}, J=14.9,7.9 \mathrm{~Hz}, 1 \mathrm{H}), 3.63(\mathrm{~s}, 3 \mathrm{H}), 3.57(\mathrm{~d}, J=14.9,5.5 \mathrm{~Hz}, 1 \mathrm{H})$, $2.56(\mathrm{~s}, 3 \mathrm{H}), 0.96(\mathrm{~m}, 1 \mathrm{H}), 0.48(\mathrm{~m}, 2 \mathrm{H}), 0.31(\mathrm{~m}, 2 \mathrm{H}) ;{ }^{13} \mathrm{C}$ NMR $(100$ $\left.\mathrm{MHz}, \mathrm{CDCl}_{3}\right): \delta 166.3,154.4,149.0,142.3,131.7,128.0,121.7,104.5$, 53.2, 51.4, 46.3, 16.4, 11.3, 3.9; MS (ESI+) calculated for $\mathrm{C}_{17} \mathrm{H}_{20} \mathrm{BrN}_{2} \mathrm{O}_{3}(\mathrm{M}+1) 379.1$, found 379.0.

(S)-26Bf: (S)-5-acetyl-4-(4-bromophenyl)-1-(cyclopropylmethyl)-6-methyl-3,4dihydropyrimidin-2(1H)-one

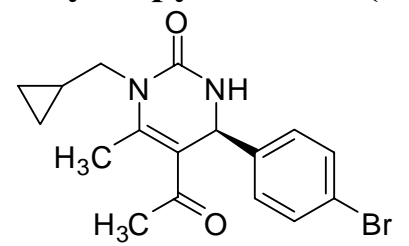

${ }^{1}$ H NMR $\left(400 \mathrm{MHz}, \mathrm{CDCl}_{3}\right): \delta 7.39(\mathrm{~d}, J=8.6 \mathrm{~Hz}, 2 \mathrm{H}), 7.14(\mathrm{~d}, J=$ $8.6 \mathrm{~Hz}, 2 \mathrm{H}), 6.95(\mathrm{~d}, J=3.1 \mathrm{~Hz}, 1 \mathrm{H}), 5.25(\mathrm{~d}, J=3.1 \mathrm{~Hz}, 1 \mathrm{H}), 3.82$ $(\mathrm{dd}, J=14.9,7.8 \mathrm{~Hz}, 1 \mathrm{H}), 3.54(\mathrm{dd}, J=14.9,6.3 \mathrm{~Hz}, 1 \mathrm{H}), 2.48(\mathrm{~s}, 3 \mathrm{H})$, $2.14(\mathrm{~s}, 3 \mathrm{H}), 0.92(\mathrm{~m}, 1 \mathrm{H}), 0.48(\mathrm{~m}, 1 \mathrm{H}), 0.39(\mathrm{~m}, 1 \mathrm{H}), 0.28(\mathrm{~m}, 1 \mathrm{H})$, $0.21(\mathrm{~m}, 1 \mathrm{H}) ;{ }^{13} \mathrm{C}$ NMR $\left(100 \mathrm{MHz}, \mathrm{CDCl}_{3}\right): \delta$ 196.3, 154.0, 148.0, $141.4,131.9,128.1,121.9,113.7,53.6,46.1,30.4,17.0,11.3,4.0,3.8$; MS (ESI+) calculated for $\mathrm{C}_{17} \mathrm{H}_{20} \mathrm{BrN}_{2} \mathrm{O}_{2}(\mathrm{M}+1) 363.1$, found 362.9 .

(S)-25Bd: (S)-methyl 4-(4-bromophenyl)-1-(furan-2-ylmethyl)-6-methyl-2-oxo-1,2,3,4tetrahydropyrimidine-5-carboxylate

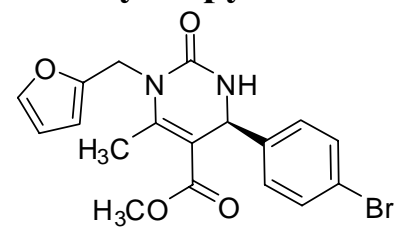

${ }^{1}$ H NMR $\left(400 \mathrm{MHz}, \mathrm{CDCl}_{3}\right): \delta 7.32(\mathrm{~d}, J=3.1 \mathrm{~Hz}, 1 \mathrm{H}), 7.31(\mathrm{~d}, J=$ $8.6 \mathrm{~Hz}, 2 \mathrm{H}), 6.96(\mathrm{~d}, J=8.6 \mathrm{~Hz}, 2 \mathrm{H}), 6.37(\mathrm{~d}, J=3.1 \mathrm{~Hz}, 1 \mathrm{H}), 6.32$ $(\mathrm{dd}, J=3.1,2.3 \mathrm{~Hz}, 1 \mathrm{H}), 6.15(\mathrm{~d}, J=3.1 \mathrm{~Hz}, 1 \mathrm{H}), 5.28(\mathrm{~d}, J=3.1 \mathrm{~Hz}$, $1 \mathrm{H}), 5.24(\mathrm{~d}, J=16.4 \mathrm{~Hz}, 1 \mathrm{H}), 4.70(\mathrm{~d}, J=16.4 \mathrm{~Hz}, 1 \mathrm{H}), 3.62(\mathrm{~s}, 3 \mathrm{H})$, $2.59(\mathrm{~s}, 3 \mathrm{H}) ;{ }^{13} \mathbf{C}$ NMR $\left(100 \mathrm{MHz}, \mathrm{CDCl}_{3}\right): \delta 166.2,153.4,150.8$, 148.8, 142.0, 131.7, 128.0, 121.6, 110.5, 108.2, 104.7, 53.4, 51.4, 38.9, 16.3; MS (ESI+) calculated for $\mathrm{C}_{18} \mathrm{H}_{18} \mathrm{BrN}_{2} \mathrm{O}_{4}(\mathrm{M}+1)$ 405.1, found 405.0.

$(S)-26 B d:(S)-5-a c e t y l-4-(4-b r o m o p h e n y l)-1-(f u r a n-2-y l m e t h y l)-6-m e t h y l-3,4-$ dihydropyrimidin-2(1H)-one

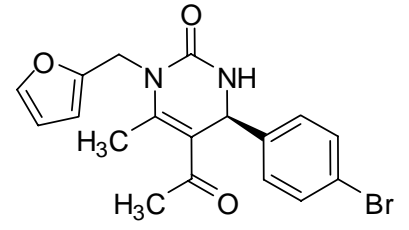

${ }^{1} \mathbf{H}$ NMR $\left(400 \mathrm{MHz}, \mathrm{CDCl}_{3}\right): \delta$ 7.28-7.34 (4H), $6.97(\mathrm{~d}, J=7.8 \mathrm{~Hz}$, $2 \mathrm{H}), 6.32(\mathrm{dd}, J=3.1,2.3 \mathrm{~Hz}, 1 \mathrm{H}), 6.16(\mathrm{~d}, J=3.1 \mathrm{~Hz}, 1 \mathrm{H}), 5.25(\mathrm{~d}, J$ $=3.1 \mathrm{~Hz}, 1 \mathrm{H}), 5.24(\mathrm{~d}, J=16.4 \mathrm{~Hz}, 1 \mathrm{H}), 4.70(\mathrm{~d}, J=16.4 \mathrm{~Hz}, 1 \mathrm{H})$, $2.52(\mathrm{~s}, 3 \mathrm{H}), 2.13(\mathrm{~s}, 3 \mathrm{H}) ; \mathrm{MS}(\mathrm{ESI}+)$ calculated for $\mathrm{C}_{18} \mathrm{H}_{18} \mathrm{BrN}_{2} \mathrm{O}_{3}$ (M+1) 389.1, found 388.9. 
$(S)$-25Be: $(S)$-methyl 4-(4-bromophenyl)-1-(2-methoxyethyl)-6-methyl-2-oxo-1,2,3,4tetrahydropyrimidine-5-carboxylate

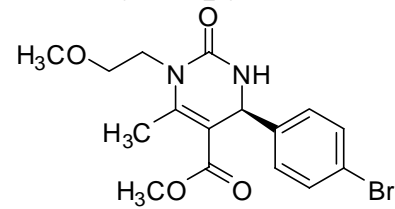

${ }^{1}$ H NMR $\left(400 \mathrm{MHz}, \mathrm{CDCl}_{3}\right): \delta 7.39(\mathrm{~d}, J=8.6 \mathrm{~Hz}, 2 \mathrm{H}), 7.14(\mathrm{~d}, J=$ $8.6 \mathrm{~Hz}, 2 \mathrm{H}), 6.42(\mathrm{~d}, J=3.1 \mathrm{~Hz}, 1 \mathrm{H}), 5.26(\mathrm{~d}, J=3.1 \mathrm{~Hz}, 1 \mathrm{H}), 4.17$ (dt, $J=14.9,4.7 \mathrm{~Hz}, 1 \mathrm{H}), 3.74(\mathrm{ddd}, J=14.9,7.8,3.9 \mathrm{~Hz}, 1 \mathrm{H}), 3.61$ (s, $3 \mathrm{H}), 3.47(\mathrm{dt}, J=10.2,4.7 \mathrm{~Hz}, 1 \mathrm{H}), 3.38(\mathrm{~m}, 1 \mathrm{H}), 3.29(\mathrm{~s}, 3 \mathrm{H}), 2.52(\mathrm{~s}$, $3 \mathrm{H}) ;{ }^{13} \mathrm{C}$ NMR $\left(100 \mathrm{MHz}, \mathrm{CDCl}_{3}\right): \delta 166.3,153.5,150.0,142.4$, $131.6,128.2,121.5,103.8,71.3,58.8,53.4,51.3,42.1,16.5$; MS (ESI+) calculated for $\mathrm{C}_{16} \mathrm{H}_{20} \mathrm{BrN}_{2} \mathrm{O}_{4}(\mathrm{M}+1)$ 383.1, found 383.0.

(S)-26Be: (S)-5-acetyl-4-(4-bromophenyl)-1-(2-methoxyethyl)-6-methyl-3,4dihydropyrimidin-2(1H)-one

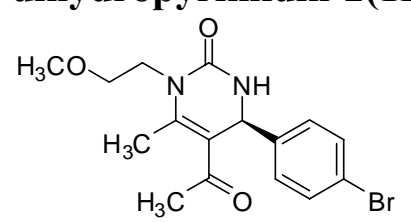

${ }^{1} \mathbf{H}$ NMR $\left(400 \mathrm{MHz}, \mathrm{CDCl}_{3}\right): \delta 7.35(\mathrm{~d}, J=8.6 \mathrm{~Hz}, 2 \mathrm{H}), 7.21(\mathrm{~d}, J=$ $3.1 \mathrm{~Hz}, 1 \mathrm{H}), 7.10(\mathrm{~d}, J=8.6 \mathrm{~Hz}, 2 \mathrm{H}), 5.18(\mathrm{~d}, J=3.1 \mathrm{~Hz}, 1 \mathrm{H}), 4.13(\mathrm{dt}$, $J=14.9,4.7 \mathrm{~Hz}, 1 \mathrm{H}), 3.70(\mathrm{ddd}, J=14.9,7.8,3.9 \mathrm{~Hz}, 1 \mathrm{H}), 3.43(\mathrm{~m}$, 1H), $3.30(\mathrm{~m}, 1 \mathrm{H}), 3.24$ (s, 3H), 2.44 (s, 3H), 2.09 (s, 3H); ${ }^{13} \mathbf{C}$ NMR $\left(100 \mathrm{MHz}, \mathrm{CDCl}_{3}\right): \delta 196.4,153.5,148.8,141.5,131.7,128.2,121.7$, $113.1,71.3,58.7,53.6,41.8,30.4,17.1$; MS (ESI+) calculated for $\mathrm{C}_{16} \mathrm{H}_{20} \mathrm{BrN}_{2} \mathrm{O}_{3}(\mathrm{M}+1) 367.1$, found 366.9.

(S)-25Bf: (S)-methyl 4-(4-bromophenyl)-6-methyl-2-oxo-1-(3,4,5-trimethoxyphenethyl)1,2,3,4-tetrahydropyrimidine-5-carboxylate

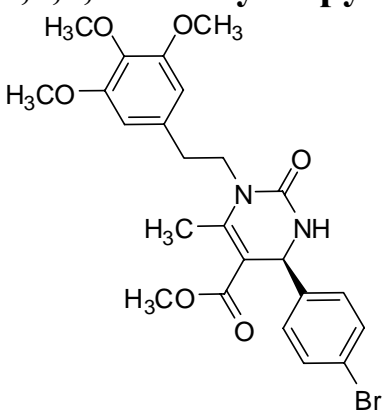

${ }^{1} \mathbf{H}$ NMR $\left(400 \mathrm{MHz}, \mathrm{CDCl}_{3}\right): \delta 7.32(\mathrm{~d}, J=8.6 \mathrm{~Hz}, 2 \mathrm{H}), 7.03(\mathrm{~d}, J=$ $8.6 \mathrm{~Hz}, 2 \mathrm{H}), 6.71(\mathrm{~d}, J=3.1 \mathrm{~Hz}, 1 \mathrm{H}), 6.38(\mathrm{~s}, 2 \mathrm{H}), 5.29(\mathrm{~d}, J=3.1 \mathrm{~Hz}$, $1 \mathrm{H}), 3.75-3.78(11 \mathrm{H}), 3.59(\mathrm{~s}, 3 \mathrm{H}), 2.75(\mathrm{~m}, 2 \mathrm{H}), 2.46(\mathrm{~s}, 3 \mathrm{H}) ;{ }^{13} \mathrm{C}$ NMR $\left(100 \mathrm{MHz}, \mathrm{CDCl}_{3}\right): \delta 166.2,153.4,153.1,148.6,142.3,133.7$, $131.5,127.73,127.65,121.4,105.5,103.7,60.6,55.9,53.0,51.2,43.9$, 36.1, 16.1; MS (ESI+) calculated for $\mathrm{C}_{24} \mathrm{H}_{28} \mathrm{BrN}_{2} \mathrm{O}_{6}(\mathrm{M}+1)$ 519.1, found 518.9 .

(R)-25Ca: (R)-methyl 1-benzyl-6-methyl-2-oxo-4-(3-(trifluoromethyl) phenyl)-1,2,3,4tetrahydropyrimidine-5-carboxylate<smiles>COC(=O)C1=C(C)N(Cc2ccccc2)C(=O)N[C@H]1c1cccc(C(F)(F)F)c1</smiles>

${ }^{1} \mathbf{H}$ NMR (400 MHz, $\left.\mathrm{CDCl}_{3}\right): \delta$ 7.09-7.56 (9H), $6.39(\mathrm{~d}, J=3.1 \mathrm{~Hz}$, $1 \mathrm{H}), 5.50(\mathrm{~d}, J=3.1 \mathrm{~Hz}, 1 \mathrm{H}), 5.23(\mathrm{~d}, J=16.4 \mathrm{~Hz}, 1 \mathrm{H}), 4.82(\mathrm{~d}, J=$ $16.4 \mathrm{~Hz}, 1 \mathrm{H}), 3.62(\mathrm{~s}, 3 \mathrm{H}), 2.46(\mathrm{~s}, 3 \mathrm{H}) ;{ }^{13} \mathbf{C ~ N M R}\left(100 \mathrm{MHz}, \mathrm{CDCl}_{3}\right)$ : $\delta 166.4,153.7,150.0,144.1,137.6,129.8,129.2,128.8,128.6,127.3$, $126.2,124.7$ (q), 123.2 (q), 104.0, 53.2, 51.4, 45.9, 16.5; MS (ESI+) calculated for $\mathrm{C}_{21} \mathrm{H}_{20} \mathrm{~F}_{3} \mathrm{~N}_{2} \mathrm{O}_{3}(\mathrm{M}+1)$ 405.1, found 405.0.

(S)-26Ca: (S)-5-acetyl-1-benzyl-6-methyl-4-(3-(trifluoromethyl)phenyl)-3,4dihydropyrimidin-2(1H)-one 
<smiles>CC(=O)C1=C(C)N(Cc2ccccc2)C(=O)NC1c1cccc(C(F)(F)F)c1</smiles>

${ }^{1} \mathbf{H}$ NMR $\left(400 \mathrm{MHz}, \mathrm{CDCl}_{3}\right): \delta$ 7.02-7.57 (10H), $5.44(\mathrm{~d}, J=3.1 \mathrm{~Hz}$, $1 \mathrm{H}), 5.25(\mathrm{~d}, J=16.4 \mathrm{~Hz}, 1 \mathrm{H}), 4.78(\mathrm{~d}, J=16.4 \mathrm{~Hz}, 1 \mathrm{H}), 2.39(\mathrm{~s}, 3 \mathrm{H})$, $2.13(\mathrm{~s}, 3 \mathrm{H}) ;{ }^{13} \mathrm{C}$ NMR $\left(100 \mathrm{MHz}, \mathrm{CDCl}_{3}\right): \delta 196.3,154.1,148.8$, $143.3,137.5,129.9,129.4,128.7,127.2,126.1,124.8$ (q), 123.2 (q), $113.1,53.4,45.8,30.4,17.2$; MS (ESI+) calculated for $\mathrm{C}_{21} \mathrm{H}_{20} \mathrm{~F}_{3} \mathrm{~N}_{2} \mathrm{O}_{2}$ (M+1) 389.1, found 389.0.

(R)-25Cb: (R)-methyl 1-ethyl-6-methyl-2-oxo-4-(3-(trifluoromethyl)phenyl)-1,2,3,4tetrahydropyrimidine-5-carboxylate<smiles>CCN1C(=O)N[C@H](c2cccc(C(F)(F)F)c2)C(C(=O)OC)=C1C</smiles>

${ }^{1}$ H NMR $\left(400 \mathrm{MHz}, \mathrm{CDCl}_{3}\right): \delta 7.43-7.55(4 \mathrm{H}), 6.54(\mathrm{~d}, J=2.4 \mathrm{~Hz}$, $1 \mathrm{H}), 5.41(\mathrm{~d}, J=2.4 \mathrm{~Hz}, 1 \mathrm{H}), 3.99(\mathrm{~m}, 1 \mathrm{H}), 3.73(\mathrm{~m} .1 \mathrm{H}), 3.65$ (s, $3 \mathrm{H}), 2.56(\mathrm{~s}, 3 \mathrm{H}), 1.22(\mathrm{t}, J=7.0 \mathrm{~Hz}, 3 \mathrm{H})$; MS (ESI+) calculated for $\mathrm{C}_{16} \mathrm{H}_{18} \mathrm{~F}_{3} \mathrm{~N}_{2} \mathrm{O}_{3}(\mathrm{M}+1)$ 343.1, found 343.0.

(S)-26Cb: (S)-5-acetyl-1-ethyl-6-methyl-4-(3-(trifluoromethyl)phenyl)-3,4dihydropyrimidin-2(1H)-one<smiles>CCN1C(=O)NC(c2cccc(C(F)(F)F)c2)C(C(C)=O)=C1C</smiles>

${ }^{1} \mathbf{H}$ NMR $\left(400 \mathrm{MHz}, \mathrm{CDCl}_{3}\right): \delta 7.37-7.55(4 \mathrm{H}), 6.73(\mathrm{~d}, J=2.4 \mathrm{~Hz}$, $1 \mathrm{H}), 5.38(\mathrm{~d}, J=2.4 \mathrm{~Hz}, 1 \mathrm{H}), 3.93(\mathrm{~m}, 1 \mathrm{H}), 3.69(\mathrm{~m} .1 \mathrm{H}), 2.50(\mathrm{~s}, 3 \mathrm{H})$, $2.16(\mathrm{~s}, 3 \mathrm{H}), 1.18(\mathrm{t}, J=7.0 \mathrm{~Hz}, 3 \mathrm{H}) ;{ }^{13} \mathbf{C ~ N M R}\left(100 \mathrm{MHz}, \mathrm{CDCl}_{3}\right): \delta$ $196.1,153.4,148.4,143.3,131.4,131.1,129.7,129.5,124.9$ (q), 123.1 (q), 113.0, 53.8, 37.7, 30.5, 16.7, 14.8; MS (ESI+) calculated for $\mathrm{C}_{16} \mathrm{H}_{18} \mathrm{~F}_{3} \mathrm{~N}_{2} \mathrm{O}_{2}(\mathrm{M}+1)$ 327.1, found 327.0.

(S)-25Cc: (S)-methyl 1-(2-ethoxy-2-oxoethyl)-6-methyl-2-oxo-4-(3(trifluoromethyl)phenyl)-1,2,3,4-tetrahydropyrimidine-5-carboxylate<smiles>CCOC(=O)CN1C(=O)NC(c2cccc(C(F)(F)F)c2)C(C(=O)OC)=C1C</smiles>

${ }^{1}$ H NMR (400 MHz, $\left.\mathrm{CDCl}_{3}\right): \delta$ 7.40-7.65 (4H), $5.90(\mathrm{~d}, J=3.1 \mathrm{~Hz}$, $1 \mathrm{H}), 5.45(\mathrm{~d}, J=3.1 \mathrm{~Hz}, 1 \mathrm{H}), 4.70(\mathrm{~d}, J=18.0 \mathrm{~Hz}, 1 \mathrm{H}), 4.35(\mathrm{~d}, J=$ $18.0 \mathrm{~Hz}, 1 \mathrm{H}), 4.21$ (q, J=7.0 Hz, 2H), $3.63(\mathrm{~s}, 3 \mathrm{H}), 2.44(\mathrm{~s}, 3 \mathrm{H}), 1.27$ $(\mathrm{t}, J=7.0 \mathrm{~Hz}, 3 \mathrm{H}) ;{ }^{13} \mathrm{C}$ NMR $\left(100 \mathrm{MHz}, \mathrm{CDCl}_{3}\right): \delta$ 169.0, 166.0, 152.9, 148.6, 144.1, 130.0, 129.3, 124.6 (q), 123.6 (q), 103.8, 61.6, 53.9 , 51.3, 44.0, 16.1, 14.0; MS (ESI+) calculated for $\mathrm{C}_{18} \mathrm{H}_{20} \mathrm{~F}_{3} \mathrm{~N}_{2} \mathrm{O}_{5}$ $(\mathrm{M}+1) 401.1$, found 400.9 .

(S)-26Cc: (S)-ethyl 2-(5-acetyl-6-methyl-2-oxo-4-(3-(trifluoromethyl)phenyl)-3,4dihydropyrimidin-1(2H)-yl)acetate 
<smiles>CCOC(=O)CN1C(=O)N[C@H](c2cccc(C(F)(F)F)c2)C(C(C)=O)=C1C</smiles>

${ }^{1}$ H NMR (400 MHz, $\left.\mathrm{CDCl}_{3}\right): \delta$ 7.40-7.62 (5H), $6.46(\mathrm{~d}, J=3.1 \mathrm{~Hz}$, $1 \mathrm{H}), 5.44(\mathrm{~d}, J=3.1 \mathrm{~Hz}, 1 \mathrm{H}), 4.69(\mathrm{~d}, J=18.0 \mathrm{~Hz}, 1 \mathrm{H}), 4.39(\mathrm{~d}, J=$ $18.0 \mathrm{~Hz}, 1 \mathrm{H}), 4.23$ (q, $J=7.0 \mathrm{~Hz}, 2 \mathrm{H}), 2.39$ (s, 3H), 2.18 (s, 3H), 1.29 $(\mathrm{t}, J=7.0 \mathrm{~Hz}, 3 \mathrm{H}) ;{ }^{13} \mathbf{C}$ NMR $\left(100 \mathrm{MHz}, \mathrm{CDCl}_{3}\right): \delta 196.3,169.0$, $152.7,146.9,143.1,141.5,130.2,129.7,125.2$ (q), 123.6 (q), 113.3, $61.7, \quad 54.5,44.1,30.6,16.9,14.1 ;$ MS (ESI+) calculated for $\mathrm{C}_{18} \mathrm{H}_{20} \mathrm{~F}_{3} \mathrm{~N}_{2} \mathrm{O}_{5}(\mathrm{M}+1) 385.1$, found 385.1.

(S)-25Cd: (S)-methyl 1-(furan-2-ylmethyl)-6-methyl-2-oxo-4-(3-(trifluoromethyl)phenyl)1,2,3,4-tetrahydropyrimidine-5-carboxylate<smiles>COC(=O)C1=C(C)N(Cc2ccco2)C(=O)N[C]1c1cccc(C(F)(F)F)c1</smiles>

${ }^{1} \mathbf{H}$ NMR $\left(400 \mathrm{MHz}, \mathrm{CDCl}_{3}\right): \delta 7.47(\mathrm{~d}, J=3.1 \mathrm{~Hz}, 1 \mathrm{H}), 7.25-7.34$ $(4 \mathrm{H}), 6.13-6.45(3 \mathrm{H}), 5.40(\mathrm{~d}, J=3.1 \mathrm{~Hz}, 1 \mathrm{H}), 5.27(\mathrm{~d}, J=16.4 \mathrm{~Hz}$, 1H), $4.71(\mathrm{~d}, J=16.4 \mathrm{~Hz}, 1 \mathrm{H}), 3.62(\mathrm{~s}, 3 \mathrm{H}), 2.60(\mathrm{~s}, 3 \mathrm{H}) ;{ }^{13} \mathbf{C}$ NMR $\left(100 \mathrm{MHz}, \mathrm{CDCl}_{3}\right): \delta 166.1,153.2,150.6,149.3,144.1,142.1,141.8$, $129.7,129.2,124.6$ (q), 123.2 (q), 110.4, 108.0, 106.8, 104.2, 53.7, 51.4, 39.0, 16.3; MS (ESI+) calculated for $\mathrm{C}_{19} \mathrm{H}_{18} \mathrm{~F}_{3} \mathrm{~N}_{2} \mathrm{O}_{4}(\mathrm{M}+1)$ 395.1, found 394.9.

(S)-26Cd: (S)-5-acetyl-1-(furan-2-ylmethyl)-6-methyl-4-(3-(trifluoromethyl)phenyl)-3,4dihydropyrimidin-2(1H)-one<smiles>CC(=O)C1=C(C)N(Cc2ccco2)C(=O)NC1c1cccc(C(F)(F)F)c1</smiles>

${ }^{1}$ H NMR $\left(400 \mathrm{MHz}, \mathrm{CDCl}_{3}\right): \delta$ 7.30-7.50 (5H), 6.64 (br., 1H), 6.12$6.30(2 \mathrm{H}), 5.37(\mathrm{~d}, J=3.1 \mathrm{~Hz}, 1 \mathrm{H}), 5.26(\mathrm{~d}, J=16.4 \mathrm{~Hz}, 1 \mathrm{H}), 4.70(\mathrm{~d}$, $J=16.4 \mathrm{~Hz}, 1 \mathrm{H}), 2.53(\mathrm{~s}, 3 \mathrm{H}), 2.15(\mathrm{~s}, 3 \mathrm{H})$; MS (ESI+) calculated for $\mathrm{C}_{19} \mathrm{H}_{18} \mathrm{~F}_{3} \mathrm{~N}_{2} \mathrm{O}_{3}(\mathrm{M}+1)$ 379.1, found 379.0.

(R)-25Ce: (R)-methyl 1-(2-methoxyethyl)-6-methyl-2-oxo-4-(3-(trifluoromethyl)phenyl)1,2,3,4-tetrahydropyrimidine-5-carboxylate

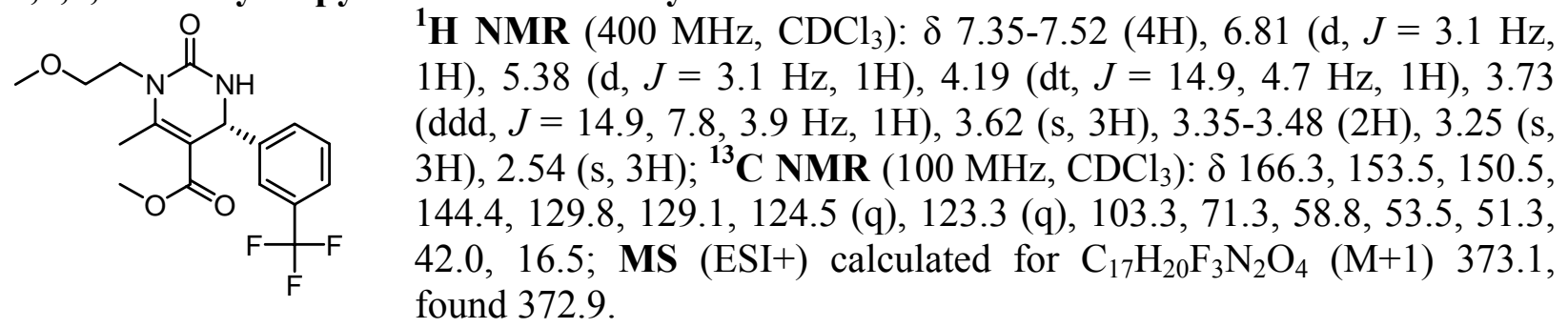

(S)-26Cf: (S)-5-acetyl-6-methyl-4-(3-(trifluoromethyl)phenyl)-1-(3,4,5trimethoxyphenethyl)-3,4-dihydropyrimidin-2(1H)-one 


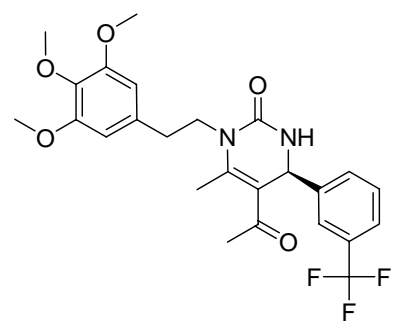

${ }^{1}$ H NMR $\left(400 \mathrm{MHz}, \mathrm{CDCl}_{3}\right): \delta$ 7.35-7.53 $(4 \mathrm{H}), 7.03(\mathrm{~d}, J=3.1 \mathrm{~Hz}$, $1 \mathrm{H}), 6.38(\mathrm{~s}, 2 \mathrm{H}), 5.40(\mathrm{~d}, J=3.1 \mathrm{~Hz}, 1 \mathrm{H}), 3.73-3.83(14 \mathrm{H}), 2.75(\mathrm{~m}$, 2H), $2.45(\mathrm{~s}, 3 \mathrm{H}), 2.13(\mathrm{~s}, 3 \mathrm{H}) ;{ }^{13} \mathbf{C}$ NMR $\left(100 \mathrm{MHz}, \mathrm{CDCl}_{3}\right): \delta 196.3$, $153.3,153.2,147.9,143.3,133.7,129.6,129.4,124.8$ (q), 123.0 (q), 113.3, 105.4, 60.7, 55.9, 53.6, 44.0, 36.1, 30.4, 16.9; MS (ESI+) calculated for $\mathrm{C}_{25} \mathrm{H}_{28} \mathrm{~F}_{3} \mathrm{~N}_{2} \mathrm{O}_{5}(\mathrm{M}+1)$ 493.2, found 493.0.

(R)-25Da: (R)-methyl 1-benzyl-4-(furan-2-yl)-6-methyl-2-oxo-1,2,3,4tetrahydropyrimidine-5-carboxylate<smiles>COC(=O)C1=C(C)N(Cc2ccccc2)C(=O)NC1c1ccco1</smiles>

${ }^{1}$ H NMR $\left(400 \mathrm{MHz}, \mathrm{CDCl}_{3}\right): \delta$ 7.15-7.35 $(6 \mathrm{H}), 6.30(\mathrm{dd}, J=3.1,1.6$ $\mathrm{Hz}, 1 \mathrm{H}), 6.09(\mathrm{~d}, J=3.1 \mathrm{~Hz}, 1 \mathrm{H}), 5.88(\mathrm{~d}, J=3.1 \mathrm{~Hz}, 1 \mathrm{H}), 5.48(\mathrm{~d}, J=$ $3.1 \mathrm{~Hz}, 1 \mathrm{H}), 5.30(\mathrm{~d}, J=16.4 \mathrm{~Hz}, 1 \mathrm{H}), 4.80(\mathrm{~d}, J=16.4 \mathrm{~Hz}, 1 \mathrm{H}), 3.68$ $(\mathrm{s}, 3 \mathrm{H}), 2.44(\mathrm{~s}, 3 \mathrm{H})$; MS $(\mathrm{ESI}+)$ calculated for $\mathrm{C}_{18} \mathrm{H}_{19} \mathrm{~N}_{2} \mathrm{O}_{4}(\mathrm{M}+1)$ 327.1, found 326.9 .

(R)-25Dd: $(R)$-methyl 4-(furan-2-yl)-1-(furan-2-ylmethyl)-6-methyl-2-oxo-1,2,3,4tetrahydropyrimidine-5-carboxylate<smiles>COC(=O)C1=C(C)N(Cc2ccco2)C(=O)NC1c1ccco1</smiles>

${ }^{1}$ H NMR $\left(400 \mathrm{MHz}, \mathrm{CDCl}_{3}\right): \delta 7.32(\mathrm{~d}, J=1.6 \mathrm{~Hz}, 1 \mathrm{H}), 7.28(\mathrm{~d}, J=$ $1.6 \mathrm{~Hz}, 1 \mathrm{H}), 6.30(\mathrm{dd}, J=3.1,1.6 \mathrm{~Hz}, 1 \mathrm{H}), 6.22(\mathrm{dd}, J=3.1,1.6 \mathrm{~Hz}$, $1 \mathrm{H}), 6.19(\mathrm{~d}, J=3.1 \mathrm{~Hz}, 1 \mathrm{H}), 6.10(\mathrm{~d}, J=3.1 \mathrm{~Hz}, 1 \mathrm{H}), 6.00(\mathrm{~d}, J=3.1$ $\mathrm{Hz}, 1 \mathrm{H}), 5.41(\mathrm{~d}, J=3.1 \mathrm{~Hz}, 1 \mathrm{H}), 5.18(\mathrm{~d}, J=16.4 \mathrm{~Hz}, 1 \mathrm{H}), 4.78(\mathrm{~d}, J$ $=16.4 \mathrm{~Hz}, 1 \mathrm{H}), 3.69(\mathrm{~s}, 3 \mathrm{H}), 2.59(\mathrm{~s}, 3 \mathrm{H})$; MS $(\mathrm{ESI}+)$ calculated for $\mathrm{C}_{16} \mathrm{H}_{17} \mathrm{~N}_{2} \mathrm{O}_{5}(\mathrm{M}+1)$ 317.1, found 316.9 .

(S)-26Dd: (S)-5-acetyl-4-(furan-2-yl)-1-(furan-2-ylmethyl)-6-methyl-3,4-dihydropyrimidin2(1H)-one<smiles>CC(=O)C1=C(C)N(Cc2ccco2)C(=O)NC1c1ccco1</smiles>

${ }^{1}$ H NMR $\left(400 \mathrm{MHz}, \mathrm{CDCl}_{3}\right): \delta$ 7.15-7.32 (3H), 6.12-6.28 (3H), 5.99 $(\mathrm{d}, J=3.1 \mathrm{~Hz}, 1 \mathrm{H}), 5.31(\mathrm{~d}, J=3.1 \mathrm{~Hz}, 1 \mathrm{H}), 5.11(\mathrm{~d}, J=16.4 \mathrm{~Hz}, 1 \mathrm{H})$, $4.73(\mathrm{~d}, J=16.4 \mathrm{~Hz}, 1 \mathrm{H}), 2.49$ (s, 3H), 2.19 (s, 3H); MS (ESI+) calculated for $\mathrm{C}_{16} \mathrm{H}_{17} \mathrm{~N}_{2} \mathrm{O}_{4}(\mathrm{M}+1)$ 301.1, found 300.9.

(S)-25Dc: (S)-methyl 1-(2-ethoxy-2-oxoethyl)-4-(furan-2-yl)-6-methyl-2-oxo-1,2,3,4tetrahydropyrimidine-5-carboxylate<smiles>CCOC(=O)CN1C(=O)N[C@@H](c2ccco2)C(C(=O)OC)=C1C</smiles>

${ }^{1}$ H NMR $\left(400 \mathrm{MHz}, \mathrm{CDCl}_{3}\right): \delta 7.31(\mathrm{~d}, J=1.6 \mathrm{~Hz}, 1 \mathrm{H}), 6.28(\mathrm{dd}, J=$ $3.1,1.6 \mathrm{~Hz}, 1 \mathrm{H}), 6.19(\mathrm{~d}, J=3.1 \mathrm{~Hz}, 1 \mathrm{H}), 5.77(\mathrm{~d}, J=3.1 \mathrm{~Hz}, 1 \mathrm{H})$, $5.47(\mathrm{~d}, J=3.1 \mathrm{~Hz}, 1 \mathrm{H}), 4.48(\mathrm{AB}, J=18.0,2 \mathrm{H}), 4.21(\mathrm{q}, J=7.0 \mathrm{~Hz}$, $2 \mathrm{H}), 3.69(\mathrm{~s}, 3 \mathrm{H}), 2.45(\mathrm{~s}, 3 \mathrm{H}), 1.27(\mathrm{t}, J=7.0 \mathrm{~Hz}, 3 \mathrm{H}) ;{ }^{13} \mathbf{C}$ NMR $(100$ $\left.\mathrm{MHz}, \mathrm{CDCl}_{3}\right): \delta 168.9,165.9,154.4,153.5,149.6,142.3,110.3,106.0$, $101.9,61.6,51.5,47.7,44.4,16.1,14.1$; MS (ESI+) calculated for $\mathrm{C}_{15} \mathrm{H}_{19} \mathrm{~N}_{2} \mathrm{O}_{6}(\mathrm{M}+1) 323.1$, found 322.8 .

(R)-26Dc: (R)-ethyl 2-(5-acetyl-4-(furan-2-yl)-6-methyl-2-oxo-3,4-dihydropyrimidin-1(2H)yl)acetate 


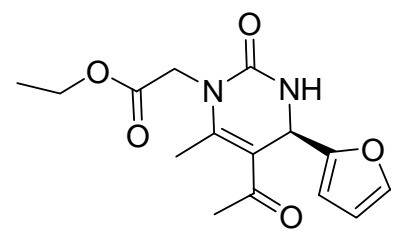

${ }^{1}$ H NMR $\left(400 \mathrm{MHz}, \mathrm{CDCl}_{3}\right): \delta 7.34(\mathrm{~d}, J=1.6 \mathrm{~Hz}, 1 \mathrm{H}), 6.29(\mathrm{dd}, J=$ $3.1,1.6 \mathrm{~Hz}, 1 \mathrm{H}), 6.23(\mathrm{~d}, J=3.1 \mathrm{~Hz}, 1 \mathrm{H}), 5.93(\mathrm{~d}, J=3.1 \mathrm{~Hz}, 1 \mathrm{H}), 5.41$ $(\mathrm{d}, J=3.1 \mathrm{~Hz}, 1 \mathrm{H}), 4.46(\mathrm{~s}, 2 \mathrm{H}), 4.21(\mathrm{q}, J=7.0 \mathrm{~Hz}, 2 \mathrm{H}), 2.37(\mathrm{~s}, 3 \mathrm{H})$, $2.23(\mathrm{~s}, 3 \mathrm{H}), 1.27(\mathrm{t}, J=7.0 \mathrm{~Hz}, 3 \mathrm{H}) ;{ }^{13} \mathbf{C ~ N M R}\left(100 \mathrm{MHz}, \mathrm{CDCl}_{3}\right): \delta$ $214.0,168.9,153.9,153.2,142.7,110.5,106.9,101.9,61.6,48.6,44.3$, 29.9, 16.6, 14.1; MS (ESI+) calculated for $\mathrm{C}_{15} \mathrm{H}_{19} \mathrm{~N}_{2} \mathrm{O}_{5}(\mathrm{M}+1)$ 307.1, found 306.9 .

(R)-26De: $(R)-5$-acetyl-4-(furan-2-yl)-1-(2-methoxyethyl)-6-methyl-3,4-dihydropyrimidin2(1H)-one<smiles>COCCN1C(=O)NC(c2ccco2)C(C(C)=O)=C1C</smiles>

${ }^{1}$ H NMR $\left(400 \mathrm{MHz}, \mathrm{CDCl}_{3}\right): \delta 7.31(\mathrm{~d}, J=1.6 \mathrm{~Hz}, 1 \mathrm{H}), 6.19(\mathrm{~d}, J=$ $3.1 \mathrm{~Hz}, 1 \mathrm{H}), 6.27(\mathrm{dd}, J=3.1,1.6 \mathrm{~Hz}, 1 \mathrm{H}), 6.13(\mathrm{~d}, J=3.1 \mathrm{~Hz}, 1 \mathrm{H})$, $5.31(\mathrm{~d}, J=3.1 \mathrm{~Hz}, 1 \mathrm{H}), 4.13(\mathrm{dt}, J=14.9,4.7 \mathrm{~Hz}, 1 \mathrm{H}), 3.73(\mathrm{ddd}, J=$ 14.9, 7.8, $3.9 \mathrm{~Hz}, 1 \mathrm{H}), 3.47(\mathrm{~m}, 1 \mathrm{H}), 3.35(\mathrm{~m}, 1 \mathrm{H}), 3.27(\mathrm{~s}, 3 \mathrm{H}), 2.48$ $(\mathrm{s}, 3 \mathrm{H}), 2.21(\mathrm{~s}, 3 \mathrm{H}) ; \mathrm{MS}(\mathrm{ESI}+)$ calculated for $\mathrm{C}_{14} \mathrm{H}_{19} \mathrm{~N}_{2} \mathrm{O}_{4}(\mathrm{M}+1)$ 279.1, found 278.9.

(S)-25De: (S)-methyl 4-(furan-2-yl)-1-(2-methoxyethyl)-6-methyl-2-oxo-1,2,3,4tetrahydropyrimidine-5-carboxylate

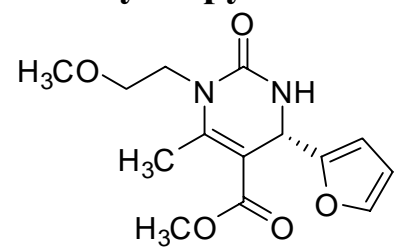

${ }^{1}$ H NMR $\left(400 \mathrm{MHz}, \mathrm{CDCl}_{3}\right): \delta 7.31(\mathrm{~d}, J=1.6 \mathrm{~Hz}, 1 \mathrm{H}), 6.24(\mathrm{dd}, J=$ $3.1,1.6 \mathrm{~Hz}, 1 \mathrm{H}), 6.10$ (d, $J=3.1 \mathrm{~Hz}, 1 \mathrm{H}), 5.93$ (br., $1 \mathrm{H}), 5.39$ (d, $J=$ $3.1 \mathrm{~Hz}, 1 \mathrm{H}), 4.14(\mathrm{dt}, J=14.9,4.7 \mathrm{~Hz}, 1 \mathrm{H}), 3.74(\mathrm{ddd}, J=14.9,7.8$, $4.7 \mathrm{~Hz}, 1 \mathrm{H}), 3.68(\mathrm{~s}, 3 \mathrm{H}), 3.50(\mathrm{dt}, J=10.2,4.7 \mathrm{~Hz}, 1 \mathrm{H}), 3.39$ (ddd, $J=$ 10.2, 7.8, 4.7 Hz, 1H), $3.28(\mathrm{~s}, 3 \mathrm{H}), 2.57(\mathrm{~s}, 3 \mathrm{H}) ;{ }^{13} \mathbf{C}$ NMR $(100 \mathrm{MHz}$, $\left.\mathrm{CDCl}_{3}\right): \delta 166.2,154.8,154.1,151.2,142.2,110.2,105.8,101.8,71.5$, $58.8,51.4,47.5,42.2,16.4$; MS (ESI+) calculated for $\mathrm{C}_{14} \mathrm{H}_{19} \mathrm{~N}_{2} \mathrm{O}_{5}$ $(\mathrm{M}+1)$ 295.1, found 274.9 .

(R)-25Ef: (R)-methyl 6-methyl-2-oxo-4-(thiophen-2-yl)-1-(3,4,5-trimethoxyphenethyl)1,2,3,4-tetrahydropyrimidine-5-carboxylate

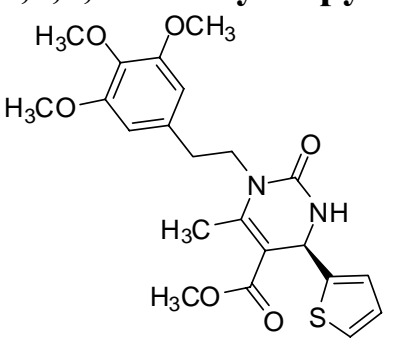

${ }^{1}$ H NMR (400 MHz, $\left.\mathrm{CDCl}_{3}\right): \delta 7.16(\mathrm{~m}, 1 \mathrm{H}), 6.88(\mathrm{~m}, 2 \mathrm{H}), 6.42(\mathrm{~s}$, $2 \mathrm{H}), 6.38(\mathrm{~d}, J=3.1 \mathrm{~Hz}, 1 \mathrm{H}), 5.63(\mathrm{~d}, J=3.1 \mathrm{~Hz}, 1 \mathrm{H}), 4.13(\mathrm{~m}, 1 \mathrm{H})$, 3.69-3.74 (10H), $3.73(\mathrm{~s}, 3 \mathrm{H}), 2.81(\mathrm{~m}, 2 \mathrm{H}), 2.49(\mathrm{~s}, 3 \mathrm{H}) ;{ }^{13} \mathbf{C}$ NMR $\left(100 \mathrm{MHz}_{\mathrm{CDCl}}\right): \delta 166.0,153.8,153.2,149.0,147.2,134.0,126.7$, 127.0, 124.6, 123.7, 105.6, 104.8, 60.8, 56.0, 51.4, 49.4, 44.3, 36.4, 16.1; MS (ESI+) calculated for $\mathrm{C}_{22} \mathrm{H}_{17} \mathrm{~N}_{2} \mathrm{O}_{6} \mathrm{~S}(\mathrm{M}+1)$ 447.2, found 446.9 .

(R)-26Ee: $(R)-5$-acetyl-1-(2-methoxyethyl)-6-methyl-4-(thiophen-2-yl)-3,4dihydropyrimidin-2(1H)-one<smiles>COCCN1C(=O)NC(c2cccs2)C(C(C)=O)=C1C</smiles>

${ }^{1} \mathbf{H}$ NMR (400 MHz, $\left.\mathrm{CDCl}_{3}\right): \delta 7.18$ (br., $\left.1 \mathrm{H}\right), 7.12(\mathrm{~d}, J=4.7 \mathrm{~Hz}, 1 \mathrm{H})$, 6.82-6.91 (2H), $5.49(\mathrm{~d}, J=3.1 \mathrm{~Hz}, 1 \mathrm{H}), 4.13(\mathrm{dt}, J=14.9,4.7 \mathrm{~Hz}$, $1 \mathrm{H}), 3.71(\mathrm{~m}, 1 \mathrm{H}), 3.46(\mathrm{~m}, 1 \mathrm{H}), 3.33(\mathrm{~m}, 1 \mathrm{H}), 3.22(\mathrm{~s}, 3 \mathrm{H}), 2.45(\mathrm{~s}$, 3H), $2.18(\mathrm{~s}, 3 \mathrm{H}) ;{ }^{13} \mathrm{C}$ NMR $\left(100 \mathrm{MHz}, \mathrm{CDCl}_{3}\right): \delta 196.1,153.7,149.0$, 146.7, 126.9, 125.2, 124.4, 113.9, 71.4, 58.7, 49.6, 42.1, 30.3, 17.0; MS (ESI+) calculated for $\mathrm{C}_{14} \mathrm{H}_{19} \mathrm{~N}_{2} \mathrm{O}_{3} \mathrm{~S}(\mathrm{M}+1)$ 295.1, found 294.8. 
(R)-26Ef: $(R)$-5-acetyl-1-(cyclopropylmethyl)-6-methyl-4-(thiophen-2-yl)-3,4dihydropyrimidin-2(1H)-one<smiles>CC(=O)C1=C(C)N(CC2CC2)C(=O)N[C@H]1c1cccs1</smiles>

${ }^{1} \mathbf{H}$ NMR $\left(400 \mathrm{MHz}, \mathrm{CDCl}_{3}\right): \delta 7.18(\mathrm{~d}, J=5.5 \mathrm{~Hz}, 1 \mathrm{H}), 6.88-6.96$ $(2 \mathrm{H}), 6.78$ (br. 1H), $5.53(\mathrm{~d}, J=3.1 \mathrm{~Hz}, 1 \mathrm{H}), 3.83$ (dd, $J=14.9,7.8 \mathrm{~Hz}$, $1 \mathrm{H}), 3.57$ (dd, $J=14.9,5.5 \mathrm{~Hz}, 1 \mathrm{H}), 2.52(\mathrm{~s}, 3 \mathrm{H}), 2.22(\mathrm{~s}, 3 \mathrm{H}), 0.96$ $(\mathrm{m}, 1 \mathrm{H}), 0.48(\mathrm{~m}, 1 \mathrm{H}), 0.41(\mathrm{~m}, 1 \mathrm{H}), 0.28(\mathrm{~m}, 2 \mathrm{H}) ;{ }^{13} \mathbf{C}$ NMR $(100$ $\left.\mathrm{MHz}, \mathrm{CDCl}_{3}\right): \delta 196.0,154.1,148.5,146.8,126.8,125.2,124.4,114.1$, $49.8, \quad 46.2, \quad 30.2, \quad 16.9, \quad 11.3,4.0 ; \quad$ MS (ESI+) calculated for $\mathrm{C}_{15} \mathrm{H}_{19} \mathrm{~N}_{2} \mathrm{O}_{2} \mathrm{~S}(\mathrm{M}+1)$ 291.1, found 291.0.

(R)-26Ea: (R)-5-acetyl-1-benzyl-6-methyl-4-(thiophen-2-yl)-3,4-dihydropyrimidin-2(1H)one<smiles>CC(=O)C1=C(C)N(Cc2ccccc2)C(=O)NC1c1cccs1</smiles>

${ }^{1}$ H NMR (400 MHz, $\left.\mathrm{CDCl}_{3}\right): \delta$ 6.88-7.29 (9H), $5.62(\mathrm{~d}, J=3.1 \mathrm{~Hz}$, $1 \mathrm{H}), 5.21(\mathrm{~d}, J=16.4 \mathrm{~Hz}, 1 \mathrm{H}), 4.85(\mathrm{~d}, J=16.4 \mathrm{~Hz}, 1 \mathrm{H}), 2.38(\mathrm{~s}, 3 \mathrm{H})$, $2.22(\mathrm{~s}, 3 \mathrm{H}) ;{ }^{13} \mathrm{C}$ NMR $\left(100 \mathrm{MHz}, \mathrm{CDCl}_{3}\right): \delta 196.0,154.2,148.8$, $146.8,137.5,128.6,127.1,126.9,126.2,125.3,124.5,114.1,49.8$, 46.0, 30.2, 17.0; MS (ESI+) calculated for $\mathrm{C}_{18} \mathrm{H}_{19} \mathrm{~N}_{2} \mathrm{O}_{2} \mathrm{~S}(\mathrm{M}+1)$ 327.1, found 326.9 .

$(R)-25 E d:(R)-m e t h y l$ 1-(furan-2-ylmethyl)-6-methyl-2-oxo-4-(thiophen-2-yl)-1,2,3,4tetrahydropyrimidine-5-carboxylate<smiles>COC(=O)C1=C(C)N(Cc2ccco2)C(=O)NC1c1cccs1</smiles>

${ }^{1}$ H NMR $\left(400 \mathrm{MHz}, \mathrm{CDCl}_{3}\right): \delta 7.28(\mathrm{~d}, J=3.1 \mathrm{~Hz}, 1 \mathrm{H}), 7.12(\mathrm{~d}, J=$ $5.5 \mathrm{~Hz}, 1 \mathrm{H}), 6.79-6.84(2 \mathrm{H}), 6.27(\mathrm{~m}, 2 \mathrm{H}), 6.15(\mathrm{~m}, 1 \mathrm{H}), 5.59(\mathrm{~d}, J=$ $3.1 \mathrm{~Hz}, 1 \mathrm{H}), 5.15(\mathrm{~d}, J=16.4 \mathrm{~Hz}, 1 \mathrm{H}), 4.76(\mathrm{~d}, J=16.4 \mathrm{~Hz}, 1 \mathrm{H}), 3.69$ $(\mathrm{s}, 3 \mathrm{H}), 2.56(\mathrm{~s}, 3 \mathrm{H})$; MS $(\mathrm{ESI}+)$ calculated for $\mathrm{C}_{16} \mathrm{H}_{17} \mathrm{~N}_{2} \mathrm{O}_{4} \mathrm{~S}(\mathrm{M}+1)$ 333.1, found 332.8 .

(S)-26Ed: (S)-5-acetyl-1-(furan-2-ylmethyl)-6-methyl-4-(thiophen-2-yl)-3,4dihydropyrimidin-2(1H)-one<smiles>CC(=O)C1=C(C)N(Cc2ccco2)C(=O)N[C@H]1c1cccs1</smiles>

${ }^{1} \mathbf{H}$ NMR $\left(400 \mathrm{MHz}, \mathrm{CDCl}_{3}\right): \delta 7.32(\mathrm{~d}, J=3.1 \mathrm{~Hz}, 1 \mathrm{H}), 7.15(\mathrm{~d}, J=$ $5.5 \mathrm{~Hz}, 1 \mathrm{H}), 6.82-6.88(2 \mathrm{H}), 6.66($ br. $1 \mathrm{H}), 6.28(\mathrm{~m}, 1 \mathrm{H}), 6.16(\mathrm{~m}, 1 \mathrm{H})$, $5.55(\mathrm{~d}, J=3.1 \mathrm{~Hz}, 1 \mathrm{H}), 5.13(\mathrm{~d}, J=16.4 \mathrm{~Hz}, 1 \mathrm{H}), 4.77(\mathrm{~d}, J=16.4$ $\mathrm{Hz}, 1 \mathrm{H}), 2.52(\mathrm{~s}, 3 \mathrm{H}), 2.20(\mathrm{~s}, 3 \mathrm{H}) ; \mathrm{MS}(\mathrm{ESI}+)$ calculated for $\mathrm{C}_{16} \mathrm{H}_{17} \mathrm{~N}_{2} \mathrm{O}_{3} \mathrm{~S}(\mathrm{M}+1) 317.1$, found 316.8 .

(R)-26Eb: $(R)-5$-acetyl-1-ethyl-6-methyl-4-(thiophen-2-yl)-3,4-dihydropyrimidin-2(1H)-one<smiles>CCN1C(=O)N[C@H](c2cccs2)C(C(C)=O)=C1C</smiles>

${ }^{1}$ H NMR $\left(400 \mathrm{MHz}, \mathrm{CDCl}_{3}\right): \delta 7.15(\mathrm{~d}, J=5.5 \mathrm{~Hz}, 1 \mathrm{H}), 6.98$ (br. $\left.1 \mathrm{H}\right)$, 6.86-6.92 (2H), $5.52(\mathrm{~d}, J=3.1 \mathrm{~Hz}, 1 \mathrm{H}), 3.89(\mathrm{~m}, 1 \mathrm{H}), 3.67(\mathrm{~m}, .1 \mathrm{H})$, $2.48(\mathrm{~s}, 3 \mathrm{H}), 2.19(\mathrm{~s}, 3 \mathrm{H}), 1.16(\mathrm{t}, J=7.0 \mathrm{~Hz}, 3 \mathrm{H}) ;{ }^{13} \mathbf{C}$ NMR (100 $\left.\mathrm{MHz}, \mathrm{CDCl}_{3}\right): \delta 195.9,153.7,148.4,146.9,126.9,125.1,124.3,113.7$, 49.6, 37.7, 30.1, 16.4, 14.8; MS (ESI+) calculated for $\mathrm{C}_{16} \mathrm{H}_{17} \mathrm{~N}_{2} \mathrm{O}_{3} \mathrm{~S}$ (M+1) 265.1, found 264.8. 
(R)-25Ee: $(R)$-methyl 1-(2-methoxyethyl)-6-methyl-2-oxo-4-(thiophen-2-yl)-1,2,3,4tetrahydropyrimidine-5-carboxylate<smiles>COCCN1C(=O)NC(c2cccs2)C(C(=O)OC)=C1C</smiles>

${ }^{1} \mathbf{H}$ NMR $\left(400 \mathrm{MHz}, \mathrm{CDCl}_{3}\right): \delta 7.14(\mathrm{~d}, J=4.7 \mathrm{~Hz}, 1 \mathrm{H}), 6.86-6.93$ $(2 \mathrm{H}), 6.41$ (br. $1 \mathrm{H}), 5.59(\mathrm{~d}, J=3.1 \mathrm{~Hz}, 1 \mathrm{H}), 4.16(\mathrm{dt}, J=14.9,4.7 \mathrm{~Hz}$, $1 \mathrm{H}), 3.73(\mathrm{~m}, 1 \mathrm{H}), 3.69(\mathrm{~s}, 3 \mathrm{H}), 3.49(\mathrm{~m}, 1 \mathrm{H}), 3.40(\mathrm{~m}, 1 \mathrm{H}), 3.24(\mathrm{~s}$, $3 \mathrm{H}), 2.53(\mathrm{~s}, 3 \mathrm{H}) ;{ }^{13} \mathbf{C}$ NMR $\left(100 \mathrm{MHz}, \mathrm{CDCl}_{3}\right): \delta 166.1,153.9,150.1$, $147.2,126.6,124.6,123.8,104.5,71.4,58.8,51.3,49.2,42.2,16.4$; MS (ESI+) calculated for $\mathrm{C}_{14} \mathrm{H}_{19} \mathrm{~N}_{2} \mathrm{O}_{4} \mathrm{~S}(\mathrm{M}+1)$ 311.1, found 310.8 .

(S)-25Fa: (S)-methyl 1-benzyl-6-methyl-2-oxo-4-phenethyl-1,2,3,4-tetrahydropyrimidine-5carboxylate<smiles>COC(=O)C1=C(C)N(Cc2ccccc2)C(=O)NC1CCc1ccccc1</smiles>

${ }^{1}$ H NMR $\left(400 \mathrm{MHz}, \mathrm{CDCl}_{3}\right): \delta$ 7.14-7.34 (10H), 6.52 (br. $\left.1 \mathrm{H}\right), 5.18$ $(\mathrm{d}, J=16.4 \mathrm{~Hz}, 1 \mathrm{H}), 4.86(\mathrm{~d}, J=16.4 \mathrm{~Hz}, 1 \mathrm{H}), 4.30(\mathrm{~m}, 1 \mathrm{H}), 3.68(\mathrm{~s}$, $3 \mathrm{H}), 2.78(\mathrm{~m}, 1 \mathrm{H}), 2.64(\mathrm{~m}, 1 \mathrm{H}), 2.39(\mathrm{~s}, 3 \mathrm{H}), 1.89(\mathrm{~m}, 2 \mathrm{H}) ;{ }^{13} \mathbf{C}$ NMR $\left(100 \mathrm{MHz}, \mathrm{CDCl}_{3}\right): \delta 166.4,149.4,141.0,138.0,128.7,128.4,128.3$, $127.2,126.2,126.0,105.0,51.3,49.7,45.9,38.3,30.8,20.9,16.3$; MS $\left(\mathrm{ESI}+\right.$ ) calculated for $\mathrm{C}_{22} \mathrm{H}_{25} \mathrm{~N}_{2} \mathrm{O}_{3}(\mathrm{M}+1) 365.1$, found 364.9 .

(S)-25Ff: (S)-methyl 1-(cyclopropylmethyl)-6-methyl-2-oxo-4-phenethyl-1,2,3,4tetrahydropyrimidine-5-carboxylate

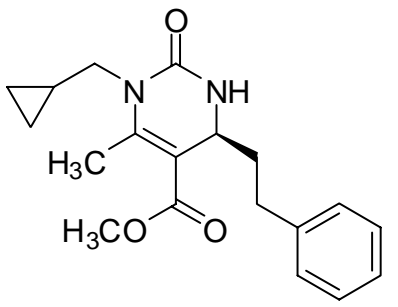

${ }^{1}$ H NMR (400 MHz, $\left.\mathrm{CDCl}_{3}\right): \delta$ 7.16-7.30 (5H), 5.85 (br. 1H), $4.34(\mathrm{~m}$, $1 \mathrm{H}), 3.72-3.68(\mathrm{~m}, 2 \mathrm{H}), 3.69(\mathrm{~s}, 3 \mathrm{H}), 2.76(\mathrm{~m}, 1 \mathrm{H}), 2.64(\mathrm{~m}, 1 \mathrm{H}), 2.29$ $(\mathrm{s}, 3 \mathrm{H}), 1.90(\mathrm{~m}, 2 \mathrm{H}), 0.96(\mathrm{~m}, 1 \mathrm{H}), 0.49(\mathrm{~m}, 2 \mathrm{H}), 0.18(\mathrm{~m}, 2 \mathrm{H}) ; \mathbf{M S}$ (ESI+) calculated for $\mathrm{C}_{19} \mathrm{H}_{25} \mathrm{~N}_{2} \mathrm{O}_{3}(\mathrm{M}+1) 329.2$, found 329.0 .

(S)-25Fd: (S)-methyl 1-(cyclopropylmethyl)-6-methyl-2-oxo-4-phenethyl-1,2,3,4tetrahydropyrimidine-5-carboxylate

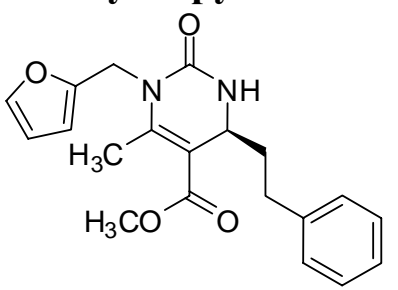

${ }^{1}$ H NMR $\left(400 \mathrm{MHz}, \mathrm{CDCl}_{3}\right): \delta$ 7.10-7.30 $(6 \mathrm{H}), 6.26(\mathrm{~m}, 2 \mathrm{H}), 6.20(\mathrm{~d}$, $J=3.1 \mathrm{~Hz}, 1 \mathrm{H}), 6.14(\mathrm{~m}, 1 \mathrm{H}), 5.59(\mathrm{~d}, J=3.1 \mathrm{~Hz}, 1 \mathrm{H}), 5.17(\mathrm{~d}, J=$ $16.4 \mathrm{~Hz}, 1 \mathrm{H}), 4.68(\mathrm{~d}, J=16.4 \mathrm{~Hz}, 1 \mathrm{H}), 3.66(\mathrm{~s}, 3 \mathrm{H}), 2.65(\mathrm{~m}, 1 \mathrm{H})$, $2.53(\mathrm{~m}, 1 \mathrm{H}), 2.49(\mathrm{~s}, 3 \mathrm{H}), 1.78(\mathrm{~m}, 2 \mathrm{H})$; MS (ESI+) calculated for $\mathrm{C}_{20} \mathrm{H}_{23} \mathrm{~N}_{2} \mathrm{O}_{4}(\mathrm{M}+1)$ 355.2, found 355.0.

(S)-26Fa: (S)-5-acetyl-1-benzyl-6-methyl-4-phenethyl-3,4-dihydropyrimidin-2(1H)-one

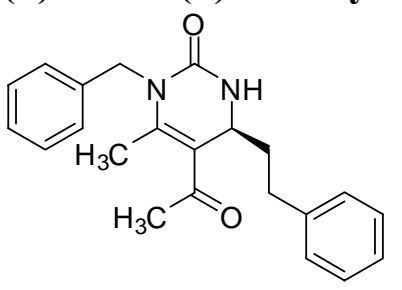

${ }^{1} \mathbf{H}$ NMR $\left(400 \mathrm{MHz}, \mathrm{CDCl}_{3}\right): \delta 7.44(\mathrm{~d}, J=3.1 \mathrm{~Hz}, 1 \mathrm{H}), 7.15-7.32$ $(10 \mathrm{H}), 5.16(\mathrm{~d}, J=16.4 \mathrm{~Hz}, 1 \mathrm{H}), 4.90(\mathrm{~d}, J=16.4 \mathrm{~Hz}, 1 \mathrm{H}), 4.22(\mathrm{~m}$, $1 \mathrm{H}), 2.78(\mathrm{~m}, 1 \mathrm{H}), 2.66(\mathrm{~m}, 1 \mathrm{H}), 2.27(\mathrm{~s}, 3 \mathrm{H}), 2.08(\mathrm{~s}, 3 \mathrm{H}), 1.92(\mathrm{~m}$, 1H), $1.76(\mathrm{~m}, 1 \mathrm{H}) ;{ }^{13} \mathbf{C}$ NMR $\left(100 \mathrm{MHz}, \mathrm{CDCl}_{3}\right): \delta$ 196.6, 154.8, $147.3,140.7,138.0,128.6,128.4,128.3,127.1,126.1,126.0,114.7$, 49.8, 45.9, 38.7, 30.7, 29.8, 16.9; MS (ESI+) calculated for $\mathrm{C}_{20} \mathrm{H}_{23} \mathrm{~N}_{2} \mathrm{O}_{4}(\mathrm{M}+1) 349.2$, found 348.9 . 
$(S)-25 F b:(S)$-methyl 1-ethyl-6-methyl-2-oxo-4-phenethyl-1,2,3,4-tetrahydropyrimidine-5carboxylate<smiles>CCN1C(=O)NC(CCc2ccccc2)C(C(=O)OC)=C1C</smiles>

${ }^{1}$ H NMR (400 MHz, $\left.\mathrm{CDCl}_{3}\right): \delta 7.15-7.31(5 \mathrm{H}), 5.62$ (br. $\left.1 \mathrm{H}\right), 4.23(\mathrm{~m}$, $1 \mathrm{H}), 3.88(\mathrm{~m}, 1 \mathrm{H}), 3.69(\mathrm{~s}, 3 \mathrm{H}), 3.68(\mathrm{~m}, .1 \mathrm{H}), 2.72(\mathrm{~m}, 1 \mathrm{H}), 2.61(\mathrm{~m}$, $1 \mathrm{H}), 2.48(\mathrm{~s}, 3 \mathrm{H}), 1.82(\mathrm{~m}, 2 \mathrm{H}), 1.19(\mathrm{t}, J=7.0 \mathrm{~Hz}, 3 \mathrm{H}) ;{ }^{13} \mathbf{C}$ NMR $\left(100 \mathrm{MHz}, \mathrm{CDCl}_{3}\right): \delta 166.6,154.2,149.2,141.1,128.4,128.3,125.9$, 104.4, 51.2, 49.6, 38.1, 37.7, 30.7, 15.8, 15.0; MS (ESI+) calculated for $\mathrm{C}_{17} \mathrm{H}_{23} \mathrm{~N}_{2} \mathrm{O}_{3}(\mathrm{M}+1)$ 303.2, found 302.9.

(S)-26Fb: (S)-5-acetyl-1-ethyl-6-methyl-4-phenethyl-3,4-dihydropyrimidin-2(1H)-one<smiles>CCN1C(=O)NC(CCc2ccccc2)C(C(C)=O)=C1C</smiles>

${ }^{1} \mathbf{H}$ NMR $\left(400 \mathrm{MHz}, \mathrm{CDCl}_{3}\right): \delta$ 7.15-7.30 $(6 \mathrm{H}), 4.14(\mathrm{~m}, 1 \mathrm{H}), 3.86(\mathrm{~m}$, $1 \mathrm{H}), 3.71(\mathrm{~m}, 1 \mathrm{H}), 2.74(\mathrm{~m}, 1 \mathrm{H}), 2.63(\mathrm{~m}, 1 \mathrm{H}), 2.36(\mathrm{~s}, 3 \mathrm{H}), 2.08(\mathrm{~s}$, $3 \mathrm{H}), 1.85(\mathrm{~m}, 1 \mathrm{H}), 1.71(\mathrm{~m}, 1 \mathrm{~h}), 1.21(\mathrm{t}, J=7.0 \mathrm{~Hz}, 3 \mathrm{H}) ;{ }^{13} \mathbf{C} \mathbf{N M R}$ $\left(100 \mathrm{MHz}, \mathrm{CDCl}_{3}\right): \delta 196.6,154.6,147.1,140.8,128.4,128.3,126.0$, 114.4, 49.6, 38.4, 37.7, 30.5, 29.7, 16.4, 14.9; MS (ESI+) calculated for $\mathrm{C}_{17} \mathrm{H}_{23} \mathrm{~N}_{2} \mathrm{O}_{2}(\mathrm{M}+1)$ 287.2, found 287.1.

(S)-25Fe: $(S)$-methyl 1-(2-methoxyethyl)-6-methyl-2-oxo-4-phenethyl-1,2,3,4tetrahydropyrimidine-5-carboxylate

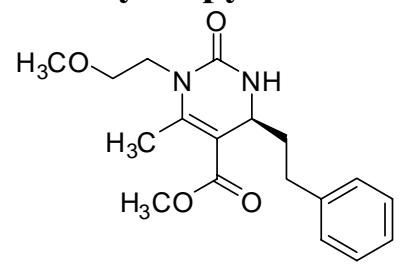

${ }^{1} \mathbf{H}$ NMR $\left(400 \mathrm{MHz}, \mathrm{CDCl}_{3}\right): \delta 7.15-7.29(\mathrm{~m}, 5 \mathrm{H}), 6.29(\mathrm{~d}, J=3.1 \mathrm{~Hz}$, $1 \mathrm{H}), 4.27(\mathrm{~m}, 1 \mathrm{H}), 4.18(\mathrm{dt}, J=14.8,4.7 \mathrm{~Hz}, 1 \mathrm{H}), 3.71(\mathrm{~m}, 1 \mathrm{H}), 3.68$ $(\mathrm{s}, 3 \mathrm{H}), 3.50(\mathrm{~m}, 2 \mathrm{H}), 3.28(\mathrm{~s}, 3 \mathrm{H}), 2.73(\mathrm{~m}, 1 \mathrm{H}), 2.61(\mathrm{~m}, 1 \mathrm{H}), 2.49(\mathrm{~s}$, $3 \mathrm{H}), 1.84(\mathrm{~m}, 2 \mathrm{H}) ;{ }^{13} \mathrm{C}$ NMR $\left(100 \mathrm{MHz}, \mathrm{CDCl}_{3}\right): \delta 166.6,154.4$, 149.9, 141.3, 128.3, 125.8, 128.3, 104.6, 71.4, 58.8, 51.2, 49.6, 42.0, 38.0, 30.6, 16.3; MS (ESI+) calculated for $\mathrm{C}_{18} \mathrm{H}_{25} \mathrm{~N}_{2} \mathrm{O}_{4}(\mathrm{M}+1)$ 333.2, found 332.9 .

(S)-26Fe: (S)-5-acetyl-1-(2-methoxyethyl)-6-methyl-4-phenethyl-3,4-dihydropyrimidin2(1H)-one

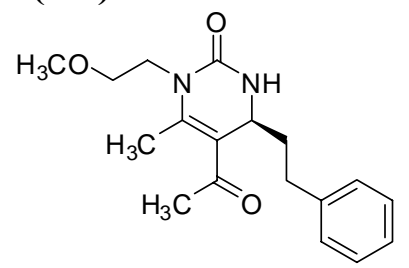

${ }^{1} \mathbf{H}$ NMR $\left(400 \mathrm{MHz}, \mathrm{CDCl}_{3}\right): \delta 7.48(\mathrm{~d}, J=3.1 \mathrm{~Hz}, 1 \mathrm{H}), 7.11-7.25(\mathrm{~m}$, $5 \mathrm{H}), 4.17(\mathrm{~m}, 2 \mathrm{H}), 3.70(\mathrm{~m}, 1 \mathrm{H}), 3.49(\mathrm{~m}, 2 \mathrm{H}), 3.22(\mathrm{~s}, 3 \mathrm{H}), 2.73(\mathrm{~m}$, $1 \mathrm{H}), 2.62(\mathrm{~m}, 1 \mathrm{H}), 2.34(\mathrm{~s}, 3 \mathrm{H}), 2.09(\mathrm{~s}, 3 \mathrm{H}), 1.86(\mathrm{~m}, 1 \mathrm{H}), 1.70(\mathrm{~m}$, $1 \mathrm{H}) ;{ }^{13} \mathrm{C}$ NMR $\left(100 \mathrm{MHz}, \mathrm{CDCl}_{3}\right): \delta$ 196.6, 154.5, 147.7, 141.0, $128.33,128.27,125.9,114.8,71.3,58.8,49.7,41.9,38.2,30.5,29.9$, 17.0; MS (ESI+) calculated for $\mathrm{C}_{18} \mathrm{H}_{25} \mathrm{~N}_{2} \mathrm{O}_{3}(\mathrm{M}+1)$ 317.2, found 316.8.

(R)-26Fd: $(R)$-5-acetyl-1-(furan-2-ylmethyl)-6-methyl-4-phenethyl-3,4-dihydropyrimidin2(1H)-one<smiles>CC(=O)C1=C(C)N(Cc2ccco2)C(=O)N[C@H]1CCc1ccccc1</smiles>

${ }^{1}$ H NMR $\left(400 \mathrm{MHz}, \mathrm{CDCl}_{3}\right): \delta$ 7.10-7.30 $(6 \mathrm{H}), 6.72(\mathrm{~d}, J=3.1 \mathrm{~Hz}$, $1 \mathrm{H}), 6.28(\mathrm{~m}, 1 \mathrm{H}), 6.14(\mathrm{~m}, 1 \mathrm{H}), 5.61(\mathrm{~d}, J=3.1 \mathrm{~Hz}, 1 \mathrm{H}), 5.16(\mathrm{~d}, J=$ $16.4 \mathrm{~Hz}, 1 \mathrm{H}), 4.71(\mathrm{~d}, J=16.4 \mathrm{~Hz}, 1 \mathrm{H}), 2.69(\mathrm{~m}, 1 \mathrm{H}), 2.54(\mathrm{~m}, 1 \mathrm{H})$, $2.38(\mathrm{~s}, 3 \mathrm{H}), 2.10(\mathrm{~s}, 3 \mathrm{H}), 1.84(\mathrm{~m}, 1 \mathrm{H}), 1.70(\mathrm{~m}, 1 \mathrm{H}) ; \mathbf{M S}(\mathrm{ESI}+)$ calculated for $\mathrm{C}_{20} \mathrm{H}_{23} \mathrm{~N}_{2} \mathrm{O}_{3}(\mathrm{M}+1)$ 339.2, found 338.9. 
(S)-26Fh: $(S)$-5-acetyl-6-methyl-4-phenethyl-1-(3,4,5-trimethoxyphenethyl)-3,4dihydropyrimidin-2(1H)-one

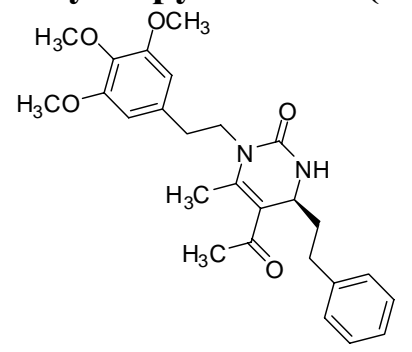

${ }^{1} \mathbf{H}$ NMR (400 MHz, $\left.\mathrm{CDCl}_{3}\right): \delta$ 7.35-7.53 (4H), $7.03(\mathrm{~d}, J=3.1 \mathrm{~Hz}$, $1 \mathrm{H}), 6.38(\mathrm{~s}, 2 \mathrm{H}), 5.40(\mathrm{~d}, J=3.1 \mathrm{~Hz}, 1 \mathrm{H}), 3.73-3.83(14 \mathrm{H}), 2.75(\mathrm{~m}$, 2H), 2.45 (s, 3H), 2.13 (s, 3H); ${ }^{13} \mathbf{C}$ NMR (100 MHz, $\left.\mathrm{CDCl}_{3}\right): \delta 196.5$, $154.2,153.2,146.8,140.5,133.9,128.8,128.6,126.4,126.1,114.7$, $105.5,60.7,55.9,49.7,43.8,38.1,36.2,30.6,29.8,16.7$; MS (ESI+) calculated for $\mathrm{C}_{26} \mathrm{H}_{33} \mathrm{~N}_{2} \mathrm{O}_{5}(\mathrm{M}+1)$ 453.2, found 452.9 .

(R)-26Fc: $(R)$-ethyl 2-(5-acetyl-6-methyl-2-oxo-4-phenethyl-3,4-dihydropyrimidin-1(2H)yl)acetate

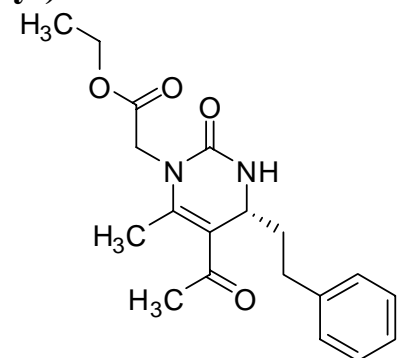

${ }^{1}$ H NMR (400 MHz, $\left.\mathrm{CDCl}_{3}\right): \delta$ 7.10-7.30 (6H), $4.58(\mathrm{~d}, J=18.0 \mathrm{~Hz}$, $1 \mathrm{H}), 4.39(\mathrm{~d}, J=18.0 \mathrm{~Hz}, 1 \mathrm{H}), 10-4.20(3 \mathrm{H}), 2.78(\mathrm{~m}, 1 \mathrm{H}), 2.65(\mathrm{~m}$, $1 \mathrm{H}), 2.26(\mathrm{~s}, 3 \mathrm{H}), 2.07(\mathrm{~s}, 3 \mathrm{H}), 2.04(\mathrm{~m}, 1 \mathrm{H}), 1.73(\mathrm{~m}, 1 \mathrm{H}), 1.22(\mathrm{t}, J=$ $7.0 \mathrm{~Hz}, 3 \mathrm{H}) ;{ }^{13} \mathbf{C}$ NMR $\left(100 \mathrm{MHz}, \mathrm{CDCl}_{3}\right): \delta 196.6,169.1,154.2$, $146.2,140.8,128.4,126.0,114.9,61.5,49.7,44.1,37.9,30.6,29.7$, 16.5, 14.0; MS (ESI+) calculated for $\mathrm{C}_{17} \mathrm{H}_{25} \mathrm{~N}_{2} \mathrm{O}_{4}(\mathrm{M}+1) 345.2$, found 345.1 


\section{Analysis of trace Pd by ICP-MS:}

General procedure for preparation of samples for analysis: Samples were digested in $4 \mathrm{~mL}$ of a $5 \%$ nitric acid, $1 \%$ hydrofluoric acid solution. Digestion was accelerated by incubation in a microwave at $120{ }^{\circ} \mathrm{C}$ for 5 minutes. Samples were then diluted to $10 \mathrm{~mL}$ with $5 \%$ nitric acid, $1 \%$ hydrofluoric acid. The ICP-MS instrument was tuned for maximum sensitivity. An external Pd standard curve was employed for a linear regression to convert raw intensities of each sample to concentration (ppb).

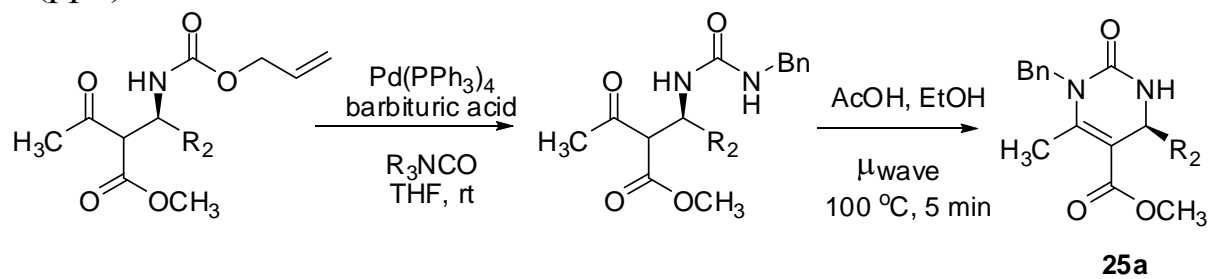

Sample 1: The reaction crude mixture containing 25a was concentrated and directly digested for analysis according to general procedure.

Sample 2: The reaction mixture was concentrated and purified by Combi-Flash ${ }^{\mathrm{TM}}$. The afforded material 25a was digested for analysis according to the general procedure.

Sample 3: The purified compound 25a (6 mg) by Combi-Flash ${ }^{\mathrm{TM}}$ was dissolved in $5 \mathrm{~mL} \mathrm{CH}_{2} \mathrm{Cl}_{2}$ and then PS-TMT resin (20mg) was added. The mixture was shaken for $2 \mathrm{~h}$ and then filtered.

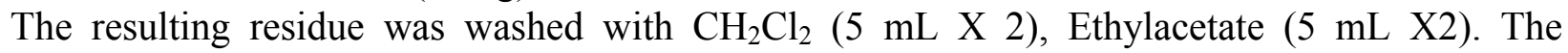
afforded compound 25a after concentration was digested for analysis according to the general procedure.

The ICP-MS analysis result is shown in the following table:

\begin{tabular}{|l|l|l|l|}
\hline & Sample weight $(\mathrm{mg})$ & {$[\mathrm{Pd}]$ in solution $(\mathrm{ppb})$} & {$[\mathrm{Pd}]$ in sample $(\mathrm{ppm})$} \\
\hline Sample 1 & 11.2 & 117 & 104 \\
\hline Sample 2 & 10.0 & 12 & 12 \\
\hline Sample 3 & 5.1 & 0.45 & 0.90 \\
\hline Blank control & - & 0.06 & - \\
\hline
\end{tabular}




\section{General procedure for synthesis of tetrahydropyrimidone:}

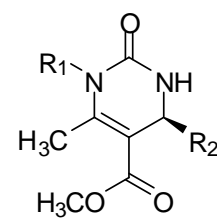

25<smiles>[R2][C@@H]1NC(=O)N([R1])C(C)=C1C(C)=O</smiles>

26

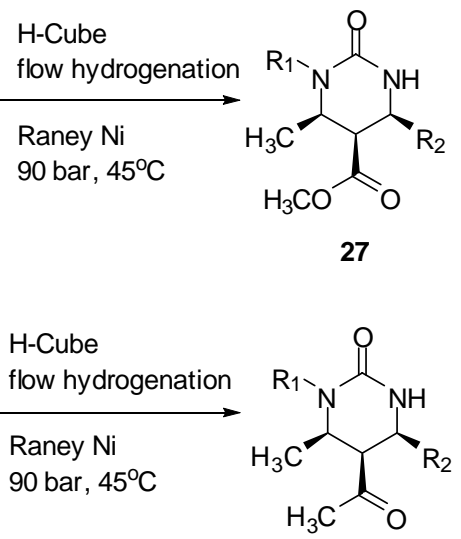

29

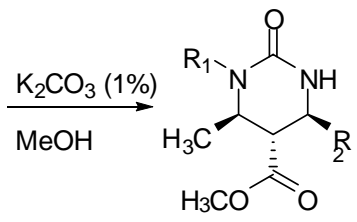

28

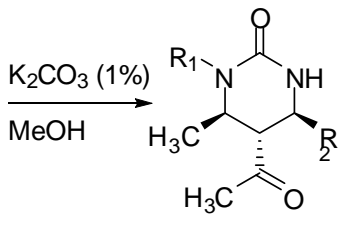

30

A $33 \mathrm{~mm}$ Ra-Ni catridge was installed in the $\mathrm{H}$-cube ${ }^{\mathrm{TM}}$. The instrumentation setup parameters were pressure at $90 \mathrm{bar}$; temperature at $45^{\circ} \mathrm{C}$ and flow rate at $0.5 \mathrm{~mL} / \mathrm{min}$. After the system hydrogen generation is stabilized (appoxmately $15 \mathrm{~min}$ ), a solution of dihydropyrimidone in $\mathrm{MeOH}$ (or $\mathrm{MeOH} /$ Ethylacetate = 9:1 for better solubility for some compounds) was injected into the system. After passing through the instrument, the reaction solution was collected, and the column was washed with the solvent $\mathrm{MeOH}$ to remove the substrate adsorbed to the catalyst. The solutions were combined and analyzed by analytical LC/MS/UV/ELSD, which showed complete conversion of the starting material. The mixture was then evaporated to dryness to afford the desired tetrahydropyrimidone product.

For tetrahydropyrimidone with ester moiety, the cis, cis-tetrahydropyrimidone $\mathbf{2 7}$ is stable upon storage. To achieve isomerization, the cis,cis-tetrahydropyrimidone 27 was dissolved in $\mathrm{MeOH}$ $(0.05 \mathrm{M})$ and catalytic amount $(1 \mathrm{~mol} \%)$ of $\mathrm{K}_{2} \mathrm{CO}_{3}$ was added. The reaction was stirred at room temperature until complete disappearance of the starting material (ca. 3 days). The mixture was then concentrated and elute through a silica plug with ethylacetate. The solution was evaporated to dryness afford the trans, trans-tetrahydropyrimidone $\mathbf{2 8}$.

For tetrahydropyrimidone with ketone moiety, the cis, cis-tetrahydropyrimidone 29 slowly epimerize at $\mathrm{C} 3$ position to produce trans, trans-tetrahydropyrimidone 30. To achieve complete isomerization, the cis,cis-tetrahydropyrimidone was dissolved in $\mathrm{MeOH}(0.05 \mathrm{M})$ and catalytic amount $(1 \mathrm{~mol} \%)$ of $\mathrm{K}_{2} \mathrm{CO}_{3}$ was added. The reaction was stirred at room temperature until complete disappearance of the starting material (ca. 12 hours). The mixture was then concentrated and elute through a silica plug with Ethyl Acetate. The solution was evaporated to dryness afford the trans, trans-tetrahydropyrimidone $\mathbf{3 0}$.

The tetrahydropyrimidone was dissolved in $800 \mu \mathrm{L}$ DMSO and purified by preparative reverse phase HPLC (fraction triggering was based on calculated exact mass). The pure fractions were combined and concentrated under reduced pressure to afford pure dihydropyrimidone. 
Determination of relative stereochemistry of tetrahydropyrimidone:
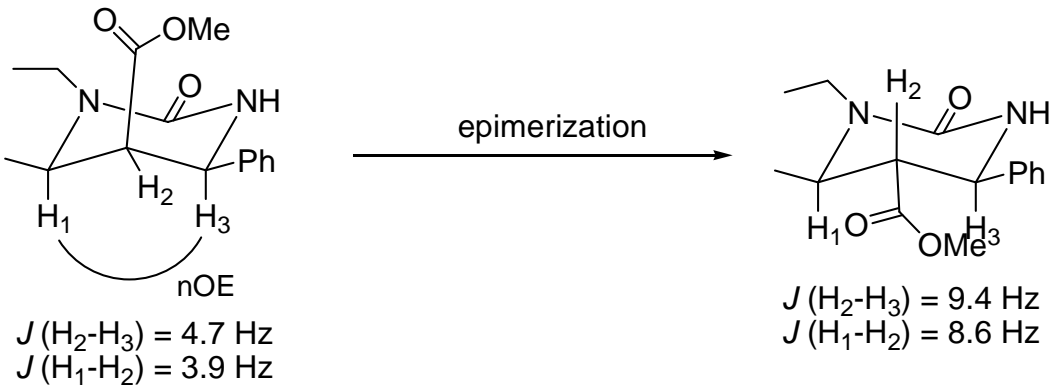

1D-selective nOe experiment was used to assign the relative stereochemistry of tetrahydropyrimidone. As shown in above figure, irradiation of $\mathrm{H} 1$ proton at $3.93 \mathrm{ppm}$ led to a $1.4 \%$ NOE enhancement of the $\mathrm{H} 3$ proton signal at $3.07 \mathrm{ppm}$, which indicated that $\mathrm{H} 1$ and $\mathrm{H} 3$ are on the same face.

The stereochemistry can be also determined by the magnitude of their vicinal coupling constants. For the cis, cis-isomer, $J\left(\mathrm{H}_{2}-\mathrm{H}_{3}\right)$ and $J\left(\mathrm{H}_{1}-\mathrm{H}_{2}\right)$ are small $(4.7 \mathrm{~Hz}$ and $3.9 \mathrm{~Hz}$ respectively). However, for the trans, trans-isomer, $J\left(\mathrm{H}_{2}-\mathrm{H}_{3}\right)$ and $J\left(\mathrm{H}_{1}-\mathrm{H}_{2}\right)$ are much bigger $(9.4$ and $8.6 \mathrm{~Hz}$ respectively).

\section{Characterization of selected compounds was listed as below:}

$(4 R, 5 R, 6 R)-27 \mathrm{Ca}:(4 R, 5 R, 6 R)-$ methyl 1-benzyl-6-methyl-2-oxo-4-(3(trifluoromethyl)phenyl) hexahydropyrimidine-5-carboxylate

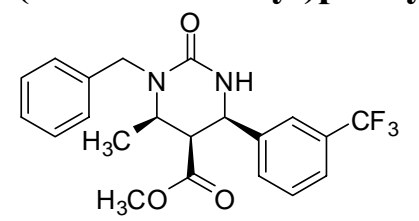

${ }^{1}$ H NMR (400 MHz, $\left.\mathrm{CDCl}_{3}\right): \delta 7.23-7.61(9 \mathrm{H}), 5.46(\mathrm{~s}, 1 \mathrm{H}), 5.15(\mathrm{~d}, J$ $=16.4 \mathrm{~Hz}, 1 \mathrm{H}), 5.00(\mathrm{~d}, J=4.7 \mathrm{~Hz}, 1 \mathrm{H}), 4.33(\mathrm{~d}, J=16.4 \mathrm{~Hz}, 1 \mathrm{H})$, 3.87 (dq, $J=7.0,3.9 \mathrm{~Hz}, 1 \mathrm{H}), 3.49$ (s, $3 \mathrm{H}), 3.09$ (dd, $J=4.7,3.9 \mathrm{~Hz}$, $1 \mathrm{H}), 1.17(\mathrm{~d}, J=7.0 \mathrm{~Hz}, 3 \mathrm{H}) ;{ }^{13} \mathbf{C}$ NMR $\left(100 \mathrm{MHz}, \mathrm{CDCl}_{3}\right): \delta 171.3$, $156.4,140.7,137.9,132.5,131.5,129.9,129.5,128.6,127.6,127.1$, 125.5 (q), 123.4 (q), 56.1, 55.6, 52.8, 52.2, 47.9, 18.7; $[\boldsymbol{\alpha}]^{\mathbf{2 3}}{ }_{\mathbf{D}}=-32.1^{\circ}$ $\left(\mathrm{c}=1.1, \mathrm{CHCl}_{3}\right)$; IR (thin film, $\left.\mathrm{cm}^{-1}\right): 3218,2930,1738,1641,1452$, 1160,701 ; HRMS $\left(\mathrm{CI}-\mathrm{NH}_{3}\right)$ calculated for $\mathrm{C}_{21} \mathrm{H}_{22} \mathrm{~F}_{3} \mathrm{~N}_{2} \mathrm{O}_{3}(\mathrm{M}+1)$ 407.1577, found 407.1560.

(4R,5S,6R)-28Ca: $(4 R, 5 S, 6 R)$-methyl 1-benzyl-6-methyl-2-oxo-4-(3(trifluoromethyl)phenyl) hexahydropyrimidine-5-carboxylate

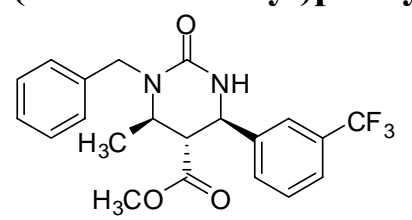

${ }^{1}$ H NMR (400 MHz, $\left.\mathrm{CDCl}_{3}\right): \delta$ 7.24-7.60 (9H), $5.22(\mathrm{~d}, J=15.7 \mathrm{~Hz}$, $1 \mathrm{H}), 5.19(\mathrm{~s}, 1 \mathrm{H}), 4.83(\mathrm{~d}, J=8.6 \mathrm{~Hz}, 1 \mathrm{H}), 4.20(\mathrm{~d}, J=15.7 \mathrm{~Hz}, 1 \mathrm{H})$, $3.78(\mathrm{dq}, J=7.0,7.8 \mathrm{~Hz}, 1 \mathrm{H}), 3.46$ (s, 3H), 3.05 (app. t, $J=8.6 \mathrm{~Hz}$, $1 \mathrm{H}), 1.11(\mathrm{~d}, J=7.0 \mathrm{~Hz}, 3 \mathrm{H}) ;{ }^{13} \mathbf{C}$ NMR $\left(100 \mathrm{MHz}, \mathrm{CDCl}_{3}\right): \delta 171.0$, $156.2,140.8,137.8,131.5,131.2,129.8,129.5,128.6,127.5,127.2$, 125.4 (q), 123.4 (q), 55.9, 55.0, 52.1, 51.9, 46.9, 18.8; $[\boldsymbol{\alpha}]^{23}{ }_{\mathbf{D}}=-22.3^{\circ}$ $\left(\mathrm{c}=1.3, \mathrm{CHCl}_{3}\right)$; IR (thin film, $\left.\mathrm{cm}^{-1}\right): 3225,2981,2925,1741,1644$, 1490, 1163; HRMS (CI-NH $)_{3}$ calculated for $\mathrm{C}_{21} \mathrm{H}_{22} \mathrm{~F}_{3} \mathrm{~N}_{2} \mathrm{O}_{3}(\mathrm{M}+1)$ 407.1577, found 407.1561. 
(4R,5S,6R)-30Ca: (4R,5S,6R)-5-acetyl-1-benzyl-6-methyl-4-(3-(trifluoromethyl)phenyl) tetrahydropyrimidin-2(1H)-one

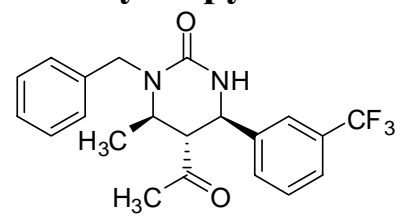

${ }^{1}$ H NMR $\left(400 \mathrm{MHz}, \mathrm{CDCl}_{3}\right): \delta$ 7.20-7.64 (9H), $5.47(\mathrm{~s}, 1 \mathrm{H}), 5.14(\mathrm{~d}, J$ $=15.6 \mathrm{~Hz}, 1 \mathrm{H}), 4.68(\mathrm{~d}, J=8.7 \mathrm{~Hz}, 1 \mathrm{H}), 4.18(\mathrm{~d}, J=15.6 \mathrm{~Hz}, 1 \mathrm{H})$, $3.78(\mathrm{dq}, J=6.3,7.8 \mathrm{~Hz}, 1 \mathrm{H}), 2.96$ (app. t, $J=8.6 \mathrm{~Hz}, 1 \mathrm{H}), 1.64$ (s, $3 \mathrm{H}), 1.07(\mathrm{~d}, J=6.3 \mathrm{~Hz}, 3 \mathrm{H}) ;{ }^{13} \mathbf{C}$ NMR $\left(100 \mathrm{MHz}, \mathrm{CDCl}_{3}\right): \delta 206.9$, $156.3,140.9,137.9,131.7,131.3,130.2,129.6,128.6,127.5,127.2$, $125.4(\mathrm{q}), 123.3(\mathrm{q}), 60.8,56.0,51.8,46.6,32.1,18.7 ;[\boldsymbol{\alpha}]^{23}{ }_{\mathbf{D}}=-20.1^{\circ}$ $\left(\mathrm{c}=1.3, \mathrm{CHCl}_{3}\right)$; IR (thin film, $\left.\mathrm{cm}^{-1}\right): 3231,3062,2981,2930,1720$, $1650,1449,1165,761$; HRMS $\left(\mathrm{CI}-\mathrm{NH}_{3}\right)$ calculated for $\mathrm{C}_{21} \mathrm{H}_{22} \mathrm{~F}_{3} \mathrm{~N}_{2} \mathrm{O}_{2}$ $(\mathrm{M}+1) 391.1628$, found 391.1613.

$(4 R, 5 R, 6 R)-27 \mathrm{Cb}:(4 R, 5 R, 6 R)$-methyl 1-ethyl-6-methyl-2-oxo-4-(3-(trifluoromethyl)phenyl) hexahydropyrimidine-5-carboxylate

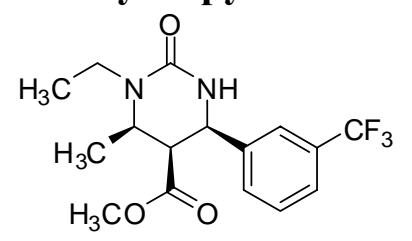

${ }^{1}$ H NMR $\left(400 \mathrm{MHz}, \mathrm{CDCl}_{3}\right): \delta$ 7.45-7.60 (4H), $5.39(\mathrm{~s}, 1 \mathrm{H}), 4.95(\mathrm{~d}, J$ $=4.7 \mathrm{~Hz}, 1 \mathrm{H}), 3.95(\mathrm{dq}, J=7.0,4.7 \mathrm{~Hz}, 1 \mathrm{H}), 3.76(\mathrm{dq}, J=14.9,7.0$ $\mathrm{Hz}, 1 \mathrm{H}), 3.48(\mathrm{~s}, 3 \mathrm{H}), 3.22(\mathrm{dq}, J=14.9,7.0 \mathrm{~Hz}, 1 \mathrm{H}), 3.11$ (app. t, $J=$ $4.7 \mathrm{~Hz}, 1 \mathrm{H}), 1.26(\mathrm{~d}, J=6.3 \mathrm{~Hz}, 3 \mathrm{H}), 1.12(\mathrm{t}, J=7.0 \mathrm{~Hz}, 3 \mathrm{H}) ;{ }^{13} \mathbf{C}$ NMR $\left(100 \mathrm{MHz}, \mathrm{CDCl}_{3}\right): \delta 168.9,155.9,144.5,140.1,129.9,129.2$, $129.1,125.1$ (q), 123.3 (q), 55.1, 51.4, 51.0, 50.3, 37.8, 17.4, 13.7; $[\boldsymbol{\alpha}]^{23}{ }_{\mathbf{D}}=-41.1^{\circ}\left(\mathrm{c}=1.1, \mathrm{CHCl}_{3}\right)$; IR (thin film, $\left.\mathrm{cm}^{-1}\right)$ : 3229, 3060, 2930, 1735, 1642, 1450, 1152, 761, 700; HRMS (CI-NH $)_{3}$ calculated for $\mathrm{C}_{16} \mathrm{H}_{20} \mathrm{~F}_{3} \mathrm{~N}_{2} \mathrm{O}_{3}(\mathrm{M}+1)$ 345.1421, found 345.1434.

$(4 R, 5 S, 6 R)-27 \mathrm{Cb}:(4 R, 5 S, 6 R)$-methyl 1-ethyl-6-methyl-2-oxo-4-(3-(trifluoromethyl)phenyl) hexahydropyrimidine-5-carboxylate

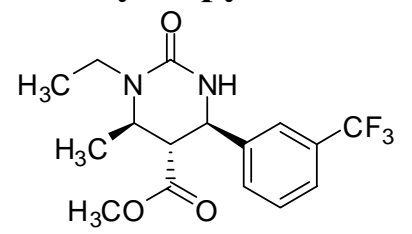

${ }^{1}$ H NMR $\left(400 \mathrm{MHz}, \mathrm{CDCl}_{3}\right): \delta$ 7.42-7.60 (4H), $4.89(\mathrm{~s}, 1 \mathrm{H}), 4.77(\mathrm{~d}, J$ $=8.6 \mathrm{~Hz}, 1 \mathrm{H}), 3.89(\mathrm{dq}, J=8.6,6.3 \mathrm{~Hz}, 1 \mathrm{H}), 3.81(\mathrm{dq}, J=14.8,7.0$ $\mathrm{Hz}, 1 \mathrm{H}), 3.53(\mathrm{~s}, 3 \mathrm{H}), 3.20(\mathrm{dq}, J=14.8,7.0 \mathrm{~Hz}, 1 \mathrm{H}), 2.77$ (app. t, $J=$ $8.6 \mathrm{~Hz}, 1 \mathrm{H}), 1.19(\mathrm{~d}, J=6.3 \mathrm{~Hz}, 3 \mathrm{H}), 1.16(\mathrm{t}, J=7.0 \mathrm{~Hz}, 3 \mathrm{H}) ;{ }^{13} \mathrm{C}$ NMR $\left(100 \mathrm{MHz}, \mathrm{CDCl}_{3}\right): \delta$ 171.2, 155.7, 144.5, 140.8, 131.2, 129.8, $129.5,125.4(\mathrm{q}), 123.4$ (q), 56.0, 55.1, 52.2, 51.8, 38.5, 19.0, 13.5; $[\mathbf{\alpha}]^{\mathbf{2 3}} \mathbf{D}=-28.8^{\circ}\left(\mathrm{c}=1.0, \mathrm{CHCl}_{3}\right) ;$ IR (thin film, $\left.\mathrm{cm}^{-1}\right): 3230,2984$, 2930, 1740, 1645, 760; HRMS $\left(\mathrm{CI}-\mathrm{NH}_{3}\right)$ calculated for $\mathrm{C}_{16} \mathrm{H}_{20} \mathrm{~F}_{3} \mathrm{~N}_{2} \mathrm{O}_{3}$ $(\mathrm{M}+1) 345.1421$, found 345.1415 .

$(4 R, 5 R, 6 R)-27 \mathrm{Ab}:(4 R, 5 R, 6 R)$-methyl 1-ethyl-6-methyl-2-oxo-4phenylhexahydropyrimidine-5-carboxylate 
<smiles>CCN1C(=O)N[C@H](c2ccccc2)[C@H](C(=O)OC)[C@H]1C</smiles>

${ }^{1}$ H NMR (400 MHz, $\left.\mathrm{CDCl}_{3}\right): \delta$ 7.26-7.34 (5H), $5.03(\mathrm{~s}, 1 \mathrm{H}), 4.86(\mathrm{~d}, J$ $=4.7 \mathrm{~Hz}, 1 \mathrm{H}), 3.94(\mathrm{dq}, J=6.3,3.9 \mathrm{~Hz}, 1 \mathrm{H}), 3.79(\mathrm{~m}, 1 \mathrm{H}), 3.45(\mathrm{~s}$, $3 \mathrm{H}), 3.20(\mathrm{~m}, 1 \mathrm{H}), 3.06$ (app. $\mathrm{t}, J=3.9 \mathrm{~Hz}, 1 \mathrm{H}), 1.26(\mathrm{~d}, J=6.3 \mathrm{~Hz}$, $3 \mathrm{H}), 1.13(\mathrm{t}, J=7.0 \mathrm{~Hz}, 3 \mathrm{H}) ;{ }^{13} \mathrm{C}$ NMR $\left(100 \mathrm{MHz}, \mathrm{CDCl}_{3}\right): \delta 168.9$, $156.0,138.8,128.6,128.3,126.1,55.3,51.3,51.2,50.2,37.6,17.4$, 13.7; IR (thin film, $\mathrm{cm}^{-1}$ ): 3215, 3063, 2980, 2928, 1736, 1644, 1490, 1451, 1162, 759, 699; MS (ESI+) calculated for $\mathrm{C}_{15} \mathrm{H}_{21} \mathrm{~N}_{2} \mathrm{O}_{3}(\mathrm{M}+1)$ 277.2, found 276.9 .

(4R,5S,6R)-28Ab: $(4 R, 5 S, 6 R)-$ methyl 1-ethyl-6-methyl-2-oxo-4phenylhexahydropyrimidine-5-carboxylate

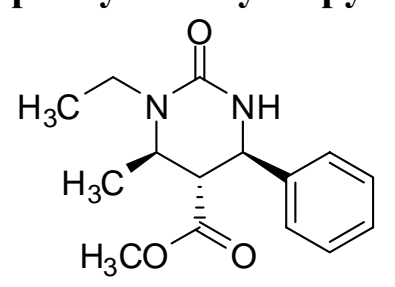

${ }^{1}$ H NMR $\left(400 \mathrm{MHz}, \mathrm{CDCl}_{3}\right): \delta$ 7.26-7.34 (5H), $4.83(\mathrm{~s}, 1 \mathrm{H}), 4.65(\mathrm{~d}, J$ $=9.4 \mathrm{~Hz}, 1 \mathrm{H}), 3.77-3.87(2 \mathrm{H}), 3.49(\mathrm{~s}, 3 \mathrm{H}), 3.19(\mathrm{~m}, 1 \mathrm{H}), 2.76($ app. $\mathrm{t}$, $J=8.6 \mathrm{~Hz}, 1 \mathrm{H}), 1.19(\mathrm{~d}, J=6.3 \mathrm{~Hz}, 3 \mathrm{H}), 1.16(\mathrm{t}, J=7.0 \mathrm{~Hz}, 3 \mathrm{H}) ;{ }^{13} \mathrm{C}$ NMR $\left(100 \mathrm{MHz}, \mathrm{CDCl}_{3}\right): \delta 171.5,155.9,139.3,128.8,128.5,126.4$, 56.4, 55.3, 52.0, 51.8, 38.2, 19.0, 13.5; IR (thin film, $\mathrm{cm}^{-1}$ ): 3225, 3066, 2926, 1740, 1646, 1453, 761; MS (ESI+) calculated for $\mathrm{C}_{15} \mathrm{H}_{21} \mathrm{~N}_{2} \mathrm{O}_{3}(\mathrm{M}+1) 277.2$, found 276.9.

(4R,5R,6R)-27Cc: $(4 R, 5 R, 6 R)-$ methyl 1-(2-ethoxy-2-oxoethyl)-6-methyl-2-oxo-4-(3(trifluoromethyl)phenyl) hexahydropyrimidine-5-carboxylate

$\mathrm{H}_{3} \mathrm{C}$

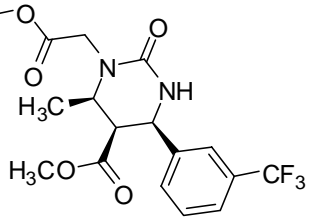

${ }^{1}$ H NMR (400 MHz, $\left.\mathrm{CDCl}_{3}\right): \delta$ 7.46-7.62 (4H), $5.22(\mathrm{~s}, 1 \mathrm{H}), 5.04(\mathrm{~d}, J$ $=5.5 \mathrm{~Hz}, 1 \mathrm{H}), 4.87(\mathrm{~d}, J=18.0 \mathrm{~Hz}, 1 \mathrm{H}), 4.16-4.24(3 \mathrm{H}), 3.71(\mathrm{~d}, J=$ $18.0 \mathrm{~Hz}, 1 \mathrm{H}), 3.47$ (s, 3H), 3.15 (app. t, $J=3.9 \mathrm{~Hz}, 1 \mathrm{H}), 1.28(\mathrm{t}, J=7.0$ $\mathrm{Hz}, 3 \mathrm{H}), 1.23(\mathrm{~d}, J=7.0 \mathrm{~Hz}, 3 \mathrm{H}) ;{ }^{13} \mathbf{C} \mathbf{N M R}\left(100 \mathrm{MHz}, \mathrm{CDCl}_{3}\right): \delta$ $170.3,168.4,156.3,144.2,139.8,129.9,129.3,128.6,125.4$ (q), 123.3 (q), 61.1, 55.4, 51.5, 51.0, 50.8, 44.3, 17.3, 14.2; MS (ESI+) calculated for $\mathrm{C}_{18} \mathrm{H}_{22} \mathrm{~F}_{3} \mathrm{~N}_{2} \mathrm{O}_{5}(\mathrm{M}+1)$ 403.2, found 402.9.

(4R,5R,6R)-27Ce: (4R,5R,6R)-methyl 1-(2-methoxyethyl)-6-methyl-2-oxo-4-(3(trifluoromethyl)phenyl) hexahydropyrimidine-5-carboxylate

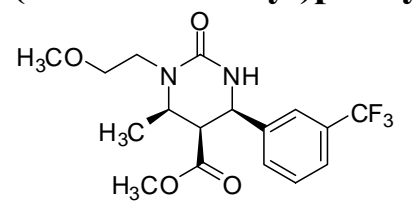

${ }^{1}$ H NMR (400 MHz, $\mathrm{CDCl}_{3}$ ): $\delta$ 7.42-7.60 (4H), 5.33 (s, 1H), 4.97 (d, J $=4.9 \mathrm{~Hz}, 1 \mathrm{H}), 4.06(\mathrm{dq}, J=6.3,3.9 \mathrm{~Hz}, 1 \mathrm{H}), 3.93(\mathrm{dt}, J=14.9,4.7 \mathrm{~Hz}$, $1 \mathrm{H}), 3.59(\mathrm{~m}, 1 \mathrm{H}), 3.48(\mathrm{~m}, 1 \mathrm{H}), 3.47(\mathrm{~s}, 3 \mathrm{H}), 3.33(\mathrm{~s}, 3 \mathrm{H}), 3.30(\mathrm{~m}$, 1H), 3.11 (app. t $J=4.7 \mathrm{~Hz}, 1 \mathrm{H}), 1.18(\mathrm{~d}, J=7.0 \mathrm{~Hz}, 3 \mathrm{H}) ;{ }^{13} \mathbf{C}$ NMR $\left(100 \mathrm{MHz}, \mathrm{CDCl}_{3}\right): \delta 168.6,156.1,140.1,129.9,129.2,127.1,126.8$, 125.1 (q), 123.3 (q), 71.9, 58.9, 55.3, 51.7, 51.4, 50.9, 42.6, 17.6; MS (ESI+) calculated for $\mathrm{C}_{17} \mathrm{H}_{22} \mathrm{~F}_{3} \mathrm{~N}_{2} \mathrm{O}_{4}(\mathrm{M}+1) 375.2$, found 375.0.

(4R,5S,6R)-28Ce: (4R,5S,6R)-methyl 1-(2-methoxyethyl)-6-methyl-2-oxo-4-(3(trifluoromethyl)phenyl) hexahydropyrimidine-5-carboxylate 


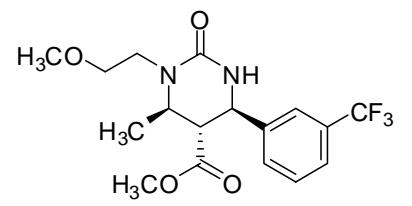

${ }^{1}$ H NMR (400 MHz, $\left.\mathrm{CDCl}_{3}\right): \delta$ 7.45-7.59 (4H), $5.03(\mathrm{~s}, 1 \mathrm{H}), 4.81$ (d, $J$ $=9.3 \mathrm{~Hz}, 1 \mathrm{H}), 3.90-4.02(2 \mathrm{H}), 3.54(\mathrm{~s}, 3 \mathrm{H}), 3.53(\mathrm{~m}, 2 \mathrm{H}), 3.35(\mathrm{~s}, 3 \mathrm{H})$, 3.32 (m, 1H), 2.74 (app. t, $J=8.6 \mathrm{~Hz}, 1 \mathrm{H}), 1.19$ (d, $J=6.3 \mathrm{~Hz}, 3 \mathrm{H})$; ${ }^{13}$ C NMR (100 MHz, $\left.\mathrm{CDCl}_{3}\right): \delta 171.2,156.0,140.9,129.9,129.5$, $129.2,128.4,125.4$ (q), 123.4 (q), 71.4, 58.9, 56.0, 55.1, 53.1, 52.1, 43.2, 19.0; MS (ESI+) calculated for $\mathrm{C}_{17} \mathrm{H}_{22} \mathrm{~F}_{3} \mathrm{~N}_{2} \mathrm{O}_{4}(\mathrm{M}+1)$ 375.2, found 374.9 .

$(4 R, 5 S, 6 R)-30 \mathrm{Ce}:(4 R, 5 S, 6 R)-5$-acetyl-1-(2-methoxyethyl)-6-methyl-4-(3(trifluoromethyl)phenyl) tetrahydropyrimidin-2(1H)-one<smiles>COCCN1C(=O)N[C@H](c2cccc(C(F)(F)F)c2)[C@H](C(C)=O)[C@H]1C</smiles>

${ }^{1}$ H NMR (400 MHz, $\left.\mathrm{CDCl}_{3}\right): \delta$ 7.42-7.66 (4H), $5.23(\mathrm{~s}, 1 \mathrm{H}), 4.72(\mathrm{~d}, J$ $=7.8 \mathrm{~Hz}, 1 \mathrm{H}), 3.89-3.97(2 \mathrm{H}), 3.51(\mathrm{~m}, 2 \mathrm{H}), 3.32(\mathrm{~s}, 3 \mathrm{H}), 3.31(\mathrm{~m}$, 1H), 2.93 (app. t, $J=7.8 \mathrm{~Hz}, 1 \mathrm{H}), 1.86(\mathrm{~s}, 3 \mathrm{H}), 1.16(\mathrm{~d}, J=6.3 \mathrm{~Hz}$, $3 \mathrm{H})$; MS (ESI+) calculated for $\mathrm{C}_{17} \mathrm{H}_{22} \mathrm{~F}_{3} \mathrm{~N}_{2} \mathrm{O}_{3}(\mathrm{M}+1)$ 359.2, found 359.0 .

(4R,5S,6R)-30Cc: ethyl 2-((4R,5S,6R)-5-acetyl-6-methyl-2-oxo-4-(3(trifluoromethyl)phenyl) tetrahydropyrimidin-1(2H)-yl)acetate

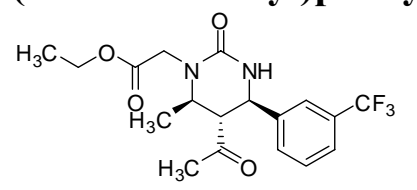

${ }^{1}$ H NMR (400 MHz, $\left.\mathrm{CDCl}_{3}\right): \delta$ 7.42-7.62 (4H), $5.42(\mathrm{~s}, 1 \mathrm{H}), 4.63(\mathrm{~d}, J$ $=10.2 \mathrm{~Hz}, 1 \mathrm{H}), 4.44(\mathrm{~d}, J=18.0 \mathrm{~Hz}, 1 \mathrm{H}), 4.13-4.20(3 \mathrm{H}), 3.92(\mathrm{~d}, J=$ $18.0 \mathrm{~Hz}, 1 \mathrm{H}), 2.96$ (app. t, $J=9.3 \mathrm{~Hz}, 1 \mathrm{H}), 1.71(\mathrm{~s}, 3 \mathrm{H}), 1.23$ (t, $J=$ $7.0 \mathrm{~Hz}, 3 \mathrm{H}), 1.11(\mathrm{~d}, J=6.3 \mathrm{~Hz}, 3 \mathrm{H})$; MS (ESI+) calculated for $\mathrm{C}_{18} \mathrm{H}_{22} \mathrm{~F}_{3} \mathrm{~N}_{2} \mathrm{O}_{4}(\mathrm{M}+1)$ 387.2, found 386.9.

(4R,5R,6R)-27Aa: $(4 R, 5 R, 6 R)-$ methyl 1-benzyl-6-methyl-2-oxo-4phenylhexahydropyrimidine-5-carboxylate<smiles>COC(=O)[C@@H]1[C@H](c2ccccc2)NC(=O)N(Cc2ccccc2)[C@@H]1C</smiles>

${ }^{1}$ H NMR (400 MHz, $\left.\mathrm{CDCl}_{3}\right): \delta$ 7.22-7.38 $(10 \mathrm{H}), 5.20(\mathrm{~s}, 1 \mathrm{H}), 5.18(\mathrm{~d}$, $J=16.4 \mathrm{~Hz}, 1 \mathrm{H}), 4.91(\mathrm{~d}, J=4.7 \mathrm{~Hz}, 1 \mathrm{H}), 4.33(\mathrm{~d}, J=16.4 \mathrm{~Hz}, 1 \mathrm{H})$, $3.86(\mathrm{dq}, J=7.0,4.7 \mathrm{~Hz}, 1 \mathrm{H}), 3.46(\mathrm{~s}, 3 \mathrm{H}), 3.05$ (app. t, $J=4.7 \mathrm{~Hz}$, $1 \mathrm{H}), 1.17(\mathrm{~d}, J=7.0 \mathrm{~Hz}, 3 \mathrm{H}) ;{ }^{13} \mathbf{C}$ NMR $\left(100 \mathrm{MHz}, \mathrm{CDCl}_{3}\right): \delta 168.9$, $156.7,138.7,138.6,128.7,128.5,128.4,127.2,126.9,126.1,55.5$, 51.4, 51.3, 50.8, 46.5, 17.4; MS (ESI+) calculated for $\mathrm{C}_{20} \mathrm{H}_{23} \mathrm{~N}_{2} \mathrm{O}_{3}$ $(\mathrm{M}+1) 339.2$, found 338.9 .

(4S,5R,6R)-27Fa: (4S,5R,6R)-methyl 1-benzyl-6-methyl-2-oxo-4phenethylhexahydropyrimidine-5-carboxylate

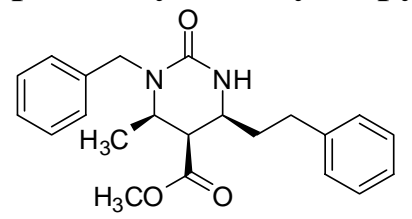

${ }^{1}$ H NMR $\left(400 \mathrm{MHz}, \mathrm{CDCl}_{3}\right): \delta$ 7.15-7.31 (10H), $5.26(\mathrm{~s}, 1 \mathrm{H}), 5.13(\mathrm{~d}$, $J=15.7 \mathrm{~Hz}, 1 \mathrm{H}), 4.24(\mathrm{~d}, J=15.7 \mathrm{~Hz}, 1 \mathrm{H}), 3.70(\mathrm{~s}, 3 \mathrm{H}), 3.64-3.68$ (2H), $2.91(\mathrm{dd}, J=4.7,3.9 \mathrm{~Hz}, 1 \mathrm{H}), 2.72(\mathrm{~m}, 2 \mathrm{H}), 1.85(\mathrm{~m}, 2 \mathrm{H}), 1.22$ $(\mathrm{d}, J=7.0 \mathrm{~Hz}, 3 \mathrm{H}) ;{ }^{13} \mathbf{C}$ NMR $\left(100 \mathrm{MHz}, \mathrm{CDCl}_{3}\right): \delta 169.7,156.3$, $140.5,138.7,128.7,128.6,128.41,128.35,127.3,126.3,51.6,50.7$, $50.6,47.9,46.9,35.4,32.2,17.3$; $\mathrm{MS}$ (ESI+) calculated for $\mathrm{C}_{22} \mathrm{H}_{27} \mathrm{~N}_{2} \mathrm{O}_{3}(\mathrm{M}+1)$ 367.2, found 367.0.

(4R,5S,6R)-30Aa: $(4 R, 5 S, 6 R)-5$-acetyl-1-benzyl-6-methyl-4-phenyltetrahydropyrimidin2(1H)-one 
<smiles>CC(=O)[C@H]1[C@@H](c2ccccc2)NC(=O)N(Cc2ccccc2)[C@H]1C</smiles>

${ }^{1}$ H NMR (400 MHz, $\left.\mathrm{CDCl}_{3}\right): \delta$ 7.18-7.34 (10H), $5.23(\mathrm{~s}, 1 \mathrm{H}), 5.10(\mathrm{~d}$, $J=15.7 \mathrm{~Hz}, 1 \mathrm{H}), 4.46(\mathrm{~d}, J=9.4 \mathrm{~Hz}, 1 \mathrm{H}), 4.17(\mathrm{~d}, J=15.7 \mathrm{~Hz}, 1 \mathrm{H})$, $3.66(\mathrm{dq}, J=8.6,6.3 \mathrm{~Hz}, 1 \mathrm{H}), 2.96(\mathrm{dd}, J=9.4,8.6 \mathrm{~Hz}, 1 \mathrm{H}), 1.55(\mathrm{~s}$, $3 \mathrm{H}), 1.01(\mathrm{~d}, J=6.3 \mathrm{~Hz}, 3 \mathrm{H}) ; \mathbf{M S}(\mathrm{ESI}+)$ calculated for $\mathrm{C}_{20} \mathrm{H}_{23} \mathrm{~N}_{2} \mathrm{O}_{2}$ $(\mathrm{M}+1)$ 323.2, found 323.0.

(4S,5S,6R)-30Fa: $(4 S, 5 S, 6 R)-5$-acetyl-1-benzyl-6-methyl-4-phenethyltetrahydropyrimidin2(1H)-one

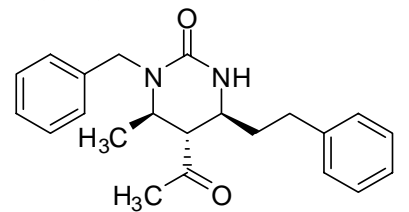

${ }^{1}$ H NMR (400 MHz, $\left.\mathrm{CDCl}_{3}\right): \delta$ 7.14-7.31 (10H), $5.94(\mathrm{~s}, 1 \mathrm{H}), 5.13(\mathrm{~d}$, $J=15.7 \mathrm{~Hz}, 1 \mathrm{H}), 4.19(\mathrm{~d}, J=15.7 \mathrm{~Hz}, 1 \mathrm{H}), 3.63-3.71(2 \mathrm{H}), 2.65-2.85$ $(3 \mathrm{H}), 2.04(\mathrm{~s}, 3 \mathrm{H}), 1.79(\mathrm{~m}, 2 \mathrm{H}), 1.17(\mathrm{~d}, J=6.3 \mathrm{~Hz}, 3 \mathrm{H})$; MS (ESI+) calculated for $\mathrm{C}_{22} \mathrm{H}_{27} \mathrm{~N}_{2} \mathrm{O}_{2}(\mathrm{M}+1)$ 351.2, found 350.9.

(4S,5R,6R)-27Fb: (4S,5R,6R)-methyl 1-ethyl-6-methyl-2-oxo-4phenethylhexahydropyrimidine-5-carboxylate<smiles>CCN1C(=O)NC(CCc2ccccc2)[C@H](C(=O)OC)[C@H]1C</smiles>

${ }^{1}$ H NMR $\left(400 \mathrm{MHz}, \mathrm{CDCl}_{3}\right): \delta$ 7.15-7.31 (5H), $4.94(\mathrm{~s}, 1 \mathrm{H}), 3.71-3.81$ $(2 \mathrm{H}), 3.70(\mathrm{~s}, 3 \mathrm{H}), 3.61(\mathrm{~m}, 1 \mathrm{H}), 3.15(\mathrm{~m}, 1 \mathrm{H}), 2.96($ app. $\mathrm{t}, J=4.7 \mathrm{~Hz}$, $1 \mathrm{H}), 2.62-2.80(2 \mathrm{H}), 1.62-1.88(2 \mathrm{H}), 1.32(\mathrm{~d}, J=6.3 \mathrm{~Hz}, 3 \mathrm{H}), 1.10(\mathrm{t}, J$ $=7.0 \mathrm{~Hz}, 3 \mathrm{H}) ;{ }^{13} \mathbf{C}$ NMR $\left(100 \mathrm{MHz}, \mathrm{CDCl}_{3}\right): \delta 170.1,155.8,140.8$, $128.8,128.6,126.5,51.9,50.9,50.5,48.1,38.5,35.7,32.6,17.7,14.0$. MS (ESI+) calculated for $\mathrm{C}_{17} \mathrm{H}_{25} \mathrm{~N}_{2} \mathrm{O}_{3}(\mathrm{M}+1)$ 305.2, found 305.0.

(4S,5S,6R)-28Fb: $(4 S, 5 S, 6 R)$-methyl 1-ethyl-6-methyl-2-oxo-4phenethylhexahydropyrimidine-5-carboxylate<smiles>CCN1C(=O)NC(CCc2ccccc2)[C@H](C(=O)OC)[C@H]1C</smiles>

${ }^{1} \mathbf{H}$ NMR $\left(400 \mathrm{MHz}, \mathrm{CDCl}_{3}\right): \delta$ 7.13-7.31 (5H), $4.75(\mathrm{~s}, 1 \mathrm{H}), 3.71-3.81$ $(2 \mathrm{H}), 3.73(\mathrm{~s}, 3 \mathrm{H}), 3.60(\mathrm{~m}, 1 \mathrm{H}), 3.15(\mathrm{~m}, 1 \mathrm{H}), 2.59-2.80(2 \mathrm{H}), 2.52$ (app. t, $J=8.6 \mathrm{~Hz}, 1 \mathrm{H}), 1.62-1.88(2 \mathrm{H}), 1.25(\mathrm{~d}, J=6.3 \mathrm{~Hz}, 3 \mathrm{H}), 1.11$ $(\mathrm{t}, J=7.0 \mathrm{~Hz}, 3 \mathrm{H}) ;$ MS (ESI+) calculated for $\mathrm{C}_{17} \mathrm{H}_{25} \mathrm{~N}_{2} \mathrm{O}_{3}(\mathrm{M}+1)$ 305.2, found 304.9.

$(4 R, 5 S, 6 R)-30 \mathrm{Ab}:(4 R, 5 S, 6 R)-5$-acetyl-1-ethyl-6-methyl-4-phenyltetrahydropyrimidin2(1H)-one<smiles>CCN1C(=O)N[C@H](c2ccccc2)[C@H](C(C)=O)[C@@H]1C</smiles>

${ }^{1}$ H NMR (400 MHz, $\left.\mathrm{CDCl}_{3}\right): \delta$ 7.26-7.38 (5H), $4.86(\mathrm{~s}, 1 \mathrm{H}), 4.42(\mathrm{~d}, J$ $=9.4 \mathrm{~Hz}, 1 \mathrm{H}), 3.76-3.84(2 \mathrm{H}), 3.24(\mathrm{~m}, 1 \mathrm{H}), 2.93$ (app. t, $J=9.4 \mathrm{~Hz}$, $1 \mathrm{H}), 1.81(\mathrm{~s}, 3 \mathrm{H}), 1.16(\mathrm{~d}, J=6.3 \mathrm{~Hz}, 3 \mathrm{H}), 1.14(\mathrm{t}, J=7.0 \mathrm{~Hz}, 3 \mathrm{H})$; MS (ESI+) calculated for $\mathrm{C}_{15} \mathrm{H}_{21} \mathrm{~N}_{2} \mathrm{O}_{2}(\mathrm{M}+1)$ 261.2, found 260.9.

$(4 S, 5 S, 6 R)-30 \mathrm{Fb}:(4 S, 5 S, 6 R)-5$-acetyl-1-ethyl-6-methyl-4-phenethyltetrahydropyrimidin2(1H)-one<smiles>CCN1C(=O)NC(CCc2ccccc2)[C@H](C(C)=O)[C@H]1C</smiles>

${ }^{1}$ H NMR (400 MHz, $\left.\mathrm{CDCl}_{3}\right): \delta$ 7.13-7.31 (5H), $5.31(\mathrm{~s}, 1 \mathrm{H}), 3.61-3.75$ $(2 \mathrm{H}), 3.54(\mathrm{~m}, 1 \mathrm{H}), 3.18(\mathrm{~m}, 1 \mathrm{H}), 2.60-2.82(2 \mathrm{H}), 2.65$ (app. t, $J=8.6$ $\mathrm{Hz}, 1 \mathrm{H}), 2.21(\mathrm{~s}, 3 \mathrm{H}), 1.67-1.88(2 \mathrm{H}), 1.24(\mathrm{~d}, J=6.3 \mathrm{~Hz}, 3 \mathrm{H}), 1.11(\mathrm{t}$, $J=7.0 \mathrm{~Hz}, 3 \mathrm{H})$; MS (ESI+) calculated for $\mathrm{C}_{17} \mathrm{H}_{25} \mathrm{~N}_{2} \mathrm{O}_{2}(\mathrm{M}+1)$ 289.2, found 288.9 . 
$(4 R, 5 R, 6 R)-27 A c:(4 R, 5 R, 6 R)-m e t h y l$ 1-(2-ethoxy-2-oxoethyl)-6-methyl-2-oxo-4phenylhexahydropyrimidine-5-carboxylate

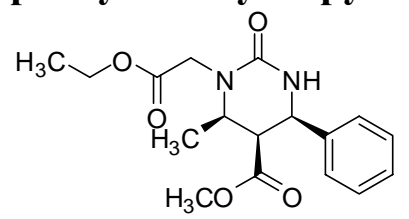

${ }^{1}$ H NMR (400 MHz, $\left.\mathrm{CDCl}_{3}\right): \delta$ 7.22-7.38 (5H), $5.17(\mathrm{~s}, 1 \mathrm{H}), 4.95(\mathrm{~d}, J$ $=4.7 \mathrm{~Hz}, 1 \mathrm{H}), 4.88(\mathrm{~d}, J=18.0 \mathrm{~Hz}, 1 \mathrm{H}), 4.17-4.23(3 \mathrm{H}), 3.70(\mathrm{~d}, J=$ $18.0 \mathrm{~Hz}, 1 \mathrm{H}), 3.44$ (s, 3H), 3.10 (app. t, $J=4.7 \mathrm{~Hz}, 1 \mathrm{H}), 1.28$ (t, $J=7.0$ $\mathrm{Hz}, 3 \mathrm{H}), 1.21(\mathrm{~d}, J=7.0 \mathrm{~Hz}, 3 \mathrm{H}) ; \mathbf{M S}$ (ESI+) calculated for $\mathrm{C}_{17} \mathrm{H}_{23} \mathrm{~N}_{2} \mathrm{O}_{5}(\mathrm{M}+1)$ 335.2, found 335.0.

(4S,5R,6R)-27Fc: (4S,5R,6R)-methyl 1-(2-ethoxy-2-oxoethyl)-6-methyl-2-oxo-4phenethylhexahydro- pyrimidine-5-carboxylate

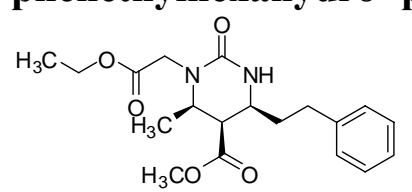

${ }^{1}$ H NMR (400 MHz, $\left.\mathrm{CDCl}_{3}\right): \delta$ 7.16-7.30 (5H), $5.22(\mathrm{~s}, 1 \mathrm{H}), 4.78(\mathrm{~d}, J$ $=18.0 \mathrm{~Hz}, 1 \mathrm{H}), 4.12-4.18(3 \mathrm{H}), 3.94(\mathrm{dq}, J=6.3,4.7 \mathrm{~Hz}, 1 \mathrm{H}), 3.70(\mathrm{~s}$, $3 \mathrm{H}), 3.67$ (d, $J=18.0 \mathrm{~Hz}, 1 \mathrm{H}), 3.00$ (app. t, $J=4.7 \mathrm{~Hz}, 1 \mathrm{H}), 2.58-2.80$ $(2 \mathrm{H}), 1.67-2.02(2 \mathrm{H}), 1.25(\mathrm{t}, J=7.0 \mathrm{~Hz}, 3 \mathrm{H}), 1.23(\mathrm{~d}, J=7.0 \mathrm{~Hz}, 3 \mathrm{H})$; MS (ESI+) calculated for $\mathrm{C}_{19} \mathrm{H}_{27} \mathrm{~N}_{2} \mathrm{O}_{5}(\mathrm{M}+1) 363.2$, found 362.9 .

(4R,5S,6R)-30Ac: ethyl 2-((4R,5S,6R)-5-acetyl-6-methyl-2-oxo-4phenyltetrahydropyrimidin-1(2H)-yl)acetate<smiles>CCOC(=O)CN1C(=O)NC(c2ccccc2)[C@H](C(C)=O)[C@H]1C</smiles>

${ }^{1}$ H NMR (400 MHz, $\left.\mathrm{CDCl}_{3}\right): \delta$ 7.27-7.36 (5H), $4.96(\mathrm{~s}, 1 \mathrm{H}), 4.53(\mathrm{~d}, J$ $=10.0 \mathrm{~Hz}, 1 \mathrm{H}), 4.48(\mathrm{~d}, J=18.0 \mathrm{~Hz}, 1 \mathrm{H}), 4.18-4.22(3 \mathrm{H}), 3.98(\mathrm{~d}, J=$ $18.0 \mathrm{~Hz}, 1 \mathrm{H}), 3.00$ (app. t, $J=10.2 \mathrm{~Hz}, 1 \mathrm{H}), 1.84$ (s, 3H), 1.29 (t, $J=$ $7.0 \mathrm{~Hz}, 3 \mathrm{H}), 1.15(\mathrm{~d}, J=6.3 \mathrm{~Hz}, 3 \mathrm{H})$; MS (ESI+) calculated for $\mathrm{C}_{17} \mathrm{H}_{23} \mathrm{~N}_{2} \mathrm{O}_{4}(\mathrm{M}+1)$ 319.2, found 318.9.

(4R,5R,6R)-27Ae: (4R,5R,6R)-methyl 1-(2-methoxyethyl)-6-methyl-2-oxo-4phenylhexahydropyrimidine-5-carboxylate<smiles>COCCN1C(=O)NC(c2ccccc2)[C@H](C(=O)OC)[C@H]1C</smiles>

${ }^{1}$ H NMR (400 MHz, $\left.\mathrm{CDCl}_{3}\right): \delta$ 7.27-7.32 (5H), $5.06(\mathrm{~s}, 1 \mathrm{H}), 4.88(\mathrm{~d}, J$ $=4.7 \mathrm{~Hz}, 1 \mathrm{H}), 4.04(\mathrm{dq}, J=6.3,3.9 \mathrm{~Hz}, 1 \mathrm{H}), 3.94(\mathrm{dt}, J=14.9,4.7 \mathrm{~Hz}$, $1 \mathrm{H}), 3.36-3.50(2 \mathrm{H}), 3.44$ (s, 3H), 3.33 (s, 3H), 3.06 (app. t, $J=3.9 \mathrm{~Hz}$, $1 \mathrm{H}), 1.28(\mathrm{~d}, J=6.3 \mathrm{~Hz}, 3 \mathrm{H}) ; \mathbf{M S}(\mathrm{ESI}+)$ calculated for $\mathrm{C}_{16} \mathrm{H}_{23} \mathrm{~N}_{2} \mathrm{O}_{4}$ $(\mathrm{M}+1)$ 307.2, found 306.9.

(4R,5S,6R)-28Ae: $(4 R, 5 S, 6 R)$-methyl 1-(2-methoxyethyl)-6-methyl-2-oxo-4phenylhexahydropyrimidine-5-carboxylate<smiles>COCCN1C(=O)N[C@@H](c2ccccc2)[C@H](C(=O)OC)[C@H]1C</smiles>

${ }^{1}$ H NMR (400 MHz, $\left.\mathrm{CDCl}_{3}\right): \delta$ 7.27-7.32 (5H), $4.94(\mathrm{~s}, 1 \mathrm{H}), 4.68(\mathrm{~d}, J$ $=9.4 \mathrm{~Hz}, 1 \mathrm{H}), 3.89-3.98(2 \mathrm{H}), 3.42-3.60(2 \mathrm{H}), 3.50(\mathrm{~s}, 3 \mathrm{H}), 3.28(\mathrm{~m}$, $1 \mathrm{H}), 3.34$ (s, 3H), 2.74 (app. t, $J=9.0 \mathrm{~Hz}, 1 \mathrm{H}), 1.18$ (d, $J=6.3 \mathrm{~Hz}$, $3 \mathrm{H})$; MS (ESI+) calculated for $\mathrm{C}_{16} \mathrm{H}_{23} \mathrm{~N}_{2} \mathrm{O}_{4}(\mathrm{M}+1)$ 307.2, found 306.9.

(4S,5R,6R)-27Fe: (4S,5R,6R)-methyl 1-(2-methoxyethyl)-6-methyl-2-oxo-4phenethylhexahydro pyrimidine-5-carboxylate 


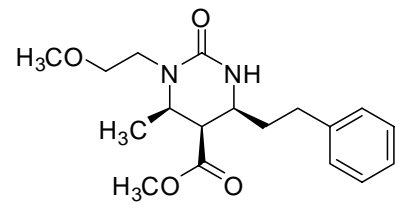

${ }^{1}$ H NMR $\left(400 \mathrm{MHz} \mathrm{CDCl}_{3}\right): \delta$ 7.12-7.30 (5H), $5.17(\mathrm{~s}, 1 \mathrm{H}), 3.84-3.93$ $(2 \mathrm{H}), 3.70(\mathrm{~s}, 3 \mathrm{H}), 3.42-3.58(3 \mathrm{H}), 3.30(\mathrm{~s}, 3 \mathrm{H}), 3.22(\mathrm{~m}, 1 \mathrm{H}), 2.97$ (app. t, $J=4.7 \mathrm{~Hz}, 1 \mathrm{H}), 2.55-2.80(2 \mathrm{H}), 1.74-1.88(2 \mathrm{H}), 1.33(\mathrm{~d}, J=$ $7.0 \mathrm{~Hz}, 3 \mathrm{H}) ;{ }^{13} \mathbf{C}$ NMR $\left(100 \mathrm{MHz}, \mathrm{CDCl}_{3}\right): \delta 169.8,155.8,140.6$, $129.0,128.5,128.34,128.30,126.2,71.8,58.8,51.7,51.6,47.7,43.1$, $35.5,33.2,32.4,17.5$; MS (ESI+) calculated for $\mathrm{C}_{17} \mathrm{H}_{27} \mathrm{~N}_{2} \mathrm{O}_{4}(\mathrm{M}+1)$ 335.2, found 334.9.

(4R,5S,6R)-28Fe: $(4 S, 5 S, 6 R)$-methyl 1-(2-methoxyethyl)-6-methyl-2-oxo-4phenethylhexahydropyrimidine-5-carboxylate<smiles>COCCN1C(=O)NC(CCc2ccccc2)[C@H](C(=O)OC)[C@H]1C</smiles>

${ }^{1}$ H NMR $\left(400 \mathrm{MHz}, \mathrm{CDCl}_{3}\right): \delta$ 7.15-7.32 (5H), $5.20(\mathrm{~s}, 1 \mathrm{H}), 3.86-3.94$ $(2 \mathrm{H}), 3.73(\mathrm{~s}, 3 \mathrm{H}), 3.43-3.58(3 \mathrm{H}), 3.32(\mathrm{~s}, 3 \mathrm{H}), 3.23(\mathrm{~m}, 1 \mathrm{H}), 2.53-$ $2.82(3 \mathrm{H}), 1.73-1.87(2 \mathrm{H}), 1.26(\mathrm{~d}, J=6.3 \mathrm{~Hz}, 3 \mathrm{H})$; MS (ESI+) calculated for $\mathrm{C}_{17} \mathrm{H}_{27} \mathrm{~N}_{2} \mathrm{O}_{4}(\mathrm{M}+1)$ 335.2, found 335.0.

(4R,5S,6R)-30Ae: $(4 R, 5 S, 6 R)-5$-acetyl-1-(2-methoxyethyl)-6-methyl-4phenyltetrahydropyrimidin-2(1H)-one<smiles>COCCN1C(=O)N[C@@H](c2ccccc2)[C@H](C(C)=O)[C@H]1C</smiles>

${ }^{1}$ H NMR (400 MHz, $\left.\mathrm{CDCl}_{3}\right): \delta$ 7.26-7.40 (5H), $5.01(\mathrm{~s}, 1 \mathrm{H}), 4.53(\mathrm{~d}, J$ $=8.6 \mathrm{~Hz}, 1 \mathrm{H}), 3.82-3.98(2 \mathrm{H}), 3.53(\mathrm{~m}, 2 \mathrm{H}), 3.34(\mathrm{~s}, 3 \mathrm{H}), 3.32(\mathrm{~m}$, 1H), 2.92 (app. t, $J=8.6 \mathrm{~Hz}, 1 \mathrm{H}), 1.75$ (s, 3H), 1.07 (d, $J=6.3 \mathrm{~Hz}$, $3 \mathrm{H})$; MS (ESI+) calculated for $\mathrm{C}_{16} \mathrm{H}_{23} \mathrm{~N}_{2} \mathrm{O}_{3}(\mathrm{M}+1)$ 291.2, found 290.8 .

(4R,5S,6R)-30Fe: $(4 S, 5 S, 6 R)-5$-acetyl-1-(2-methoxyethyl)-6-methyl-4phenethyltetrahydropyrimidin-2(1H)-one

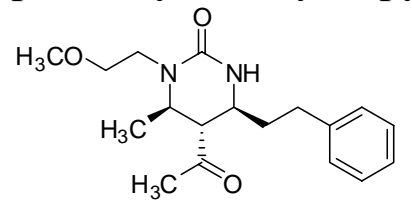

${ }^{1}$ H NMR (400 MHz, $\left.\mathrm{CDCl}_{3}\right): \delta$ 7.11-7.31 (5H), 5.27 (s, 1H), $3.90(\mathrm{dt}, J$ $=14.9,4.7 \mathrm{~Hz}, 1 \mathrm{H}), 3.80(\mathrm{~m}, 1 \mathrm{H}), 3.48-3.56(3 \mathrm{H}), 3.29(\mathrm{~s}, 3 \mathrm{H}), 3.19$ $(\mathrm{m}, 1 \mathrm{H}), 2.78(\mathrm{~m}, 1 \mathrm{H}), 2.56-2.6(2 \mathrm{H}), 1.73-1.88(2 \mathrm{H}), 1.28(\mathrm{~d}, J=6.3$ $\mathrm{Hz}, 3 \mathrm{H})$; MS (ESI+) calculated for $\mathrm{C}_{18} \mathrm{H}_{27} \mathrm{~N}_{2} \mathrm{O}_{3}(\mathrm{M}+1)$ 319.2, found 318.9 .

(4R,5S,6R)-28Ah: (4R,5S,6R)-methyl 6-methyl-2-oxo-4-phenyl-1-(3,4,5trimethoxyphenethyl)- hexahydropyrimidine-5-carboxylate

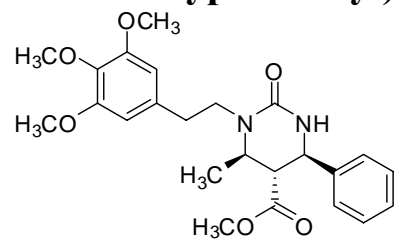

${ }^{1}$ H NMR $\left(400 \mathrm{MHz}, \mathrm{CDCl}_{3}\right): \delta 7.25-7.35(7 \mathrm{H}), 4.83(\mathrm{~s}, 1 \mathrm{H}), 4.60(\mathrm{~d}, J$ $=8.6 \mathrm{~Hz}, 1 \mathrm{H}), 3.88-4.04(2 \mathrm{H}), 3.86(\mathrm{~s}, 6 \mathrm{H}), 3.82(\mathrm{~s}, 3 \mathrm{H}), 3.80(\mathrm{~s}, 3 \mathrm{H})$, 3.31 (ddd, $J=14.1,10.2,6.3 \mathrm{~Hz}, 1 \mathrm{H}), 2.90$ (ddd, $J=13.3,8.6,5.5 \mathrm{~Hz}$, 1H), 2.79 (m, 1H), 2.77 (app. t, $J=8.6 \mathrm{~Hz}, 1 \mathrm{H}), 1.20(\mathrm{~d}, J=7.0 \mathrm{~Hz}$, $3 \mathrm{H})$; MS (ESI+) calculated for $\mathrm{C}_{24} \mathrm{H}_{31} \mathrm{~N}_{2} \mathrm{O}_{6}(\mathrm{M}+1)$ 443.2, found 442.8 . 


\section{(S)-methyl 1-benzyl-3-(3-methoxy-3-oxopropanoyl)-6-methyl-2-oxo-4-phenyl-1,2,3,4-} tetrahydropyrimidine-5-carboxylate

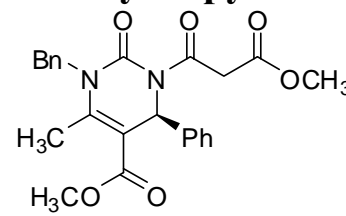

A round-bottom flask was equipped with stir bar and purged with argon.

Dihydropyrimidone 31a (1.0 mmol, $350 \mathrm{mg})$, benzene $(10 \mathrm{~mL})$ and methyl 3-chloro-3-oxo-propionate $(270 \mu \mathrm{L}, 2.0 \mathrm{mmol})$ was added successively. The reaction mixture was heated to reflux for 1 hour and cooled to room temperature. Saturated $\mathrm{NaHCO}_{3}(10.0 \mathrm{~mL})$ and ethyl acetate $(10 \mathrm{~mL})$ was added to quench the reaction. The organic layer was separated and washed with water and brine. The solution was dried over anhydrous sodium sulfate, filtered and concentrated under reduced pressure. The resulting residue was subjected to column chromatography on silica gel to afford the amide product. Yield: $420 \mathrm{mg}, 92 \% .{ }^{1} \mathbf{H}$ NMR $\left(400 \mathrm{MHz}, \mathrm{CDCl}_{3}\right): \delta$ 7.28-7.09 (m, 8H), $6.83(\mathrm{~s}, 1 \mathrm{H}), 6.73(\mathrm{~d}, J=7.2 \mathrm{~Hz}, 2 \mathrm{H}), 5.23(\mathrm{~d}, J=16.4$ $\mathrm{Hz}, 1 \mathrm{H}), 4.62(\mathrm{~d}, J=16.4 \mathrm{~Hz}, 1 \mathrm{H}), 4.02(\mathrm{~d}, J=16.4 \mathrm{~Hz}, 1 \mathrm{H}), 3.92$ (d, $J=$ $16.4 \mathrm{~Hz}, 1 \mathrm{H}), 3.72(\mathrm{~s}, 6 \mathrm{H}), 2.45(\mathrm{~s}, 3 \mathrm{H}) .{ }^{13} \mathrm{C}$ NMR $\left(75.0 \mathrm{MHz}, \mathrm{CDCl}_{3}\right)$ : $\delta 168.0,167.3,165.8,152.8,149.4,138.8,136.4,128.8,128.2,127.6$, 126.7, 126.5, 110.3, 52.6, 52.1, 51.1, 47.0, 45.1, 16.4. IR (neat, $\mathrm{cm}^{-1}$ ): 3020, 2952, 1742, 1695, 1635, 1436, 1385, 1215, 1166. HRMS: calc'd for $(\mathrm{M}+\mathrm{H})^{+} \mathrm{C}_{14} \mathrm{H}_{25} \mathrm{~N}_{2} \mathrm{O}_{6}$ : 437.1534; found: 437.1578 .

(S)-methyl 1-benzyl-3-(2-diazo-3-methoxy-3-oxopropanoyl)-6-methyl-2-oxo-4-phenyl-

\section{1,2,3,4-tetrahydropyrimidine-5-carboxylate (32a)}

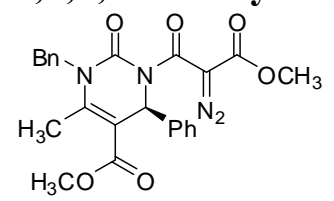

Into a round-bottom flask equipped with stir bar was added amide (724 $\mathrm{mg}, \quad 1.61 \mathrm{mmol}$ ) obtained from previous reaction. $p$ Acetamidobenzenesulfonyl azide ( $p$-ABSA, $772 \mathrm{mg}, 3.22 \mathrm{mmol}$ ) and $\mathrm{CH}_{2} \mathrm{Cl}_{2}(20 \mathrm{~mL})$ was added successively. The reaction solution was cooled in ice bath and DBU $(721 \mu \mathrm{L}, 4.8 \mathrm{mmol})$ was added. The reaction mixture was stirred at room temperature for overnight and quenched with $5 \% \mathrm{KOH}$ aqueous solution. The organic layer was separated and washed with water and brine. The organic solution was dried over sodium sulfate, filtered and concentrated under reduced pressure. The residue was subjected to column chromatography on slice gel to afford the desired product 32a. Yield: $689 \mathrm{mg}, 90 \%$. ${ }^{1} \mathbf{H}$ NMR $\left(400 \mathrm{MHz}, \mathrm{CDCl}_{3}\right): \delta 7.21$ $(\mathrm{m}, 5 \mathrm{H}), 7.08(\mathrm{~m}, 3 \mathrm{H}), 6.74(\mathrm{~d}, J=7.6 \mathrm{~Hz}, 2 \mathrm{H}), 6.30(\mathrm{~s}, 1 \mathrm{H}), 5.29(\mathrm{~d}, J=$ $16.4 \mathrm{~Hz}, 1 \mathrm{H}), 4.58(\mathrm{~d}, J=16.4 \mathrm{~Hz}, 1 \mathrm{H}), 3.78(\mathrm{~s}, 3 \mathrm{H}), 3.75(\mathrm{~s}, 3 \mathrm{H}), 2.50(\mathrm{~s}$, $3 \mathrm{H}) .{ }^{13} \mathrm{C}$ NMR $\left(75.0 \mathrm{MHz}, \mathrm{CDCl}_{3}\right): \delta 165.9,163.4,161.5,152.7,149.8$, $139.0,136.6,128.7,128.1,127.5,127.0,126.7,111.3,54.1,52.6,52.1$, 46.9, 16.5. IR (neat, $\mathrm{cm}^{-1}$ ): 3370, 3020, 2953, 2138, 1720, 1690, 1648, 1495, 1435, 1384, 1278, 1215, 1125, 740. HRMS: calc'd for $(\mathrm{M}+\mathrm{H})^{+}$ $\mathrm{C}_{14} \mathrm{H}_{24} \mathrm{~N}_{4} \mathrm{O}_{6}$ : 463.1539 ; found: 463.1577 . 


\section{General procedure for intermolecular dipolar cycloaddition}<smiles>COC(=O)C(=N)C(=O)N1C(=O)N(Cc2ccccc2)C(C)=C(C(=O)OC)C1[PH2+]</smiles>

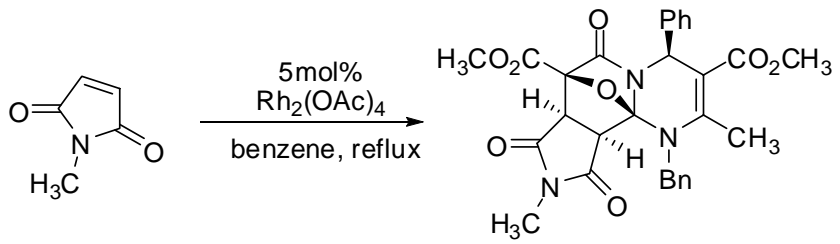

An oven dried $17 \times 110 \mathrm{~mm}$ round bottom reaction vessel was charged with $\mathrm{Rh}_{2}(\mathrm{OAc})_{4}(2.5 \mathrm{mg}$, $0.005 \mathrm{mmol}$ ), diazoamide 32a (45 $\mathrm{mg}, 0.10 \mathrm{mmol})$, methyl maleimide $(34 \mathrm{mg}, 0.30 \mathrm{mmol})$ and anhydrous benzene $(2 \mathrm{~mL})$ successively. The solution was heated to reflux and stirred under nitrogen for $1.0 \mathrm{~h}$. The reaction mixture was filtered through a silica plug via elution with ethyl acetate. The filtrate was concentrated under reduced pressure and subjected to the flash chromatography over silica gel (elution with $15-30 \%$ ethyl acetate in hexanes) to give the desired bicycle product $\mathbf{3 4 a}$ (50 $\mathrm{mg}, 92 \%$ yield).
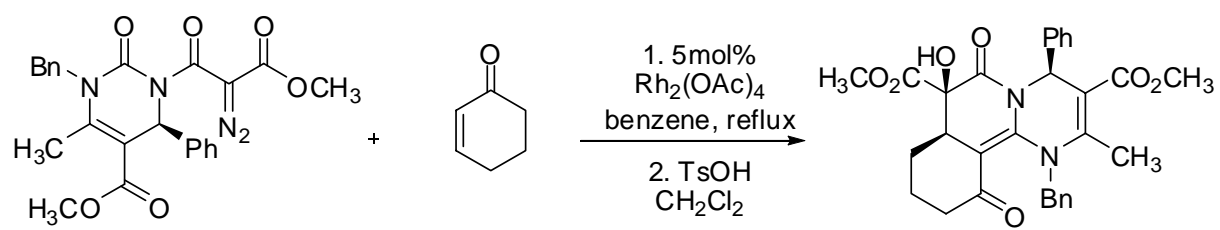

An oven dried $17 \times 110 \mathrm{~mm}$ round bottom reaction vessel was charged with $\mathrm{Rh}_{2}(\mathrm{OAc})_{4}(2.5 \mathrm{mg}$, $0.005 \mathrm{mmol}$ ), diazoamide 32a (45 $\mathrm{mg}, 0.10 \mathrm{mmol})$, methyl maleimide $(34 \mathrm{mg}, 0.30 \mathrm{mmol})$ and anhydrous benzene $(2 \mathrm{~mL})$ seccessively. The solution was heated to reflux and stirred under nitrogen for $1.0 \mathrm{~h}$. The reaction mixture was filtered through a silica plug via elution with ethyl acetate. The filtrate was concentrated under reduced pressure and was treated with $p$-toluene sulfonic acid $(5 \mathrm{mg})$ and $\mathrm{CH}_{2} \mathrm{Cl}_{2}(2 \mathrm{~mL})$. The reaction mixture was stirred at room temperature for overnight. The filtrate was concentrated under reduced pressure and directly subjected to the flash chromatography over silica gel (elution with $30-50 \%$ ethyl acetate in hexanes) to afford the desired tertiary alcohol 36h (42 mg, 80\% yield). 
General procedure of NOE experiment to determine the relative stereochemistry of dipolar cycloaddition product.

The ${ }^{1} \mathrm{H}-\mathrm{NMR}$ of $\mathbf{3 4 c}(10 \mathrm{mg})$ was aquired. NOESY1D was chosen on Varian Unity 400 NMR. $\mathrm{H}_{\mathrm{a}}$ at $5.49 \mathrm{ppm}$ was chosen to be irradiated. The spinner was turned off and the lock level was adjusted to be $>70$ with the spinner off. The experiment was run for $2 \mathrm{~h}$. The observed NOE of $\mathrm{H}_{\mathrm{b}}$ confirmed the close proximity to $\mathrm{H}_{\mathrm{a}}$.<smiles></smiles>
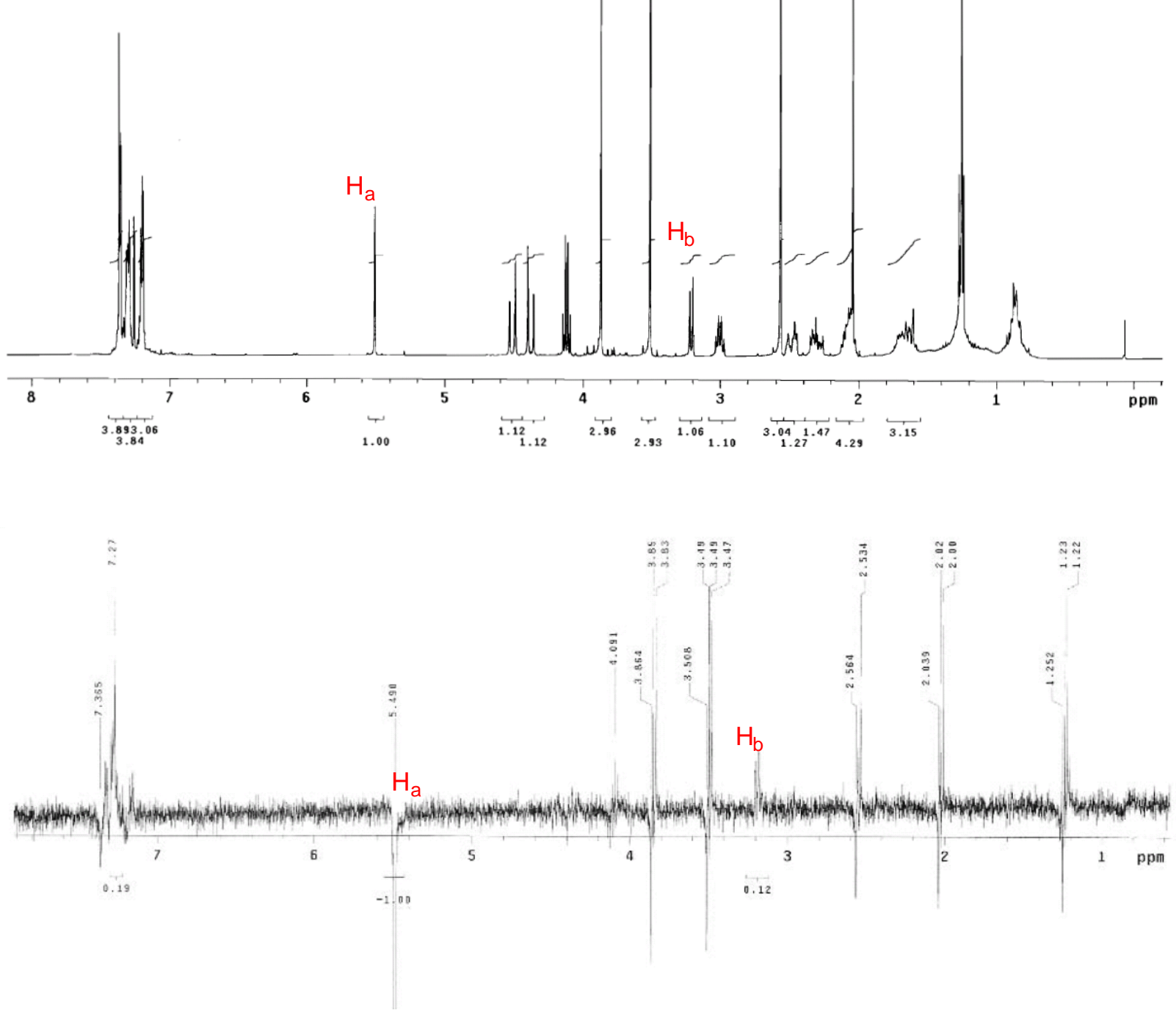
The ${ }^{1} \mathrm{H}-\mathrm{NMR}$ of $\mathbf{3 4 g}$ (10 mg) was aquired. NOESY1D was chosen on Varian Unity 400 NMR. $\mathrm{H}_{\mathrm{a}}$ at $5.49 \mathrm{ppm}$ was chosen to be irradiated. The spinner was turned off and the lock level was adjusted to be $>70$ with the spinner off. The experiment was run for $2 \mathrm{~h}$. The observed NOE of $\mathrm{H}_{\mathrm{b}}$ confirmed the close proximity to $\mathrm{H}_{\mathrm{a}}$.
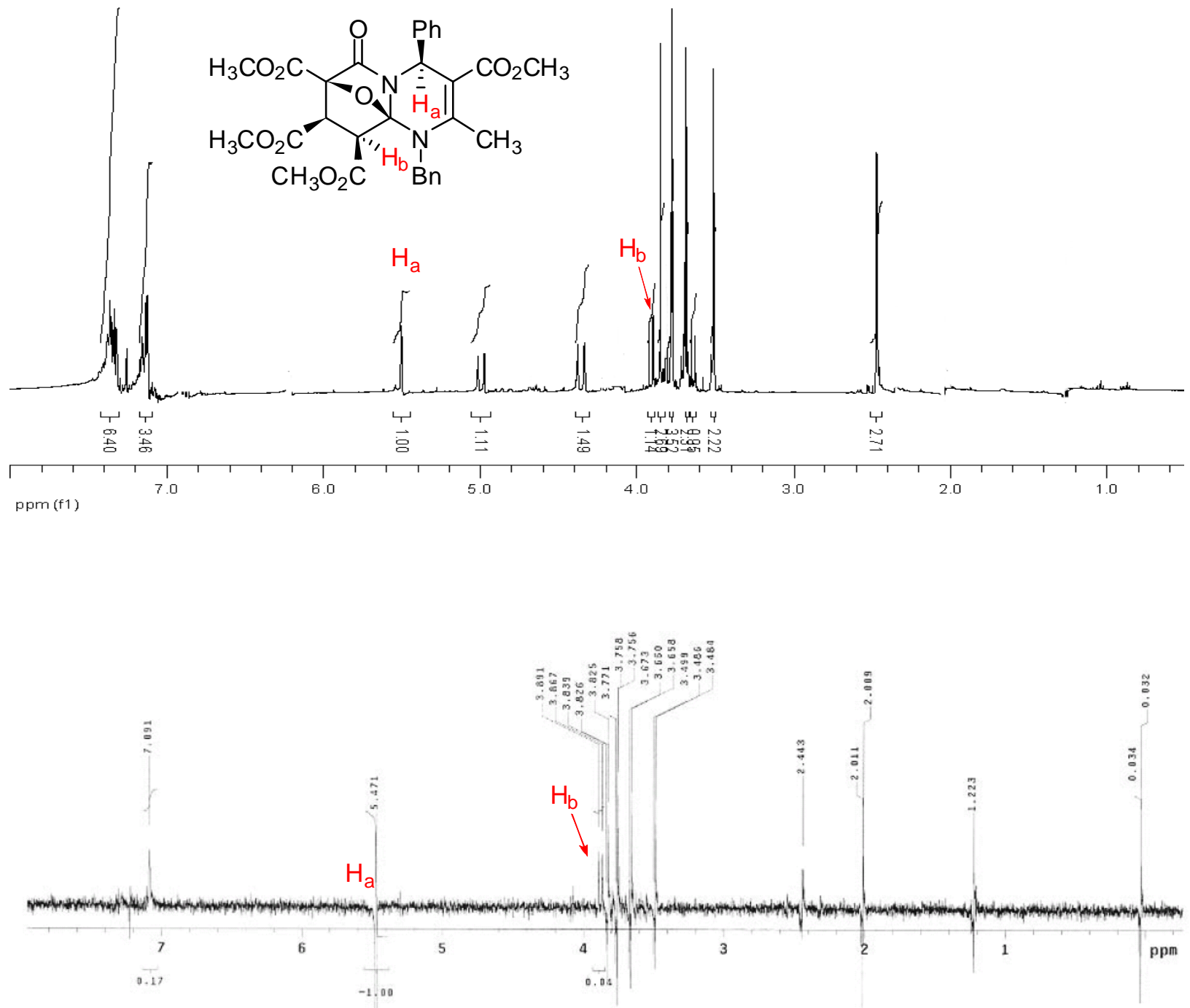


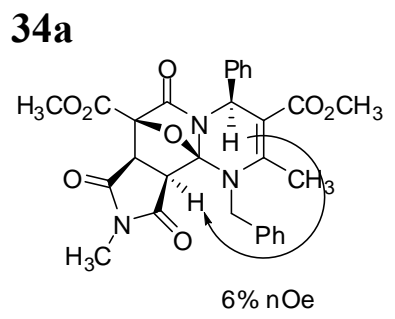

Yield: $25 \mathrm{mg}, 92 \% .{ }^{1} \mathbf{H}$ NMR $\left(400 \mathrm{MHz}, \mathrm{CDCl}_{3}\right): \delta 7.22(\mathrm{~m}, 4 \mathrm{H}), 7.12$ $(\mathrm{m}, 3 \mathrm{H}), 7.10(\mathrm{~m}, 3 \mathrm{H}), 5.52(\mathrm{~s}, 1 \mathrm{H}), 4.48(\mathrm{~s}, 2 \mathrm{H}), 3.83(\mathrm{~s}, 3 \mathrm{H}), 3.65(\mathrm{~d}$, $J=6.8 \mathrm{~Hz}, 1 \mathrm{H}), 3.59$ (d, $J=6.8 \mathrm{~Hz}, 1 \mathrm{H}), 3.45$ (s, 3H), $2.94(\mathrm{~s}, 3 \mathrm{H})$, $2.53(\mathrm{~s}, 3 \mathrm{H}) .{ }^{13} \mathrm{C}$ NMR $\left(75.0 \mathrm{MHz}, \mathrm{CDCl}_{3}\right): \delta 171.8,171.5,166.1$, 164.6, 161.7, 153.3, 139.3, 137.2, 128.7, 128.2, 127.9, 127.8, 103.9, 100.1, 85.1, 54.4, 53.2, 51.1, 49.9, 48.6, 47.4, 25.6, 16.5. IR (neat, $\mathrm{cm}^{-}$ $\left.{ }^{1}\right)$ : 3470, 2954, 1752, 1708, 1592, 1408, 1285, 1138, 737. HRMS: calc'd for $(\mathrm{M}+\mathrm{Na})^{+} \mathrm{C}_{29} \mathrm{H}_{27} \mathrm{~N}_{3} \mathrm{O}_{8} \mathrm{Na}$ : 568.1696; found: 568.1750.

35a The reaction was run in $0.05 \mathrm{mmol}$ scale.

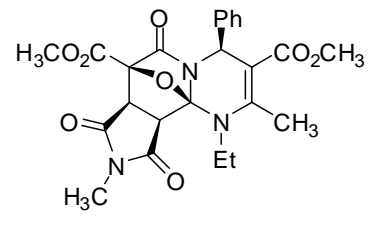

Yield: $23 \mathrm{mg}, 93 \% .{ }^{1} \mathbf{H}$ NMR (400 MHz, $\left.\mathrm{CDCl}_{3}\right): \delta 7.28(\mathrm{~m}, 2 \mathrm{H}), 7.15$ $(\mathrm{m}, 3 \mathrm{H}), 5.52(\mathrm{~s}, 1 \mathrm{H}), 3.96(\mathrm{~d}, J=20 \mathrm{~Hz}, 1 \mathrm{H}), 3.87(\mathrm{~s}, 3 \mathrm{H}), 3.76(\mathrm{~d}, J$ $=6.8 \mathrm{~Hz}, 1 \mathrm{H}), 3.55(\mathrm{~s}, 3 \mathrm{H}), 3.22(\mathrm{~m}, 1 \mathrm{H}), 2.96(\mathrm{~d}, \mathrm{~J}=20 \mathrm{~Hz}, 1 \mathrm{H}), 2.94$ $(\mathrm{s}, 3 \mathrm{H}), 2.58(\mathrm{~s}, 3 \mathrm{H}) \cdot{ }^{13} \mathrm{C}$ NMR $\left(75.0 \mathrm{MHz}, \mathrm{CDCl}_{3}\right): \delta 171.8,171.2$, 166.3, 164.2, 152.9, 140.3, 128.3, 127.8, 127.4, 103.9, 99.8, 85.0, 54.0, 53.3, 51.1, 50.0, 48.4, 38.7, 29.7, 25.5, 16.7, 15.9. HRMS: calc'd for $(\mathrm{M}+\mathrm{H})^{+} \mathrm{C}_{34} \mathrm{H}_{29} \mathrm{~N}_{3} \mathrm{O}_{8}$ : 607.1955; found: 607.1930 .

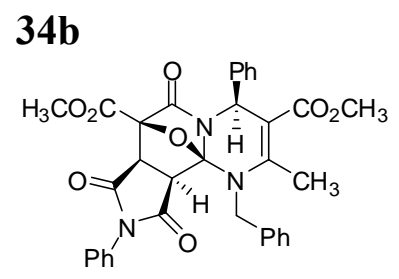

Yield: $27 \mathrm{mg}, 90 \% .{ }^{1} \mathbf{H}$ NMR $\left(400 \mathrm{MHz}, \mathrm{CDCl}_{3}\right): \delta 7.45(\mathrm{~m}, 3 \mathrm{H}), 7.32-$ $7.20(\mathrm{~m}, 12 \mathrm{H}), 5.65(\mathrm{~s}, 1 \mathrm{H}), 4.63(\mathrm{~d}, J=16 \mathrm{~Hz}, 1 \mathrm{H}), 4.55(\mathrm{~d}, J=16$ $\mathrm{Hz}, 1 \mathrm{H}), 3.91(\mathrm{~s}, 3 \mathrm{H}), 3.84(\mathrm{~d}, J=7.2 \mathrm{~Hz}, 1 \mathrm{H}), 3.76(\mathrm{~d}, J=7.2 \mathrm{~Hz}$, $1 \mathrm{H}), 3.54(\mathrm{~s}, 3 \mathrm{H}), 2.60(\mathrm{~s}, 3 \mathrm{H}) .{ }^{13} \mathbf{C} \mathbf{N M R}\left(75.0 \mathrm{MHz}, \mathrm{CDCl}_{3}\right): \delta 171.0$, $170.6,166.1,164.5,161.6,153.3,139.3,137.2,131.0,129.4,129.3$, 128.7, 128.3, 128.0, 127.9, 127.8, 126.1, 104.1, 100.4, 85.5, 54.5, 53.3, 51.2, 49.9, 48.5, 47.4, 16.5. IR (neat, $\mathrm{cm}^{-1}$ ): 3392, 3062, 2954, 1766, 1719, 1591, 1408, 1383, 1286, 1197, 1140, 737, 698. HRMS: calc'd for $(\mathrm{M}+\mathrm{Na})^{+} \mathrm{C}_{34} \mathrm{H}_{29} \mathrm{~N}_{3} \mathrm{O}_{8} \mathrm{Na}$ : 630.1852; found: 630.1870 .

$34 c$

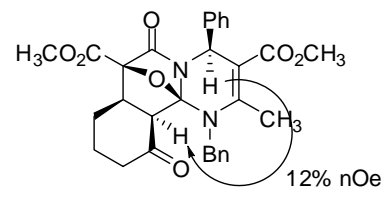

Yield: $45 \mathrm{mg}, 85 \% .{ }^{1} \mathbf{H}$ NMR (400 $\left.\mathrm{MHz}, \mathrm{CDCl}_{3}\right): \delta 7.40-7.18(\mathrm{~m}$, $10 \mathrm{H}), 5.50(\mathrm{~s}, 1 \mathrm{H}), 4.51(\mathrm{~d}, J=15.2 \mathrm{~Hz}, 1 \mathrm{H}), 4.38(\mathrm{~d}, J=15.2 \mathrm{~Hz}$, $1 \mathrm{H}), 3.84$ (s, 3H), 3.49 (s, 3H), 3.20 (d, $J=8.0 \mathrm{~Hz}, 1 \mathrm{H}), 3.0(\mathrm{dd}, J=$ 8.0, $5.6 \mathrm{~Hz}, 1 \mathrm{H}), 2.58(\mathrm{~s}, 3 \mathrm{H}), 2.54(\mathrm{~m}, 1 \mathrm{H}), 2.32(\mathrm{~m}, 1 \mathrm{H}), 2.07(\mathrm{~m}$, $2 \mathrm{H}), 1.60(\mathrm{~m}, 2 \mathrm{H})$. HRMS: calc'd for $(\mathrm{M}+\mathrm{H})^{+} \mathrm{C}_{30} \mathrm{H}_{31} \mathrm{~N}_{2} \mathrm{O}_{7}: 531.2053$; found: 531.2080 .

34d

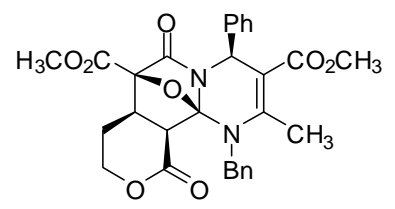

Yield: $46 \mathrm{mg}, 86 \% .{ }^{1} \mathbf{H}$ NMR $\left(400 \mathrm{MHz}, \mathrm{CDCl}_{3}\right): \delta 7.36(\mathrm{~m}, 6 \mathrm{H}), 7.30$ (m, 3H), $7.21(\mathrm{~m}, 3 \mathrm{H}), 5.59$ (s, 1H), $4.66(\mathrm{~d}, J=16 \mathrm{~Hz}, 1 \mathrm{H}), 4.61(\mathrm{~d}, J$ $=16 \mathrm{~Hz}, 1 \mathrm{H}), 4.55(\mathrm{~m}, 1 \mathrm{H}), 4.28(\mathrm{~m}, 1 \mathrm{H}), 3.87(\mathrm{~s}, 3 \mathrm{H}), 3.60(\mathrm{~d}, \mathrm{~J}=8.4$ $\mathrm{Hz}, 1 \mathrm{H}), 3.54$ (s, 3H), $3.03(\mathrm{~m}, 1 \mathrm{H}), 2.57$ (s, 3H), $2.21(\mathrm{~m}, 1 \mathrm{H}), 1.97$ $(\mathrm{m}, 1 \mathrm{H}) .{ }^{13} \mathrm{C}$ NMR $\left(75.0 \mathrm{MHz}, \mathrm{CDCl}_{3}\right): \delta 166.5,163.4,153.9,139.9$, $137.8,128.9,128.6,128.5,128.2,128.0,127.9,126.2,103.8,101.7$, $87.5,66.8,54.8,53.4,51.2,47.4,47.1,39.5,24.6,16.9$. IR (neat, $\mathrm{cm}^{-}$ 
$\left.{ }^{1}\right):$ 3404, 2954, 1732, 1581, 1408, 1266, 1266, 1107, 737, 700. HRMS: calc'd for $(\mathrm{M}+\mathrm{H})^{+} \mathrm{C}_{29} \mathrm{H}_{29} \mathrm{~N}_{2} \mathrm{O}_{8}$ : 533.1924; found: 533.1976.

$34 f$

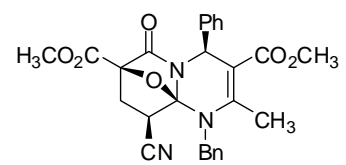

Yield: $43 \mathrm{mg}, 90 \% .{ }^{1} \mathbf{H}$ NMR $\left(400 \mathrm{MHz}, \mathrm{CDCl}_{3}\right): \delta 7.38(\mathrm{~m}, 5 \mathrm{H}), 7.34$ (m, 1H), $7.21(\mathrm{~m}, 4 \mathrm{H}), 5.54(\mathrm{~s}, 1 \mathrm{H}) 5.15(\mathrm{~d}, J=16.4 \mathrm{~Hz}, 1 \mathrm{H}), 4.84(\mathrm{~d}$, $J=16.4 \mathrm{~Hz}, 1 \mathrm{H}), 3.87(\mathrm{~s}, 3 \mathrm{H}), 3.58(\mathrm{t}, J=10.2 \mathrm{~Hz}, 1 \mathrm{H}), 3.54(\mathrm{~s}, 3 \mathrm{H})$, $2.70(\mathrm{~d}, J=10.2 \mathrm{~Hz}, 2 \mathrm{H}), 2.59$ (s, 3H). ${ }^{13} \mathbf{C ~ N M R}\left(75.0 \mathrm{MHz}, \mathrm{CDCl}_{3}\right)$ : $\delta 175.2,166.2,166.1,152.5,139.4,137.4,129.1,128.5,128.1,128.0$, $117.4,104.4,100.3,84.4,54.6,53.6,51.4,48.1,35.1,34.8,17.1$. IR (neat, $\mathrm{cm}^{-1}$ ): 3387, 3062, 2954, 2245, 2193, 1764, 1703, 1593, 1408, 1286, 1123, 737, 700. HRMS: calc'd for $(\mathrm{M})^{+} \mathrm{C}_{27} \mathrm{H}_{25} \mathrm{~N}_{3} \mathrm{O}_{6}$ : 487.1743; found: 487.1716.

Yield: $52 \mathrm{mg}, 90 \%{ }^{1}{ }^{1} \mathbf{H}$ NMR $\left(400 \mathrm{MHz}, \mathrm{CDCl}_{3}\right): \delta 7.36(\mathrm{~m}, 6 \mathrm{H}), 7.13$ $(\mathrm{m}, 4 \mathrm{H}), 5.50(\mathrm{~s}, 1 \mathrm{H}), 4.99(\mathrm{~d}, J=16 \mathrm{~Hz}, 1 \mathrm{H}), 4.35(\mathrm{~d}, J=16 \mathrm{~Hz}, 1 \mathrm{H})$, $3.90(\mathrm{~d}, J=9.6 \mathrm{~Hz}, 1 \mathrm{H}), 3.85(\mathrm{~s}, 3 \mathrm{H}), 3.78(\mathrm{~s}, 3 \mathrm{H}), 3.68(\mathrm{~s}, 3 \mathrm{H}), 3.64$ $(\mathrm{d}, J=9.6 \mathrm{~Hz}, 1 \mathrm{H}), 3.51(\mathrm{~s}, 3 \mathrm{H}), 2.47(\mathrm{~s}, 3 \mathrm{H}) .{ }^{13} \mathbf{C}$ NMR $(75.0 \mathrm{MHz}$, $\left.\mathrm{CDCl}_{3}\right): \delta 171.3,168.2,167.8,166.3,163.2,152.3,139.4,137.5$, $128.9,128.5,128.3,128.1,127.9,126.0,105.1,99.9,85.9,55.0,53.2$, 53.0, 52.7, 51.2, 49.6, 47.7, 17.1. IR (neat, $\mathrm{cm}^{-1}$ ): 3455, 3032, 2953, 1746, 1701, 1436, 1408, 1265, 1210, 1139, 737, 702. HRMS: calc'd for $(\mathrm{M}+\mathrm{Na})^{+} \mathrm{C}_{30} \mathrm{H}_{30} \mathrm{~N}_{2} \mathrm{O}_{10} \mathrm{Na}: 601.1798$; found: 601.1837 .

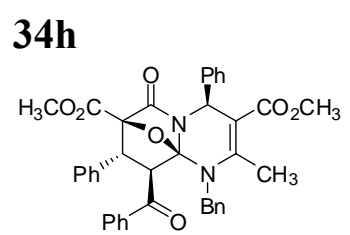

Yield: $56 \mathrm{mg}, 87 \% .{ }^{1} \mathbf{H}$ NMR $\left(400 \mathrm{MHz}, \mathrm{CDCl}_{3}\right): \delta$ 7.80-7.14 (m, $20 \mathrm{H}), 5.41(\mathrm{~s}, 1 \mathrm{H}), 5.18(\mathrm{~d}, J=14.8 \mathrm{~Hz}, 1 \mathrm{H}), 4.60(\mathrm{~d}, J=14.8 \mathrm{~Hz}$, $1 \mathrm{H}), 4.48(\mathrm{~d}, J=3.2 \mathrm{~Hz}, 1 \mathrm{H}), 4.24(\mathrm{~d}, J=3.2 \mathrm{~Hz}, 1 \mathrm{H}), 3.43(\mathrm{~s}, 3 \mathrm{H})$, $3.42(\mathrm{~s}, 3 \mathrm{H}), 1.64(\mathrm{~s}, 3 \mathrm{H}) .{ }^{13} \mathrm{C}$ NMR $\left(75.0 \mathrm{MHz}, \mathrm{CDCl}_{3}\right): \delta 194.0$, $166.0,165.7,163.4,151.5,140.7,138.4,137.7,137.4,133.9,129.2$, $129.2,129.0,128.3,128.2,128.1,128.0,127.9,127.7,104.5,100.8$, $89.5,62.1,55.6,52.7,51.8,51.1,47.3,21.3,16.3$. IR (neat, $\mathrm{cm}^{-1}$ ): 3367, 3056, 2952, 1700, 1625, 1495, 1434, 1265, 1119, 738, 704. HRMS: calc'd for $(\mathrm{M}+\mathrm{H})^{+} \mathrm{C}_{39} \mathrm{H}_{35} \mathrm{~N}_{2} \mathrm{O}_{7}$ : 643.2444; found: 643.2488.

34i (2 : $1 \mathrm{dr}$, major diastereomer was reported)

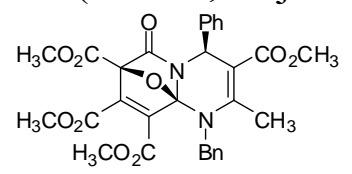

Yield: $51 \mathrm{mg}, 90 \% .{ }^{1} \mathbf{H}$ NMR $\left(400 \mathrm{MHz}, \mathrm{CDCl}_{3}\right): \delta 7.40-7.29(\mathrm{~m}, 5 \mathrm{H})$, $7.11(\mathrm{~m}, 3 \mathrm{H}), 6.91(\mathrm{t}, J=7.6 \mathrm{~Hz}, 2 \mathrm{H}), 6.76(\mathrm{~s}, 1 \mathrm{H}), 6.25(\mathrm{~d}, J=7.2 \mathrm{~Hz}$, $2 \mathrm{H}), 5.14(\mathrm{~d}, J=15.2 \mathrm{~Hz}, 1 \mathrm{H}), 4.79(\mathrm{~d}, J=15.2 \mathrm{~Hz}, 1 \mathrm{H}), 3.93(\mathrm{~s}, 3 \mathrm{H})$, 3.87 (s, 3H), 3.83 (s, 3H), 3.76 (s, 3H), 2.44 (s, 3H). ${ }^{13}$ C NMR (75.0 $\left.\mathrm{MHz}, \mathrm{CDCl}_{3}\right): \delta 171.3,166.9,164.8,164.4,163.7,158.6,154.1,151.8$, $149.1,136.9,133.9,129.2,129.0,128.8,128.5,128.3,126.4,124.8$, $119.0,90.3,56.0,55.9,55.8,53.2,52.7,52.4,18.0$. IR (neat, $\mathrm{cm}^{-1}$ ): 3057, 2954, 1721, 1525, 1434, 1265, 1048, 738, 701. HRMS: calc'd 
for $(\mathrm{M}+\mathrm{H})^{+} \mathrm{C}_{30} \mathrm{H}_{29} \mathrm{~N}_{2} \mathrm{O}_{10}$ : 577.1822; found: 577.1830.

36a

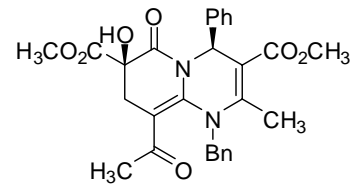

36b

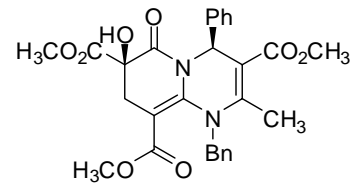

Yield: 45mg, 90\%. ${ }^{\mathbf{1}} \mathbf{H}$ NMR (400 MHz, $\left.\mathrm{CDCl}_{3}\right): \delta 7.39(\mathrm{~m}, 3 \mathrm{H}), 7.12$ $(\mathrm{m}, 2 \mathrm{H}), 7.04(\mathrm{t}, J=7.2 \mathrm{~Hz}, 1 \mathrm{H}), 6.99(\mathrm{~s}, 1 \mathrm{H}), 6.86(\mathrm{t}, J=8.0 \mathrm{~Hz}$, $2 \mathrm{H}), 6.12$ (d, $J=7.6 \mathrm{~Hz}, 2 \mathrm{H}), 4.74(\mathrm{~d}, J=15.2 \mathrm{~Hz}, 1 \mathrm{H}), 4.44(\mathrm{~s}, 1 \mathrm{H})$, $4.13(\mathrm{~d}, J=15.2 \mathrm{~Hz}, 1 \mathrm{H}), 3.81(\mathrm{~s}, 3 \mathrm{H}), 3.73(\mathrm{~s}, 3 \mathrm{H}), 3.39(\mathrm{~d}, J=14.4$ $\mathrm{Hz}, 1 \mathrm{H}), 3.04(\mathrm{~d}, J=14.4 \mathrm{~Hz}, 1 \mathrm{H}), 2.38(\mathrm{~s}, 3 \mathrm{H}), 2.27(\mathrm{~s}, 3 \mathrm{H}) .{ }^{13} \mathrm{C}$ NMR (75.0 MHz, $\left.\mathrm{CDCl}_{3}\right): \delta 194.5,170.5,169.2,165.8,153.2,146.8$, $138.4,135.2$, 129.2, 128.5, 128.4, 128.1, 127.7, 126.1, 114.3, 90.9, 76.3, 56.1, 53.5, 52.2, 50.6, 32.5, 29.1, 18.0. IR (neat, $\mathrm{cm}^{-1}$ ): 3417 , 3057, 2953, 1740, 1712, 1634, 1495, 1434, 1265, 1138, 737, 703. HRMS: calc'd for $(\mathrm{M}+\mathrm{H})^{+} \mathrm{C}_{28} \mathrm{H}_{29} \mathrm{~N}_{2} \mathrm{O}_{7}$ : 505.1975; found: 505.2010.

Yield: $45 \mathrm{mg}, 87 \% .{ }^{1} \mathbf{H}$ NMR (400 MHz, $\left.\mathrm{CDCl}_{3}\right): \delta 7.37(\mathrm{~m}, 3 \mathrm{H}), 7.20$ $(\mathrm{m}, 2 \mathrm{H}), 7.05(\mathrm{t}, J=7.6 \mathrm{~Hz}, 1 \mathrm{H}), 6.94(\mathrm{~s}, 1 \mathrm{H}), 6.87(\mathrm{t}, J=7.6 \mathrm{~Hz}$, $2 \mathrm{H}), 6.16(\mathrm{~d}, J=7.6 \mathrm{~Hz}, 2 \mathrm{H}), 4.75(\mathrm{~d}, J=15.2 \mathrm{~Hz}, 1 \mathrm{H}), 4.34(\mathrm{~s}, 1 \mathrm{H})$, $4.23(\mathrm{~d}, J=15.2 \mathrm{~Hz}, 1 \mathrm{H}), 3.80(\mathrm{~s}, 3 \mathrm{H}), 3.76(\mathrm{~s}, 3 \mathrm{H}), 3.74(\mathrm{~s}, 3 \mathrm{H}), 3.54$ $(\mathrm{d}, J=14.4 \mathrm{~Hz}, 1 \mathrm{H}), 2.92(\mathrm{~d}, J=14.4 \mathrm{~Hz}, 1 \mathrm{H}), 2.35(\mathrm{~s}, 3 \mathrm{H}) .{ }^{13} \mathrm{C}$ NMR (75.0 MHz, $\left.\mathrm{CDCl}_{3}\right): \delta 170.3,166.4,165.9,164.5,152.9,147.5$, $138.4,135.3,129.1,128.6,128.3,128.0,127.8,126.0,81.6,76.1$, 56.3, 53.4, 52.2, 51.7, 50.6, 31.6, 17.9. IR (neat, $\mathrm{cm}^{-1}$ ): 3411, 3058, 2952, 1738, 1689, 1588, 1435, 1264, 1130, 738, 701. HRMS: calc'd for $(\mathrm{M}+\mathrm{Na})^{+} \mathrm{C}_{28} \mathrm{H}_{28} \mathrm{~N}_{2} \mathrm{O}_{8} \mathrm{Na}$ : 543.1743; found: 543.1755.

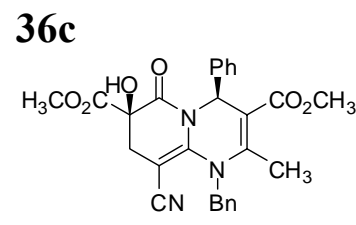

Yield: $46 \mathrm{mg}, 95 \% .{ }^{1} \mathbf{H}$ NMR $\left(400 \mathrm{MHz}, \mathrm{CDCl}_{3}\right): \delta 7.35(\mathrm{~m}, 3 \mathrm{H})$, $7.11(\mathrm{t}, J=6.8 \mathrm{~Hz}, 1 \mathrm{H}), 7.02(\mathrm{~m}, 2 \mathrm{H}), 6.97(\mathrm{t}, J=7.6 \mathrm{~Hz}, 2 \mathrm{H}), 6.89$ $(\mathrm{s}, 1 \mathrm{H}), 6.36(\mathrm{~d}, J=7.6 \mathrm{~Hz}, 2 \mathrm{H}), 5.11(\mathrm{~d}, J=16 \mathrm{~Hz}, 1 \mathrm{H}), 4.86(\mathrm{~d}, J=$ $16 \mathrm{~Hz}, 1 \mathrm{H}), 4.27$ (s, 1H), 3.83 (s, 3H), 3.77 (s, 3H), 3.04 (d, $J=15.2$ $\mathrm{Hz}, 1 \mathrm{H}), 2.90(\mathrm{~d}, J=15.2 \mathrm{~Hz}, 1 \mathrm{H}), 2.30(\mathrm{~s}, 3 \mathrm{H}) .{ }^{13} \mathbf{C}$ NMR (75.0 $\left.\mathrm{MHz}, \mathrm{CDCl}_{3}\right): \delta 168.9,165.6,151.0,149.1,137.7,134.9,131.1$, $129.2,128.9,128.4,128.1,127.6,125.9,119.4,113.7,75.0,60.7$, 53.9, 53.8, 52.3, 50.4, 32.3, 17.6. HRMS: calc'd for $(\mathrm{M}+\mathrm{H})^{+}$ $\mathrm{C}_{34} \mathrm{H}_{33} \mathrm{~N}_{2} \mathrm{O}_{7}: 581.2288$; found: 581.2296.

Yield: $48 \mathrm{mg}, 84 \%$. ${ }^{1} \mathbf{H}$ NMR $\left(400 \mathrm{MHz}, \mathrm{CDCl}_{3}\right): \delta 7.29(\mathrm{t}, J=7.6$ $\mathrm{Hz}, 1 \mathrm{H}), 7.21-6.97(\mathrm{~m}, 8 \mathrm{H}), 6.85$ (t, $J=7.6 \mathrm{~Hz}, 2 \mathrm{H}), 6.75$ (d, $J=7.6$ $\mathrm{Hz}, 2 \mathrm{H}), 6.74(\mathrm{~s}, 1 \mathrm{H}), 6.22$ (d, $J=7.6 \mathrm{~Hz}, 2 \mathrm{H}), 4.59$ (d, $J=14.8 \mathrm{~Hz}$, $1 \mathrm{H}), 4.50(\mathrm{~s}, 1 \mathrm{H}), 4.31(\mathrm{~d}, J=14.8 \mathrm{~Hz}, 1 \mathrm{H}), 4.27(\mathrm{~s}, 1 \mathrm{H}), 3.68$ (s, 3H), 3.66 (s, 3H), 2.49 (s, 3H), 1.99 (s, 3H). ${ }^{13}$ C NMR $(75.0 \mathrm{MHz}$, $\mathrm{CDCl}_{3}$ ): $\delta$ 194.0. IR (neat, $\mathrm{cm}^{-1}$ ): 3403, 3055, 2953, 1738, 1708, 
1636, 1405, 1265, 1131, 738, 702. HRMS: calc'd for $(\mathrm{M}+\mathrm{H})^{+}$ $\mathrm{C}_{34} \mathrm{H}_{33} \mathrm{~N}_{2} \mathrm{O}_{7}$ : 581.2288; found: 581.2296.

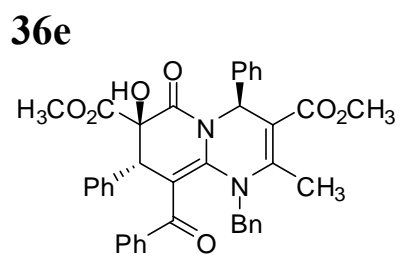

Yield: 60mg, 95\%. ${ }^{1} \mathbf{H}$ NMR (400 MHz, $\left.\mathrm{CDCl}_{3}\right): \delta 7.34(\mathrm{~m}, 2 \mathrm{H})$, 7.27-7.14 (m, 10H), $7.06(\mathrm{~m}, 3 \mathrm{H}), 6.86(\mathrm{~m}, 4 \mathrm{H}), 6.28(\mathrm{~d}, J=7.2 \mathrm{~Hz}$, $2 \mathrm{H}), 4.46(\mathrm{~m}, 2 \mathrm{H}), 4.21(\mathrm{~s}, 1 \mathrm{H}), 3.71(\mathrm{~s}, 3 \mathrm{H}), 3.68(\mathrm{~s}, 3 \mathrm{H}), 2.43(\mathrm{~s}$, $3 \mathrm{H}) .{ }^{13} \mathrm{C}$ NMR $\left(75.0 \mathrm{MHz}, \mathrm{CDCl}_{3}\right): \delta 192.3,170.2,169.4,152.7$, $139.5,137.7,134.3,131.7,130.1,128.9,128.5,128.3,128.2,128.0$, $127.9,127.7,127.5,113.1,93.7,78.7,55.2,53.2,52.0,51.8,46.0$, 17.0. HRMS: calc'd for $(\mathrm{M}+\mathrm{H})^{+} \mathrm{C}_{34} \mathrm{H}_{33} \mathrm{~N}_{2} \mathrm{O}_{7}$ : 581.2288; found: 581.2296.

36f The reaction was run in $0.050 \mathrm{mmol}$ scale.

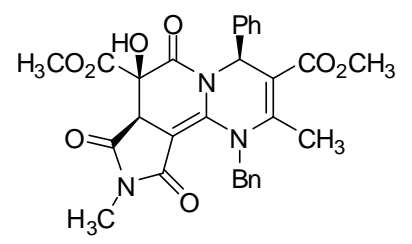

Yield: $20 \mathrm{mg}, 72 \% .{ }^{1} \mathbf{H}$ NMR $\left(400 \mathrm{MHz}, \mathrm{CDCl}_{3}\right): \delta$ 7.35-7.27 (m, $5 \mathrm{H}), 7.12(\mathrm{~m}, 3 \mathrm{H}), 6.70(\mathrm{~d}, J=7.6 \mathrm{~Hz}, 2 \mathrm{H}), 6.49(\mathrm{~s}, 1 \mathrm{H}), 5.22(\mathrm{~d}, J=$ $16 \mathrm{~Hz}, 1 \mathrm{H}), 4.63(\mathrm{~d}, \mathrm{~J}=16 \mathrm{~Hz}, 1 \mathrm{H}), 4.10(\mathrm{~s}, 1 \mathrm{H}), 3.95(\mathrm{~s}, 3 \mathrm{H}), 3.77$ (s, 3H), 2.99 (s, 3H), 2.44 (s, 3H). ${ }^{13} \mathbf{C}$ NMR $\left(75.0 \mathrm{MHz}, \mathrm{CDCl}_{3}\right)$ : $\delta 176.1,172.7,171.0,168.8,167.7,165.7,152.6,149.1,138.1,135.9$, $129.2,129.1,128.9,128.5,128.3,126.6,126.4,111.0,84.6,82.2$, 54.3, 54.0, 52.3, 47.7, 32.2, 25.2, 16.4. HRMS: calc'd for $(\mathrm{M}+\mathrm{H})^{+}$ $\mathrm{C}_{34} \mathrm{H}_{33} \mathrm{~N}_{2} \mathrm{O}_{7}$ : 581.2288; found: 581.2296.

$36 \mathrm{~g}$ The reaction was run in $0.050 \mathrm{mmol}$ scale.

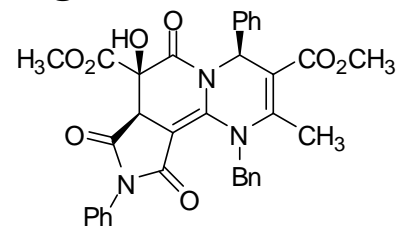

Yield: $22 \mathrm{mg}, 72 \% .{ }^{1} \mathbf{H}$ NMR $\left(400 \mathrm{MHz}, \mathrm{CDCl}_{3}\right): \delta$ 7.46-7.24 (m, $8 \mathrm{H}), 7.15-7.06(\mathrm{~m}, 5 \mathrm{H}), 6.70(\mathrm{~d}, J=7.6 \mathrm{~Hz}, 2 \mathrm{H}), 6.54(\mathrm{~s}, 1 \mathrm{H}), 5.26$ (d, J = $16 \mathrm{~Hz}, 1 \mathrm{H}), 4.61(\mathrm{~d}, \mathrm{~J}=16 \mathrm{~Hz}, 1 \mathrm{H}), 4.55(\mathrm{br}, 1 \mathrm{H}), 3.96(\mathrm{~s}$, 3H), 3.90 (s, 1H), 3.79 (s, 3H), 2.46 (s, 3H). ${ }^{13} \mathbf{C}$ NMR (75.0 MHz, $\left.\mathrm{CDCl}_{3}\right): \delta 175.1,171.3,168.7,165.7,164.7,149.1,139.5,138.1$, $135.9,131.9,129.7,129.3,128.9,128.5,128.3,128.2,127.7,126.7$, 126.6, 126.4, 111.1, 105.0, 82.6, 54.1, 52.9, 52.3, 47.8, 47.0, 32.5, 16.4. HRMS: calc'd for $(\mathrm{M}+\mathrm{H})^{+} \mathrm{C}_{34} \mathrm{H}_{33} \mathrm{~N}_{2} \mathrm{O}_{7}$ : 581.2288; found: 581.2296 .

36h The reaction was run in $0.050 \mathrm{mmol}$ scale.

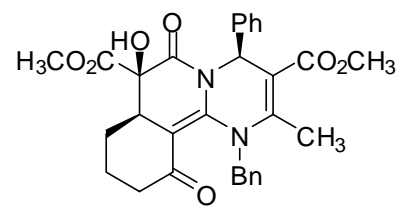

Yield: $21 \mathrm{mg}, 80 \% .{ }^{1} \mathbf{H}$ NMR $\left(400 \mathrm{MHz}, \mathrm{CDCl}_{3}\right): \delta 7.23(\mathrm{~m}, 2 \mathrm{H})$, 7.17-7.09 (m, 4H), $7.0(\mathrm{t}, J=7.6 \mathrm{~Hz}, 2 \mathrm{H}), 6.75(\mathrm{~s}, 1 \mathrm{H}), 6.71(\mathrm{~d}, \mathrm{~J}=7.6$ $\mathrm{Hz}, 2 \mathrm{H}), 4.90(\mathrm{~d}, J=15.6 \mathrm{~Hz}, 1 \mathrm{H}), 4.32(\mathrm{~s}, 1 \mathrm{H}), 4.03(\mathrm{~d}, J=15.6 \mathrm{~Hz}$, $1 \mathrm{H}), 3.73(\mathrm{~s}, 3 \mathrm{H}), 3.67(\mathrm{~s}, 3 \mathrm{H}), 3.75(\mathrm{~s}, 3 \mathrm{H}), 3.03(\mathrm{~m}, 1 \mathrm{H}), 2.57(\mathrm{~m}$, $1 \mathrm{H}), 2.48(\mathrm{~s}, 3 \mathrm{H}), 2.31(\mathrm{~m}, 2 \mathrm{H}), 2.08(\mathrm{~m}, 1 \mathrm{H}), 1.75(\mathrm{~m}, 1 \mathrm{H}), 1.56(\mathrm{~m}$, 1H). ${ }^{13}$ C NMR $\left(75.0 \mathrm{MHz}, \mathrm{CDCl}_{3}\right): \delta 195.7,168.7,167.4,155.8$, $144.7,139.3,136.5,128.9,128.7,128.6,128.4,127.7,127.6,127.0$, $126.5,110.0,99.8,75.4,56.2,54.3,53.0,51.8,43.7,42.0,24.7,22.8$, 17.2. IR (neat, $\left.\mathrm{cm}^{-1}\right): 3452,2952,1694,1555,1435,1387,1264,908$, 
734. HRMS: calc'd for $(\mathrm{M}+\mathrm{H})^{+} \mathrm{C}_{30} \mathrm{H}_{31} \mathrm{~N}_{2} \mathrm{O}_{7}$ : 531.2131; found: 531.2154 .

36i The reaction was run in $0.050 \mathrm{mmol}$ scale.

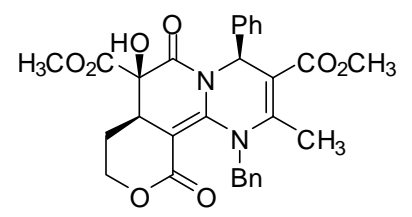

Yield: $39 \mathrm{mg}, 73 \% .{ }^{1} \mathbf{H}$ NMR $\left(400 \mathrm{MHz}, \mathrm{CDCl}_{3}\right): \delta 7.41(\mathrm{~m}, 2 \mathrm{H})$, 7.31-7.10 (m, 5H), $7.01(\mathrm{~s}, 1 \mathrm{H}), 6.87(\mathrm{t}, J=7.6 \mathrm{~Hz}, 2 \mathrm{H}), 6.14(\mathrm{~d}, J=$ $7.6 \mathrm{~Hz}, 2 \mathrm{H}), 4.75(\mathrm{~d}, J=14.8 \mathrm{~Hz}, 1 \mathrm{H}), 4.50(\mathrm{~d}, J=14.8 \mathrm{~Hz}, 1 \mathrm{H}), 4.41$ $(\mathrm{m}, 1 \mathrm{H}), 4.20(\mathrm{~m}, 1 \mathrm{H}), 3.96(\mathrm{~s}, 3 \mathrm{H}), 3.78(\mathrm{~s}, 3 \mathrm{H}), 3.45(\mathrm{~m}, 1 \mathrm{H}), 2.71$ $(\mathrm{m}, 1 \mathrm{H}), 2.61(\mathrm{~m}, 1 \mathrm{H}), 1.93(\mathrm{~m}, 1 \mathrm{H}), 1.60(\mathrm{~m}, 1 \mathrm{H}) .{ }^{13} \mathbf{C}$ NMR $(75.0$ $\left.\mathrm{MHz}, \mathrm{CDCl}_{3}\right): \delta 173.6,169.3,166.3,163.7,161.6,154.3,147.7$, $138.9,134.9,129.2,128.9,128.6,128.5,127.6,126.7,126.4,126.2$, 113.9, 104.9, 77.8, 67.6, 54.3, 52.2, 46.9, 37.7, 16.3. HRMS: calc'd for $(\mathrm{M}+\mathrm{H})^{+} \mathrm{C}_{34} \mathrm{H}_{33} \mathrm{~N}_{2} \mathrm{O}_{7}$ : 581.2288; found: 581.2296.

\section{Methyl-2-((2R,3S,4R)-3-(dimethyl(phenyl)silyl)-4-methyltetrahydrofuran-2-yl)acetate (39)}

$\mathrm{H}_{3} \mathrm{C} \quad \mathrm{Si}\left(\mathrm{CH}_{3}\right)_{2} \mathrm{Ph}$ Into a round-bottom flask equipped with stir bar was added 1,3,5-trioxane (901 $\mathrm{mg}, 10 \mathrm{mmol})$ and dichloromethane $(5.0 \mathrm{~mL})$. The solution was cooled to $-20 \mathrm{C}$ and stirred for 10 minutes. The solution of $(S)$-silane $\mathbf{3 8}$ (5.25 g, $20 \mathrm{mmol})$ in $\mathrm{CH}_{2} \mathrm{Cl}_{2}(3 \mathrm{~mL})$ was added slowly and the reaction solution was stirred overnight. Saturated $\mathrm{NaHCO}_{3}$ aqueous solution (3.0 $\mathrm{mL}$ ) was added to quench the reaction. The organic layer was separated and the aqueous layer was washed with ethylacetate. The organic layers were combined and dried over sodium sulfat, filtered and concentrated. The resulting residue was subjected to column chromatography on silica gel ( $10 \%$ EtOAc in hexanes as eluant) to give the product. Yield: $5.54 \mathrm{~g}$, 90\%. ${ }^{1} \mathbf{H}$ NMR $\left(400 \mathrm{MHz}, \mathrm{CDCl}_{3}\right): \delta 7.38(\mathrm{~m}, 2 \mathrm{H}), 7.23(\mathrm{~m}, 3 \mathrm{H}), 4.14$ (ddd, $J=9.6,9.6,3.2 \mathrm{~Hz}, 1 \mathrm{H}), 3.64$ (dd, $J=8.4,7.2 \mathrm{~Hz}, 1 \mathrm{H}), 3.51$ (s, $3 \mathrm{H}), 3.18(\mathrm{dd}, J=8.0,7.2 \mathrm{~Hz}, 1 \mathrm{H}), 2.22-2.16(\mathrm{dd}, J=15.2,8.4 \mathrm{~Hz}, 1 \mathrm{H})$, 2.11-2.08 (m, 1H), $0.82(\mathrm{~d}, J=6.4 \mathrm{~Hz}), 0.25(\mathrm{~s}, 3 \mathrm{H}), 0.24(\mathrm{~s}, 3 \mathrm{H}) .{ }^{13} \mathbf{C}$ NMR $\left(75.0 \mathrm{MHz}, \mathrm{CDCl}_{3}\right): \delta 171.5,137.0,133.6,129.2,127.8,78.7,74.7$, 51.4, 41.4, 39.8, 37.4, 18.7, - 4.0, - 4.6. IR (neat, $\mathrm{cm}^{-1}$ ): 3459, 3069, 2955, 1741, 1433, 1377, 1253, 1111, 1026, 831, 736, 702. HRMS: calc'd for $(\mathrm{M}+\mathrm{Na})^{+} \mathrm{C} 16 \mathrm{H} 24 \mathrm{NaO} 3 \mathrm{Si}: 315.1392$; found: 315.1740 .

\section{$(S, E)$-methyl 6-hydroxy-5-methylhex-3-enoate (40)}

C A solution of furan $39(2.92 \mathrm{~g}, 10 \mathrm{mmol})$ in $200 \mathrm{~mL}$ of $\mathrm{CH}_{2} \mathrm{Cl}_{2}(0.5 \mathrm{M})$ at $-78{ }^{\circ} \mathrm{C}$ was treated with a solution of $\mathrm{SbCl}_{5}$ in $\mathrm{CH}_{2} \mathrm{Cl}_{2}(1 \mathrm{M}, 12 \mathrm{~mL}, 12.0$ $\mathrm{mmol}$ ). The resulting light yellow reaction solution was warmed to $-50{ }^{\circ} \mathrm{C}$ and stirred for $12 \mathrm{~h}$. The reaction was diluted by the addition of $100 \mathrm{~mL}$ of distilled water. The resulting white precipitate suspension was filtered through a short plug of celite and washed with $\mathrm{CH}_{2} \mathrm{Cl}_{2}$. The filtrate was collected and the organic layer was separated. The aqueous layer was extracted with $\mathrm{CH}_{2} \mathrm{Cl}_{2}(3 \times 100 \mathrm{~mL})$. The organic layer was combined and dried over $\mathrm{MgSO}_{4}$, filtered and concentrated in vacuo. Chromatography on silica gel $(40 \%$ EtOAc in hexanes eluant) gave desired primary 
alcohol. Yield: $1.37 \mathrm{~g}, 87 \%$. ${ }^{\mathbf{1}} \mathbf{H}$ NMR $\left(400 \mathrm{MHz}, \mathrm{CDCl}_{3}\right): \delta 5.54(\mathrm{~m}, 1 \mathrm{H})$, $5.38(\mathrm{~m}, 1 \mathrm{H}), 3.61(\mathrm{~s}, 3 \mathrm{H}), 3.35(\mathrm{~m}, 2 \mathrm{H}), 2.99(\mathrm{~d}, J=7.2 \mathrm{~Hz}, 2 \mathrm{H}), 2.52(\mathrm{br}$, 1H), 2.29(m, 1H), 0.94(d, $J=7.2 \mathrm{~Hz}, 3 \mathrm{H}) .{ }^{13} \mathbf{C ~ N M R}\left(75.0 \mathrm{MHz}, \mathrm{CDCl}_{3}\right)$ : $\delta 172.4,136.9,122.4,66.9,51.7,39.3,37.6,16.0$. IR (neat, $\mathrm{cm}^{-1}$ ): 3417 , 2957, 2929, 2874, 1736, 1437, 1260, 1205, 1168, 1035, 972. HRMS: calc'd for $(\mathrm{M}+\mathrm{H})^{+} \mathrm{C}_{8} \mathrm{H}_{15} \mathrm{O}_{3}$ : 159.1021; found: 159.1018 .

\section{$(S, E)$-methyl 5-methyl-6-(tosyloxy)hex-3-enoate}

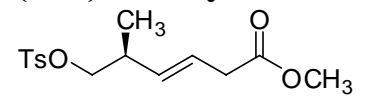

Into a round-bottom flask equipped with stir bar was charged with alcohol 40 (1.16 g, $7.3 \mathrm{mmol}$ ), DMAP (90 mg, $0.73 \mathrm{mmol})$, pyridine (1.31 mL, 16 mmol) and $\mathrm{CH}_{2} \mathrm{Cl}_{2}(7.0 \mathrm{~mL})$ successively. The reaction solution was cooled at $0{ }^{\circ} \mathrm{C}$. A solution of $p$-toluenesulfonyl chloride (1.54 g, 8.0 mmol) in $\mathrm{CH}_{2} \mathrm{Cl}_{2}(3 \mathrm{~mL})$ was added. The reaction was stirred at room temperature for 5 hours. The solution was concentrated in vacuo and the crude mixture was subjected to column chromatography on silica gel to afford the product. Yield: $2.1 \mathrm{~g}, 92 \% .{ }^{1} \mathbf{H} \mathbf{N M R}\left(400 \mathrm{MHz}, \mathrm{CDCl}_{3}\right): \delta$ $7.70(\mathrm{~d}, J=8 \mathrm{~Hz}, 2 \mathrm{H}), 7.28(\mathrm{~d}, J=8 \mathrm{~Hz}, 2 \mathrm{H}), 5.53(\mathrm{~m}, 1 \mathrm{H}), 5.30(\mathrm{~m}, 1 \mathrm{H})$, $3.82(\mathrm{~m}, 2 \mathrm{H}), 3.61(\mathrm{~s}, 3 \mathrm{H}), 2.93(\mathrm{~d}, J=6.8 \mathrm{~Hz}, 2 \mathrm{H}), 2.45(\mathrm{~m}, 1 \mathrm{H}), 2.38(\mathrm{~s}$, $3 \mathrm{H}), 0.93(\mathrm{~d}, J=6.8 \mathrm{~Hz}, 3 \mathrm{H}) .{ }^{13} \mathbf{C} \mathbf{N M R}\left(75.0 \mathrm{MHz}, \mathrm{CDCl}_{3}\right): \delta 171.8$, $144.6,133.9,132.9,129.7,127.7,123.3,73.8,51.6,37.5,35.9,21.4,16.1$. IR (neat, $\mathrm{cm}^{-1}$ ): 2955, 1737, 1598, 1437, 1359, 1175, 972, 816, 667. HRMS: calc'd for $(\mathrm{M}+\mathrm{H})^{+} \mathrm{C}_{15} \mathrm{H}_{21} \mathrm{O}_{5} \mathrm{~S}$ : 313.1110; found: 313.1108 .

\section{$(S, E)$-methyl 6-azido-5-methylhex-3-enoate}

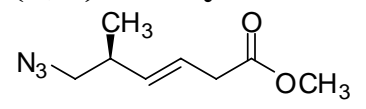

To a round-bottom flask was charged with tosyl protected alcohol $(1.87 \mathrm{~g}$, $6.0 \mathrm{mmol})$, sodium azide $(3.89 \mathrm{~g}, 60 \mathrm{mmol})$, and DMF $(50 \mathrm{~mL})$. The reaction mixture was stirred at $50{ }^{\circ} \mathrm{C}$ for $6 \mathrm{~h}$. Water $(20 \mathrm{~mL})$ and $\mathrm{CH}_{2} \mathrm{Cl}_{2}$ $(20 \mathrm{~mL})$ were added to the reaction solution. The organic layer was separated and aqueous layer was washed with $\mathrm{CH}_{2} \mathrm{Cl}_{2}(3 \times 20 \mathrm{~mL})$. The organic layers were combined, dried over sodium sulfate, filtered and concentrated in vacuo. The residue was subjected column chromatography to afford the azide product. Yield: $1.0 \mathrm{~g}, 91 \%{ }^{1} \mathbf{H}$ NMR $\left(400 \mathrm{MHz}, \mathrm{CDCl}_{3}\right): \delta 5.62(\mathrm{~m}, 1 \mathrm{H}), 5.40(\mathrm{~m}, 1 \mathrm{H}), 3.62(\mathrm{~s}, 3 \mathrm{H}), 3.12(\mathrm{~m}$, 2H), $3.00(\mathrm{~d}, J=7.2 \mathrm{~Hz}, 2 \mathrm{H}), 2.40(\mathrm{~m}, 1 \mathrm{H}), 1.00(\mathrm{~d}, J=7.2 \mathrm{~Hz}, 3 \mathrm{H}) .{ }^{13} \mathbf{C}$ NMR (75.0 MHz, $\left.\mathrm{CDCl}_{3}\right): \delta 171.9,135.9,122.7,56.8,51.6,37.6,36.9$, 17.4. IR (neat, $\mathrm{cm}^{-1}$ ): 3614, 3544, 2957, 2198, 1740, 1439, 1209, 1171, 972, 689. HRMS: calc'd for $(M)^{+} \mathrm{C}_{8} \mathrm{H}_{13} \mathrm{~N}_{3} \mathrm{O}_{2}$ : 183.1008; found: 183.1012 .

\section{$(S, E)$-methyl 6-amino-5-methylhex-3-enoate}<smiles>COC(=O)C/C=C/C(C)CN</smiles>

To a round-bottom flask equipped with stir bar was charged with azide $(1.00 \mathrm{~g}, 5.45 \mathrm{mmol})$, polymer supported triphenylphosphine resin (10.9 mmol), water $(0.25 \mathrm{~mL}, 13.6 \mathrm{mmol})$ and THF $(2 \mathrm{~mL})$ subsequently. The reaction mixture was stirred at room temperature for $5 \mathrm{~h}$. Resin was filtered and washed with THF $(3 \times 5 \mathrm{~mL})$. The THF solution was dried over sodium sulfate and filtered. The filtrate was concentrated in vacuo 
and residue was subjected to chromatography (100\% EtOAc as eluant) to afford the primary amine. Yield: $703 \mathrm{mg}, 82 \%$. ${ }^{1} \mathbf{H}$ NMR $(400 \mathrm{MHz}$, $\left.\mathrm{CDCl}_{3}\right): \delta 5.58(\mathrm{~m}, 1 \mathrm{H}), 5.34(\mathrm{~m}, 1 \mathrm{H}), 3.64(\mathrm{~s}, 3 \mathrm{H}), 3.02(\mathrm{~d}, J=6.8 \mathrm{~Hz}$, 2H), $2.58(\mathrm{dd}, J=12.4,5.6 \mathrm{~Hz}, 1 \mathrm{H}), 2.47$ (dd, $J=12.4,5.6 \mathrm{~Hz}, 1 \mathrm{H}), 2.13$ $(\mathrm{m}, 1 \mathrm{H}), 0.94(\mathrm{~d}, J=6.8 \mathrm{~Hz}, 3 \mathrm{H}) .{ }^{13} \mathbf{C}$ NMR $\left(75.0 \mathrm{MHz}, \mathrm{CDCl}_{3}\right): \delta 172.4$, 138.0, 122.2, 51.7, 47.7, 40.4, 37.8, 17.6. IR (neat, $\mathrm{cm}^{-1}$ ): 3367, 2957, 1736, 1651, 1168, 732. HRMS: calc'd for $(\mathrm{M}+1)^{+} \mathrm{C} 8 \mathrm{H} 15 \mathrm{NO}_{2}$ : 158.1136; found: 158.1181 .

\section{$(S, E)$-methyl 6-isocyanato-5-methylhex-3-enoate (41)}

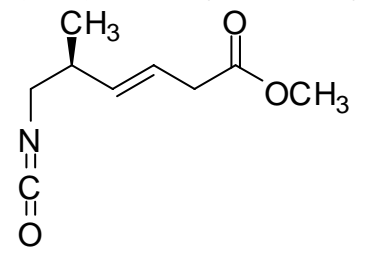

To a round-bottom flask equipped with stir bar was charged with primary amine $(510 \mathrm{mg}, 3.24 \mathrm{mmol})$ and $\mathrm{CH}_{2} \mathrm{Cl}_{2}(5.0 \mathrm{~mL})$. The $5.0 \mathrm{~mL}$ saturated $\mathrm{NaHCO}_{3}$ solution was added. Phosgene solution $(15.5 \mathrm{~mL}, 8.1 \mathrm{mmol}$, $1.92 \mathrm{M}$ in toluene) was added into the bottom $\mathrm{CH}_{2} \mathrm{Cl}_{2}$ layer with syringe. The solution was stirred at 600 revolutions per min at room temperature for 10 minutes. The bottom organic layer was separated. The aqueous layer was extracted with $\mathrm{CH}_{2} \mathrm{Cl}_{2}(3 \times 5 \mathrm{~mL})$. The organic layers were combined and dried over sodium sulfate, filtered and concentrated in vacuo and dried under high vacuum to give the product. ${ }^{1} \mathrm{H}-\mathrm{NMR}$ showed $100 \%$ conversion of the start material. Crude 41 was used directly in next step without further purification. Yield: $590 \mathrm{mg}, 99 \% .{ }^{1} \mathbf{H}$ NMR (400 $\left.\mathrm{MHz}, \mathrm{CDCl}_{3}\right): \delta 5.78(\mathrm{~m}, 1 \mathrm{H}), 5.53(\mathrm{~m}, 1 \mathrm{H}), 3.79(\mathrm{~s}, 3 \mathrm{H}), 3.23(\mathrm{~d}, J=6.4$ $\mathrm{Hz}, 2 \mathrm{H}), 3.18(\mathrm{~d}, J=6.4 \mathrm{~Hz}, 2 \mathrm{H}), 2.52(\mathrm{~m}, 1 \mathrm{H}), 1.16(\mathrm{~d}, J=6.4 \mathrm{~Hz}, 3 \mathrm{H})$. ${ }^{13}$ C NMR $\left(75.0 \mathrm{MHz}, \mathrm{CDCl}_{3}\right): \delta 171.7,135.4,128.2,123.7,51.5,48.1$, 38.4, 37.6, 21.2, 17.1. IR (neat, $\mathrm{cm}^{-1}$ ): 2959, 2263, 1740, 1439, 1196, 973.

(5S,E)-methyl-6-(3-((1R)-2-(methoxycarbonyl)-3-oxo-1-phenylbutyl)ureido)-5-methylhex-3enoate

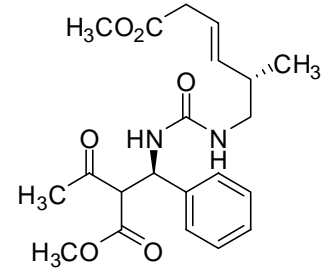

To a round-bottom flask was charged with tetrakis(triphenylphosphine) palladium (504 mg, $0.43 \mathrm{mmol})$ and THF $(1.50 \mathrm{~mL})$. The solution was stirred under nitrogen at room temperature. Isocyanate $\mathbf{4 2}$ (400 mg, 2.18 mmol) in THF ( $2 \mathrm{~mL})$ was added into palladium solution. The solution of 5a $(1.33 \mathrm{~g}, 4.36 \mathrm{mmol})$ and 1,3-dimetnylbarbituric acid (375 $\mathrm{mg}, 2.4$ $\mathrm{mmol})$ in THF $(1 \mathrm{~mL})$ was added to the pallasium solution slowly. The reaction was stirred at room temperature for 1 hour. The reaction solution was concentrated and was chromatographed on silica gel (eluted with 20$50 \%$ ethylacetate in hexanes) to afford the desired product. Yield: 710 mg, 80\%. ${ }^{1} \mathbf{H}$ NMR (400 MHz, $\mathrm{CDCl}_{3}$, both isomers were reported): $\delta$ $7.30-7.20(\mathrm{~m}, 10 \mathrm{H}), 6.16(\mathrm{~d}, J=9.6 \mathrm{~Hz}, 1 \mathrm{H}), 5.77(\mathrm{~m}, 2 \mathrm{H}), 5.61-5.46(\mathrm{~m}$, $3 \mathrm{H}), 5.31(\mathrm{~m}, 2 \mathrm{H}), 4.92(\mathrm{t}, 1 \mathrm{H}), 4.86(\mathrm{t}, 1 \mathrm{H}), 4.06(\mathrm{~d}, J=4.8 \mathrm{~Hz}, 1 \mathrm{H})$, $4.01(\mathrm{~d}, J=7,2 \mathrm{~Hz}, 1 \mathrm{H}), 3.71(\mathrm{~s}, 6 \mathrm{H}), 3.64(\mathrm{~s}, 3 \mathrm{H}), 3.59(\mathrm{~s}, 3 \mathrm{H}), 3.26(\mathrm{~m}$, 2H), $3.04(\mathrm{~m}, 4 \mathrm{H}), 2.87-2.77(\mathrm{~m}, 2 \mathrm{H}), 2.36(\mathrm{~s}, 3 \mathrm{H}), 2.30(\mathrm{~m}, 2 \mathrm{H}), 2.18(\mathrm{~s}$, $3 \mathrm{H}), 0.99(\mathrm{~d}, J=6.4 \mathrm{~Hz}, 3 \mathrm{H}), 0.96(\mathrm{~d}, J=6.8 \mathrm{~Hz}, 3 \mathrm{H}) .{ }^{13} \mathbf{C}$ NMR $(75.0$ $\left.\mathrm{MHz}, \mathrm{CDCl}_{3}\right): \delta 203.5,201.8,172.8,172.9,169.5,167.9,157.2,157.1$, $140.2,140.0,137.9,137.8,128.6,127.6,127.4,126.5,126.0,123.0$, $122.9,64.3,64.0,53.5,52.6,52.2,52.0,45.6,45.4,37.55,37.53,37.48$, 
37.44, 30.1, 28.8, 17.5, 17.4. IR (neat, $\mathrm{cm}^{-1}$ ): 3371, 2953, 1755, 1700, 1683, 1558, 1436, 1380, 1241, 1216, 1173, 1120, 755. HRMS: calc'd for $(\mathrm{M}+1)^{+} \mathrm{C}_{21} \mathrm{H}_{29} \mathrm{~N}_{2} \mathrm{O}_{6}$ : 405.1947; found: 405.1998 .

(S)-methyl 1-((R,E)-6-methoxy-2-methyl-6-oxohex-3-enyl)-6-methyl-2-oxo-4-phenyl-1,2,3,4tetrahydropyrimidine-5-carboxylate (42)

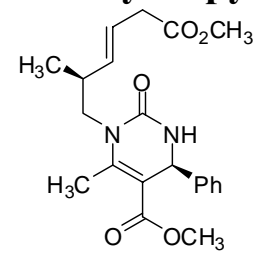

A heavy-walled microwave vessel was equipped with stir bar and charged with urea $(100 \mathrm{mg}, 0.25 \mathrm{mmol})$ and $\mathrm{EtOH} / \mathrm{AcOH}$ solution $(0.1 \mathrm{~mL}$, $\mathrm{EtOH}: \mathrm{AcOH}=1: 3)$. The reaction was irradiated in microwave at $130{ }^{\circ} \mathrm{C}$ for 5 minutes. The residue was directly subject to column chromatography on silica gel to afford the product 42 . Yield: $71 \mathrm{mg}, 75 \%$. ${ }^{1} \mathbf{H}$ NMR (400 $\left.\mathrm{MHz}, \mathrm{CDCl}_{3}\right): \delta 7.27(\mathrm{~m}, 5 \mathrm{H}), 5.94(\mathrm{~s}, 1 \mathrm{H}), 5.45(\mathrm{~m}, 1 \mathrm{H}), 5.37(\mathrm{~m}, 1 \mathrm{H})$, $5.35(\mathrm{~m}, 1 \mathrm{H}), 4.04(\mathrm{~m}, 1 \mathrm{H}), 3.65(\mathrm{~s}, 3 \mathrm{H}), 3.63(\mathrm{~s}, 3 \mathrm{H}), 3.38(\mathrm{~m}, 1 \mathrm{H}), 2.88$ $(\mathrm{dd}, J=13.2,6.0 \mathrm{~Hz}, 1 \mathrm{H}), 2.82(\mathrm{dd}, J=13.2,6.0 \mathrm{~Hz}, 1 \mathrm{H}), 2.50(\mathrm{~s}, 3 \mathrm{H})$, $2.43(\mathrm{~m}, 1 \mathrm{H}), 0.90(\mathrm{~d}, J=6.0 \mathrm{~Hz}, 3 \mathrm{H}) .{ }^{13} \mathbf{C}$ NMR $\left(75.0 \mathrm{MHz}, \mathrm{CDCl}_{3}\right)$ : $\delta 172.4,166.9,153.8,149.5,143.4,136.6,128.8,127.9,126.4,122.7$, 104.4, 53.8, 51.9, 51.6, 47.5, 38.0, 17.8, 16.7. IR (neat, $\mathrm{cm}^{-1}$ ): 3242, 2955, 2927, 1739, 1691, 1620, 1435, 1387, 1249, 1214, 1190, 1092, 970, 756. HRMS: calc'd for $(\mathrm{M}+\mathrm{H})^{+} \mathrm{C}_{21} \mathrm{H}_{27} \mathrm{~N}_{2} \mathrm{O}_{5}$ : 387.1842; found: 387.1856 .

(S)-methyl-1-((S,E)-6-methoxy-2-methyl-6-oxohex-3-enyl)-3-(3-methoxy-3-oxopropanoyl)6-methyl-2-oxo-4-phenyl-1,2,3,4-tetrahydropyrimidine-5-carboxylate

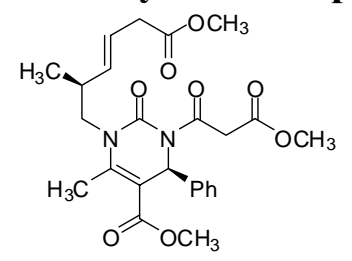

To a round-bottom flask equipped with stir bar was charged with dihydropyrimidone $42(53 \mathrm{mg}, 0.137 \mathrm{mmol})$, benzene $(2 \mathrm{~mL})$ and methyl 3-chloro-3-oxo-propionate $(30 \mu \mathrm{L}, 0.274 \mathrm{mmol})$ successively. The reaction mixture was heated to reflux for 1 hour and cooled to room temperature. Saturated $\mathrm{NaHCO}_{3}(5.0 \mathrm{~mL})$ and ethyl acetate $(5 \mathrm{~mL})$ was added. The organic layer was separated and washed with water and brine. The solution was dried over anhydrous sodium sulfate, filtered and concentrated in vacuo. The resulting residue was subjected to column chromatography on silica gel to afford the amide product. Yield: $60 \mathrm{mg}$, 90\%. ${ }^{1} \mathbf{H}$ NMR $\left(400 \mathrm{MHz}, \mathrm{CDCl}_{3}\right): \delta 7.26(\mathrm{~m}, 5 \mathrm{H}), 6.76(\mathrm{~s}, 1 \mathrm{H}), 5.22(\mathrm{~m}$, $1 \mathrm{H}), 5.02(\mathrm{~m}, 1 \mathrm{H}), 4.03(\mathrm{dd}, J=14.4,7.2 \mathrm{~Hz}, 1 \mathrm{H}), 3.91(\mathrm{~s}, 2 \mathrm{H}), 3.74(\mathrm{~s}$, $3 \mathrm{H}), 3.62(\mathrm{~s}, 3 \mathrm{H}), 3.42(\mathrm{~s}, 3 \mathrm{H}), 3.23(\mathrm{dd}, \mathrm{J}=14,7.2 \mathrm{~Hz}, 1 \mathrm{H}), 2.77$ (dd, $J$ $=13.2,6.0 \mathrm{~Hz}, 1 \mathrm{H}), 2.71(\mathrm{dd}, J=13.2,6.0 \mathrm{~Hz}, 1 \mathrm{H}), 2.51(\mathrm{~s}, 3 \mathrm{H}), 2.12(\mathrm{~m}$, $1 \mathrm{H}), 0.60(\mathrm{~d}, J=6.0 \mathrm{~Hz}, 3 \mathrm{H}) .{ }^{13} \mathbf{C}$ NMR $\left(75.0 \mathrm{MHz}, \mathrm{CDCl}_{3}\right): \delta 172.4$, $167.8,167.2,165.9,152.1,149.1,138.5,135.5,128.5,127.8,125.9$, $122.2,109.7,53.4,52.7,52.0,51.8,48.3,37.5,36.3,16.8,16.3$. IR (neat, $\left.\mathrm{cm}^{-1}\right): 2956,2926,1741,1700,1634,1435,1385,1340,1258,1166,758$. HRMS: calc'd for $(\mathrm{M}+\mathrm{H})^{+} \mathrm{C}_{25} \mathrm{H}_{31} \mathrm{~N}_{2} \mathrm{O}_{8}$ : 487.2002; found: 487.2053.

(S)-methyl-3-(2-diazo-3-methoxy-3-oxopropanoyl)-1-((S,E)-6-methoxy-2-methyl-6-oxohex3-enyl)-6-methyl-2-oxo-4-phenyl-1,2,3,4-tetrahydropyrimidine-5-carboxylate 43 


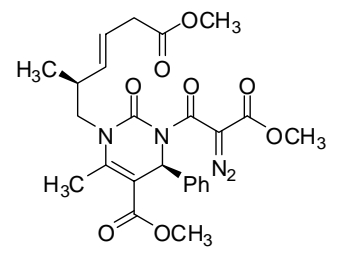

To a round-bottom flask equipped with stir bar was charged with amide (53 mg, $0.11 \mathrm{mmol}$ ) obtained from previous reaction. $p$ Acetamidobenzenesulfonyl azide ( $p$-ABSA, $31.4 \mathrm{mg}, 0.13 \mathrm{mmol}$ ) and methlene chloride $(2.0 \mathrm{~mL})$ was added subsequently. The reaction solution was cooled in ice bath and triethylamine $(36.4 \mu \mathrm{L}, 0.26 \mathrm{mmol})$ was added. The reaction mixture was stirred at room temperature for overnight and the solution was concentrated in vacuo and the residue was directly subjected to column chromatography on slice gel to afford the desired product 43. Yield: $50 \mathrm{mg}, 90 \% .{ }^{1} \mathbf{H}$ NMR (400 MHz, $\left.\mathrm{CDCl}_{3}\right): \delta 7$. $27(\mathrm{~m}, 5 \mathrm{H}), 6.26(\mathrm{~s}, 1 \mathrm{H}), 5.28(\mathrm{~m}, 1 \mathrm{H}), 5.04(\mathrm{~m}, 1 \mathrm{H}), 3.99(\mathrm{dd}, J=14.0$, $6.8 \mathrm{~Hz}, 1 \mathrm{H}), 3.76(\mathrm{~s}, 3 \mathrm{H}), 3.74(\mathrm{~s}, 3 \mathrm{H}), 3.64(\mathrm{~s}, 3 \mathrm{H}), 3.25(\mathrm{dd}, J=14.0$, $6.8 \mathrm{~Hz}, 1 \mathrm{H}), 2.80(\mathrm{~m}, 2 \mathrm{H}), 2.56(\mathrm{~s}, 3 \mathrm{H}), 2.14(\mathrm{~m}, 1 \mathrm{H}), 0.58(\mathrm{~d}, J=7.2 \mathrm{~Hz}$, $3 \mathrm{H}) .{ }^{13} \mathrm{C}$ NMR $\left(75.0 \mathrm{MHz}, \mathrm{CDCl}_{3}\right): \delta 172.2,165.9,163.0,161.3,152.0$, $149.5,138.8,135.6,128.5,127.8,126.1,122.0,110.4,53.4,52.3,51.9$, 51.7, 48.5, 37.6, 36.3, 16.8, 16.3. IR (neat, $\mathrm{cm}^{-1}$ ): 2950, 2135, 1740, 1700, 1630, 1380, 1155. HRMS: calc'd for $(\mathrm{M}+\mathrm{H})^{+} \mathrm{C}_{25} \mathrm{H}_{29} \mathrm{~N}_{4} \mathrm{O}_{8}$ : 513.1907; found: 513.1868 .
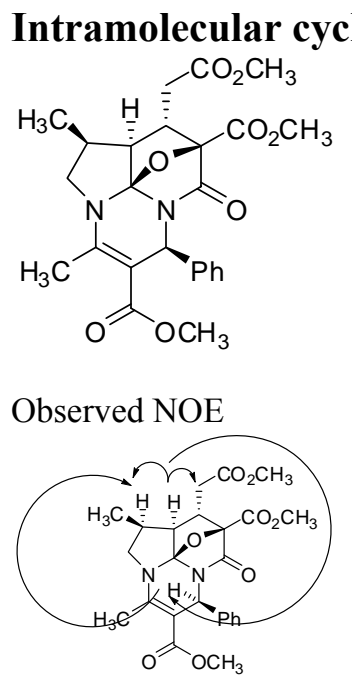

To a round-bottom flask equipped with a stir bar was charged with diazo amide 43 (30 mg, $0.06 \mathrm{mmol}), \mathrm{Rh}_{2}(\mathrm{OAc})_{4}(1.5 \mathrm{mg}, 0.003 \mathrm{mmol})$ and anhydrous benzene $(3 \mathrm{~mL})$. The reaction solution was refluxed overnight under Ar. The resulting solution was concentrated in vacuo and the residue was directly subjected to column chromatography on silica gel to afford the final bicycle product 44. Yield: $23 \mathrm{mg}, 80 \%$. ${ }^{1} \mathbf{H}$ NMR (400 $\left.\mathrm{MHz}, \mathrm{CDCl}_{3}\right): \delta 7.19(\mathrm{~m}, 5 \mathrm{H}), 6.03(\mathrm{~s}, 1 \mathrm{H}), 3.70(\mathrm{dd}, J=14,6.0 \mathrm{~Hz}, 1 \mathrm{H})$, $3.57(\mathrm{~s}, 1 \mathrm{H}), 3.51(\mathrm{~s}, 3 \mathrm{H}), 3.35(\mathrm{~s}, 3 \mathrm{H}), 2.88(\mathrm{dd}, J=13.2,3.6 \mathrm{~Hz}), 2.63$ (dd, $J=13.2,11.2 \mathrm{~Hz}, 1 \mathrm{H}), 2.44(\mathrm{~s}, 3 \mathrm{H}), 2.42(\mathrm{dd}, J=14,6.4 \mathrm{~Hz}, 1 \mathrm{H})$, $2.25(\mathrm{dd}, \mathrm{J}=14,6.4 \mathrm{~Hz}, 1 \mathrm{H}), 1.07(\mathrm{~d}, J=6.8 \mathrm{~Hz}, 3 \mathrm{H}) 0.89(\mathrm{~m}, 1 \mathrm{H}) .{ }^{13} \mathbf{C}$ NMR (75.0 MHz, $\left.\mathrm{CDCl}_{3}\right): \delta 173.1,172.2,170.2,166.7,164.7,147.7$, $141.9,131.7,128.7,128.3,127.6,127.2,104.9,96.6,78.8,55.4,53.5$, 53.3, 53.1, 50.9, 37.9, 34.8, 29.9, 21.9, 16.3. IR (neat, $\mathrm{cm}^{-1}$ ): 3445, 2953, 1739, 1689, 1576, 1433, 1247, 1166, 1124, 1029, 759. HRMS: calc'd for $(\mathrm{M}+\mathrm{H})^{+} \mathrm{C}_{25} \mathrm{H}_{29} \mathrm{~N}_{2} \mathrm{O}_{8}$ : 485.1846; found: 485.1880 . 


\section{References and Notes}

1) a) Petrini, M.; Mecozzi T. J. Org. Chem. 1999, 64, 8970-8972. b) Murry, J. A.; Frantz D. E.; Soheili A.; Tillyer, R.; Grabowski, E. J. J.; Reider, P. J. J. Am. Chem. Soc. 2001, 123, 96969697.

2) Gleim, C. E. J. Am. Chem. Soc. 1954, 76, 107-111.

3) Lou, S.; Taoka, B. M.; Ting, A.; Schaus, S. E. J. Am. Chem. Soc. 2005, 127, 11256-11257.

4) a) Gerald, O. D.; Gert, P. V. J. Am. Chem. Soc. 1963, 85, 2697-2702; b) George, D. H.; Richard, D. H.; David, W. C. J. Org. Chem. 1983, 48, 41190-4122.

5) Uraguchi, D.; Terada, M. J. Am. Chem. Soc. 2004, 126, 5356-5357.

6) Song, J.; Wang, Y.; Deng, L. J. Am. Chem. Soc. 2006, 128, 6048-6049.

7) Ting, A.; Lou, S.; Schaus, S. E. Org. Lett. 2006, 8, 2003-2006. 


\section{Selected NMR Spectra:}<smiles>CCN1C(=O)NC(c2ccccc2)C(C(C)=O)=C1C</smiles>
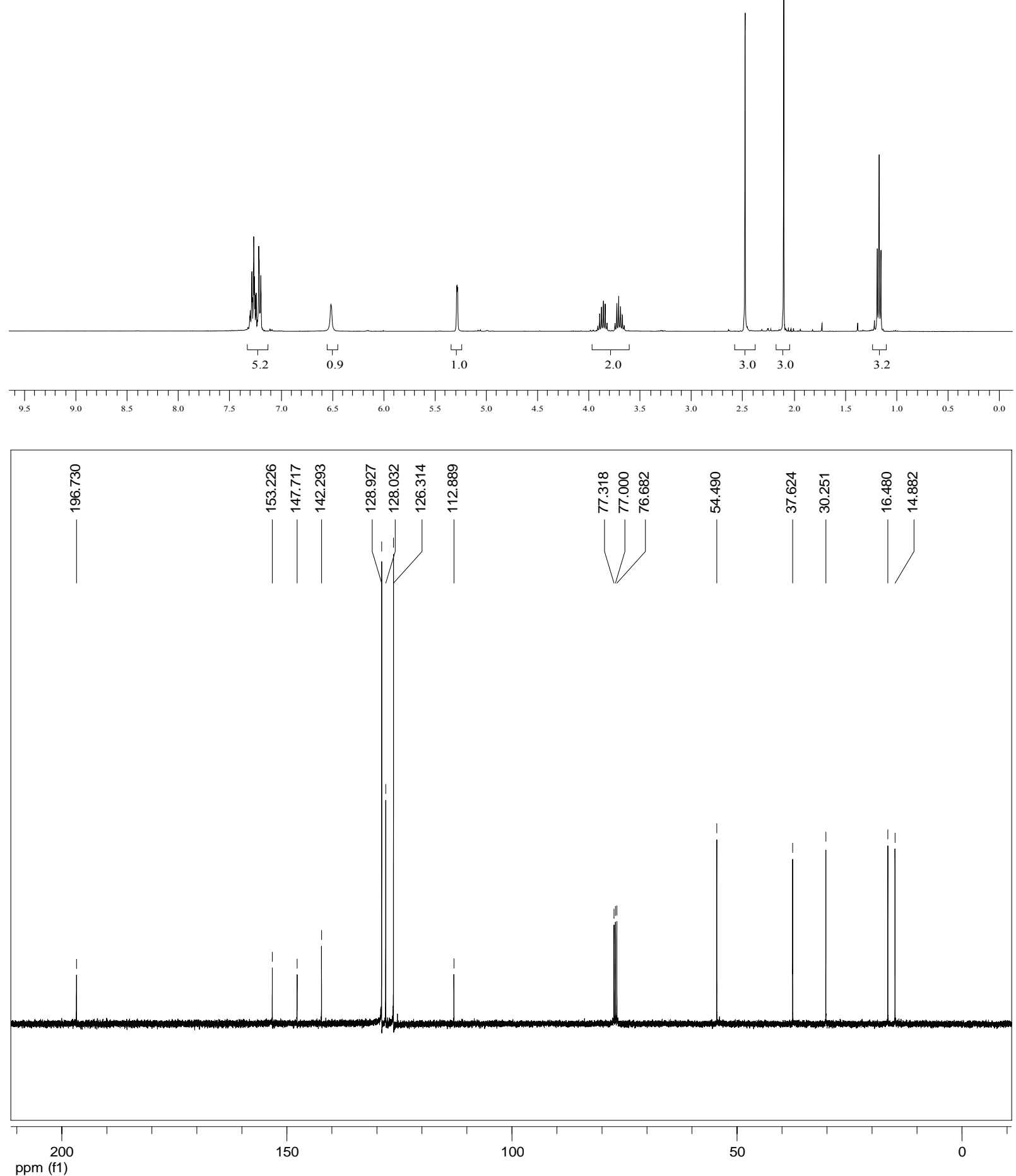
<smiles>CCOC(=O)CN1C(=O)N[C@H](CCc2ccccc2)C(C(=O)OC)=C1C</smiles>
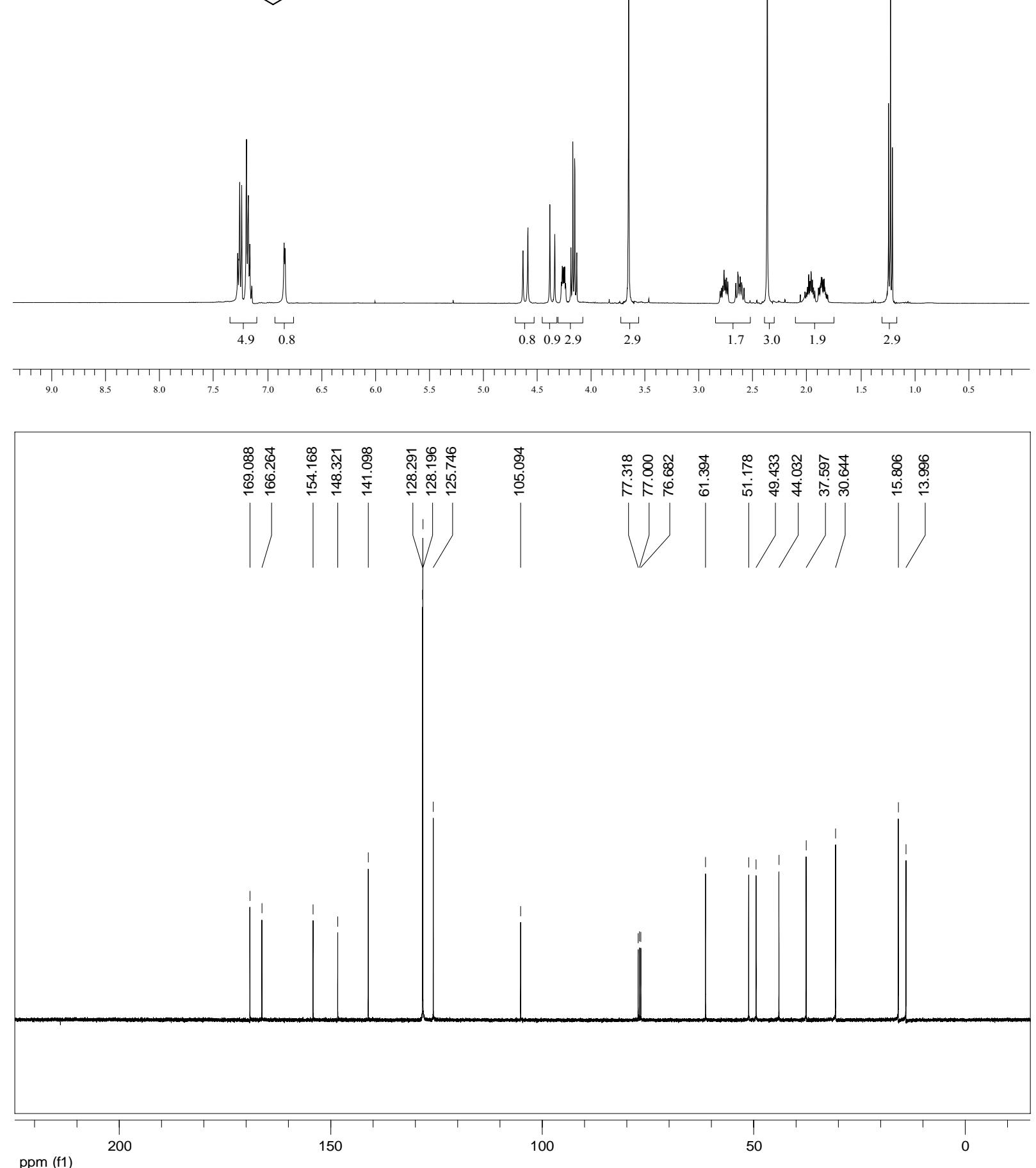
<smiles>COC(=O)C1=C(C)N(CC2CC2)C(=O)N[C@H]1c1ccccc1</smiles>
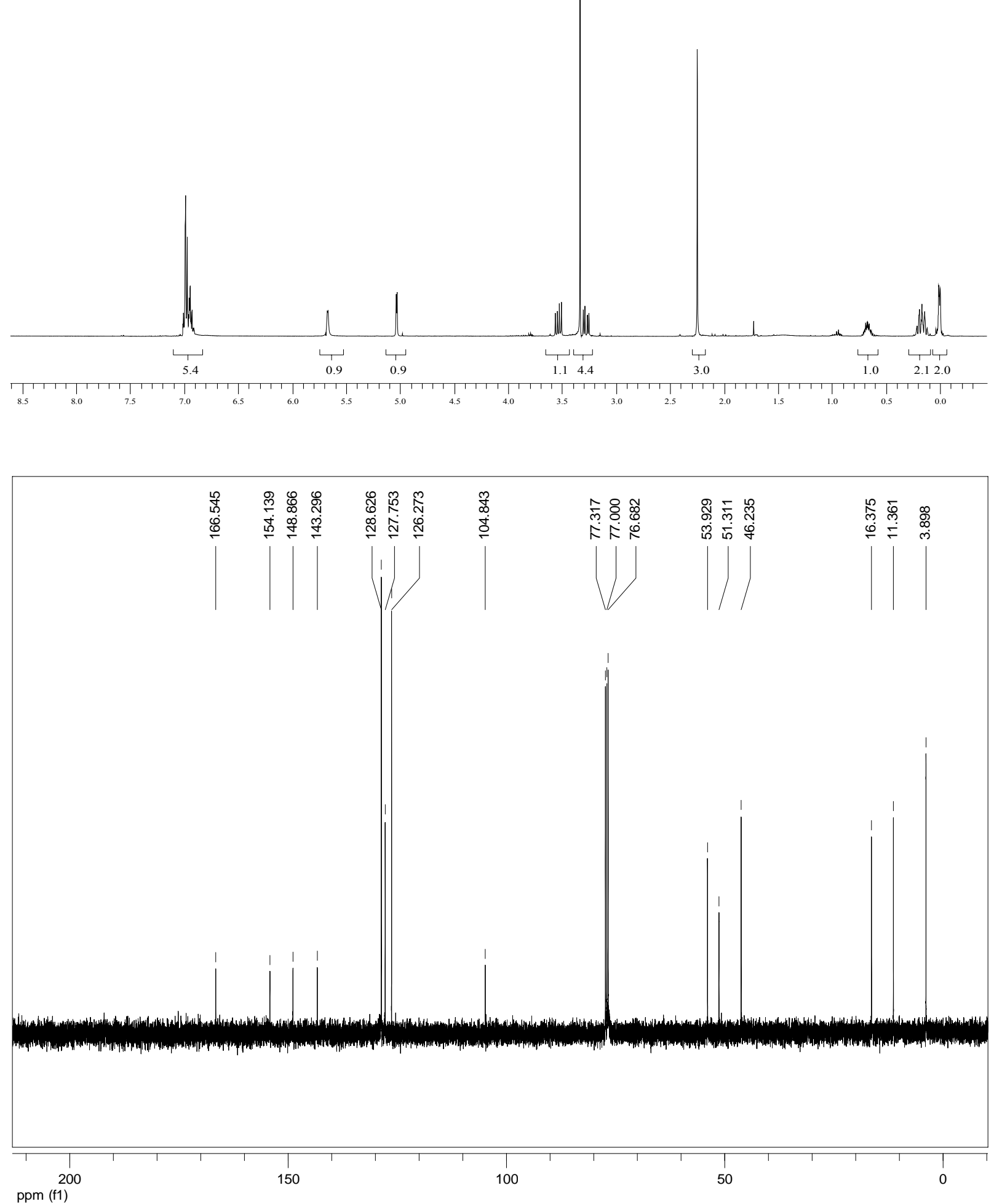
<smiles>COCCN1C(=O)NC(c2ccc(Br)cc2)C(C(=O)OC)=C1C</smiles>

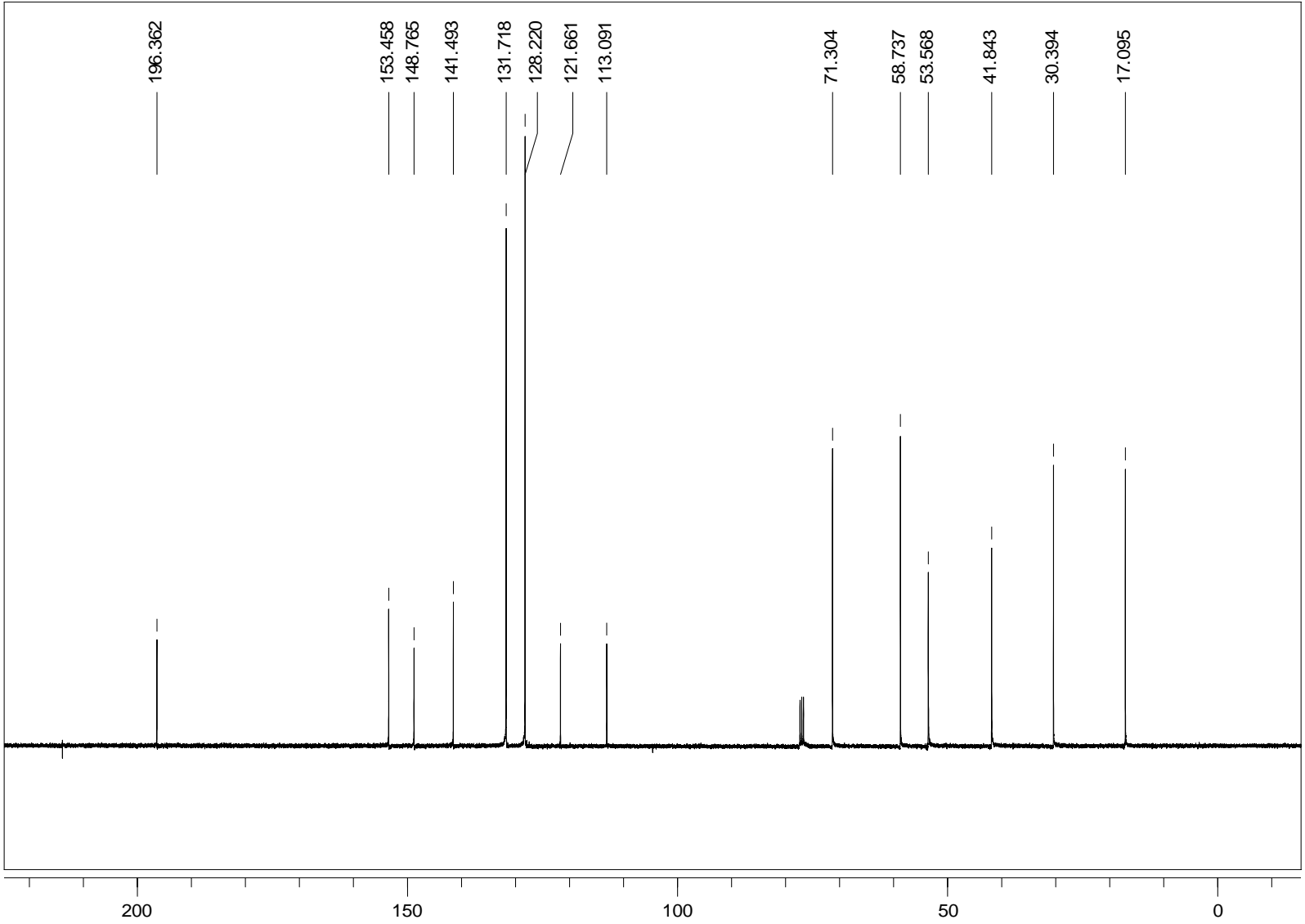

ppm (f1) 
<smiles>COC(=O)C1=C(C)N(Cc2ccco2)C(=O)N[C@H]1c1ccccc1</smiles>
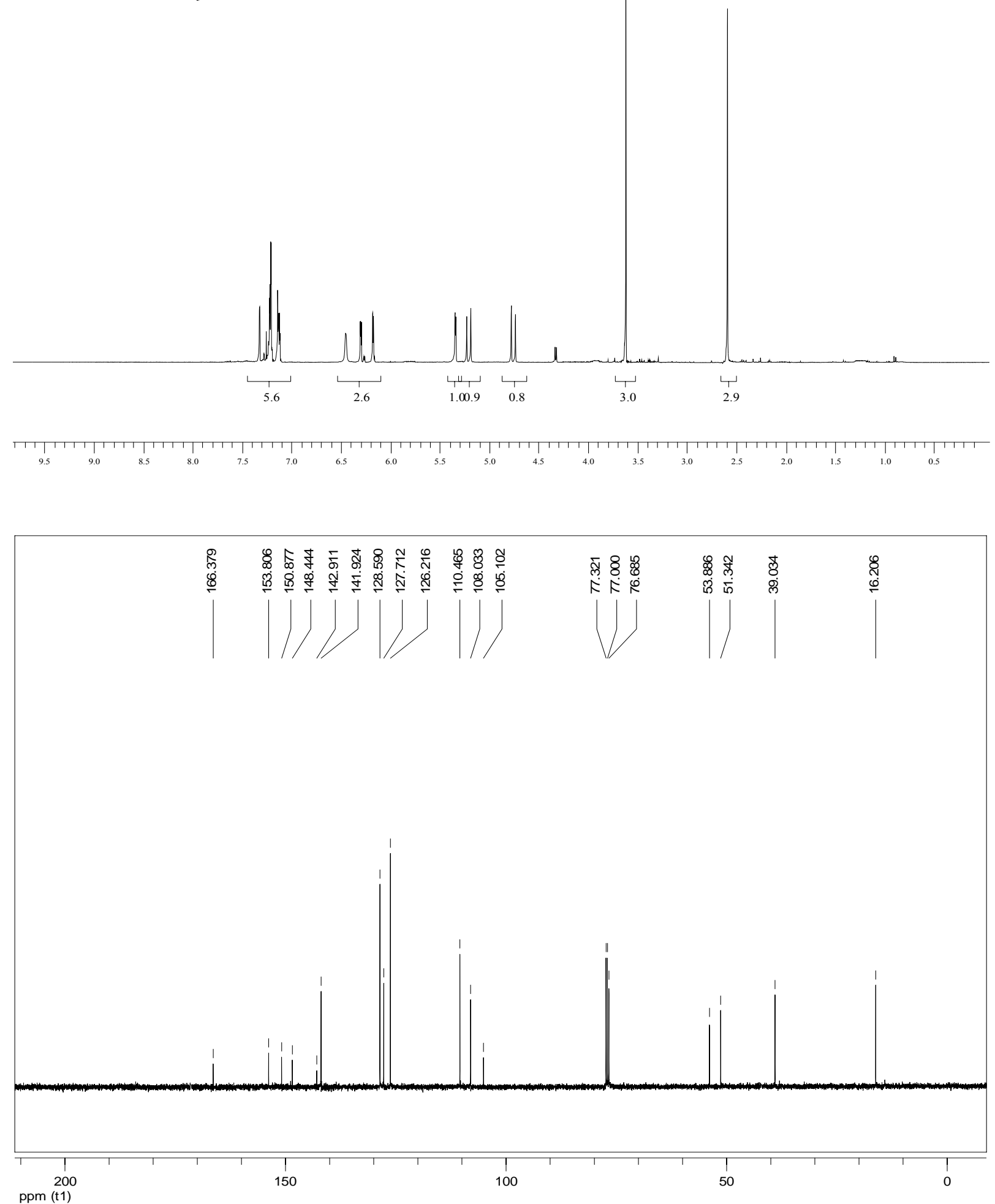
<smiles>COC(=O)C1=C(C)N(CCc2cc(OC)c(OC)c(OC)c2)C(=O)N[C@H]1c1cccs1</smiles>
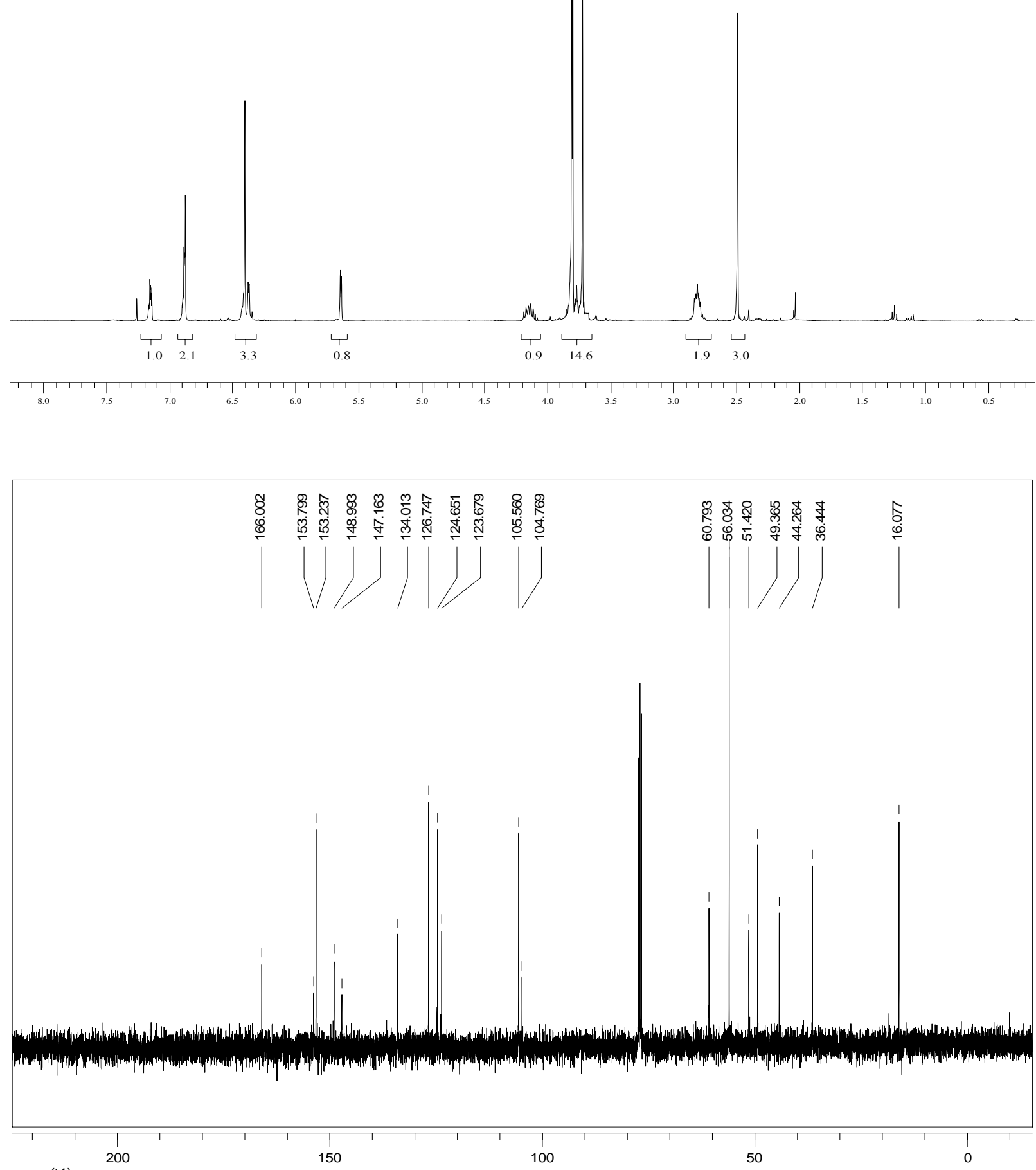

ppm (t1) 
<smiles>COCCN1C(=O)N[C@@H](c2ccco2)C(C(=O)OC)=C1C</smiles>
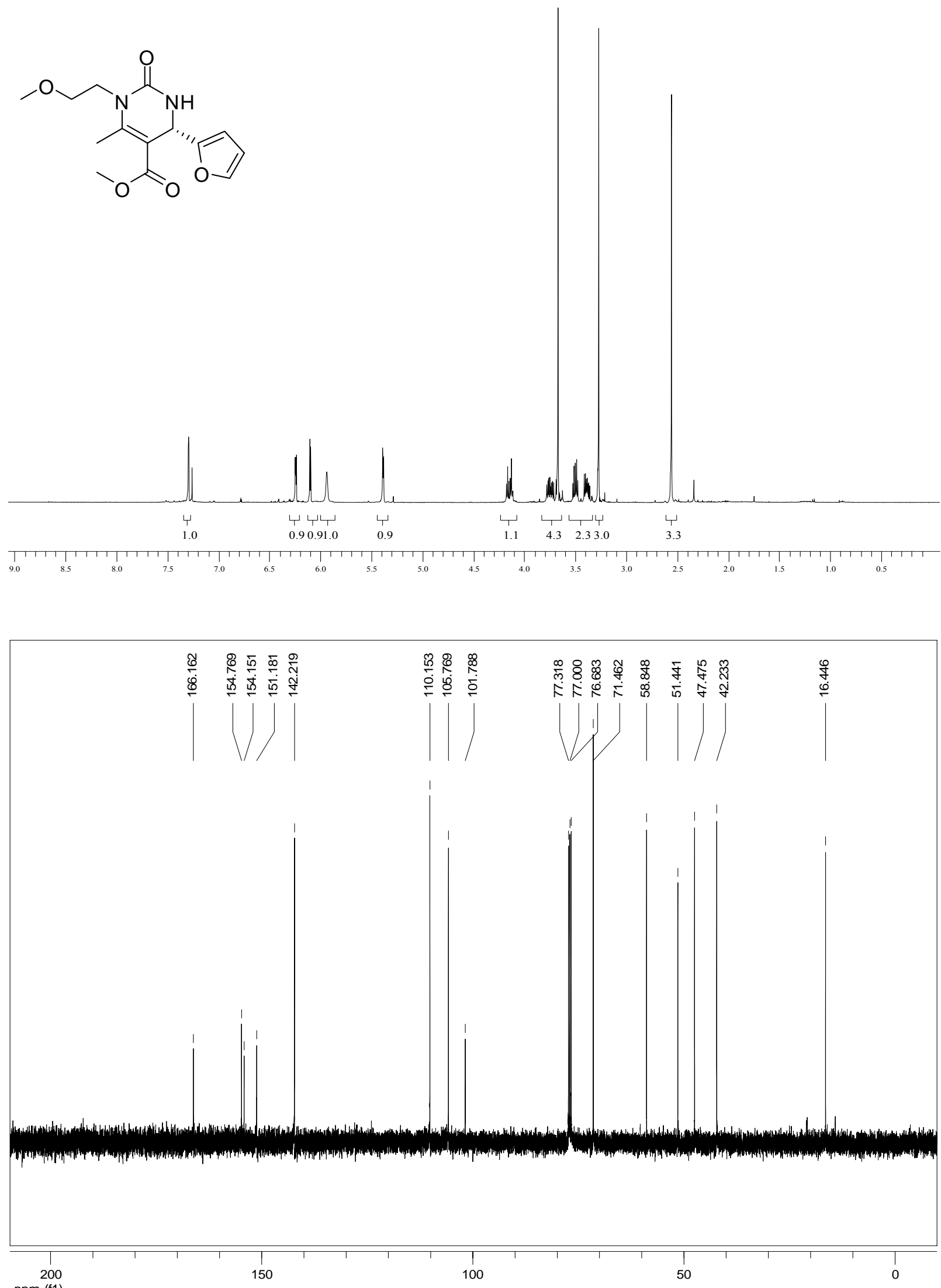

ppm (f1) 
<smiles>COC(=O)[C@H]1[C@@H](c2cccc(C(F)(F)F)c2)NC(=O)N1Cc1ccccc1</smiles>
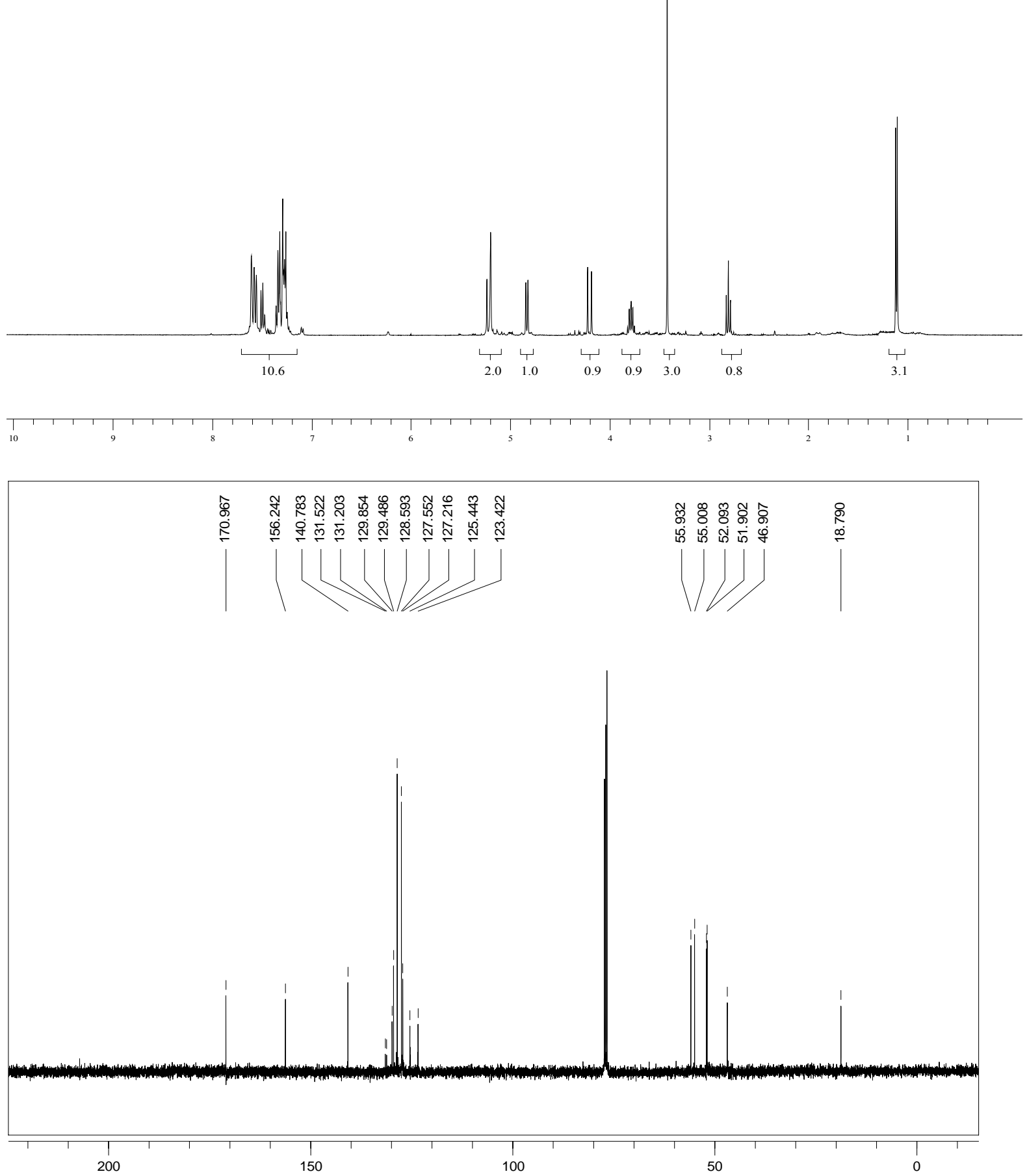

ppm (t1) 
<smiles>CC(=O)[C@H]1[C@@H](c2cccc(C(F)(F)F)c2)NC(=O)N(Cc2ccccc2)[C@H]1C</smiles>
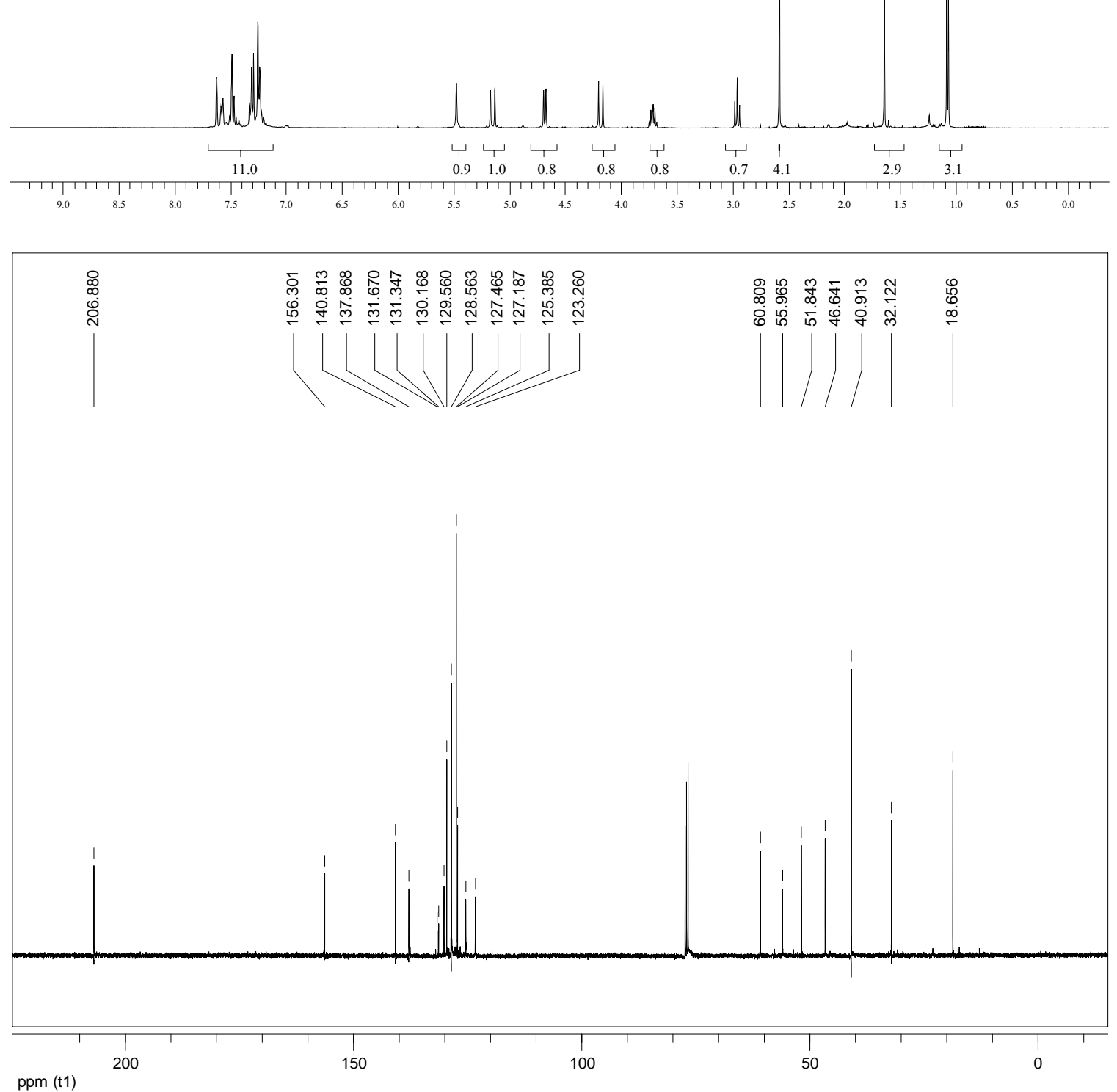
<smiles>CCN1C(=O)N[C@@H](c2cccc(C(F)(F)F)c2)[C@H](C(=O)OC)[C@@H]1C</smiles>

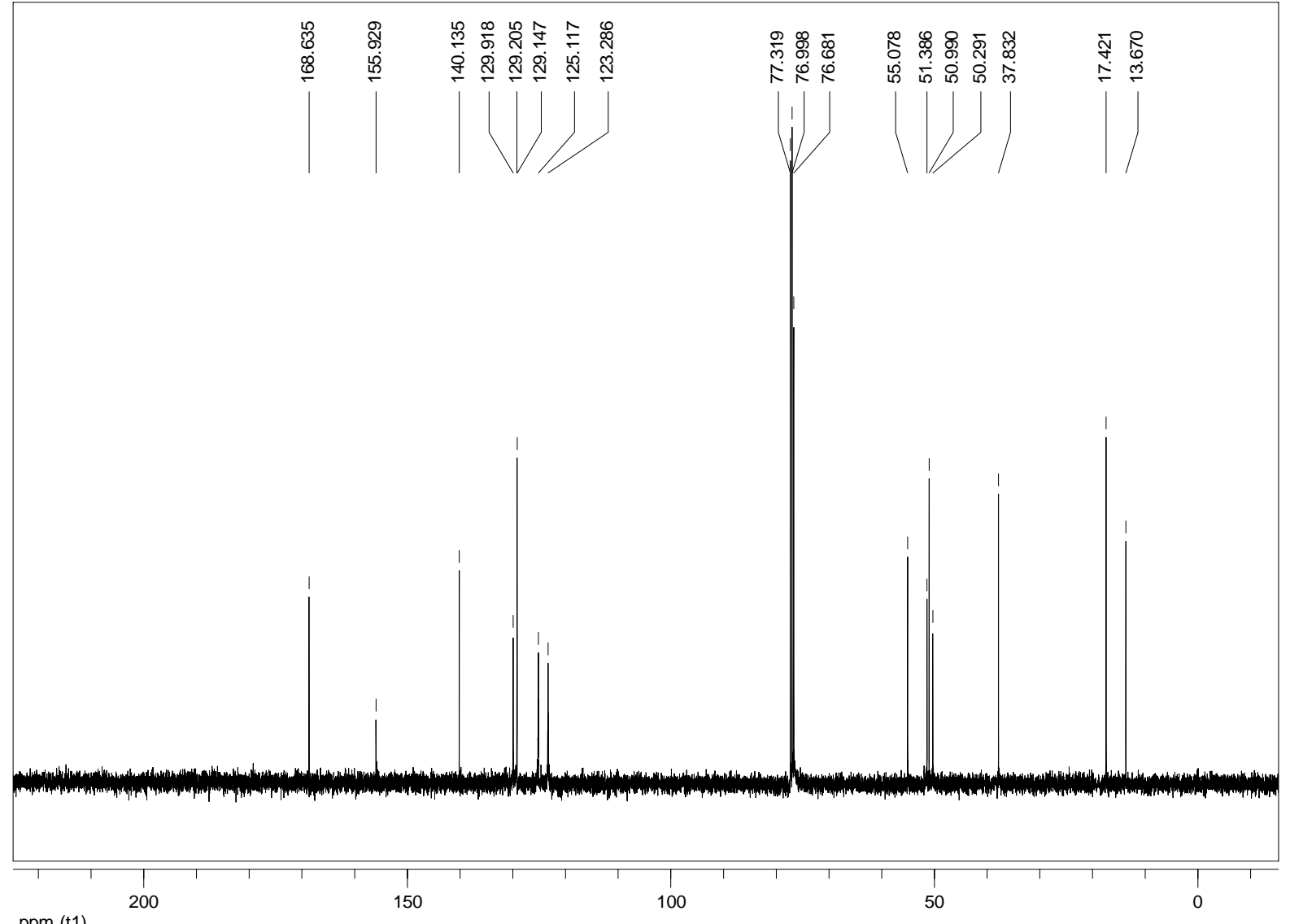


<smiles>CCN1C(=O)N[C@@H](c2cccc(C(F)(F)F)c2)[C@H](C(=O)OC)[C@@H]1C</smiles>
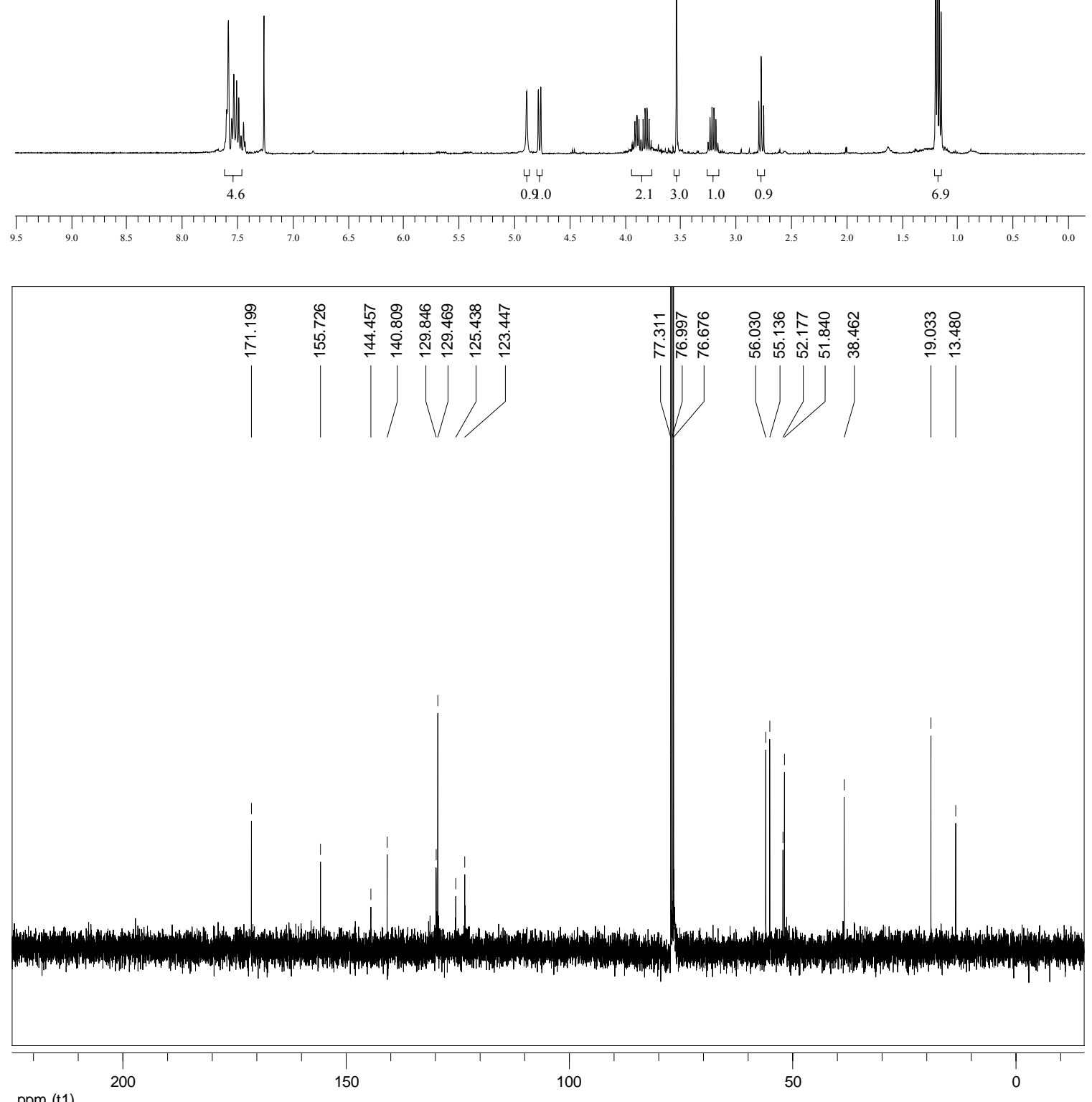

ppm (t1) 
<smiles>CCN1C(=O)N[C@@H](c2ccccc2)[C@H](C(=O)OC)[C@@H]1C</smiles>

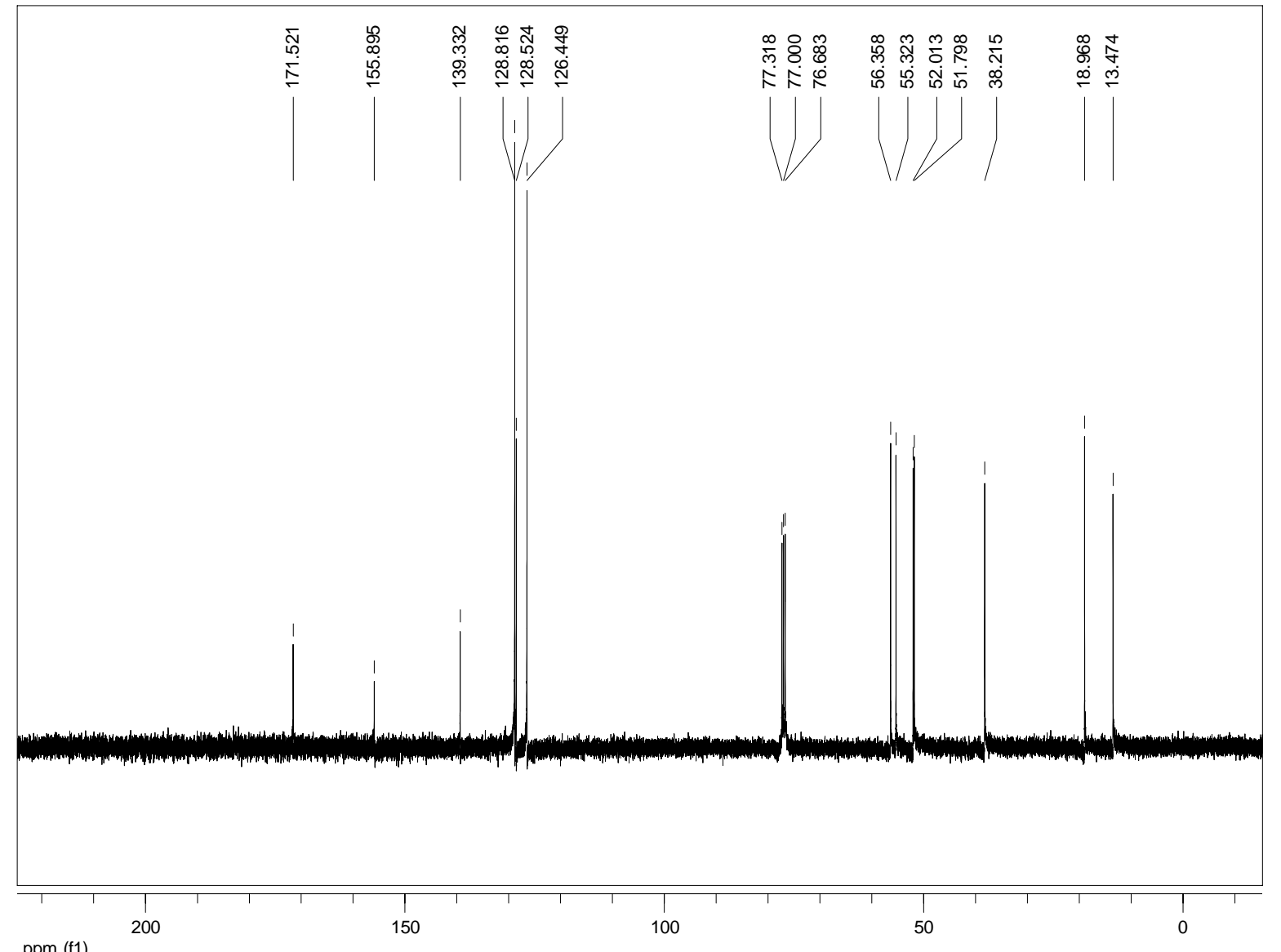

ppm (f1) 

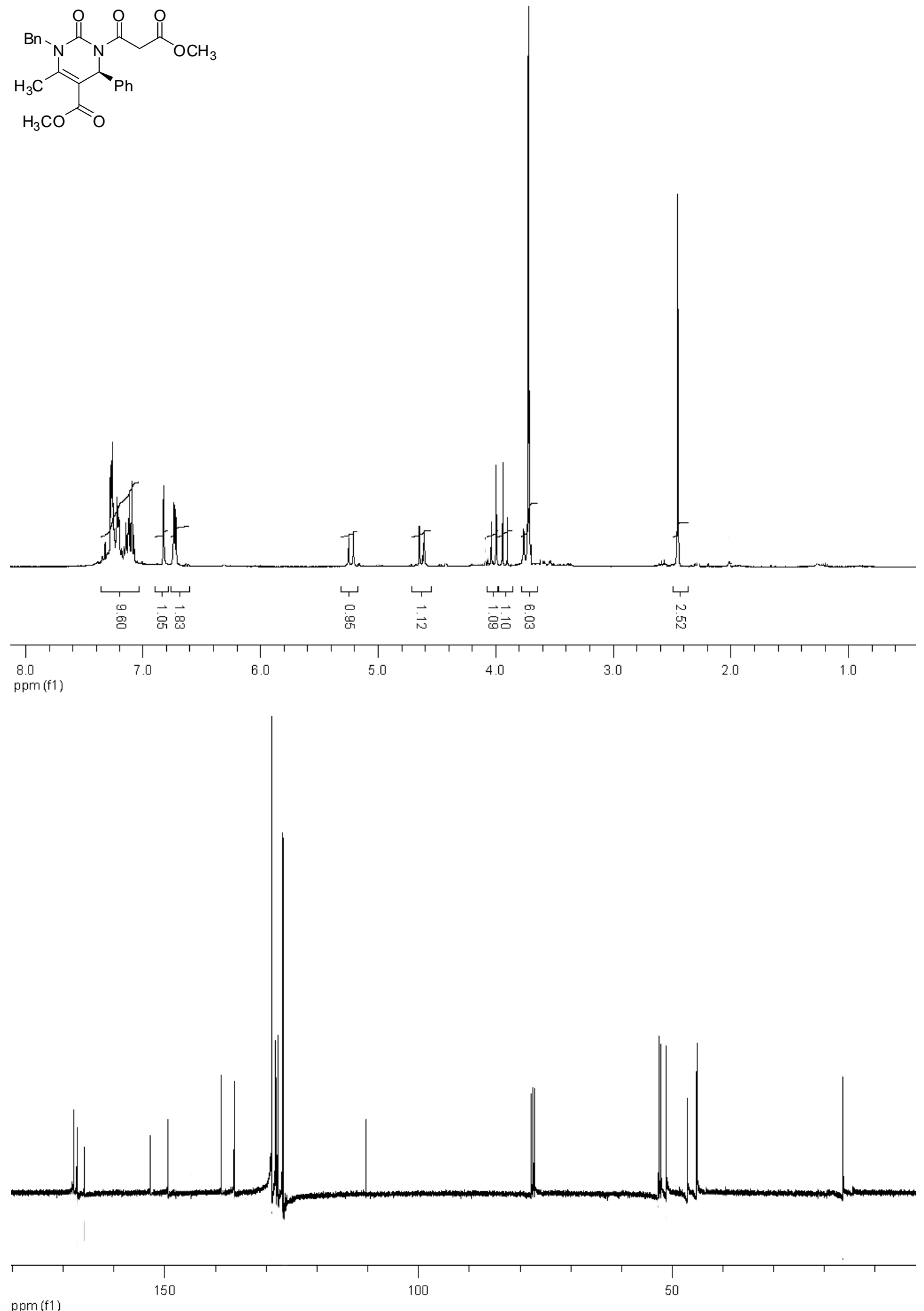

ppm (f1) 

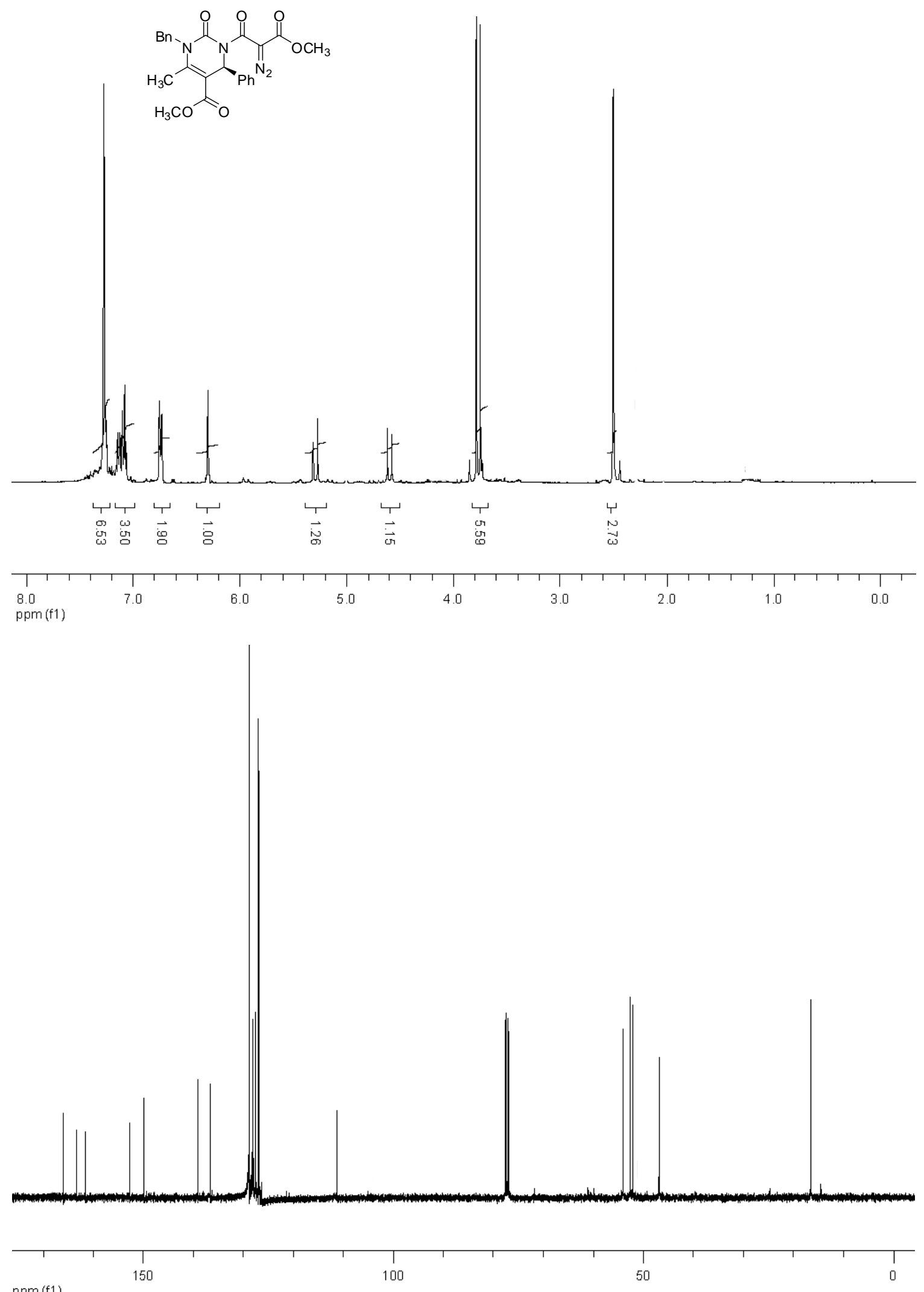

ppm (f1) 
<smiles>COC(=O)C1=C(C)N(Cc2ccccc2)[C@@]23OC1(C(=O)OC)C(=O)N2C(c1ccccc1)[C@H]1C(=O)N(C)C(=O)[C@H]13</smiles>
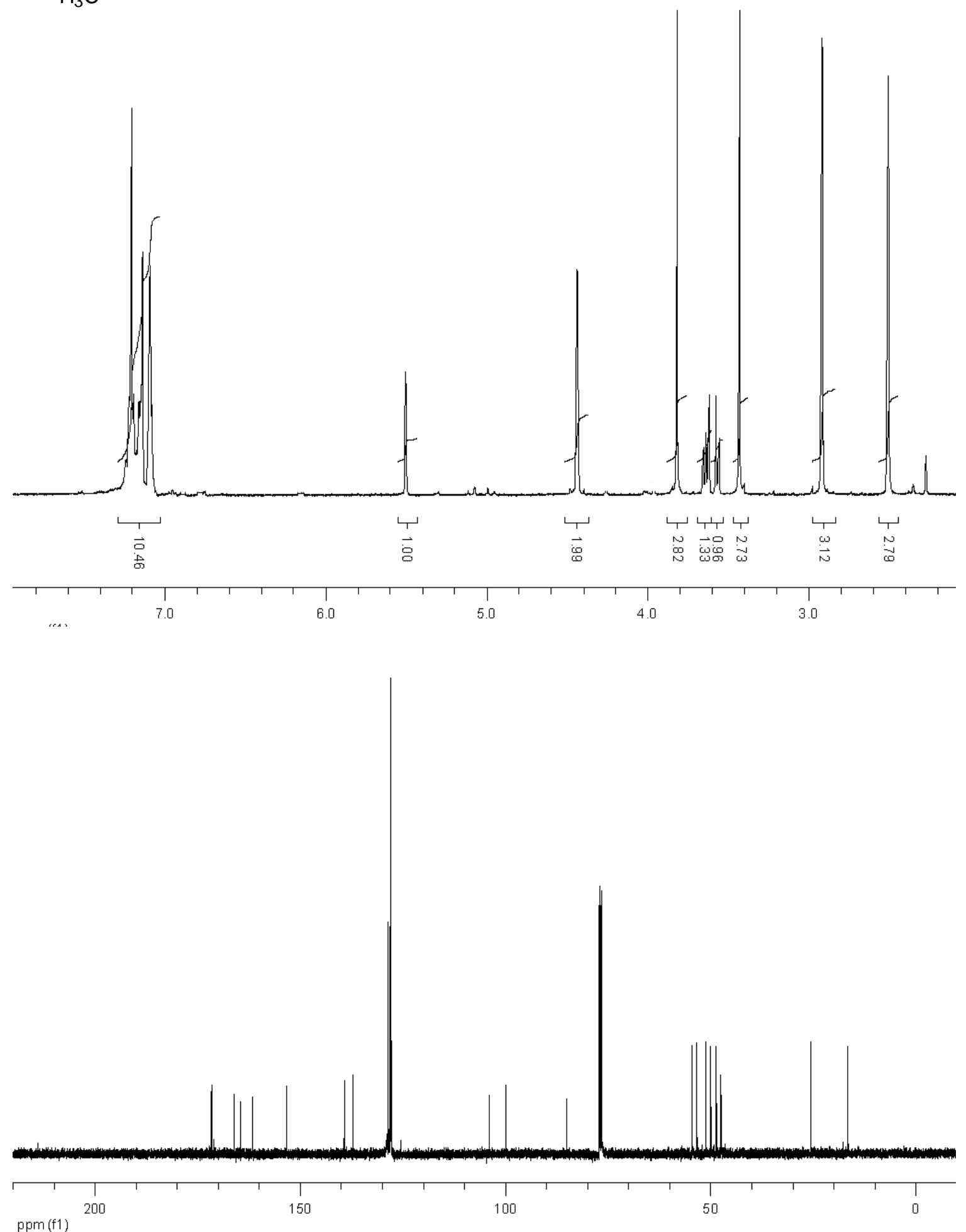
<smiles>COC(=O)C12O[C@]3([C@@H]4C(=O)N(c5ccccc5)C(=O)[C@@H]4C(c4ccccc4)N3C1=O)N(Cc1ccccc1)C(C)=C2C(C)=O</smiles>
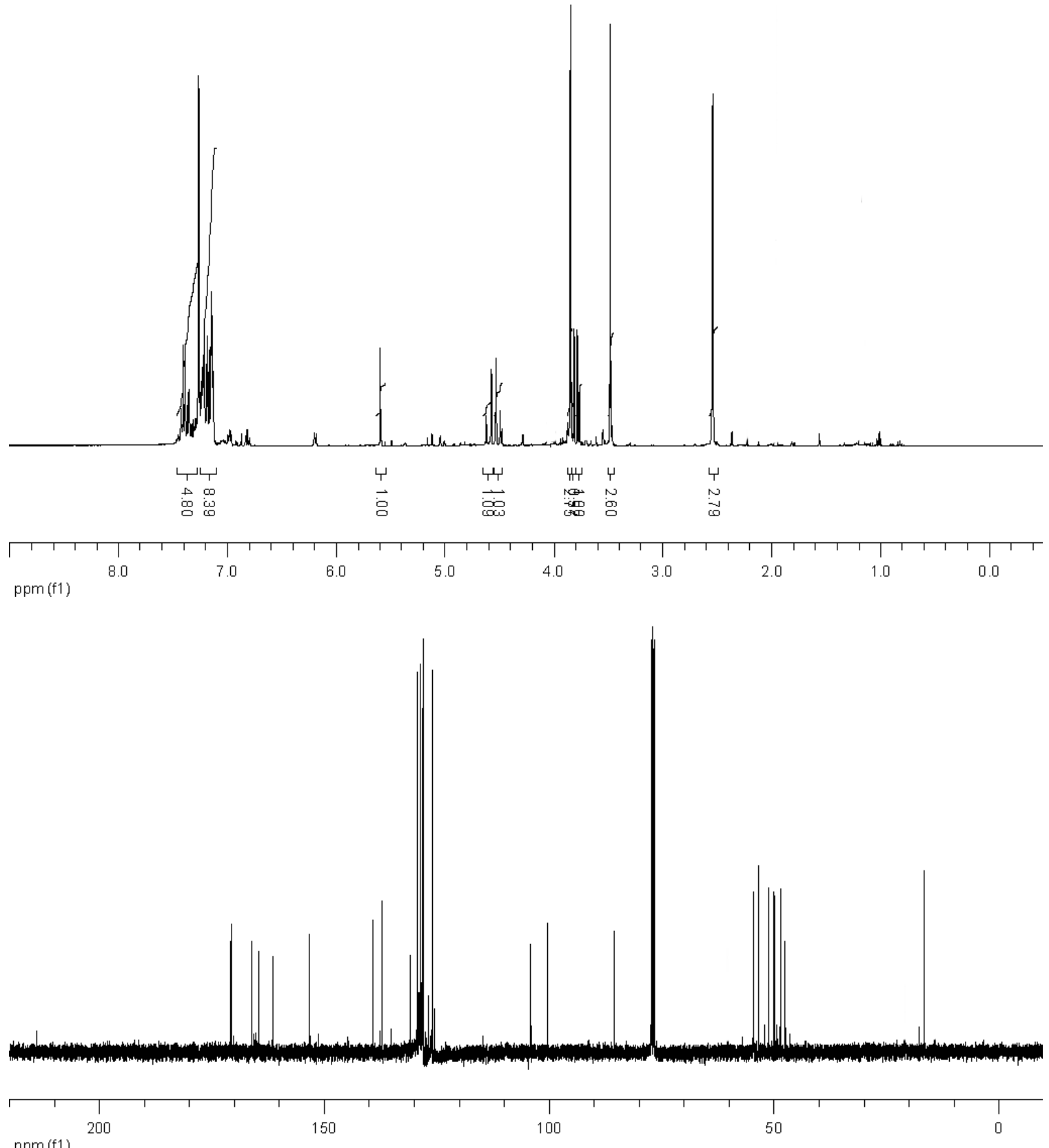
ppm (f1) 
<smiles>COCC1(C(=O)OC)C[C@H](C#N)C2N3C(=O)[C@@H](c4ccccc4)C(C(=O)OC)=C(C)N(Cc4ccccc4)C23O1</smiles>
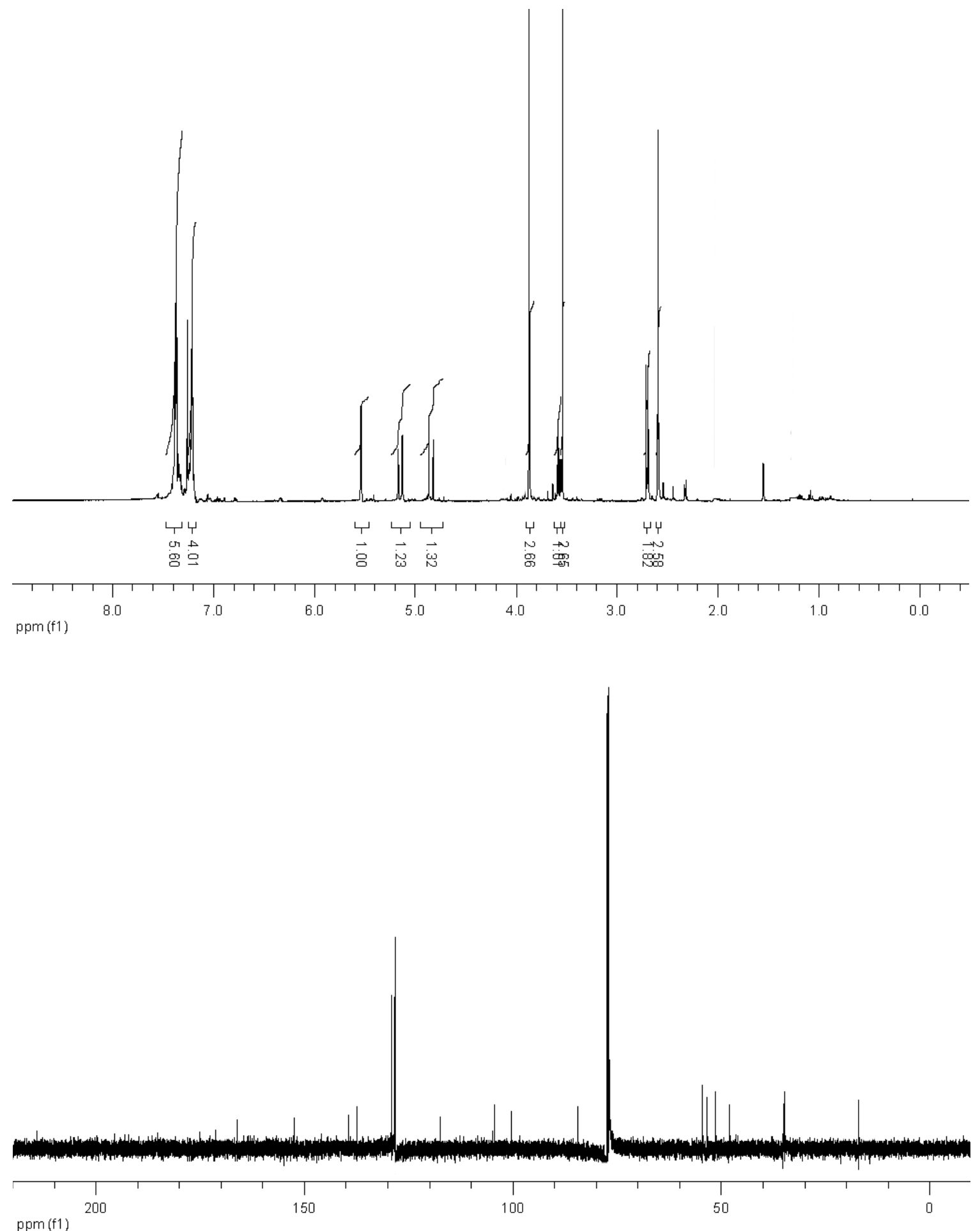
<smiles>COC(=O)[C@@]1(O)CC(C(C)=O)=C2N(Cc3ccccc3)C(C)=C(C(C)=O)[C@H](c3ccccc3)N2C1=O</smiles>

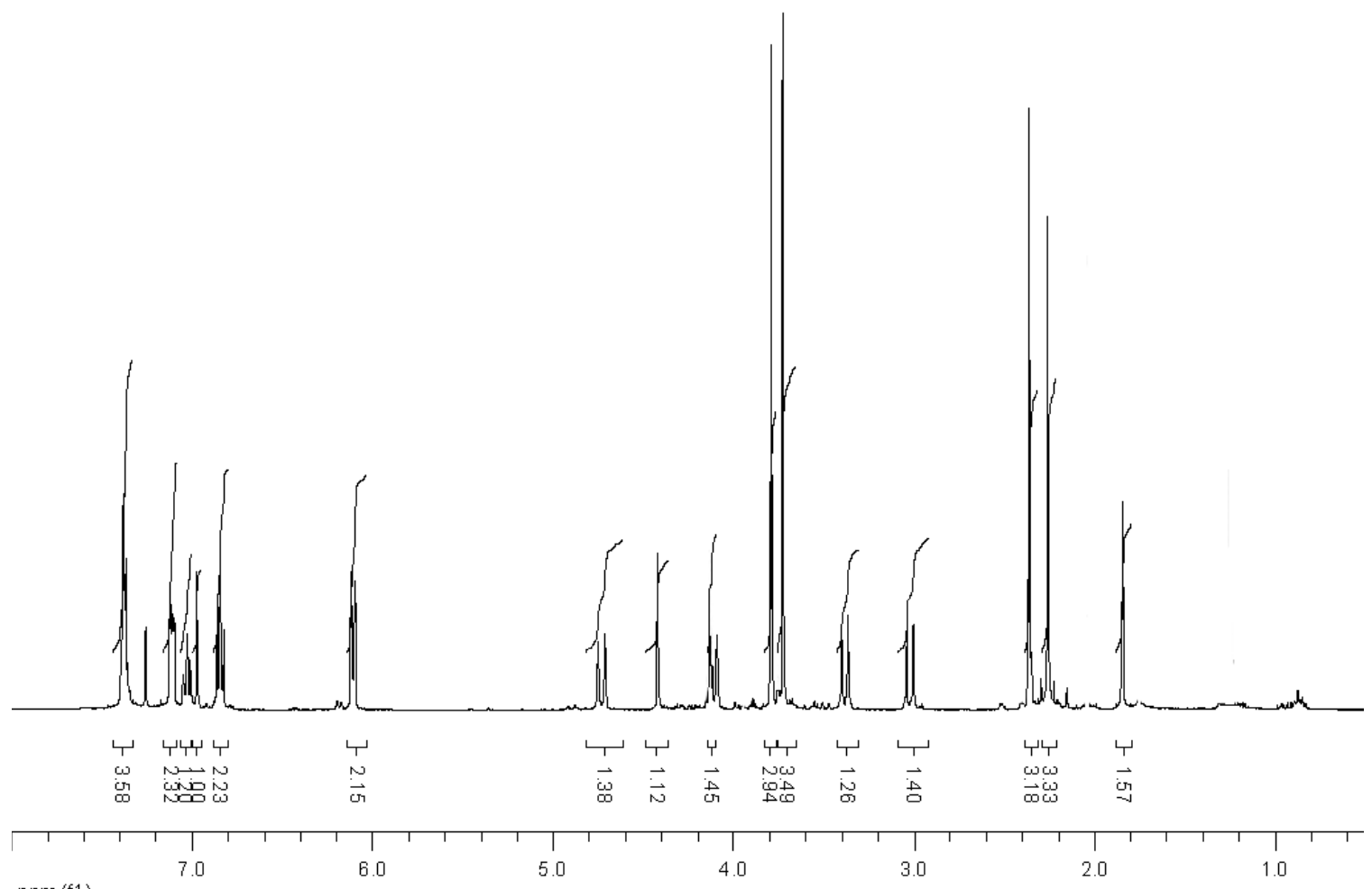
ppm (f1)

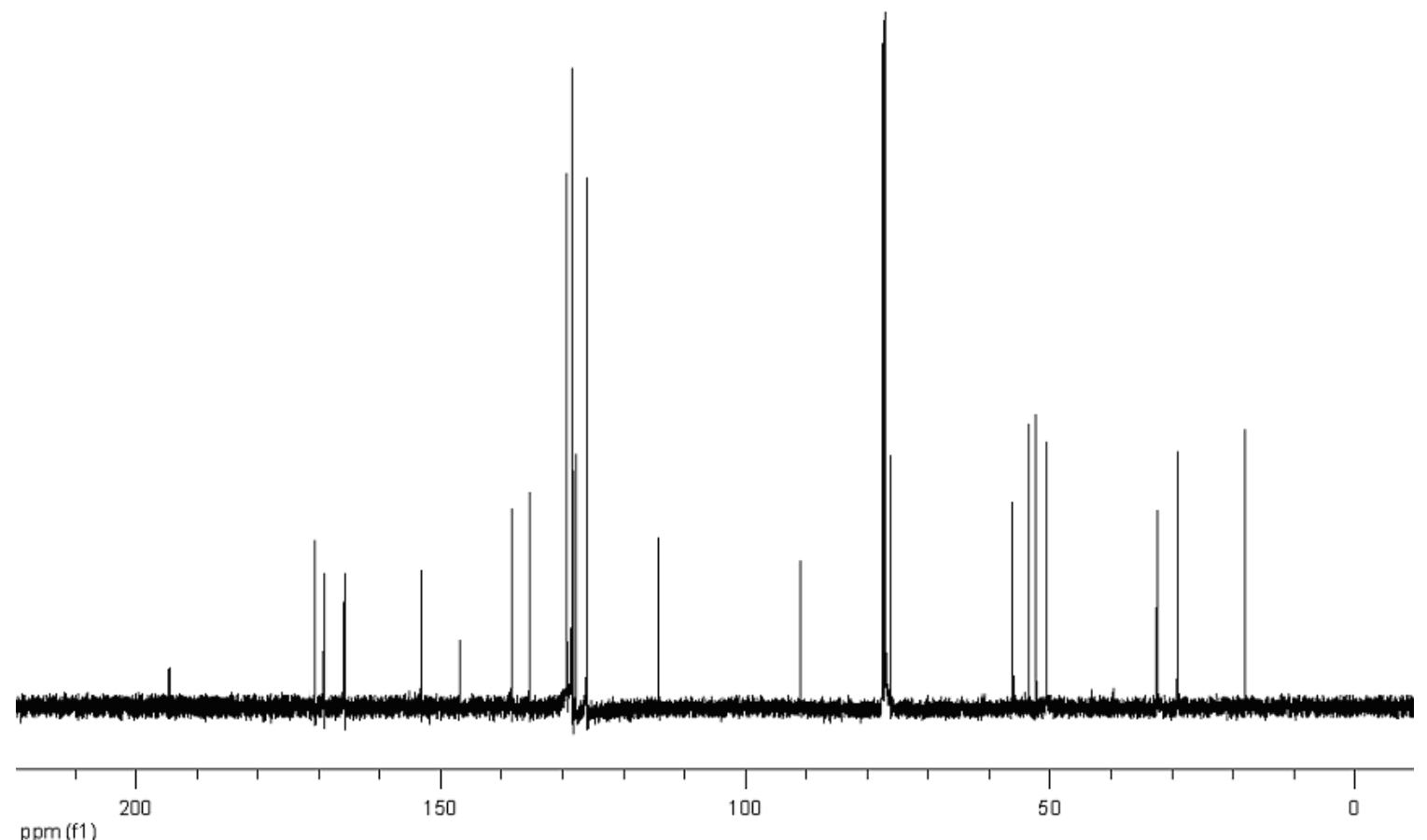
ppm (f1) 
<smiles>COC(=O)C1=C2N(Cc3ccccc3)C(C)=C(C(=O)OC)[C@H](c3ccccc3)N2C(=O)C(O)(C(=O)OC)C1=O</smiles>

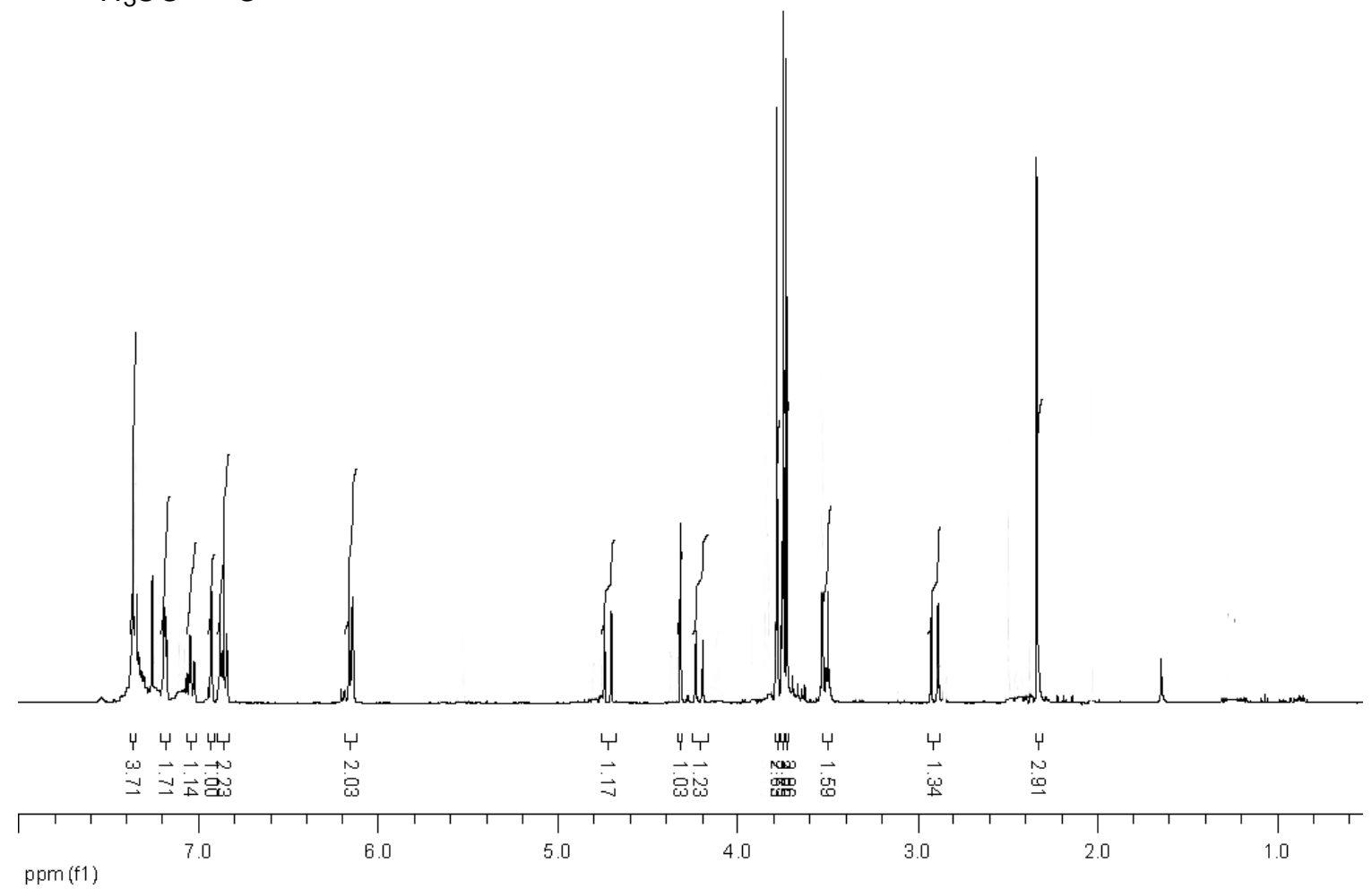

ppm (f1)
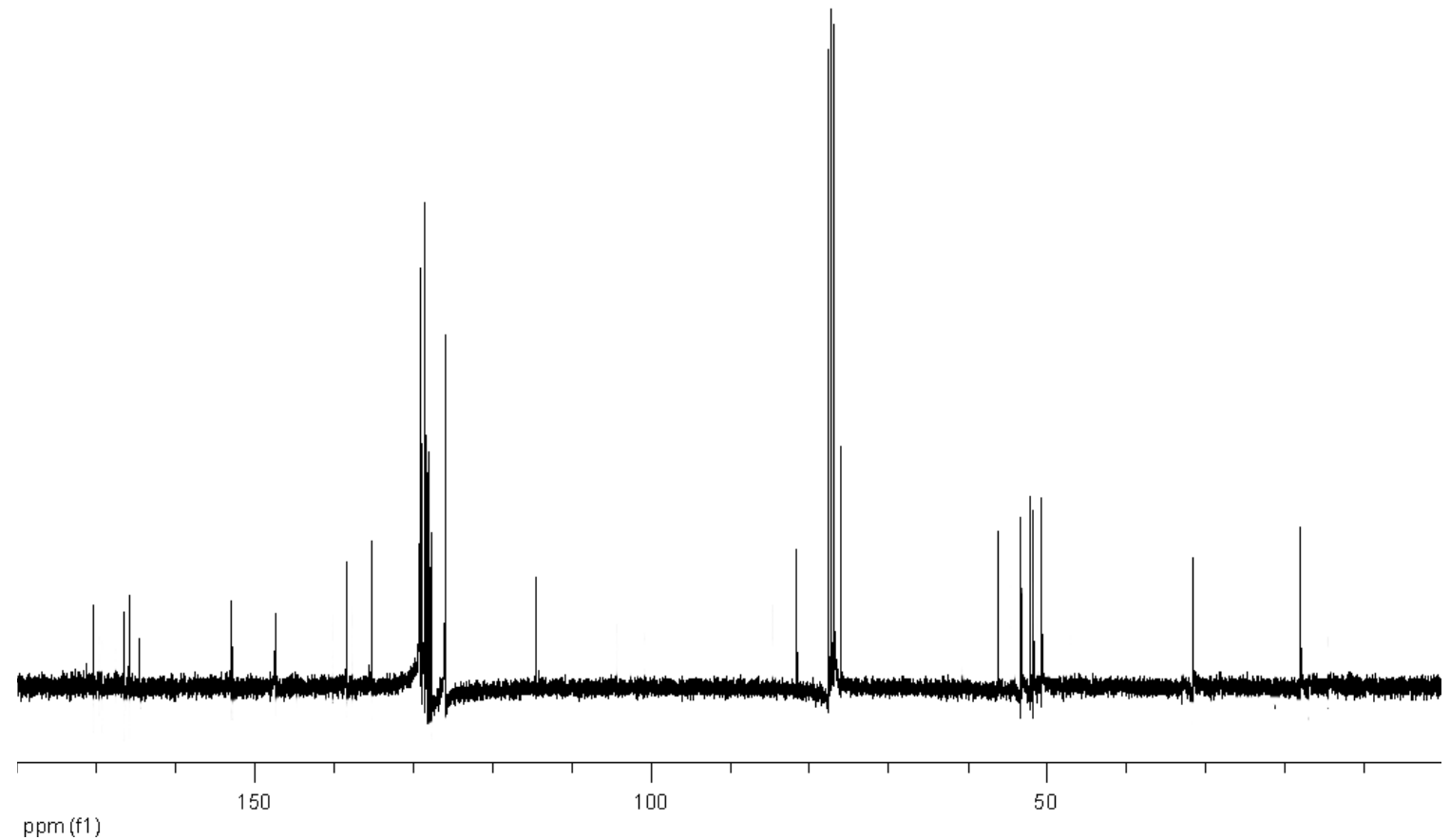

150

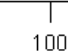

50

ppm (f1) 


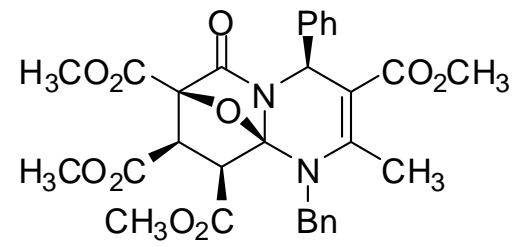

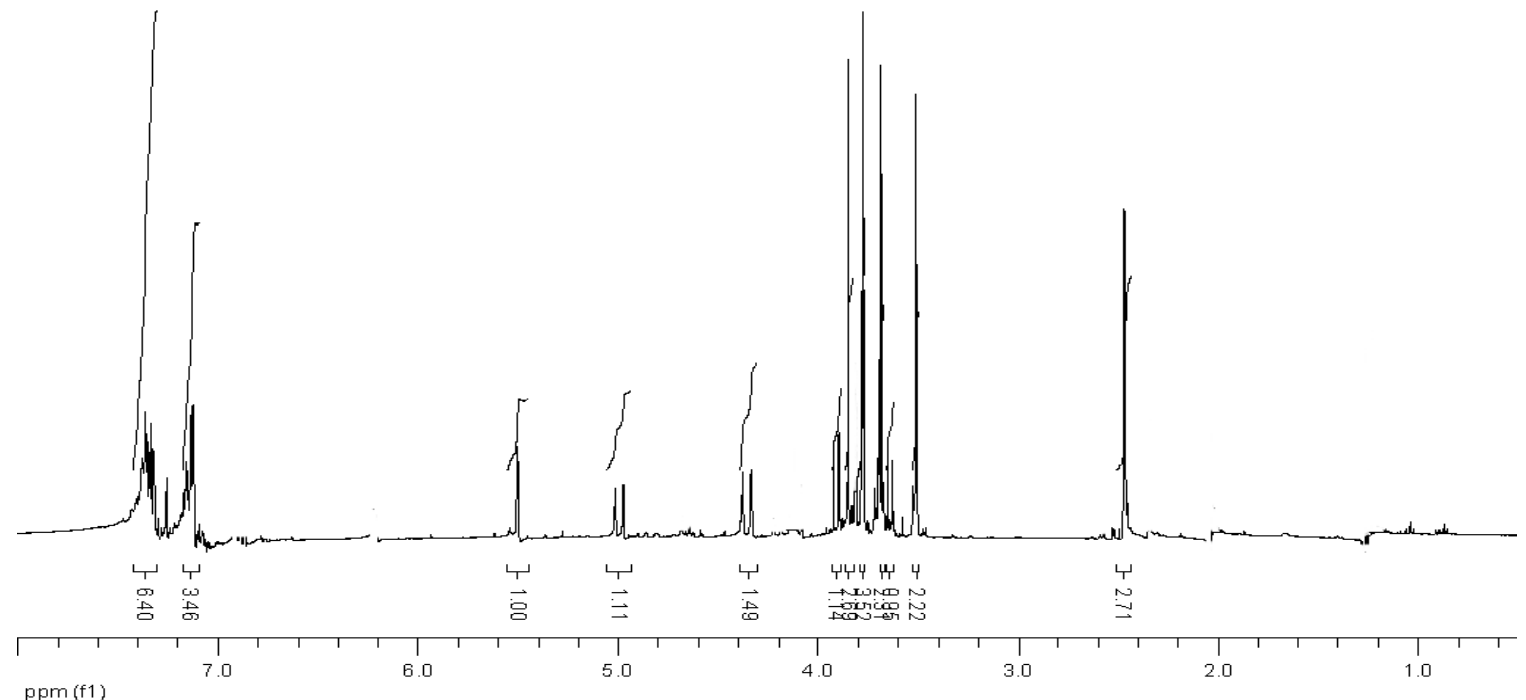

ppm (f1)

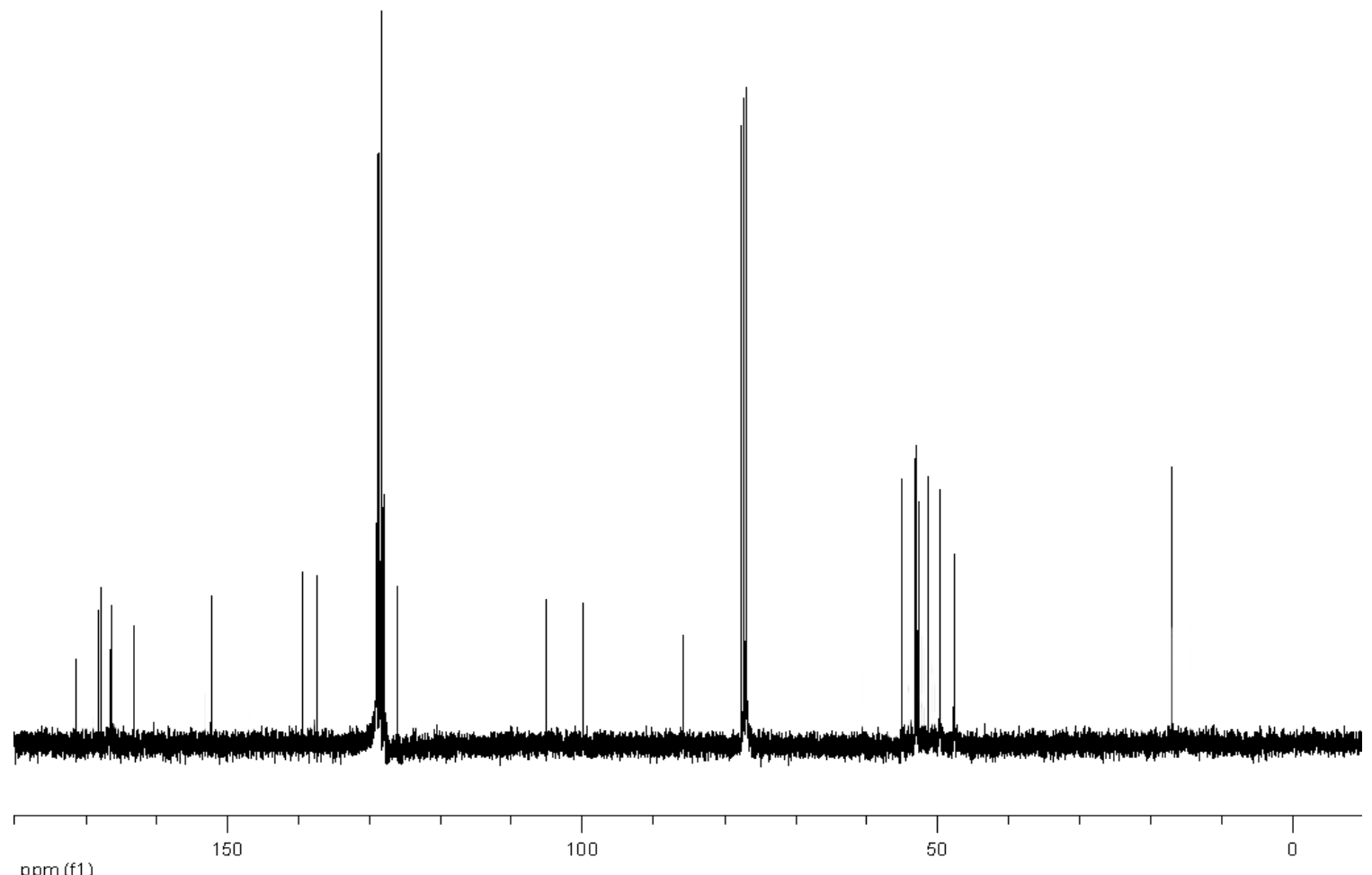


<smiles></smiles>
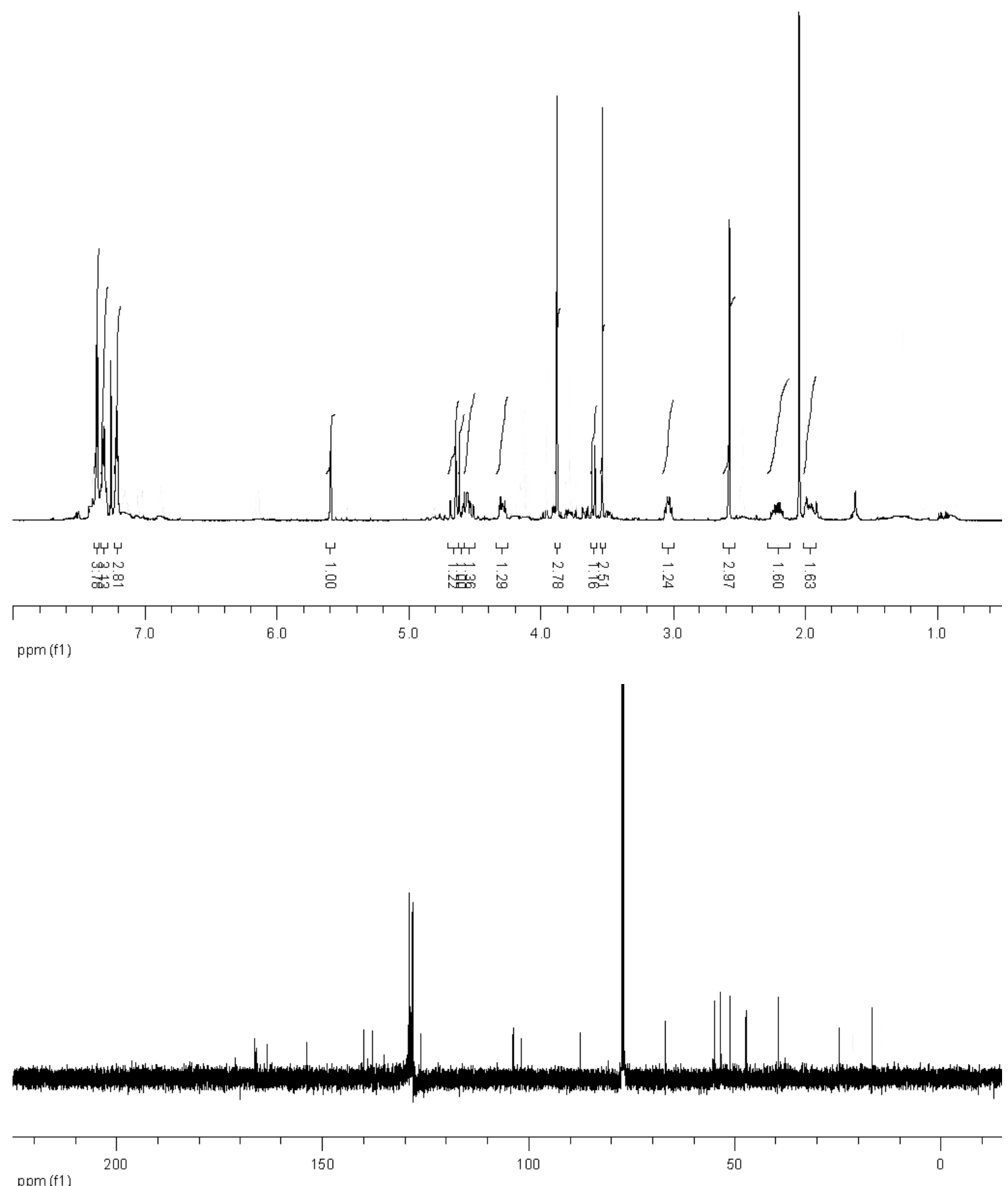
<smiles>CC(=O)C1=C(C)N(Cc2ccccc2)C2=C3C(=O)CCC[C@H]3[C@@](O)(C(C)=O)C(=O)N2[C@H]1c1ccccc1</smiles>
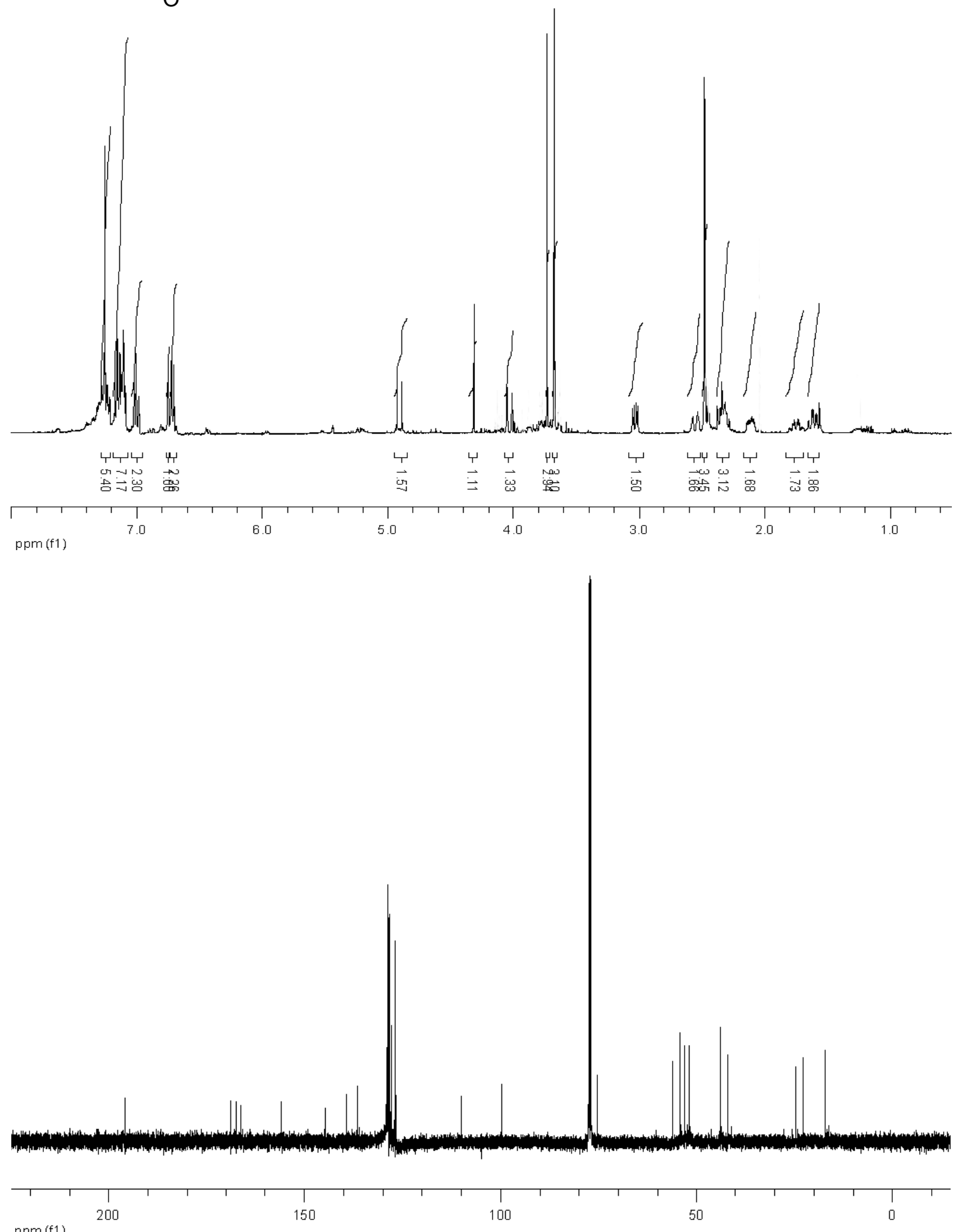

ppm (f1) 


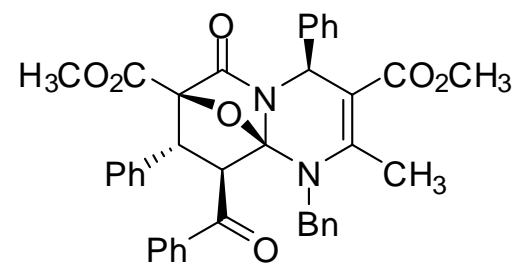
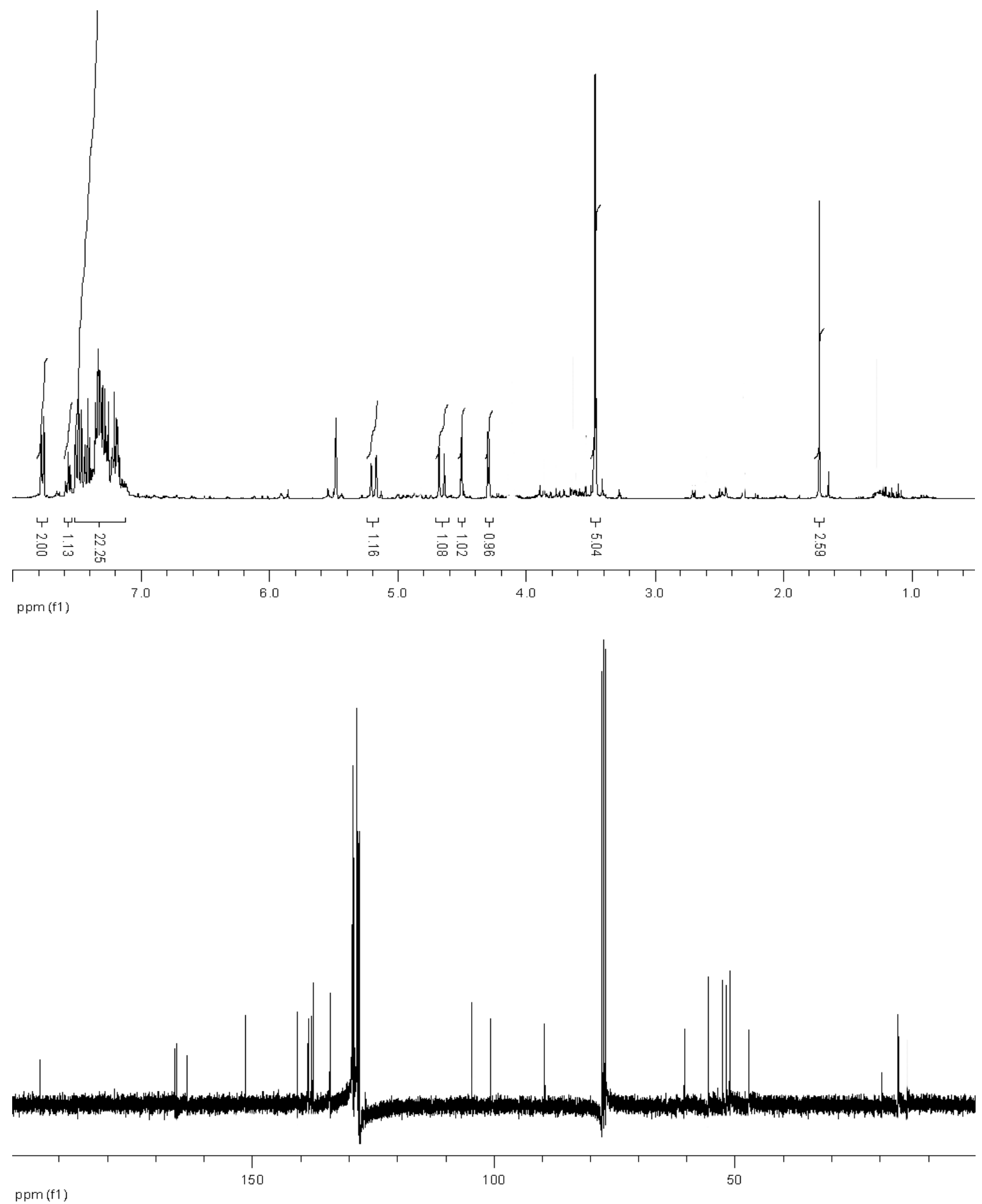
<smiles></smiles>
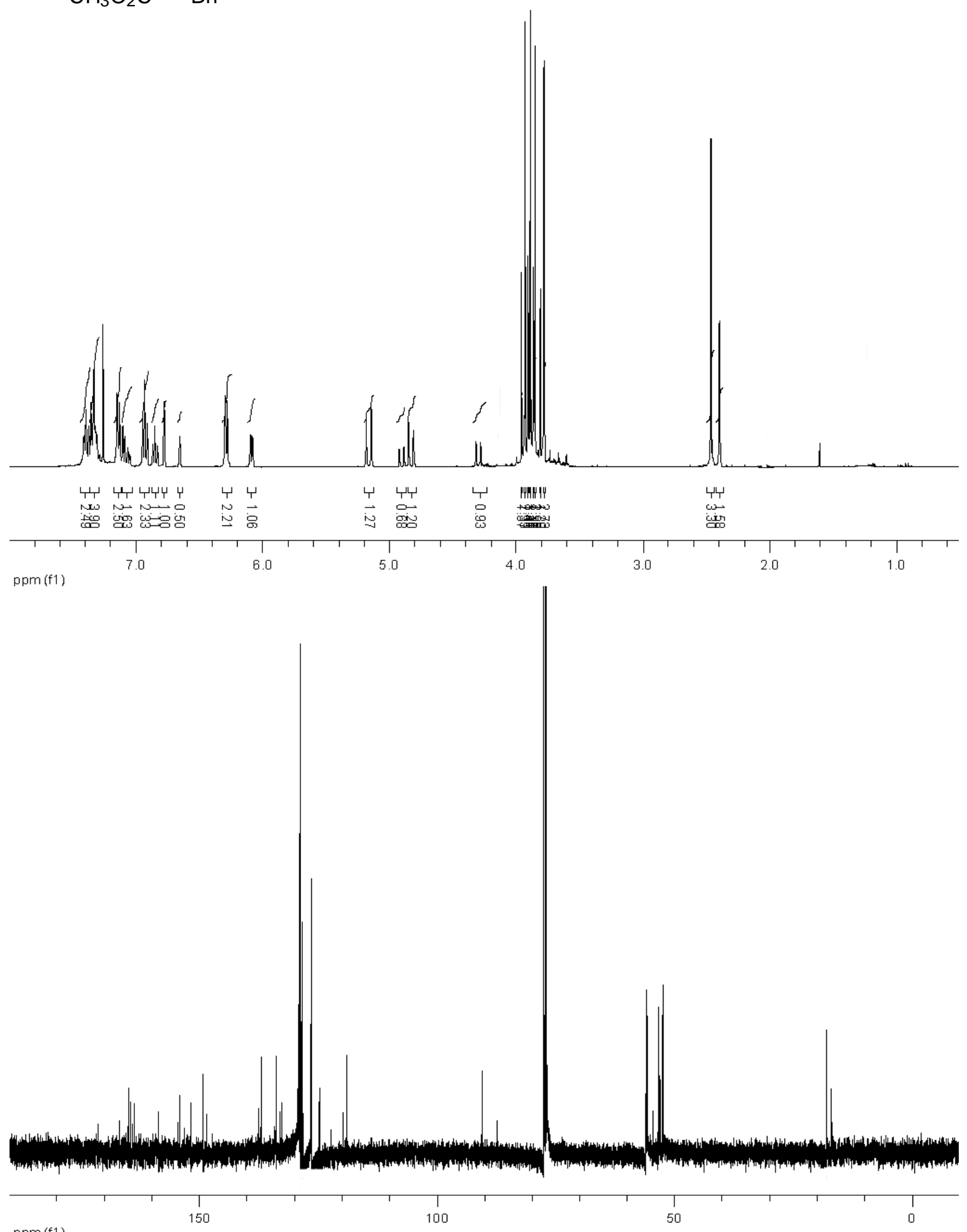
<smiles>COC(=O)C1=C(C)N(Cc2ccccc2)C2=C(C#N)C[C@](O)(C(=O)OC)C(=O)N2C1c1ccccc1</smiles>
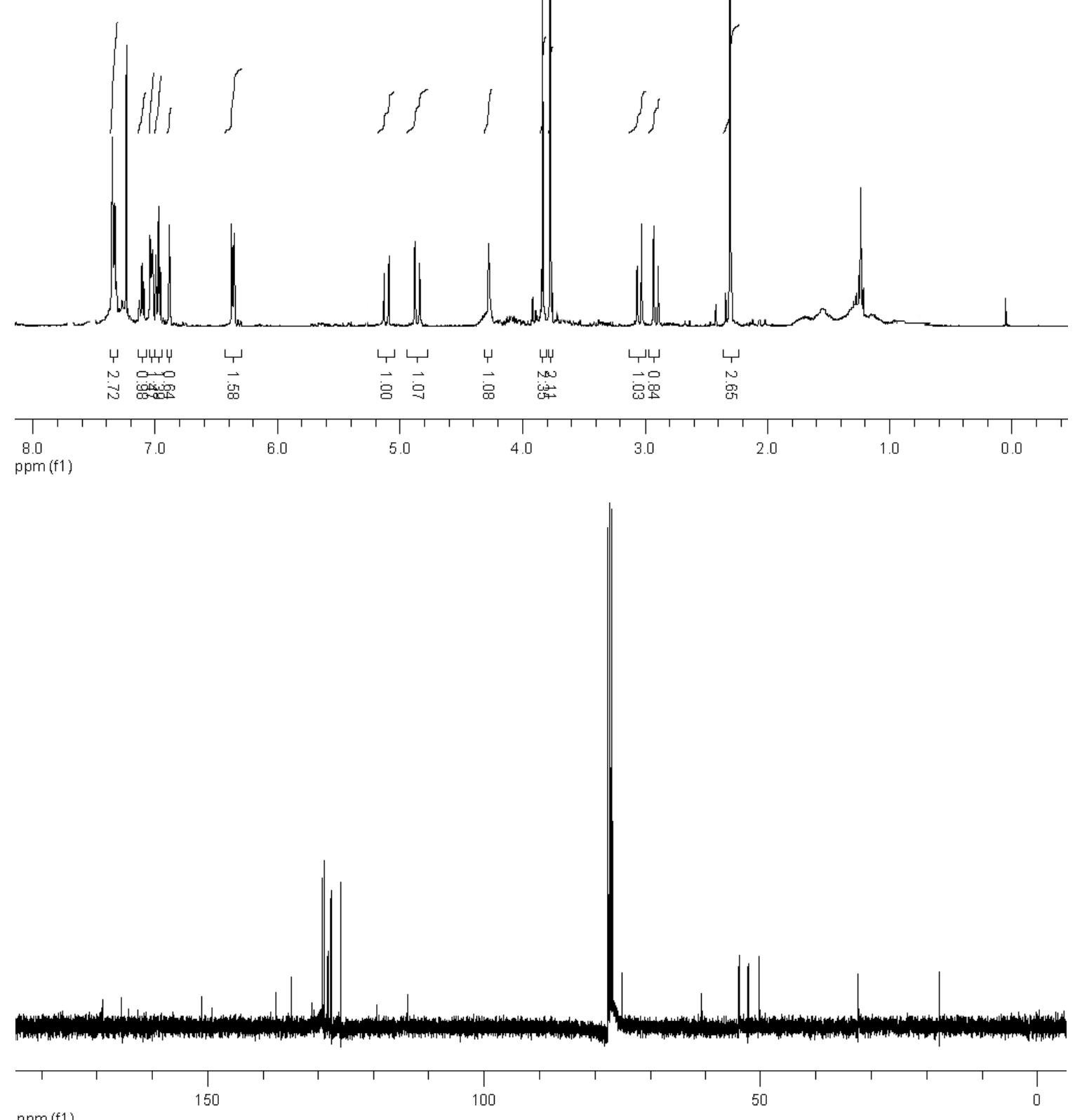

ppm (f1) 

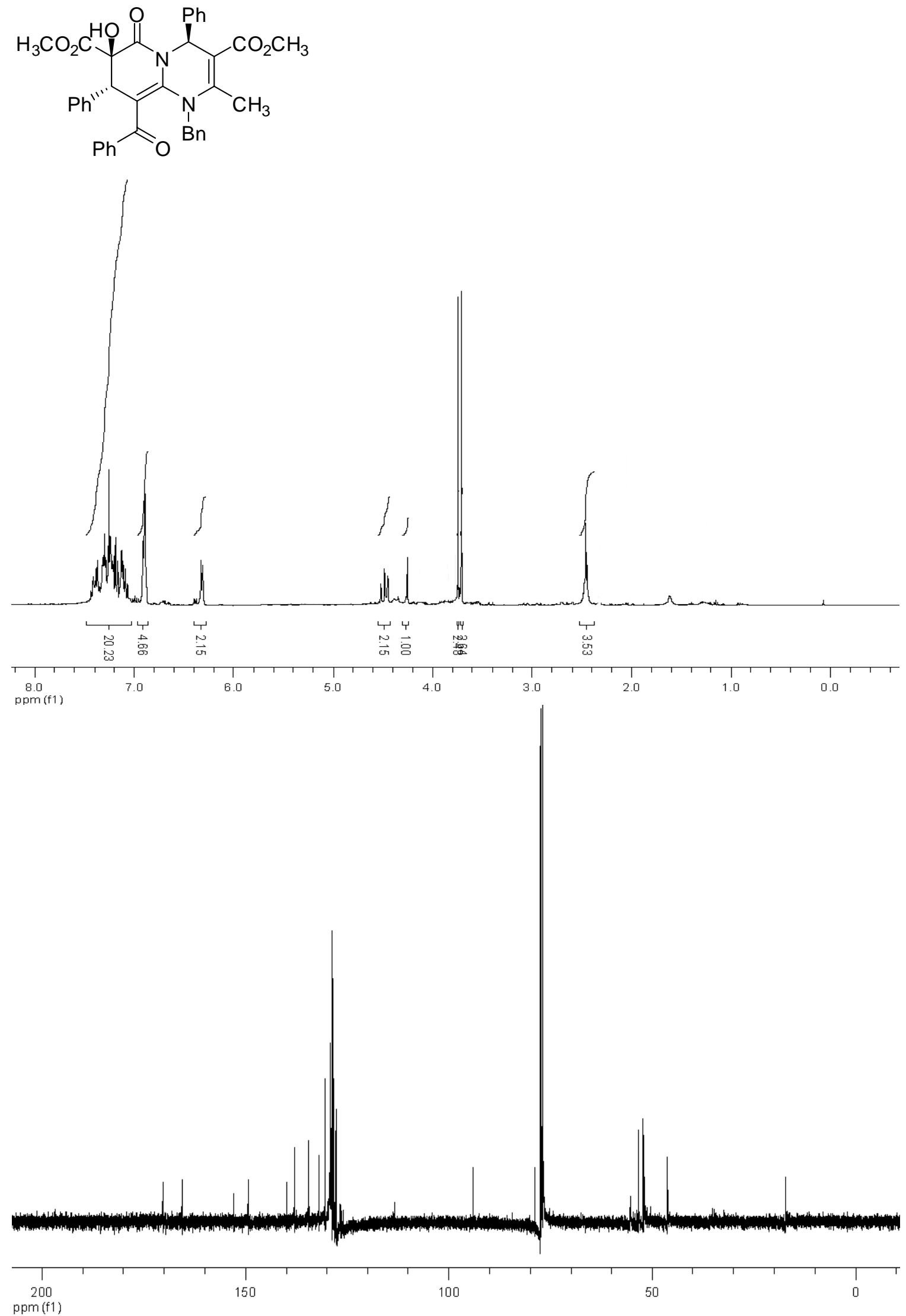

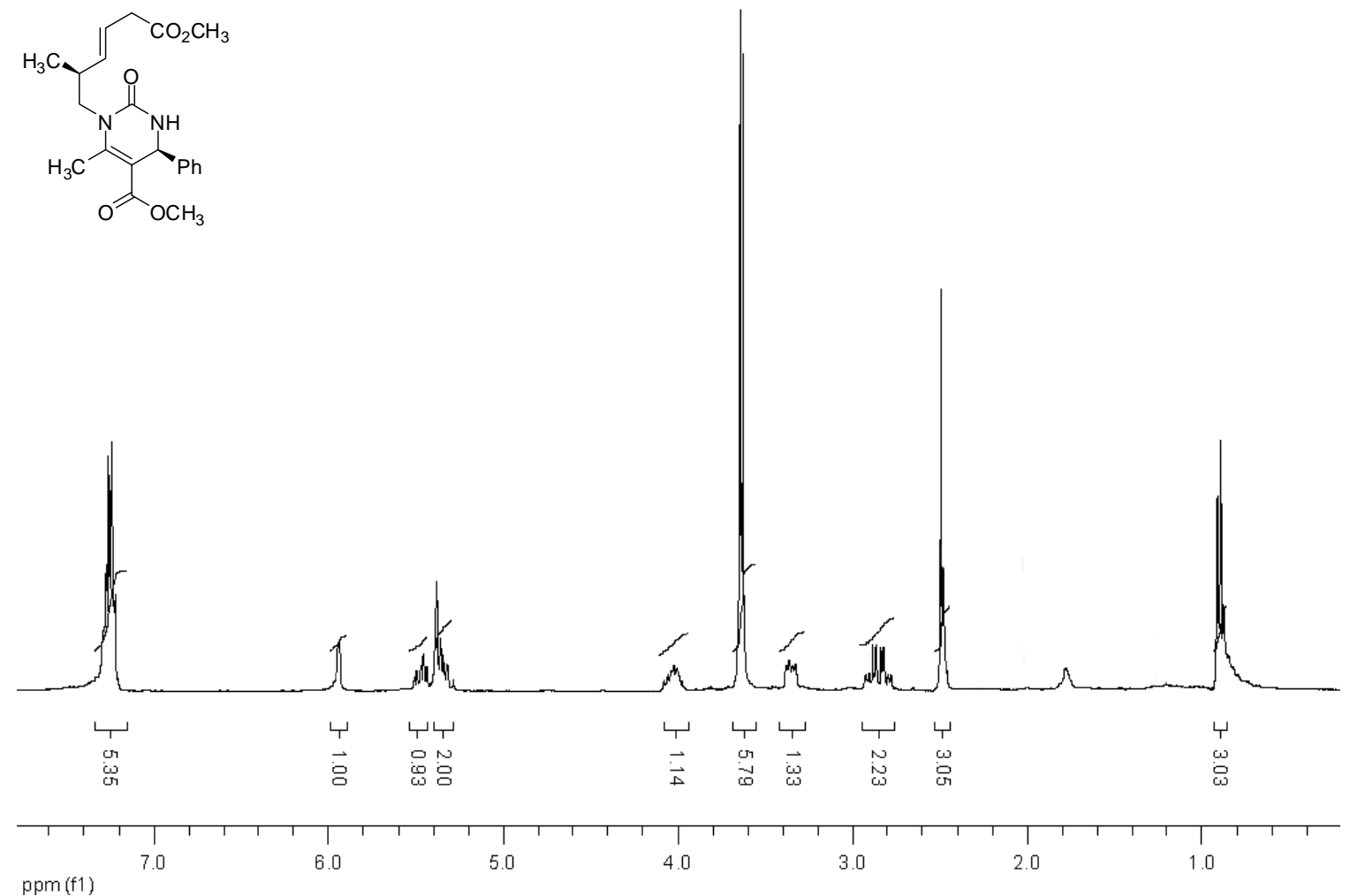

ppm (f1)

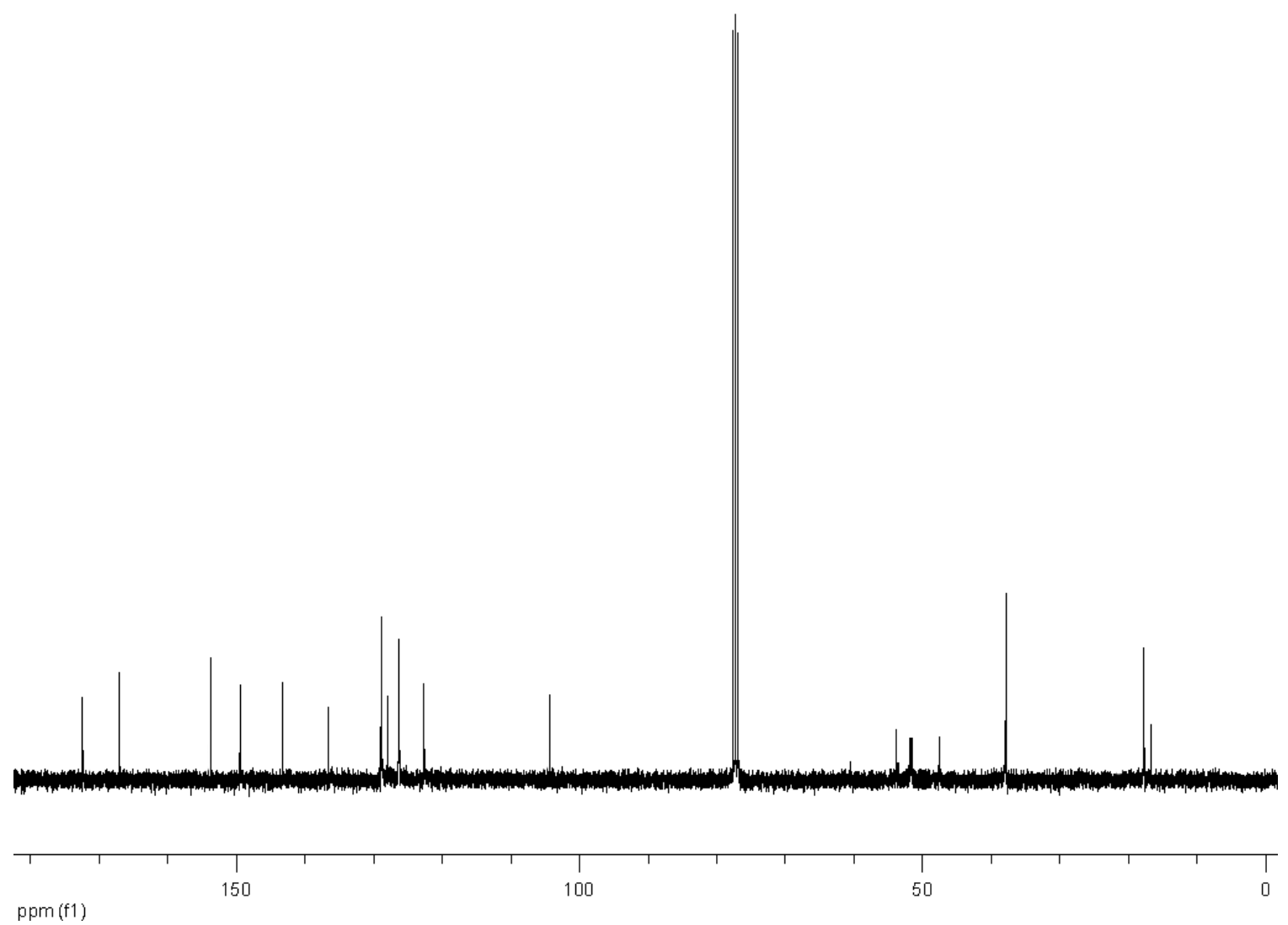



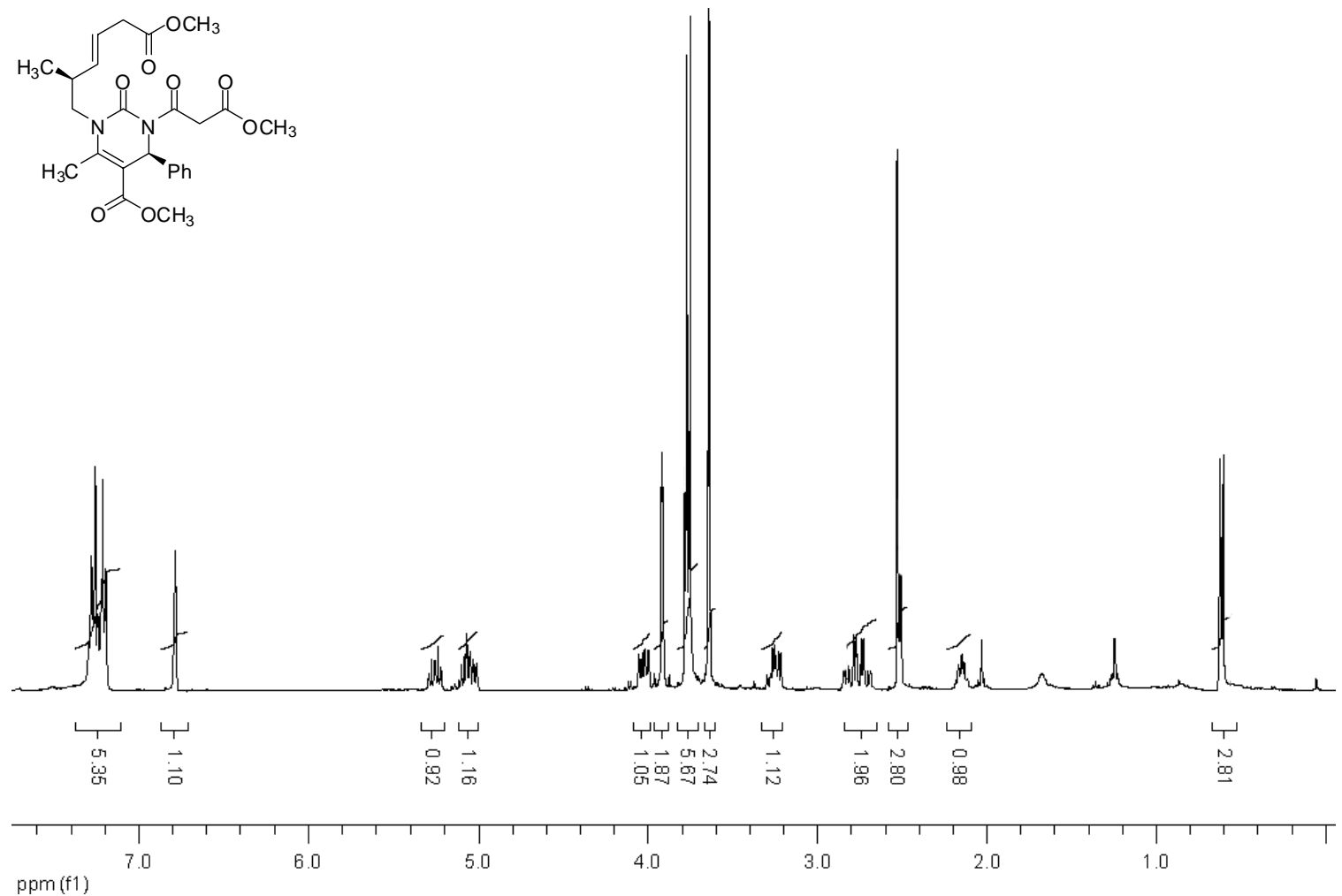

ppm (f

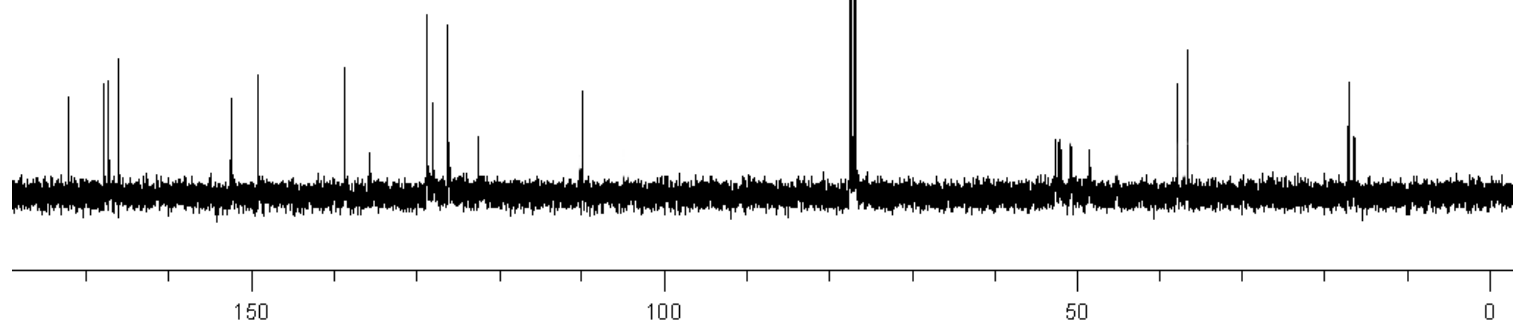
ppm (f1) 


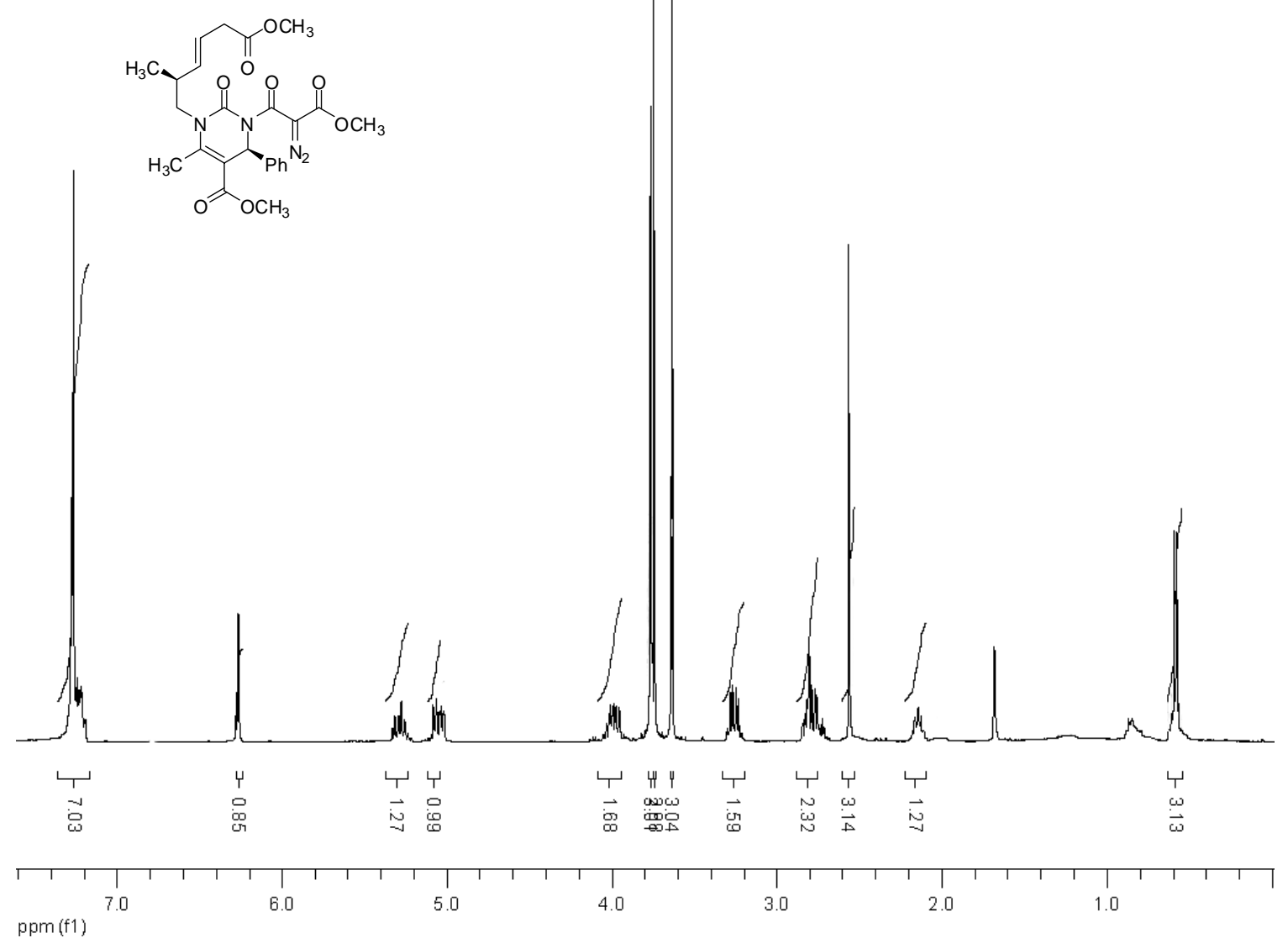
ppm (f1)

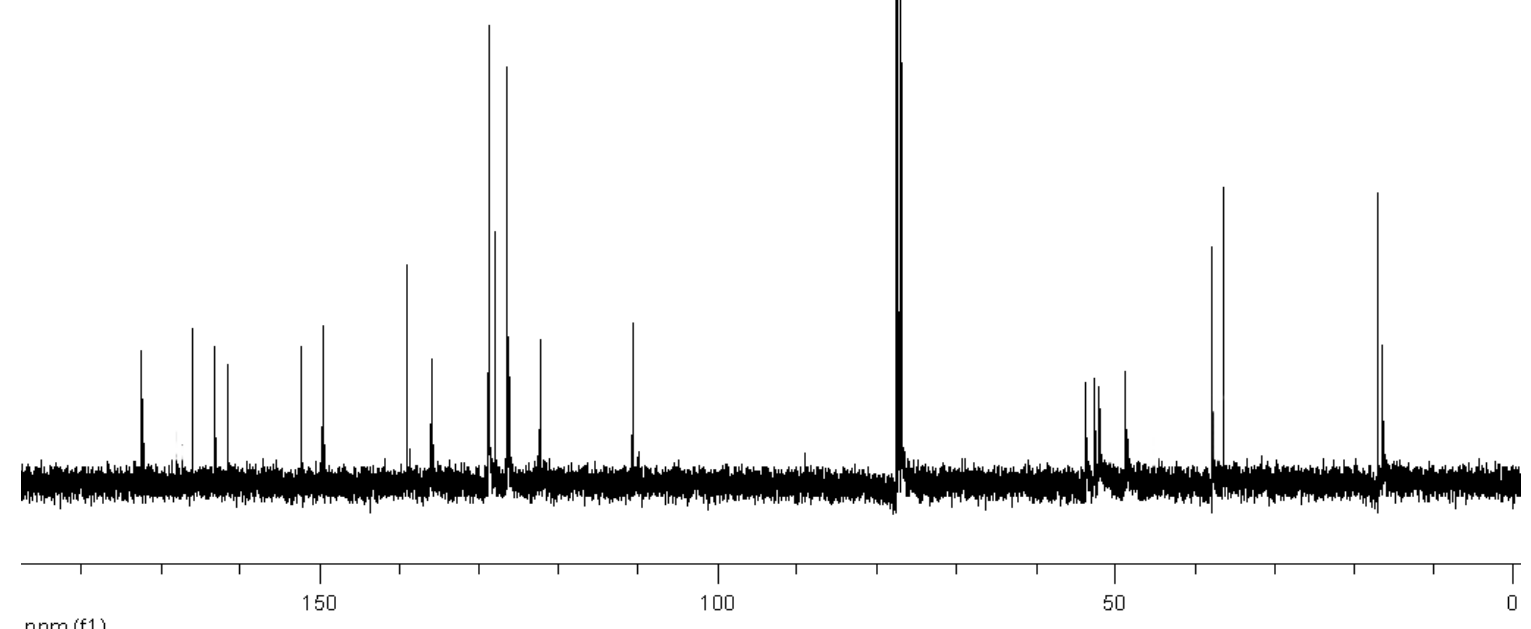

ppm (f1) 

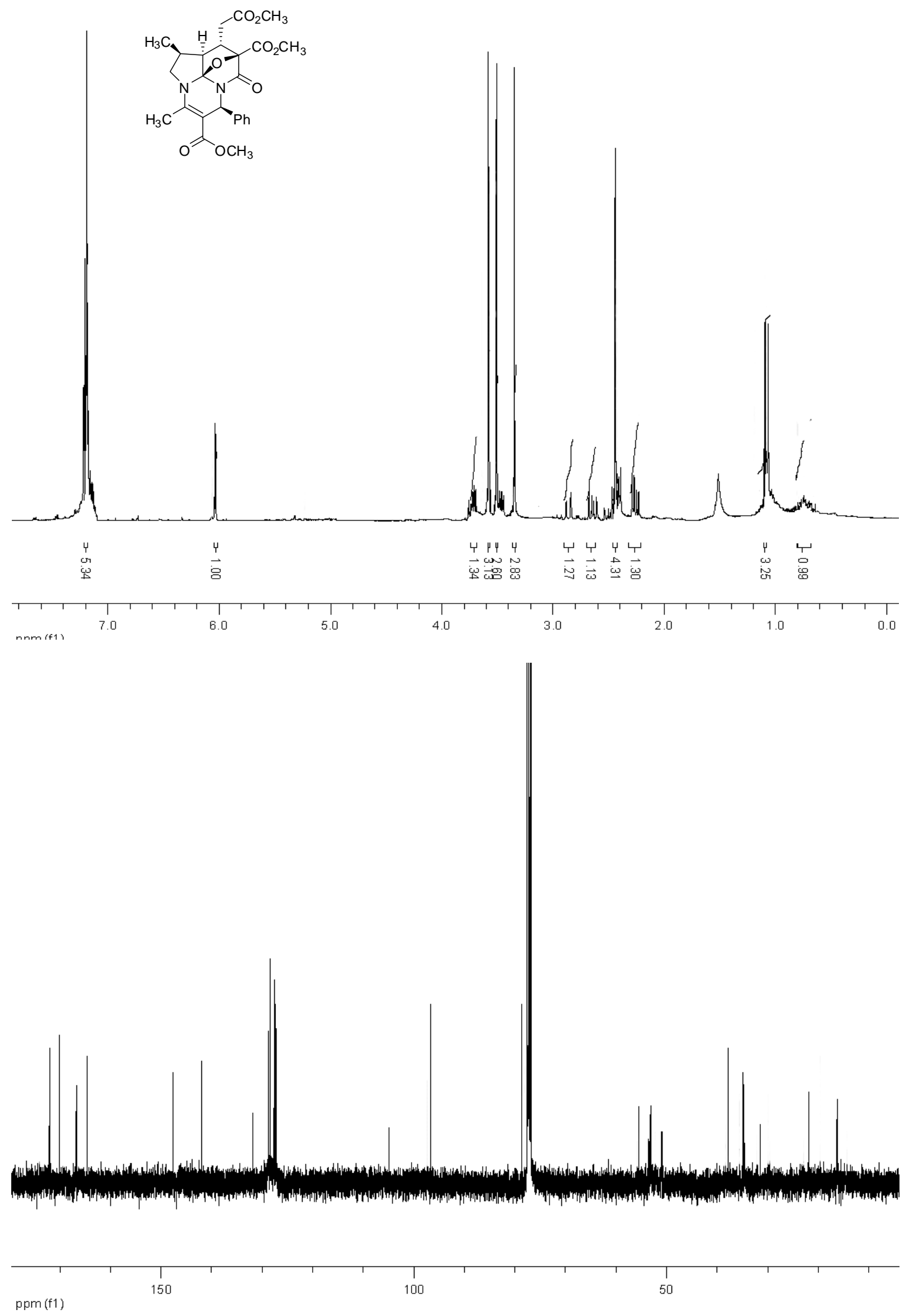

ppm (f1) 
Table 2, entry 1: 8a Chiralcel ${ }^{\circledR}$ OD-H Column, Hexane:IPA = 98:2, $1.0 \mathrm{~mL} / \mathrm{min}, 210 \mathrm{~nm}$

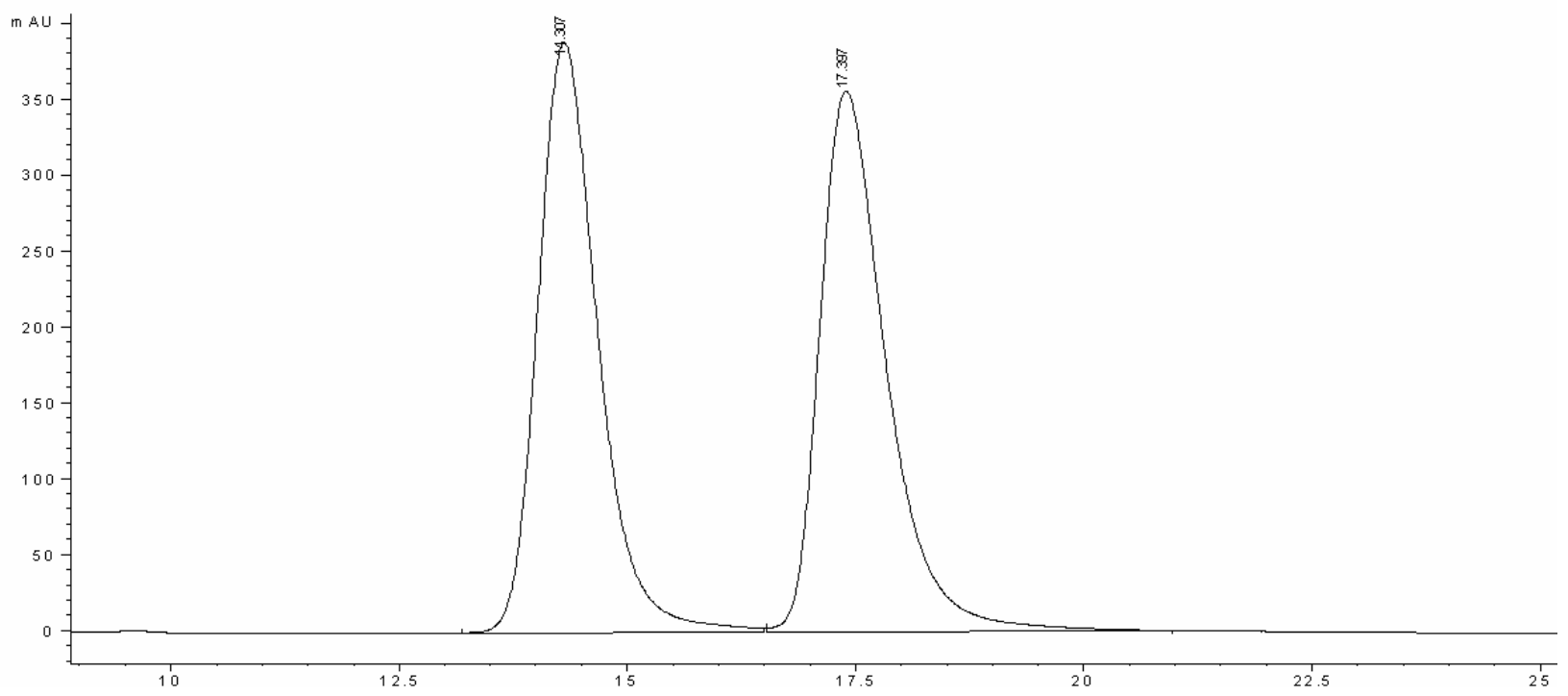

Signal 3: MWD1 C, Sig=210,8 Ref $=360,100$

Peak RetTime Type Width Area Height Area \# [min] [min] [mAU*s] [mAU]

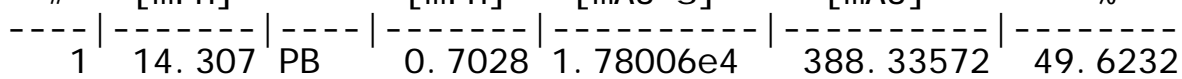

$\begin{array}{lllllll}1 & 14.307 & \text { PB } & 0.7028 & 1.78006 \mathrm{e} 4 & 388.33572 & 49.6232 \\ 2 & 17.397 & \text { B B } & 0.7685 & 1.80710 \mathrm{e} 4 & 355.84894 & 50.3768\end{array}$

Totals: $\quad 3.58716 \mathrm{e} 4 \quad 744.18466$
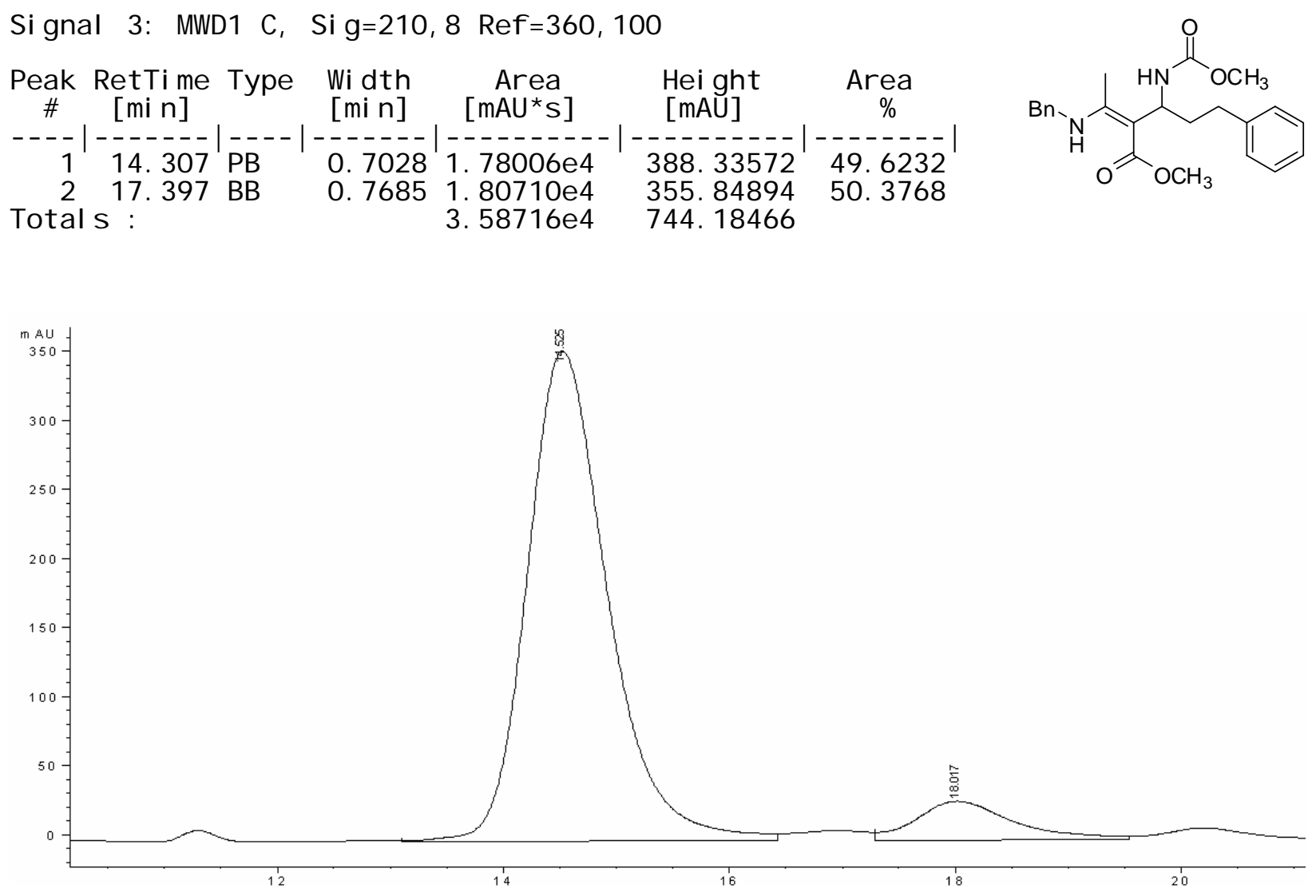

Signal 3: MWD1 C, Sig=210,8 Ref $=360,100$

Peak RetTime Type Width Area Height Area \# $[\mathrm{min}] \quad[\mathrm{min}] \quad\left[\mathrm{mAU}^{*} \mathrm{~s}\right] \quad[\mathrm{mAU}] \quad \%$

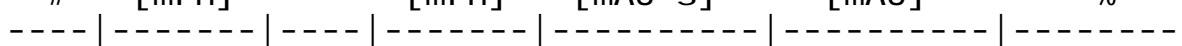
$114.525 \mathrm{VB}$
$0.7573 \quad 1.75369 \mathrm{e} 4 \quad 354.37503$
95.1304

$218.017 \mathrm{MM}$

$\begin{array}{lll}0.7035 & 897.69598 & 21.26670\end{array}$

4.8696

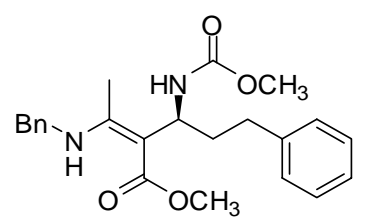

Totals: $1.84346 \mathrm{e} 4 \quad 375.641735$ 
Table 2, entry 2: 8b Chiralcel $^{\circledR}$ OD Column, Hexane:IPA $=98: 2,1.0 \mathrm{~mL} / \mathrm{min}, 210 \mathrm{~nm}$

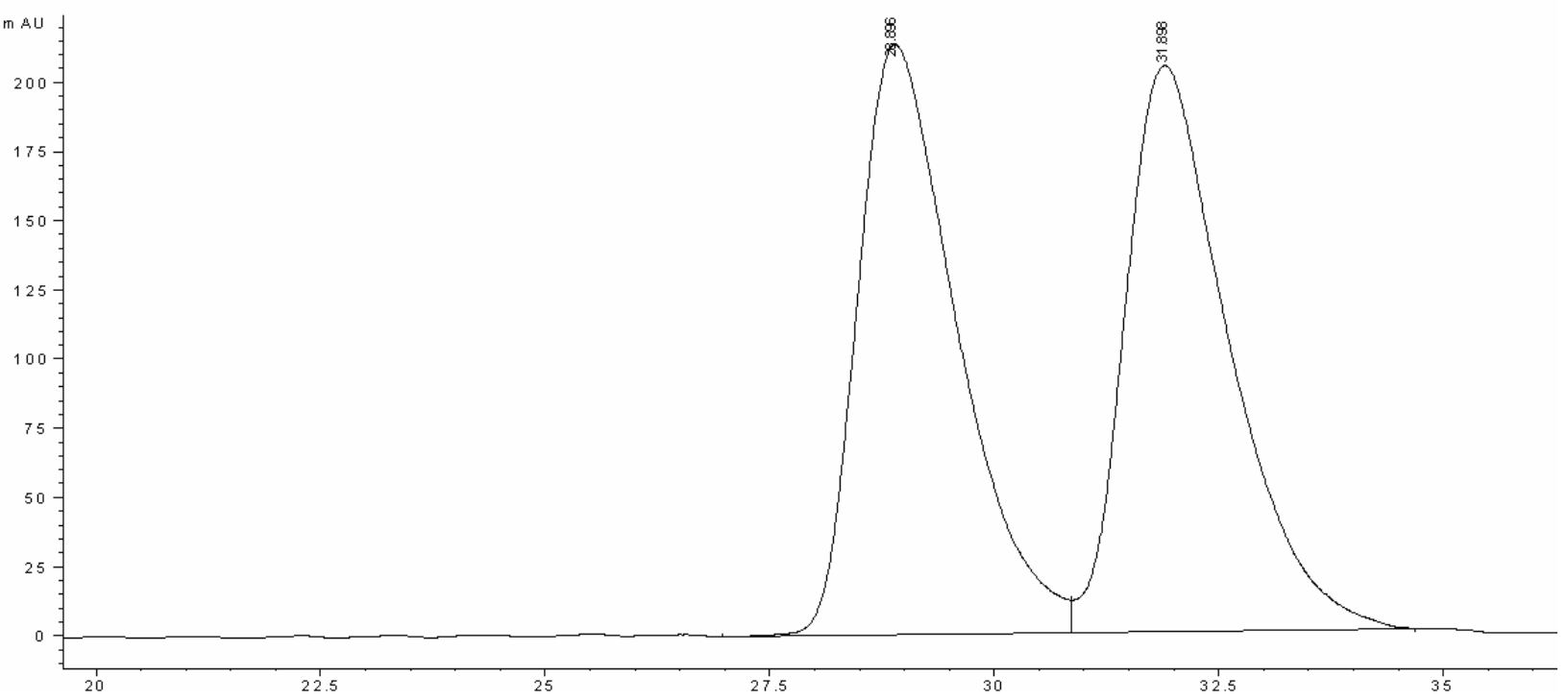

Signal 3: MWD1 C, Si $g=210,8$ Ref $=360,100$

Peak RetTime Type Width Area Height Area \# $[\mathrm{min}] \quad[\mathrm{min}] \quad\left[\mathrm{mAU}^{*} \mathrm{~s}\right] \quad[\mathrm{mAU}] \quad \ldots$

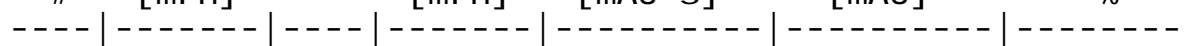

$128.896 \mathrm{VV} \quad 1.18251 .64025 \mathrm{e} 4 \quad 213.46295 \quad 49.9663$

$2 \quad 31.898 \mathrm{VB} \quad 1.18461 .64246 \mathrm{e} 4 \quad 204.59958 \quad 50.0337$

Totals :

3. 28270 e4 418.06253
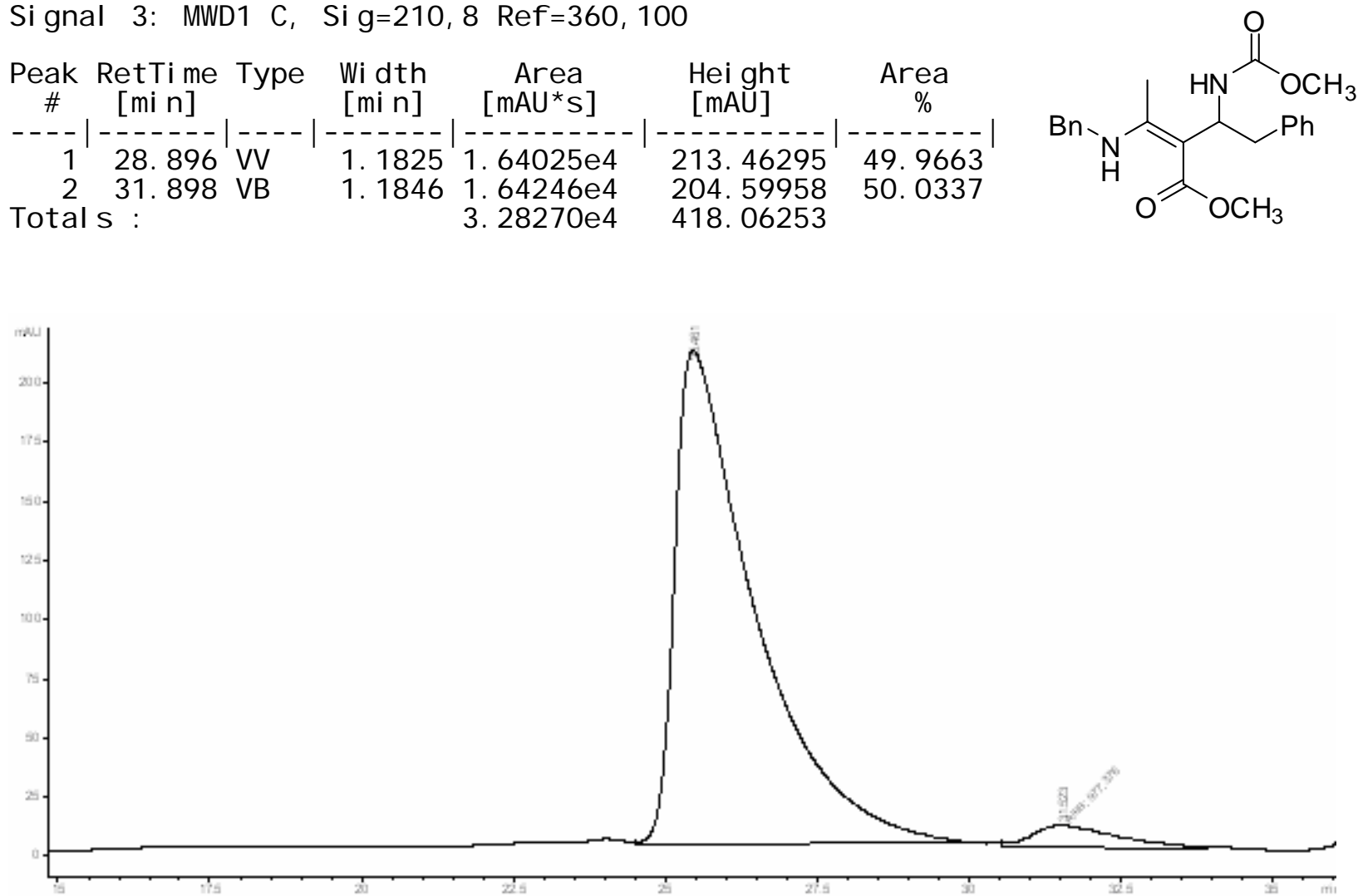

Signal 3: MWD1 C, Si $g=210,8$ Ref $=360,100$

Peak RetTime Type Width Area Height Area

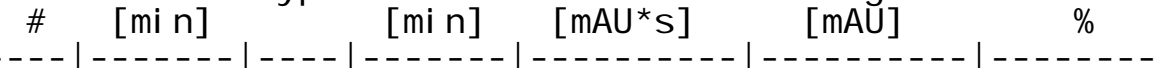

$\begin{array}{lllllll}1 & 25.461 & \mathrm{VB} & 1.2892 & 1.92477 \mathrm{e} & 208.84921 & 95.1675\end{array}$

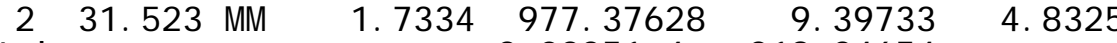

Totals:

2. 02251 e4 218.24654<smiles>COC(=O)N[C@@H](Cc1ccccc1)C(C(=O)OC)=C(C)NCc1ccccc1</smiles> 
Table 2, entry 3: 8c Chiralcel OD Column, Hexane:IPA = 99:1, $1.0 \mathrm{~mL} / \mathrm{min}, 210 \mathrm{~nm}$

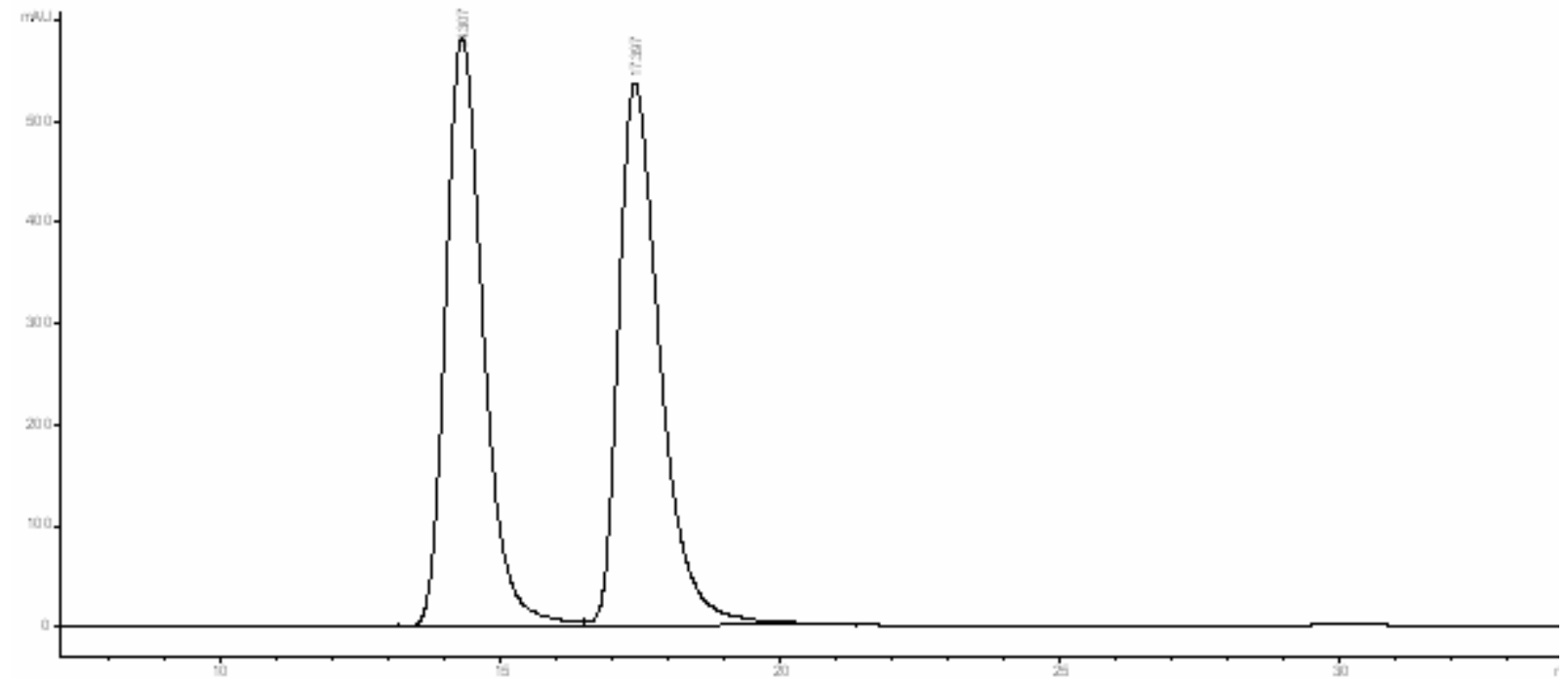

Signal 4: MWD1 D, Si $g=230,16$ Ref $=360,100$
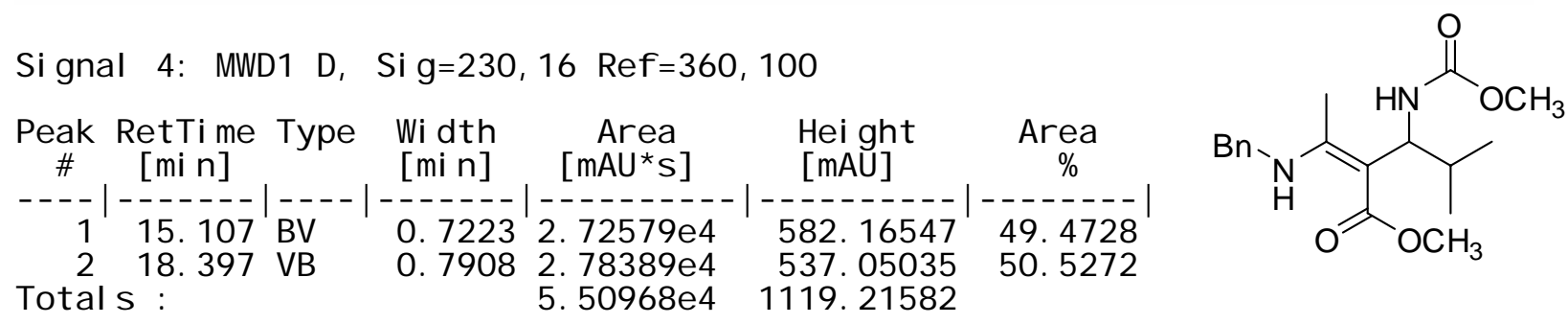

Totals:
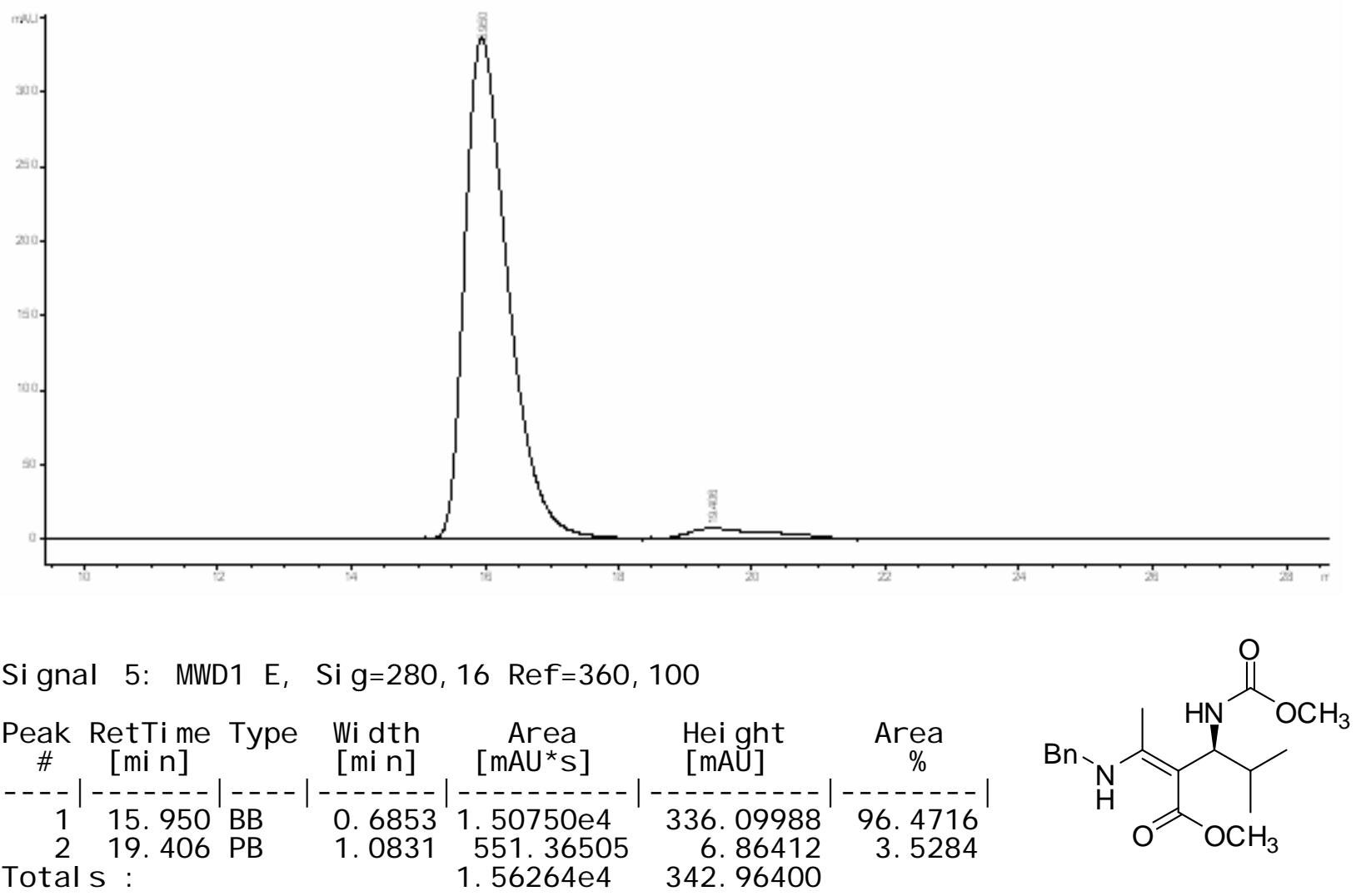
Table 2, entry 4: 8d Chiralcel OD Column, Hexane:IPA = 95:5, $1.0 \mathrm{~mL} / \mathrm{min}, 210 \mathrm{~nm}$

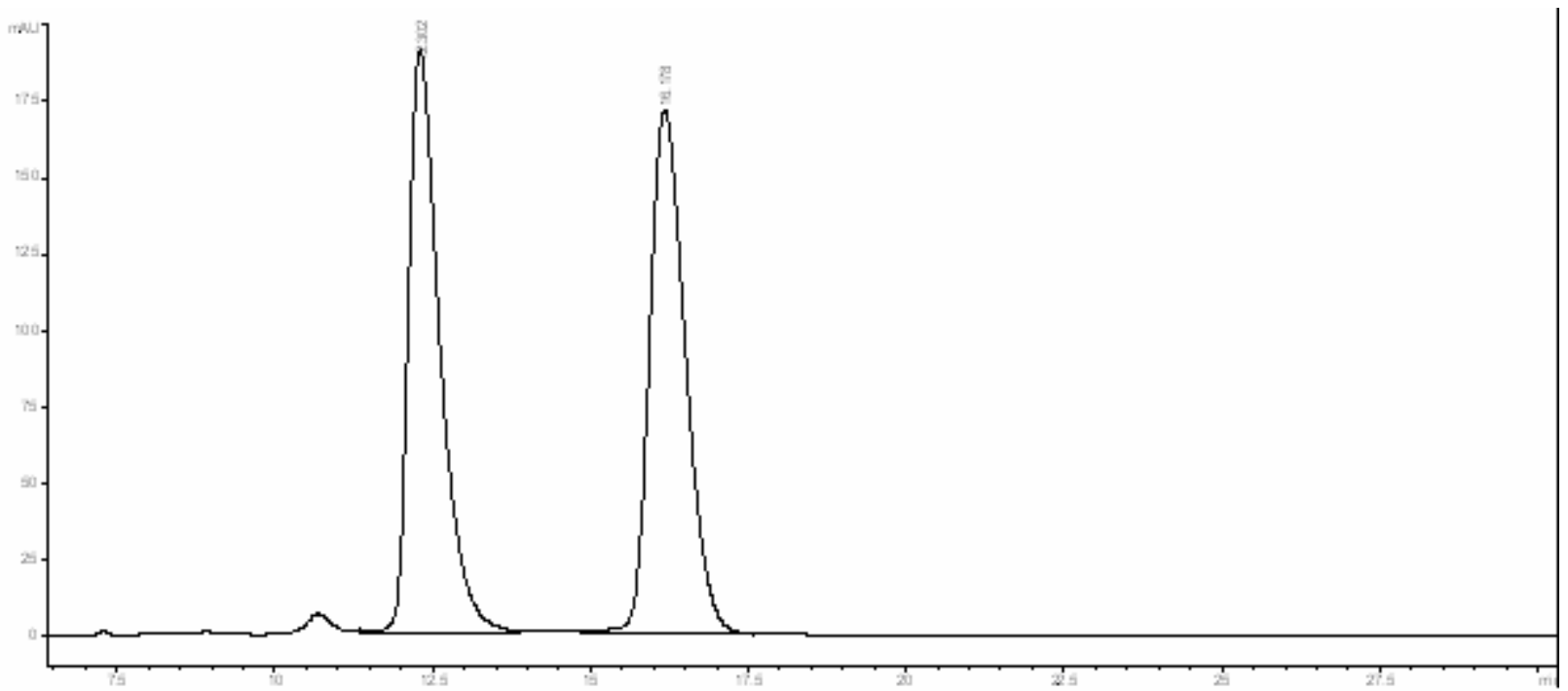

Signal 1: MWD1 A, Sig=250,100 Ref $=360,100$

Peak RetTime Type Width Area Height Area

\# $[\mathrm{min}] \quad[\mathrm{min}] \quad\left[\mathrm{mAU}^{*} \mathrm{~s}\right] \quad[\mathrm{mAU}] \quad \%$

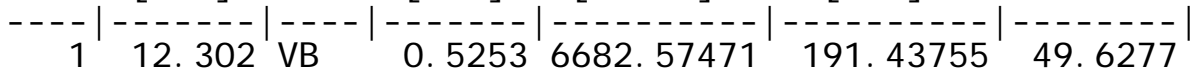

$\begin{array}{lllllll}2 & 16.178 & V B & 0.61176782 .82910 & 171.39934 & 50.3723\end{array}$

Totals:<smiles>COC(=O)NC(C(C)=C(C)NCc1ccccc1)C1CCCCC1</smiles>

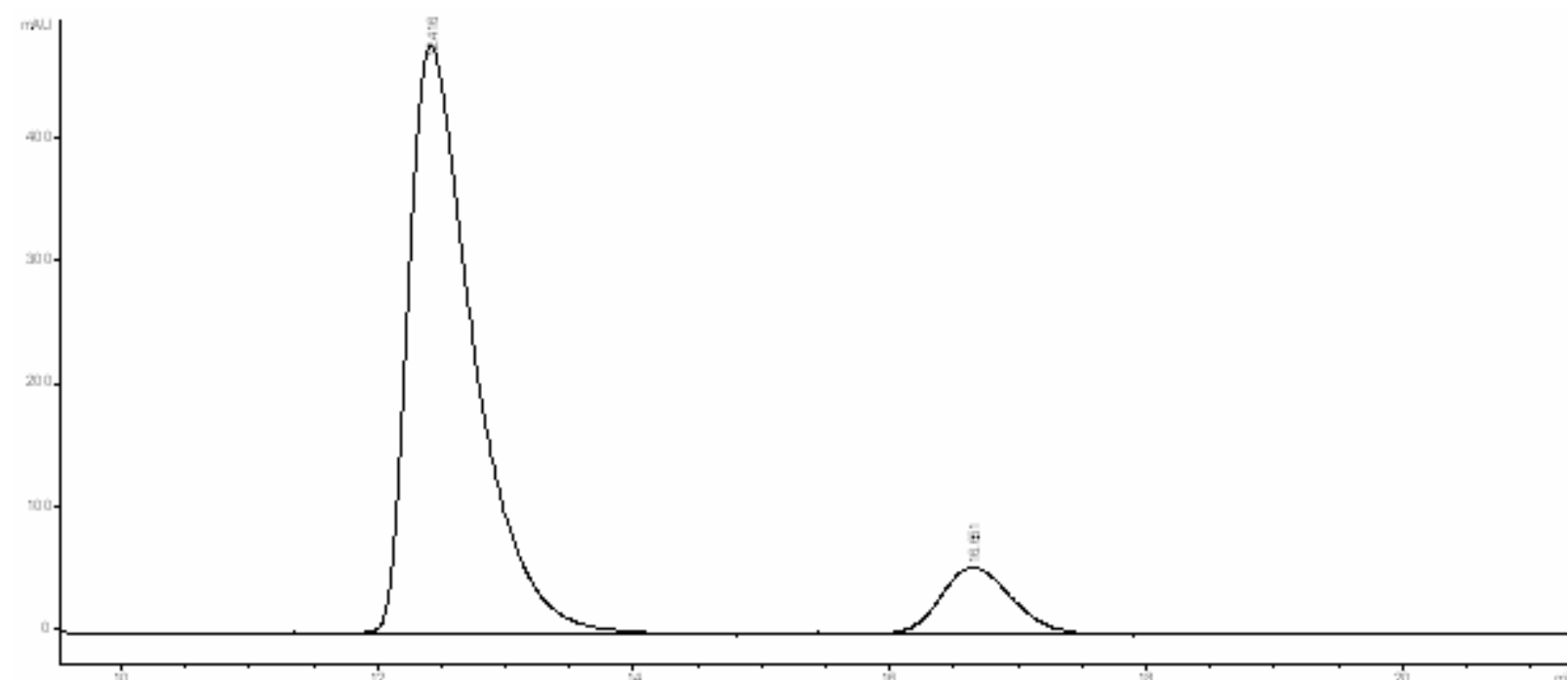

Signal 5: MWD1 E, Sig=280,16 Ref $=360,100$

\begin{tabular}{|c|c|c|c|c|c|c|}
\hline Peak & $\begin{array}{l}\text { Ret Ti me } \\
{[\mathrm{mi} n]}\end{array}$ & Type & $\begin{array}{l}\text { Wi } d t h \\
{[\mathrm{mi} n]}\end{array}$ & $\begin{array}{c}\operatorname{Area} \\
{\left[\mathrm{mA} U^{*} \mathrm{~s}\right]}\end{array}$ & $\begin{array}{l}\text { Height } \\
{[\mathrm{mAU}]}\end{array}$ & $\begin{array}{c}\operatorname{Ar} \underset{\%}{e} a \\
\%\end{array}$ \\
\hline $\begin{array}{c}\cdots \\
1 \\
2 \\
\text { Tot al }\end{array}$ & $\begin{array}{l}12.416 \\
16.651 \\
s:\end{array}$ & $\begin{array}{l}B B \\
M M\end{array}$ & $\begin{array}{ll}0.5344 \\
0.5344\end{array}$ & $\begin{array}{l}1.70128 \mathrm{e} 4 \\
1486.31702 \\
1.84991 \mathrm{e} 4\end{array}$ & $\begin{array}{r}478.94916 \\
46.35049 \\
525.29965\end{array}$ & $\begin{array}{r}91.9655 \\
8.0345\end{array}$ \\
\hline
\end{tabular}<smiles></smiles> 
Table 2, entry 5: 8e Chiralcel OD Column, Hexane:IPA = 95:5, $1.0 \mathrm{~mL} / \mathrm{min}, 210 \mathrm{~nm}$

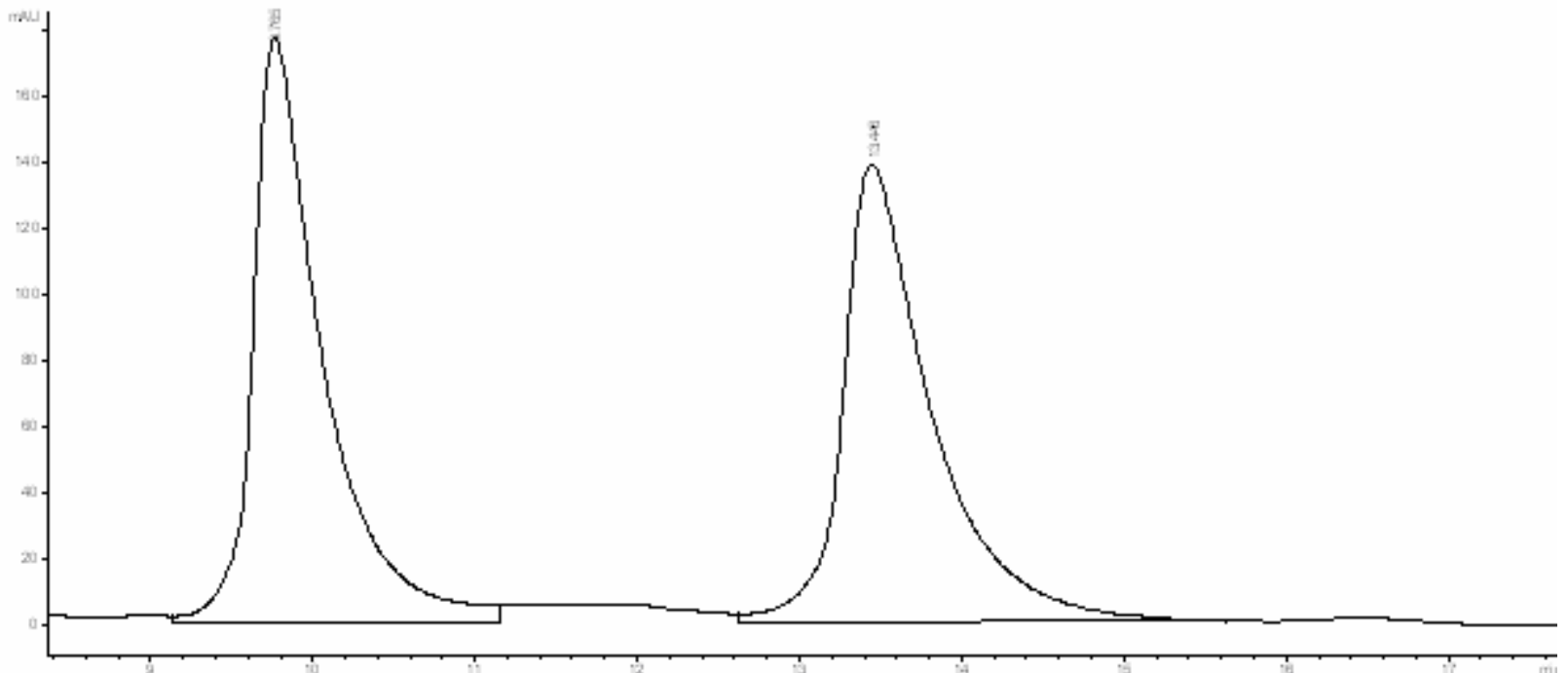

Signal 3: MWD1 C, Sig=210,8 Ref $=360,100$

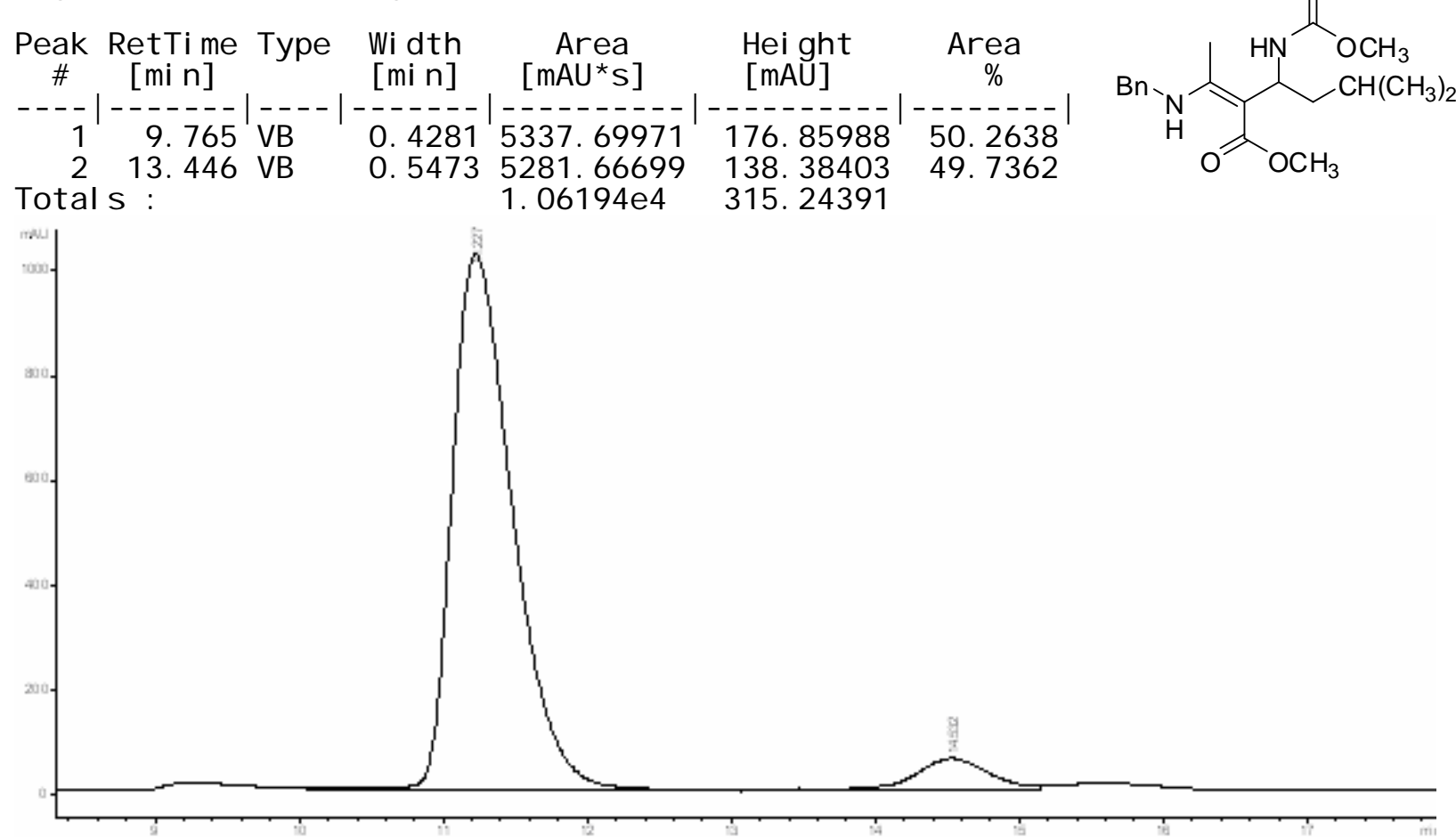

Signal 3: MWD1 C, Sig $=210,8$ Ref $=360,100$

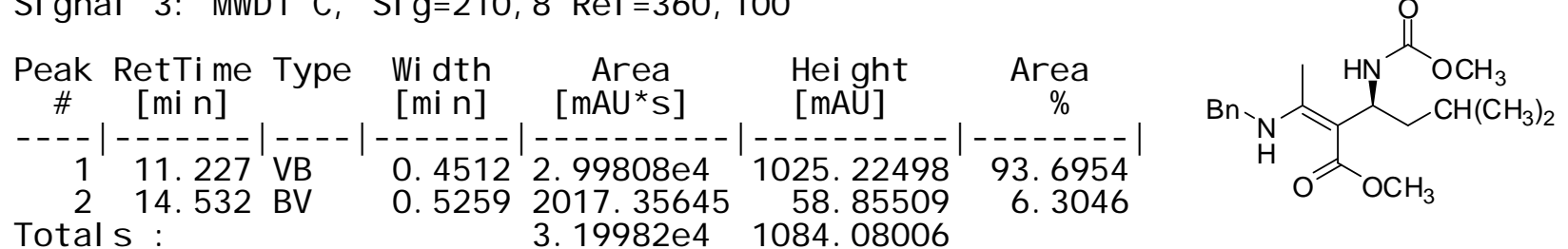


Table 2, entry 6: 8f Chiralcel OD-H Column, Hexane:IPA = 95:5, $1.0 \mathrm{~mL} / \mathrm{min}, 214 \mathrm{~nm}$

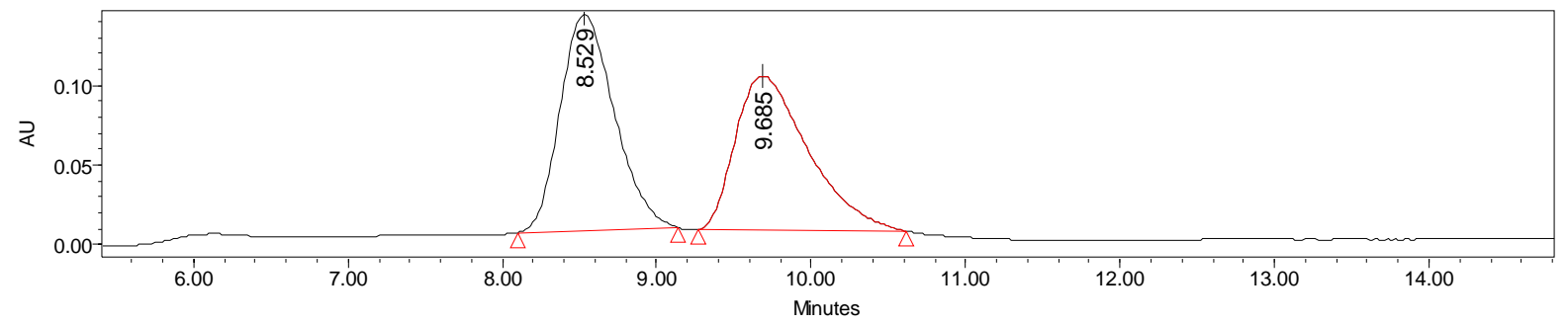

\begin{tabular}{|r|r|r|r|r|r|}
\hline & Retention Time & Area & \% Area & Height & \% Height \\
\hline 1 & 8.529 & 3296168 & 51.05 & 135324 & 58.37 \\
\hline 2 & 9.685 & 3160706 & 48.95 & 96498 & 41.63 \\
\hline
\end{tabular}
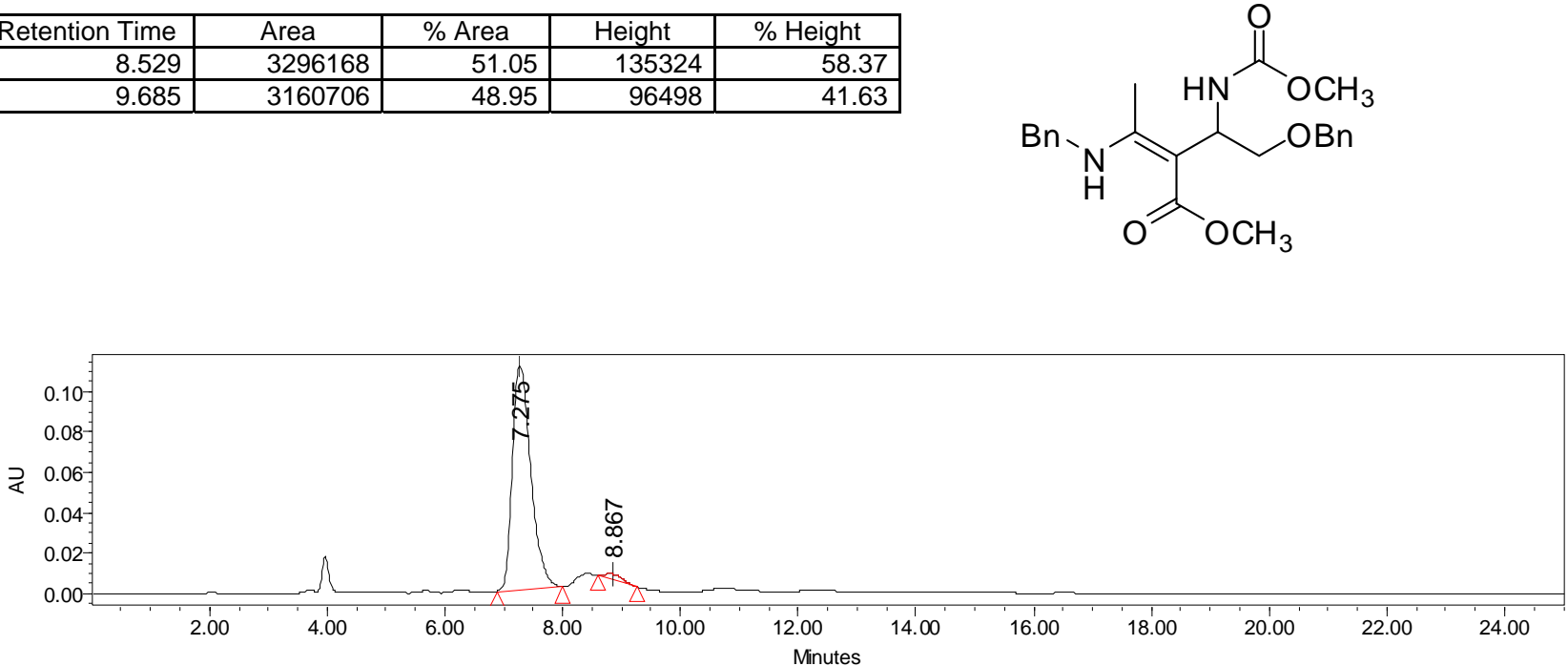

\begin{tabular}{|r|r|r|r|r|r|}
\hline & Retention Time & \multicolumn{1}{c|}{ Area } & \% Area & Height & \% Height \\
\hline 1 & 7.275 & 2416630 & 97.72 & 111002 & 97.54 \\
\hline 2 & 8.867 & 56435 & 2.28 & 2794 & 2.46 \\
\hline
\end{tabular}<smiles>COC(=O)N[C@@H](COCc1ccccc1)/C(C(=O)OC)=C(\C)NCc1ccccc1</smiles> 
Table 2, entry 8: 8h Chiralcel OD Column, Hexane:IPA = 95:5, $1.0 \mathrm{~mL} / \mathrm{min}, 214 \mathrm{~nm}$

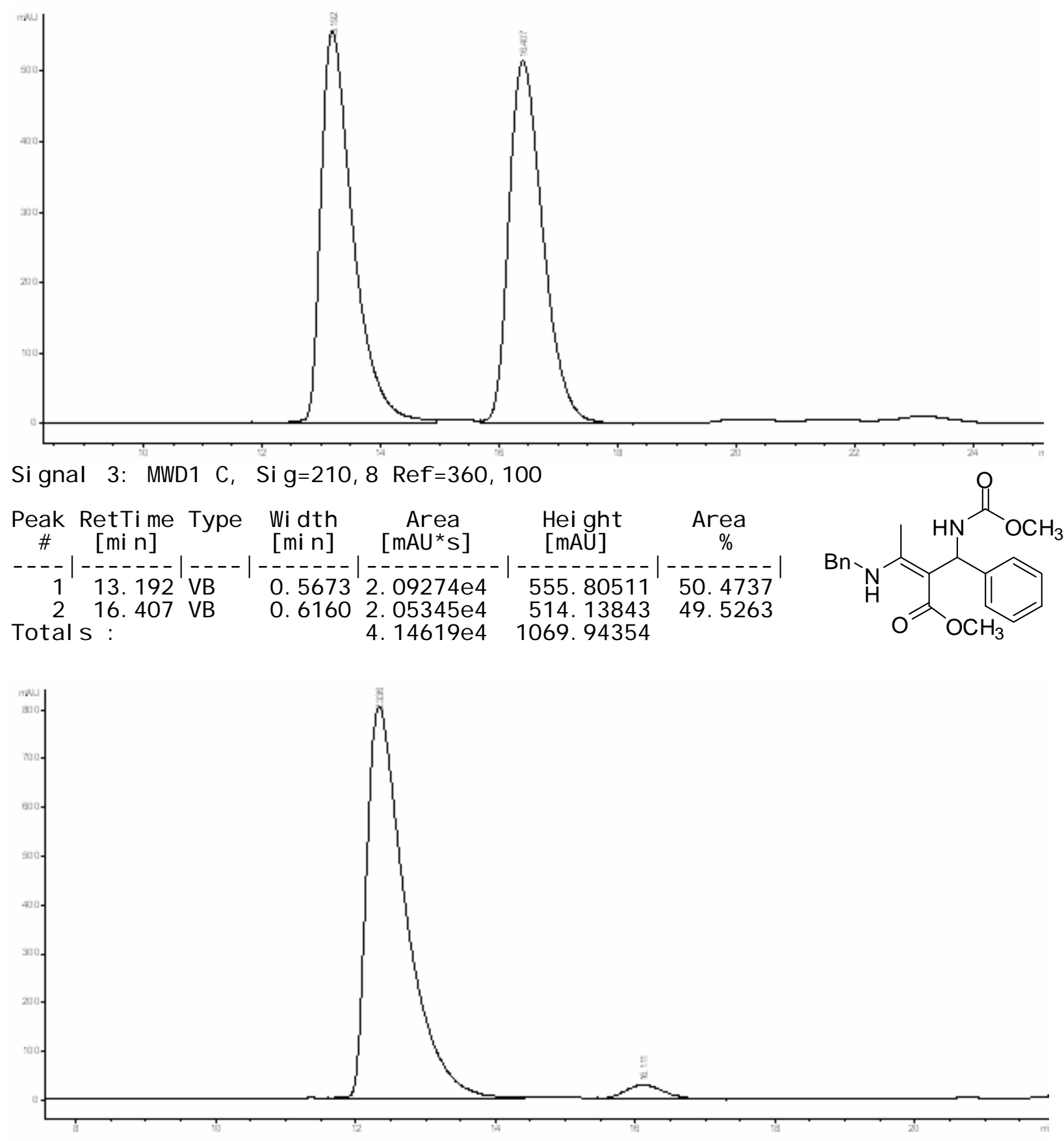

Signal 3: MWD1 C, Sig $=210,8$ Ref $=360,100$

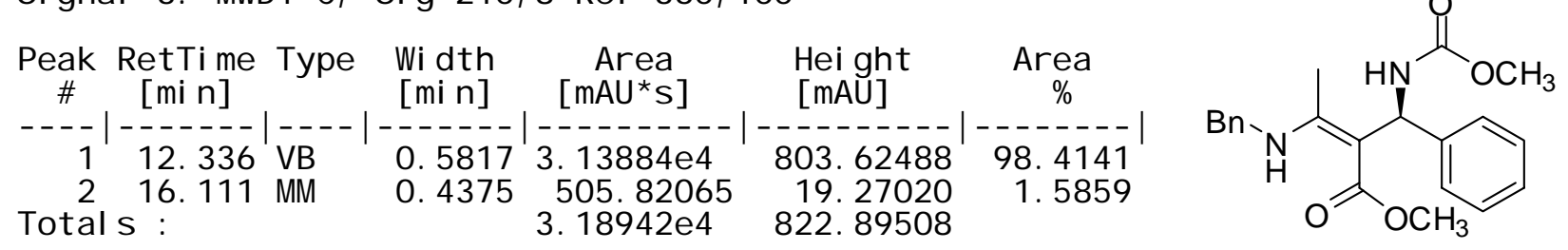


Table 2, entry 9: 8i Chiralcel OD Column, Hexane:IPA = 95:5, $1.0 \mathrm{~mL} / \mathrm{min}, 214 \mathrm{~nm}$

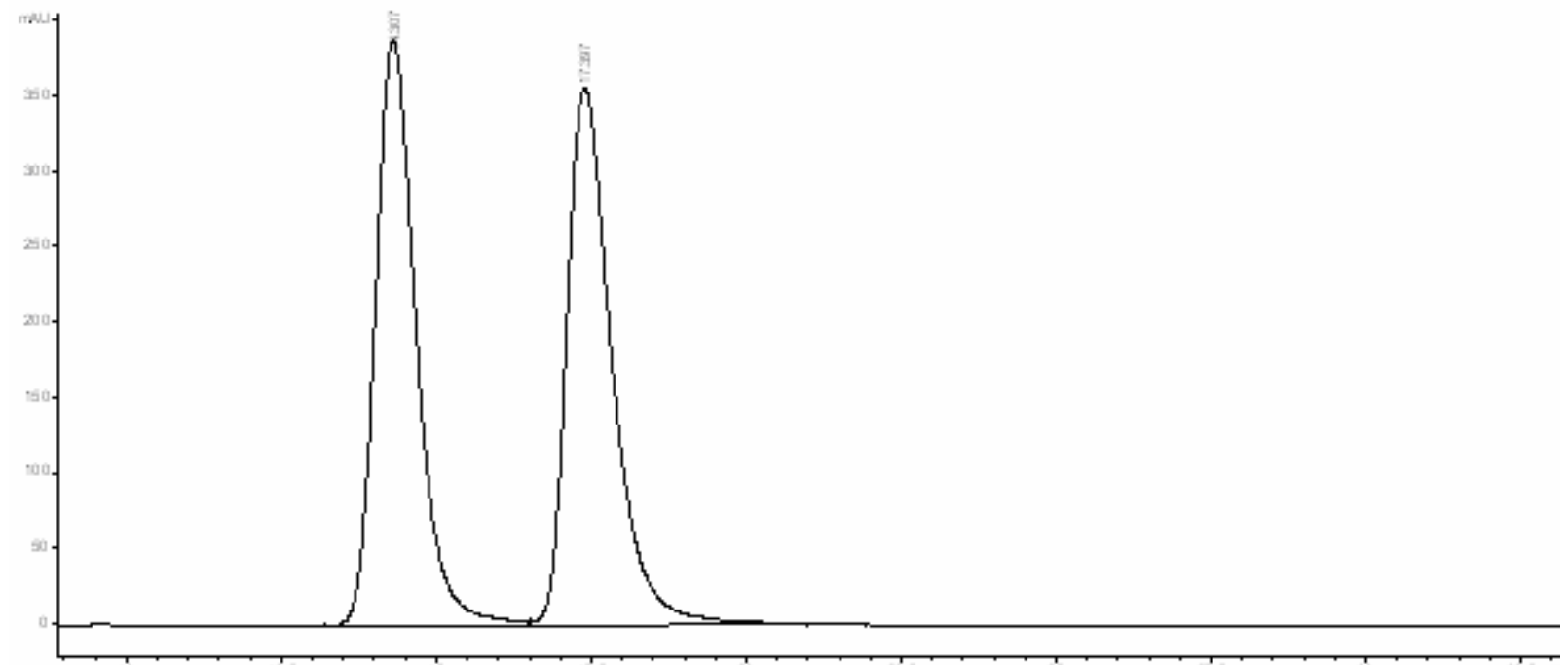

Signal 3: MWD1 C, Sig=210,8 Ref $=360,100$

Peak RetTime Type Width Area Height Area

\# $[\mathrm{min}] \quad[\mathrm{min}] \quad\left[\mathrm{mAU}^{*} \mathrm{~s}\right] \quad[\mathrm{mAU}] \quad \%$

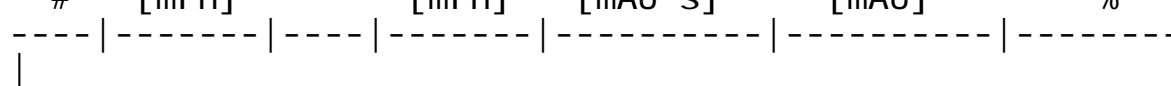

\begin{tabular}{|c|c|c|c|}
\hline $\begin{array}{llll}1 & 14.307 & \mathrm{~PB} \\
2 & 17.397 & \mathrm{BB} \\
\mathrm{a} / \mathrm{s} & : & \end{array}$ & $\begin{array}{l}0.7028 \\
0.7685\end{array}$ & $\begin{array}{l}1.78006 \mathrm{e} 4 \\
1.80710 \mathrm{e} 4 \\
3.58716 \mathrm{e} 4\end{array}$ & $\begin{array}{l}388.33572 \\
355.84894 \\
744.18466\end{array}$ \\
\hline
\end{tabular}<smiles>COC(=O)NC(C(C)=C(C)NCc1ccccc1)c1ccc(Br)cc1</smiles>

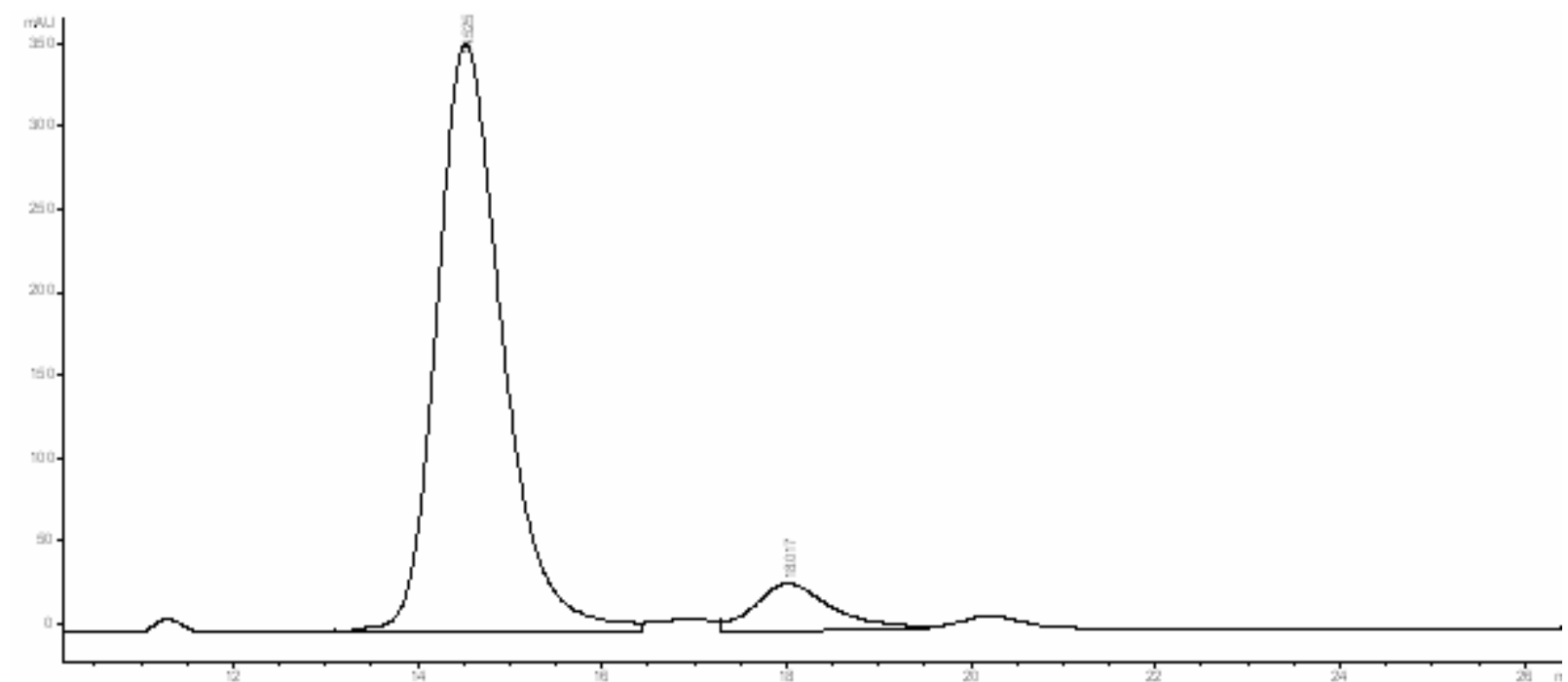

Signal 3: MWD1 C, Sig=210,8 Ref $=360,100$

Peak RetTime Type Width Area Height Area

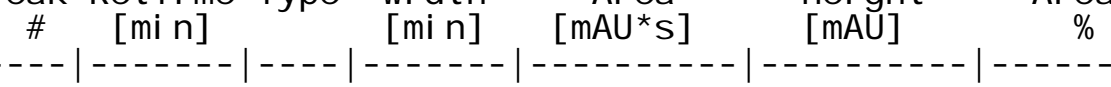
|

$114.525 \mathrm{VB}$

$218.017 \mathrm{MM}$

$0.7573 \quad 1.75369 e 4 \quad 354.37503$

95.1304 Totals:

$0.7035 \quad 897.69598 \quad 21.26670$

4.8696<smiles>COC(=O)N[C@@H](C(C(=O)OC)=C(C)NCc1ccccc1)c1ccc(Br)cc1</smiles> 
Table 2, entry 10: 8j Chiralcel OD Column, Hexane:IPA = 95:5, $1.0 \mathrm{~mL} / \mathrm{min}, 214 \mathrm{~nm}$

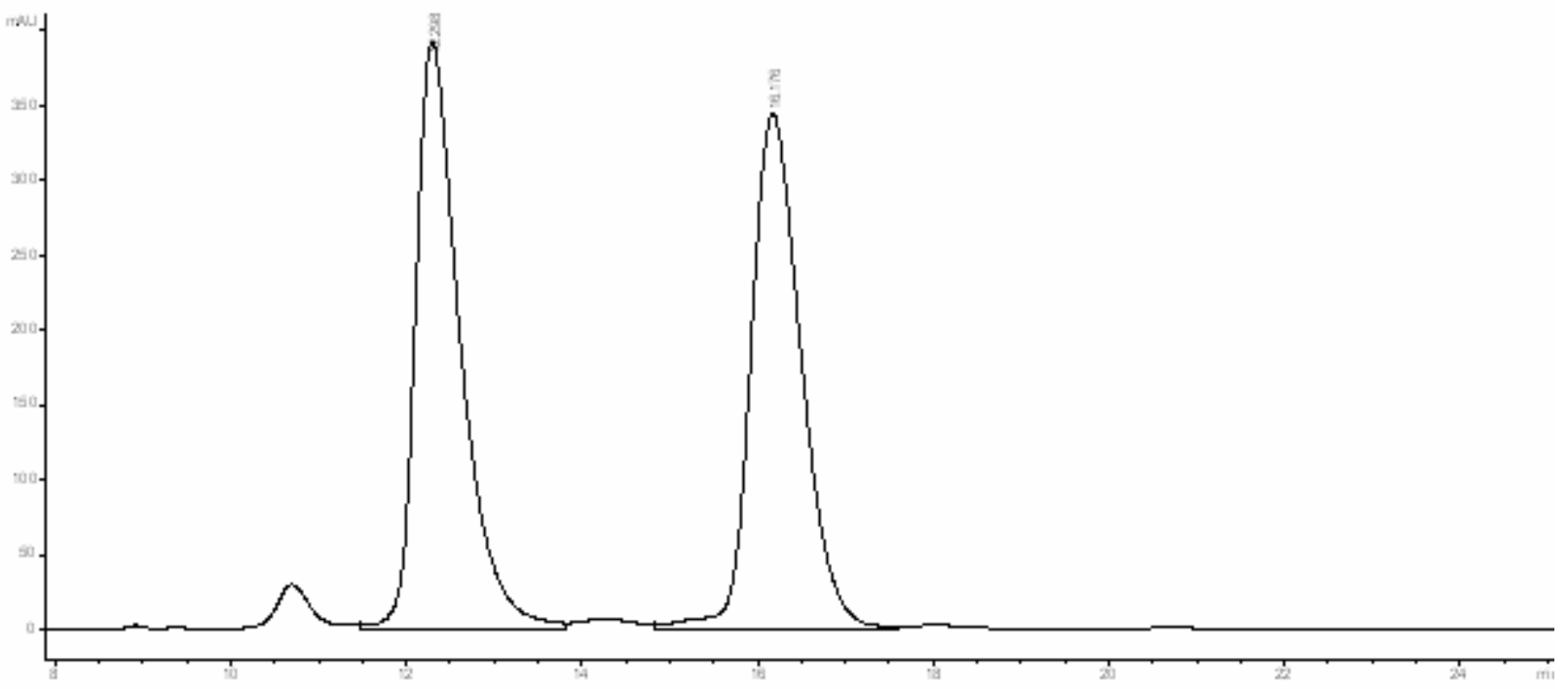

Signal 3: MWD1 C, Sig $=210,8$ Ref $=360,100$

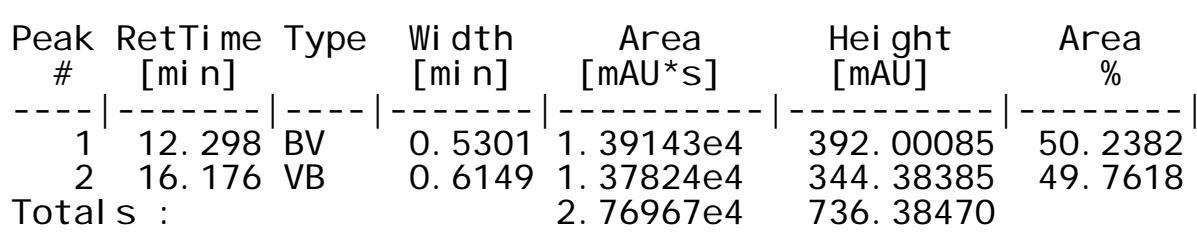<smiles>COC(=O)NC(C(C)=C(C)NCc1ccccc1)c1cccc(F)c1</smiles>

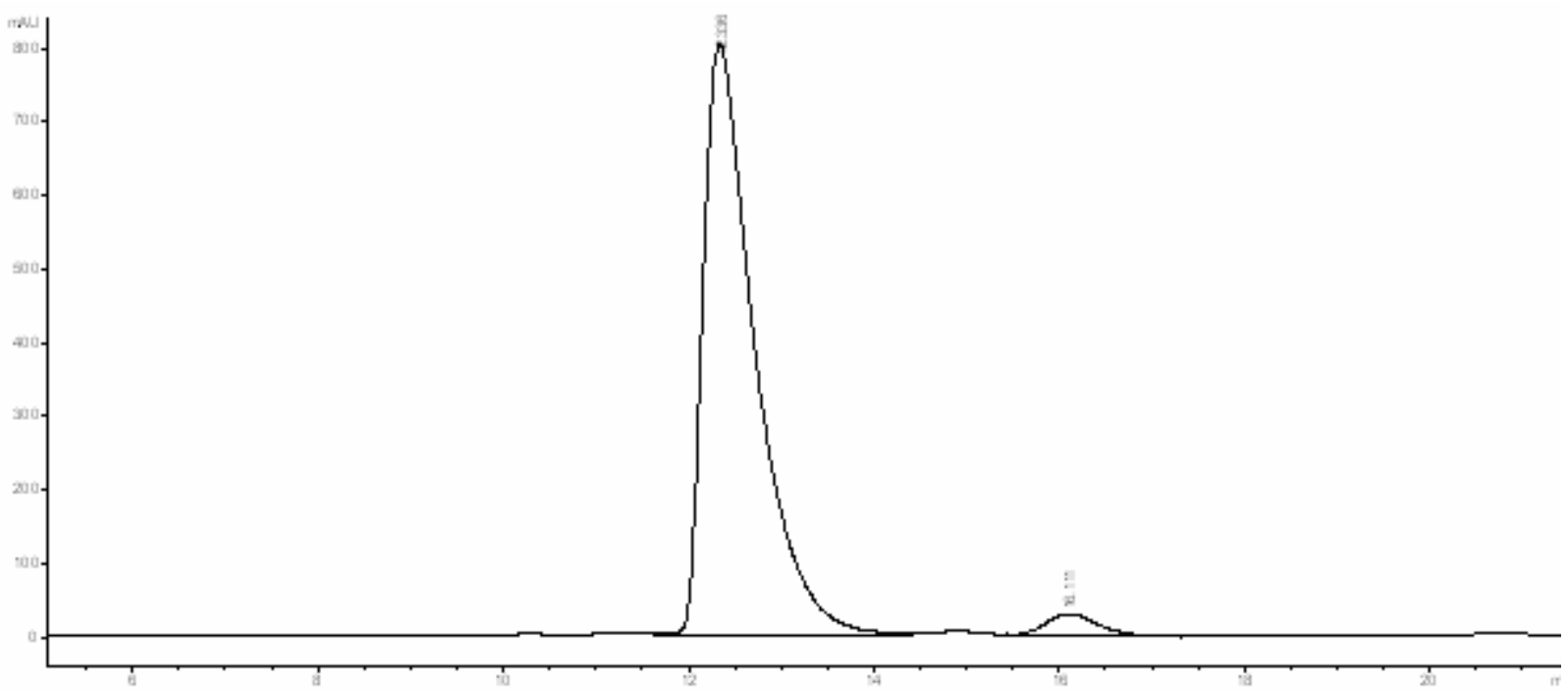

Signal 3: MWD1 C, Sig=210,8 Ref $=360,100$

\begin{tabular}{|c|c|c|c|c|c|c|}
\hline $\begin{array}{c}\text { Peak } \\
\#\end{array}$ & $\begin{array}{l}\text { Ret Ti me } \\
{\left[\begin{array}{lll}\mathrm{mi} & \mathrm{n}\end{array}\right]}\end{array}$ & Type & $\begin{array}{l}\text { Width } \\
{\left[\begin{array}{ll}\mathrm{mi} & \mathrm{n}\end{array}\right.}\end{array}$ & $\begin{array}{c}\text { Area } \\
{\left[\mathrm{mAU}^{*} \mathrm{~s}\right]}\end{array}$ & $\begin{array}{l}\text { Height } \\
{[\mathrm{mAUU}]}\end{array}$ & $\begin{array}{c}\text { Area } \\
\%\end{array}$ \\
\hline Tot ${ }^{\frac{1}{2}}$ & $\begin{array}{r}12.336 \\
16.111\end{array}$ & $\begin{array}{l}\text { VB } \\
M M\end{array}$ & $\begin{array}{l}0.5817 \\
0.5187\end{array}$ & $\begin{array}{l}3.13884 \mathrm{e} 4 \\
752.69019 \\
3.21411 \mathrm{e} 4\end{array}$ & $\begin{array}{r}803.62488 \\
24.18504 \\
827.80992\end{array}$ & $\begin{array}{r}97.6582 \\
2.3418\end{array}$ \\
\hline
\end{tabular}<smiles>COC(=O)NC(C(C)=C(C)NCc1ccccc1)c1cccc(F)c1</smiles> 
Table 2, entry 11: 8k Chiralcel OD Column, Hexane:IPA = 95:5, $1.0 \mathrm{~mL} / \mathrm{min}, 214 \mathrm{~nm}$

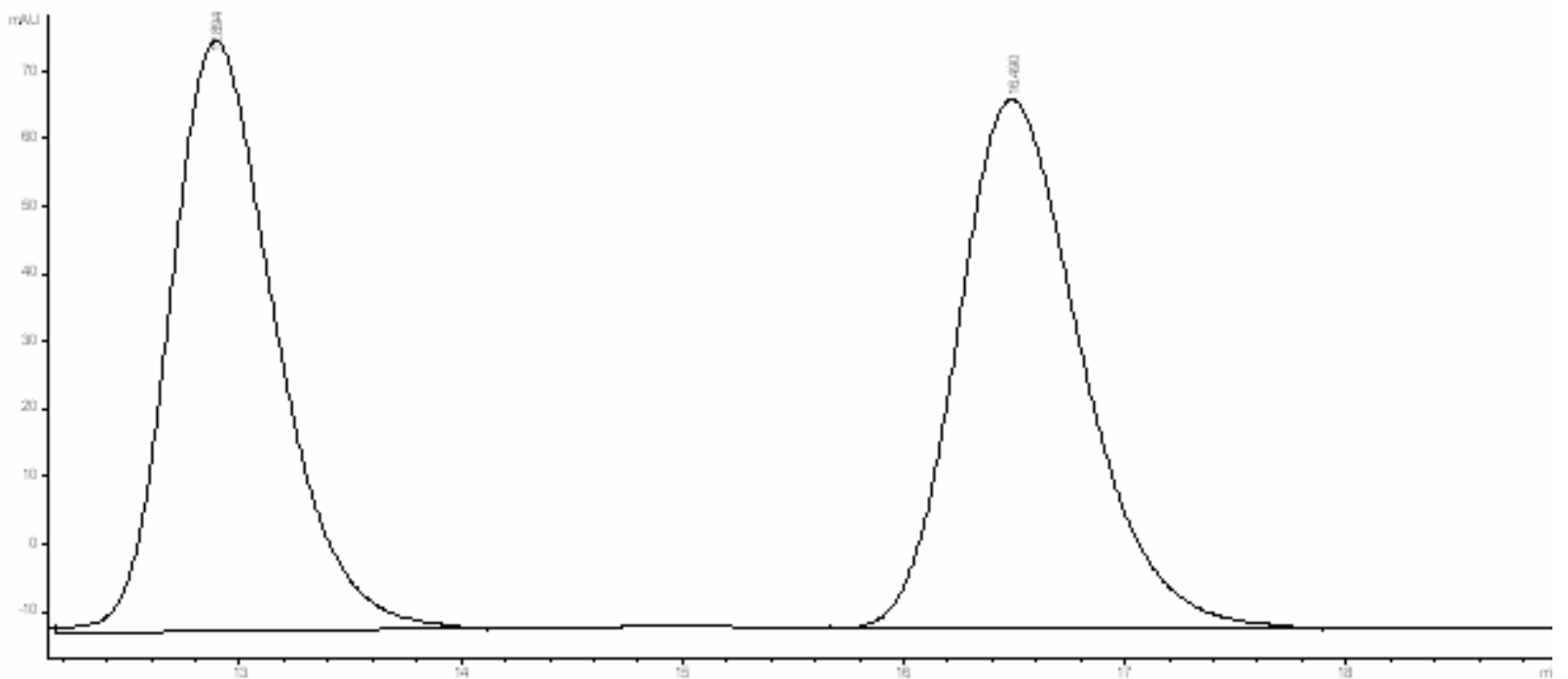

Signal 3: MWD1 C, Sig=210,8 Ref $=360,100$
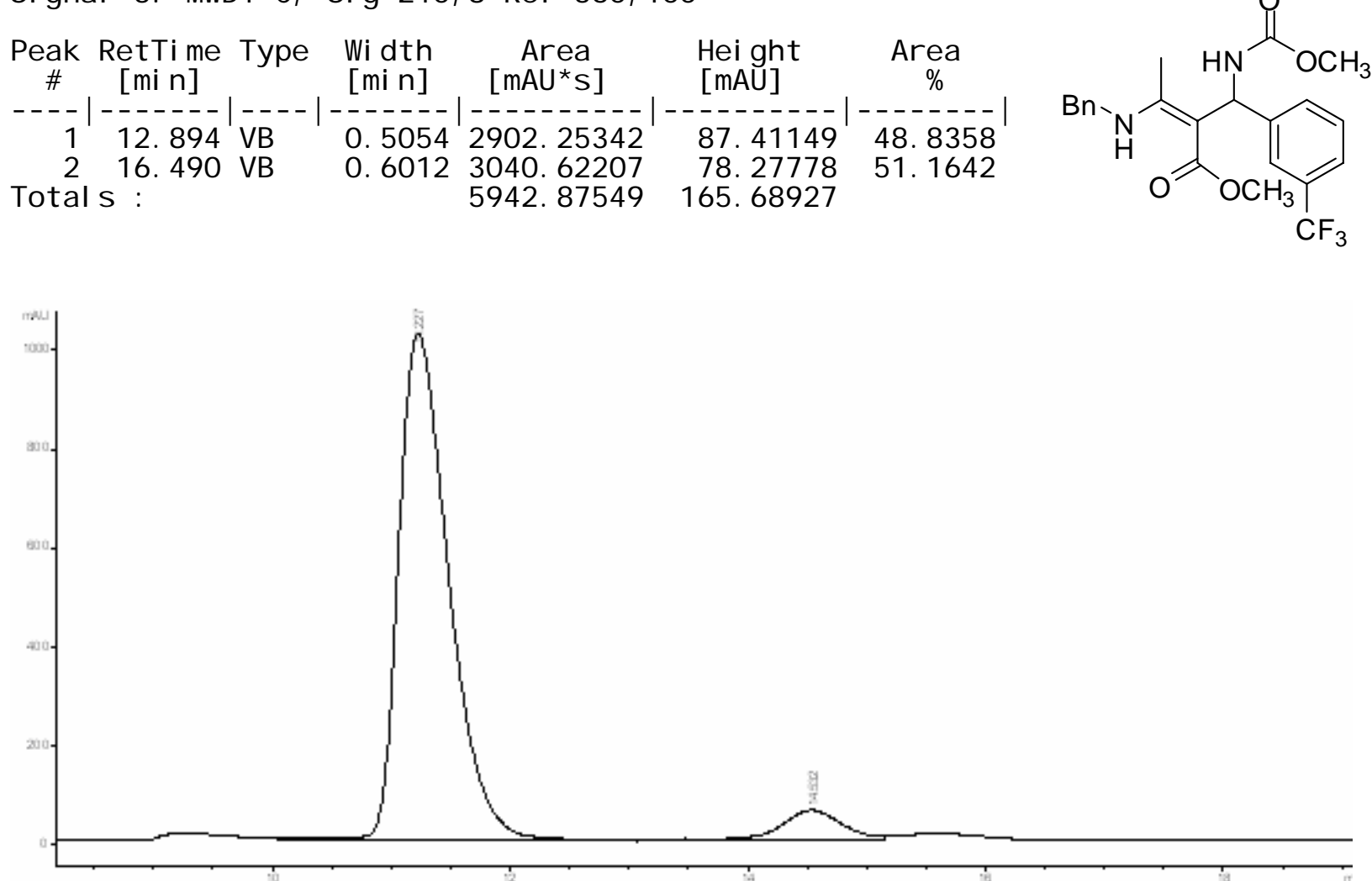

Signal 3: MWD1 C, Sig=210,8 Ref $=360,100$
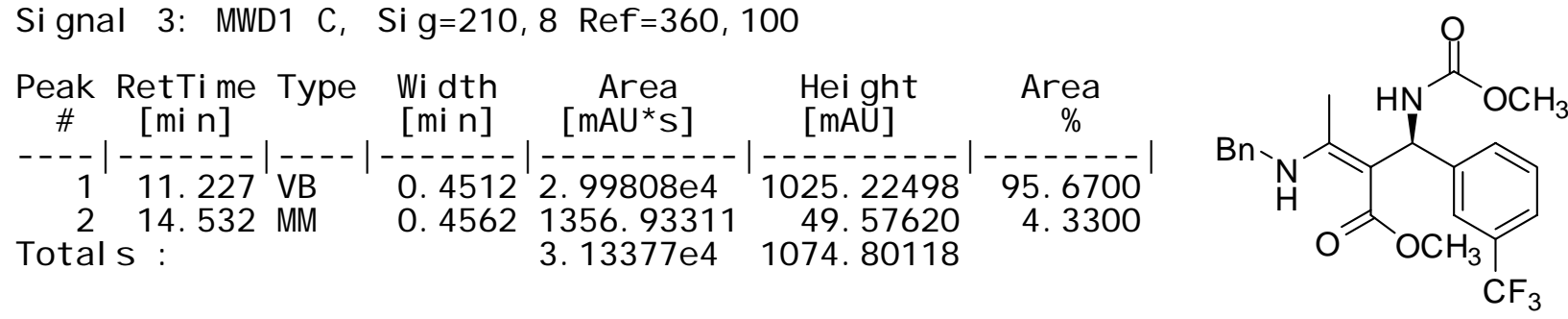
Table 2, entry 12: 81 Chiralcel OD Column, Hexane:IPA = 95:5, $1.0 \mathrm{~mL} / \mathrm{min}, 214 \mathrm{~nm}$

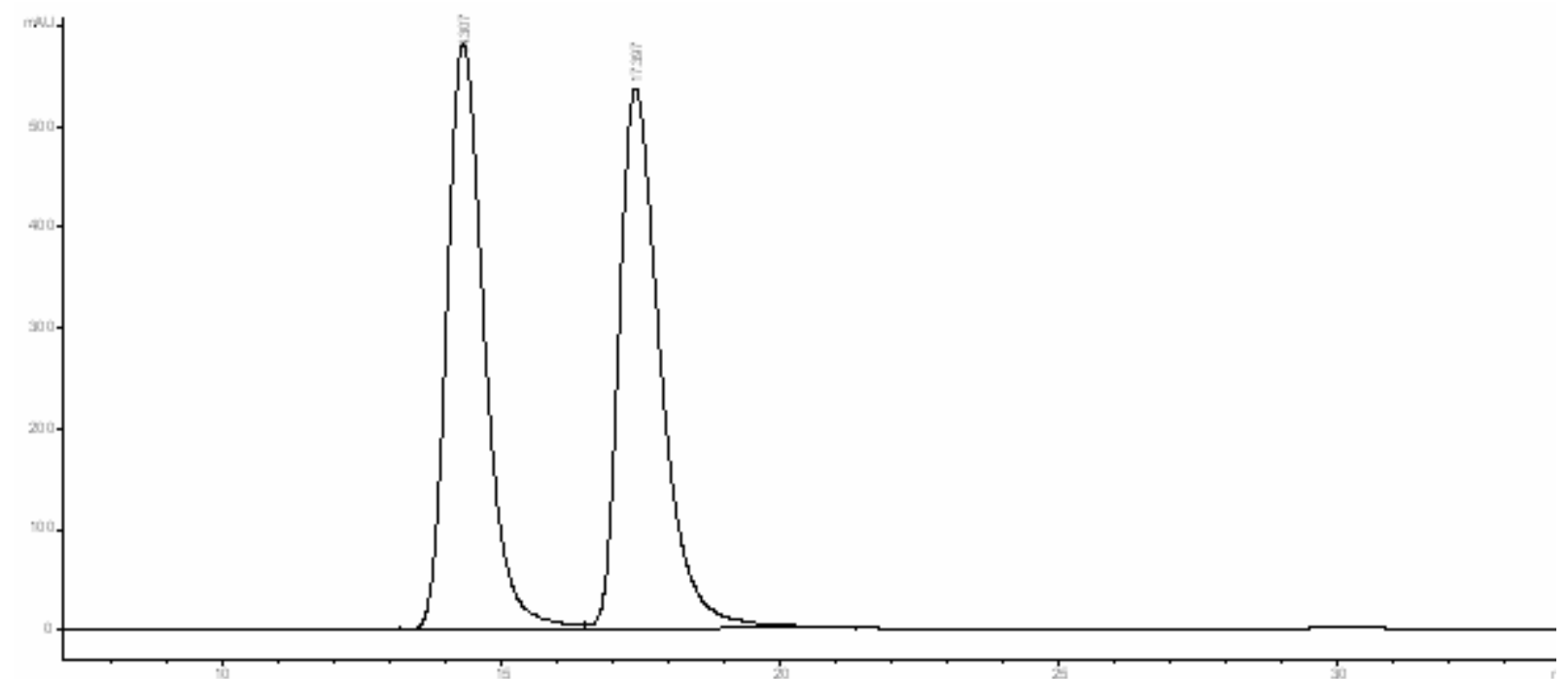

Signal 4: MWD1 D, Sig=230,16 Ref $=360,100$
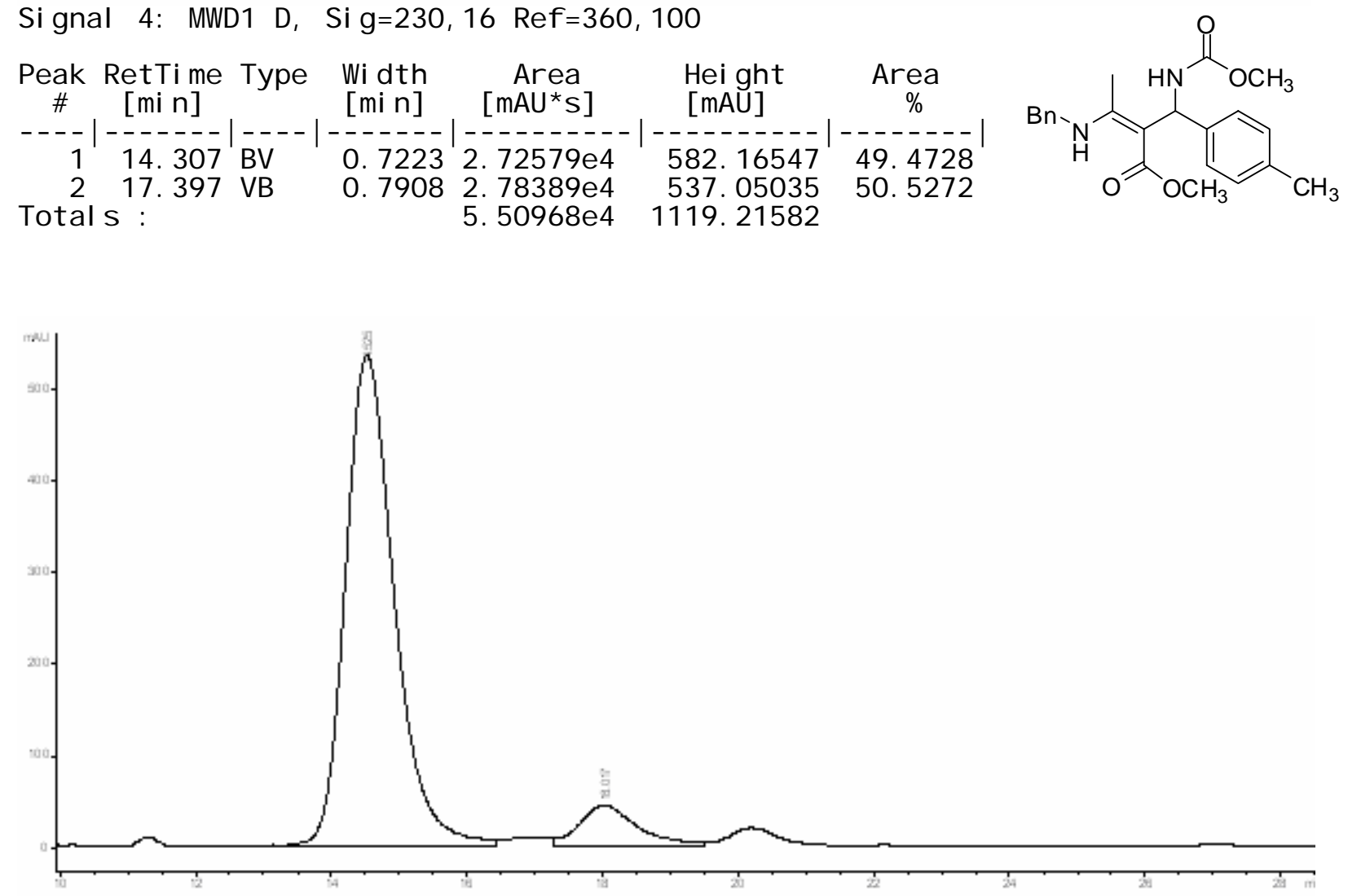

Signal 4: MWD1 $D$, Si $g=230,16$ Ref $=360,100$
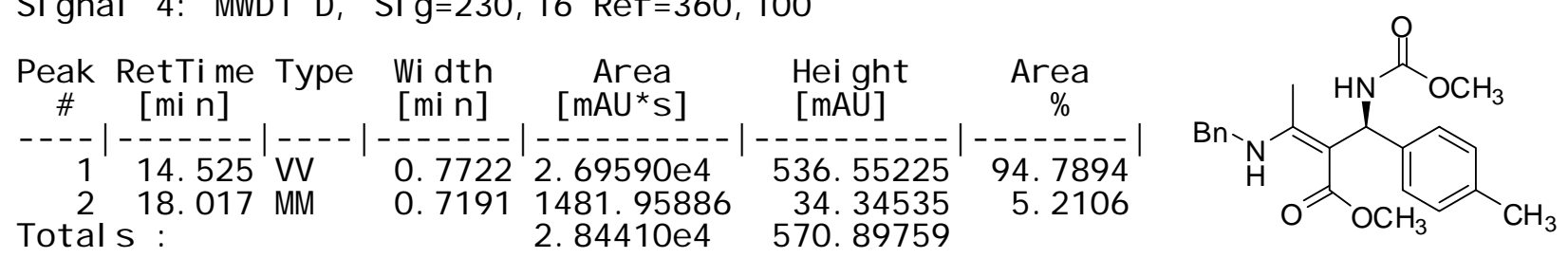
Table 2, entry 13: 8m Chiralcel OD Column, Hexane:IPA = 95:5, $1.0 \mathrm{~mL} / \mathrm{min}, 214 \mathrm{~nm}$

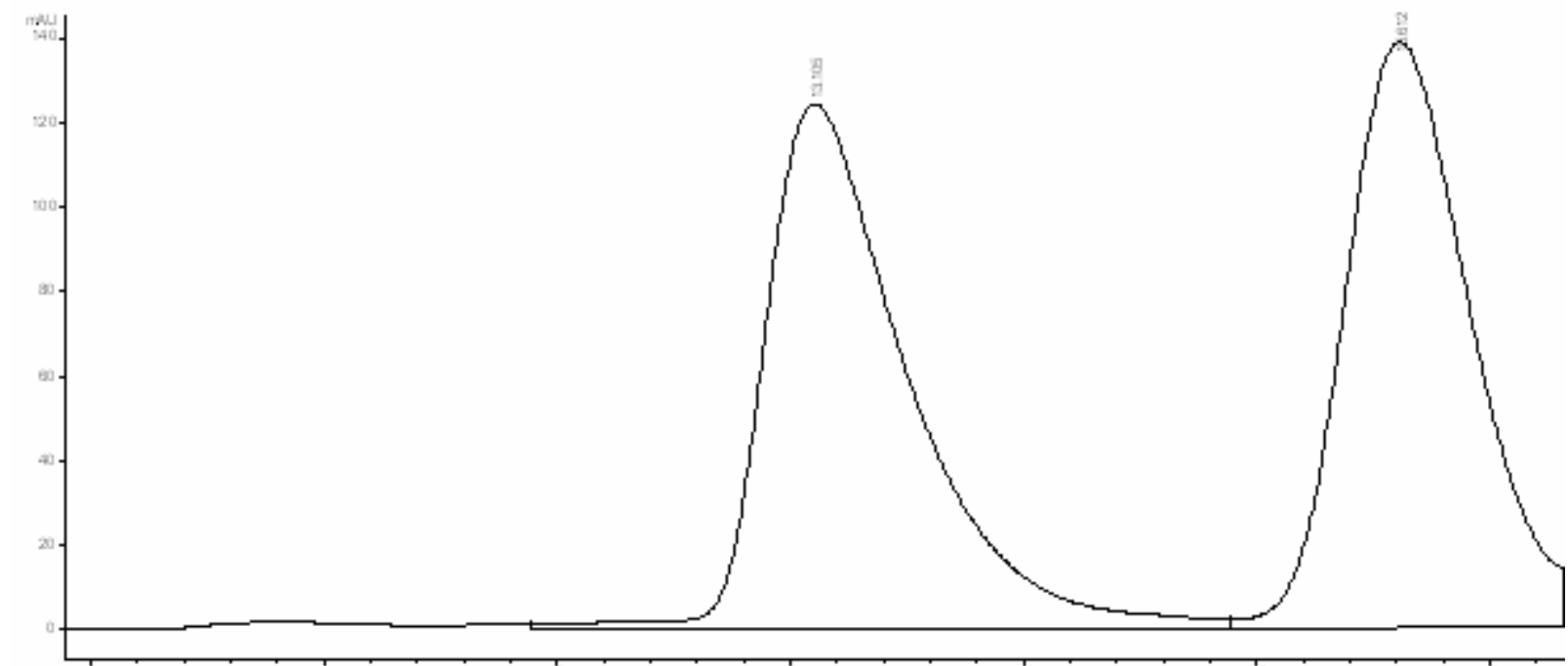

Signal 5: MWD1 E, Si $g=280,16$ Ref $=360,100$

Peak RetTime Type Width Area Height Area \# [min] [min] [mAU*s] [mAU]

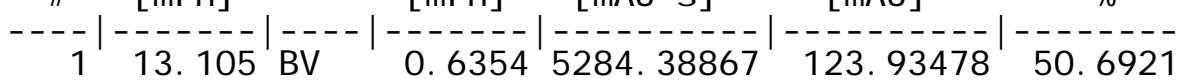

\section{$\begin{array}{lllll}2 & 15.612 \mathrm{VV} & 0.5772 & 5140.09668 & 138.42728\end{array}$}

Totals:
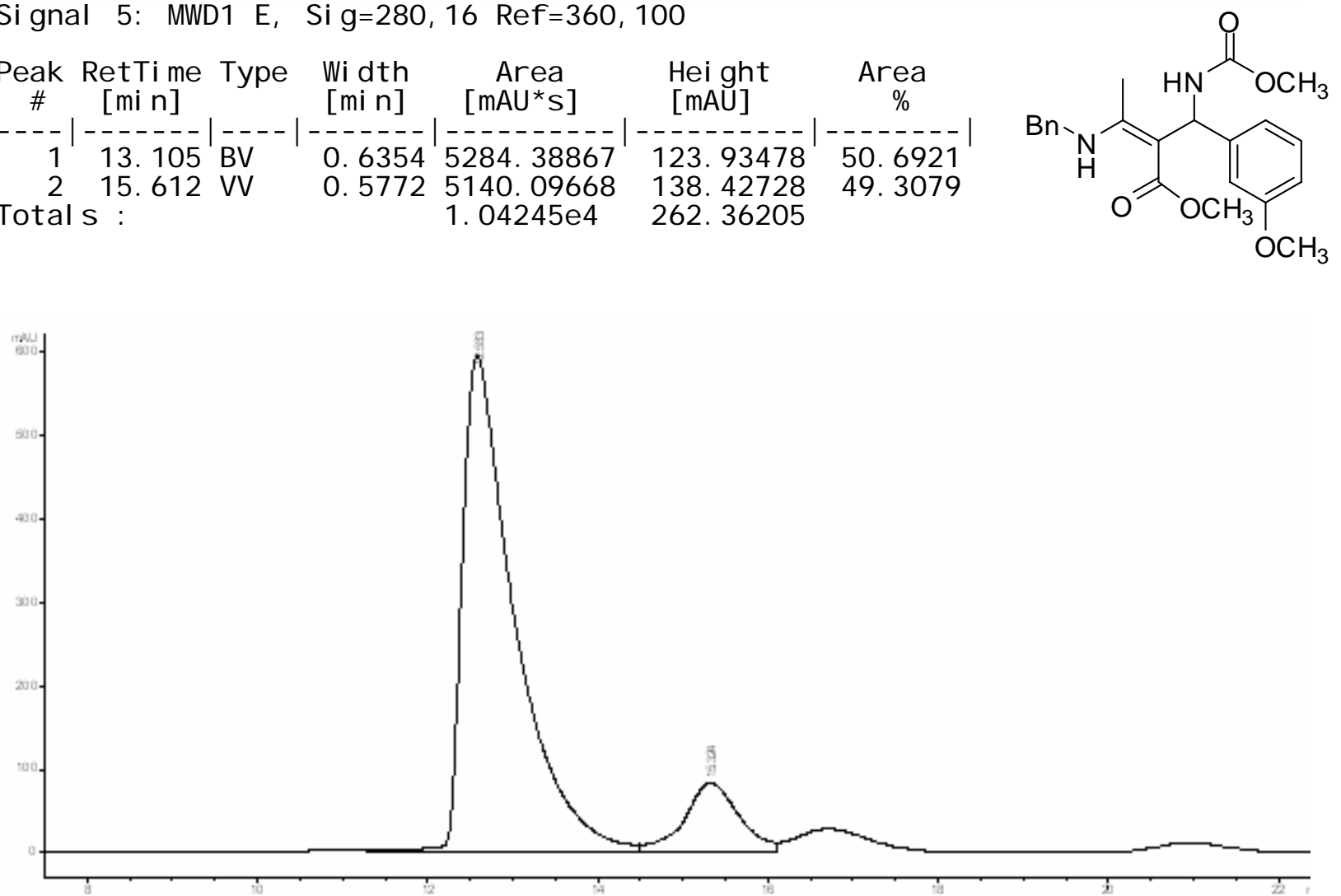

Signal 4: MWD1 D, Si $g=230,16$ Ref $=360,100$

Peak RetTime Type Width Area Height Area \# [min] [min] [mAU*s] [mAU] \%

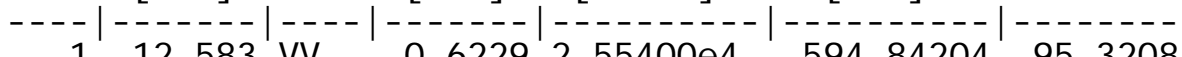

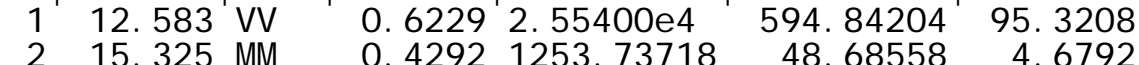
Totals: 2. $67937 e 4 \quad 643.52762$<smiles>COC(=O)N[C@@H](C(C)=NCc1ccccc1)c1cccc(OC)c1</smiles> 
Table 2, entry 14: 8n Chiralcel OD Column, Hexane:IPA = 95:5, $1.0 \mathrm{~mL} / \mathrm{min}, 214 \mathrm{~nm}$

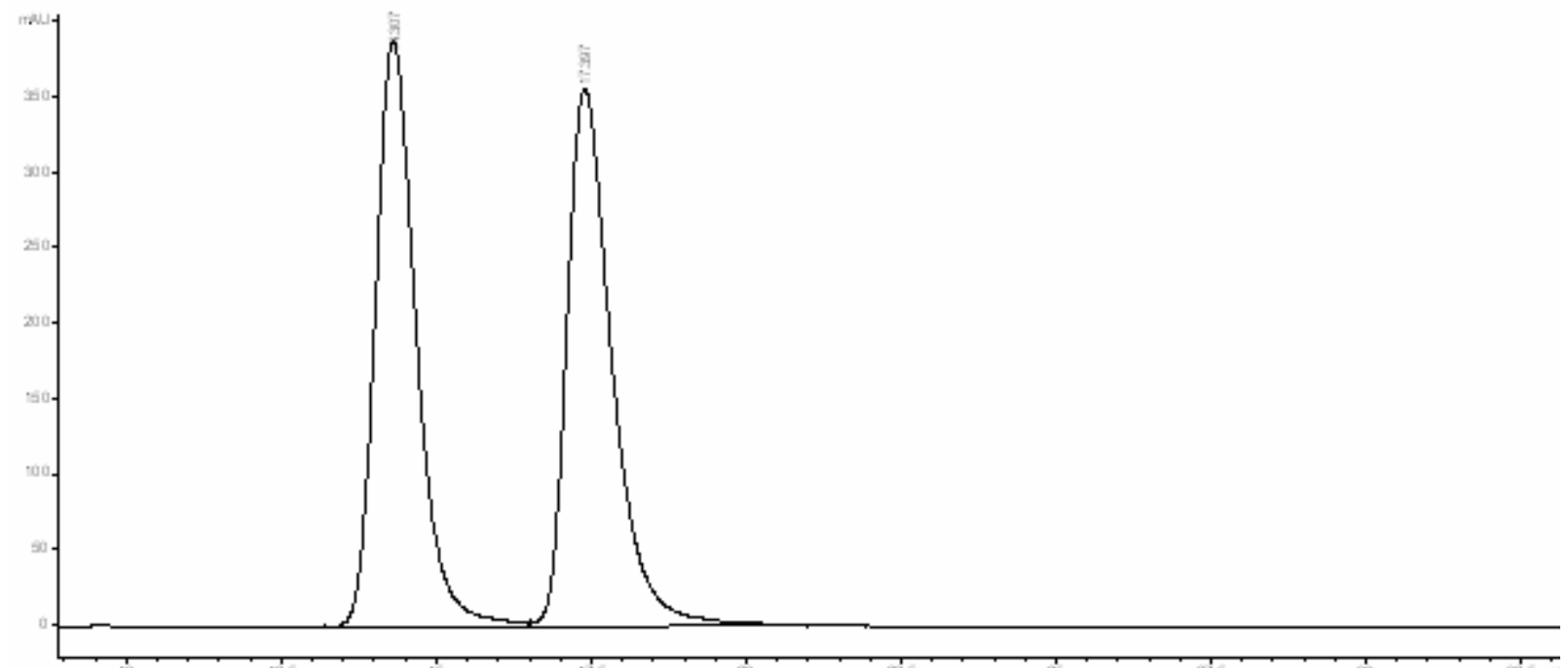

Signal 3: MWD1 C, Sig=210,8 Ref $=360,100$

Peak RetTime Type Width Area Height Area \# [min] [min] [mAU*s] [mAU]

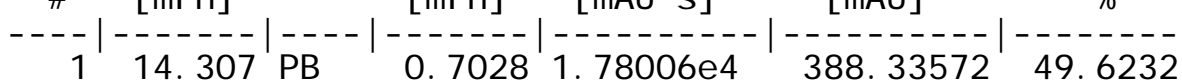

$\begin{array}{lllllll}2 & 17.397 & B B & 0.7685 & 1.80710 e 4 & 355.84894 & 50.3768\end{array}$

Totals:

3. $58716 \mathrm{e} 4 \quad 744.18466$
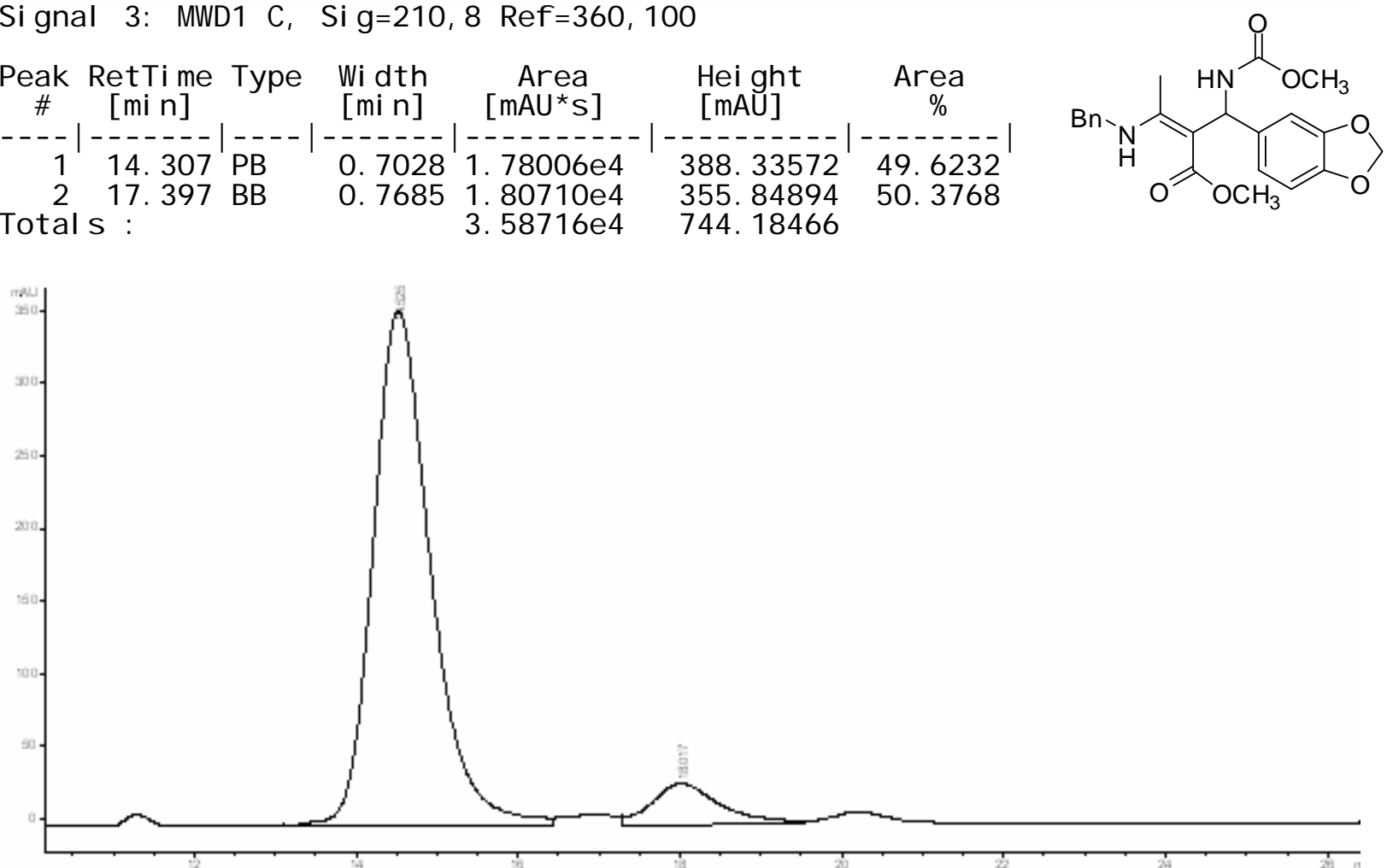

Signal 3: MWD1 C, Sig=210,8 Ref $=360,100$

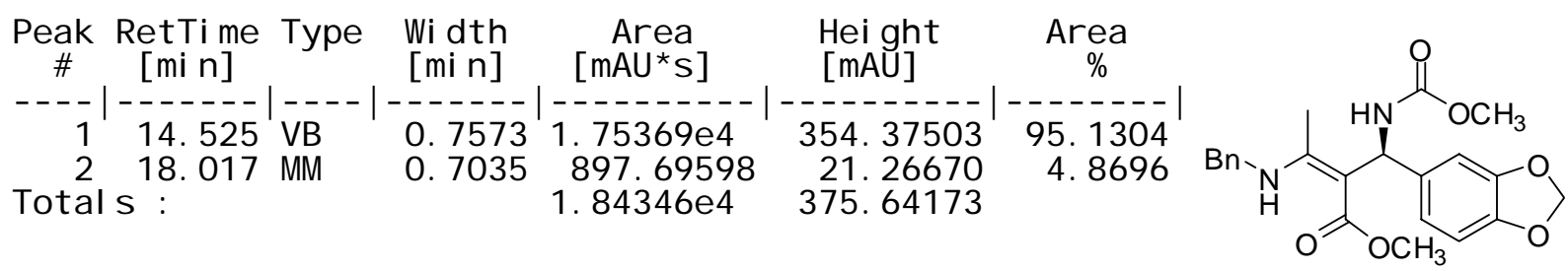


Table 2, entry 15: 8o Chiralcel OD Column, Hexane:IPA = 95:5, $1.0 \mathrm{~mL} / \mathrm{min}, 214 \mathrm{~nm}$

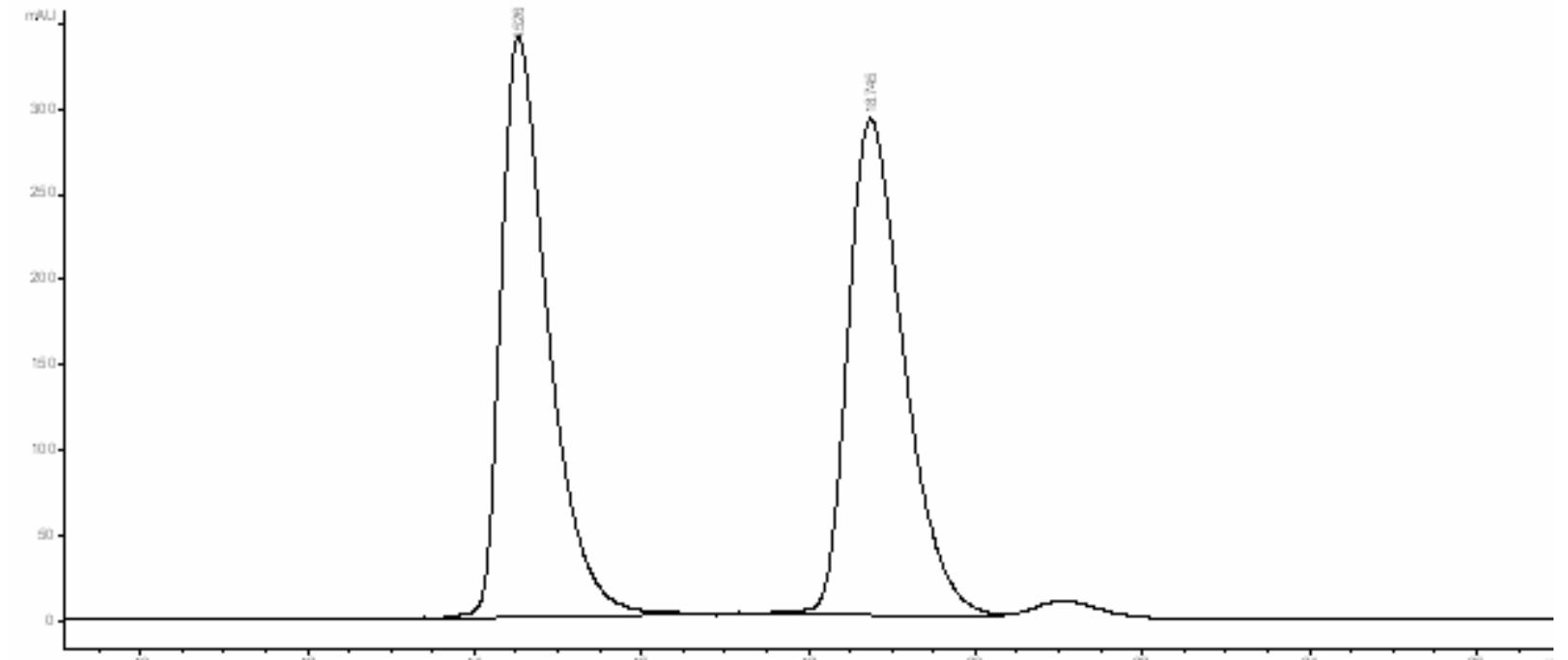

Signal 3: MWD1 C, Sig=210,8 Ref $=360,100$

Peak RetTime Type Width Area Height Area $\#$ [min] [mi $n] \quad\left[m A U^{*} s\right] \quad[m A U] \quad \%$

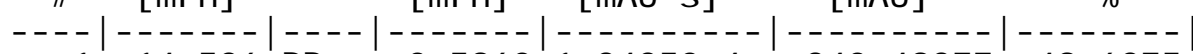
$\begin{array}{lllllll}1 & 14.526 \mathrm{~PB} & 0.5960 & 1.34250 e 4 & 340.49377 & 49.6275\end{array}$ $\begin{array}{lllllll}2 & 18.745 & B V & 0.7158 & 1.36265 e 4 & 291.27304 & 50.3725\end{array}$ Tot als:<smiles>COC(=O)NC(C(C)=C(C)NCc1ccccc1)c1ccco1</smiles>

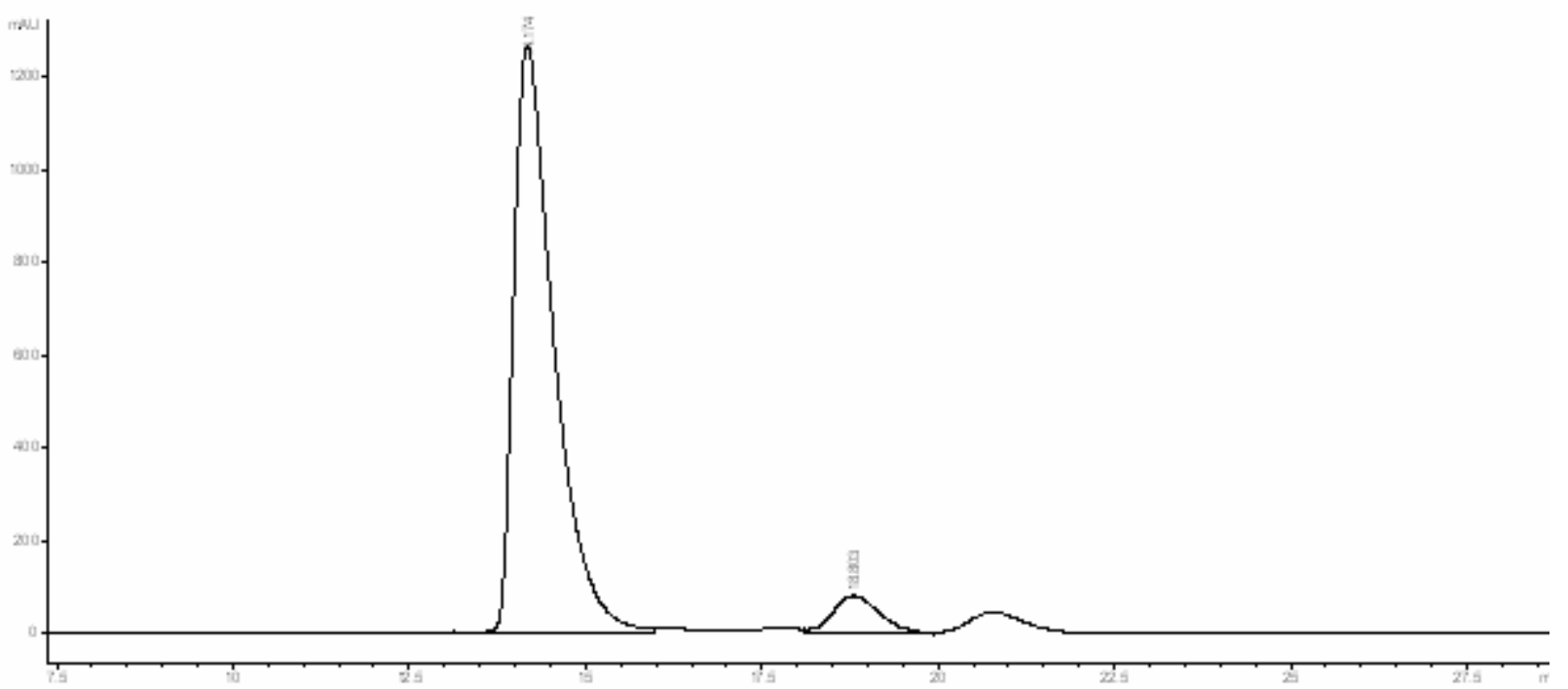

Signal 3: MWD1 C, Sig $=210,8$ Ref $=360,100$

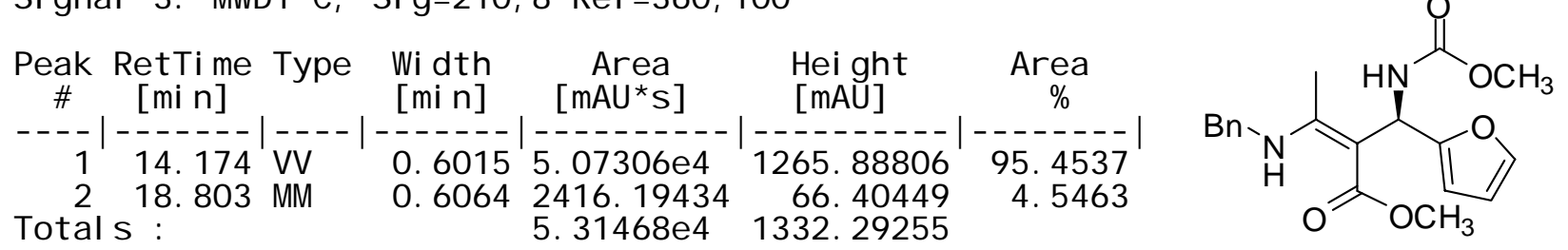


Table 2, entry 16: 8p Chiralcel OD Column, Hexane:IPA = 95:5, $1.0 \mathrm{~mL} / \mathrm{min}, 214 \mathrm{~nm}$

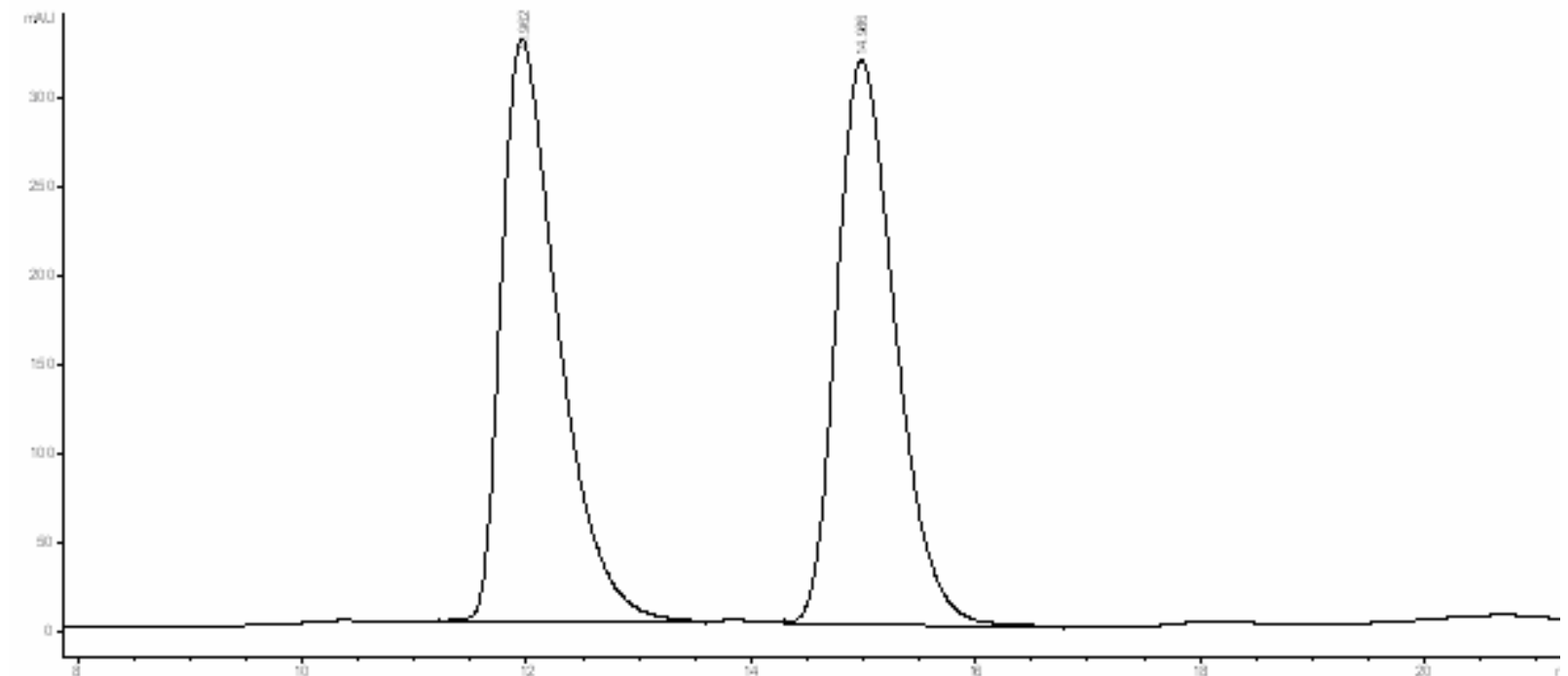

Signal 3: MWD1 C, Sig=210,8 Ref $=360,100$

Peak RetTime Type Width Area Height Area

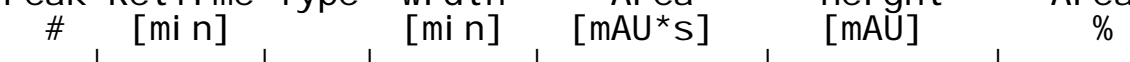

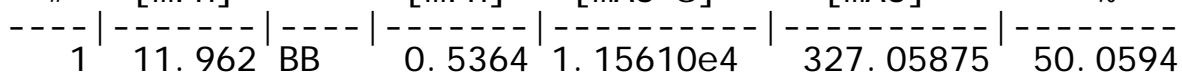

214.986 VP

Totals:

$0.56401 .15336 \mathrm{e} 4 \quad 317.35126$

2. $30945 e 4 \quad 644.41000$<smiles>COC(=O)NC(C(=O)OC)C(NCc1ccccc1)C(C)=O</smiles>

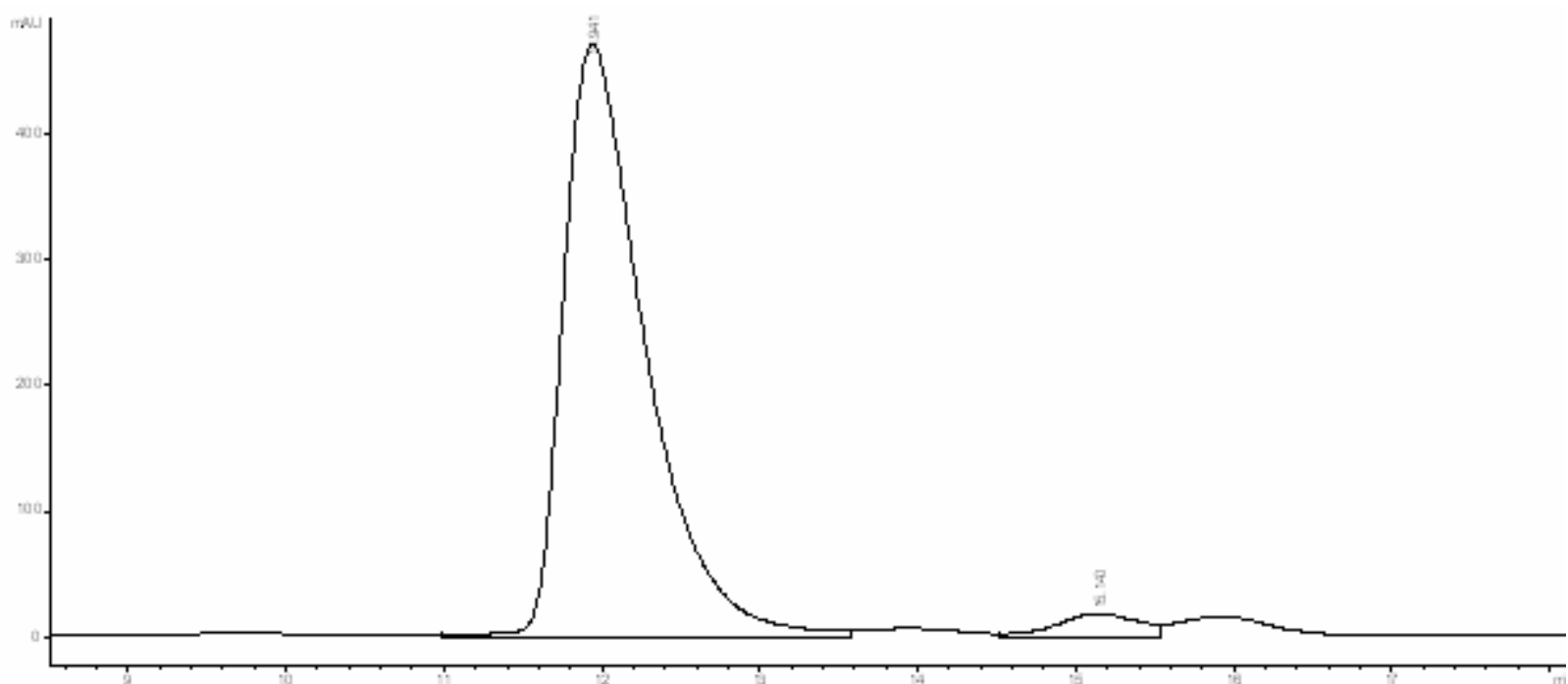

Signal 3: MWD1 C, Sig=210,8 Ref $=360,100$

Peak RetTime Type Width Area Height Area \# [min] [min] [mAU*s] [mAU]

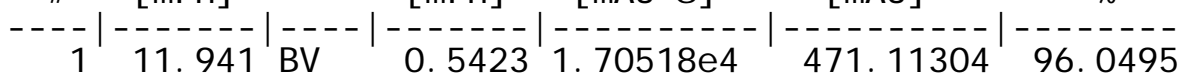
2 15.140 VV
0.5471
701.33539
3. 9505

18.63303
489.74607

Totals:

$1.77532 \mathrm{e}$<smiles>COC(=O)N[C@H](C(C(=O)OC)=C(C)NCc1ccccc1)c1cccs1</smiles> 
Table 2, entry 17: 9a Chiralcel OD-H Column, Hexane:IPA = 95:5, $1.0 \mathrm{~mL} / \mathrm{min}, 214 \mathrm{~nm}$

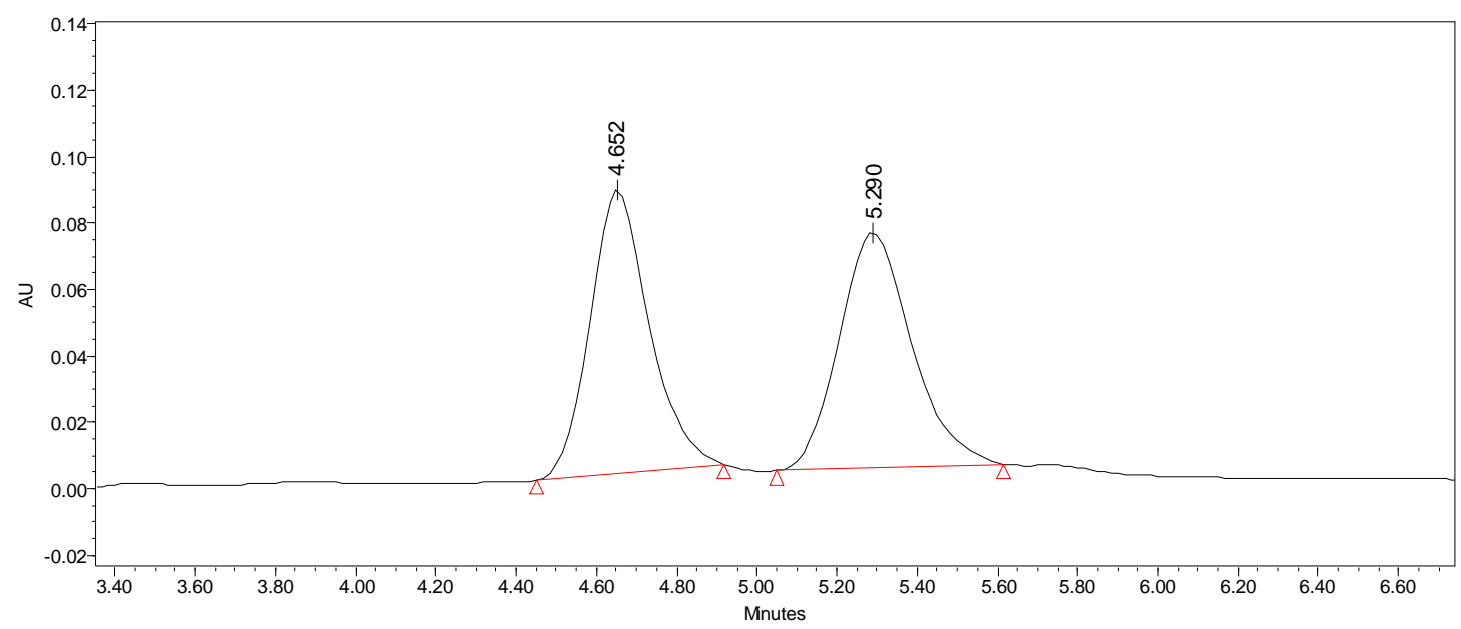

\begin{tabular}{|c|c|c|c|c|c|}
\hline & $\begin{array}{c}\text { RT } \\
(\mathrm{min})\end{array}$ & $\begin{array}{c}\text { Area } \\
\left(\mathrm{V}^{*} \mathrm{sec}\right)\end{array}$ & $\%$ Area & $\begin{array}{c}\text { Height } \\
(\mathrm{V})\end{array}$ & $\begin{array}{c}\% \\
\text { Height }\end{array}$ \\
\hline 1 & 4.652 & 876148 & 49.65 & 85432 & 54.61 \\
\hline 2 & 5.290 & 888515 & 50.35 & 70999 & 45.39 \\
\hline
\end{tabular}<smiles>C=CCOC(=O)NC(CCc1ccccc1)/C(C(=O)OC)=C(\C)NCc1ccccc1</smiles>

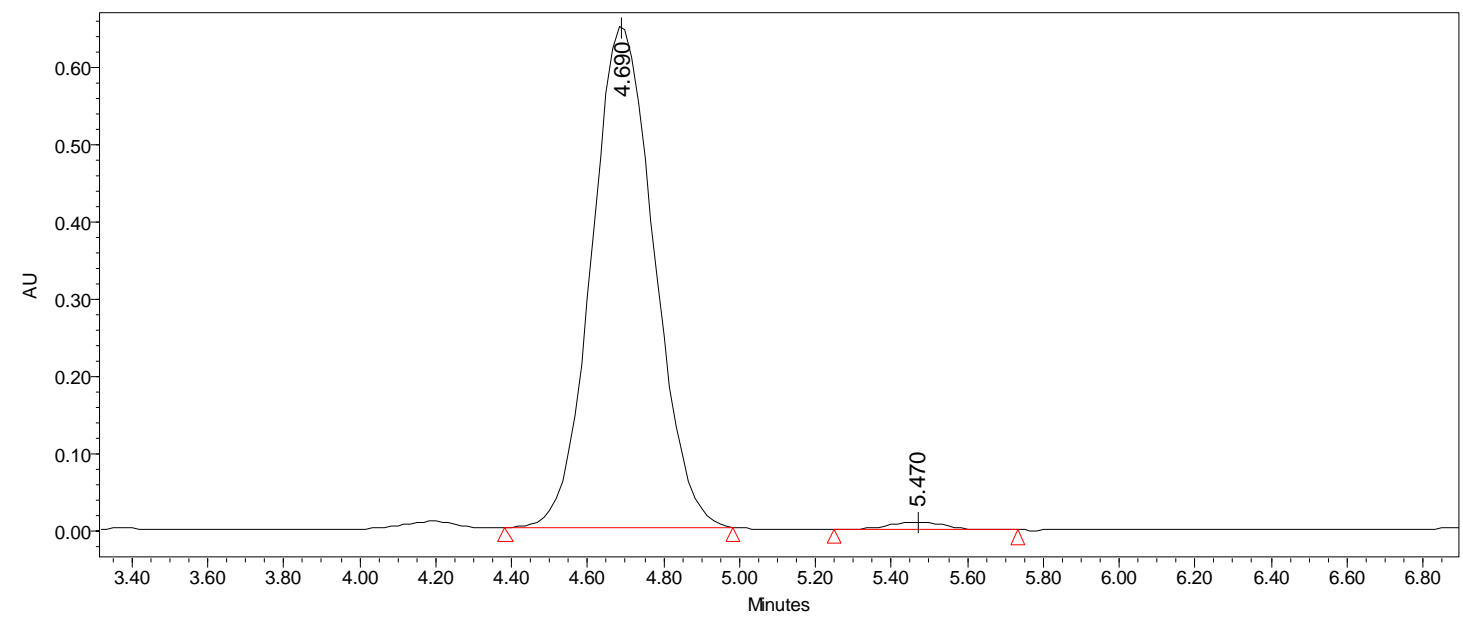

\begin{tabular}{|r|c|r|r|r|r|}
\hline & $\begin{array}{c}\text { RT } \\
(\mathrm{min})\end{array}$ & $\begin{array}{c}\text { Area } \\
\left(\mathrm{V}^{*} \mathrm{sec}\right)\end{array}$ & $\%$ Area & $\begin{array}{c}\text { Height } \\
(\mathrm{V})\end{array}$ & $\begin{array}{c}\% \\
\text { Height }\end{array}$ \\
\hline 1 & 4.690 & 1814117 & 95.15 & 292059 & 96.78 \\
\hline 2 & 5.470 & 92544 & 4.85 & 9725 & 3.22 \\
\hline
\end{tabular}<smiles>C=CCOC(=O)N[C@@H](CCc1ccccc1)/C(C(=O)OC)=C(\C)NCc1ccccc1</smiles> 
Table 2, entry 18: 9b Chiralcel OD Column, Hexane:IPA = 95:5, $1.0 \mathrm{~mL} / \mathrm{min}), 214 \mathrm{~nm}$

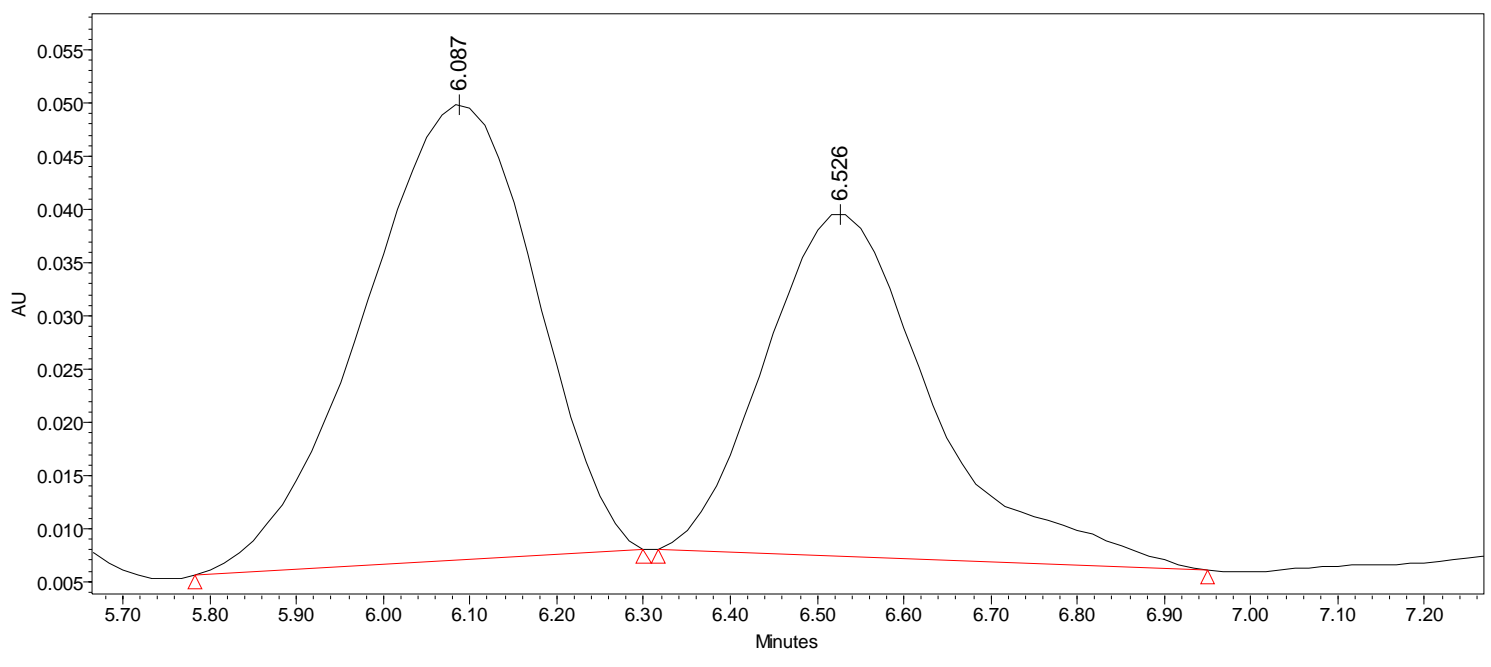

\begin{tabular}{|c|c|c|c|c|c|}
\hline & $\begin{array}{c}\text { RT } \\
(\mathrm{min})\end{array}$ & $\begin{array}{c}\text { Area } \\
\left(\mathrm{V}^{*} \mathrm{sec}\right)\end{array}$ & $\%$ Area & $\begin{array}{c}\text { Height } \\
(\mathrm{V})\end{array}$ & $\begin{array}{c}\% \\
\text { Height }\end{array}$ \\
\hline 1 & 6.087 & 465895 & 51.98 & 37987 & 54.06 \\
\hline 2 & 6.526 & 430398 & 48.02 & 32278 & 45.94 \\
\hline
\end{tabular}
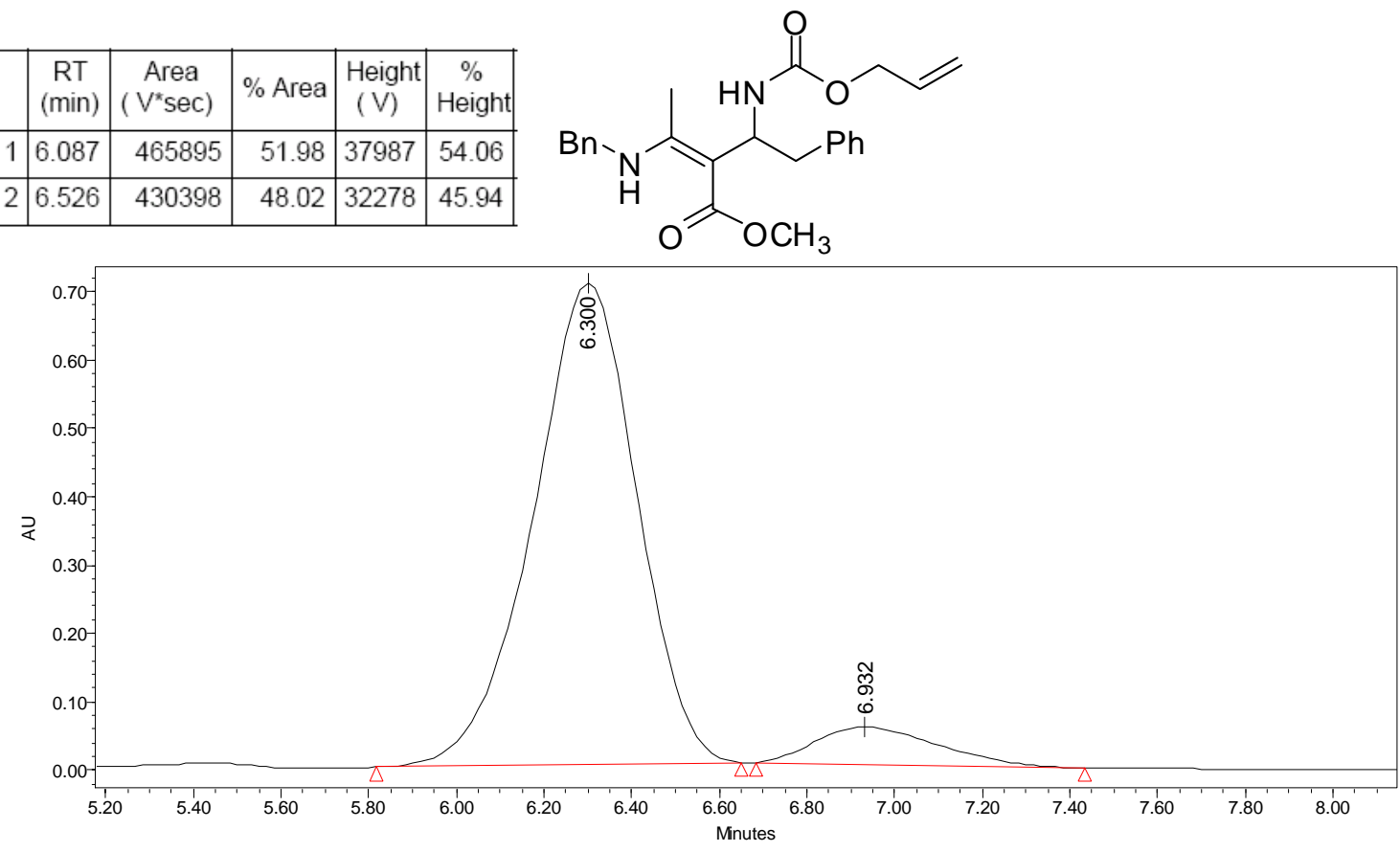

\begin{tabular}{|c|c|c|r|r|r|}
\hline & $\begin{array}{c}\mathrm{RT} \\
(\mathrm{min})\end{array}$ & $\begin{array}{c}\text { Area } \\
\left(\mathrm{V}^{*} \mathrm{sec}\right)\end{array}$ & \% Area & $\begin{array}{c}\text { Height } \\
(\mathrm{V})\end{array}$ & $\begin{array}{c}\% \\
\text { Height }\end{array}$ \\
\hline 1 & 6.300 & 11571825 & 94.46 & 704336 & 94.17 \\
\hline 2 & 6.932 & 678335 & 5.54 & 43631 & 5.83 \\
\hline
\end{tabular}<smiles>C=CCOC(=O)N[C@@H](Cc1ccccc1)/C(C(=O)OC)=C(\C)NCc1ccccc1</smiles> 
Table 2, entry 19: 9b: Chiralcel OD-H Column, Hexane:IPA = 96:4, $1.0 \mathrm{~mL} / \mathrm{min}), 214 \mathrm{~nm}$

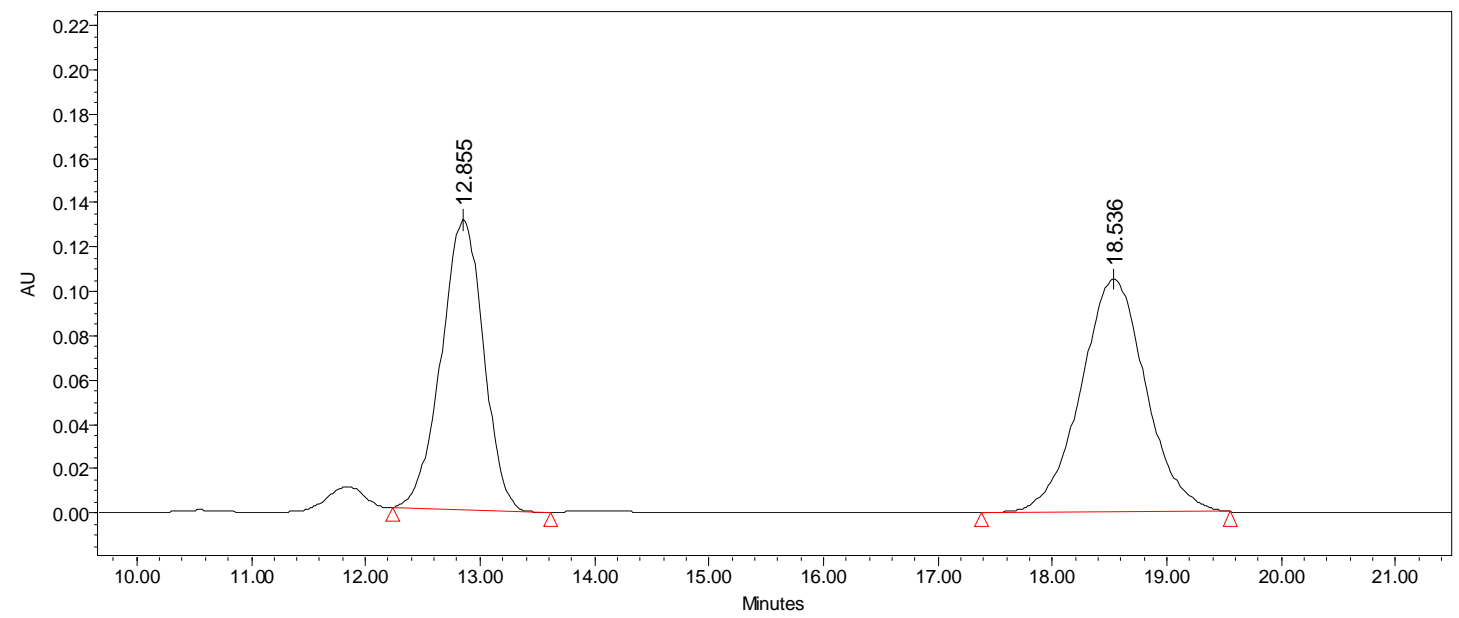

\begin{tabular}{|c|c|c|c|c|c|}
\hline & $\begin{array}{c}\text { RT } \\
(\mathrm{min})\end{array}$ & $\begin{array}{c}\text { Area } \\
\left(\mathrm{V}^{*} \mathrm{sec}\right)\end{array}$ & $\%$ Area & $\begin{array}{c}\text { Height } \\
(\mathrm{V})\end{array}$ & $\begin{array}{c}\% \\
\text { Height }\end{array}$ \\
\hline 1 & 12.855 & 3348474 & 48.05 & 130732 & 56.81 \\
\hline 2 & 18.536 & 3620718 & 51.95 & 99377 & 43.19 \\
\hline
\end{tabular}
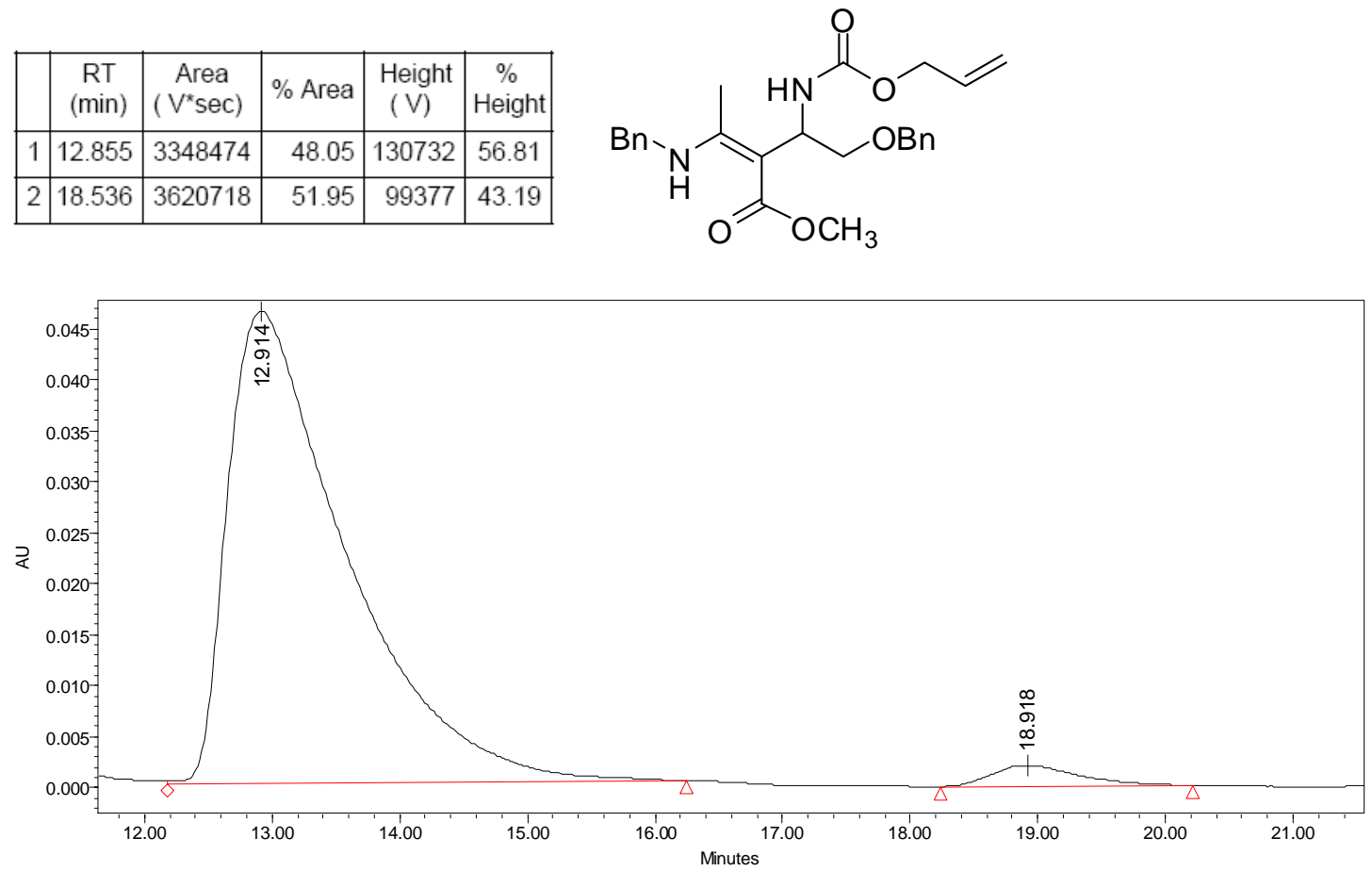

\begin{tabular}{|l|c|r|r|r|r|}
\hline & $\begin{array}{c}\text { RT } \\
(\mathrm{min})\end{array}$ & $\begin{array}{c}\text { Area } \\
\left(\mathrm{V}^{*} \mathrm{sec}\right)\end{array}$ & $\%$ Area & $\begin{array}{l}\text { Height } \\
(\mathrm{V})\end{array}$ & $\begin{array}{c}\% \\
\text { Height }\end{array}$ \\
\hline 1 & 12.913 & 1971004 & 95.32 & 38702 & 94.99 \\
\hline 2 & 18.918 & 96792 & 4.68 & 2041 & 5.01 \\
\hline
\end{tabular}<smiles>C=CCOC(=O)N[C@@H](COc1ccccc1)/C(C(=O)OC)=C(\C)NCc1ccccc1</smiles> 
Table 2, entry 20: 9d Chiralcel OD Column, Hexane:IPA = 96:4, $1.0 \mathrm{~mL} / \mathrm{min}), 214 \mathrm{~nm}$

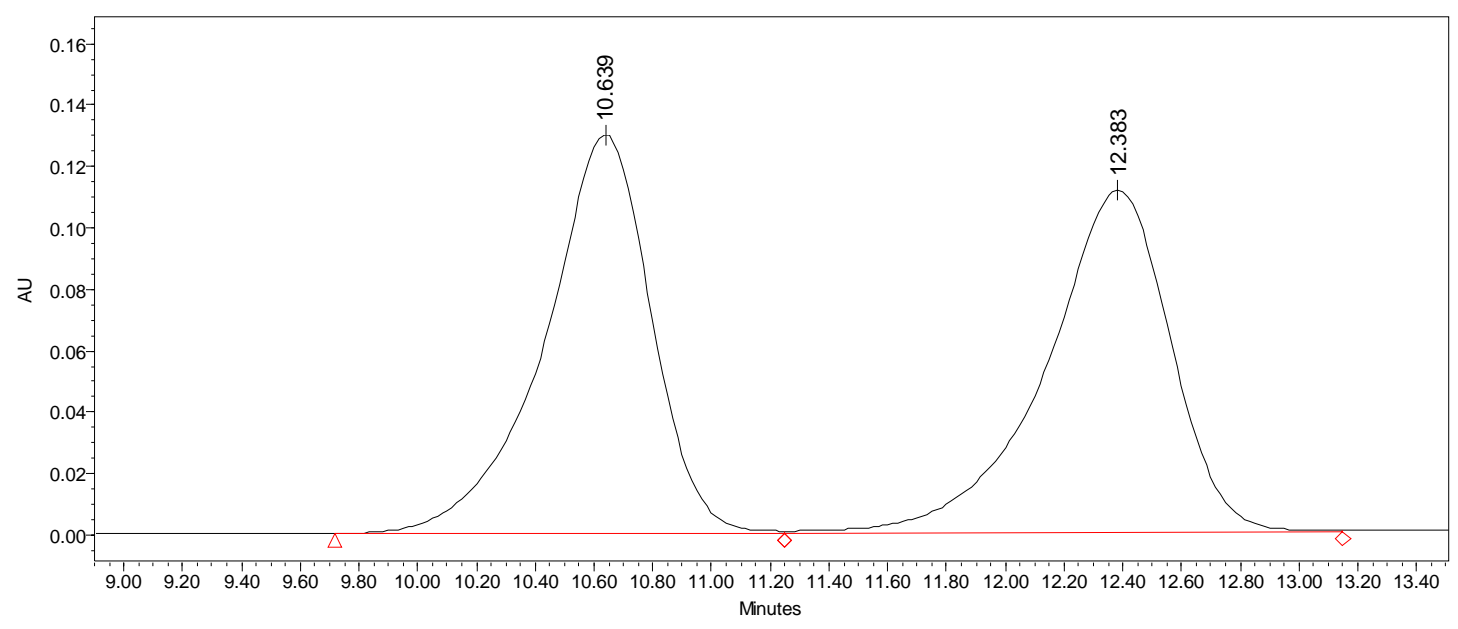

\begin{tabular}{|c|c|c|c|c|c|}
\hline & $\begin{array}{c}\text { RT } \\
(\mathrm{min})\end{array}$ & $\begin{array}{c}\text { Area } \\
\left(\mathrm{V}^{*} \text { sec }\right)\end{array}$ & $\%$ Area & $\begin{array}{c}\text { Height } \\
(\mathrm{V})\end{array}$ & $\begin{array}{c}\% \\
\text { Height }\end{array}$ \\
\hline 1 & 10.639 & 3196304 & 50.79 & 128849 & 54.06 \\
\hline 2 & 12.383 & 3096997 & 49.21 & 109476 & 45.94 \\
\hline
\end{tabular}<smiles>C=CCOC(=O)NC(CC(C)C)/C(C(=O)OC)=C(\C)NCc1ccccc1</smiles>

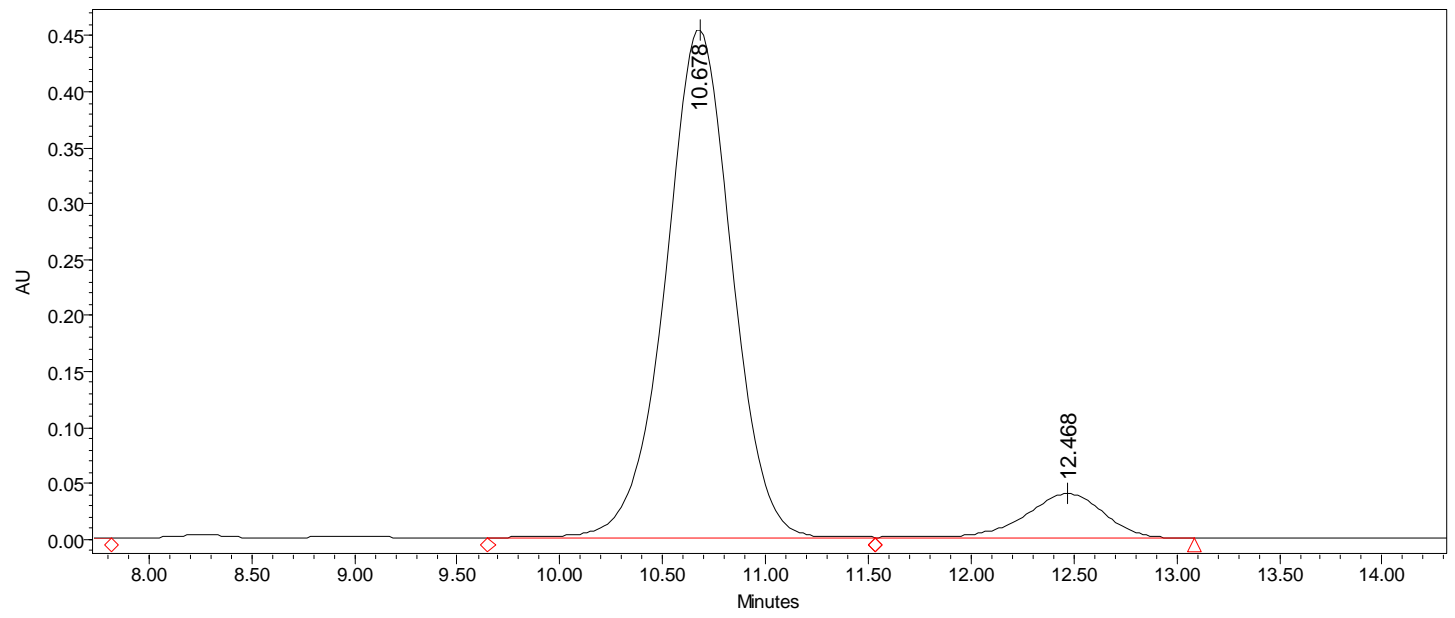

\begin{tabular}{|c|c|c|r|r|r|}
\hline & $\begin{array}{c}\mathrm{RT} \\
(\mathrm{min})\end{array}$ & $\begin{array}{c}\text { Area } \\
\left(\mathrm{V}^{*} \mathrm{sec}\right)\end{array}$ & $\%$ Area & $\begin{array}{c}\text { Height } \\
(\mathrm{V})\end{array}$ & $\begin{array}{c}\% \\
\text { Height }\end{array}$ \\
\hline 1 & 10.678 & 9848331 & 93.90 & 450095 & 93.53 \\
\hline 2 & 12.468 & 640300 & 6.10 & 31158 & 6.47 \\
\hline
\end{tabular}<smiles>C=CCOC(=O)N[C@@H](CC(C)C)/C(C(=O)OC)=C(\C)NCc1ccccc1</smiles> 
Table 2, entry 21: 9e Chiralcel OD-H Column, Hexane:IPA = 96:4, $1.0 \mathrm{~mL} / \mathrm{min}$ ), 214nm

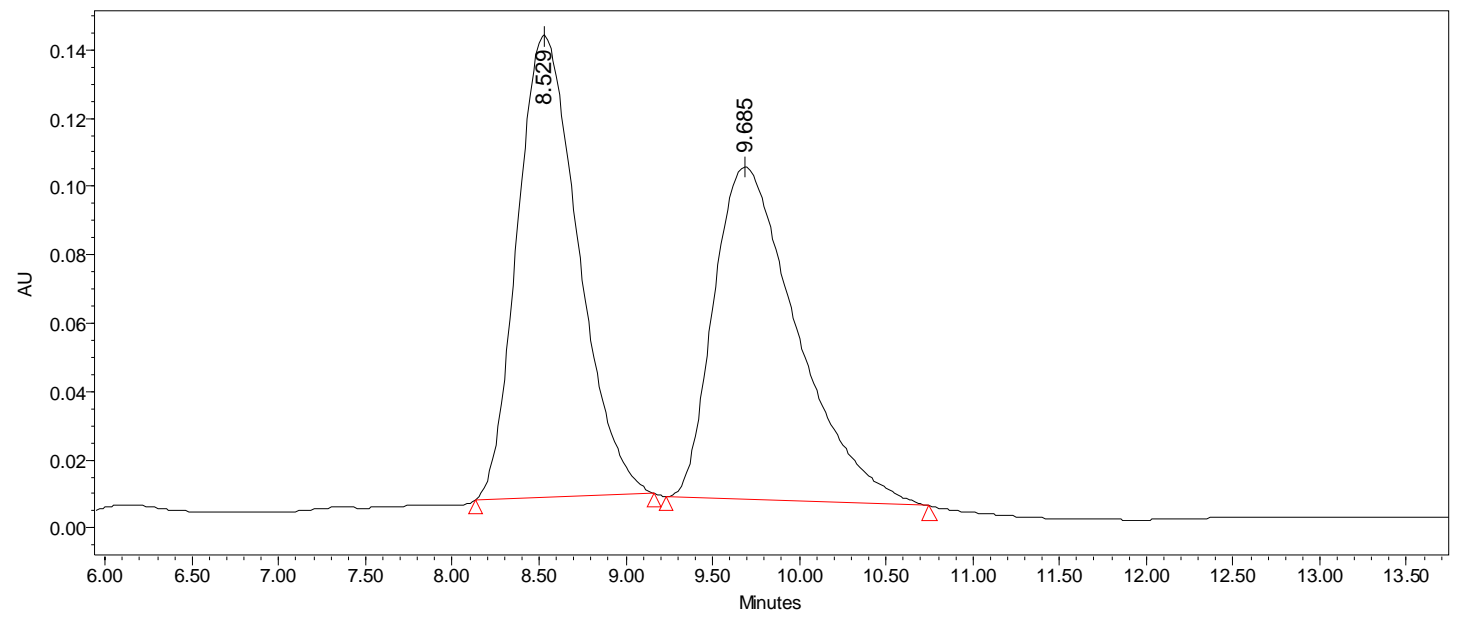

\begin{tabular}{|c|c|c|c|c|c|}
\hline & $\begin{array}{c}\mathrm{RT} \\
(\mathrm{min})\end{array}$ & $\begin{array}{c}\text { Area } \\
\left(\mathrm{V}^{*} \mathrm{sec}\right)\end{array}$ & $\%$ Area & $\begin{array}{c}\text { Height } \\
(\mathrm{V})\end{array}$ & $\begin{array}{c}\% \\
\text { Height }\end{array}$ \\
\hline 1 & 8.529 & 3297843 & 50.30 & 135167 & 58.13 \\
\hline 2 & 9.685 & 3258773 & 49.70 & 97339 & 41.87 \\
\hline
\end{tabular}
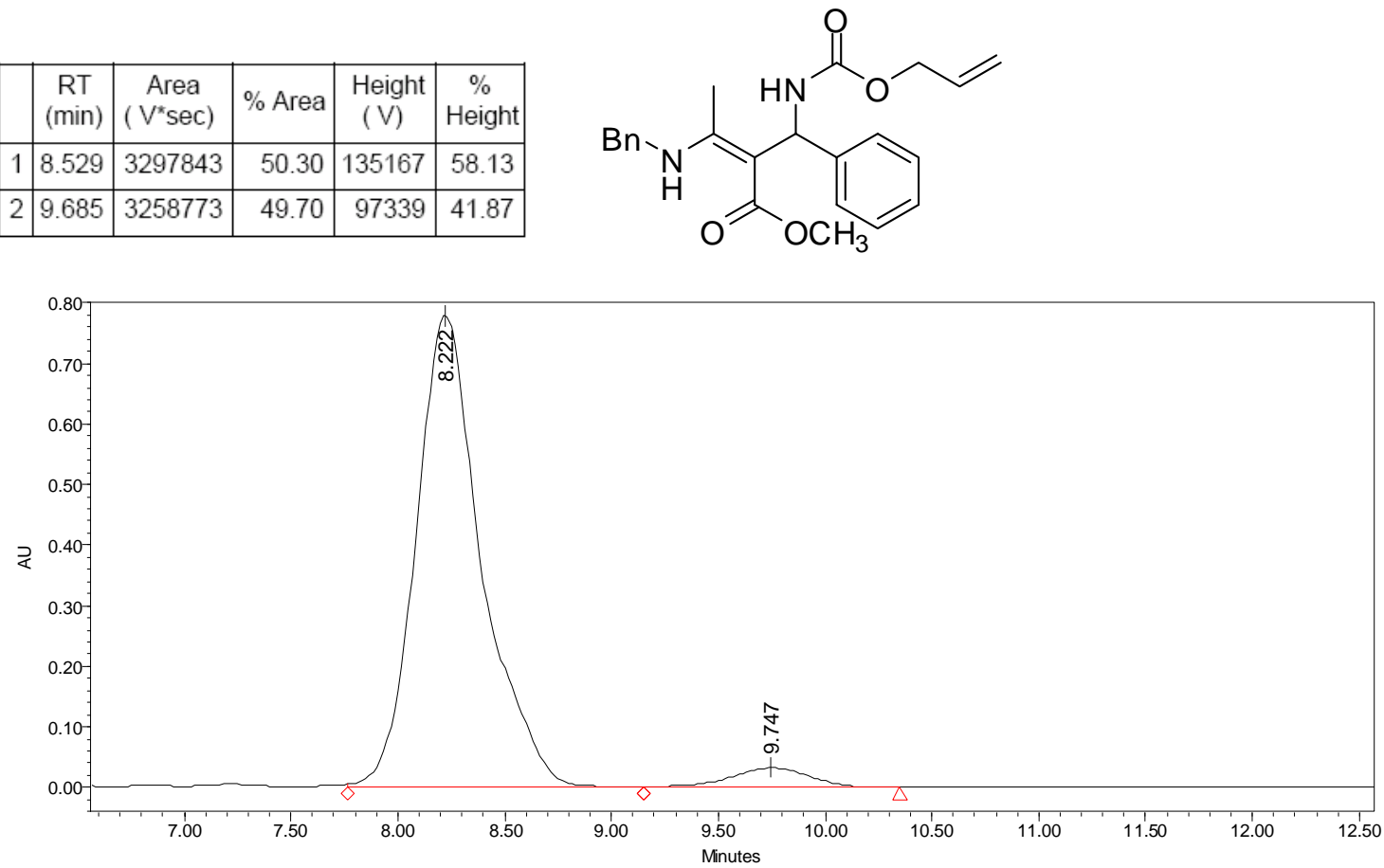

\begin{tabular}{|l|c|r|r|r|r|}
\hline & $\begin{array}{c}\text { RT } \\
(\mathrm{min})\end{array}$ & $\begin{array}{c}\text { Area } \\
\left(\mathrm{V}^{*} \mathrm{sec}\right)\end{array}$ & $\%$ Area & $\begin{array}{c}\text { Height } \\
(\mathrm{V})\end{array}$ & $\begin{array}{c}\% \\
\text { Height }\end{array}$ \\
\hline 1 & 8.222 & 15672155 & 95.62 & 763261 & 96.18 \\
\hline 2 & 9.747 & 717286 & 4.38 & 30310 & 3.82 \\
\hline
\end{tabular}<smiles>C=CCOC(=O)N[C@@H](/C(C(=O)OC)=C(\C)NCc1ccccc1)c1ccccc1</smiles> 
Table 2, entry 22: 9f Chiralcel OD-H Column, Hexane:IPA = 96:4, $1.0 \mathrm{~mL} / \mathrm{min}), 214 \mathrm{~nm}$
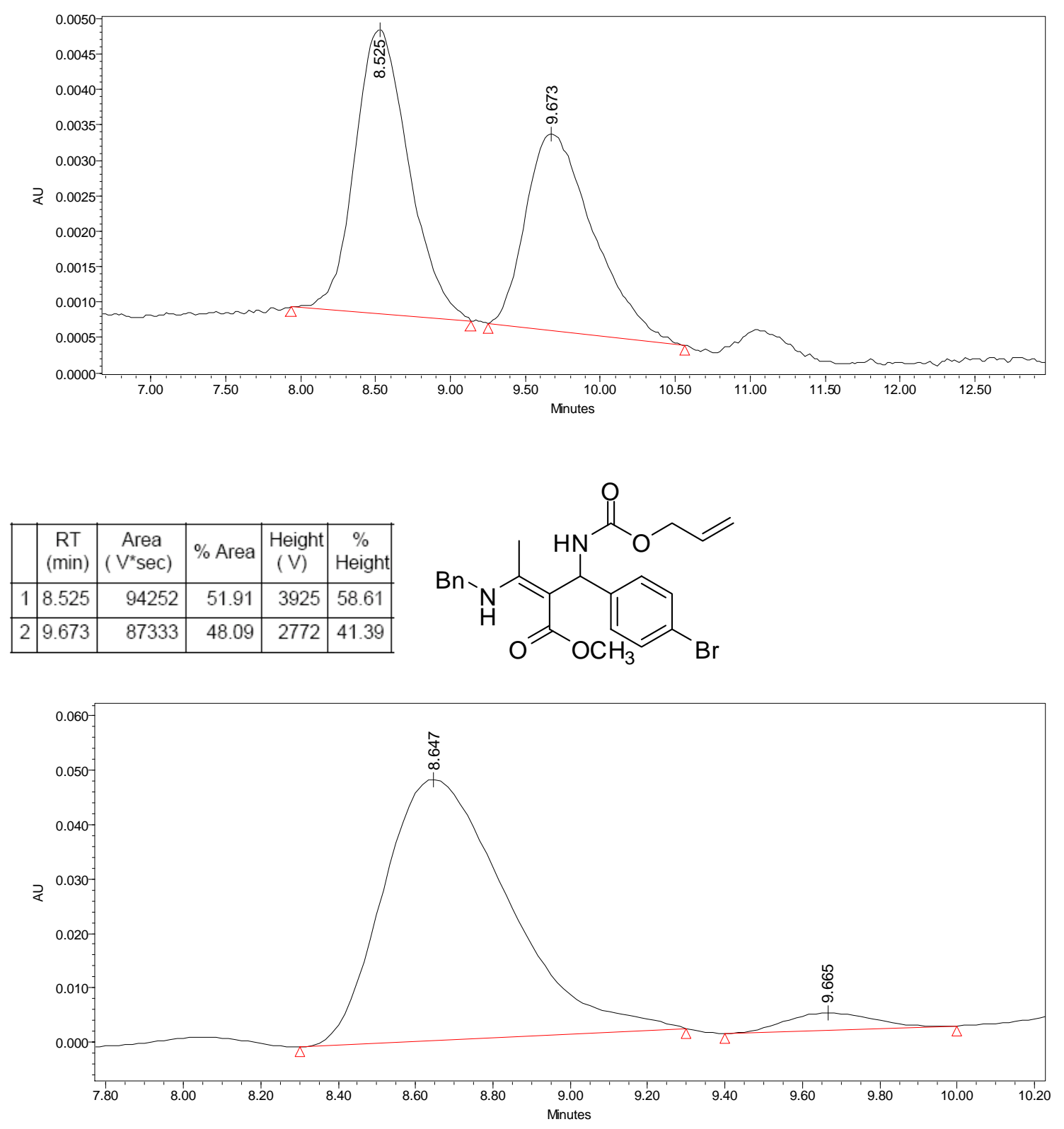

\begin{tabular}{|l|c|r|r|r|r|}
\hline & $\begin{array}{c}\text { RT } \\
(\mathrm{min})\end{array}$ & $\begin{array}{c}\text { Area } \\
\left(\mathrm{V}^{*} \mathrm{sec}\right)\end{array}$ & \% Area & $\begin{array}{c}\text { Height } \\
(\mathrm{V})\end{array}$ & $\begin{array}{c}\% \\
\text { Height }\end{array}$ \\
\hline 1 & 8.647 & 872794 & 95.01 & 44638 & 93.71 \\
\hline 2 & 9.665 & 45864 & 4.99 & 2998 & 6.29 \\
\hline
\end{tabular}<smiles>C=CCOC(=O)N[C@H](/C(C(=O)OC)=C(\C)NCc1ccccc1)c1ccc(Br)cc1</smiles> 
Table 2, entry 23: 9g: Chiralcel OD-H Column, Hexane:IPA = 96:4, $1.0 \mathrm{~mL} / \mathrm{min}), 214 \mathrm{~nm}$

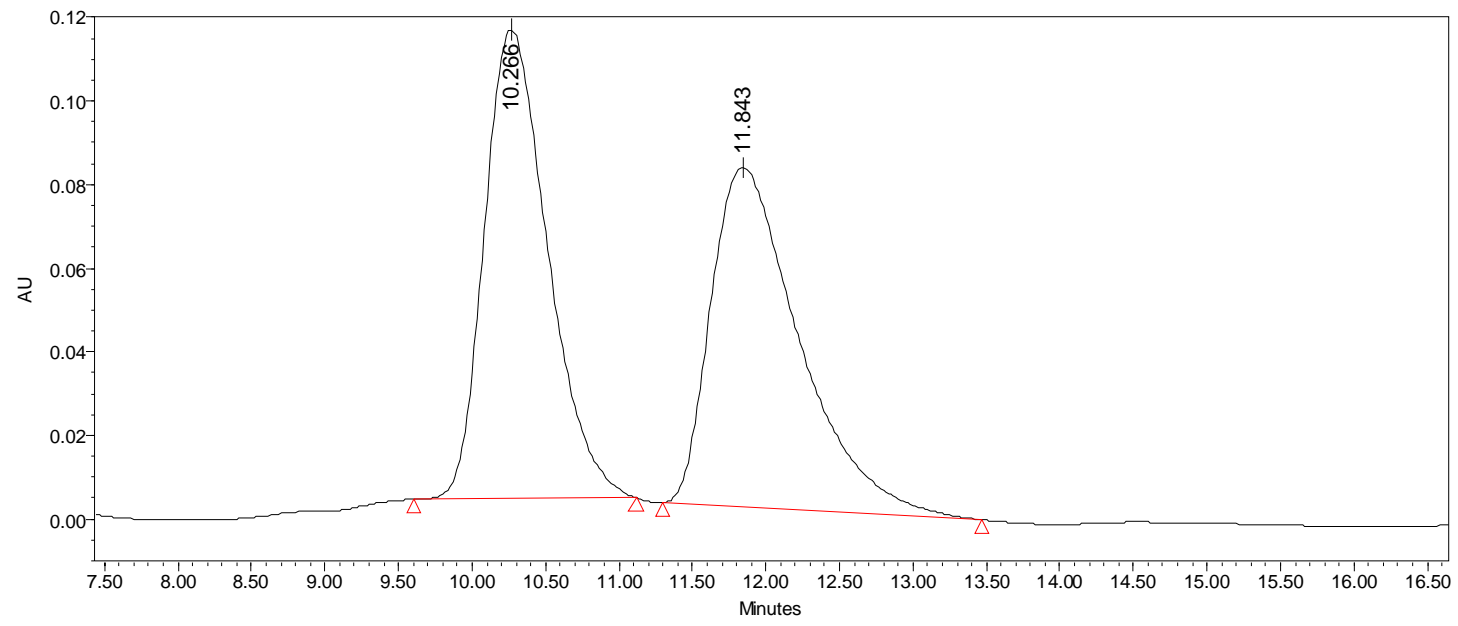

\begin{tabular}{|c|c|c|c|c|c|}
\hline & $\begin{array}{c}\text { RT } \\
(\mathrm{min})\end{array}$ & $\begin{array}{c}\text { Area } \\
\left(\mathrm{V}^{*} \text { sec }\right)\end{array}$ & $\%$ Area & $\begin{array}{c}\text { Height } \\
(\mathrm{V})\end{array}$ & $\begin{array}{c}\% \\
\text { Height }\end{array}$ \\
\hline 1 & 10.266 & 3377499 & 49.82 & 112269 & 58.01 \\
\hline 2 & 11.843 & 3401448 & 50.18 & 81262 & 41.99 \\
\hline
\end{tabular}<smiles>C=CCOC(=O)NC(/C(C(=O)OC)=C(\C)NCc1ccccc1)c1cccc(F)c1</smiles>

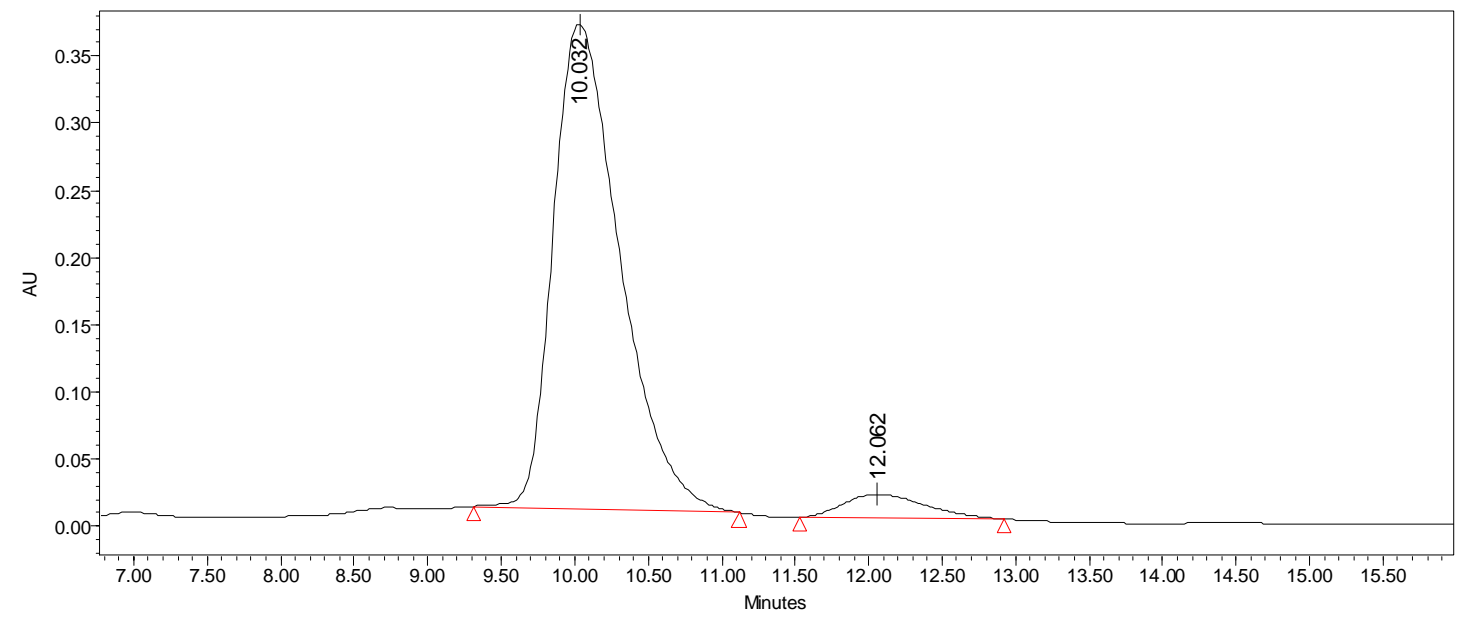

\begin{tabular}{|l|c|r|r|r|r|}
\hline & $\begin{array}{c}\mathrm{RT} \\
(\mathrm{min})\end{array}$ & $\begin{array}{c}\text { Area } \\
\left(\mathrm{V}^{\star} \mathrm{sec}\right)\end{array}$ & $\%$ Area & $\begin{array}{c}\text { Height } \\
(\mathrm{V})\end{array}$ & $\begin{array}{c}\% \\
\text { Height }\end{array}$ \\
\hline 1 & 10.032 & 11394006 & 95.08 & 360809 & 95.59 \\
\hline 2 & 12.062 & 590118 & 4.92 & 16664 & 4.41 \\
\hline
\end{tabular}<smiles>C=CCOC(=O)NC(/C(C(=O)O)=C(\C)NCc1ccccc1)c1cccc(F)c1</smiles> 
Table 2, entry 23: 9h: Chiralcel OD-H Column, Hexane:IPA = 95:5, $1.0 \mathrm{~mL} / \mathrm{min}), 214 \mathrm{~nm}$
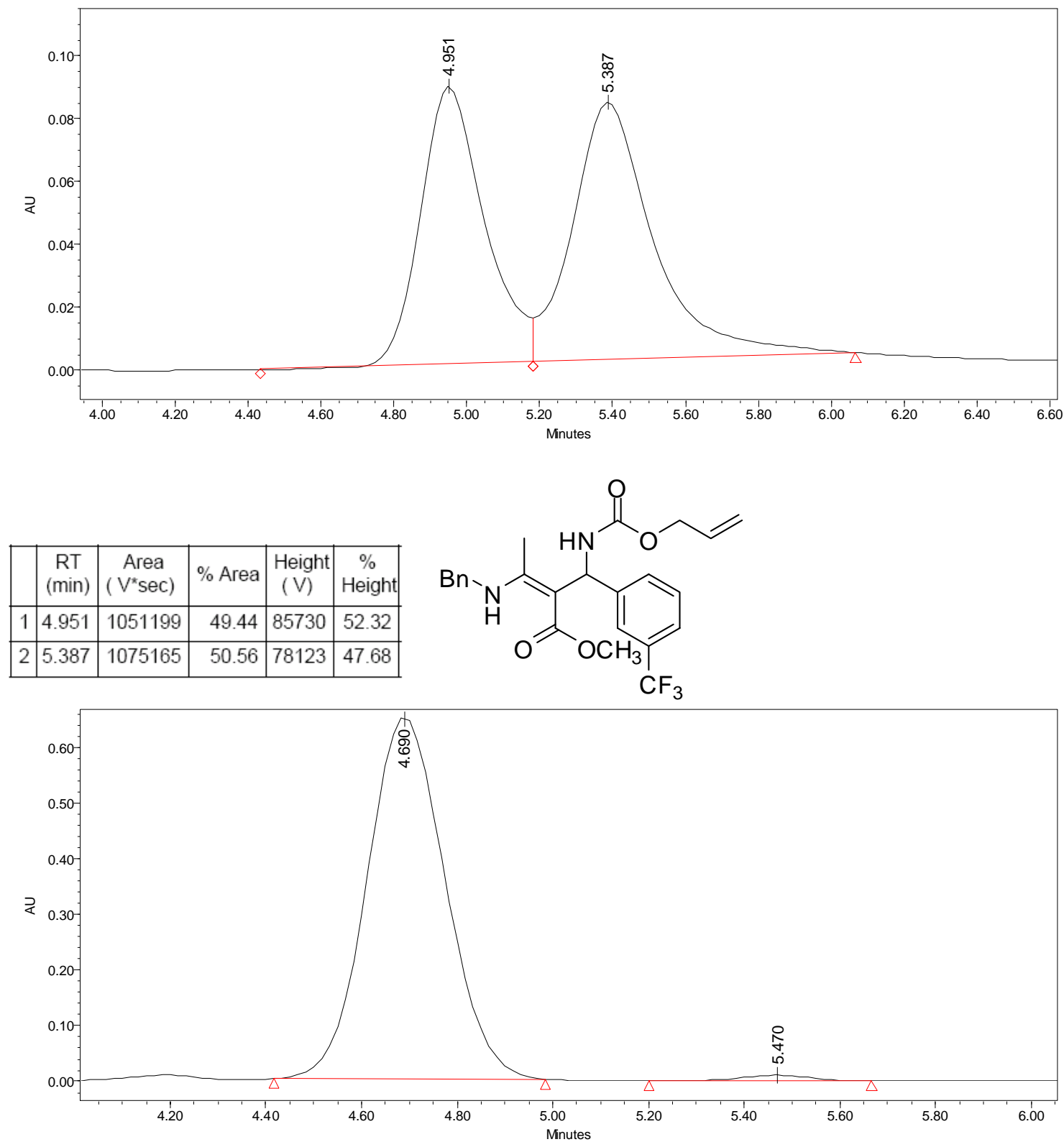

\begin{tabular}{|c|c|c|r|r|r|}
\hline & $\begin{array}{c}\text { RT } \\
(\mathrm{min})\end{array}$ & $\begin{array}{c}\text { Area } \\
\left(\mathrm{V}^{*} \mathrm{sec}\right)\end{array}$ & $\%$ Area & $\begin{array}{c}\text { Height } \\
(\mathrm{V})\end{array}$ & $\begin{array}{c}\% \\
\text { Height }\end{array}$ \\
\hline 1 & 4.690 & 2310065 & 95.57 & 343964 & 97.06 \\
\hline 2 & 5.470 & 107183 & 4.43 & 10419 & 2.94 \\
\hline
\end{tabular}<smiles>C=CCOC(=O)N[C@H](C(C(=O)OC)=C(C)NCc1ccccc1)c1cccc(C(F)(F)F)c1</smiles> 
Table 2, entry 23: 9i: Chiralcel OD-H Column, Hexane:IPA = 98:2, $1.0 \mathrm{~mL} / \mathrm{min}), 214 \mathrm{~nm}$

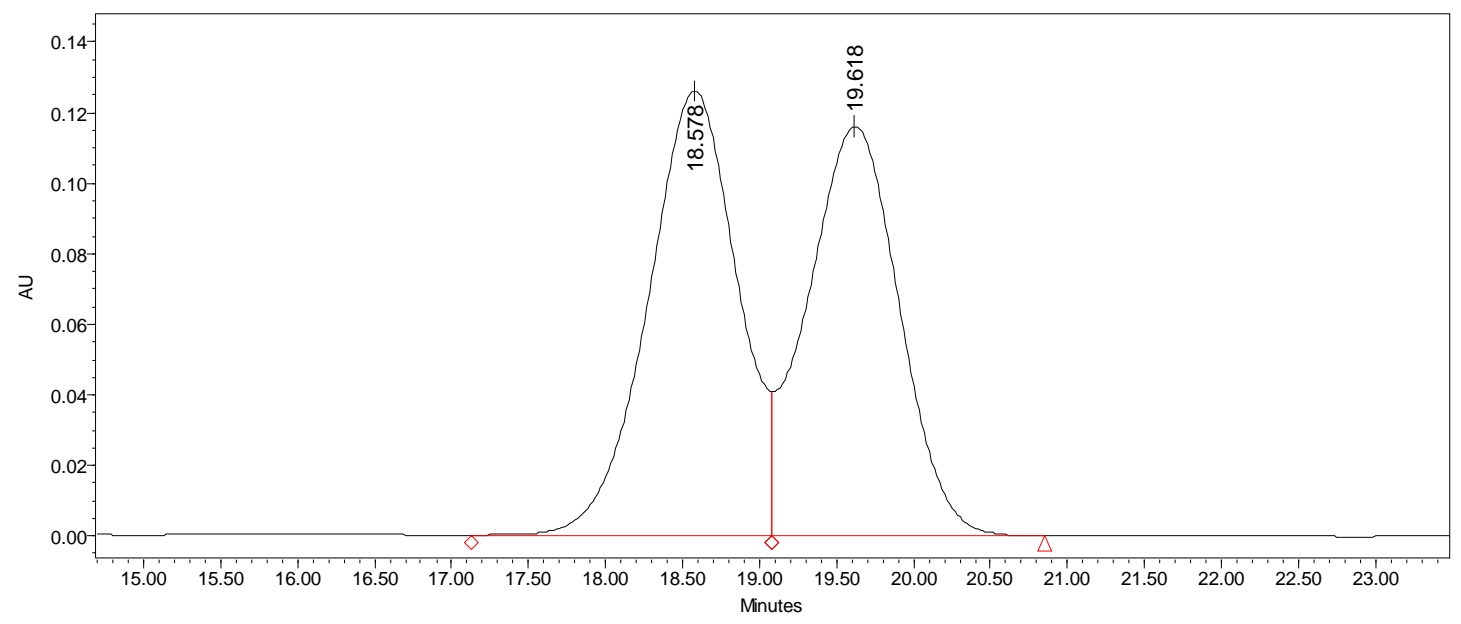

\begin{tabular}{|c|c|c|c|c|c|}
\hline & $\begin{array}{c}\mathrm{RT} \\
(\mathrm{min})\end{array}$ & $\begin{array}{c}\text { Area } \\
\left(\mathrm{V}^{*} \mathrm{sec}\right)\end{array}$ & \% Area & $\begin{array}{c}\text { Height } \\
(\mathrm{V})\end{array}$ & $\begin{array}{c}\% \\
\text { Height }\end{array}$ \\
\hline 1 & 18.578 & 5124119 & 51.48 & 126195 & 52.08 \\
\hline 2 & 19.618 & 4830160 & 48.52 & 116109 & 47.92 \\
\hline
\end{tabular}
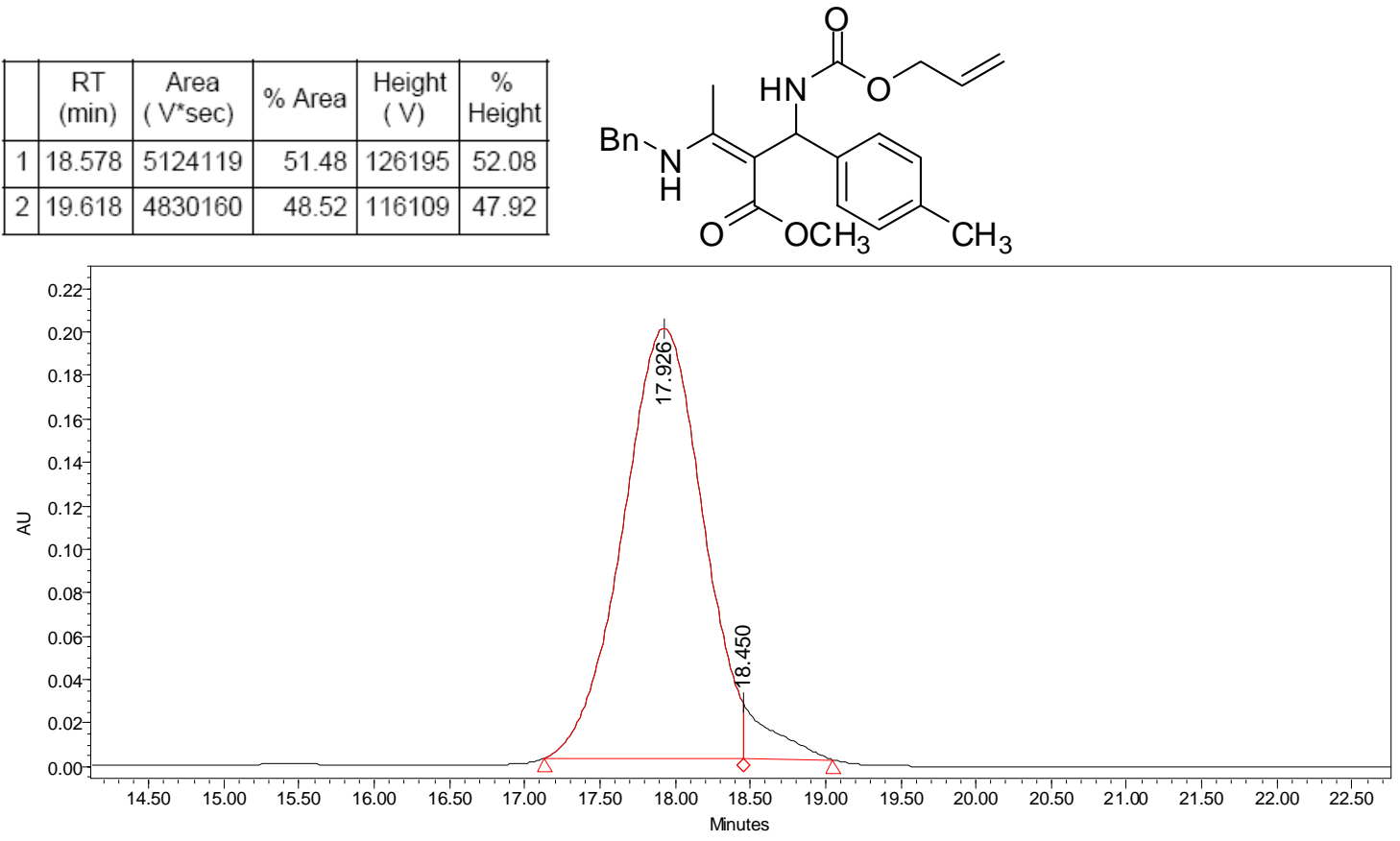

\begin{tabular}{|c|c|c|r|r|c|}
\hline & $\begin{array}{c}\text { RT } \\
(\mathrm{min})\end{array}$ & $\begin{array}{c}\text { Area } \\
\left(\mathrm{V}^{*} \mathrm{sec}\right)\end{array}$ & $\%$ Area & $\begin{array}{c}\text { Height } \\
(\mathrm{V})\end{array}$ & $\begin{array}{c}\% \\
\text { Height }\end{array}$ \\
\hline 1 & 17.926 & 7266103 & 95.27 & 197906 & 88.34 \\
\hline 2 & 18.450 & 360450 & 4.73 & 26132 & 11.66 \\
\hline
\end{tabular}<smiles>C=CCOC(=O)N[C@H](C(C(=O)OC)=C(C)NCc1ccccc1)c1ccc(C)cc1</smiles> 
Table 2, entry 26: 9j: Chiralcel OD Column, Hexane:IPA = 96:4, $1.0 \mathrm{~mL} / \mathrm{min}), 214 \mathrm{~nm}$

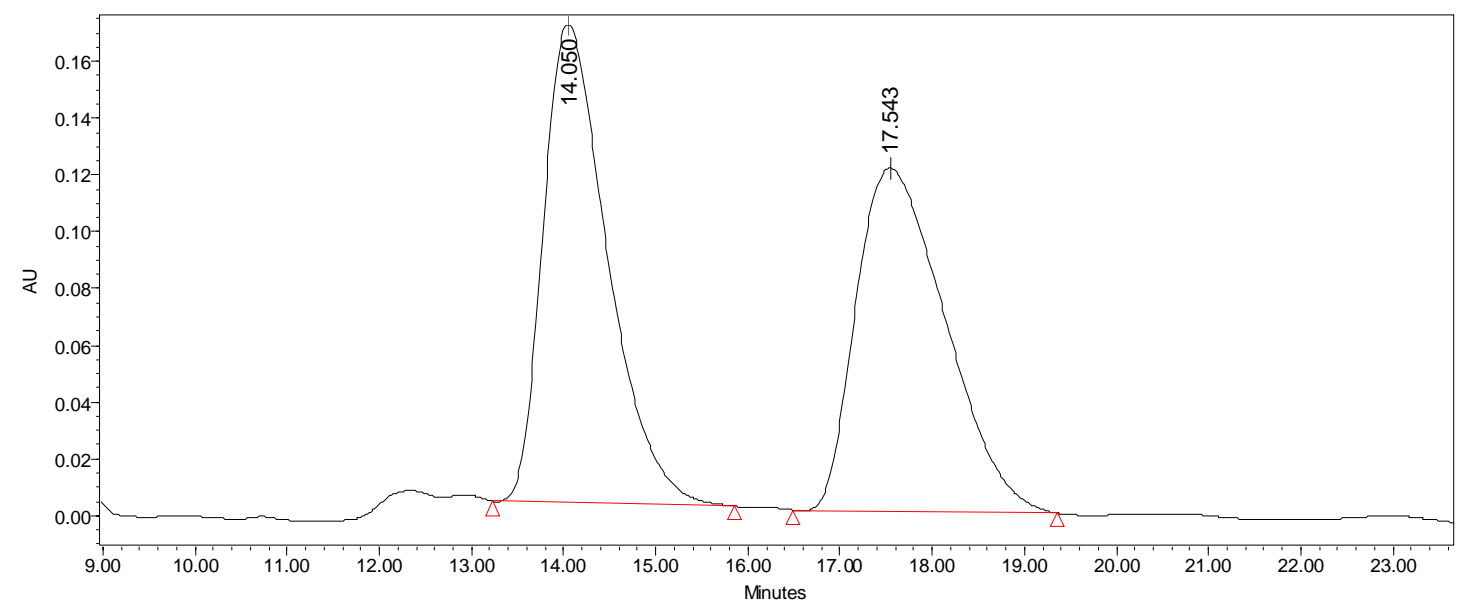

\begin{tabular}{|c|c|c|c|c|c|}
\hline & $\begin{array}{c}\mathrm{RT} \\
(\mathrm{min})\end{array}$ & $\begin{array}{c}\text { Area } \\
\left(\mathrm{V}^{*} \text { sec }\right)\end{array}$ & $\%$ Area & $\begin{array}{c}\text { Height } \\
(\mathrm{V})\end{array}$ & $\begin{array}{c}\% \\
\text { Height }\end{array}$ \\
\hline 1 & 14.050 & 8177719 & 50.11 & 168532 & 58.25 \\
\hline 2 & 17.543 & 8143325 & 49.89 & 120806 & 41.75 \\
\hline
\end{tabular}<smiles>C=CCOC(=O)NC(/C(C)=N/Cc1ccccc1)c1cccc(OC)c1</smiles>

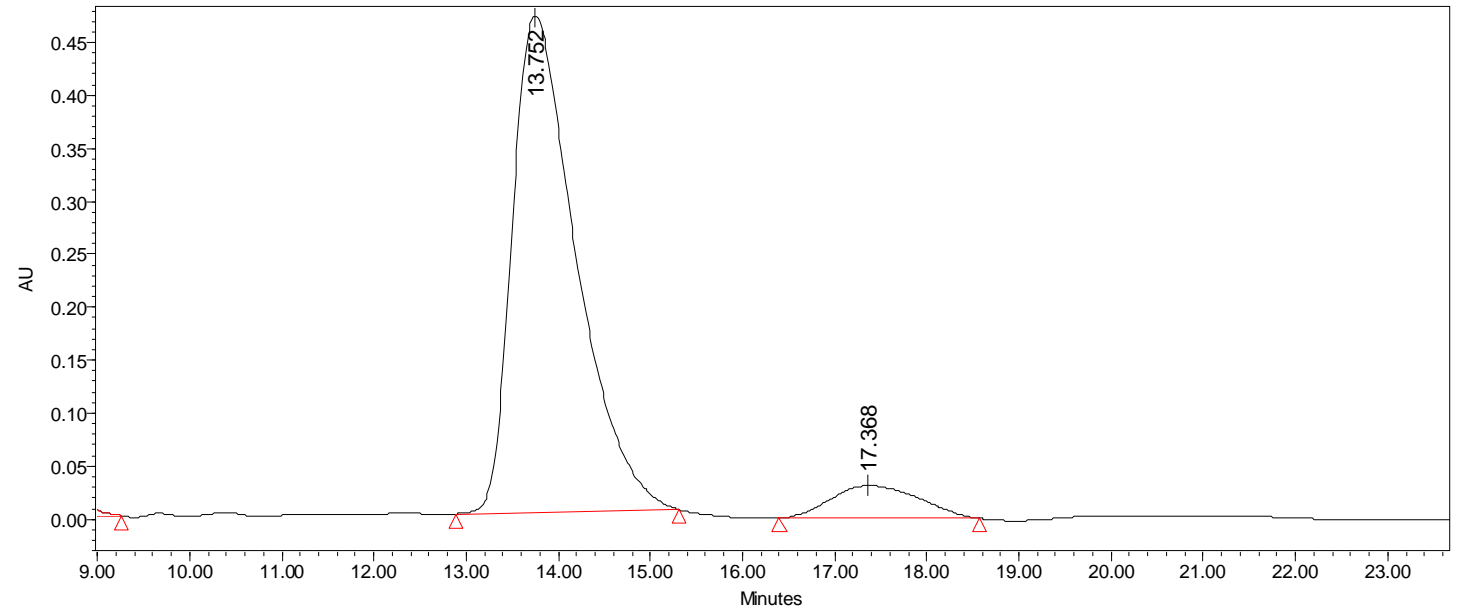

\begin{tabular}{|l|c|c|r|r|r|}
\hline & $\begin{array}{c}\mathrm{RT} \\
(\mathrm{min})\end{array}$ & $\begin{array}{c}\text { Area } \\
\left(\mathrm{V}^{*} \mathrm{sec}\right)\end{array}$ & $\%$ Area & $\begin{array}{l}\text { Height } \\
(\mathrm{V})\end{array}$ & $\begin{array}{c}\% \\
\text { Height }\end{array}$ \\
\hline 1 & 13.752 & 22617004 & 94.45 & 468467 & 95.27 \\
\hline 2 & 17.368 & 1330253 & 5.55 & 23251 & 4.73 \\
\hline
\end{tabular}<smiles>C=CCOC(=O)N[C@H](C(C)=C(C)NCc1ccccc1)c1cccc(OC)c1</smiles> 
Table 2, entry 27: 9k: Chiralcel OD Column, Hexane:IPA = 95:5, $1.0 \mathrm{~mL} / \mathrm{min}), 214 \mathrm{~nm}$

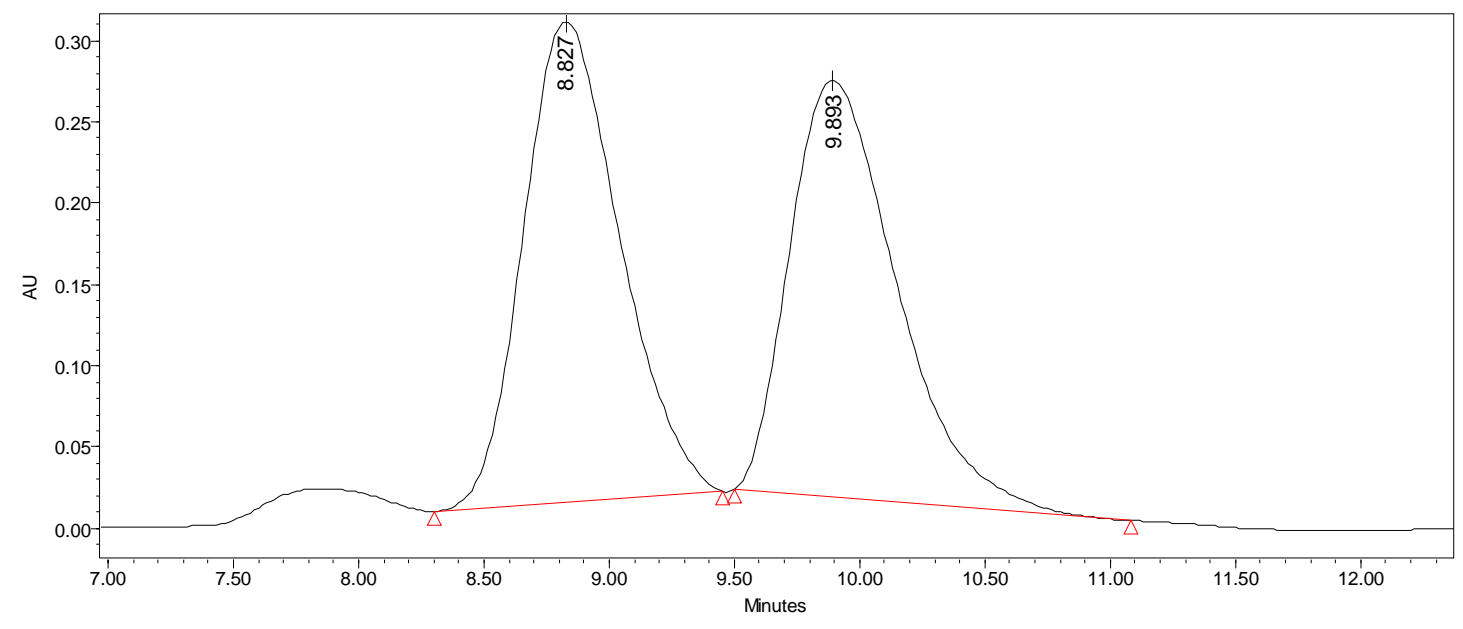

\begin{tabular}{|c|c|c|c|c|c|}
\hline & $\begin{array}{c}\text { RT } \\
(\mathrm{min})\end{array}$ & $\begin{array}{c}\text { Area } \\
\left(\mathrm{V}^{*} \text { sec }\right)\end{array}$ & $\%$ Area & $\begin{array}{c}\text { Height } \\
(\mathrm{V})\end{array}$ & $\begin{array}{c}\% \\
\text { Height }\end{array}$ \\
\hline 1 & 8.827 & 7913302 & 50.91 & 295774 & 53.55 \\
\hline 2 & 9.893 & 7631471 & 49.09 & 256508 & 46.45 \\
\hline
\end{tabular}<smiles>C=CCOC(=O)NC(/C(C(=O)OC)=C(\C)NCc1ccccc1)c1ccc2c(c1)OCO2</smiles>

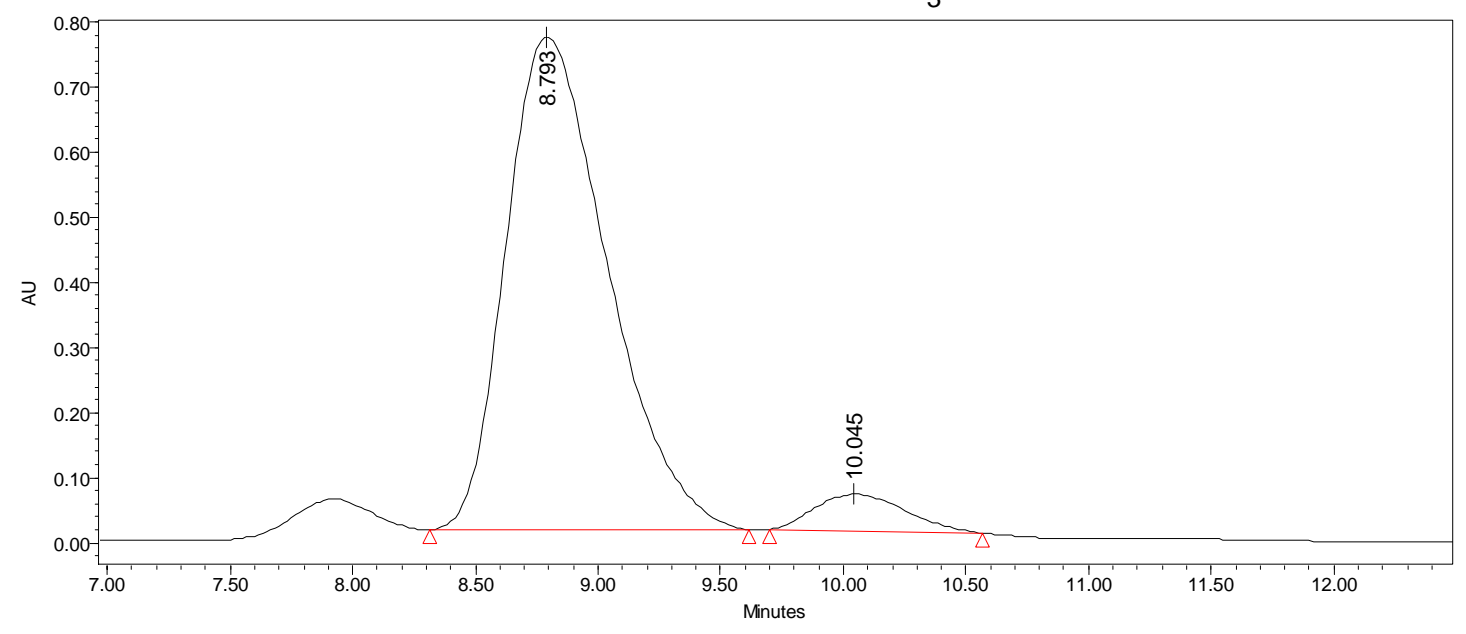

\begin{tabular}{|c|c|c|r|r|r|}
\hline & $\begin{array}{c}\text { RT } \\
(\mathrm{min})\end{array}$ & $\begin{array}{c}\text { Area } \\
\left(\mathrm{V}^{*} \mathrm{sec}\right)\end{array}$ & $\%$ Area & $\begin{array}{c}\text { Height } \\
(\mathrm{V})\end{array}$ & $\begin{array}{c}\% \\
\text { Height }\end{array}$ \\
\hline 1 & 8.793 & 21534704 & 93.19 & 753728 & 92.82 \\
\hline 2 & 10.045 & 1572904 & 6.81 & 58323 & 7.18 \\
\hline
\end{tabular}<smiles>C=CCOC(=O)N[C@H](/C(C(=O)OC)=C(\C)NCc1ccccc1)c1ccc2c(c1)OCO2</smiles> 
Table 2, entry 28: 9l Chiralcel OD-H Column, Hexane:IPA = 96:4, $1.0 \mathrm{~mL} / \mathrm{min}), 214 \mathrm{~nm}$

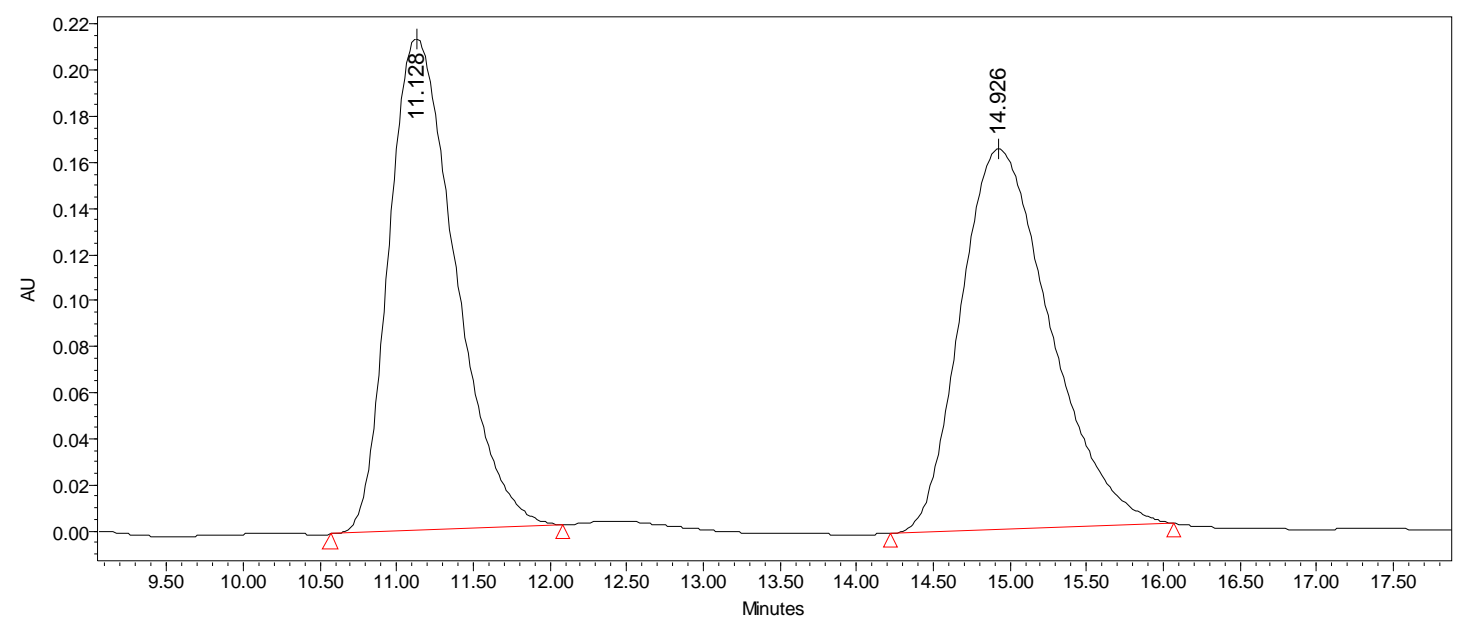

\begin{tabular}{|c|c|c|c|c|c|}
\hline & $\begin{array}{c}\mathrm{RT} \\
(\mathrm{min})\end{array}$ & $\begin{array}{c}\text { Area } \\
\left(\mathrm{V}^{*} \mathrm{sec}\right)\end{array}$ & $\%$ Area & $\begin{array}{c}\text { Height } \\
(\mathrm{V})\end{array}$ & $\begin{array}{c}\% \\
\text { Height }\end{array}$ \\
\hline 1 & 11.128 & 6445389 & 48.89 & 213794 & 56.40 \\
\hline 2 & 14.926 & 6736995 & 51.11 & 165267 & 43.60 \\
\hline
\end{tabular}
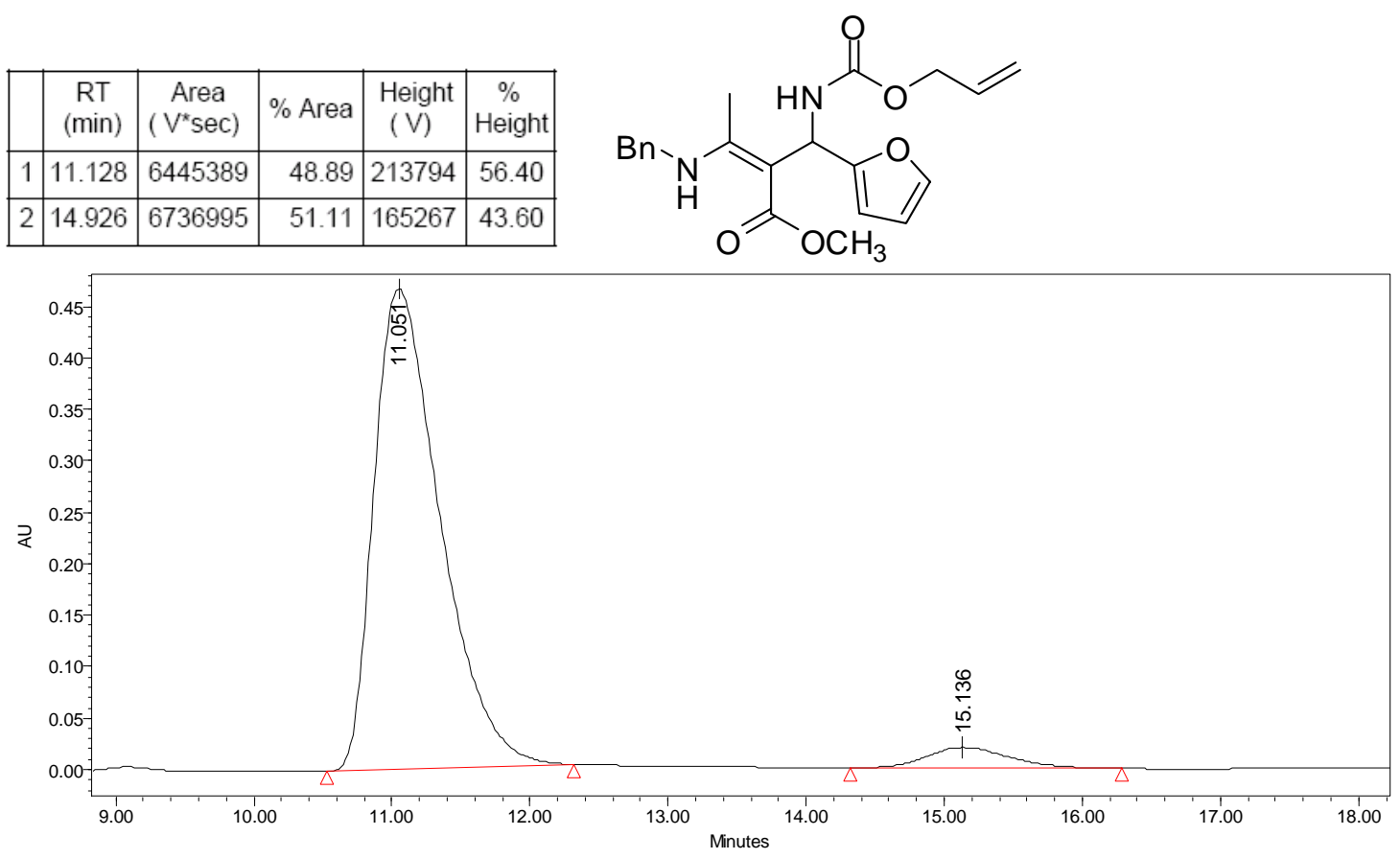

\begin{tabular}{|l|c|r|r|r|r|}
\hline & $\begin{array}{c}\mathrm{RT} \\
(\mathrm{min})\end{array}$ & $\begin{array}{c}\text { Area } \\
\left(\mathrm{V}^{*} \mathrm{sec}\right)\end{array}$ & $\%$ Area & $\begin{array}{l}\text { Height } \\
(\mathrm{V})\end{array}$ & $\begin{array}{c}\% \\
\text { Height }\end{array}$ \\
\hline 1 & 11.051 & 15564079 & 95.04 & 467654 & 95.91 \\
\hline 2 & 15.136 & 812265 & 4.96 & 19934 & 4.09 \\
\hline
\end{tabular}<smiles>C=CCOC(=O)N[C@H](C(C(=O)OC)=C(C)NCc1ccccc1)c1ccco1</smiles> 
Table 3, entry 1: 12a ChiralPak AD Column, Hexanes : IPA $=95: 5,1.0 \mathrm{~mL} / \mathrm{min}, 210 \mathrm{~nm}$

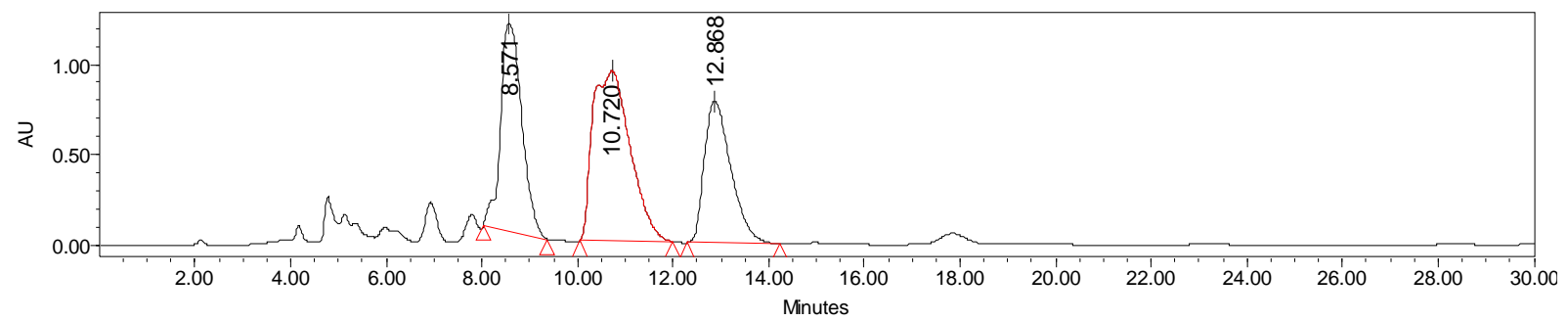

\begin{tabular}{|r|r|r|r|r|r|}
\hline & Retention Time & Area & \% Area & Height & \% Height \\
\hline 1 & 8.571 & 35599661 & 30.44 & 1143644 & 39.94 \\
\hline 2 & 10.720 & 51037670 & 43.64 & 936775 & 32.72 \\
\hline 3 & 12.868 & 30318025 & 25.92 & 782922 & 27.34 \\
\hline
\end{tabular}
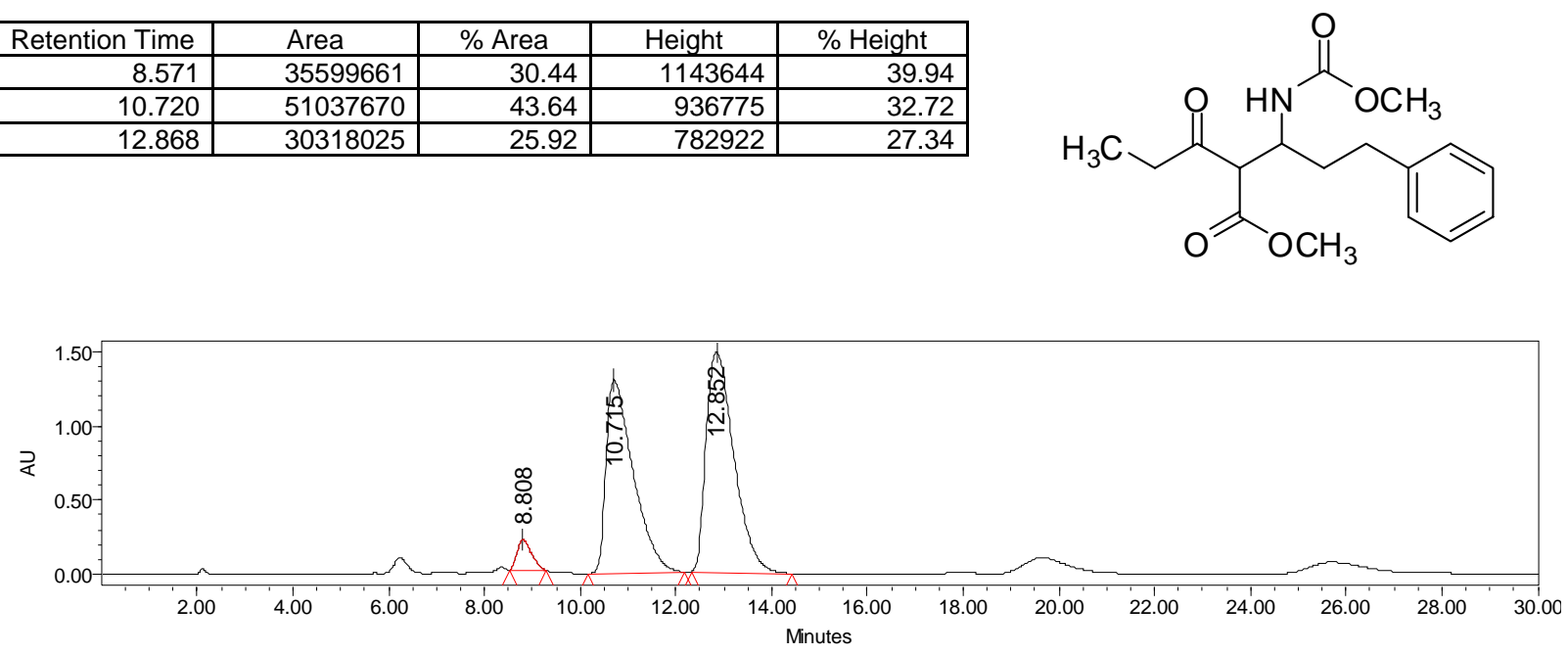

\begin{tabular}{|r|r|r|r|r|r|}
\hline & Retention Time & \multicolumn{1}{c|}{ Area } & \% Area & \multicolumn{1}{c|}{ Height } & \% Height \\
\hline 1 & 8.808 & 3027340 & 2.65 & 170544 & 5.77 \\
\hline 2 & 10.715 & 51362548 & 44.94 & 1301694 & 44.06 \\
\hline 3 & 12.852 & 59897434 & 52.41 & 1482263 & 50.17 \\
\hline
\end{tabular}<smiles>CCC(=O)C(C(=O)OC)[C@H](CCc1ccccc1)NC(=O)OC</smiles> 
Table 3, entry 2: 12b ChiralPak AD Column, Hexanes : IPA $=95: 5,1.0 \mathrm{~mL} / \mathrm{min}, 210 \mathrm{~nm}$

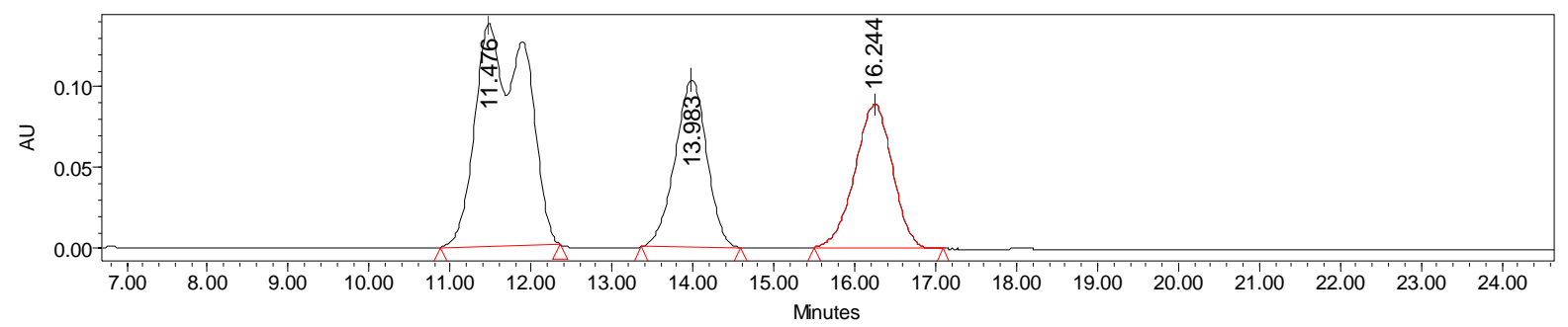

\begin{tabular}{|r|r|r|r|r|r|}
\hline & Retention Time & Area & \% Area & Height & \% Height \\
\hline 1 & 11.476 & 5971766 & 51.14 & 137702 & 41.71 \\
\hline 2 & 13.983 & 2845412 & 24.37 & 103676 & 31.40 \\
\hline 3 & 16.244 & 2860656 & 24.50 & 88761 & 26.89 \\
\hline
\end{tabular}<smiles>COC(=O)NC(CCc1ccccc1)C(C(=O)OC)C(=O)c1ccccc1</smiles>

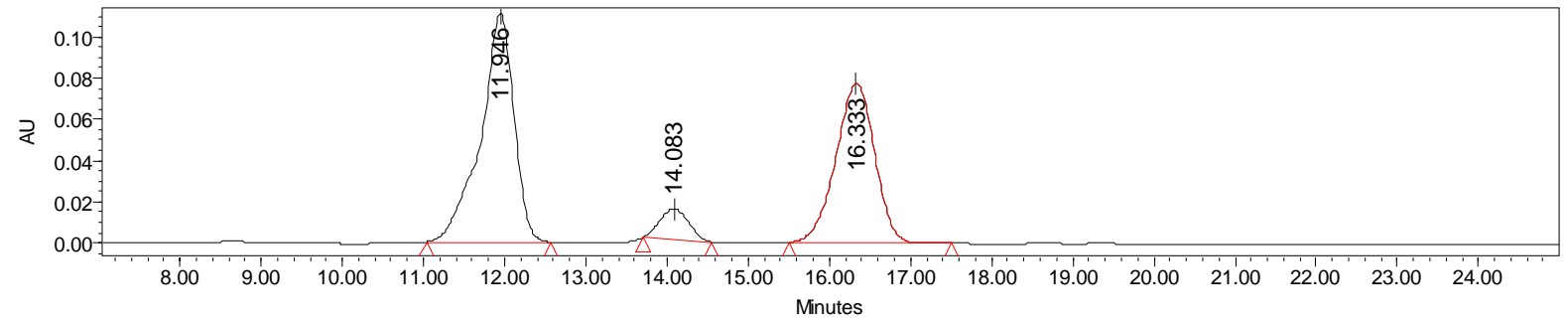

\begin{tabular}{|r|r|r|r|r|r|}
\hline & Retention Time & \multicolumn{1}{c|}{ Area } & \% Area & Height & \multicolumn{1}{c|}{ \% Height } \\
\hline 1 & 11.946 & 3173892 & 51.96 & 110771 & 54.52 \\
\hline 2 & 14.083 & 367489 & 6.02 & 14882 & 7.32 \\
\hline 3 & 16.333 & 2567259 & 42.03 & 77523 & 38.16 \\
\hline
\end{tabular}<smiles>COC(=O)N[C@@H](CCc1ccccc1)C(C(=O)OC)C(=O)c1ccccc1</smiles> 
Table 3, entry 3: 12c ChiralPak AD Column, Hexanes : IPA = 95:5, $1.0 \mathrm{~mL} / \mathrm{min}, 210 \mathrm{~nm}$

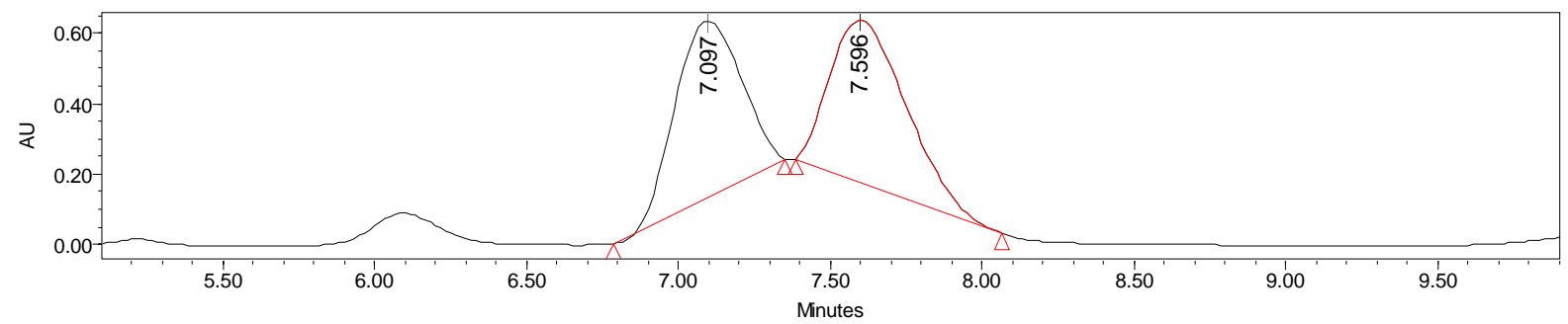

\begin{tabular}{|r|r|r|r|r|r|}
\hline & Retention Time & Area & \% Area & Height & \% Height \\
\hline 1 & 7.097 & 7658981 & 48.45 & 500272 & 52.05 \\
\hline 2 & 7.596 & 8147998 & 51.55 & 460944 & 47.95 \\
\hline
\end{tabular}<smiles>COCC(=O)C(C(=O)OC)C(CCc1ccccc1)NC(=O)OC</smiles>

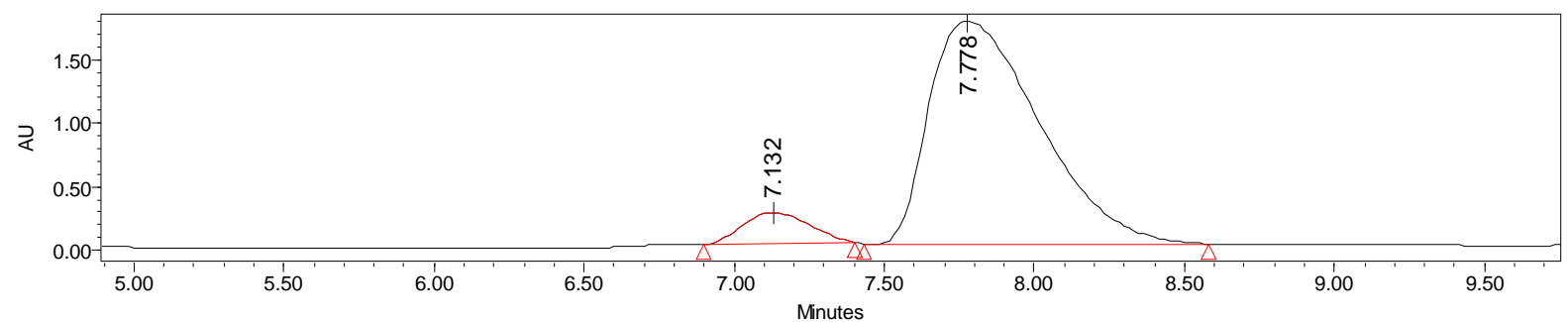

\begin{tabular}{|r|r|r|r|r|r|}
\hline & Retention Time & \multicolumn{1}{c|}{ Area } & \% Area & Height & \% Height \\
\hline 1 & 7.132 & 2620430 & 5.52 & 197982 & 10.15 \\
\hline 2 & 7.778 & 44860951 & 94.48 & 1751940 & 89.85 \\
\hline
\end{tabular}<smiles>COCC(=O)C(C(=O)OC)[C@H](CCc1ccccc1)NC(=O)OC</smiles> 
Table 4, entry 1: 14a ChiralPak AD-H Column, Hexane:IPA = 95:5, $1.0 \mathrm{~mL} / \mathrm{min}, 214 \mathrm{~nm}$ racemate:

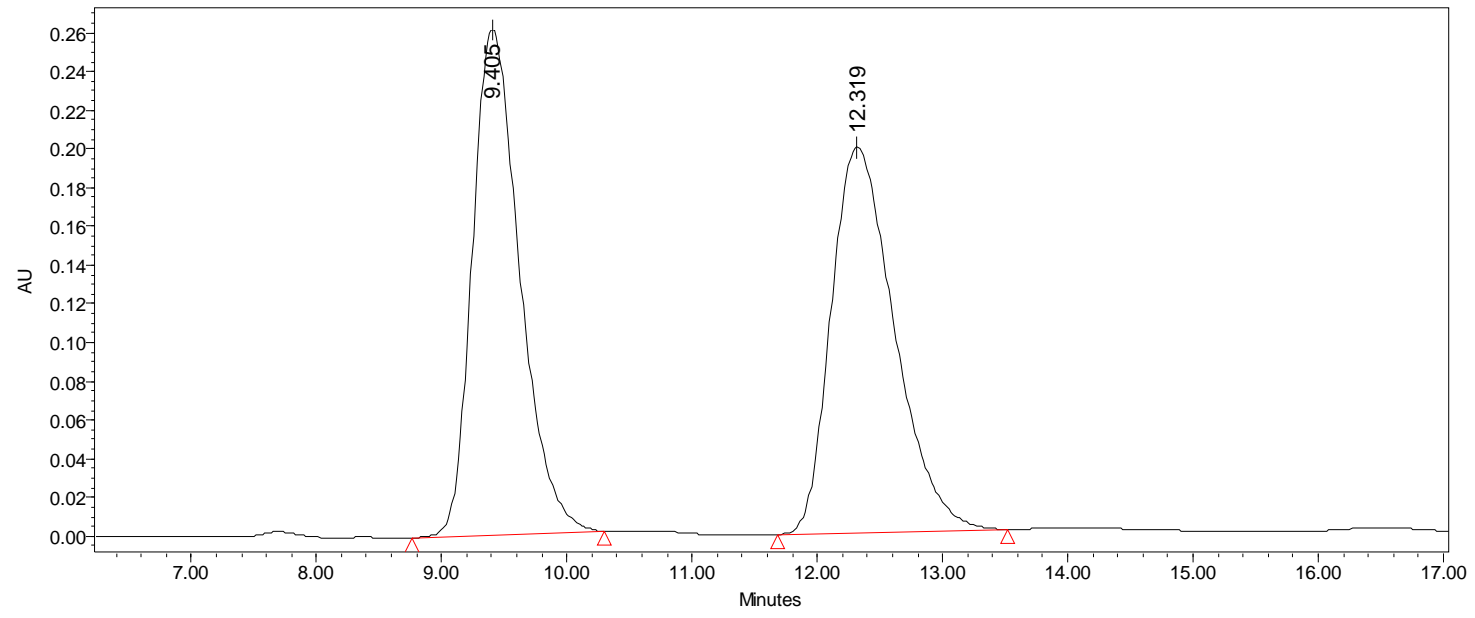

\begin{tabular}{|c|c|c|c|c|c|}
\hline & $\begin{array}{c}\text { RT } \\
(\mathrm{min})\end{array}$ & $\begin{array}{c}\text { Area } \\
\left(\mathrm{V}^{*} \mathrm{sec}\right)\end{array}$ & $\%$ Area & $\begin{array}{c}\text { Height } \\
(\mathrm{V})\end{array}$ & $\begin{array}{c}\% \\
\text { Height }\end{array}$ \\
\hline 1 & 9.405 & 6883509 & 49.63 & 261359 & 56.79 \\
\hline 2 & 12.319 & 6986151 & 50.37 & 198898 & 43.21 \\
\hline
\end{tabular}<smiles>C=CCOC(=O)NC(CCc1ccccc1)C(C(C)=O)C(C)=O</smiles>

product from cinchonine catalyzed reaction:

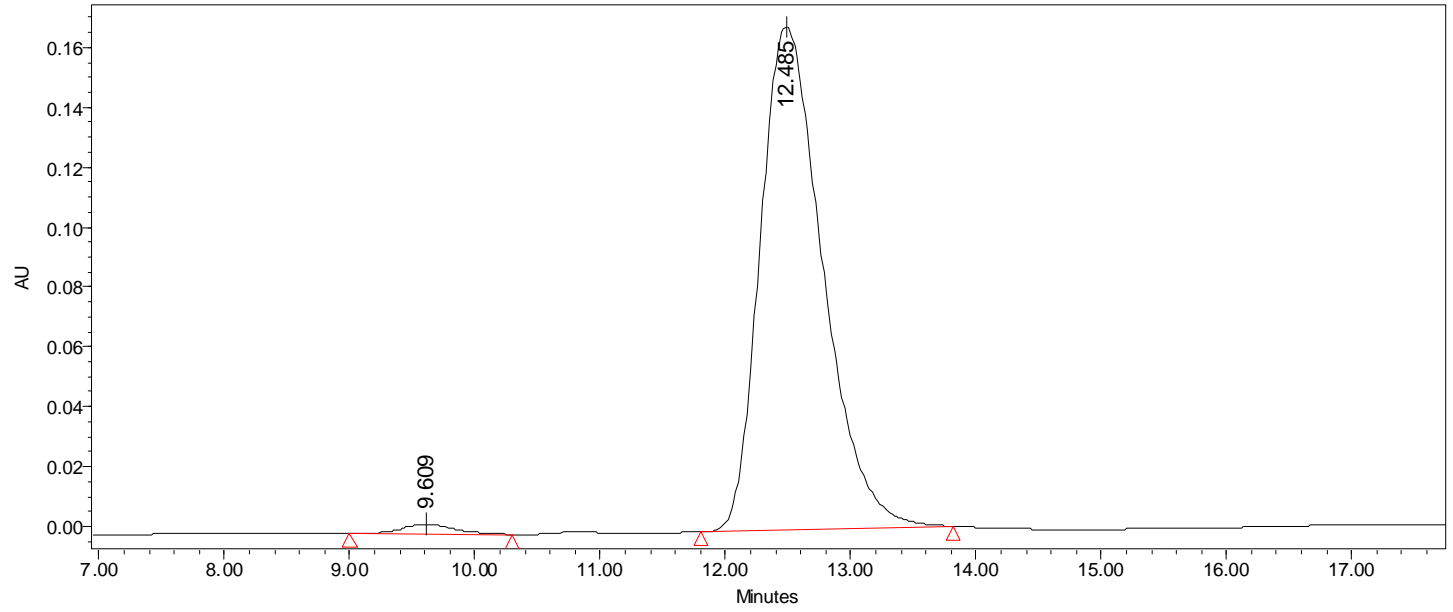

\begin{tabular}{|c|c|c|r|r|r|}
\hline & $\begin{array}{c}\text { RT } \\
(\mathrm{min})\end{array}$ & $\begin{array}{c}\text { Area } \\
\left(\mathrm{V}^{*} \mathrm{sec}\right)\end{array}$ & $\%$ Area & $\begin{array}{c}\text { Height } \\
(\mathrm{V})\end{array}$ & $\begin{array}{c}\% \\
\text { Height }\end{array}$ \\
\hline 1 & 9.609 & 101774 & 4.81 & 3405 & 3.46 \\
\hline 2 & 12.485 & 2014827 & 95.19 & 94927 & 96.54 \\
\hline
\end{tabular}<smiles>C=CCOC(=O)N[C@@H](CCc1ccccc1)C(C(C)=O)C(C)=O</smiles>

product from cinchonidine catalyzed reaction 


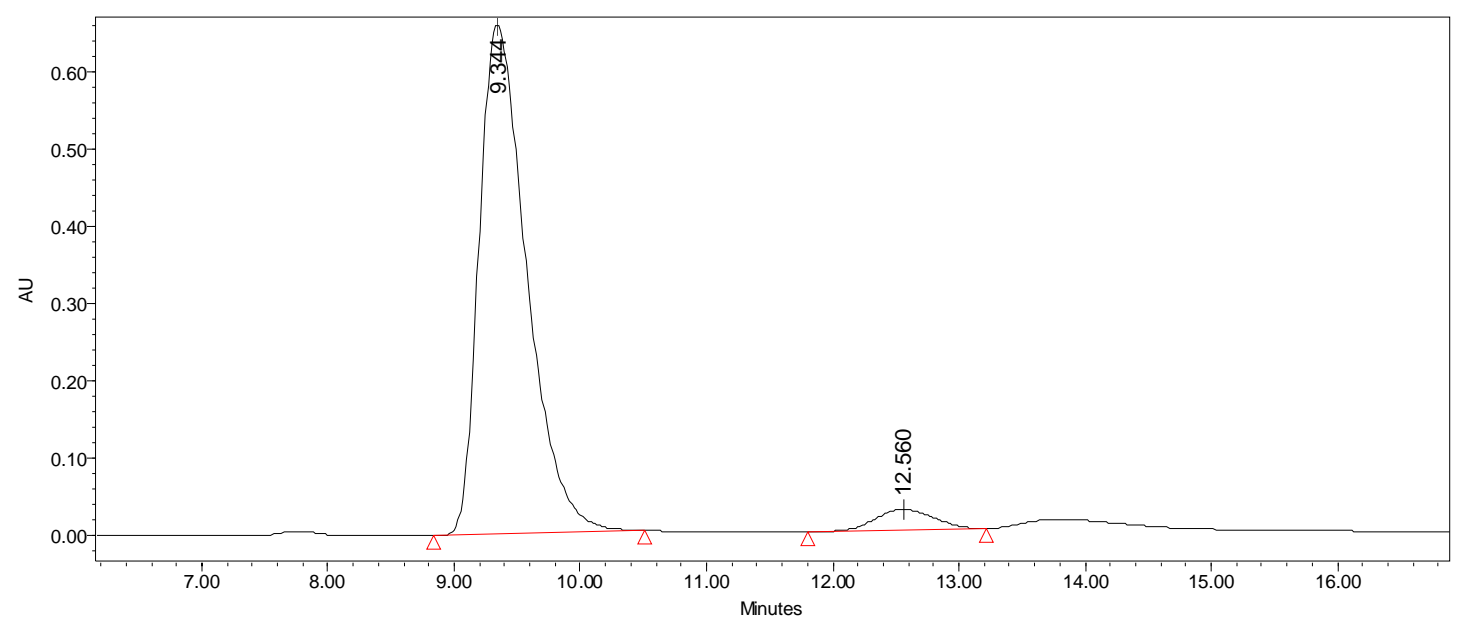

\begin{tabular}{|l|r|r|r|r|r|}
\hline & $\begin{array}{c}\mathrm{RT} \\
(\mathrm{min})\end{array}$ & $\begin{array}{c}\text { Area } \\
\left(\mathrm{V}^{*} \mathrm{sec}\right)\end{array}$ & $\%$ Area & $\begin{array}{c}\text { Height } \\
(\mathrm{V})\end{array}$ & $\begin{array}{c}\% \\
\text { Height }\end{array}$ \\
\hline 1 & 9.344 & 16477273 & 95.19 & 651267 & 96.11 \\
\hline 2 & 12.560 & 832041 & 4.81 & 26365 & 3.89 \\
\hline
\end{tabular}<smiles>C=CCOC(=O)N[C@@H](CCc1ccccc1)C(C(C)=O)C(C)=O</smiles>

Table 4, entry 2: 14b ChiralPak AD-H Column, Hexane:IPA = 95:5, $1.0 \mathrm{~mL} / \mathrm{min}, 214 \mathrm{~nm}$ racemate:

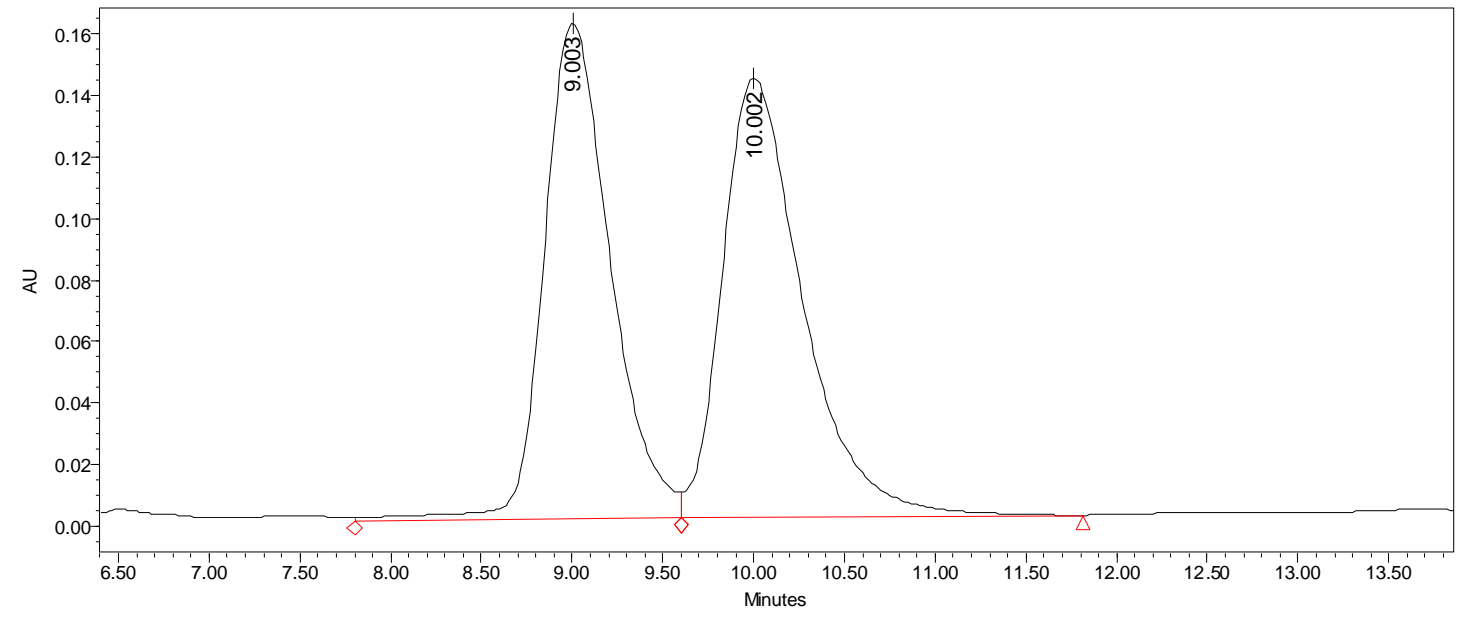

\begin{tabular}{|c|r|c|c|c|c|}
\hline & $\begin{array}{c}\text { RT } \\
(\mathrm{min})\end{array}$ & $\begin{array}{c}\text { Area } \\
\left(\mathrm{V}^{*} \mathrm{sec}\right)\end{array}$ & \% Area & $\begin{array}{c}\text { Height } \\
(\mathrm{V})\end{array}$ & $\begin{array}{c}\% \\
\text { Height }\end{array}$ \\
\hline 1 & 9.003 & 4151860 & 48.76 & 161224 & 53.03 \\
\hline 2 & 10.002 & 4363063 & 51.24 & 142827 & 46.97 \\
\hline
\end{tabular}<smiles>C=CCOC(=O)NC(c1ccccc1)C(C(C)=O)C(C)=O</smiles>

product from cinchonine catalyzed reaction 


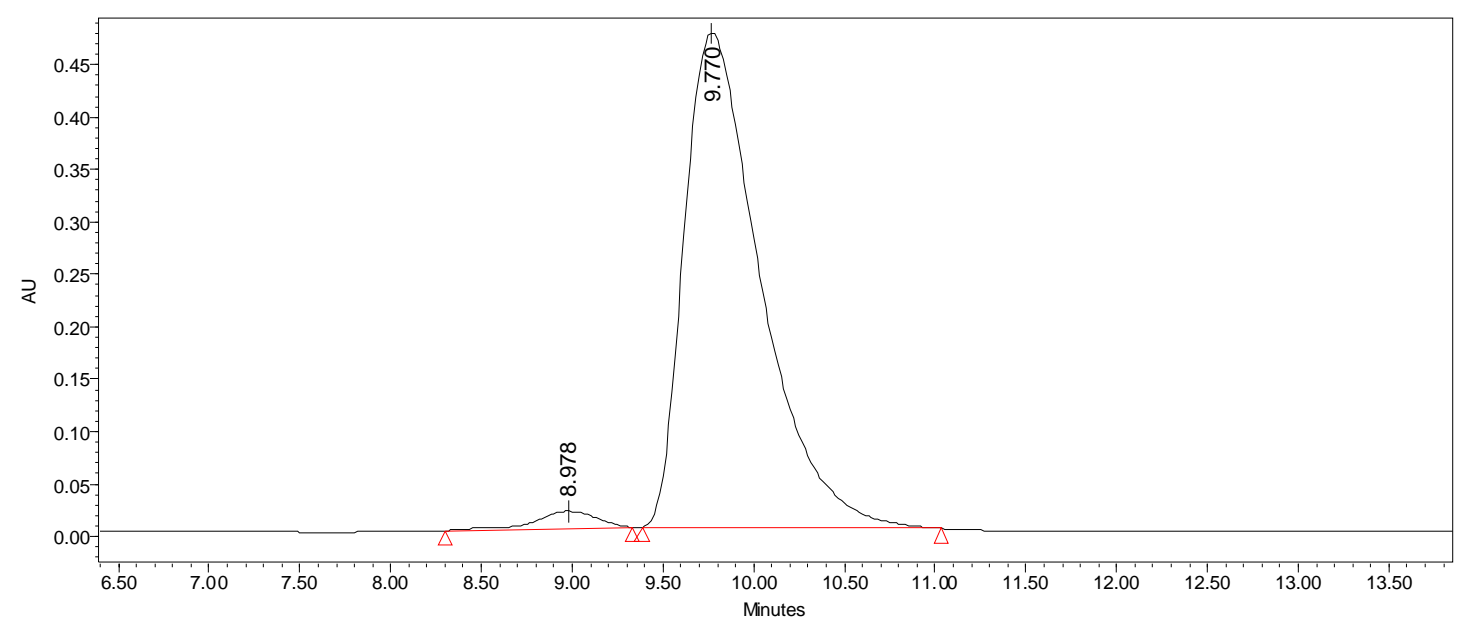

\begin{tabular}{|l|c|c|r|r|r|}
\hline & $\begin{array}{c}\text { RT } \\
(\mathrm{min})\end{array}$ & $\begin{array}{c}\text { Area } \\
\left(\mathrm{V}^{*} \mathrm{sec}\right)\end{array}$ & $\%$ Area & $\begin{array}{l}\text { Height } \\
(\mathrm{V})\end{array}$ & $\begin{array}{c}\% \\
\text { Height }\end{array}$ \\
\hline 1 & 8.978 & 362089 & 3.38 & 15759 & 3.70 \\
\hline 2 & 9.770 & 10361201 & 96.62 & 410654 & 96.30 \\
\hline
\end{tabular}<smiles>C=CCOC(=O)N[C@H](c1ccccc1)C(C(C)=O)C(C)=O</smiles>

Product from cinchonidine catalyzed reaction:

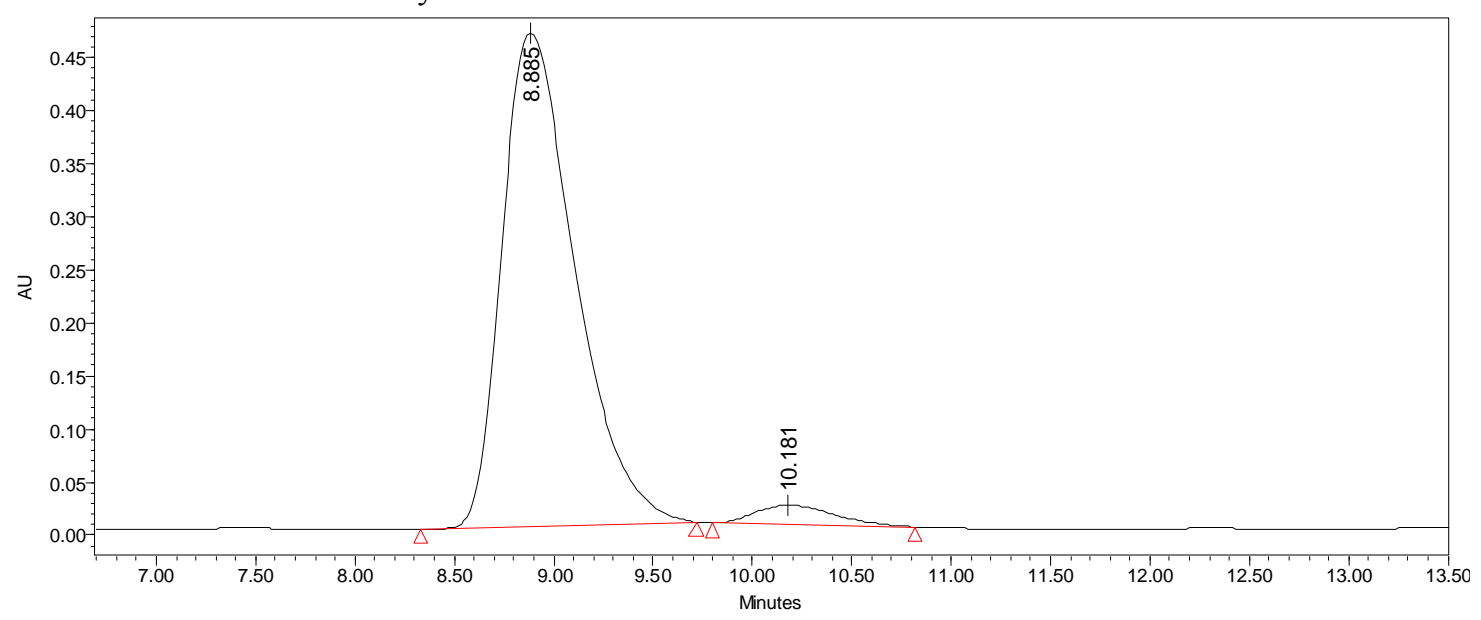

\begin{tabular}{|l|r|r|r|r|r|}
\hline & $\begin{array}{c}\mathrm{RT} \\
(\mathrm{min})\end{array}$ & $\begin{array}{c}\text { Area } \\
\left(\mathrm{V}^{*} \mathrm{sec}\right)\end{array}$ & $\%$ Area & $\begin{array}{c}\text { Height } \\
(\mathrm{V})\end{array}$ & $\begin{array}{c}\% \\
\text { Height }\end{array}$ \\
\hline 1 & 8.885 & 10898695 & 95.66 & 448933 & 96.12 \\
\hline 2 & 10.181 & 494035 & 4.34 & 18127 & 3.88 \\
\hline
\end{tabular}<smiles>C=CCOC(=O)N[C@H](c1ccccc1)C(C(C)=O)C(C)=O</smiles> 
Table 4, entry 3: 14c: ChiralPak AD-H Column, Hexane:IPA = 95:5, $1.0 \mathrm{~mL} / \mathrm{min}, 214 \mathrm{~nm}$ racemate:

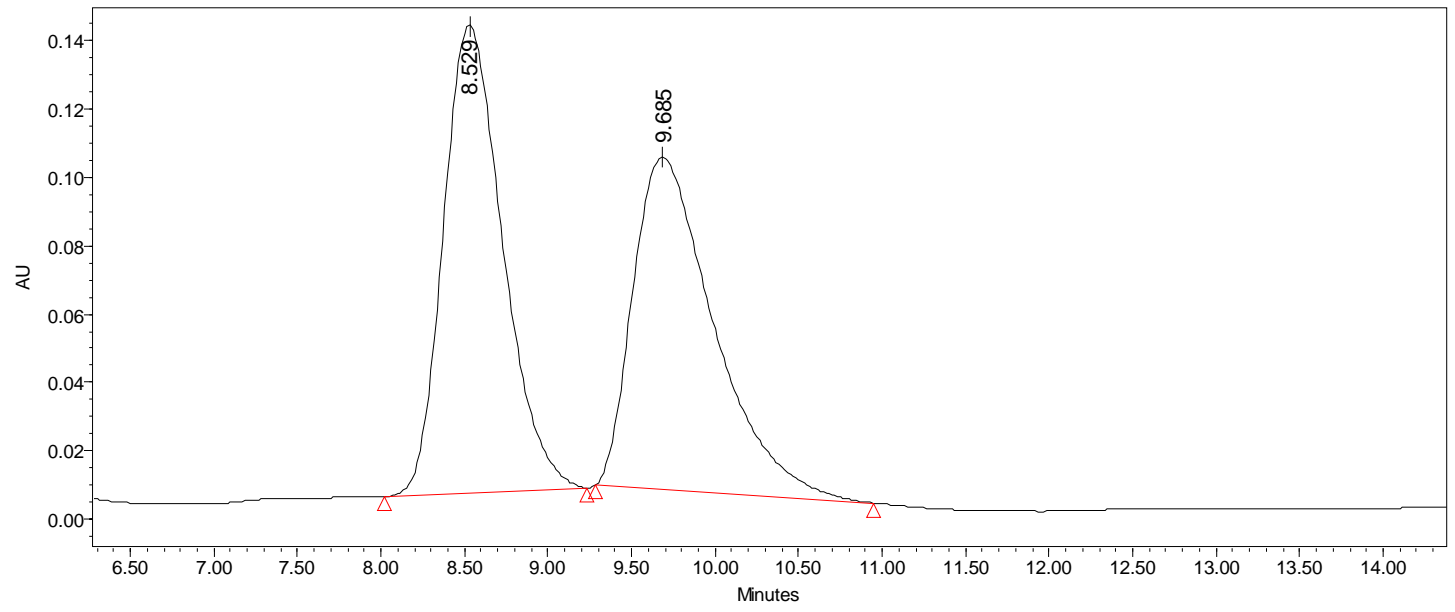

\begin{tabular}{|c|c|c|c|c|c|}
\hline & $\begin{array}{c}\text { RT } \\
(\mathrm{min})\end{array}$ & $\begin{array}{c}\text { Area } \\
\left(\mathrm{V}^{*} \mathrm{sec}\right)\end{array}$ & $\%$ Area & $\begin{array}{c}\text { Height } \\
(\mathrm{V})\end{array}$ & $\begin{array}{c}\% \\
\text { Height }\end{array}$ \\
\hline 1 & 8.529 & 3379670 & 50.84 & 136473 & 58.46 \\
\hline 2 & 9.685 & 3268586 & 49.16 & 96968 & 41.54 \\
\hline
\end{tabular}<smiles>C=CCOC(=O)NC(c1ccc(Br)cc1)C(C(C)=O)C(C)=O</smiles>

product from cinchonine catalyzed reaction:

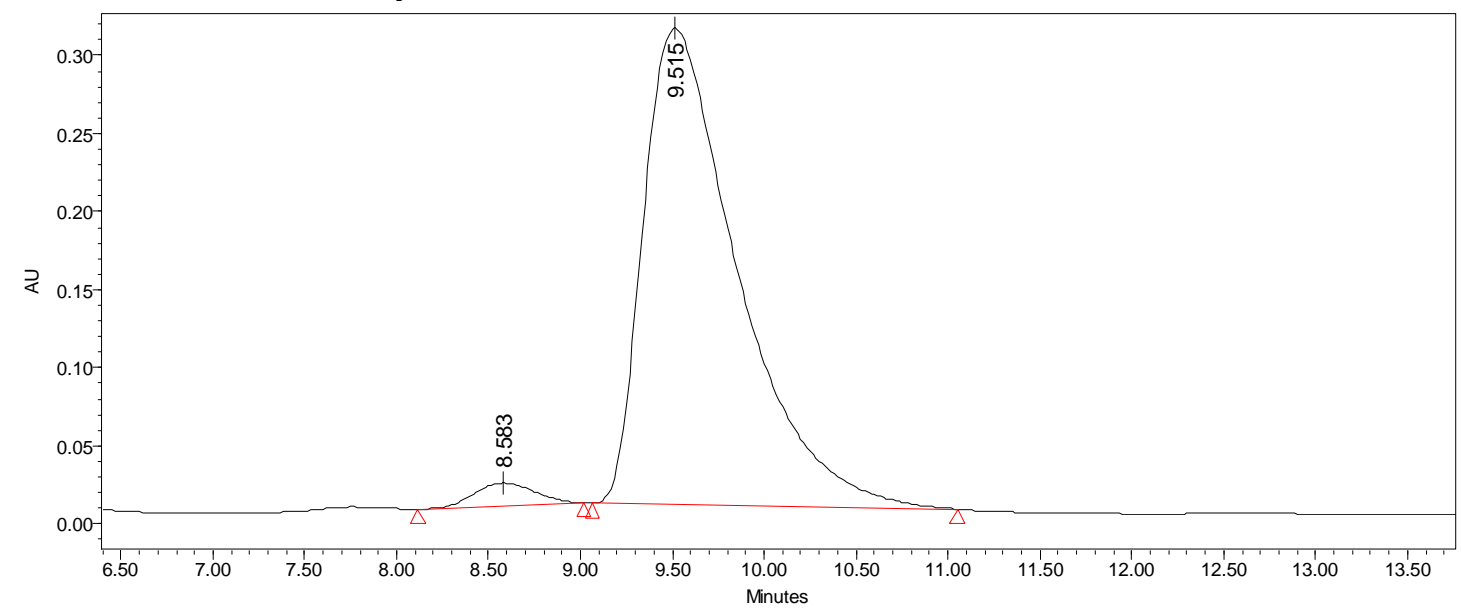

\begin{tabular}{|c|c|c|r|c|c|}
\hline & $\begin{array}{c}\text { RT } \\
(\mathrm{min})\end{array}$ & $\begin{array}{c}\text { Area } \\
\left(\mathrm{V}^{*} \mathrm{sec}\right)\end{array}$ & $\%$ Area & $\begin{array}{c}\text { Height } \\
(\mathrm{V})\end{array}$ & $\begin{array}{c}\% \\
\text { Height }\end{array}$ \\
\hline 1 & 9.200 & 502169 & 4.41 & 26707 & 8.00 \\
\hline 2 & 9.515 & 10893180 & 95.59 & 307283 & 92.00 \\
\hline
\end{tabular}<smiles>C=CCOC(=O)N[C@H](c1ccc(Br)cc1)C(C(C)=O)C(C)=O</smiles> 
Product from cinchonidine catalyzed reaction:

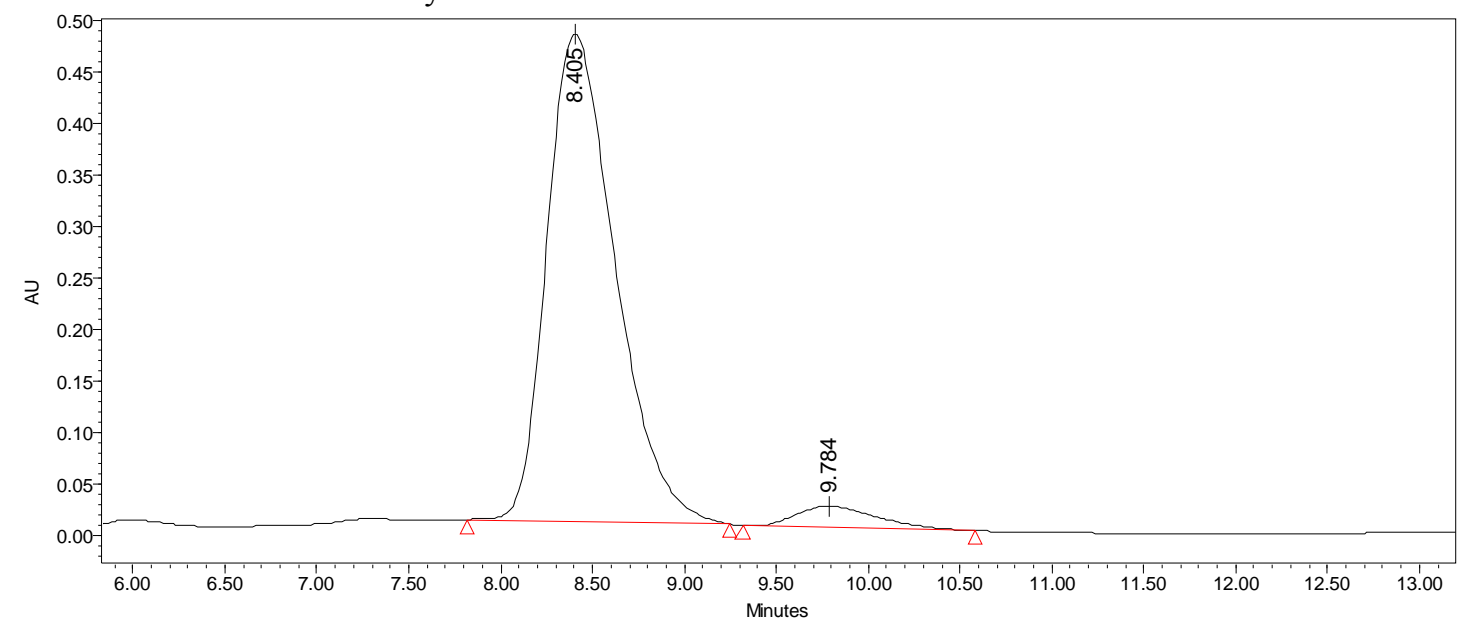

\begin{tabular}{|c|c|r|r|r|r|}
\hline & $\begin{array}{c}\mathrm{RT} \\
(\mathrm{min})\end{array}$ & $\begin{array}{c}\text { Area } \\
\left(\mathrm{V}^{*} \mathrm{sec}\right)\end{array}$ & $\%$ Area & $\begin{array}{c}\text { Height } \\
(\mathrm{V})\end{array}$ & $\begin{array}{c}\% \\
\text { Height }\end{array}$ \\
\hline 1 & 8.405 & 12278245 & 95.08 & 474816 & 95.85 \\
\hline 2 & 9.784 & 636015 & 4.92 & 20562 & 4.15 \\
\hline
\end{tabular}<smiles>C=CCOC(=O)N[C@H](c1ccc(Br)cc1)C(C(C)=O)C(C)=O</smiles>

Table 4, entry 4: 14d ChiralPak AD-H Column, Hexane:IPA = 95:5, $1.0 \mathrm{~mL} / \mathrm{min}, 214 \mathrm{~nm}$ racemate
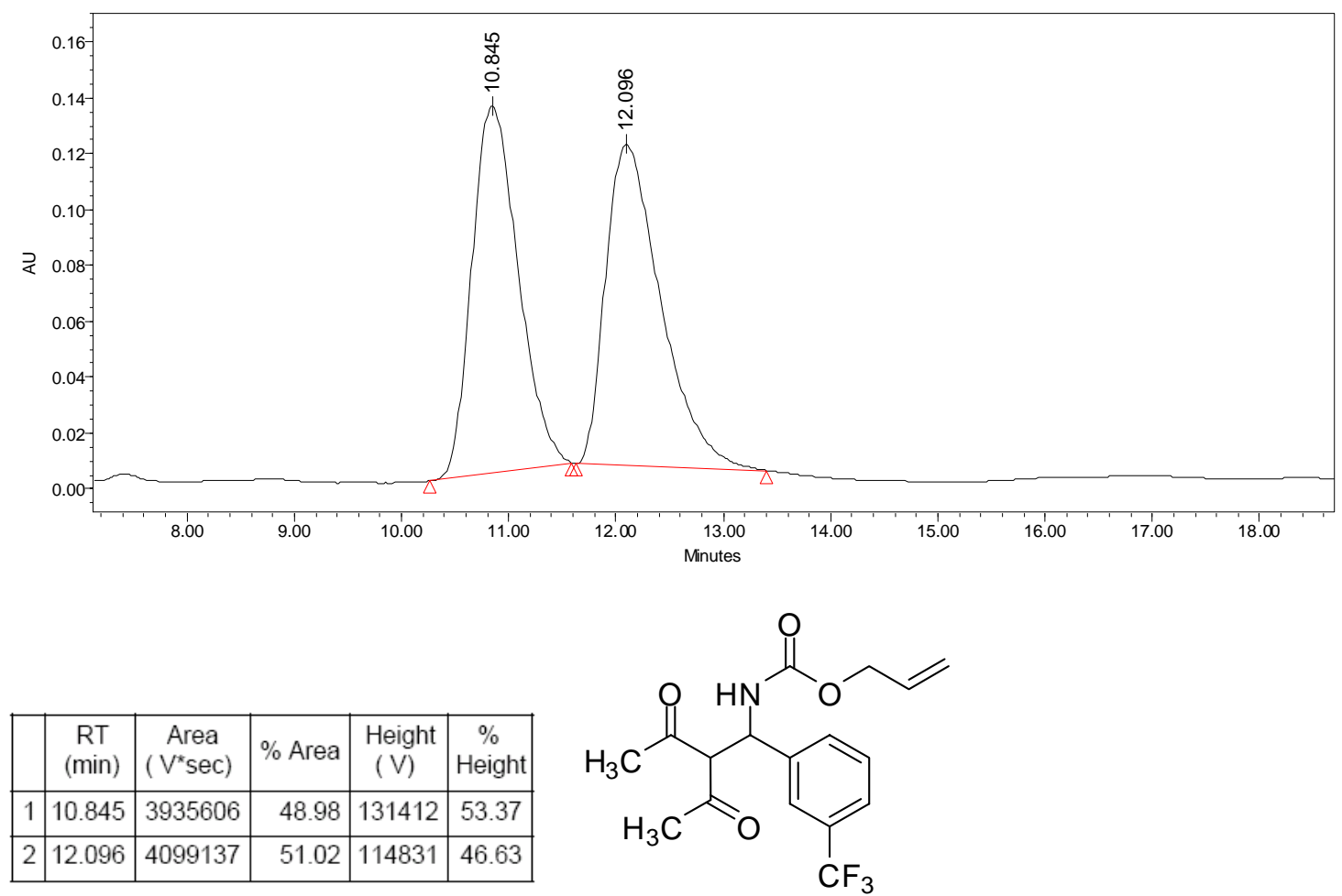
Product from cinchonine catalyzed reaction:

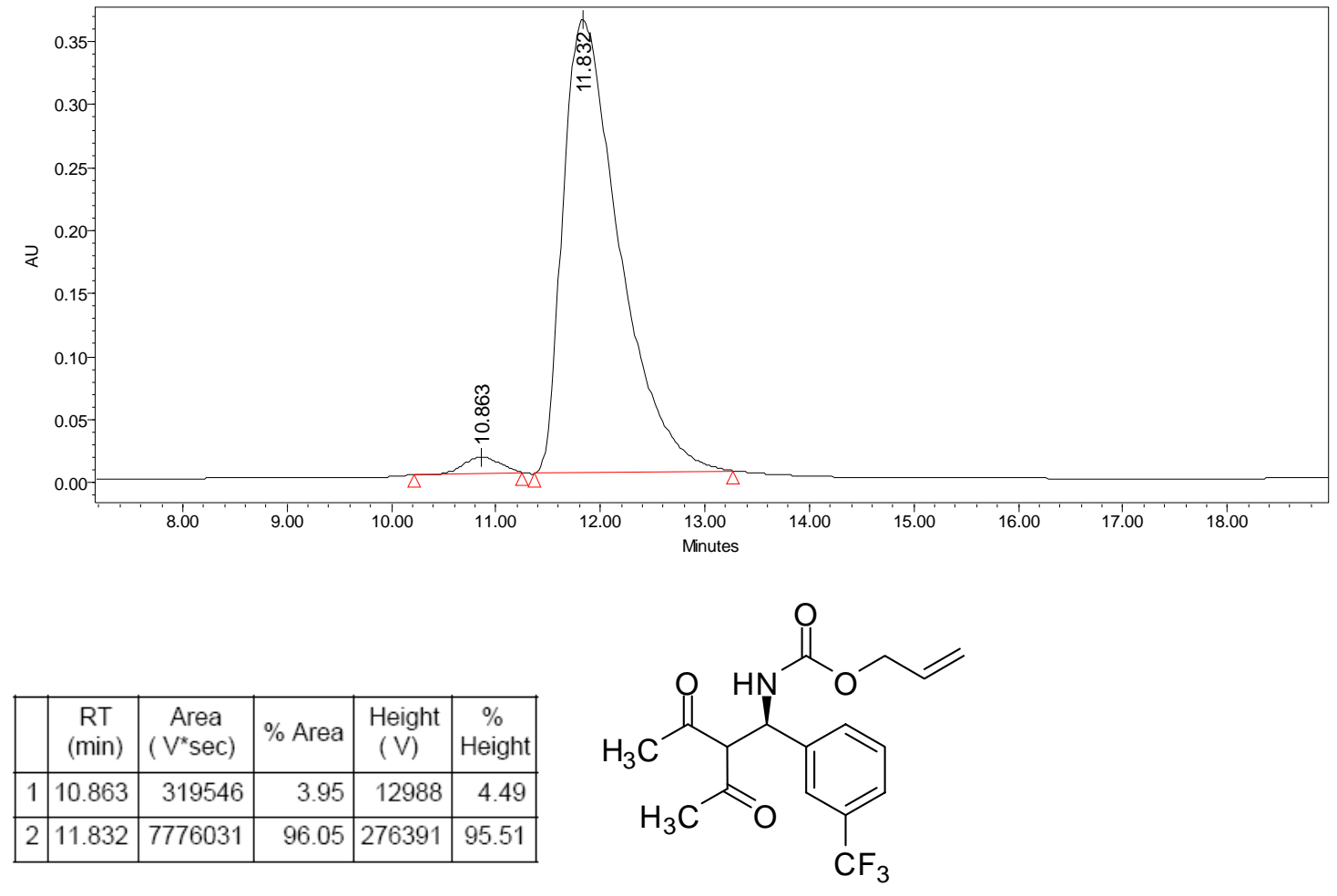

Product from cinchonidine catalyzed reaction:
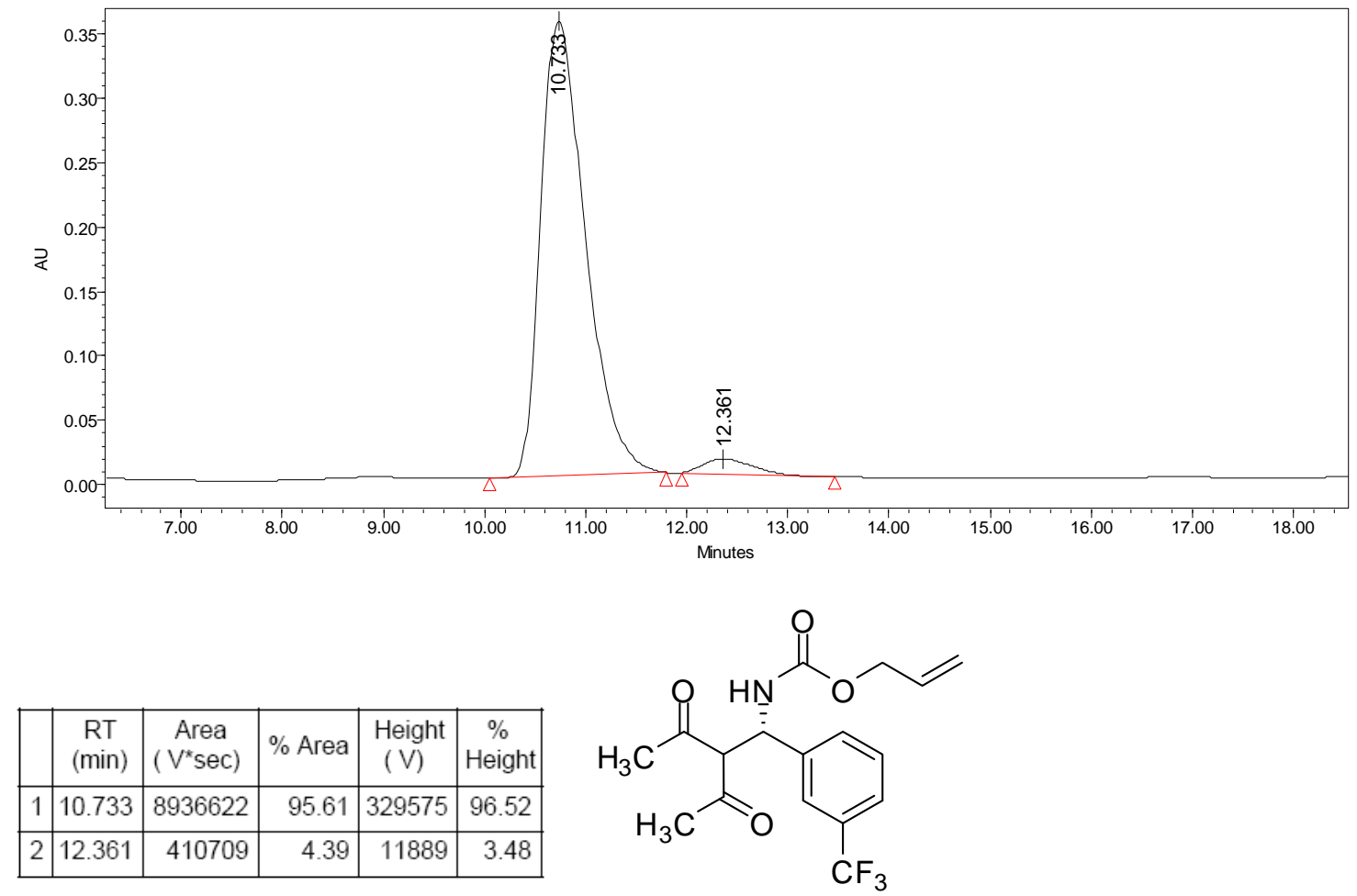
Table 4, entry 5: 14e: ChiralPak AD-H Column, Hexane:IPA = 95:5, $1.0 \mathrm{~mL} / \mathrm{min}, 214 \mathrm{~nm}$ racemate:

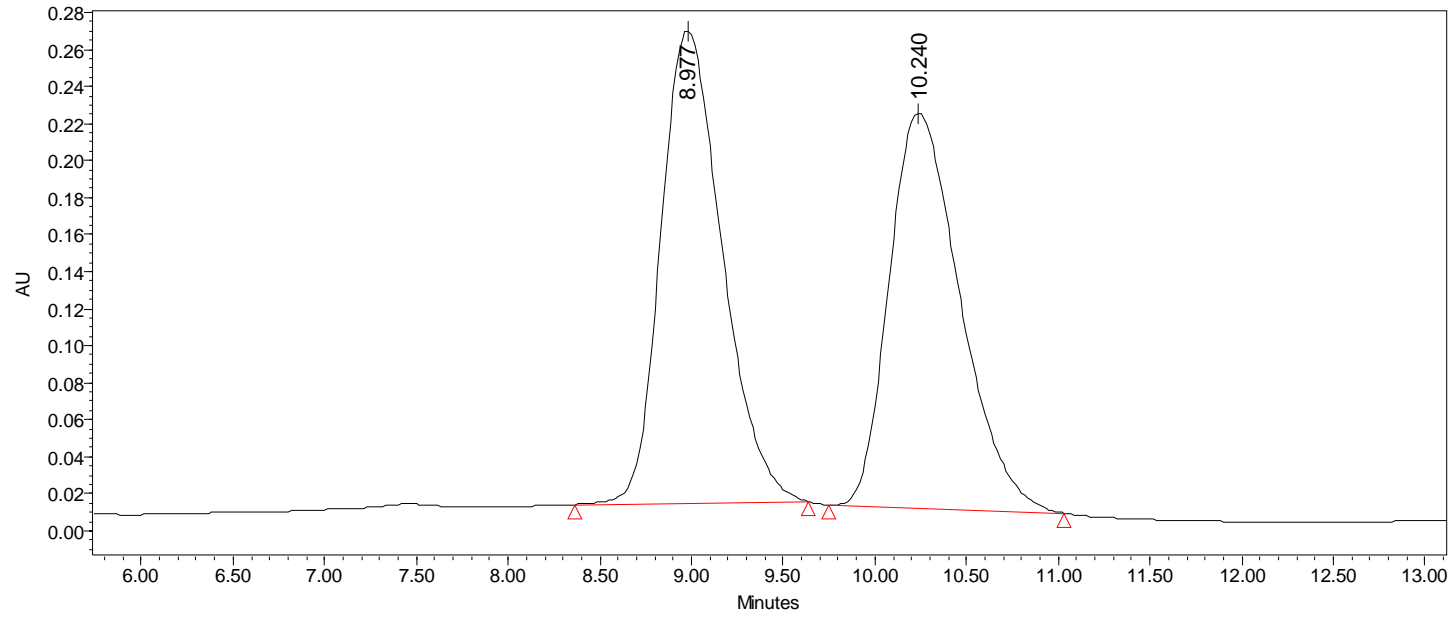

\begin{tabular}{|c|c|c|c|c|c|}
\hline & $\begin{array}{c}\text { RT } \\
(\mathrm{min})\end{array}$ & $\begin{array}{c}\text { Area } \\
\left(\mathrm{V}^{*} \mathrm{sec}\right)\end{array}$ & $\%$ Area & $\begin{array}{c}\text { Height } \\
(\mathrm{V})\end{array}$ & $\begin{array}{c}\% \\
\text { Height }\end{array}$ \\
\hline 1 & 8.977 & 5944284 & 50.83 & 255437 & 54.46 \\
\hline 2 & 10.240 & 5749151 & 49.17 & 213569 & 45.54 \\
\hline
\end{tabular}<smiles>C=CCOC(=O)NC(c1ccco1)C(C(C)=O)C(C)=O</smiles>

product from cinchonine catalyzed reaction:

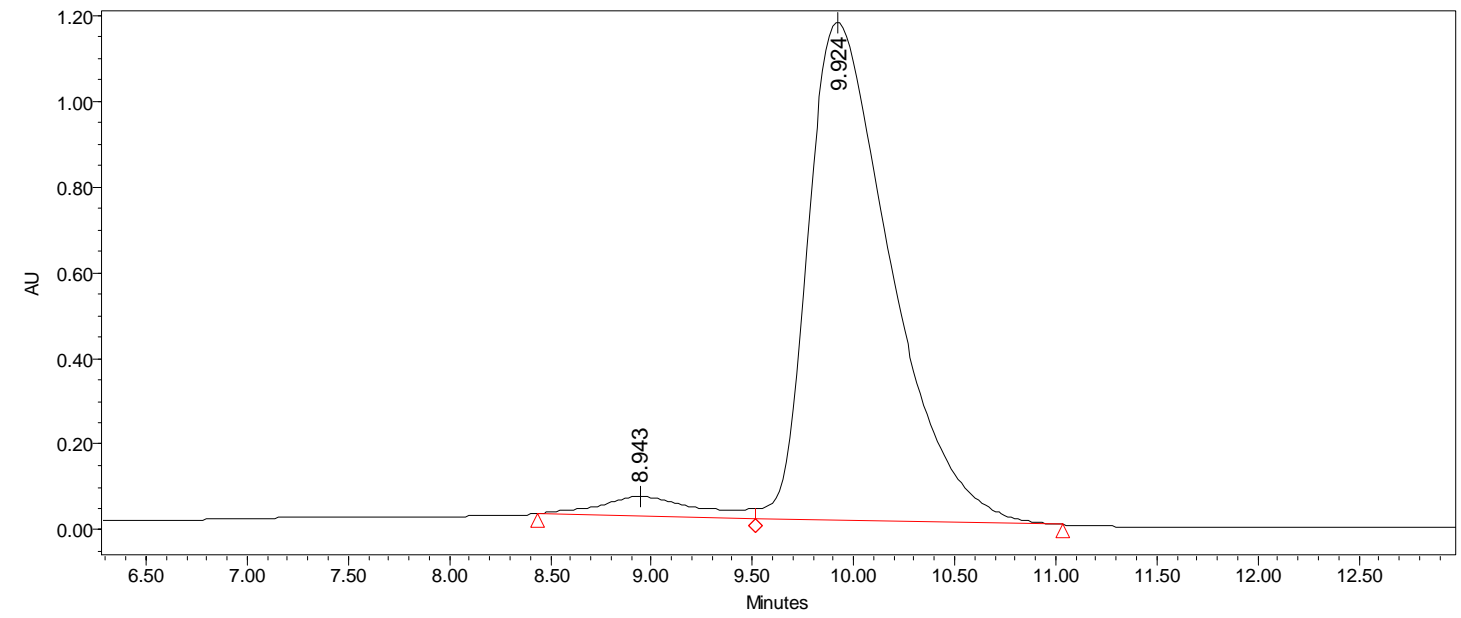

\begin{tabular}{|l|c|c|r|r|r|}
\hline & $\begin{array}{c}\text { RT } \\
(\mathrm{min})\end{array}$ & $\begin{array}{c}\text { Area } \\
\left(\mathrm{V}^{*} \mathrm{sec}\right)\end{array}$ & $\%$ Area & $\begin{array}{c}\text { Height } \\
(\mathrm{V})\end{array}$ & $\begin{array}{c}\% \\
\text { Height }\end{array}$ \\
\hline 1 & 8.943 & 1541772 & 4.47 & 45041 & 3.74 \\
\hline 2 & 9.924 & 32976201 & 95.53 & 1160416 & 96.26 \\
\hline
\end{tabular}<smiles>C=CCOC(=O)N[C@H](c1ccco1)C(C(C)=O)C(C)=O</smiles>

Product from cinchonidine catalyzed reaction: 

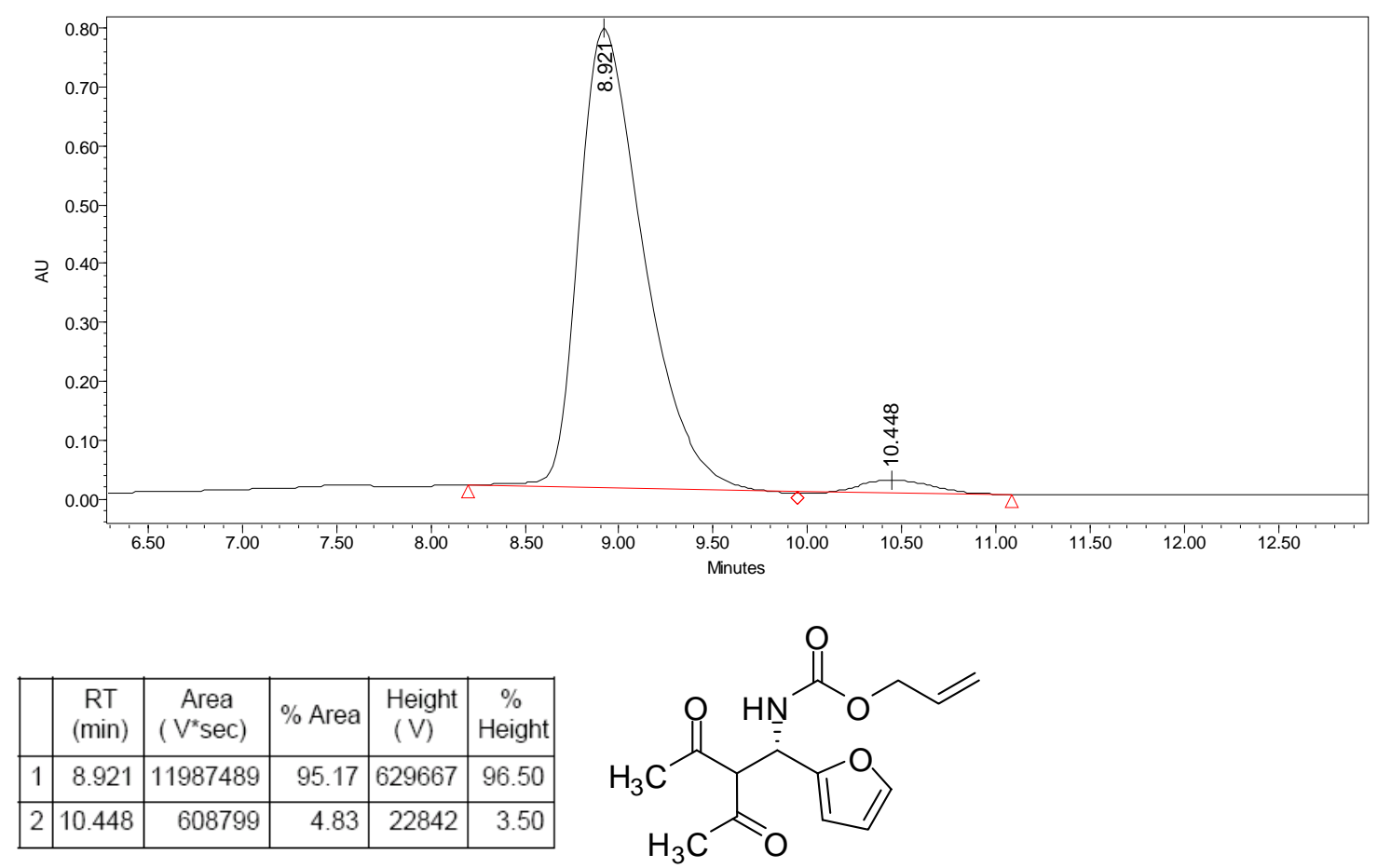

Table 4, entry 6: 14f: ChiralPak AD-H Column, Hexane:IPA = 95:5, $1.0 \mathrm{~mL} / \mathrm{min}, 214 \mathrm{~nm}$ racemate:

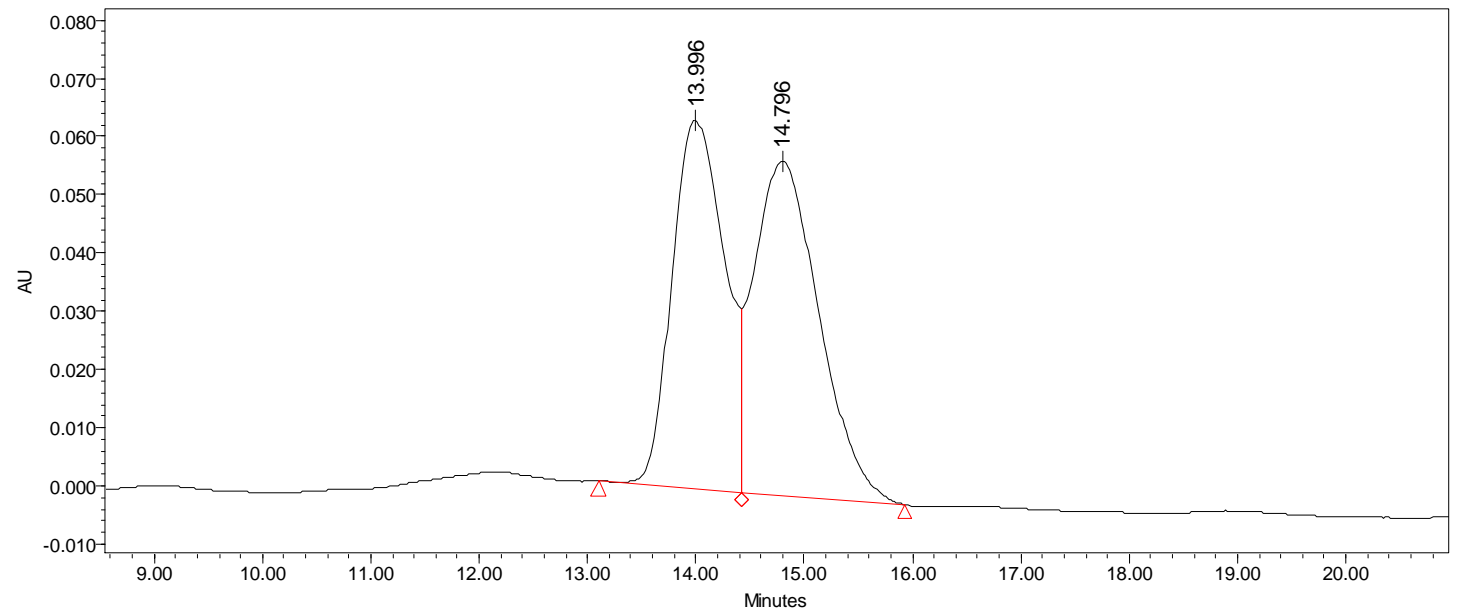

\begin{tabular}{|c|c|c|c|c|c|}
\hline & $\begin{array}{c}\text { RT } \\
(\mathrm{min})\end{array}$ & $\begin{array}{c}\text { Area } \\
\left(\mathrm{V}^{*} \mathrm{sec}\right)\end{array}$ & $\%$ Area & $\begin{array}{c}\text { Height } \\
(\mathrm{V})\end{array}$ & $\begin{array}{c}\% \\
\text { Height }\end{array}$ \\
\hline 1 & 13.996 & 2227650 & 48.97 & 63238 & 52.42 \\
\hline 2 & 14.796 & 2321700 & 51.03 & 57397 & 47.58 \\
\hline
\end{tabular}<smiles>C=CCOC(=O)NC(c1cccs1)C(C(C)=O)C(C)=O</smiles> 
product from cinchonine catalyzed reaction:

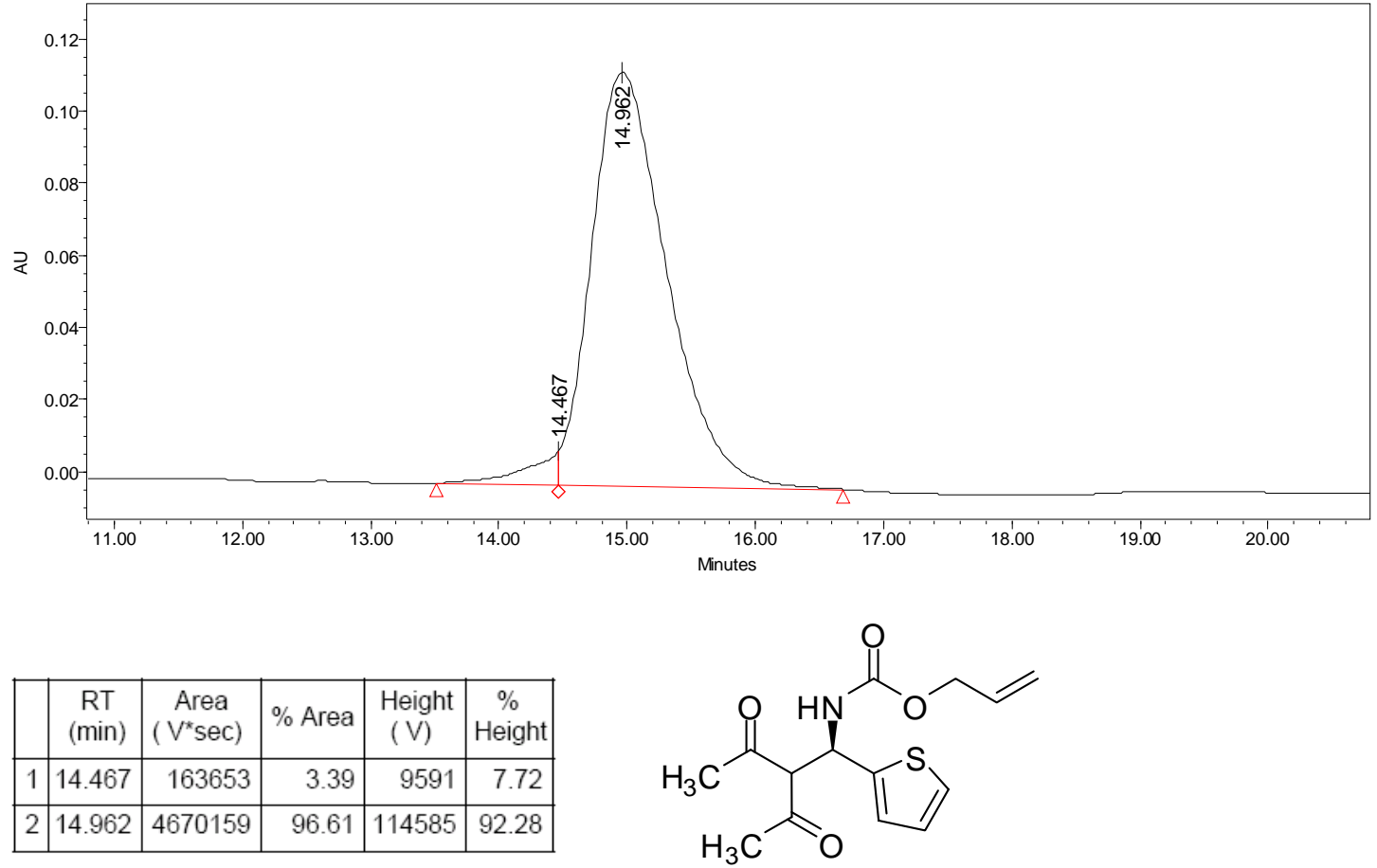

Product from cinchonidine catalyzed reaction:

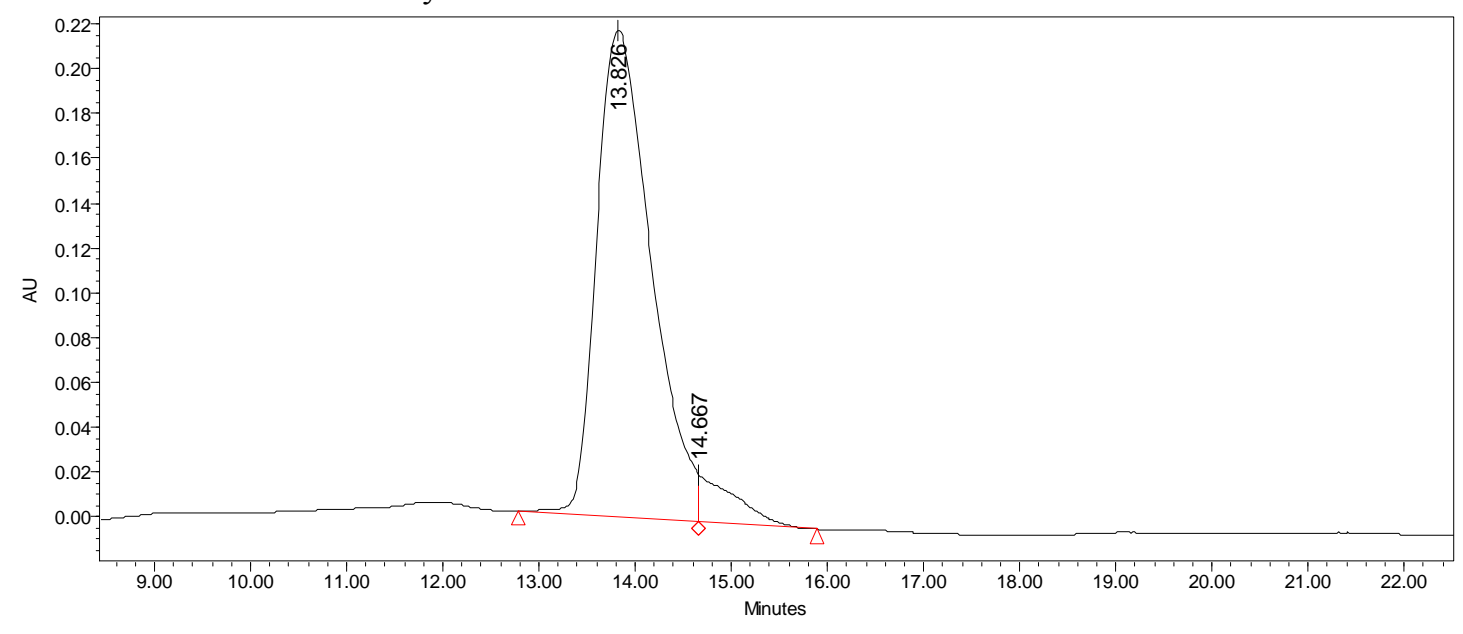

\begin{tabular}{|c|c|c|r|r|r|}
\hline & $\begin{array}{c}\text { RT } \\
(\mathrm{min})\end{array}$ & $\begin{array}{c}\text { Area } \\
\left(\mathrm{V}^{*} \mathrm{sec}\right)\end{array}$ & \% Area & $\begin{array}{c}\text { Height } \\
(\mathrm{V})\end{array}$ & $\begin{array}{c}\% \\
\text { Height }\end{array}$ \\
\hline 1 & 13.826 & 8628497 & 95.42 & 216674 & 92.63 \\
\hline 2 & 14.783 & 413864 & 4.58 & 17246 & 7.37 \\
\hline
\end{tabular}<smiles>C=CCOC(=O)N[C@@H](C(=O)C(C)=O)c1cccs1</smiles> 
Table 5, entry 1: 18a Chiralcel OD-H Column, Hexanes : IPA $=90: 10,1.0 \mathrm{~mL} / \mathrm{min}, 214 \mathrm{~nm}$

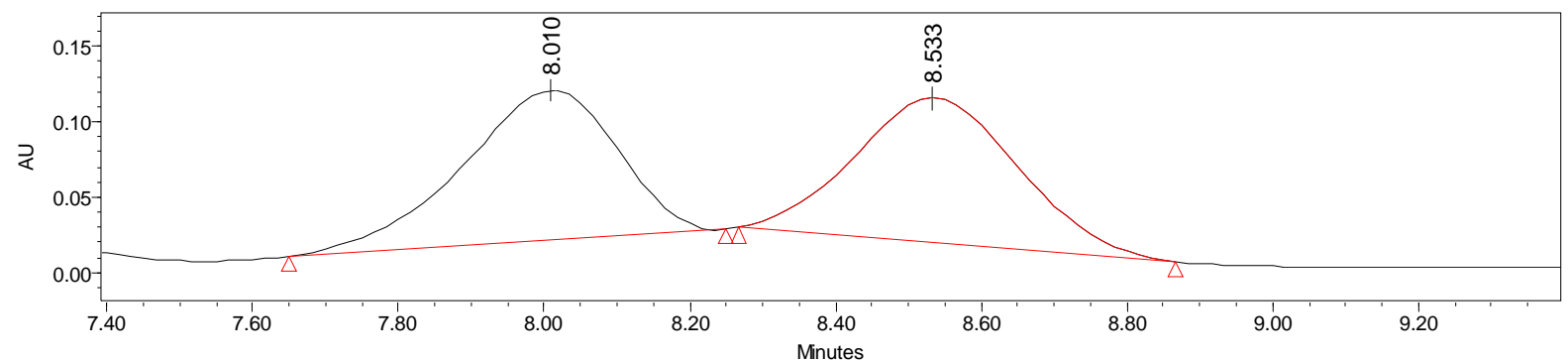

\begin{tabular}{|r|r|r|r|r|r|}
\hline & Retention Time & Area & \% Area & Height & \% Height \\
\hline 1 & 8.010 & 1463811 & 49.33 & 99404 & 50.92 \\
\hline 2 & 8.533 & 1503734 & 50.67 & 95819 & 49.08 \\
\hline
\end{tabular}
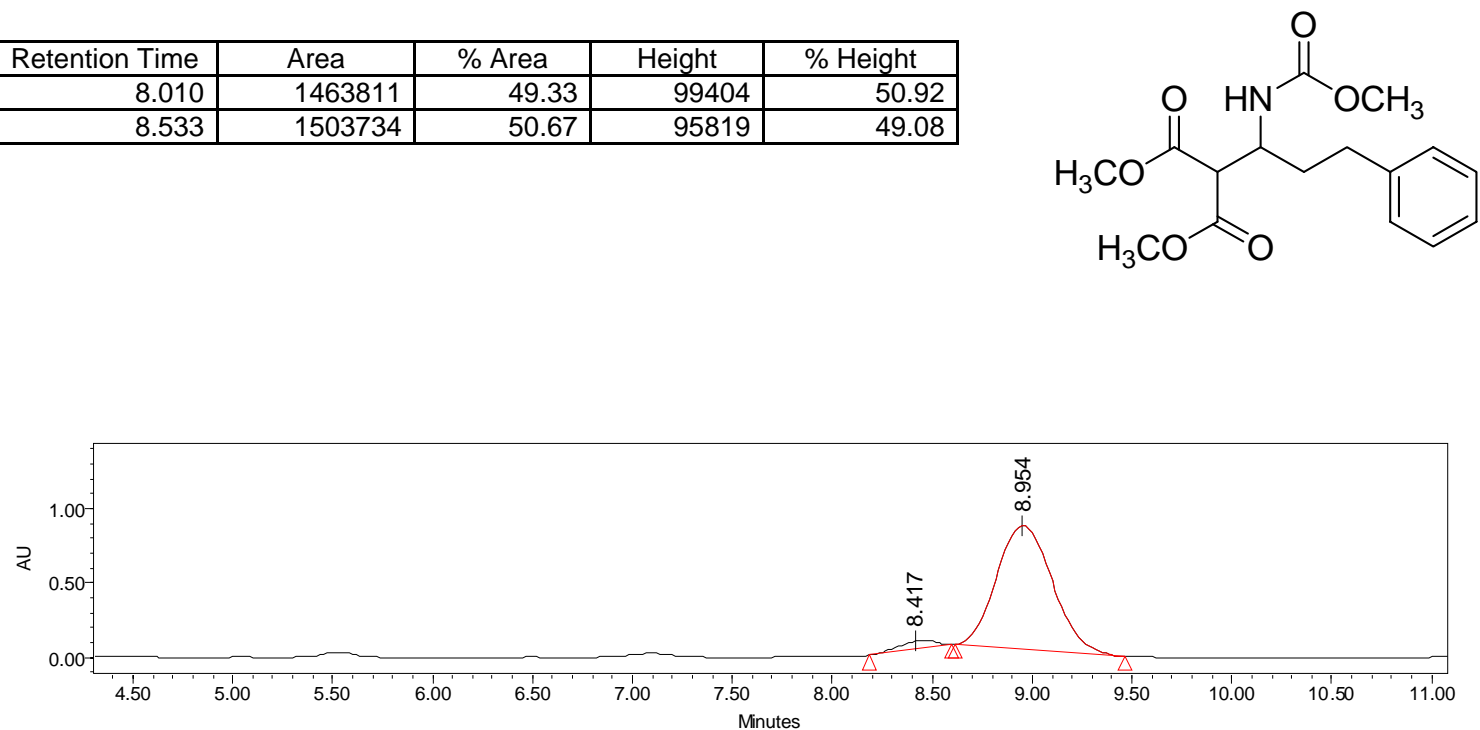

\begin{tabular}{|r|r|r|r|r|r|}
\hline & Retention Time & \multicolumn{1}{c|}{ Area } & \% Area & Height & \multicolumn{1}{c|}{ \% Height } \\
\hline 1 & 8.417 & 680281 & 4.05 & 52824 & 5.97 \\
\hline 2 & 8.954 & 16137457 & 95.95 & 831547 & 94.03 \\
\hline
\end{tabular}<smiles>COC(=O)N[C@@H](C(=O)OC)C(CCc1ccccc1)C(=O)OC</smiles> 
Table 5, entry 2: 18b Chiralcel OD-H Column, Hexanes : IPA $=95: 5,1.0 \mathrm{~mL} / \mathrm{min}, 214 \mathrm{~nm}$

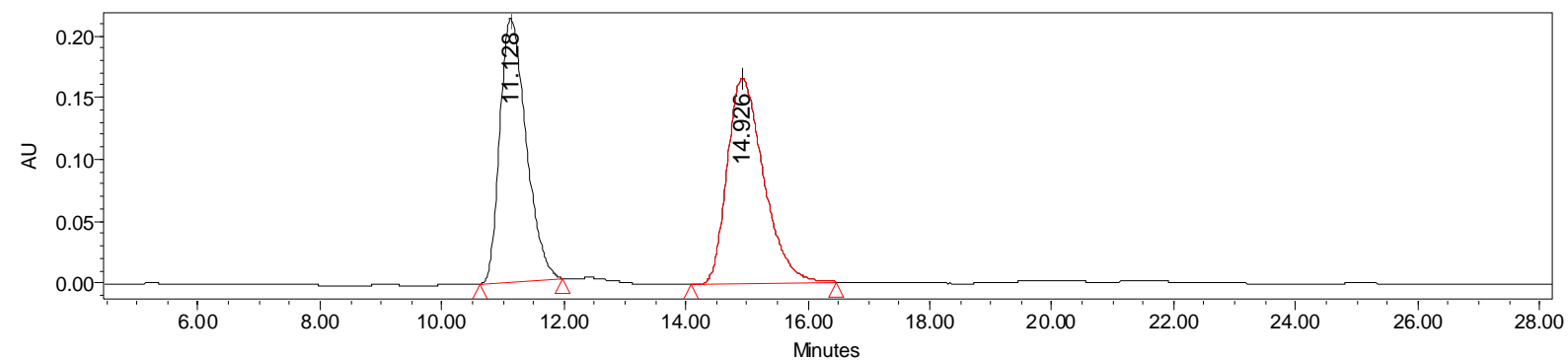

\begin{tabular}{|r|r|r|r|r|r|}
\hline & Retention Time & \multicolumn{1}{|c|}{ Area } & \% Area & Height & \% Height \\
\hline 1 & 11.128 & 6378008 & 48.01 & 213152 & 56.17 \\
\hline 2 & 14.926 & 6907536 & 51.99 & 166305 & 43.83 \\
\hline
\end{tabular}
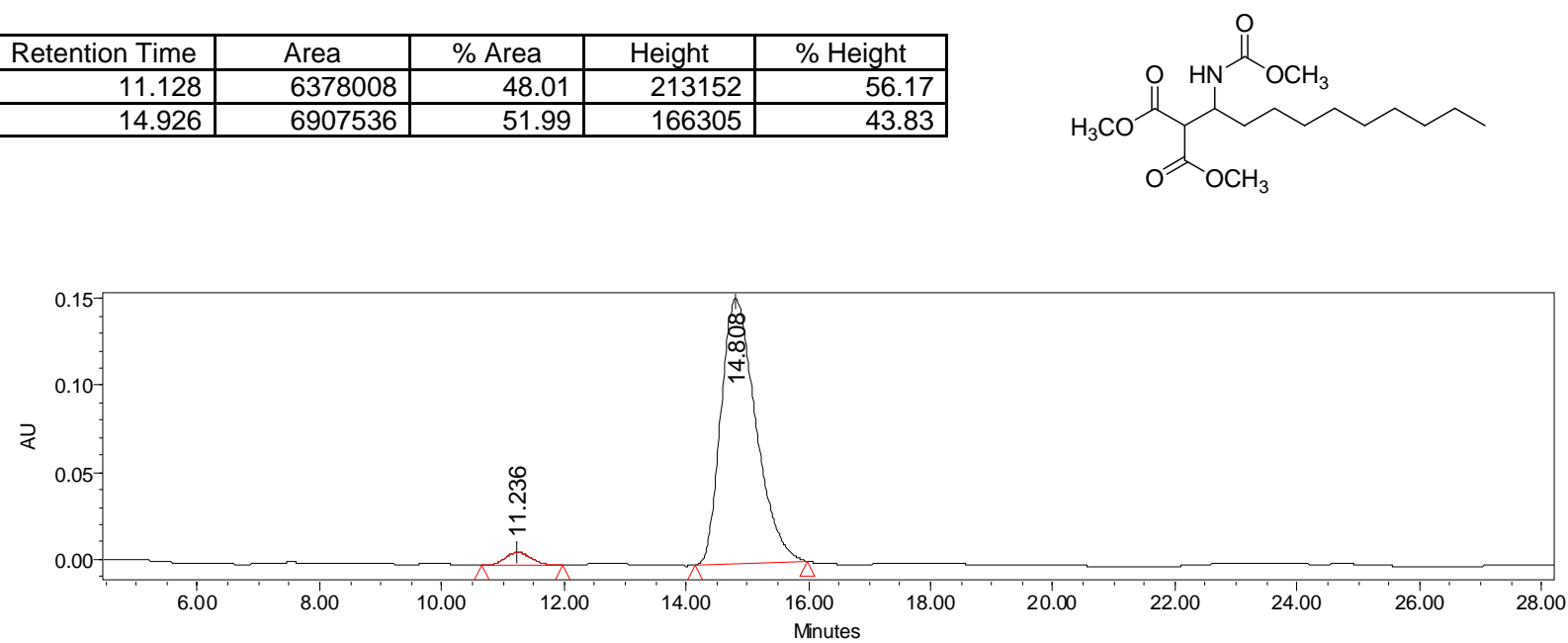

\begin{tabular}{|r|r|r|r|r|r|}
\hline & Retention Time & \multicolumn{1}{c|}{ Area } & \% Area & Height & \% Height \\
\hline 1 & 11.236 & 229648 & 3.64 & 7513 & 4.69 \\
\hline 2 & 14.808 & 6076306 & 96.36 & 152797 & 95.31 \\
\hline
\end{tabular}<smiles>CCCCCCCCCC(NC(=O)OC)C(C(=O)OC)C(=O)OC</smiles> 
Table 5, entry 1: 18c Chiralcel OD-H Column, Hexanes : IPA = 99: 1, $1.0 \mathrm{~mL} / \mathrm{min}, 214 \mathrm{~nm}$ The racemate was made by using cinchonine and cinchonidine mixture (1:1) as catalyst.

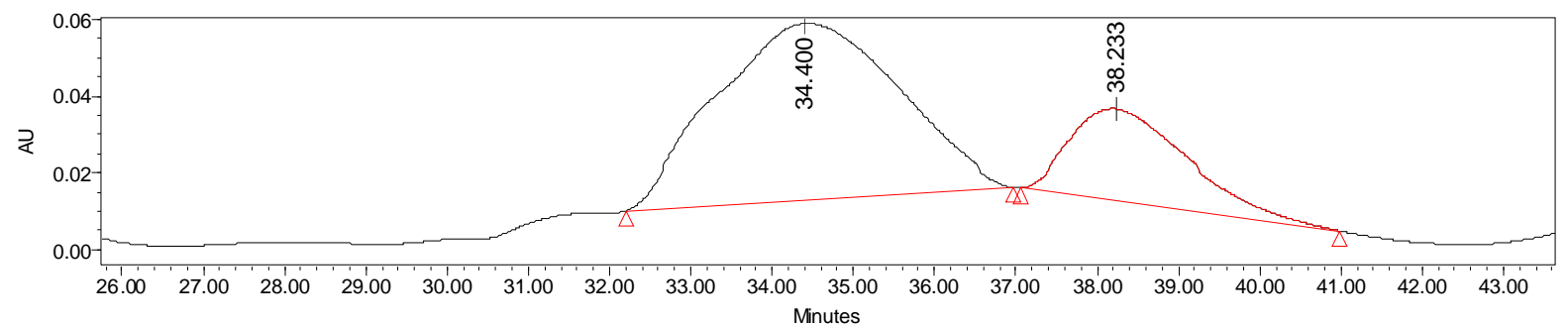

\begin{tabular}{|r|r|r|r|r|r|}
\hline & Retention Time & Area & \% Area & Height & \% Height \\
\hline 1 & 34.400 & 7173001 & 74.77 & 45859 & 65.78 \\
\hline 2 & 38.233 & 2419882 & 25.23 & 23856 & 34.22 \\
\hline
\end{tabular}<smiles>COC(=O)NC(COCc1ccccc1)C(C(=O)OC)C(=O)OC</smiles>

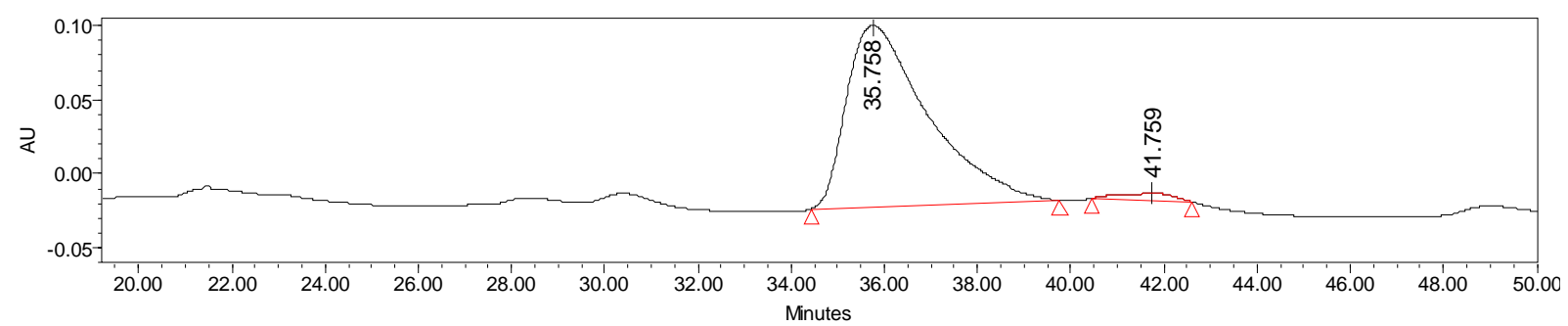

\begin{tabular}{|r|r|r|r|r|r|}
\hline & Retention Time & \multicolumn{1}{|c|}{ Area } & \% Area & Height & \multicolumn{1}{c|}{ \% Height } \\
\hline 1 & 35.758 & 14903084 & 97.48 & 122168 & 96.08 \\
\hline 2 & 41.759 & 385975 & 2.52 & 4984 & 3.92 \\
\hline
\end{tabular}<smiles>COC(=O)N[C@@H](COCc1ccccc1)C(C(=O)OC)C(=O)OC</smiles> 
Table 5, entry 4: 18d Chiralcel OD-H Column, Hexanes : IPA = $95: 5,1.0 \mathrm{~mL} / \mathrm{min}, 214 \mathrm{~nm}$

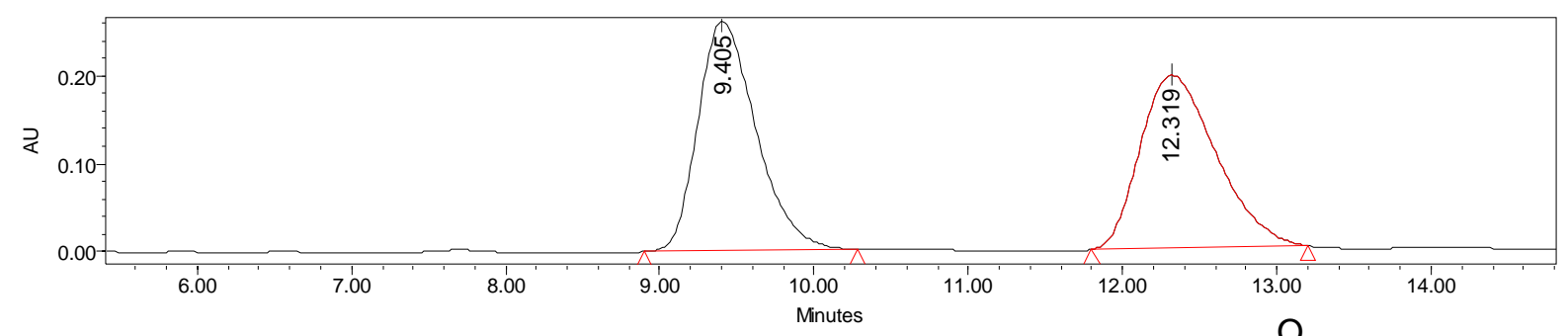

\begin{tabular}{|r|r|r|r|r|r|}
\hline & Retention Time & Area & \% Area & Height & \% Height \\
\hline 1 & 9.405 & 6843810 & 50.38 & 260828 & 57.02 \\
\hline 2 & 12.319 & 6741755 & 49.62 & 196593 & 42.98 \\
\hline
\end{tabular}<smiles>COC(=O)NC(c1ccccc1)C(C(=O)OC)C(=O)OC</smiles>

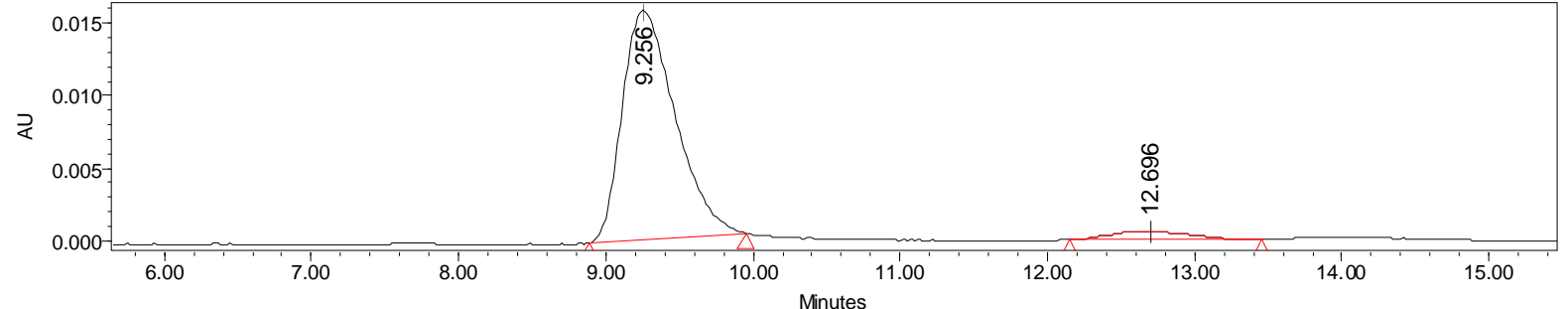

\begin{tabular}{|r|r|r|r|r|r|}
\hline & Retention Time & \multicolumn{1}{c|}{ Area } & \% Area & Height & \% Height \\
\hline 1 & 9.256 & 393835 & 95.42 & 15813 & 96.64 \\
\hline 2 & 12.696 & 18921 & 4.58 & 550 & 3.36 \\
\hline
\end{tabular}<smiles>COC(=O)N[C@@H](c1ccccc1)C(C(=O)OC)C(=O)OC</smiles> 
Table 5, entry 5: 18f Chiralcel OD-H Column, Hexanes : IPA = $95: 5,1.0 \mathrm{~mL} / \mathrm{min}, 214 \mathrm{~nm}$

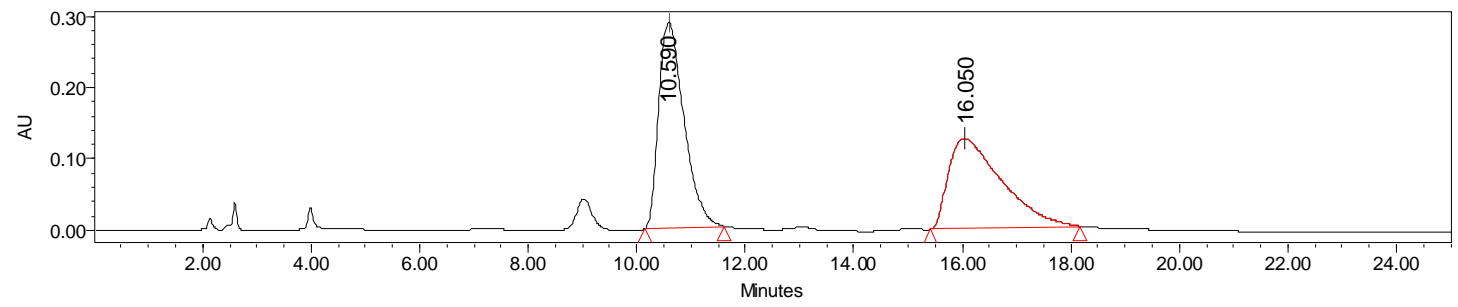

\begin{tabular}{|r|r|r|r|r|r|}
\hline & Retention Time & Area & \% Area & Height & \% Height \\
\hline 1 & 10.590 & 9329761 & 52.05 & 288479 & 69.52 \\
\hline 2 & 16.050 & 8594885 & 47.95 & 126487 & 30.48 \\
\hline
\end{tabular}<smiles>COC(=O)NC(c1ccc(Br)cc1)C(C(=O)OC)C(=O)OC</smiles>

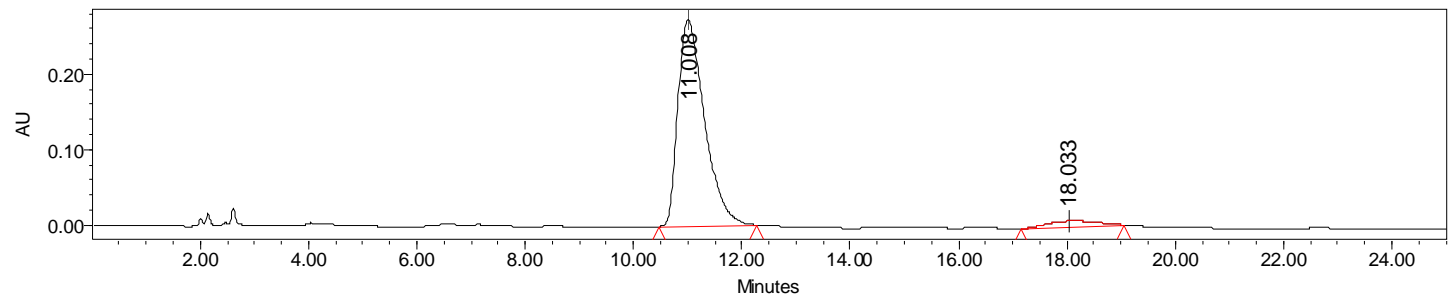

\begin{tabular}{|r|r|r|r|r|r|}
\hline & Retention Time & \multicolumn{1}{c|}{ Area } & \multicolumn{1}{c|}{ \% Area } & Height & \% Height \\
\hline 1 & 11.008 & 9281155 & 95.03 & 272826 & 97.31 \\
\hline 2 & 18.033 & 485713 & 4.97 & 7542 & 2.69 \\
\hline
\end{tabular}<smiles>COC(=O)N[C@@H](c1ccc(Br)cc1)C(C(=O)OC)C(=O)OC</smiles> 
Table 5, entry 6: 18g Chiralcel OD-H Column, Hexanes : IPA $=90: 10,1.0 \mathrm{~mL} / \mathrm{min}, 214 \mathrm{~nm}$

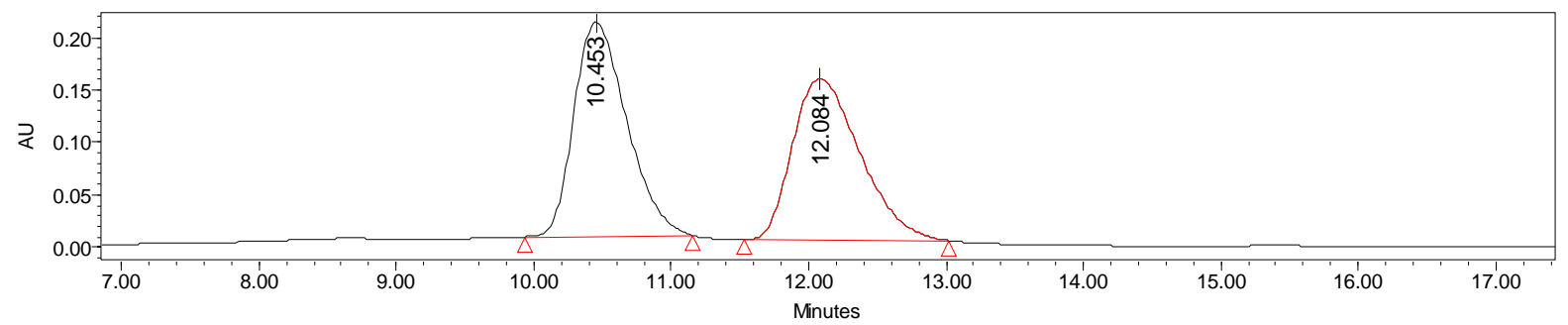

\begin{tabular}{|r|r|r|r|r|r|}
\hline & Retention Time & Area & \% Area & Height & \% Height \\
\hline 1 & 10.453 & 5435790 & 50.77 & 204090 & 56.93 \\
\hline 2 & 12.084 & 5270499 & 49.23 & 154403 & 43.07 \\
\hline
\end{tabular}<smiles>COC(=O)NC(c1ccc2c(c1)OCO2)C(C(=O)OC)C(=O)OC</smiles>

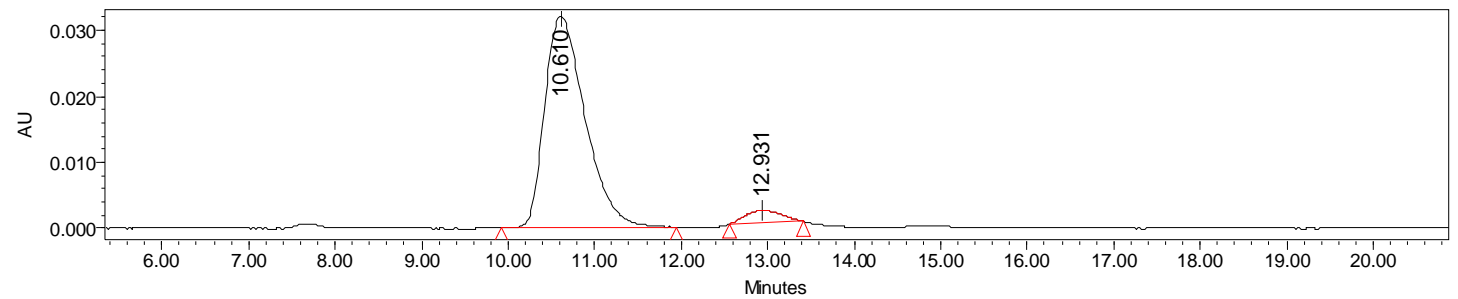

\begin{tabular}{|r|r|r|r|r|r|}
\hline & Retention Time & \multicolumn{1}{c|}{ Area } & \% Area & Height & \% Height \\
\hline 1 & 10.610 & 1039420 & 95.08 & 31964 & 94.56 \\
\hline 2 & 12.931 & 53732 & 4.92 & 1838 & 5.44 \\
\hline
\end{tabular}<smiles>COC(=O)N[C@@H](c1ccc2c(c1)OCO2)C(C(=O)OC)C(=O)OC</smiles> 
Table 5, entry 6: 19a $(R, R)$-Whelk-O 1 Column, Hexanes : IPA $=95: 5,1.0 \mathrm{~mL} / \mathrm{min}, 210 \mathrm{~nm}$ The racemate was made using 1:1 cinchonine and cinchonidine as catalyst.

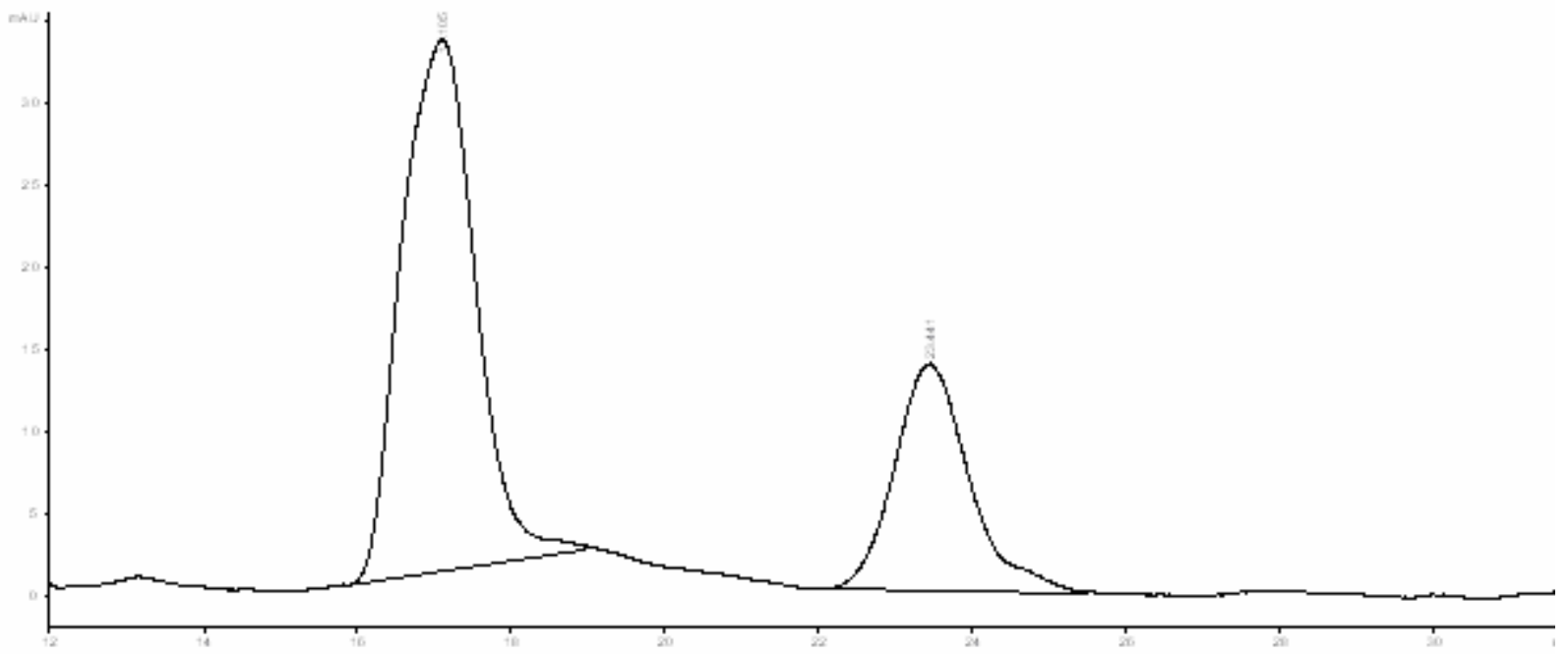

Signal 3: MWD1 C, Sig=210,8 Ref $=360,100$
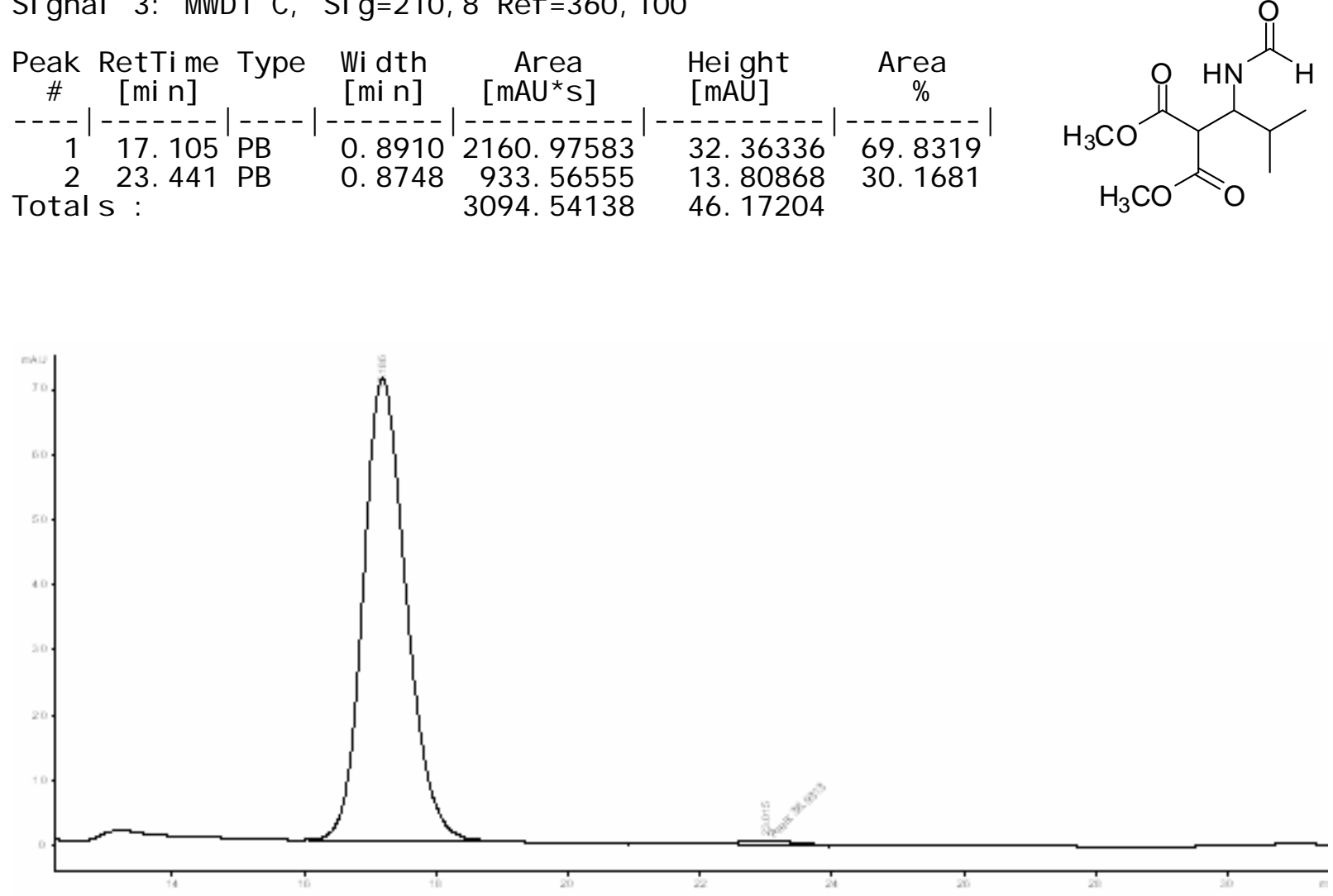

Signal 3: MWD1 C, Sig $=210,8$ Ref $=360,100$

\begin{tabular}{|c|c|c|c|c|c|c|}
\hline $\begin{array}{c}\text { Peak } \\
\#\end{array}$ & $\begin{array}{l}\text { Ret Ti me } \\
{[\mathrm{mi} \mathrm{n}]}\end{array}$ & Type & $\begin{array}{l}\text { Wi dt h } \\
{[\mathrm{min}]}\end{array}$ & $\begin{array}{c}\text { Area } \\
{\left[\mathrm{mAU}^{*} \mathrm{~s}\right]}\end{array}$ & $\begin{array}{l}\text { Height } \\
{[\mathrm{mAU}]}\end{array}$ & $\begin{array}{c}\operatorname{Area} \\
\%\end{array}$ \\
\hline Tot ${ }^{2}$ & $\begin{array}{l}17.186 \\
23.015 \\
s \quad:\end{array}$ & $\begin{array}{l}- \\
B B \\
M M\end{array}$ & $\begin{array}{l}0.6851 \\
0.9304\end{array}$ & $\begin{array}{r}3206.17090 \\
38.93131 \\
3245.10221\end{array}$ & $\begin{array}{r}70.96543 \\
6.97410 \mathrm{e}-1 \\
71.66284\end{array}$ & $\begin{array}{r}98.8003 \\
1.1997\end{array}$ \\
\hline
\end{tabular}<smiles>COC(=O)C(C(=O)OC)[C@H](NC=O)C(C)C</smiles> 
Table 5, entry 7: 19b $(R, R)$-Whelk-O 1 Column, Hexanes : IPA $=95: 5,1.0 \mathrm{~mL} / \mathrm{min}, 210 \mathrm{~nm}$

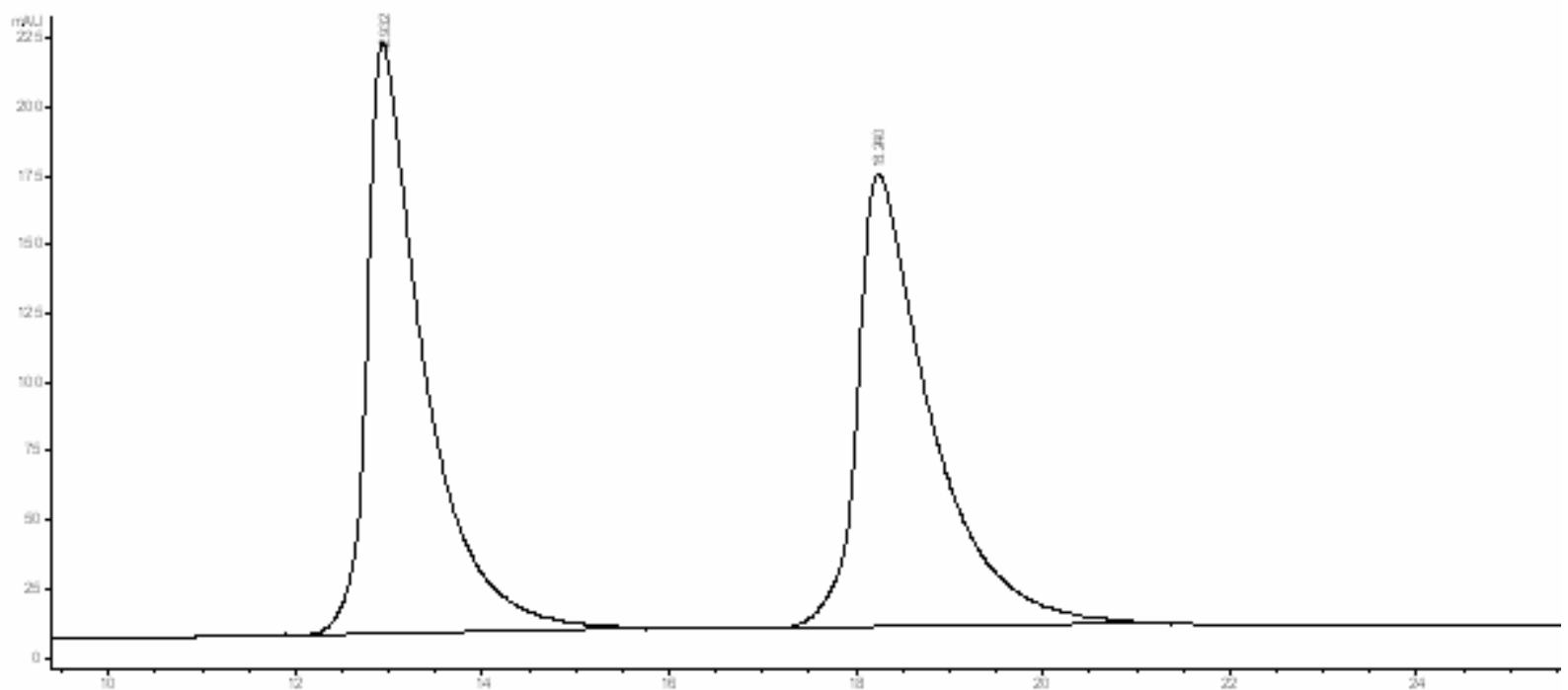

Signal 3: MWD1 C, Sig=210,8 Ref $=360,100$

Peak RetTime Type Width Area Height Area

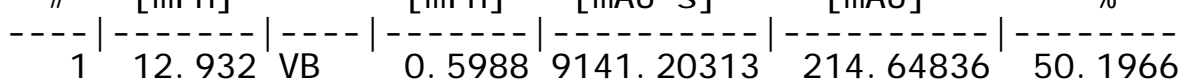

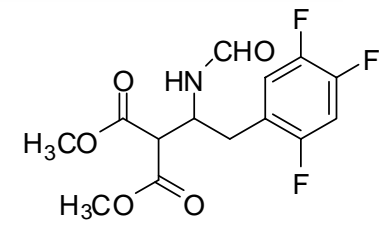

Totals:

$0.77569069 .60156 \quad 164.92244$

49.8034

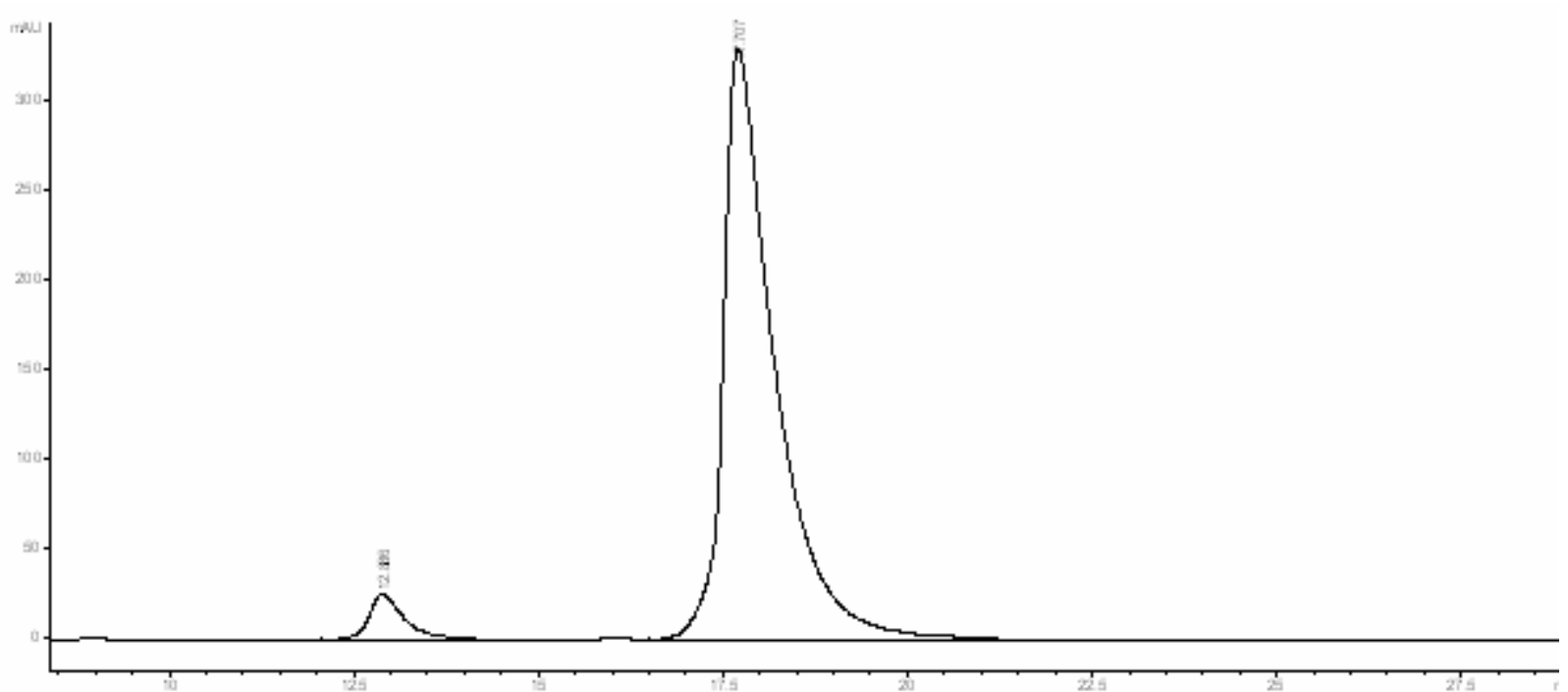

Signal 3: MWD1 C, Sig=210,8 Ref $=360,100$

Peak RetTime Type Width Area Height Area $\# \quad[\mathrm{mi}]$
$\ldots$ $112.886 \mathrm{MM} \quad 0.5063 \quad 764.06006 \quad 25.15283 \quad 4.4035$

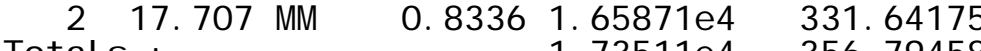
Totals:

$1.73511 \mathrm{e} 4356.79458$<smiles>COC(=O)C(C(=O)OC)[C@H](Cc1cc(F)c(F)cc1F)NC=O</smiles> 
Table 5, entry 8: 19c ChiralPak AD-H Column, Hexanes : IPA = $95: 5,1.0 \mathrm{~mL} / \mathrm{min}, 214 \mathrm{~nm}$

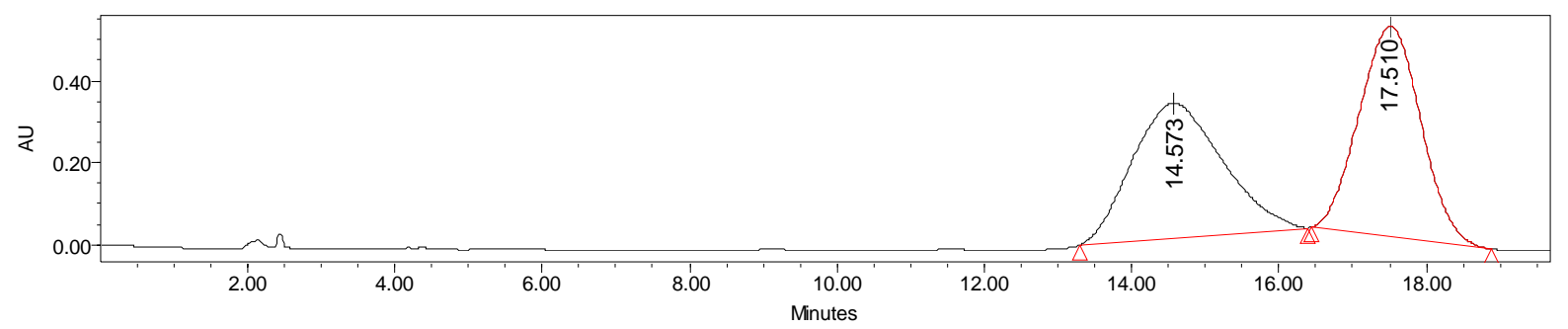

\begin{tabular}{|r|r|r|r|r|r|}
\hline & Retention Time & Area & \% Area & Height & \% Height \\
\hline 1 & 14.573 & 27872347 & 48.12 & 328928 & 39.07 \\
\hline 2 & 17.510 & 30044979 & 51.88 & 512947 & 60.93 \\
\hline
\end{tabular}<smiles>COC(=O)C(C(=O)OC)C(NC=O)c1ccccc1</smiles>

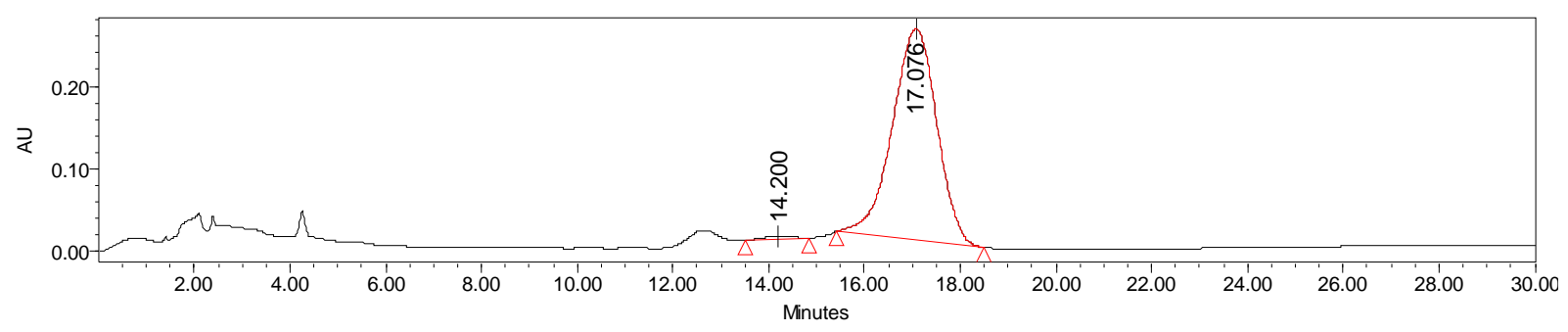

\begin{tabular}{|r|r|r|r|r|r|}
\hline & Retention Time & \multicolumn{1}{|c|}{ Area } & \% Area & Height & \% Height \\
\hline 1 & 14.200 & 149852 & 0.90 & 3457 & 1.33 \\
\hline 2 & 17.076 & 16503858 & 99.10 & 255850 & 98.67 \\
\hline
\end{tabular}<smiles>COC(=O)C(C(=O)OC)[C@H](NC=O)c1ccccc1</smiles> 
Table 6, entry 1: 21a ChiralPak AD-H Column, Hexanes : IPA = $95: 5,1.0 \mathrm{~mL} / \mathrm{min}, 210 \mathrm{~nm}$

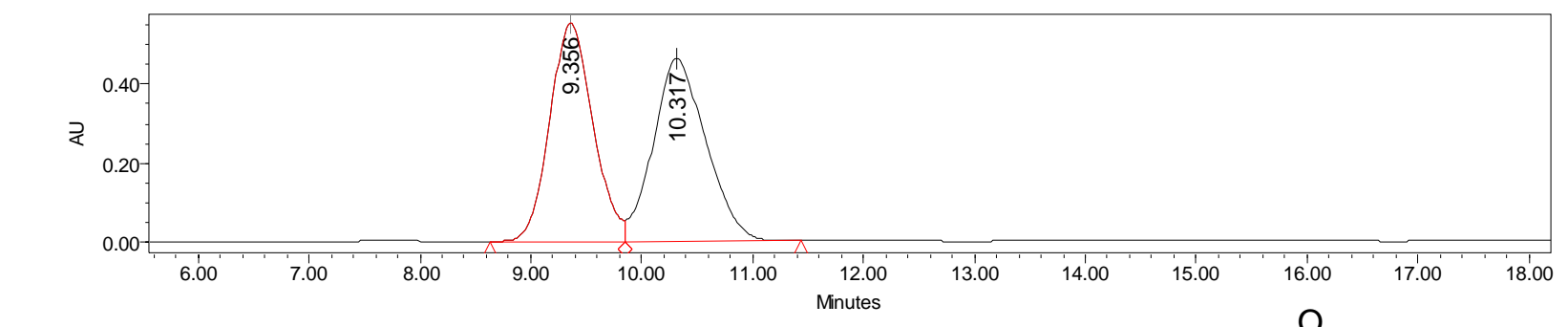

\begin{tabular}{|r|r|r|r|r|r|}
\hline & Retention Time & Area & \% Area & Height & \% Height \\
\hline 1 & 9.356 & 14975266 & 49.55 & 553879 & 54.39 \\
\hline 2 & 10.317 & 15248228 & 50.45 & 464435 & 45.61 \\
\hline
\end{tabular}

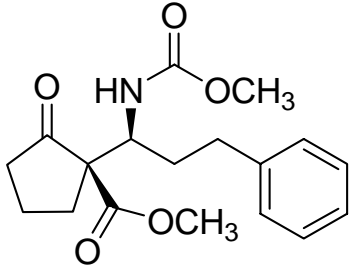

single diastereomer racemate

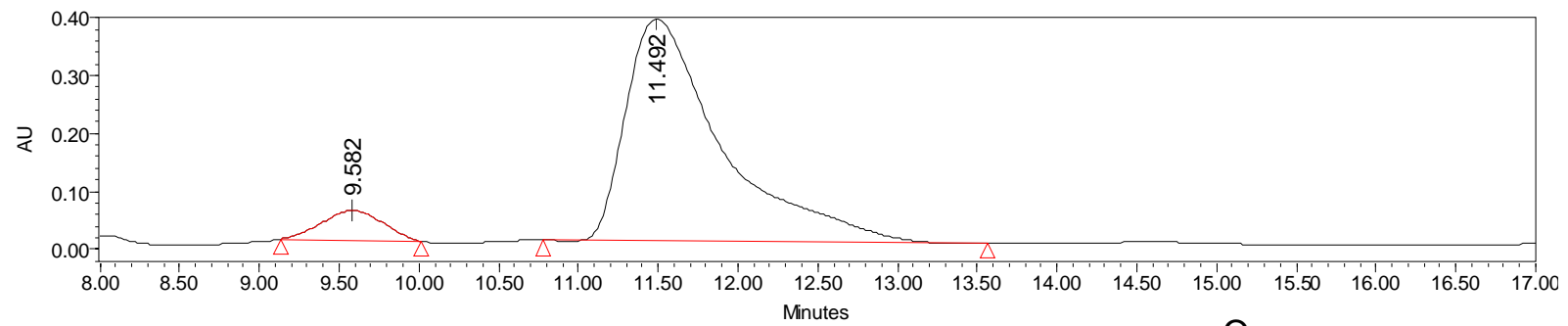

\begin{tabular}{|r|r|r|r|r|r|}
\hline & Retention Time & \multicolumn{1}{c|}{ Area } & \% Area & Height & \multicolumn{1}{c|}{ \% Height } \\
\hline 1 & 9.582 & 869767 & 5.12 & 40325 & 9.50 \\
\hline 2 & 11.492 & 16103967 & 94.88 & 383974 & 90.50 \\
\hline
\end{tabular}<smiles>COC(=O)N[C@@H](CCc1ccccc1)[C@]1(C(=O)OC)CCCC1=O</smiles> 
Table 6, entry 2: 21b ChiralPak AD-H Column, Hexanes : IPA = $95: 5,1.0 \mathrm{~mL} / \mathrm{min}, 210 \mathrm{~nm}$

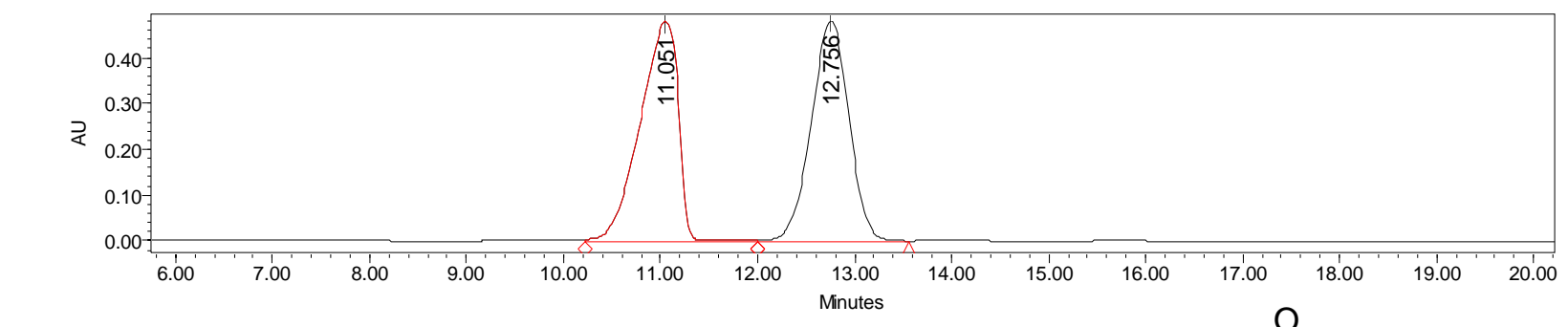

\begin{tabular}{|r|r|r|r|r|r|}
\hline & Retention Time & Area & \% Area & Height & \% Height \\
\hline 1 & 11.051 & 13087448 & 50.26 & 479244 & 49.96 \\
\hline 2 & 12.756 & 12950289 & 49.74 & 480020 & 50.04 \\
\hline
\end{tabular}<smiles>COC(=O)N[C@@H](CCc1ccccc1)C1(C(C)=O)CCCC1=O</smiles>

single diastereomer racemate

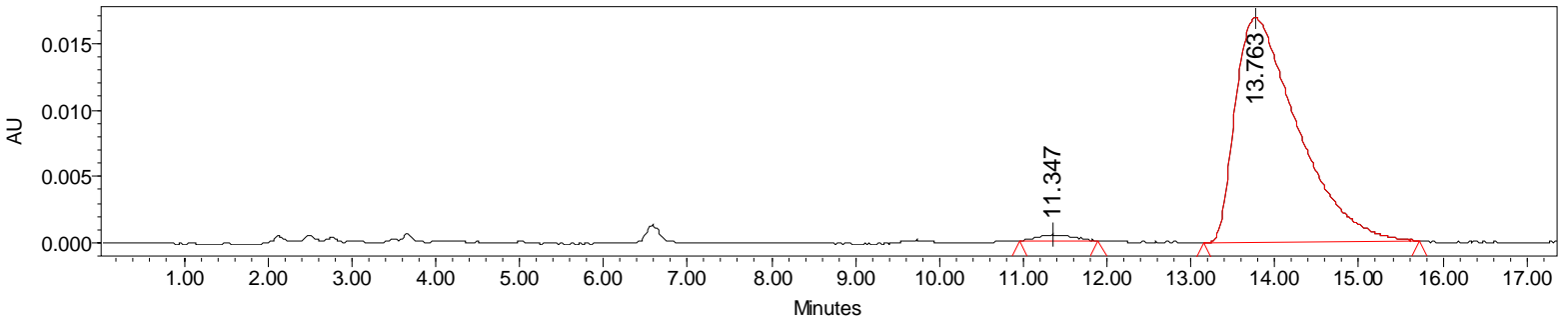

\begin{tabular}{|r|r|r|r|r|r|}
\hline & Retention Time & \multicolumn{1}{c|}{ Area } & \% Area & Height & \% Height \\
\hline 1 & 11.347 & 13257 & 1.50 & 456 & 2.63 \\
\hline 2 & 13.763 & 870111 & 98.50 & 16859 & 97.37 \\
\hline
\end{tabular}<smiles>COC(=O)N[C@@H](CCc1ccccc1)[C@]1(C(C)=O)CCCC1=O</smiles> 
Table 6, entry 3: 21c ChiralPak AD-H Column, Hexanes : IPA = 95:5, $1.0 \mathrm{~mL} / \mathrm{min}, 210 \mathrm{~nm}$

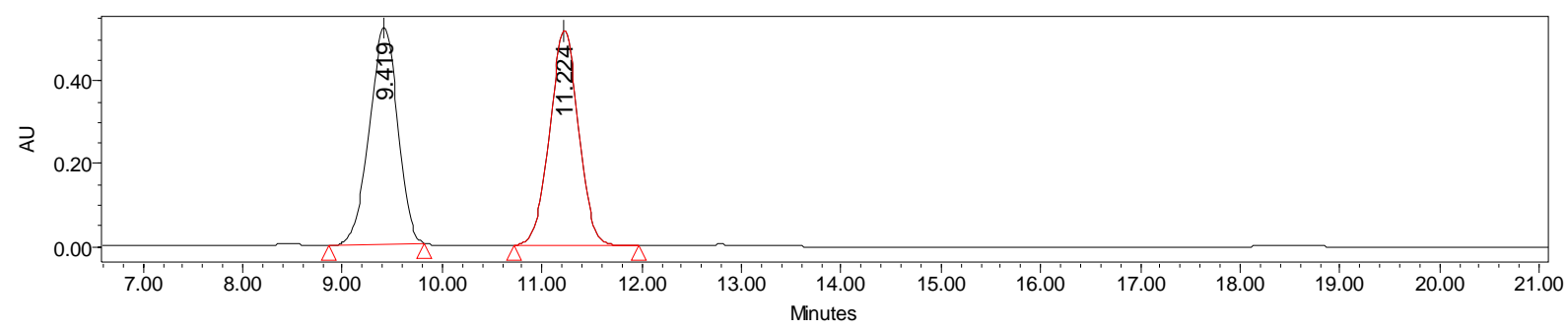

\begin{tabular}{|r|r|r|r|r|r|}
\hline & Retention Time & Area & \% Area & Height & \% Height \\
\hline 1 & 9.419 & 10344631 & 49.64 & 522368 & 50.25 \\
\hline 2 & 11.224 & 10493345 & 50.36 & 517214 & 49.75 \\
\hline
\end{tabular}

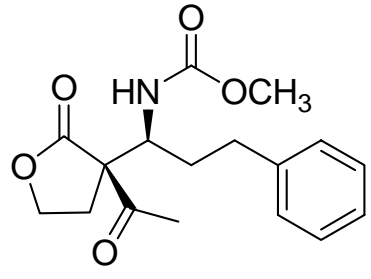

single diastereomer racemate

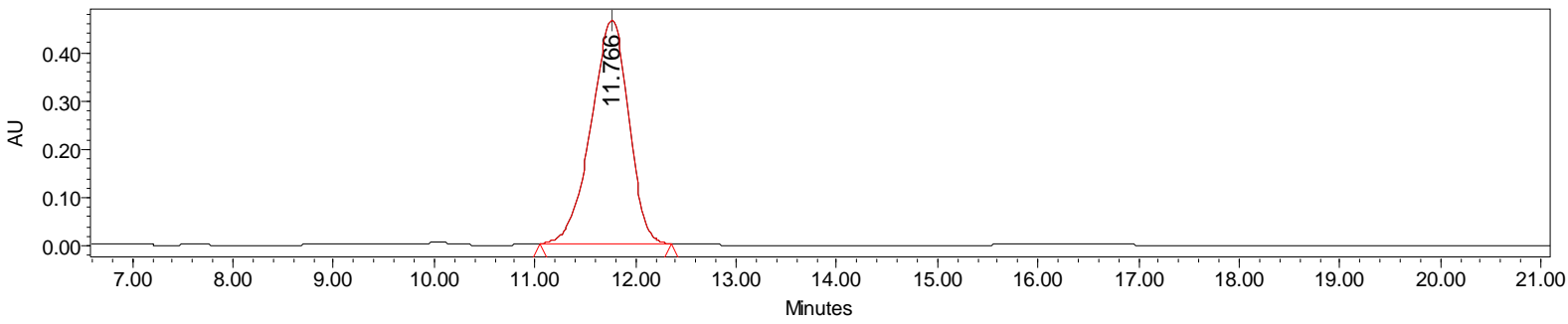

\begin{tabular}{|l|r|r|r|r|r|}
\hline & Retention Time & Area & \% Area & Height & \% Height \\
\hline 1 & 11.766 & 12044302 & 100.00 & 462534 & 100.00 \\
\hline
\end{tabular}<smiles>COC(=O)N[C@@H](CCc1ccccc1)C1(C(C)=O)CCOC1=O</smiles> 
Table 7, entry 1: 23a $(R, R)$-Whelk-O 1 Column, Hexanes : IPA $=95: 5,1.0 \mathrm{~mL} / \mathrm{min}, 210 \mathrm{~nm}$

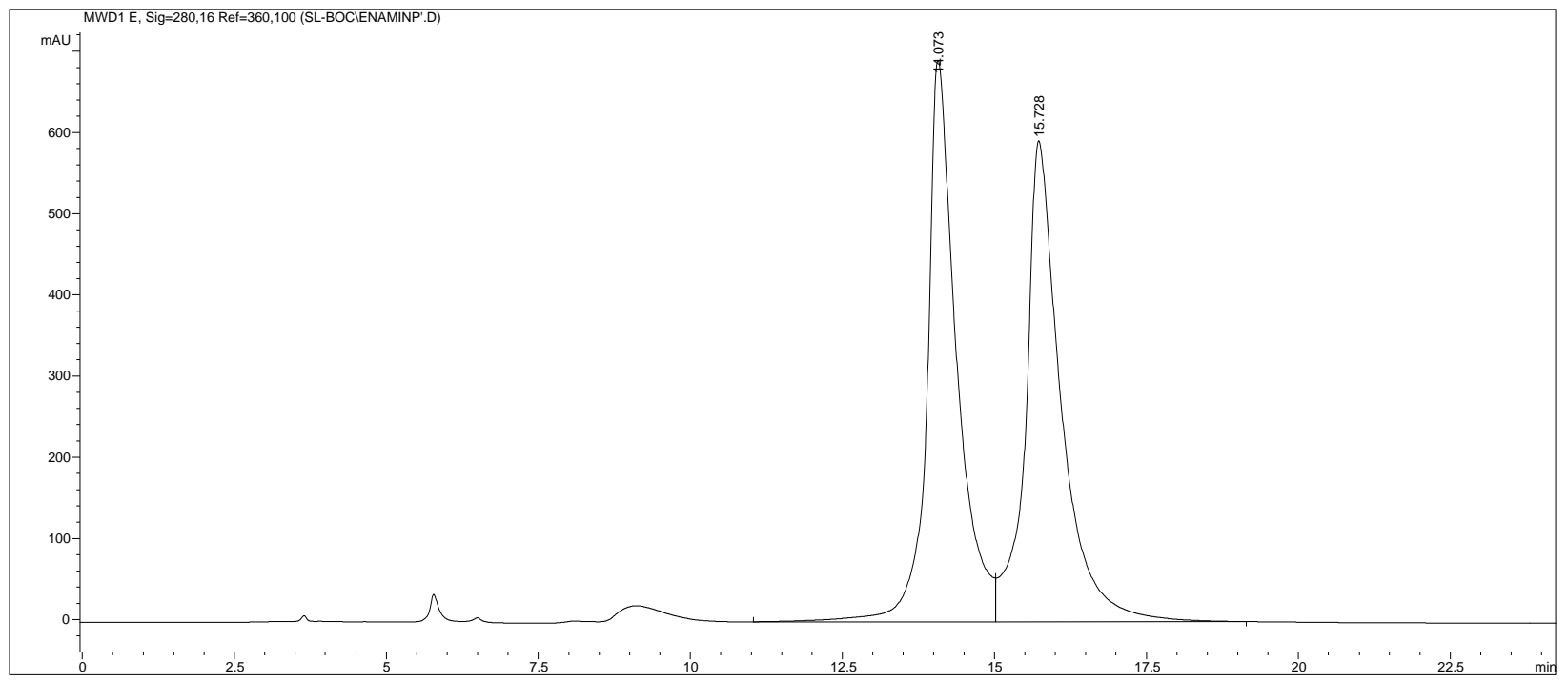

Signal 5: MWD1 E, Sig=280,16 Ref $=360,100$

Peak RetTime Type Width Area Height Area $\# \quad[\mathrm{~min}] \quad[\mathrm{min}] \quad\left[\mathrm{mAU}^{*} \mathrm{~s}\right] \quad[\mathrm{mAU}] \quad \%$

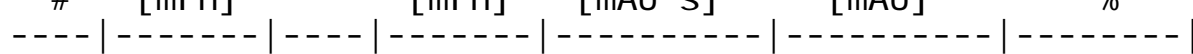

$$
114.073 \mathrm{BV}
$$

$2 \quad 15.728$ VB

Totals:

0.48132 .35352 e 4

0.56882 .40134 e 4

2. $40134 \mathrm{e} \quad 592.56567$

4. 75486 e 4 1283.71082<smiles>CCOC(=O)NC(C(C(=O)OC)=C(C)NCc1ccccc1)c1ccccc1</smiles>

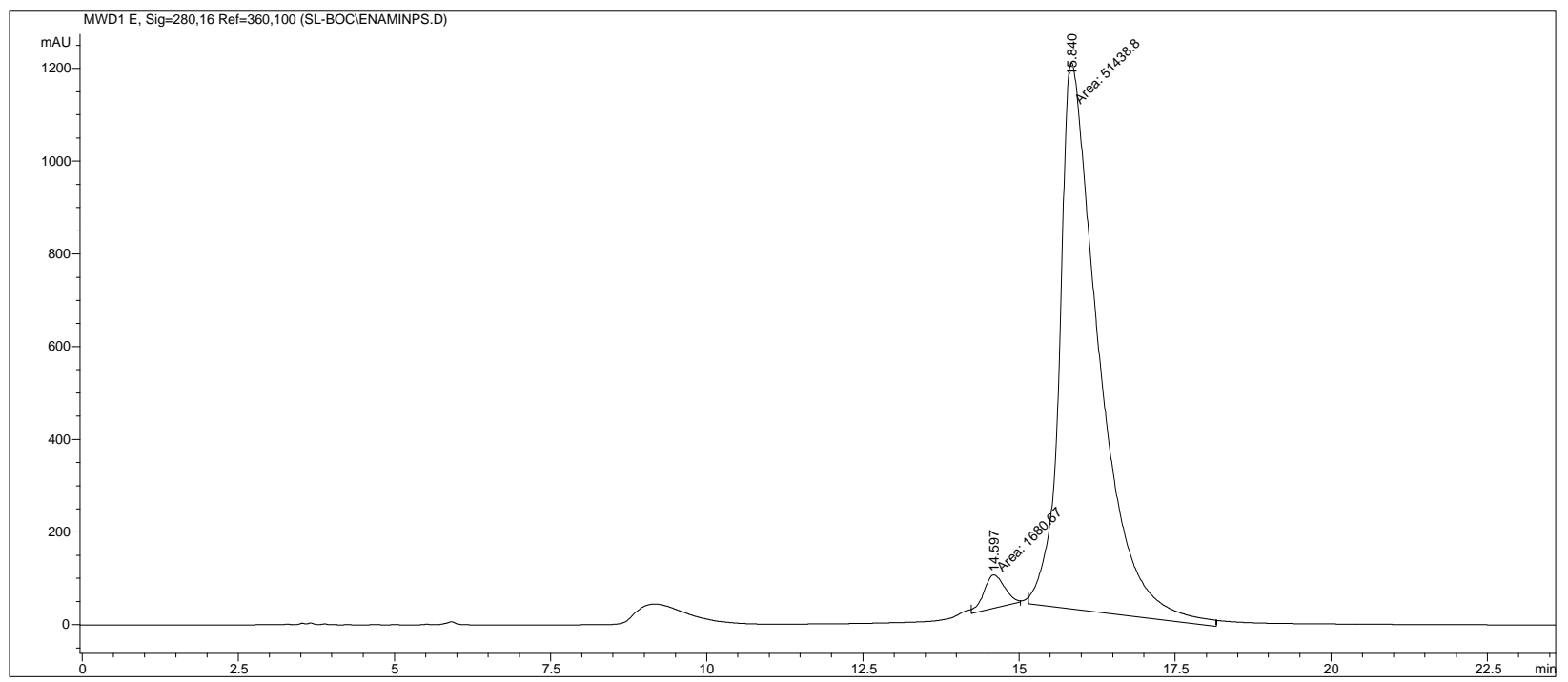

Signal 5: MWD1 E, Sig=280,16 Ref $=360,100$

Peak RetTime Type Width Area $\quad$ Height Area

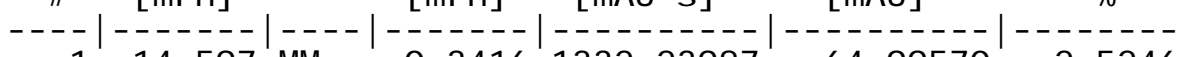

$\begin{array}{lllllll}1 & 14.597 & \text { MM } & 0.3416 & 1332.23987 & 64.99570 & 2.5246\end{array}$

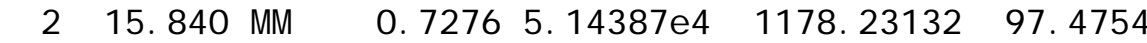

Totals:

5. 27710 e 1243.22702<smiles>CCCOC(=O)N[C@@H](C(C(=O)OC)=C(C)NCc1ccccc1)c1ccccc1</smiles> 
Table 7, entry 2: 23b ChiralPak AD-H Column, Hexanes : IPA = $99: 1,1.0 \mathrm{~mL} / \mathrm{min}, 214 \mathrm{~nm}$

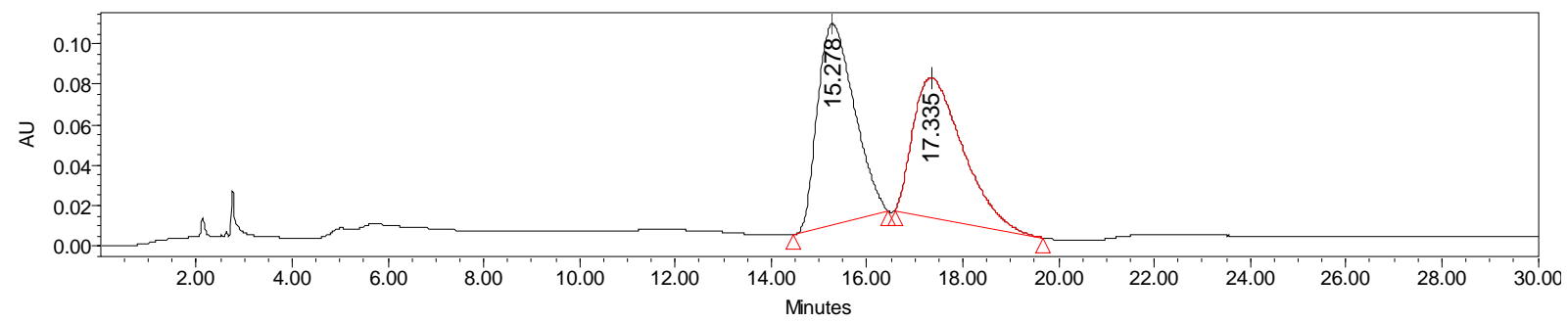

\begin{tabular}{|r|r|r|r|r|r|}
\hline & Retention Time & Area & \% Area & Height & \% Height \\
\hline 1 & 15.278 & 5299120 & 51.55 & 99708 & 59.04 \\
\hline 2 & 17.335 & 4981084 & 48.45 & 69166 & 40.96 \\
\hline
\end{tabular}<smiles>CC(=O)OC(=O)NC(c1ccccc1)C(C(C)=O)C(C)=O</smiles>

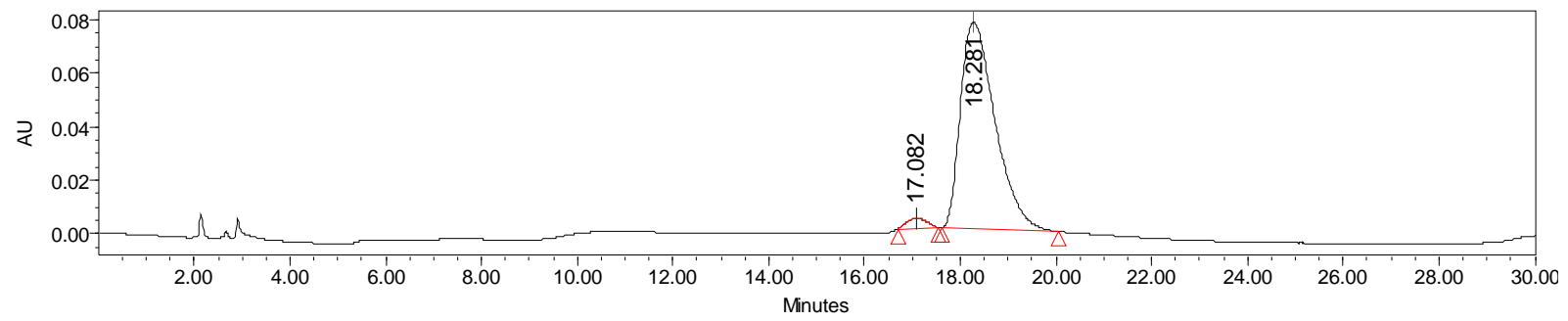

\begin{tabular}{|r|r|r|r|r|r|}
\hline & Retention Time & \multicolumn{1}{c|}{ Area } & \% Area & Height & \multicolumn{1}{c|}{ \% Height } \\
\hline 1 & 17.082 & 106140 & 2.66 & 3634 & 4.49 \\
\hline 2 & 18.281 & 3889009 & 97.34 & 77385 & 95.51 \\
\hline
\end{tabular}<smiles>CC(=O)OC(=O)N[C@H](c1ccccc1)C(C(C)=O)C(C)=O</smiles> 
Table 7, entry 3: 23c ChiralPak AD-H Column, Hexanes : IPA = $85: 15,1.0 \mathrm{~mL} / \mathrm{min}, 214 \mathrm{~nm}$

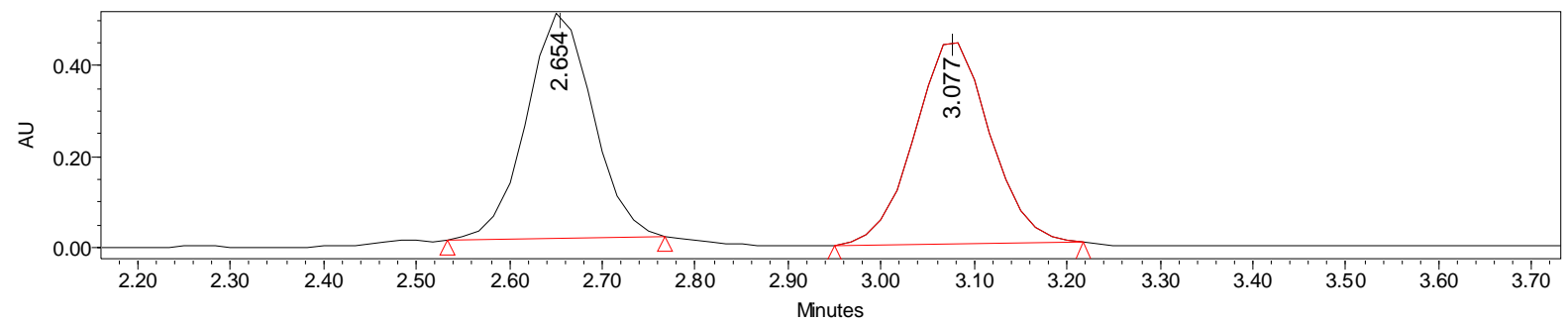

\begin{tabular}{|r|r|r|r|r|r|}
\hline & Retention Time & \multicolumn{1}{|c|}{ Area } & \% Area & Height & \% Height \\
\hline 1 & 2.654 & 2472204 & 49.35 & 489383 & 52.43 \\
\hline 2 & 3.077 & 2537691 & 50.65 & 443965 & 47.57 \\
\hline
\end{tabular}<smiles>COC(=O)NC(c1ccccc1)C(C(=O)OC)C(=O)OC</smiles>

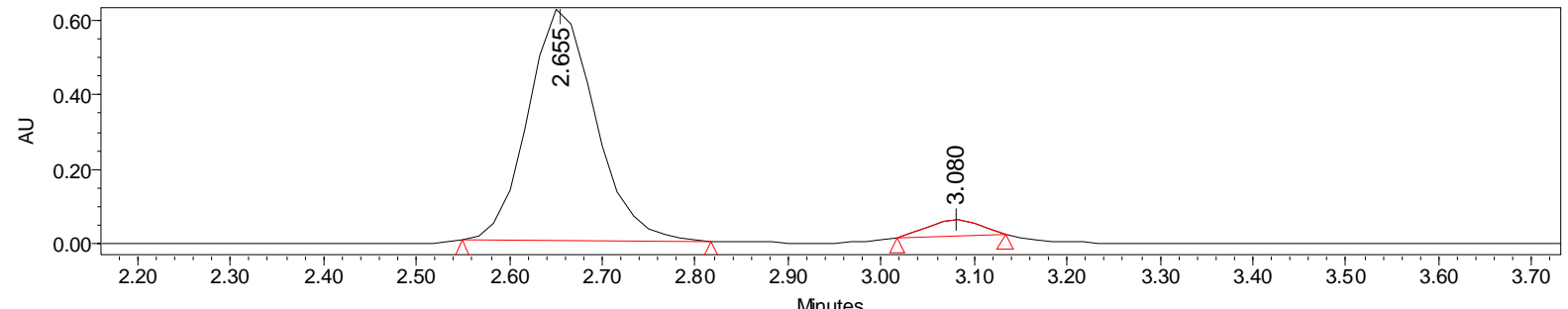

\begin{tabular}{|r|r|r|r|r|r|}
\hline & Retention Time & \multicolumn{1}{|c|}{ Area } & \% Area & Height & \% Height \\
\hline 1 & 2.655 & 3151303 & 94.81 & 617516 & 93.55 \\
\hline 2 & 3.080 & 172429 & 5.19 & 42584 & 6.45 \\
\hline
\end{tabular}<smiles>CCCCOC(=O)N[C@H](c1ccccc1)C(C(=O)OC)C(=O)OC</smiles> 
Table 7, entry 5: 23f $(R, R)$-Whelk-O 1 column, Hexanes : IPA $=98: 2,1.0 \mathrm{~mL} / \mathrm{min}, 210 \mathrm{~nm}$

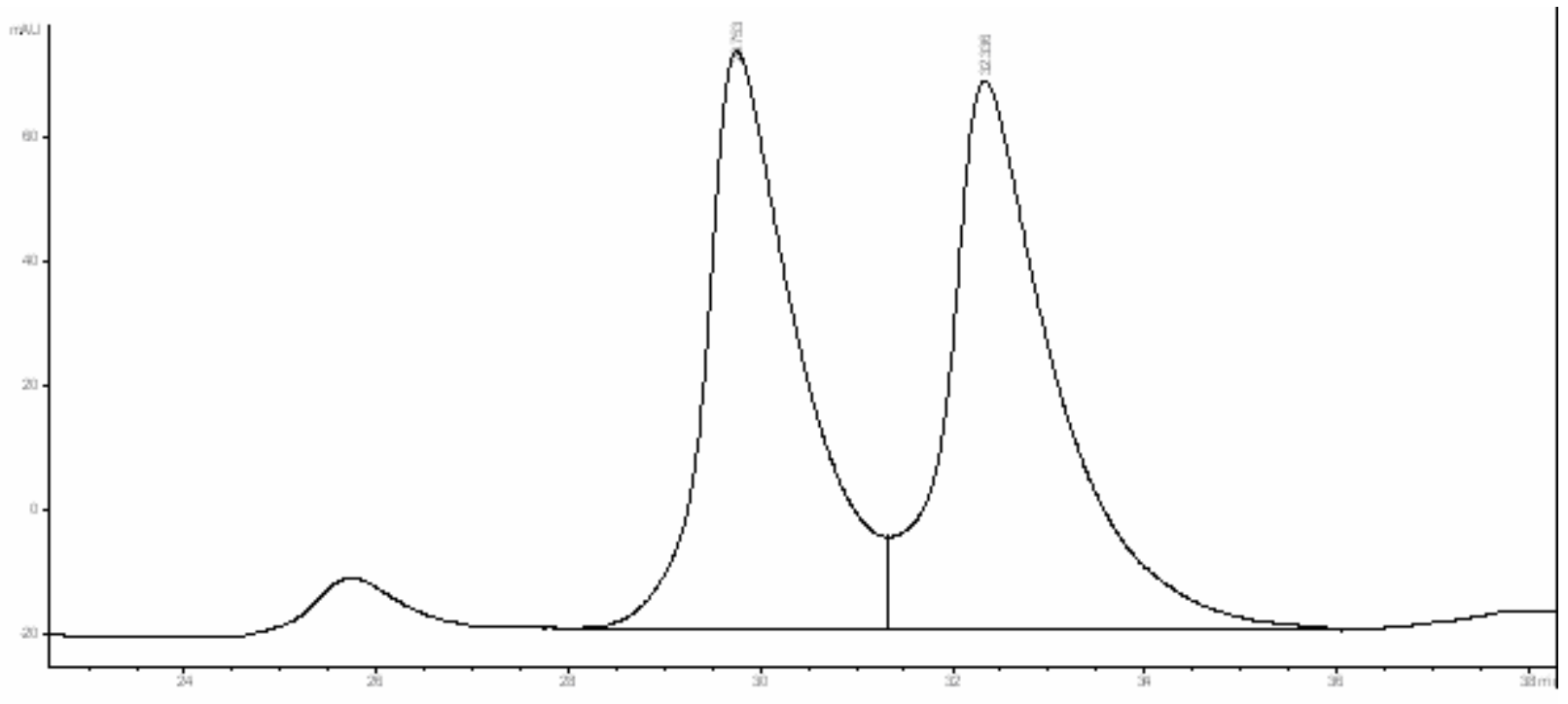

Signal 3: MWD1 C, Sig=210,8 Ref $=360,100$
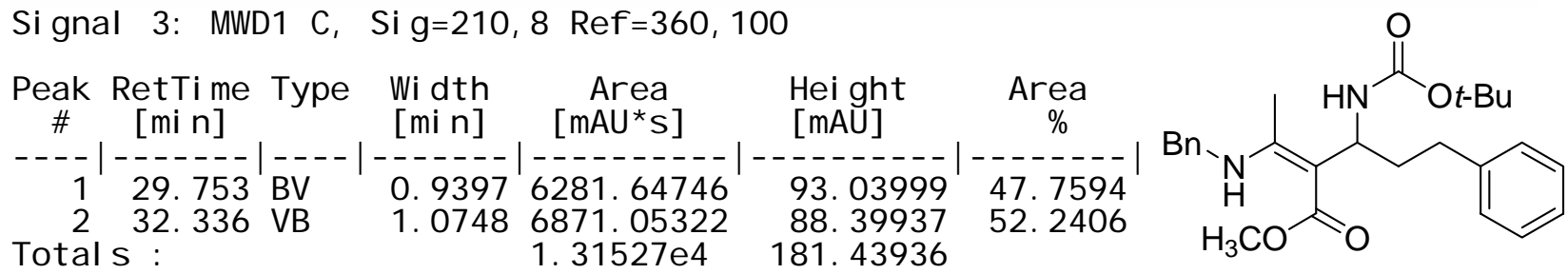

Totals:

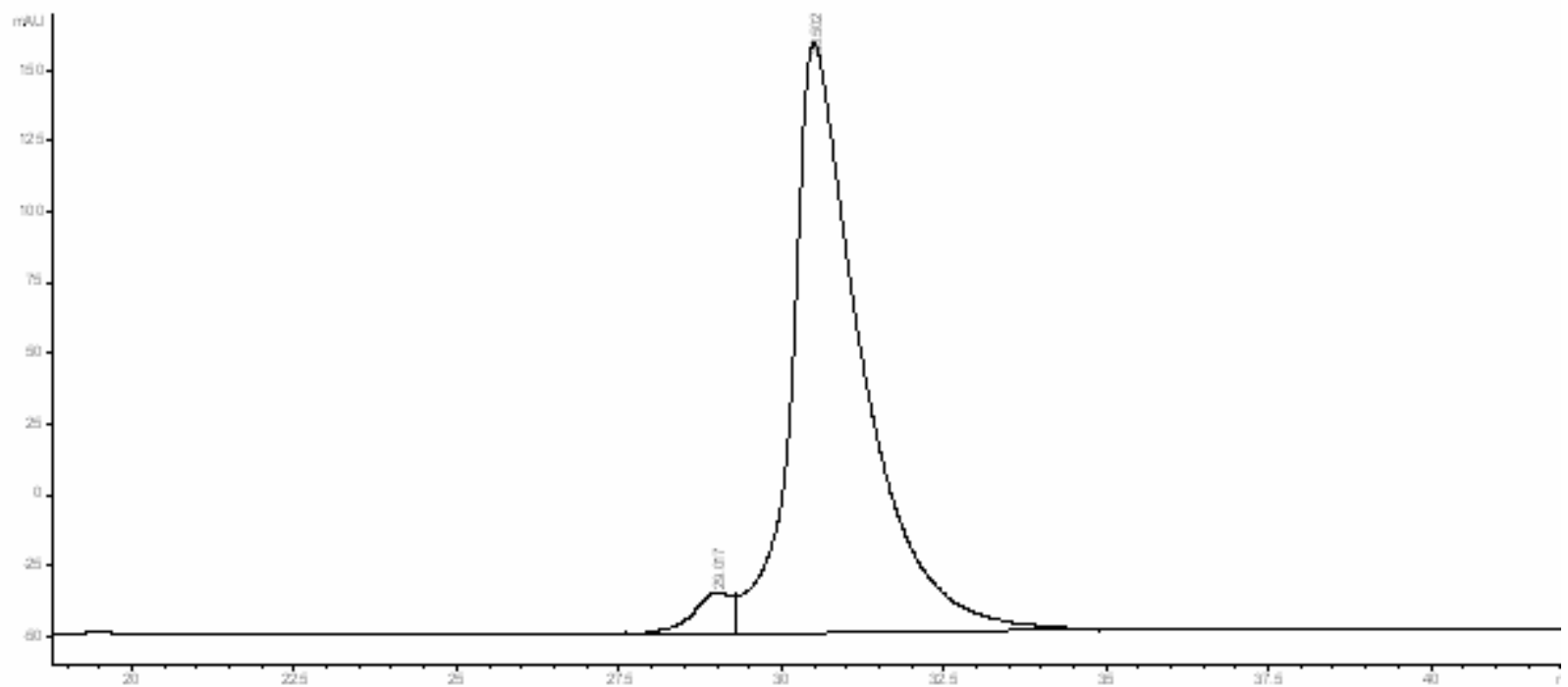

Signal 3: MWD1 C, Sig=210,8 Ref $=360,100$

\begin{tabular}{|c|c|c|c|c|c|c|}
\hline$\underset{\#}{\text { Peak }}$ & $\begin{array}{c}\text { Ret Ti me } \\
{[\mathrm{mi} n]}\end{array}$ & Type & $\begin{array}{l}\text { Wi dt h } \\
{[\mathrm{mi} \mathrm{n}]}\end{array}$ & $\begin{array}{c}\text { Area } \\
{[m A \cup * s]}\end{array}$ & $\begin{array}{l}\text { Height } \\
\text { [mAU] }\end{array}$ & $\begin{array}{c}\operatorname{Area} \\
\%\end{array}$ \\
\hline $\begin{array}{c}\frac{1}{2} \\
\text { Tot al }\end{array}$ & $\begin{array}{r}29.017 \\
30.502 \\
S \quad:\end{array}$ & $\begin{array}{l}\text { B V } \\
\text { VB }\end{array}$ & $\begin{array}{l}0.5994 \\
1.0436\end{array}$ & $\begin{array}{r}603.57031 \\
1.55606 \mathrm{e} 4 \\
1.61642 \mathrm{e} 4\end{array}$ & $\begin{array}{r}14.15732 \\
208.27594 \\
222.43326\end{array}$ & $\begin{array}{r}3.7340 \\
96.2660\end{array}$ \\
\hline
\end{tabular}<smiles>CCOC(=O)N[C@@H](CCc1ccccc1)C(C(=O)OC)=C(C)NCc1ccccc1</smiles> 
Table 7, entry 4: 23e $(R, R)$-Whelk-O 1 column, Hexanes : IPA $=95: 5,1.0 \mathrm{~mL} / \mathrm{min}, 210 \mathrm{~nm}$

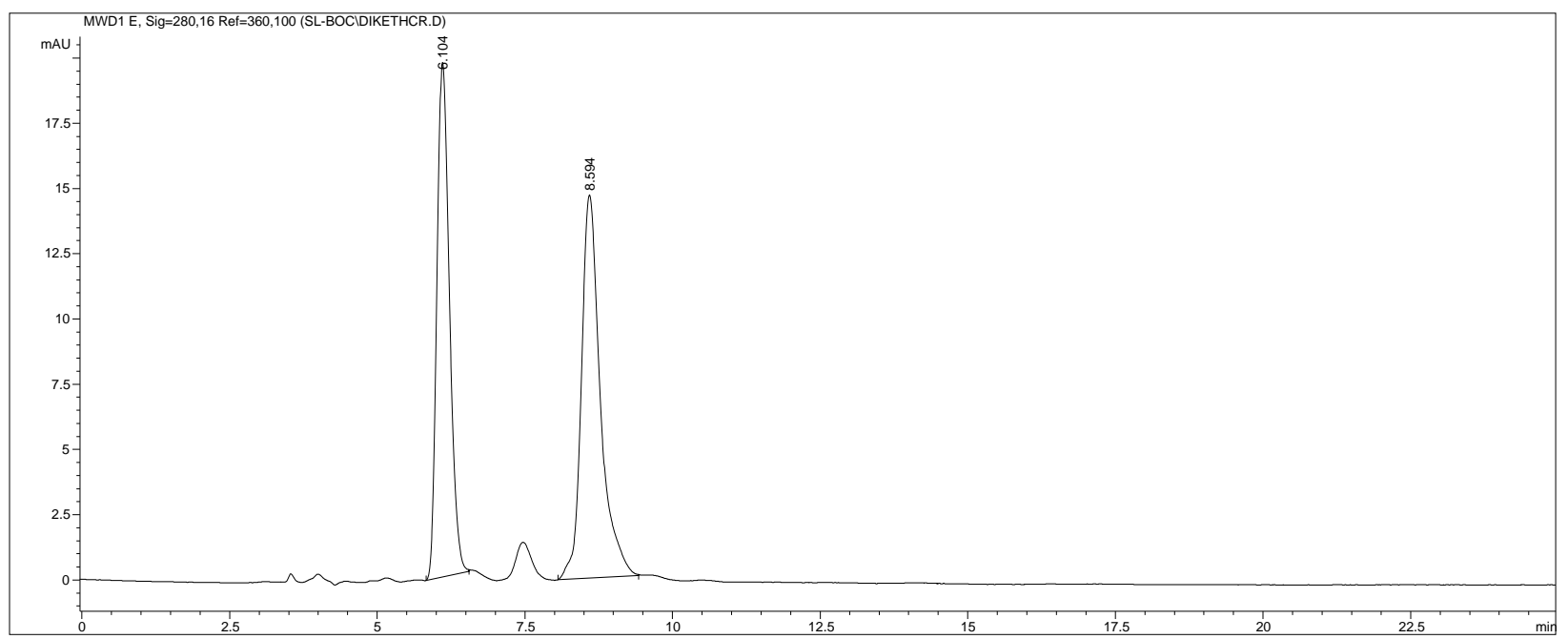

Signal 5: MWD1 E, Sig=280,16 Ref $=360,100$

Peak RetTime Type Width Area Height Area

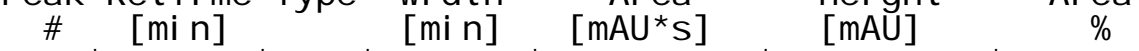

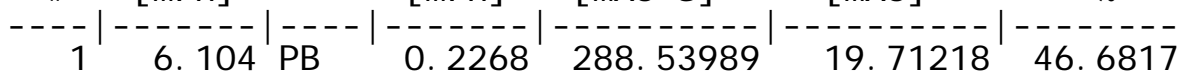

$\begin{array}{llllll}1 & 6.104 & & \end{array}$

Totals:

$618.10095 \quad 34.38703$
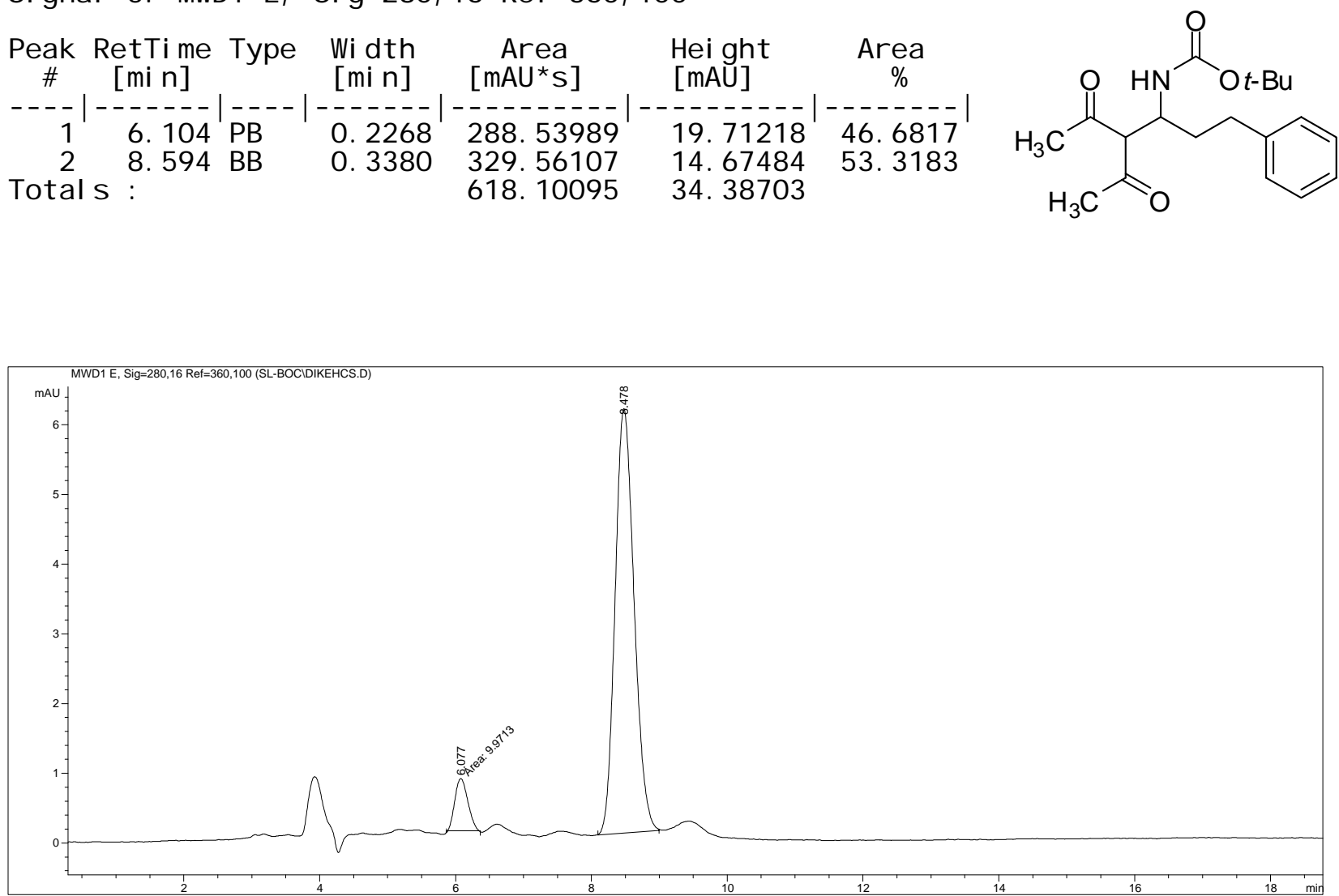

Signal 5: MWD1 $E, \stackrel{4}{4}^{2} i g=280,16$ Ref $=360,100$

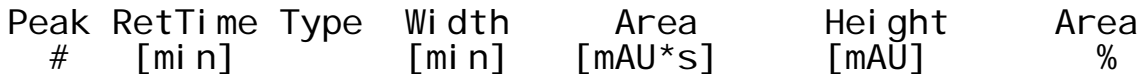

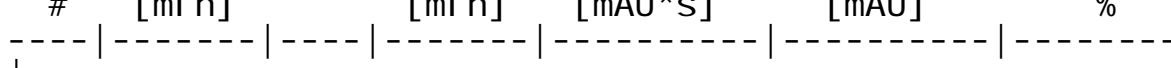

।

$1 \quad 6.077$ MM $\quad 0.1817 \quad 9.97134 \quad 5.98081 \mathrm{e}-1$

$2 \quad 8.478$ MM $\quad 0.3380 \quad 124.81108$ 6.15501

Tot als

6. 75309

4. 9655

95.0345

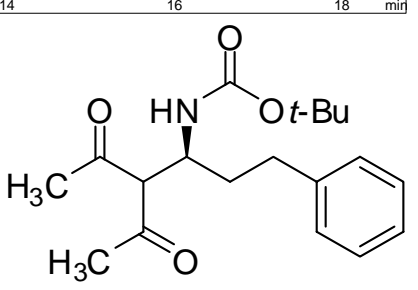




\section{Selected Chiral HPLC trace for dihydropyrimidones:}<smiles>CCN1C(=O)N[C@@H](c2ccccc2)C(C(=O)OC)=C1C</smiles>

ChiralPak OD Column, Hexane:IPA $=90: 10,1.0 \mathrm{~mL} / \mathrm{min}$

$S$ enatiomer: $\mathrm{t}_{\mathrm{r}}=7.0 \mathrm{~min}, R$ enatiomer: $\mathrm{t}_{\mathrm{r}}=8.2 \mathrm{~min}$.

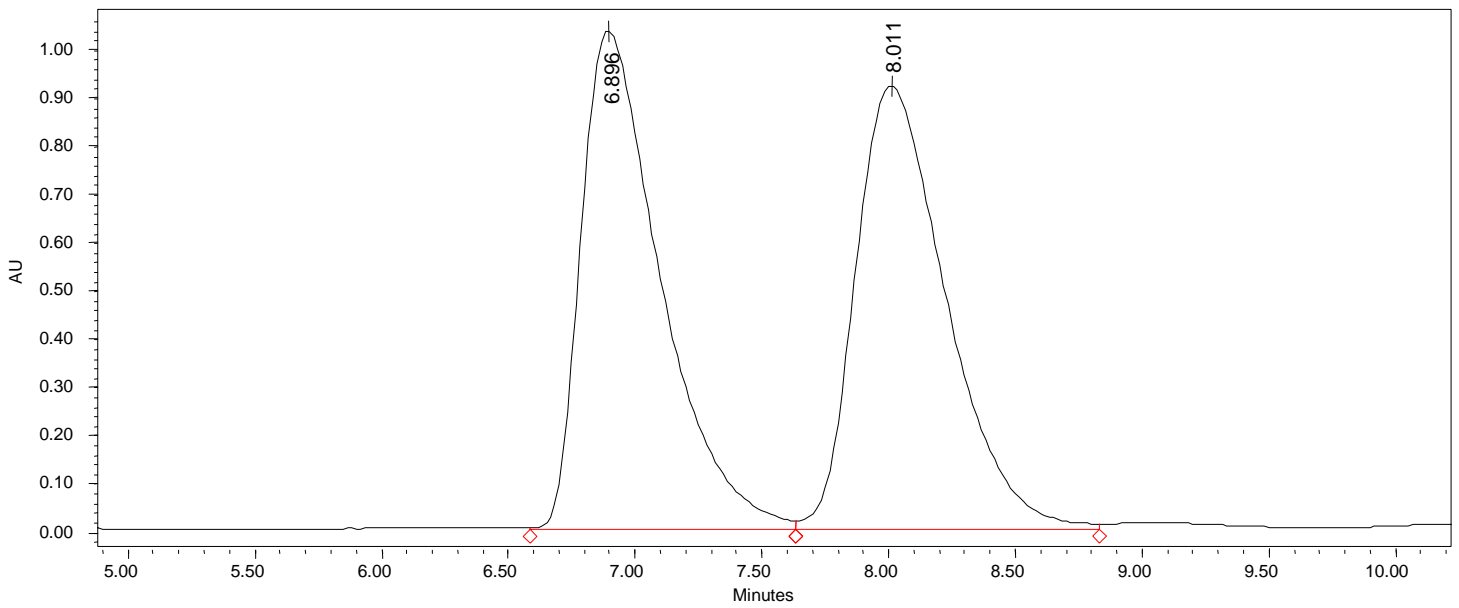

\begin{tabular}{|r|r|r|l|r|r|r|r|}
\hline & Name & Retention Time & Peak Type & Area & \% Area & \multicolumn{1}{|c|}{ Height } & \% Height \\
\hline 1 & & 6.896 & Unknown & 22527771 & 49.66 & 1034122 & 52.92 \\
\hline 2 & & 8.011 & Unknown & 22840439 & 50.34 & 919986 & 47.08 \\
\hline
\end{tabular}

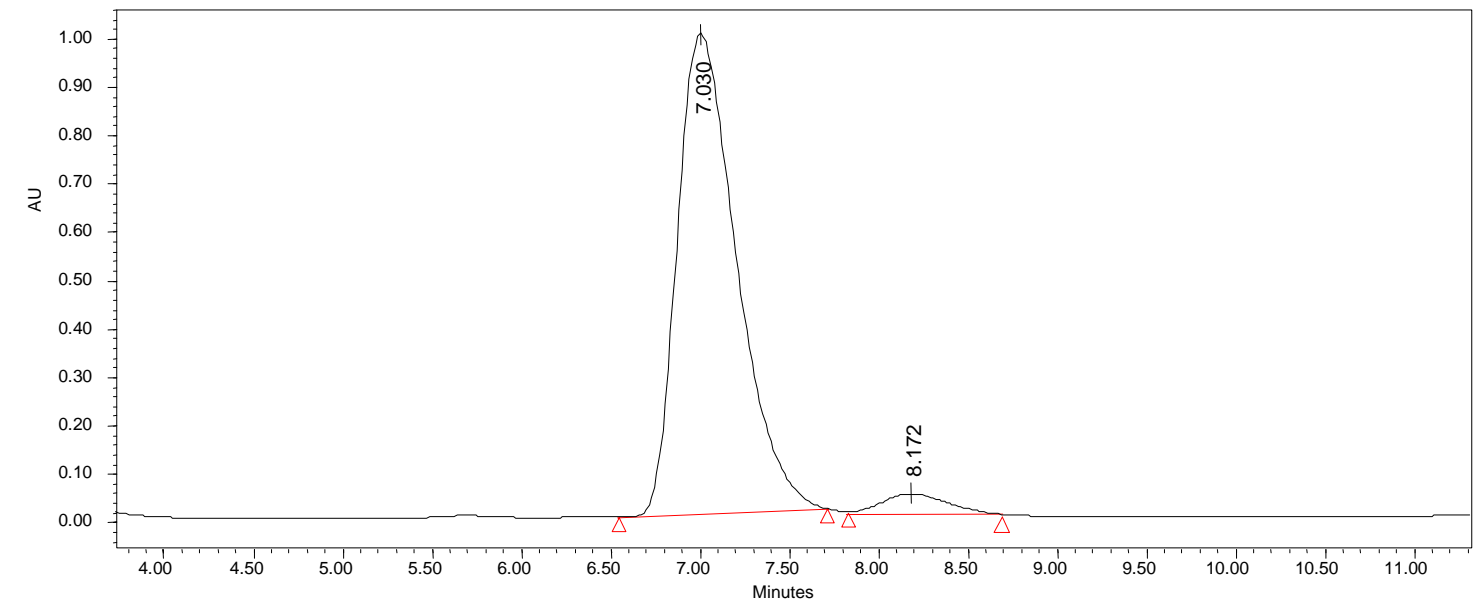

\begin{tabular}{|r|r|r|l|r|r|r|r|}
\hline & Name & Retention Time & Peak Type & \multicolumn{1}{c|}{ Area } & \% Area & \multicolumn{1}{c|}{ Height } & \% Height \\
\hline 1 & & 7.030 & Unknown & 21805965 & 95.54 & 1025283 & 96.11 \\
\hline 2 & & 8.172 & Unknown & 1017946 & 4.46 & 41498 & 3.89 \\
\hline
\end{tabular}




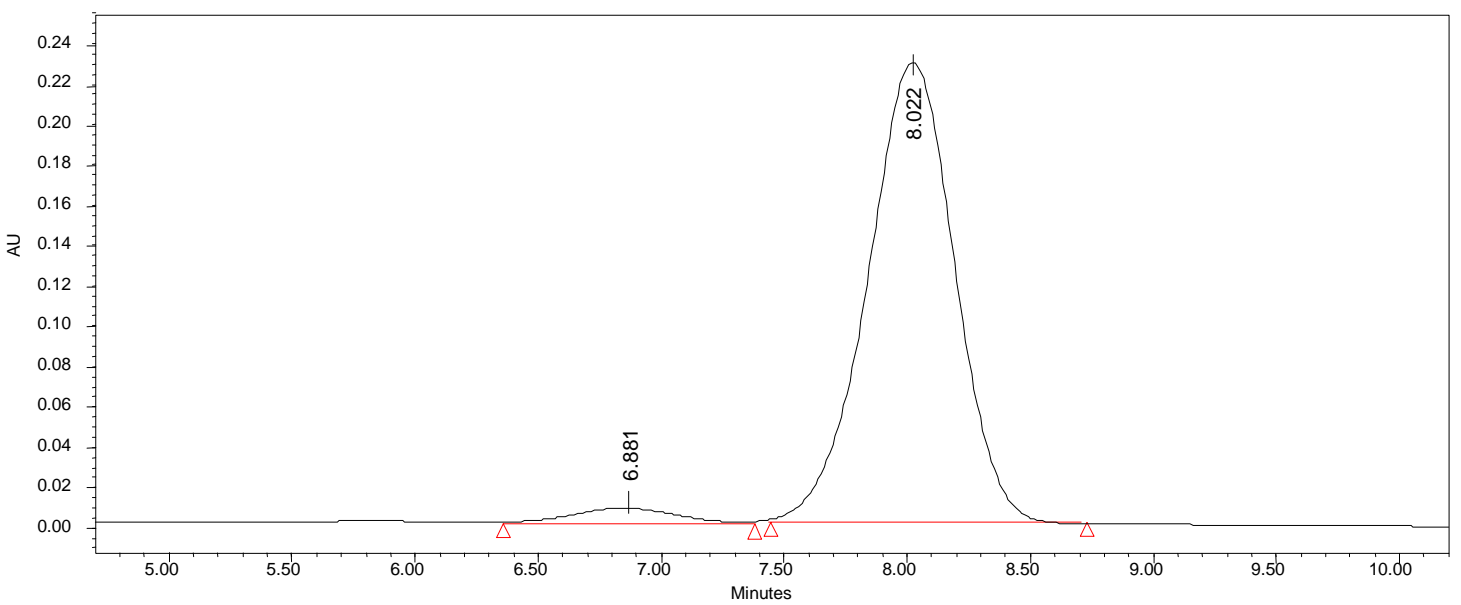

\begin{tabular}{|r|r|r|l|r|r|r|r|}
\hline & Name & Retention Time & Peak Type & \multicolumn{1}{c|}{ Area } & \% Area & \multicolumn{1}{c|}{ Height } & \% Height \\
\hline 1 & & 6.881 & Unknown & 217012 & 3.89 & 10903 & 4.77 \\
\hline 2 & & 8.022 & Unknown & 5361720 & 96.11 & 217669 & 95.23 \\
\hline
\end{tabular}<smiles>CC(=O)C1=C(C)N(Cc2ccccc2)C(=O)NC1c1ccc(Br)cc1</smiles>

ChiralPak OD Column, Hexane:IPA $=90: 10,1.0 \mathrm{~mL} / \mathrm{min}$ $S$ enatiomer: $\mathrm{t}_{\mathrm{r}}=12.3 \mathrm{~min}, R$ enatiomer: $\mathrm{t}_{\mathrm{r}}=21.2 \mathrm{~min}$.

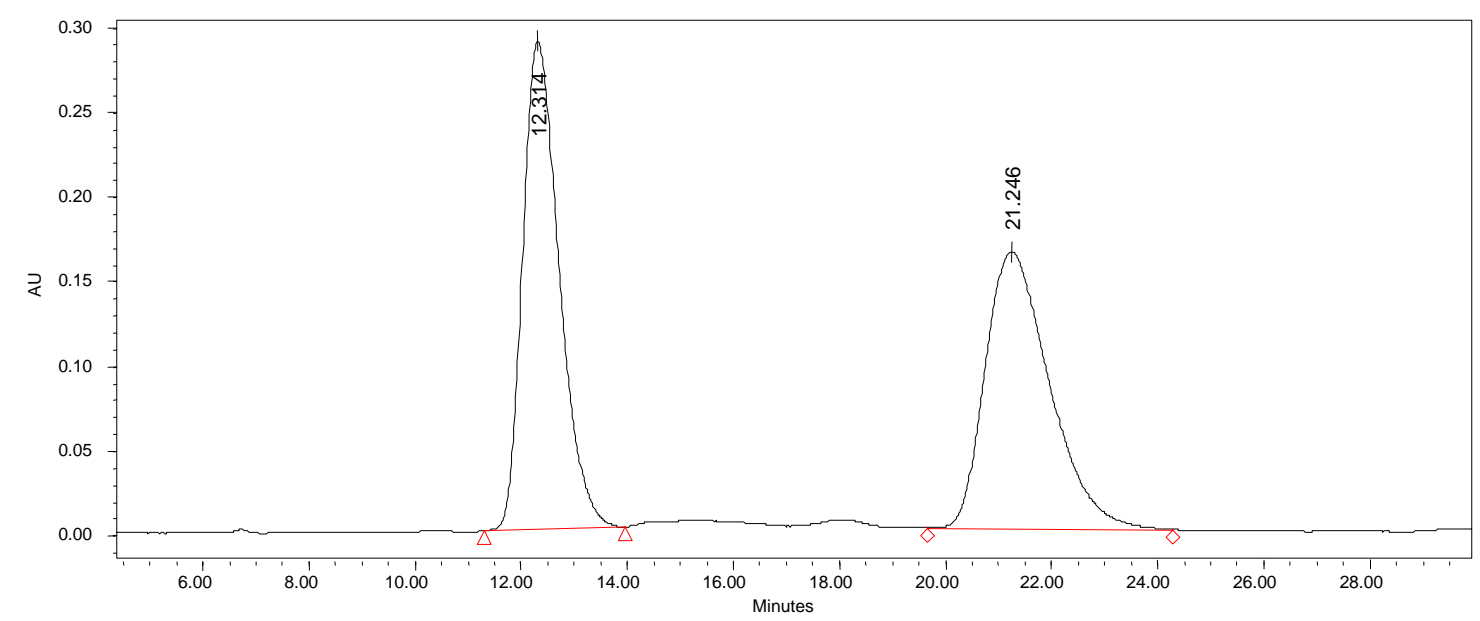

\begin{tabular}{|l|c|r|l|r|r|r|r|}
\hline & Name & $\begin{array}{c}\text { Retention } \\
\text { Time }\end{array}$ & Peak Type & Area & \% Area & Height & $\%$ Height \\
\hline 1 & & 12.314 & Unknown & 13769284 & 49.83 & 288247 & 63.78 \\
\hline 2 & & 21.246 & Unknown & 13865260 & 50.17 & 163696 & 36.22 \\
\hline
\end{tabular}




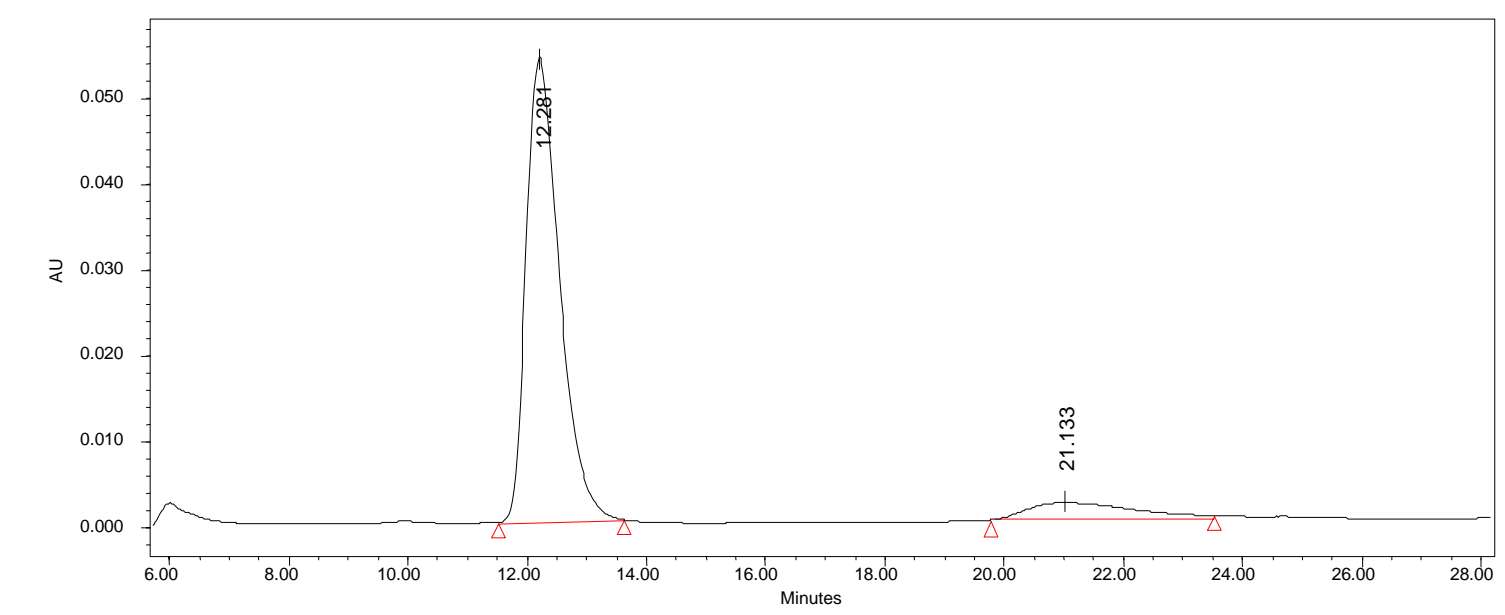

\begin{tabular}{|r|r|r|l|r|r|r|r|}
\hline & Name & Retention Time & Peak Type & \multicolumn{1}{c|}{ Area } & \% Area & \multicolumn{1}{c|}{ Height } & \multicolumn{1}{c|}{ Height } \\
\hline 1 & & 12.281 & Unknown & 2639700 & 95.03 & 56193 & 97.21 \\
\hline 2 & & 21.133 & Unknown & 138054 & 4.97 & 1613 & 2.79 \\
\hline
\end{tabular}

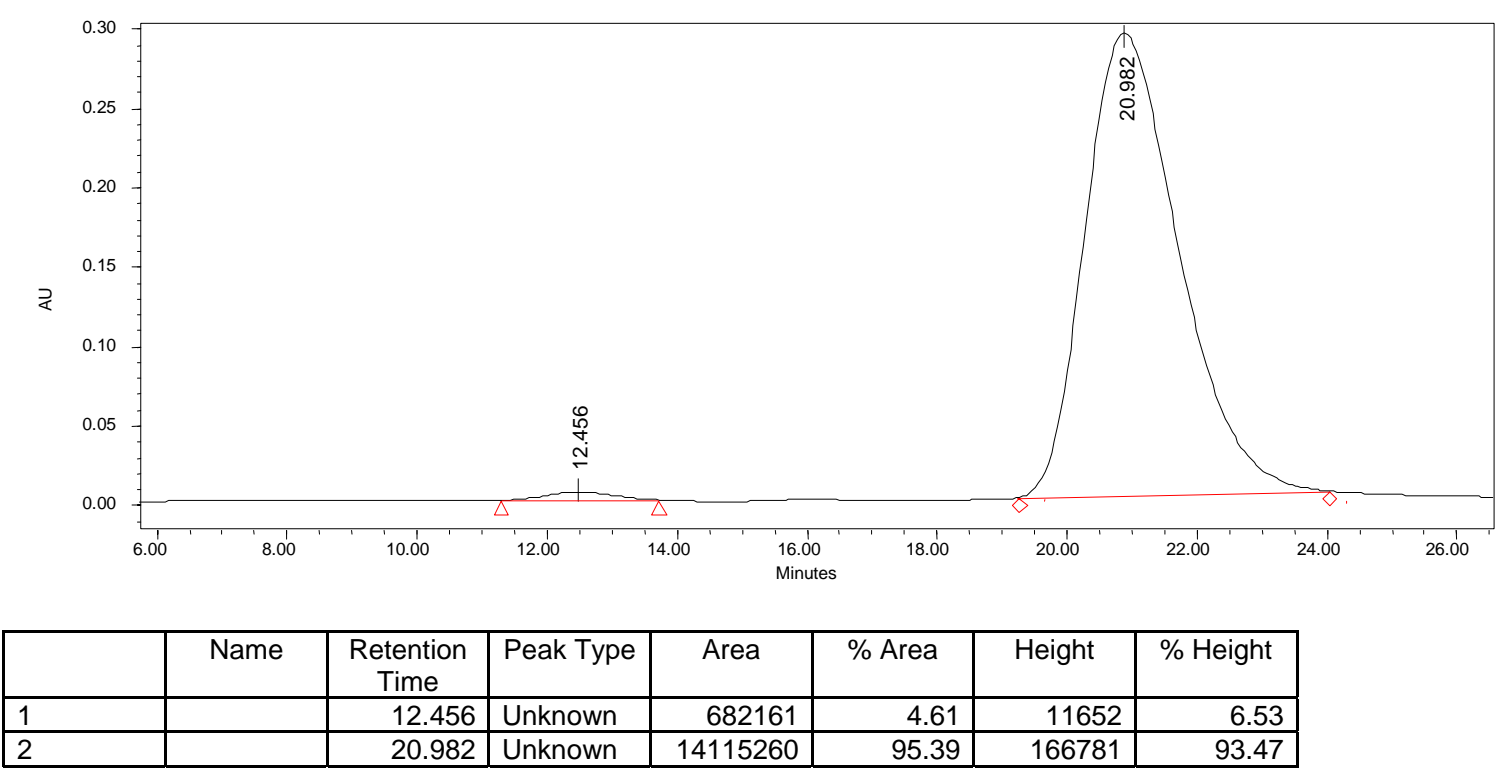


$\mathrm{H}_{3} \mathrm{C}$<smiles>COC(=O)CN1C(=O)NC(CCc2ccccc2)C(C(=O)OC)=C1C</smiles>

ChiralPak OD Column, Hexane:IPA $=90: 10,1.0 \mathrm{~mL} / \mathrm{min}$ $S$ enatiomer: $\mathrm{t}_{\mathrm{r}}=12.4$ min, $R$ enatiomer: $\mathrm{t}_{\mathrm{r}}=17.8 \mathrm{~min}$.

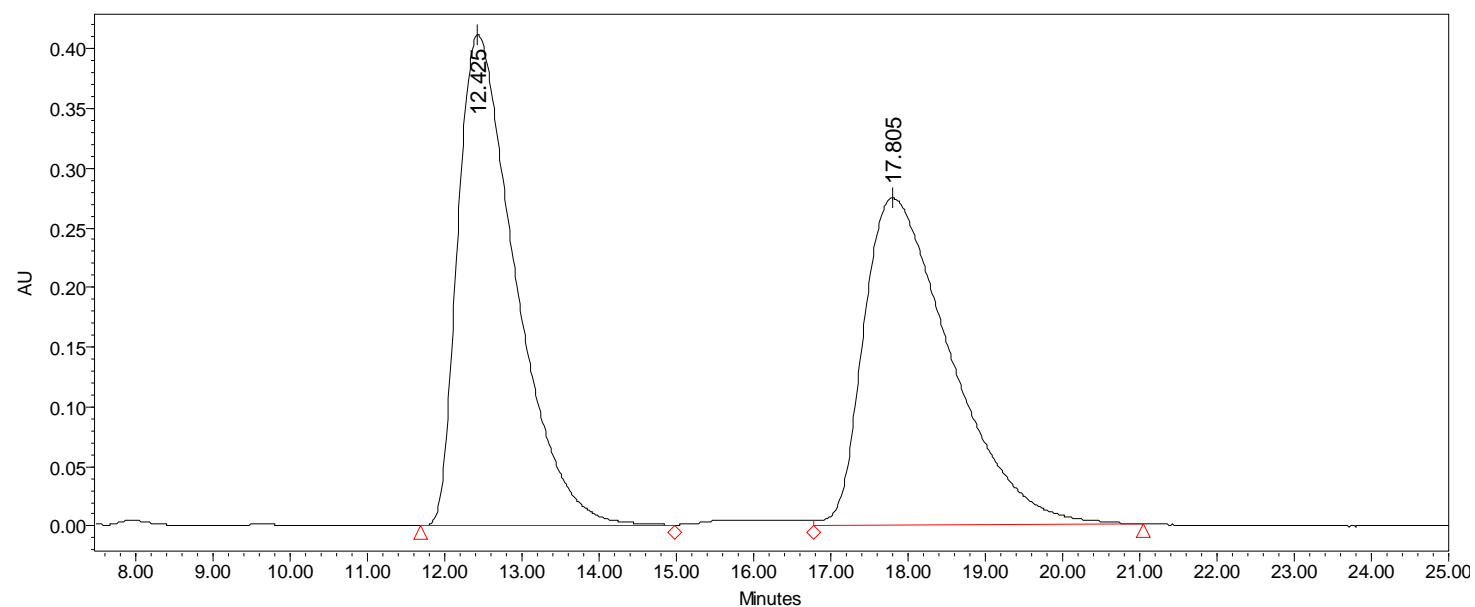

\begin{tabular}{|l|r|r|l|r|r|r|r|}
\hline & Name & $\begin{array}{c}\text { Retention } \\
\text { Time }\end{array}$ & Peak Type & Area & \% Area & Height & \% Height \\
\hline 1 & & 12.425 & Unknown & 21405274 & 49.96 & 410967 & 60.05 \\
\hline 2 & & 17.805 & Unknown & 21438518 & 50.04 & 273382 & 39.95 \\
\hline
\end{tabular}

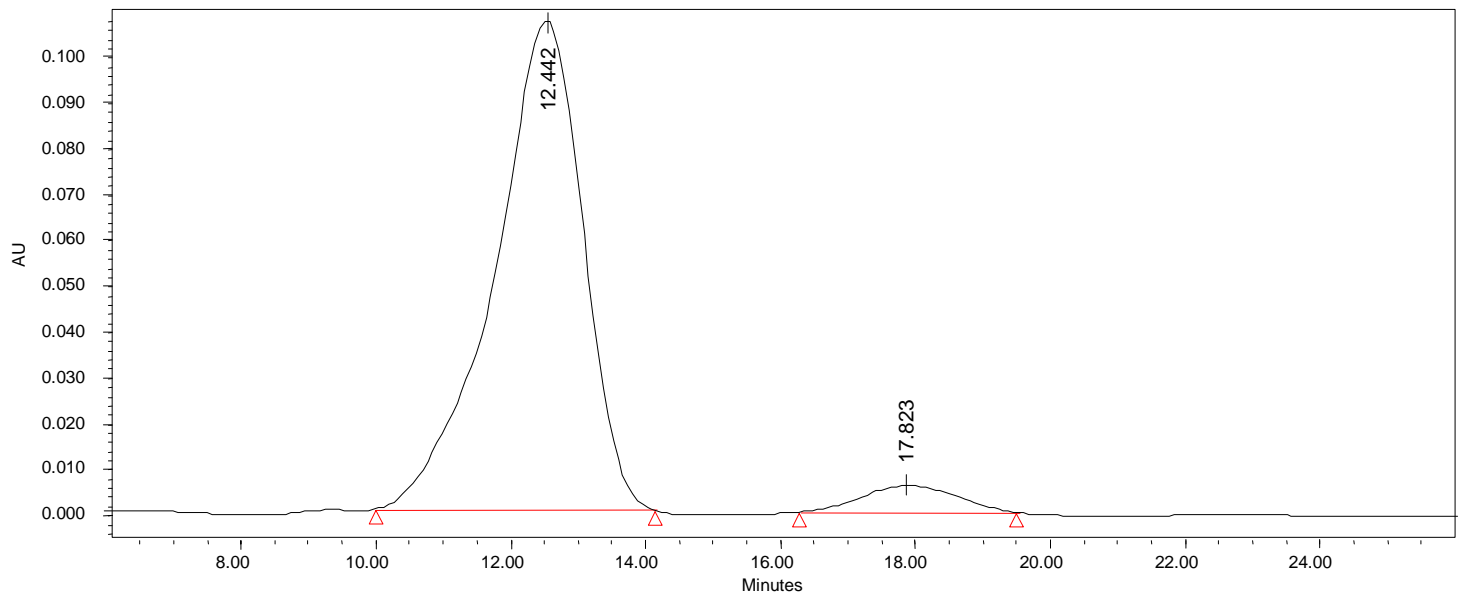

\begin{tabular}{|l|r|r|l|r|r|r|r|}
\hline & Name & $\begin{array}{c}\text { Retention } \\
\text { Time }\end{array}$ & Peak Type & \multicolumn{1}{c|}{ Area } & \% Area & Height & $\%$ Height \\
\hline 1 & & 12.442 & Unknown & 5542406 & 94.81 & 106907 & 94.03 \\
\hline 2 & & 17.823 & Unknown & 303397 & 5.19 & 6788 & 5.97 \\
\hline
\end{tabular}


<smiles>COCCN1C(=O)N[C@H](c2cccc(C(F)(F)F)c2)C(C(C)=O)=C1C</smiles>

ChiralPak OD Column, Hexane:IPA $=90: 10,1.0 \mathrm{~mL} / \mathrm{min}$ $S$ enatiomer: $\mathrm{t}_{\mathrm{r}}=12.3 \mathrm{~min}, R$ enatiomer: $\mathrm{t}_{\mathrm{r}}=21.2 \mathrm{~min}$.

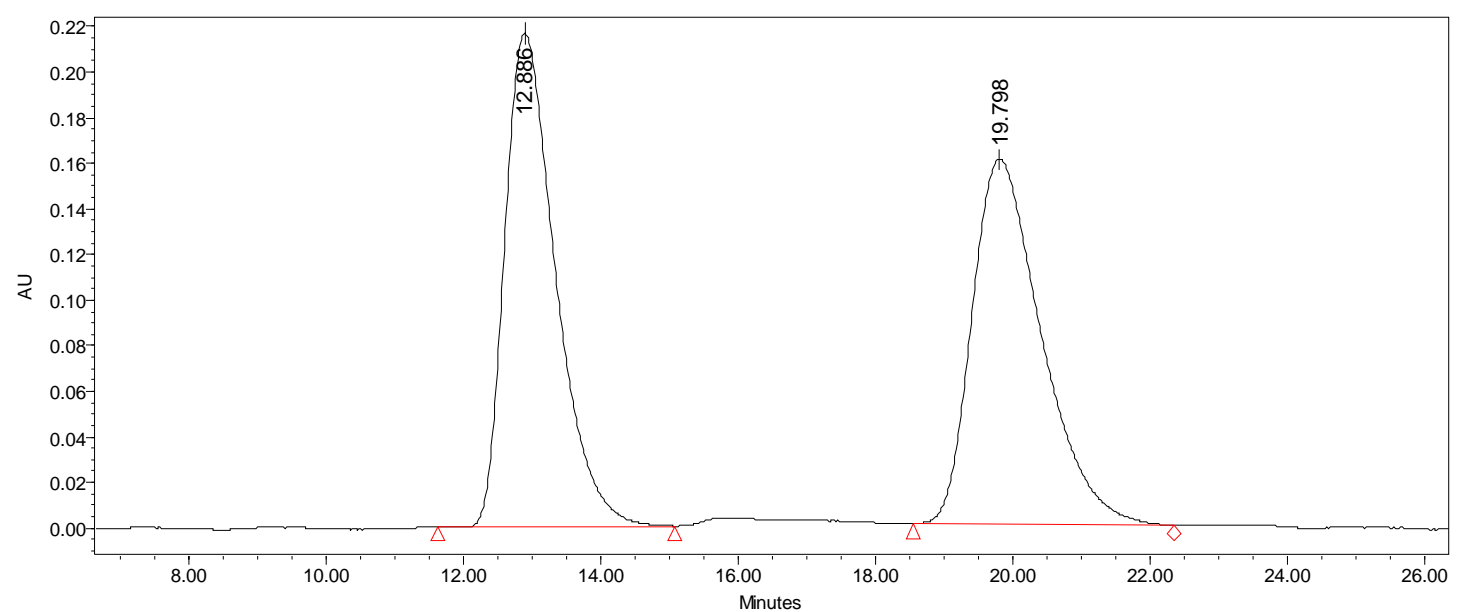

\begin{tabular}{|l|r|r|l|r|r|r|r|}
\hline & Name & $\begin{array}{c}\text { Retention } \\
\text { Time }\end{array}$ & Peak Type & Area & \% Area & Height & \% Height \\
\hline 1 & & 12.886 & Unknown & 11150354 & 49.63 & 216447 & 57.43 \\
\hline 2 & & 19.798 & Unknown & 11318370 & 50.37 & 160425 & 42.57 \\
\hline & & & & & & & \\
\hline
\end{tabular}

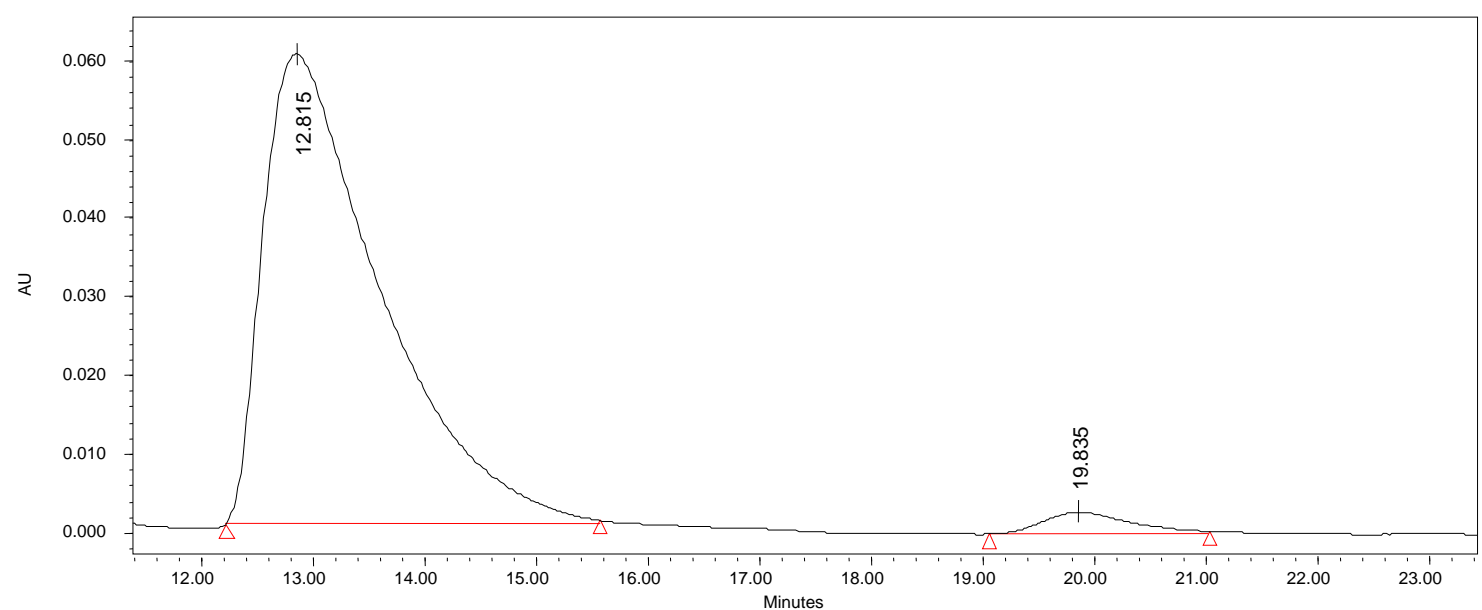

\begin{tabular}{|r|r|r|l|r|r|r|r|}
\hline & Name & Retention Time & Peak Type & \multicolumn{1}{c|}{ Area } & \% Area & \multicolumn{1}{c|}{ Height } & \multicolumn{1}{c|}{ Height } \\
\hline 1 & & 12.815 & Unknown & 3215193 & 95.28 & 62303 & 94.75 \\
\hline 2 & & 19.835 & Unknown & 159275 & 4.72 & 3452 & 5.25 \\
\hline
\end{tabular}



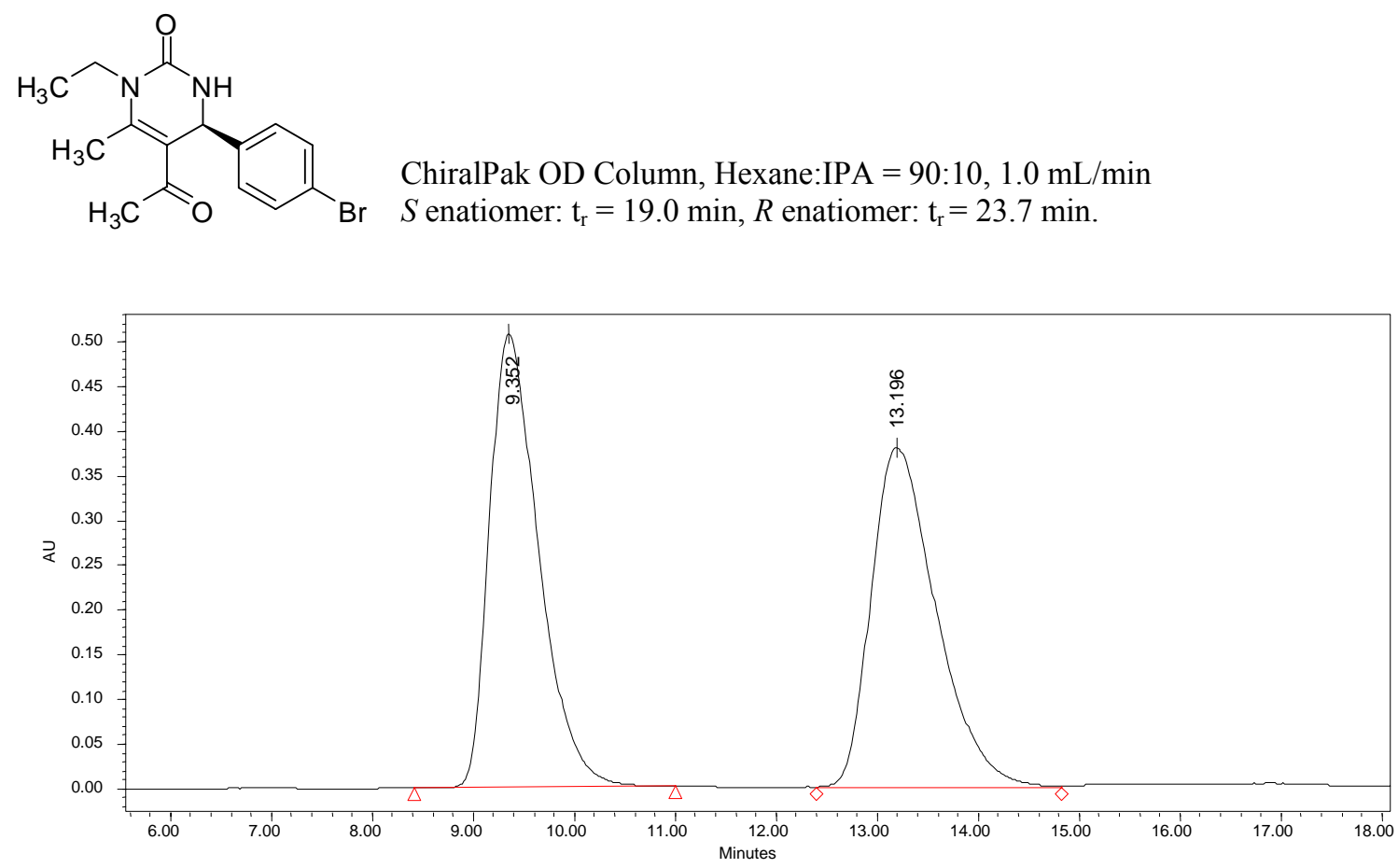

\begin{tabular}{|r|r|r|l|r|r|r|r|}
\hline & Name & Retention Time & Peak Type & Area & \% Area & Height & \% Height \\
\hline 1 & & 9.352 & Unknown & 17116607 & 50.19 & 506720 & 57.18 \\
\hline 2 & & 13.196 & Unknown & 16987538 & 49.81 & 379454 & 42.82 \\
\hline
\end{tabular}

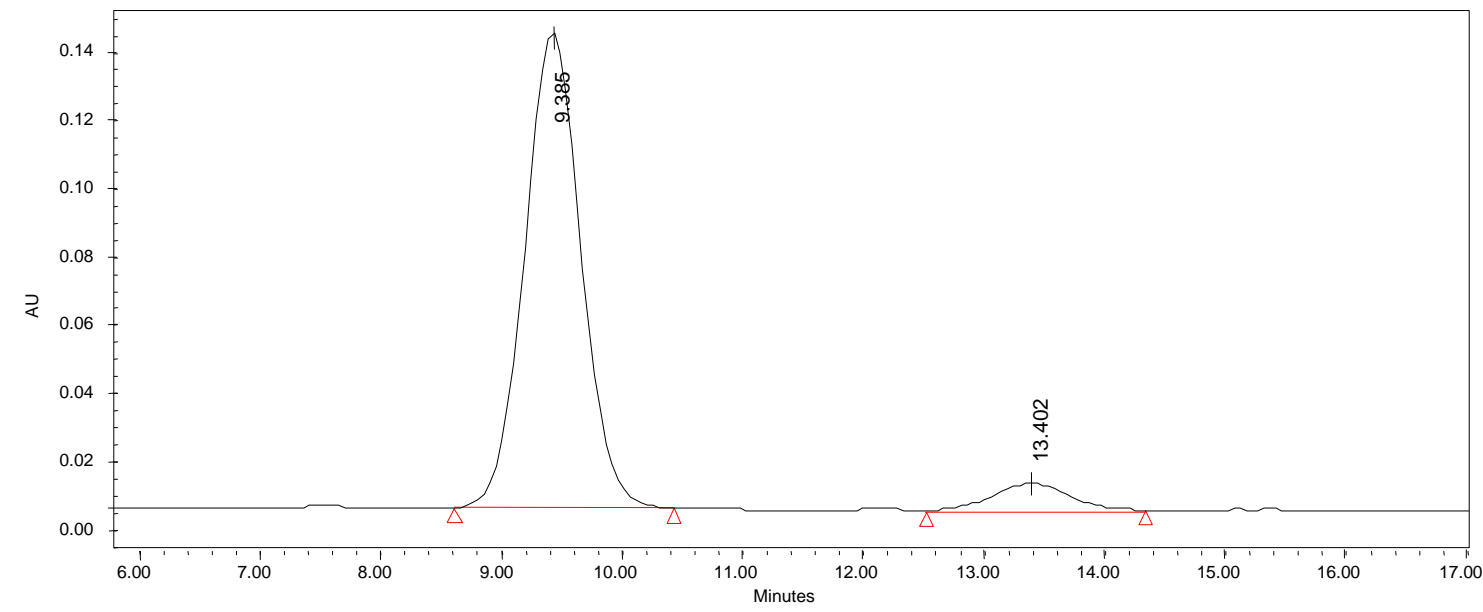

\begin{tabular}{|l|r|r|l|r|r|r|r|}
\hline & Name & $\begin{array}{c}\text { Retention } \\
\text { Time }\end{array}$ & Peak Type & \multicolumn{1}{c|}{ Area } & \% Area & Height & \% Height \\
\hline 1 & & 9.385 & Unknown & 4910625 & 94.83 & 144390 & 92.93 \\
\hline 2 & & 13.402 & Unknown & 267720 & 5.17 & 10985 & 7.07 \\
\hline
\end{tabular}




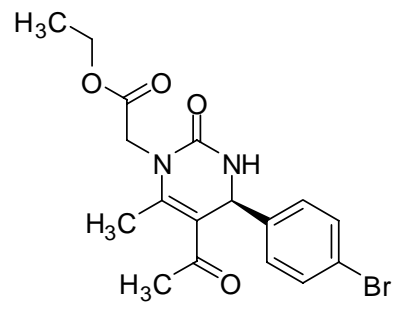

ChiralPak OD Column, Hexane:IPA $=90: 10,1.0 \mathrm{~mL} / \mathrm{min}$ $S$ enatiomer: $\mathrm{t}_{\mathrm{r}}=19.0 \mathrm{~min}, R$ enatiomer: $\mathrm{t}_{\mathrm{r}}=23.7 \mathrm{~min}$.

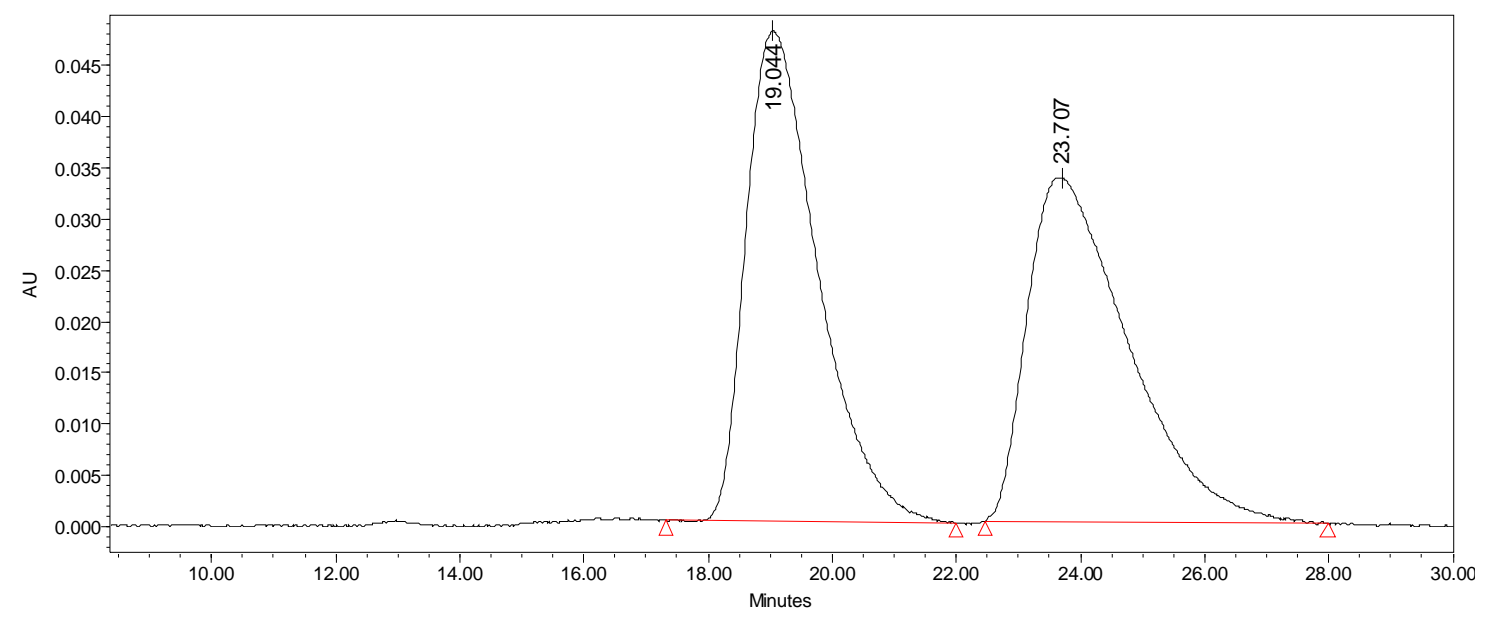

\begin{tabular}{|l|c|c|l|r|r|r|r|}
\hline & Name & $\begin{array}{c}\text { Retention } \\
\text { Time }\end{array}$ & Peak Type & Area & \% Area & Height & $\%$ Height \\
\hline 1 & & 19.044 & Unknown & 3861068 & 50.45 & 47797 & 58.78 \\
\hline 2 & & 23.707 & Unknown & 3792001 & 49.55 & 33523 & 41.22 \\
\hline
\end{tabular}

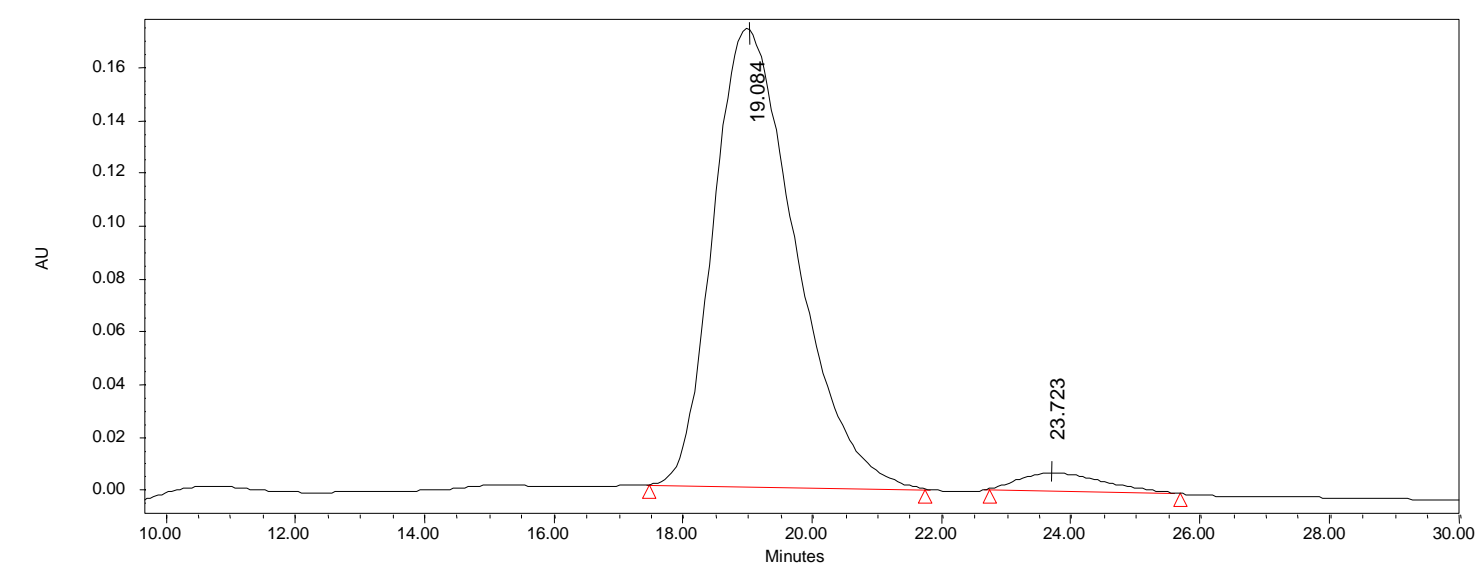

\begin{tabular}{|l|r|r|l|r|r|r|r|}
\hline & Name & $\begin{array}{c}\text { Retention } \\
\text { Time }\end{array}$ & Peak Type & Area & \% Area & Height & \% Height \\
\hline 1 & & 19.084 & Unknown & 13655220 & 95.23 & 168269 & 95.31 \\
\hline 2 & & 23.723 & Unknown & 683980 & 4.77 & 8280 & 4.69 \\
\hline
\end{tabular}

